


\section{ADVANCED APPLICATIONS OF RAPID PROTOTYPING TECHNOLOGY IN MODERN ENGINEERING}

Edited by Muhammad Enamul Hoque 


\section{Contributors}

Raphael Olszewski, Hervé Reychler, Vaibhav Bagaria, Darshana Rasalkar, Jami Ilyas, Shalini Jain, Yanpu Liu, Libin Zhou, Philippe Abdel-Sayed, Ludwig Karl Von Segesser, Touati Youcef, Aoudia Hania, Arab Ali Cherif, David Papo, Kentaro Iwami, Norihiro Umeda, Tiago M. Fernandez-Carames, Miguel González-López, Carlos J. Escudero, Luis Castedo, Michel Jezequel, Amer Baghdadi, Atif Raza Jafri, Sheng Lu, Yong-Qing Xu, Jose Fernando Mangili Jr., Leonimer Melo, Jose Augusto Coeve Florino, Zhengying Wei, Yves Blaquière, Yvon Savaria, Walder André, Grzegorz Budzik, Elisângela Freitas, Jorge Silva, Pedro Noritomi, Takuma Nakano, Fumihito Arai, Andrew Taylor, Giovanni Biglino, Silvia Schievano, Jiasheng Dong, Dong Han, Zhe-Yuan Yu, Hua Xu, Gang Chai, Shen Guo-Xiong, Song-Tao Ai, De Jun Cao, Frederico Assis De Salles

\section{(c) The Editor(s) and the Author(s) 2011}

The moral rights of the and the author(s) have been asserted.

All rights to the book as a whole are reserved by INTECH. The book as a whole (compilation) cannot be reproduced, distributed or used for commercial or non-commercial purposes without INTECH's written permission.

Enquiries concerning the use of the book should be directed to INTECH rights and permissions department (permissions@intechopen.com).

Violations are liable to prosecution under the governing Copyright Law.

\section{(c)) BY}

Individual chapters of this publication are distributed under the terms of the Creative Commons Attribution 3.0 Unported License which permits commercial use, distribution and reproduction of the individual chapters, provided the original author(s) and source publication are appropriately acknowledged. If so indicated, certain images may not be included under the Creative Commons license. In such cases users will need to obtain permission from the license holder to reproduce the material. More details and guidelines concerning content reuse and adaptation can be foundat http://www.intechopen.com/copyright-policy.html.

\section{Notice}

Statements and opinions expressed in the chapters are these of the individual contributors and not necessarily those of the editors or publisher. No responsibility is accepted for the accuracy of information contained in the published chapters. The publisher assumes no responsibility for any damage or injury to persons or property arising out of the use of any materials, instructions, methods or ideas contained in the book.

First published in Croatia, 2011 by INTECH d.o.o.

eBook (PDF) Published by IN TECH d.o.o.

Place and year of publication of eBook (PDF): Rijeka, 2019.

IntechOpen is the global imprint of IN TECH d.o.o.

Printed in Croatia

Legal deposit, Croatia: National and University Library in Zagreb

Additional hard and PDF copies can be obtained from orders@intechopen.com

Advanced Applications of Rapid Prototyping Technology in Modern Engineering Edited by Muhammad Enamul Hoque

p. $\mathrm{cm}$.

ISBN 978-953-307-698-0

eBook (PDF) ISBN 978-953-51-5588-1 


\section{We are IntechOpen, the world's largest scientific publisher of Open Access books.}

\section{$3,250+$}

\section{1}

Countries delivered to

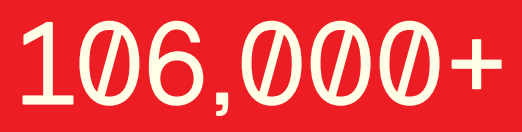

International authors and editors

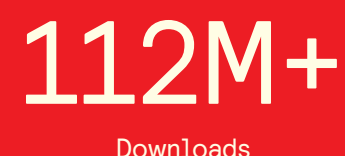

Downloads

Our authors are among the

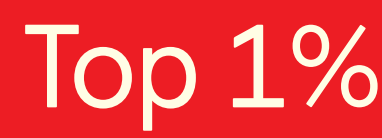

most cited scientists

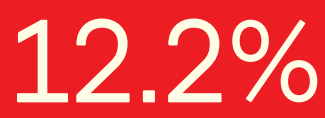

Contributors from top 500 universities

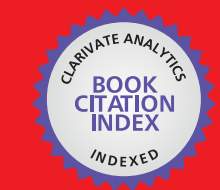

WEB OF SCIENCE ${ }^{\mathrm{M}}$

Selection of our books indexed in the Book Citation Index in Web of Science ${ }^{\mathrm{TM}}$ Core Collection (BKCI)

\section{Interested in publishing with us? Contact book.department@intechopen.com}

Numbers displayed above are based on latest data collected.

For more information visit www.intechopen.com 



\section{Meet the editor}

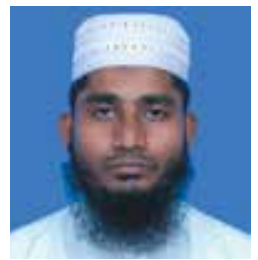

Dr. Muhammad Enamul Hoque is an Associate Professor in the Department of Mechanical, Materials \& Manufacturing Engineering and serves as Head of the Bioengineering Research Group at the University of Nottingham Malaysia Campus. He received his bachelor degree in Materials and Metallurgical Engineering in 1998 from BUET, Bangladesh, then M Eng and PhD in Bioengineering from NUS, Singapore in 2003 and 2007, respectively. So far, he has authored two books, co-authored three book chapters and edited two books. He has also published more than 60 technical papers in referred journals and international conference proceedings. He is a member of InTech's Scientific Board and an invited lead guest editor of the International Journal of Materials and Mechanical Engineering and serves as a technical reviewer for about 10 international journals. He is a member of several professional scientific societies including Tissue Engineering and Regenerative Medicine International Society (TERMIS), and Tissue Engineering Society Malaysia (TESMA). His research interests include the areas of Biomaterials, Biofuel, Tissue Engineering, Stem Cells, Nanomaterials and Composite Materials. 



\section{Contents}

\section{Preface XI}

Chapter 1 Medical Applications of Rapid Prototyping

- A New Horizon 1

Vaibhav Bagaria, Darshana Rasalkar,

Shalini Jain Bagaria and Jami llyas

Chapter 2 The Use of Rapid Prototyping in Clinical Applications 21

Giovanni Biglino, Silvia Schievano and Andrew M. Taylor

Chapter 3 Circulation Type Blood Vessel Simulator

Made by Microfabrication 41

Takuma Nakano and Fumihito Arai

Chapter 4 Rapid Prototyping for Training Purposes

in Cardiovascular Surgery $\mathbf{6 1}$

Philippe Abdel-Sayed and Ludwig Karl von Segesser

Chapter 5 Rapid Prototyping in Biomedical Engineering $\mathbf{7 5}$

Kentaro Iwami and Norihiro Umeda

Chapter 6 Usage of Rapid Prototyping Technique in Customized

Craniomaxillofacial Bone Tissue Engineering Scaffold 91

Dong Han, Jiasheng Dong, De Jun Cao, Zhe-Yuan Yu,

Hua Xu, Gang Chai, Shen Guo-Xiong and Song-Tao Ai

Chapter 7 Use of Rapid Prototyping

and 3D Reconstruction in Veterinary Medicine

103

Elisângela Perez de Freitas, Pedro Yoshito Noritomi

and Jorge Vicente Lopes da Silva

Chapter 8 Rapid Prototyping in Correction

of Craniofacial Skeletal Deformities 119

Libin Zhou and Yanpu Liu 
Chapter 9 Application of a Novel Patient - Specific Rapid Prototyping Template in Orthopedics Surgery

Sheng Lu, Yong-qing Xu and Yuan-zhi Zhang

Chapter 10 Rapid Prototyping Applied to Maxillofacial Surgery 153

Marcos Vinícius Marques Anchieta,

Marcelo Marques Quaresma and Frederico Assis de Salles

Chapter 11 Clinical Applications of Rapid Prototyping Models

in Cranio-Maxillofacial Surgery 173

Olszewski Raphael and Reychler Hervé

Chapter 12 A Wafer-Scale Rapid Electronic

Systems Prototyping Platform 207

Walder André, Yves Blaquière and Yvon Savaria

Chapter 13 Rapid Prototyping for Mobile Robots

Embedded Control Systems 225

Leonimer Flavio de Melo, Jose FernandoMangili Junior

and Jose Augusto Coeve Florino

Chapter 14 ASIP Design and Prototyping

for Wireless Communication Applications 243

Atif Raza Jafri, Amer Baghdadi and Michel Jezequel

Chapter 15 Rapid Prototyping

for Evaluating Vehicular Communications 267

Tiago M. Fernández-Caramés, Miguel González-López,

Carlos J. Escudero and Luis Castedo

Chapter 16 Position Location Technique in Wireless Sensor Network Using Rapid Prototyping Algorithm 291

Touati Youcef, Aoudia Hania, Ali-Cherif Arab and Mohamed Demri

Chapter 17 Application of RP and Manufacturing to Water-Saving Emitters 307

Zhengying Wei

Chapter 18 The Use of the Rapid Prototyping Method for the Manufacture and Examination of Gear Wheels 339

Grzegorz Budzik 


\section{Preface}

Rapid prototyping (RP) technology has been widely known and appreciated due to its flexible and customized manufacturing capabilities. The widely studied RP techniques include stereolithography apparatus (SLA), selective laser sintering (SLS), threedimensional printing (3DP), fused deposition modeling (FDM), 3D plotting, solid ground curing (SGC), multiphase jet solidification (MJS), laminated object manufacturing (LOM). Different techniques are associated with different materials and/or processing principles and thus are devoted to specific applications. RP technology has no longer been only for prototype building rather has been extended for real industrial manufacturing solutions. Today, the RP technology has contributed to almost all engineering areas that include mechanical, materials, industrial, aerospace, electrical and most recently biomedical engineering. This book aims to present the advanced development of RP technologies in various engineering areas as the solutions to the real world engineering problems.

\section{Dr. Md Enamul Hoque}

Associate Professor

Department of Mechanical, Materials \& Manufacturing Engineering University of Nottingham Malaysia Campus Jalan Broga, Semenyih Selangor Darul Ehsan 



\title{
Medical Applications of Rapid Prototyping - A New Horizon
}

\author{
Vaibhav Bagaria ${ }^{1}$, Darshana Rasalkar², Shalini Jain Bagaria ${ }^{3}$ and Jami Ilyas ${ }^{4}$ \\ ${ }^{1}$ Senior Consultant Orthopaedic and Joint Replacement surgeon. Dept of Orthopaedic \\ Surgery. Columbia Asia Hospital, Ghaziabad, NCR Delhi \\ 2Department of Diagnostic Radiology and Organ Imaging, The Chinese University of \\ Hong Kong, Prince of Wales Hospital, \\ ${ }^{3}$ Consultant Gynecologist and Laparoscopic Surgeon, ORIGYN Clinic, Ghaziabad \\ ${ }^{4}$ Department of Orthopaedics, Royal Perth Hospital, Perth WA \\ 1,3India \\ ${ }^{2}$ Hongkong \\ ${ }^{4}$ Australia
}

\section{Introduction}

Rapid Prototyping is a promising powerful technology that has the potential to revolutionise certain spheres in the ever changing and challenging field of medical science. The process involves building of prototypes or working models in relatively short time to help create and test various design features, ideas, concepts, functionality and in certain instances outcome and performance. The technology is also known by several other names like digital fabrication, 3D printing, solid imaging, solid free form fabrication, layer based manufacturing, laser prototyping, free form fabrication, and additive manufacturing. The history of use of this technique can be traced back to sixties and its foundation credited to engineering Prof Herbert Voelcker who devised basic tools of mathematics that described the three dimensional aspects of the objects and resulted in the mathematical and algorithmic theories for solid modelling and fabrication. However the true impetus came in 1987 through the work of Carl Deckard, a university of Texas researcher who developed layered manufacturing and printed $3 \mathrm{D}$ model by utilizing laser light for fusing the metal powder in solid prototypes, single layer at a time. The first patent of an apparatus for production of 3D objects by stereolithography was awarded to Charles Hull whom many believe to be father of Rapid prototyping industry.

Since its first use in industrial design process, Rapid prototyping has covered vast territories right form aviation sector to the more artful sculpture designing. The use of Rapid prototyping for medical applications although still in early days has made impressive strides. Its use in orthopaedic surgery, maxillo-facial and dental reconstruction, preparation of scaffold for tissue engineering and as educational tool in fields as diverse as obstetrics and gynecology and forensic medicine to plastic surgery has now gained wide acceptance and is likely to have far reaching impact on how complicated cases are treated and various conditions taught in medical schools. 


\section{Steps in production of rapid prototyping models}

The various steps in production of an RP model include-

1. Imaging using CT scan or MRI scan

2. Acquisition of DIACOM files.

3. Conversion of DIACOM into. STL files.

4. Evaluation of the design

5. Surgical planning and superimposition if desired

6. Additive Manufacturing and creation of model

7. Validation of the model.

In short, the procedure involves getting a CT scan or MRI scan of the patient. It is preferable that the CT scan is of high slice calibre and that slice thickness is of $1-2 \mathrm{~mm}$. Most of the MRI and CT software give output in form of digital imaging and communication in medicine format popularly known as DIACOM image format.

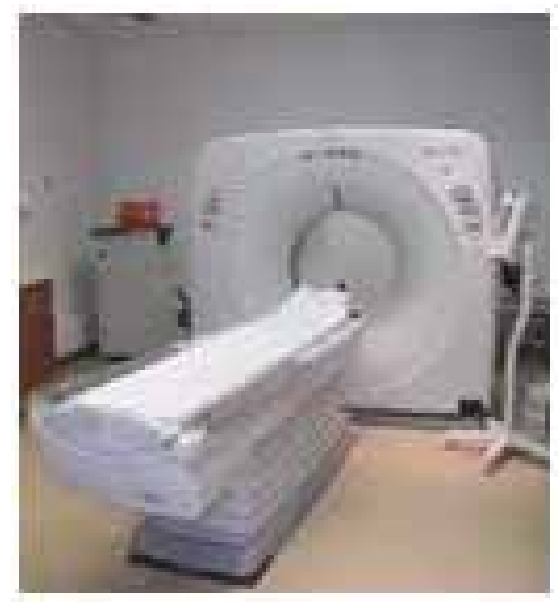

Fig. 1. CT Scan Machine

Acquisition of DIACOM files and conversion to .STL file format: After the data is exported in DIACOM file format, it needs to be converted into a file format which can be processed for computing and manufacturing process. In most cases the desired file format for Rapid manufacturing is .STL or sterolithographic file format. The conversion requires specialised softwares like MIMICS, 3D Doctors, AMIRA. These softwares process the data by segmentation using threshold technique which takes into the account the tissue density. This ensures that at the end of the segmentation process, there are pixels with value equal to or higher than the threshold value. A good model production requires a good segmentation with good resolution and small pixels.

Softwares available for conversion:

MIMICS by Materialise (http://www.materialise.com/mt.asp?mp=mm_main)

Analyse by the Clinique Mayo

Amira http://amira.zib.de/

3D Doctor (http://www.ablesw.com/3d-doctor/)

BioBuild by Anatomics (http://www.qmi.asn.au/anatomics/)

SliceOmatic by TomoVision (http://www.tomovision.com/tomo_prod_sliceo.htm) 


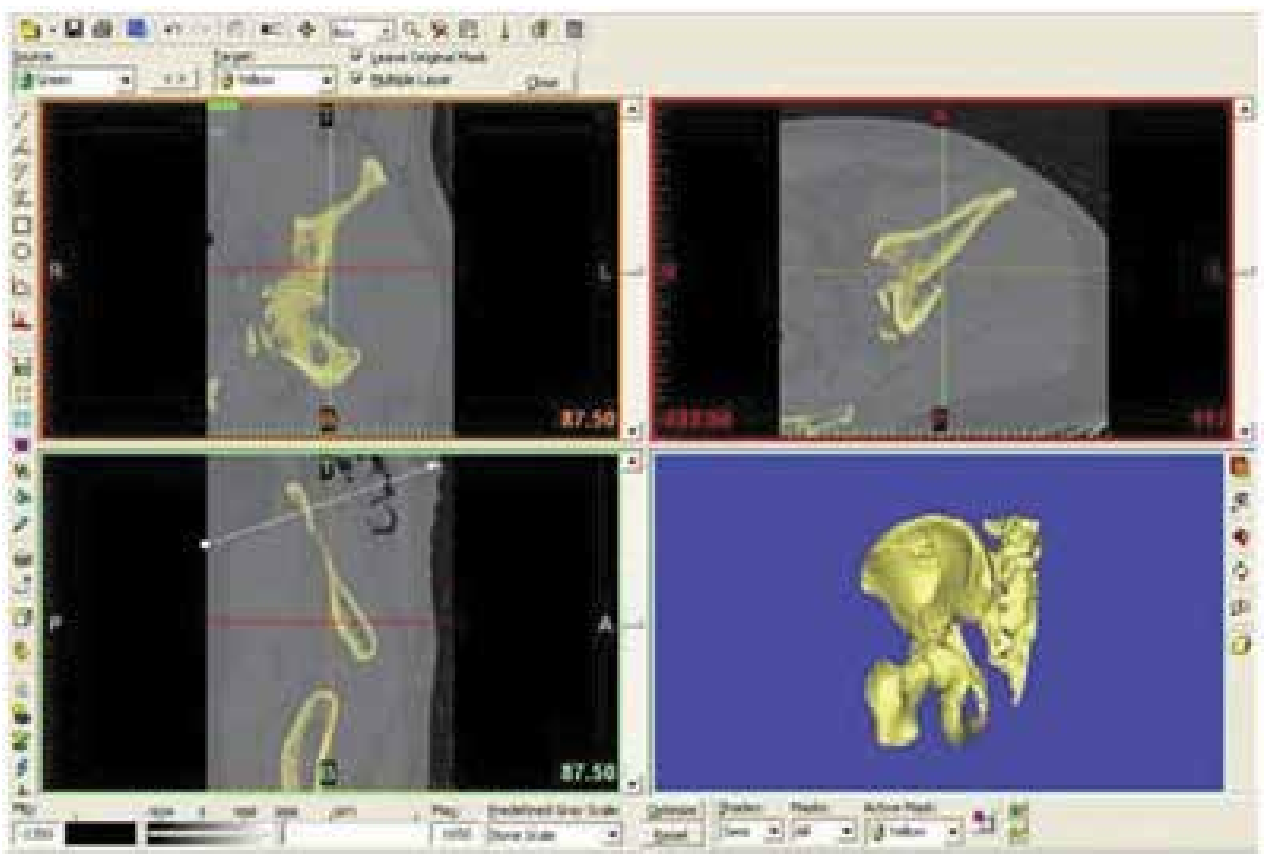

Fig. 2. Segmentation using the software

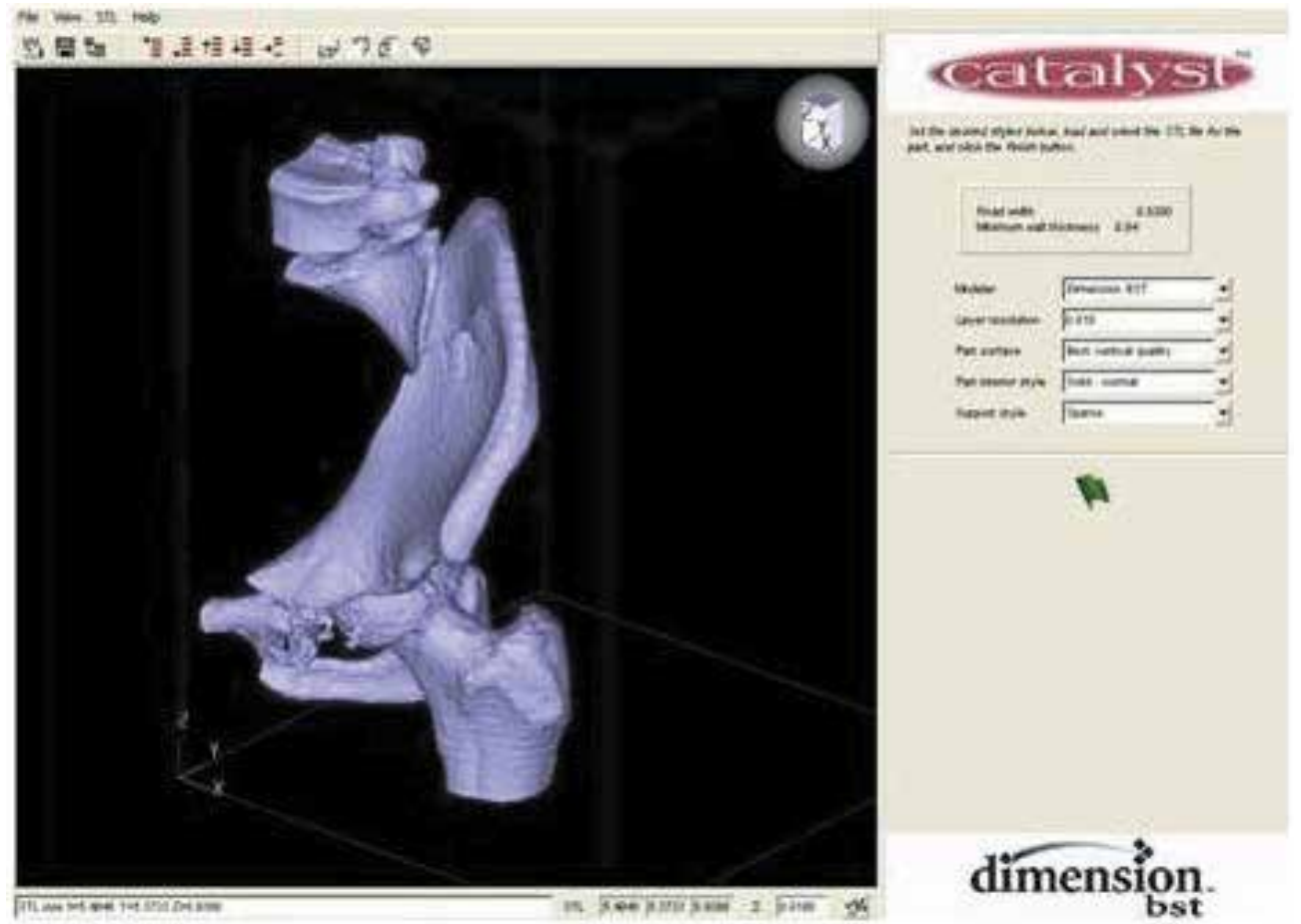

Fig. 3. Designing using CAD software 
Evaluation of design and surgical planning: This step requires combined effort of surgeon, bio engineer and in some cases radiologist. It is important that unnecessary data is discarded and the data that is useful is retained. This decreases the time required for creating the model and also the material required and hence cost of production.

Sometimes this data can be sent directly to machine for the production of model especially when the purpose of model is to teach students. The real use however is in surgical planning in which it is critical that the surgeon and designer brain storm to create the final prototype. There may be a need to incorporate other objects such as fixation devices, prosthesis and implants. The step may involve a surgical simulation carried out by the surgeon and creation of templates or jigs. This may require in addition to the existing converting softwares, computer aided designing softwares like Pro- Engineer, Auto CAD or Turbo CAD.

Additive manufacturing and production of the model: There are various technologies available to create the RP model including stereolithography, selective laser sentring, laminated object manufacturing (LOM), fused deposition modelling (FDM), Solid Ground Curing (SGC) and Ink Jet printing techniques. The choice of the technology depends on the need for accuracy, finish, surface appearance, number of desired colours, strength and property of the materials. It also takes a bit of innovation and planning to orient the part during production so as to ensure that minimum machine running time is taken. The model can also be made on different scale to original size like 1: 0.5, this ensures a faster turnaround time for production and sometimes especially for teaching purpose this may be convenient and sufficient.

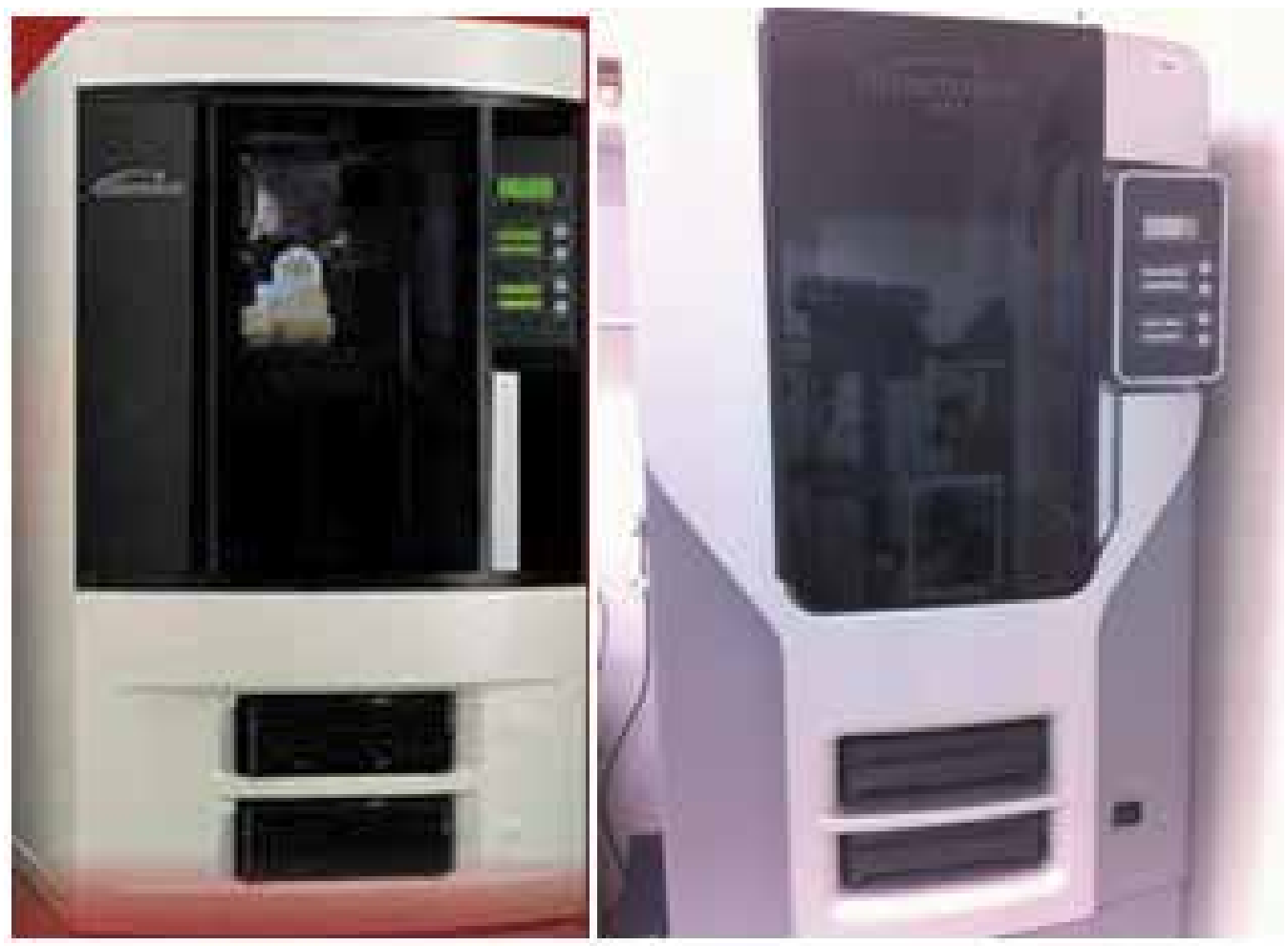

Fig. 4. Various types of Rapid Prototyping Machine 
Validation of the model: Once the model is ready, it needs to evaluated and validated y the team and in particular surgeon so as to ensure that it is correct and serves the purpose.

\section{Rapid prototyping applications}

1. Orthopaedic and Spinal Surgery

2. Maxillofacial and Dental Surgeries

3. Oncology and Reconstruction surgeries

4. Customised joint replacement Prosthesis

5. Patient Specific Instrumentation

6. Patient Specific Orthoses

7. Implant design Testing and Validation

8. Teaching Tool - Orthopaedics, Congenital Defects, Obstetrics, Dental, Maxillofacial.

Table 1. Key Medical speciality areas in which Rapid Prototyping is currently used:

\section{Surgical simulation and virtual planning}

The importance of preoperative templating is well known to surgeons. Especially in difficult cases it gives the surgeon an opportunity to plan complex surgery accurately before actual performance. Advanced technologies like digital templating, computer aided surgical simulation; patient matched instrumentation and use of customized patient specific jigs are increasingly gaining ground. Once the entire process of model generated is accomplished, the surgeon can study the fracture configuration or the deformity that he wants to manage Different surgical options and modalities can be thought of and even be simulated upon the model. In the next stage, the surgeon can contour the desired implant according to bony anatomy. Often as in the complex cases involving acetabulum, calcaneum and other periarticular area contouring the implant in three planes is usually necessary. The fixation hardware can thus be pre-planned, pre-contoured and prepositioned. Once the implant is contoured, computer generated inter-positioning templates or jigs can be used for easy, accurate, preplanning of the screw trajectories and osteotomies. Finally the surgeon can also accurately measure the screw sizes that he desires to use in the surgery thus saving valuable intraoperative time. The model could also be referred to intra operatively should a help is required in understanding the orientation during the surgery.

1. Better understanding of the fracture configuration or disease pathology.

2. Helped to achieve near anatomical reduction

3. Reduced the surgical time

4. Decreased intra-operative blood loss

5. Decreased the requirement of anaesthetic dosage

Table 2. Advantages of Rapid Prototyping

\subsection{Illustrative cases}

Case 1 - Acetabular Fracture

Mr Y, a 29-year-old male, with a history of fall from a 20-ft height presented in the casualty department with multiple fractures. There was no history of head injury and his spine 


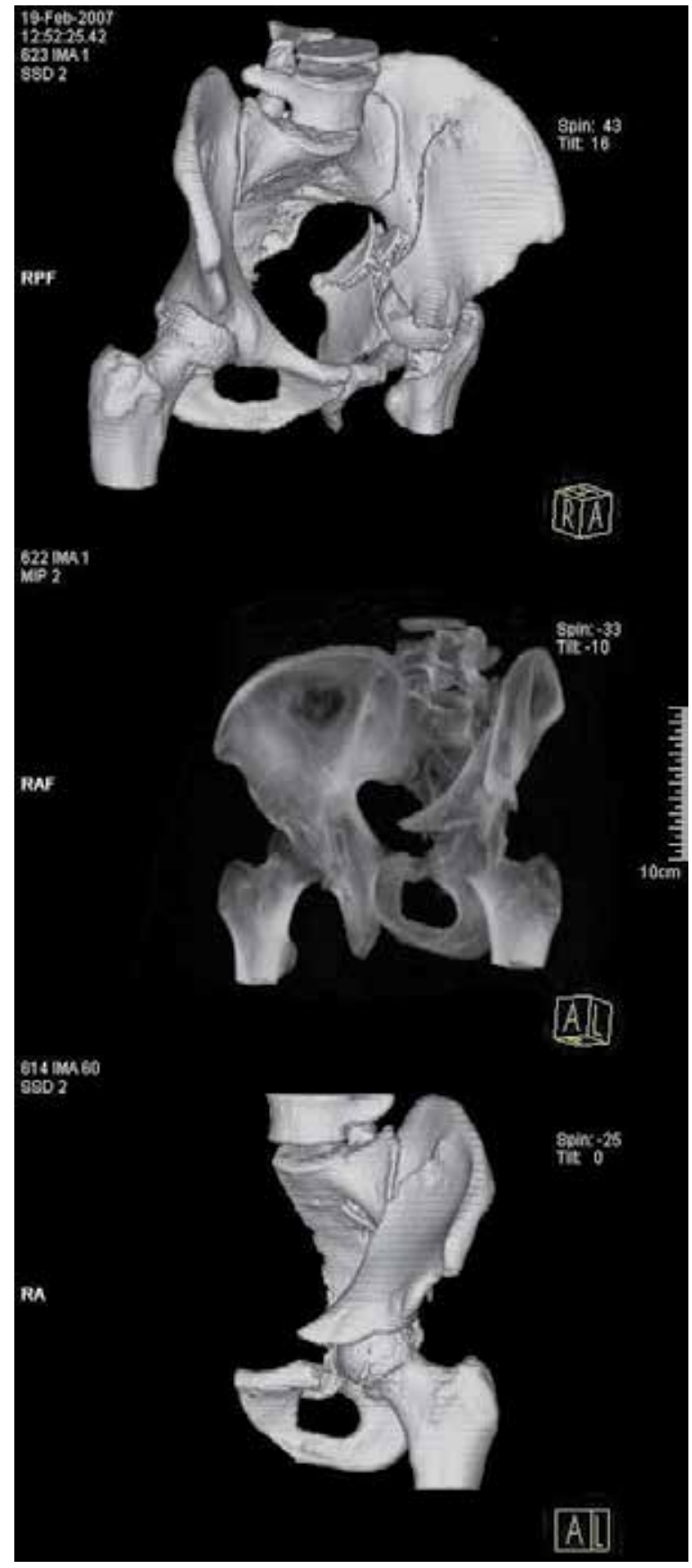

Fig. 5. A: Preoperative Xrays - Judet's obturator view.

Fig. 5. B, C: CT scan showing a vertical displaced a fracture involving iliac blade starting $3 \mathrm{~cm}$ below the iliac crest and extending forward reaching up to the acetabular roof and triradiate cartilage, involving both anterior and posterior column. There is also a mild protrusion of the femoral head and the fracture line extension was present till the superior pubic rami. 
screening was normal. Other fractures included grade IIIb open fractures of the lower third of the right humerus, left volar Barton fracture, and a bicolumnar fracture of the acetabulum on the left side. His vitals were stable and after appropriate stabilization, a CT scan of the pelvis was taken.

The CT scan showed a vertical displaced fracture involving the iliac blade starting $3 \mathrm{~cm}$ below the iliac crest and extending forward, reaching up to the acetabular roof and triradiate cartilage, involving both anterior and posterior columns. There was a mild protrusion of the femoral head and the fracture line extension was present till the superior pubic rami [Figure 5A, B, C].

The preoperative planning before surgery of the acetabulum comprised sequential steps: 3D reconstruction and segmentation of CT scan data], surgical simulation, template design, sizing and alignment of the implant and production of the templates using the RP technology [Figure 6]. CT scanning of all sections was done with 1-mm-thick slices.

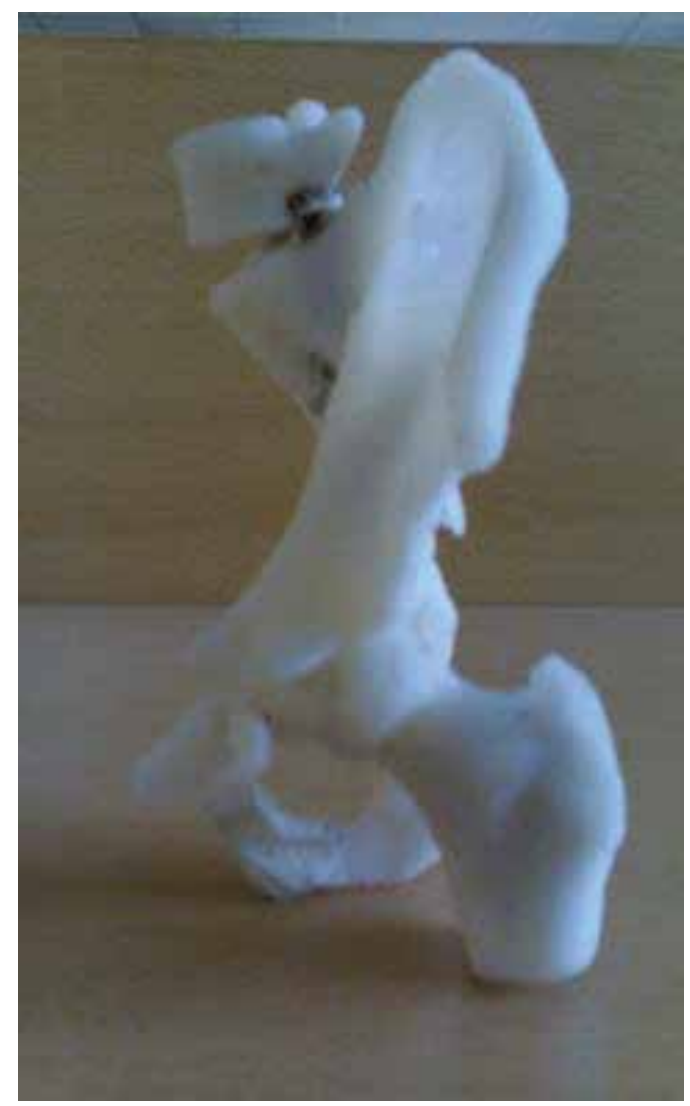

Fig. 6. Rapid-prototyping (RP) Model of fractured acetabulum using a RP machine.

For the preoperative planning process, template was used to contour a 4.5 -mm-thick reconstruction plate. The screw sizes were determined preoperatively and the position of the plate and holes was also decided and marked with indelible ink on the 3D model. An ilioinguinal approach was used for anteriorly exposing the fracture site. The total surgical time required was $3 \mathrm{~h} 10 \mathrm{~min}$. Of this, the instrumentation took only $20 \mathrm{~min}$. The blood loss 
during the procedure was $600 \mathrm{ml}$ and the patient was transfused one unit of whole blood. Next morning, a haemoglobin check was done which was in the normal range and no postoperative transfusion was given. Post operative period was uneventful and normal postoperative rehabilitation protocol was followed.

The postoperative evaluation was carried out using radiographs and CT scans. Computerassisted analyses were carried out for judging the accuracy of the reduction and sizing of the implants [Figures 7,8].

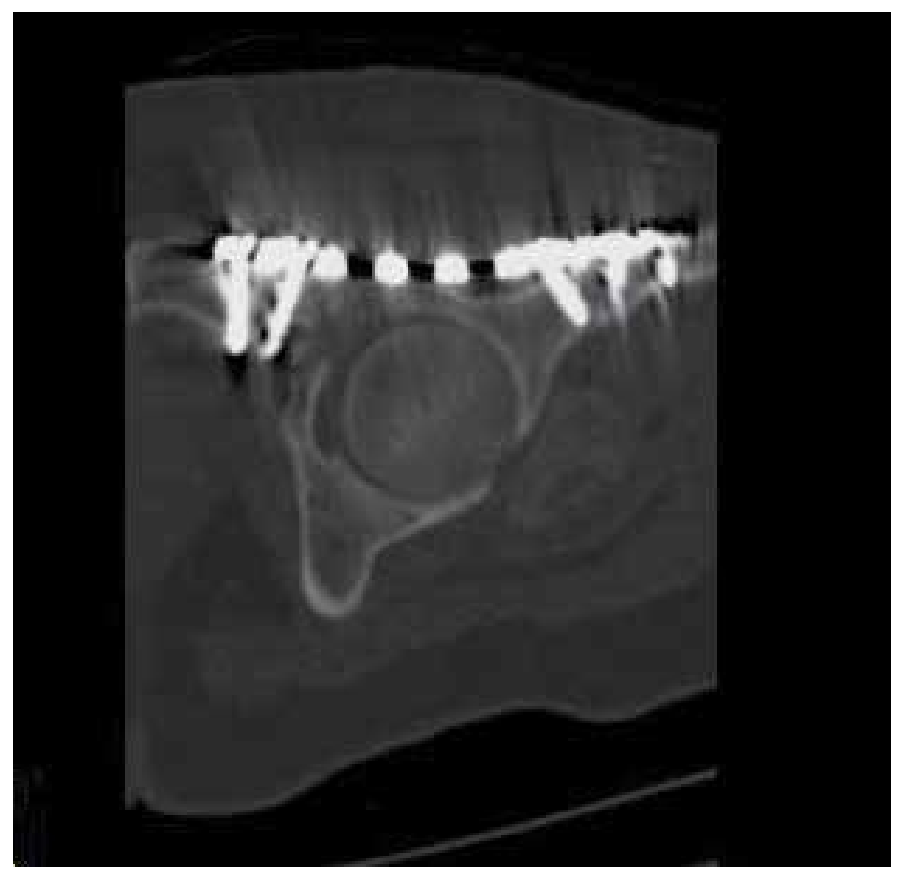

Fig. 7. Postoperative Judets view (obturator view) of Acetabulum.

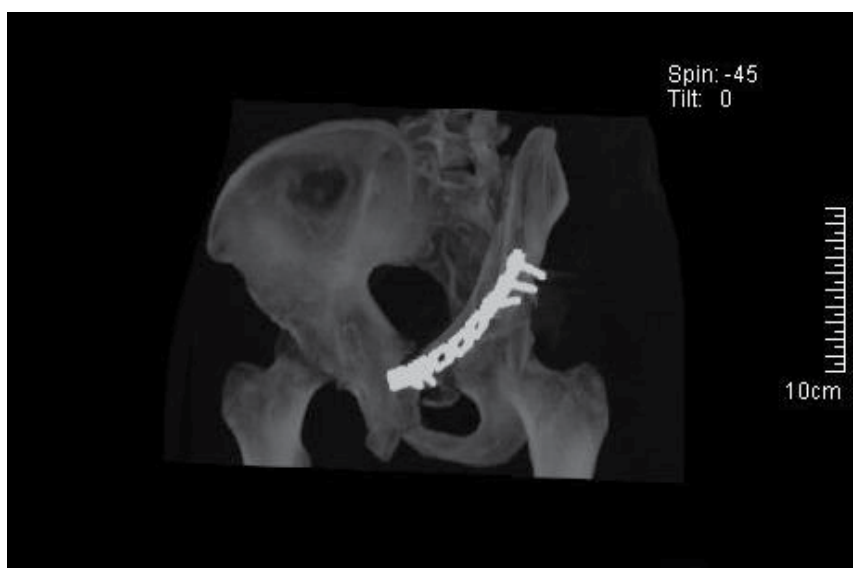

Fig. 8. Axial sections CT images along the plate showing the well contoured plate and fracture reduction. 


\section{Case 2: Calcaneal Fracture}

A 16-year-old male was admitted with a history of fall from a 12-ft height 2 days after injury. He had sustained a type IIB Sanders' classification closed calcaneal fracture. Spine screening and other examinations were normal. After the swelling decreased as proven by the appearance of wrinkles on day 8 , surgery was planned. A CT scan was done [FIG 9] and a 3D model of the calcaneum was made using the RP technique. The 3D model showed the fracture lines clearly and helped plan the surgery [Figures 10].

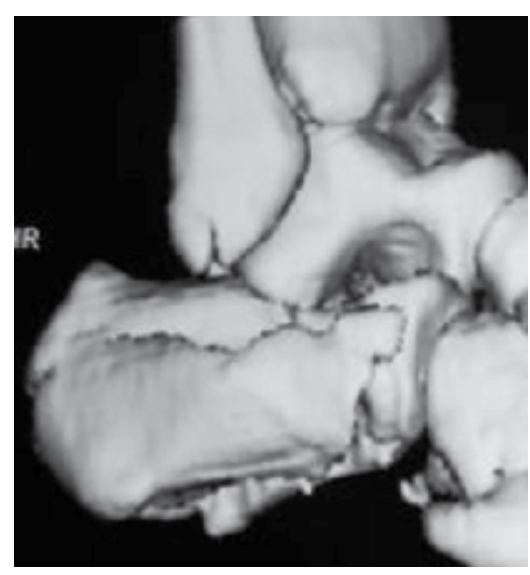

Fig. 9. Fracture Calcaneum CT scan image reconstruction.

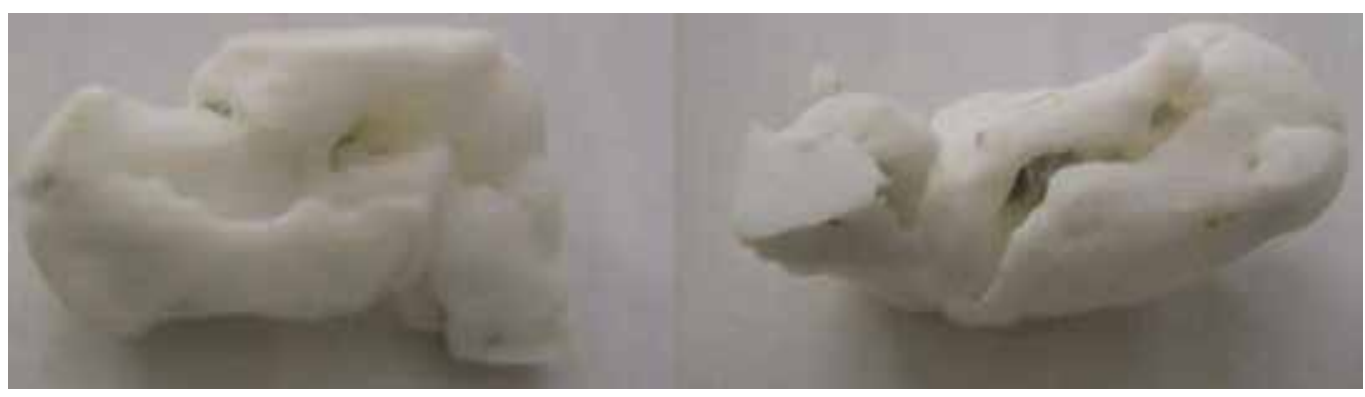

Fig. 10. Fracture Calcaneum Rapid prototype Model.

An open reduction and internal fixation was done using a lateral approach. The subtalar joint was anatomically reduced and a stable fixation was done. Postoperative radiographs [Figure 11] revealed an acceptable fracture reduction and the patient was mobilized at 6 weeks. At 2-year follow-up he is ambulating well without any pain and disability. 


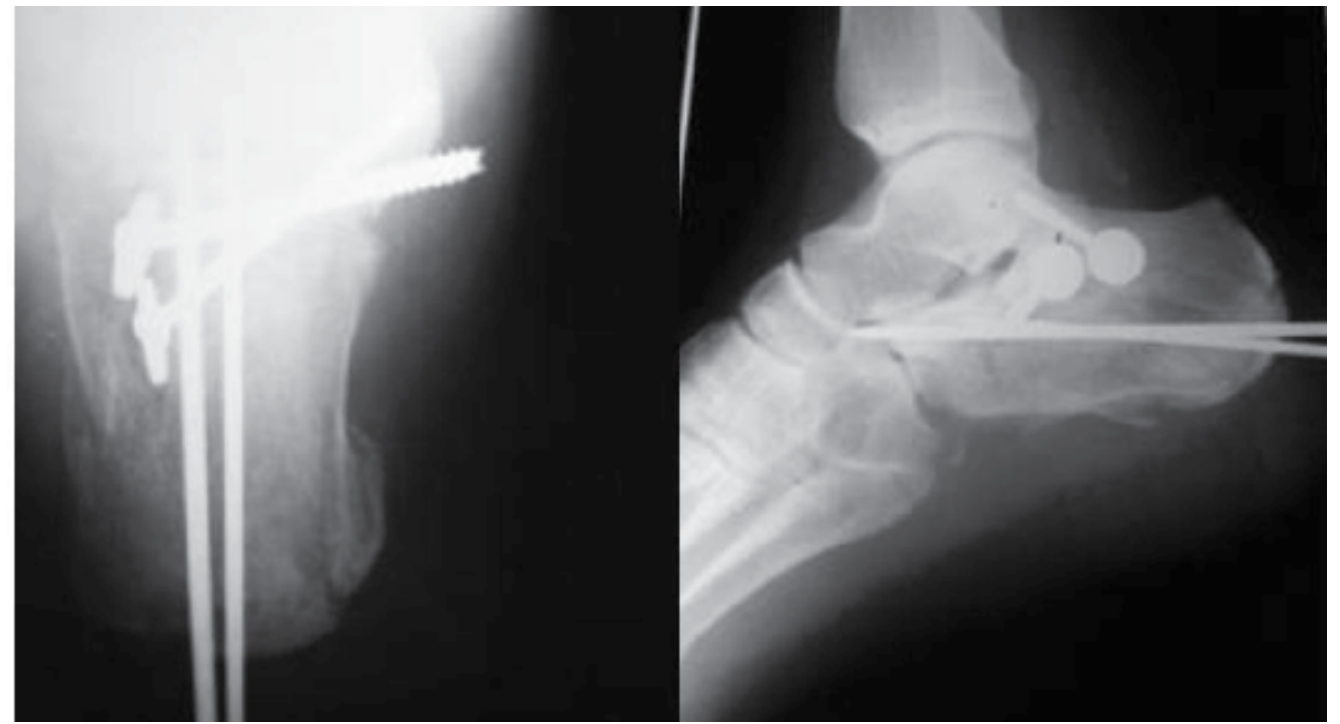

Fig. 11. ORIF done for fracture calcaneum showing good reduction.

\section{Case 3: Hoffa's Fracture}

An 18-year-old male was brought to the emergency department with a head injury, and an injury to right knee and ankle. After stabilization, radiographs that were taken revealed a right Hoffa's fracture involving the posteromedial femoral condyle and an open ankle dislocation. His right knee CT scan was done and the data was used to make a 3D model depicting the fracture pattern. The model was used to study the fracture pattern, for the possible reduction manoeuvre, and to decide the screw trajectory and length.
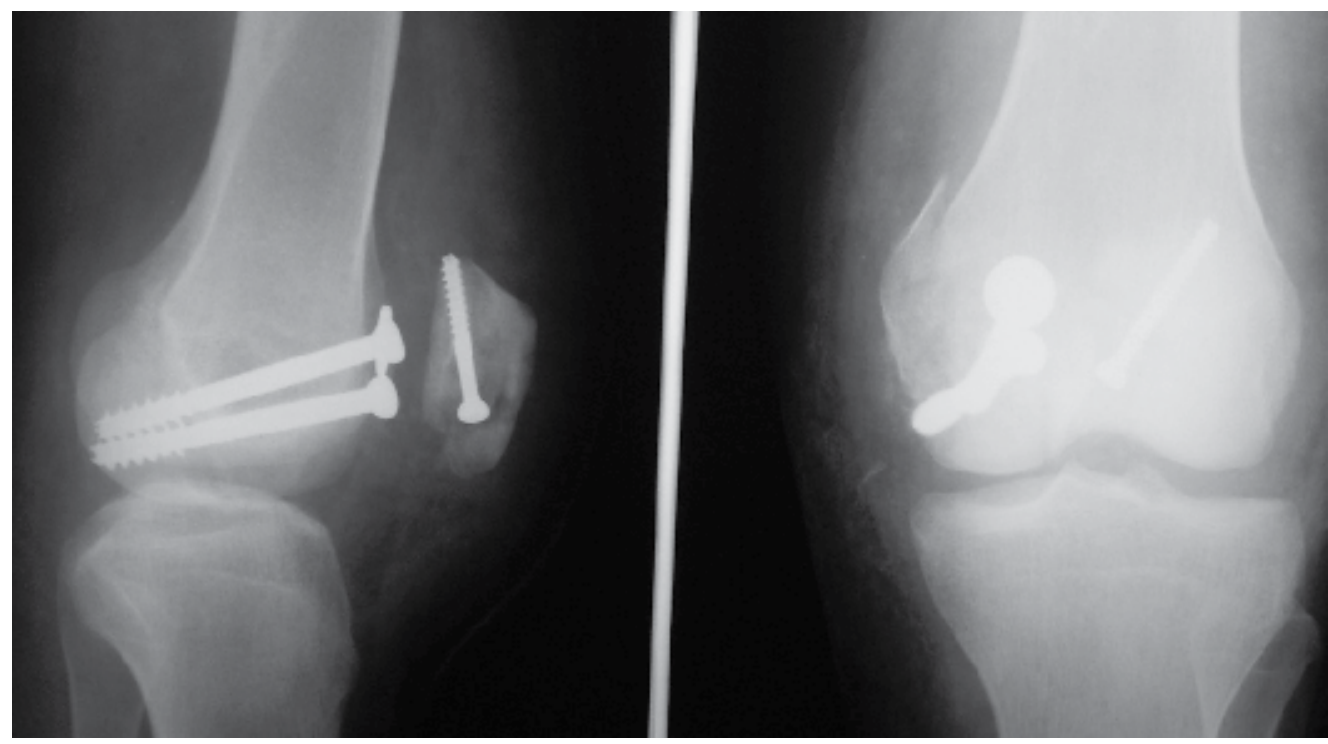

Fig. 12. Hoffas fracture fixation done with aid of surgical simulation on RP model showing anatomic reduction. 
A median parapatellar approach was used to expose the fracture pattern and then fixation was done along the planned trajectory using two 6.5 CC screws [Figure 12]. Non weight bearing knee mobilization was started at 6 weeks. At 3-month follow-up, the patient is ambulating with a walker.

Case 4: Complex Spinal Deformity

A 3 year old child with scoliosis and D6 hemi vertebrae who was posted for a corrective surgery a 3 D Model was created (Fig 13, 14,15) using Rapid Prototyping technique. The model helped understand the complex anatomy and planning hemi-vertebrae resection anteriorly. The surgeon felt it immensely useful in providing preoperative rehearsal with a 360 degree visualisation of pedicles and planning entry point, screw trajectories and screw length.

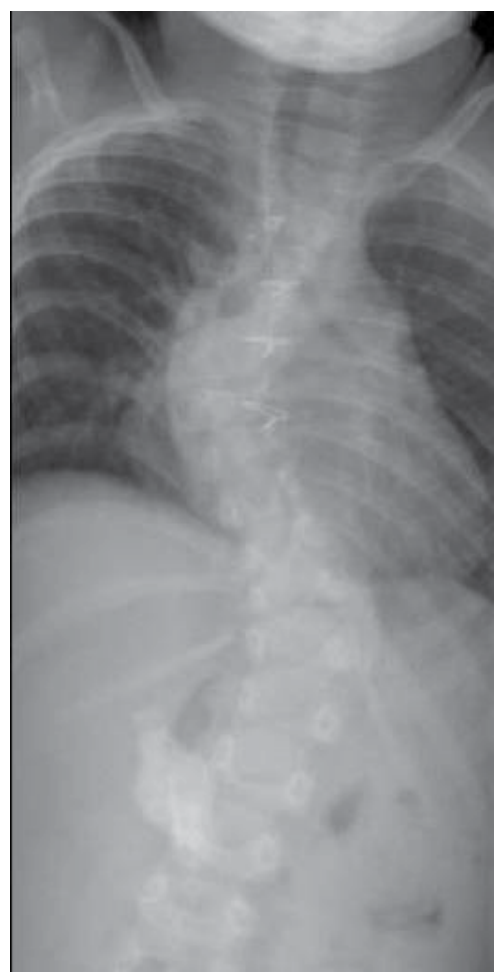

Fig. 13. Xray picture of congenital Scoliosis 


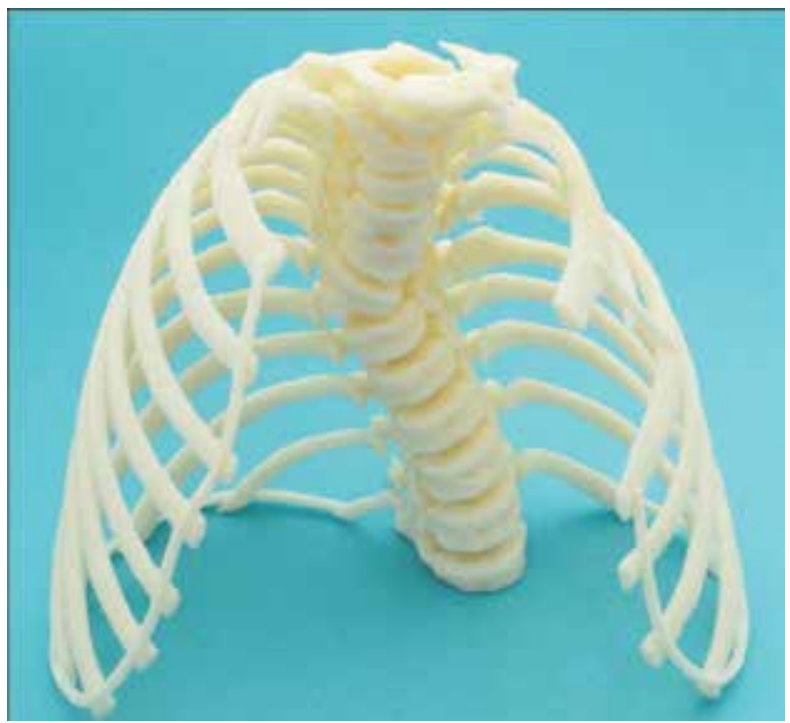

Fig. 14. RP model of congenital scoliosis.

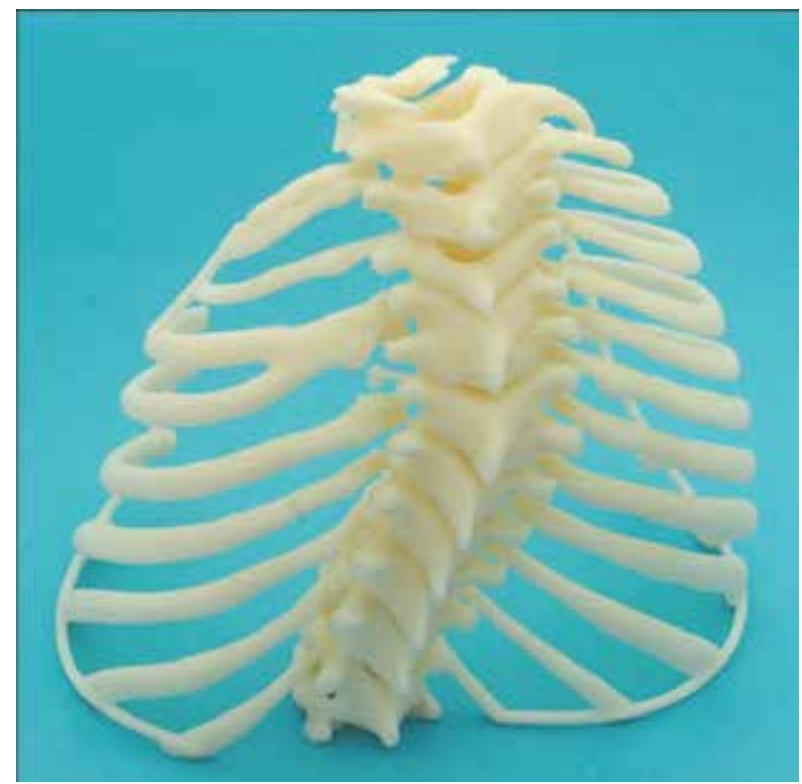

Fig. 15. RP model of Congenital scoliosis as seen from back.

Case 5: Acetabular defect reconstruction before THR

Complex adult reconstruction like those requiring total hip replacement in case of defects on the acetabular side require extensive planning and also various customised inventory. $3 \mathrm{~d}$ modelling helps to plan and also design additional implants. The case described here had acetabular defect secondary to hip infection (FIG 16, 17). A 3D model using RP was made and an acetabular cage/ antiprotrusion ring designed for the same (FIG 18). The surgery in 
this case went smoothly and surgeon felt that the time required and inventory on table was also reduced.

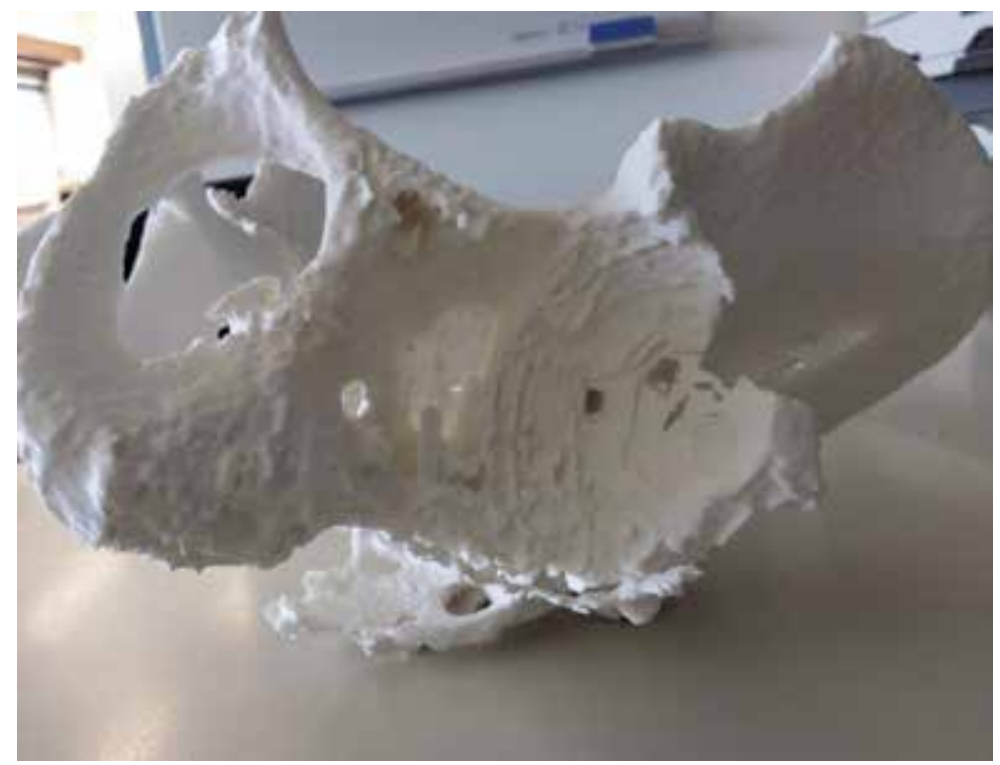

Fig. 16. RP model showing acetabular defect in patient scheduled to undergo total hip replacement.

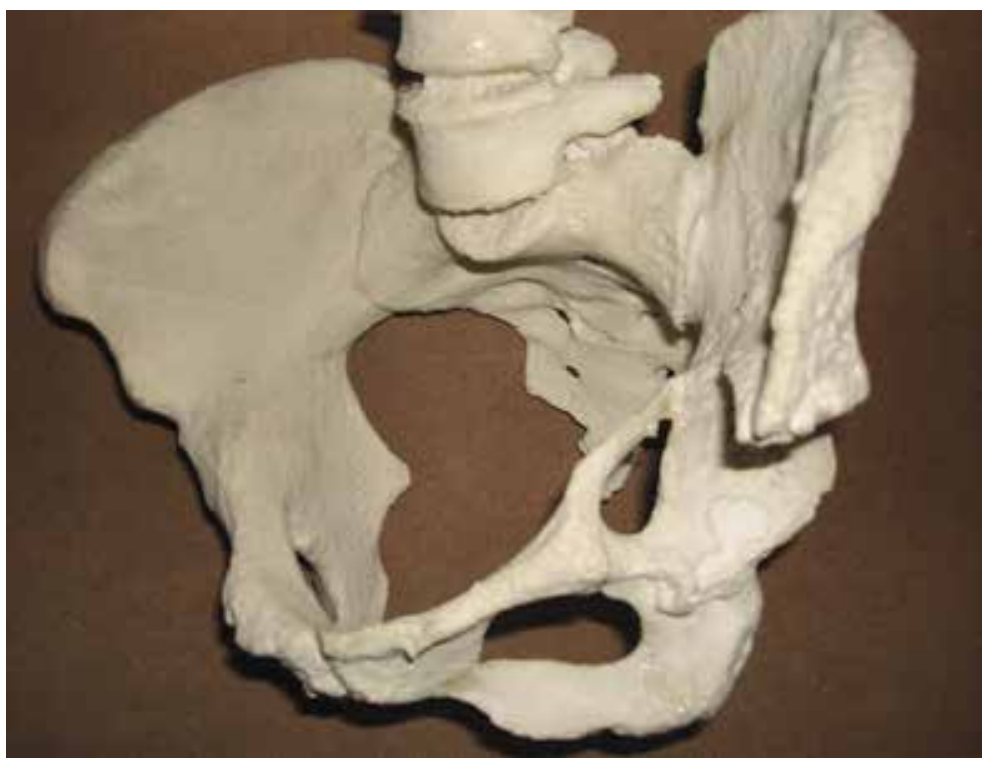

Fig. 17. RP model of acetabulum as seen from front. 


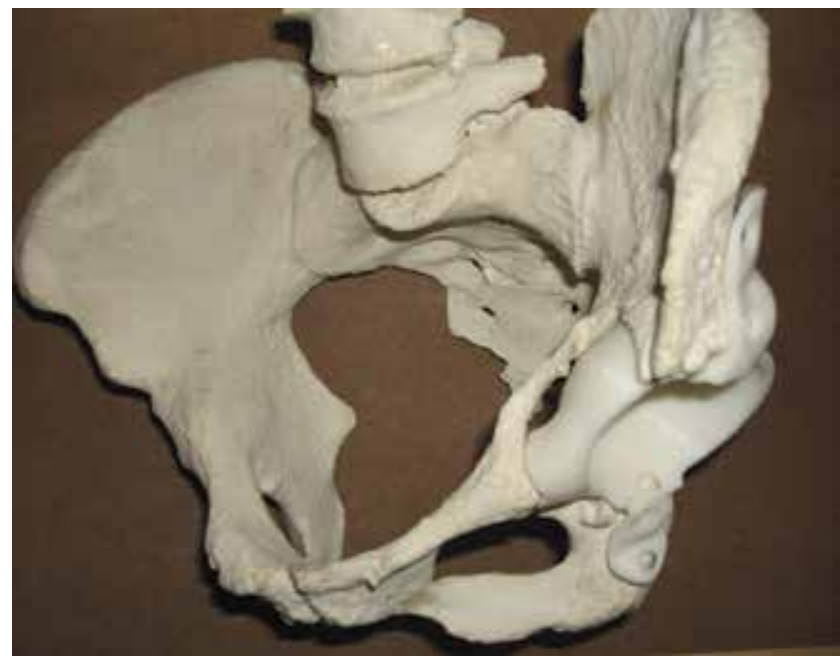

Fig. 18. Designing and planning the use of anti protrusion ring on the acetabular side before Total hip Replacement.

\section{Patient specific implants/instruments}

\subsection{Designing patient specific knee and hip instrumentation and implants}

Knee replacement surgery has gained wide spread popularity for managing arthritic cases. It repairs damage and relieves pain in patients with severe osteoarthritis or knee injury. The process involves removing diseased cartilage and bone from the surfaces of the knee joint-the thigh bone, shin bone, and kneecap--and replacing them with an artificial joint made from a combination of metal and plastic. A partial knee replacement can also be performed on one part of the joint. Typically, a surgeon chooses an artificial joint from several options of different sizes. However, the sizes available are limited and usually do not take into the account racial, gender or morphological factors in account. Although the limited sizes available have been used successfully for several years in past, there is growing number of surgeons who believe that the outcome may be better if the implants and instruments are designed based on patients anatomy and demand vis a vis the functional outcome. Recent years have shown some acceptability for gender specific implants and high flexion knee (catering to the functional need of deep flexion).

\subsection{Patient specific instrumentation}

Conventional knee replacement is carried out using jigs that take standard bone cuts depending on the planned size of implants. Patient specific instrument use preoperative planning to design jigs to ensure accurate bone cuts. Most of these systems use planning based on mechanical axis and for the purpose a long film X-rays, MRI or CT is used. CAD software then helps to simulate the surgical procedure and appropriate amount of bone resections and the degree of rotation in which the prosthesis should be implanted is determined. The calculations and drawings are then sent to surgeon for final approval. (Figure 19, 20) After the planning is done, the jigs are prepared customised to the patient anatomy and incorporating the planned resections and appropriate rotations. An appropriate size is also mentioned and provided the surgeon. The surgeon however has flexibility to intraoperatively switch to conventional procedure or to use different size. 


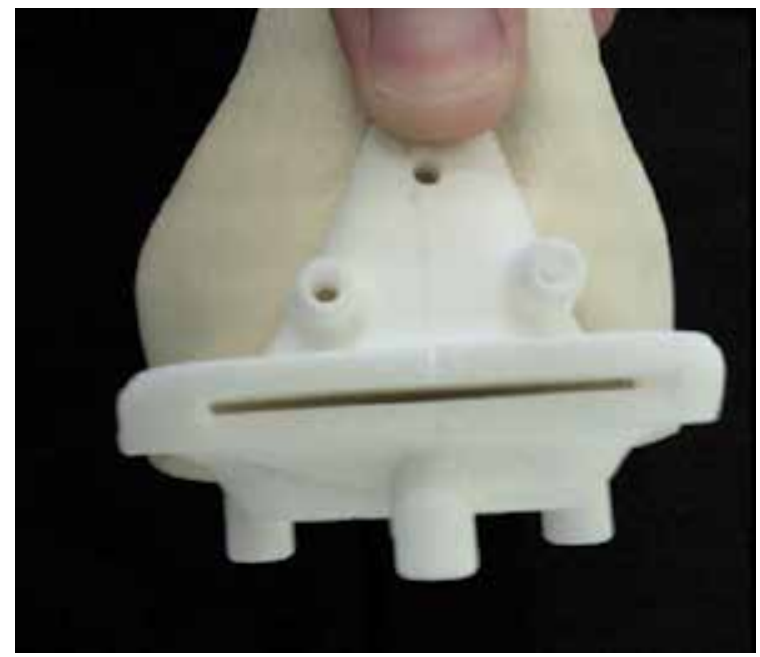

Fig. 19. Patient specific Instrumentation used to make distal femoral cut.

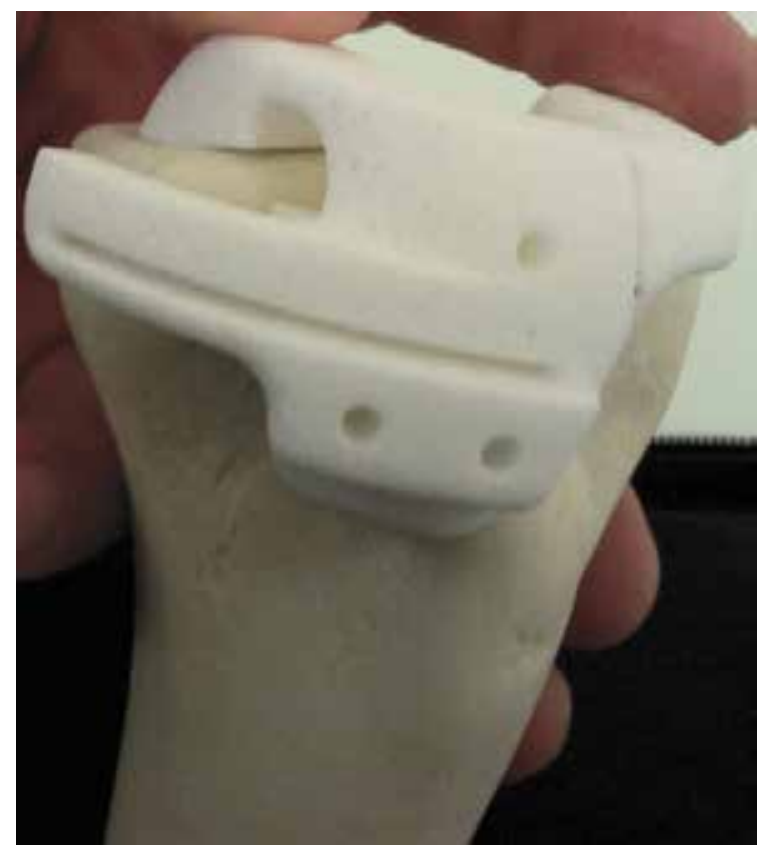

Fig. 20. Patient specific jig being used for tibial cut during total knee replacement.

Benefits:

- Eliminate as many as 22 steps in the surgical procedure with patient match alignment that potentially can achieve a better outcome for the patient. Since the instruments are specifically designed according to patient dimension, the implant is likely to fit better, at the same time the system is versatile enough to allow the surgeon to take intraoperative decisions as deemed necessary. 
- Reduce set-up, surgery and clean up time with shorter surgeries and less instrumentation.

- Patient specific alignment may lead to better patient outcomes and lowered risk of complications such as DVT due to lack of violation of the IM canal. There are potential risks with any surgery.

- Eliminating as many as 22 steps shortens surgery time, meaning the patient needs less time under anaesthesia.

\subsection{Patient specific implant}

In order to design patient specific implant - a MRI or CT scan of the patient is taken and DIACOM images acquired. After converting these into STL files, the planning stage starts. The team usually comprises of surgeon and bio engineer who identify the area where the cartilage is worn off. An exact negative topography is then generated and a customized knee implant is created which would replace only the areas of the cartilage defect, this could be unicompartmental or bicompartmental or in the case of hip or any other joint only the damaged area (fig 21, 22). The model and its negative are then used to create patient specific instrumentation and jigs that would resect only the desired part as described above.

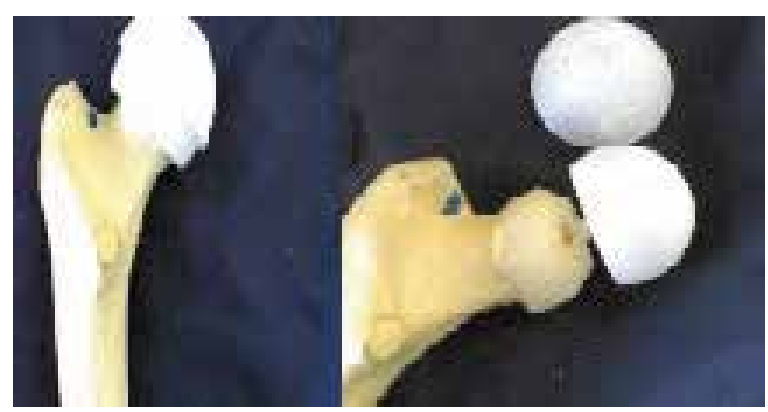

Fig. 21. RP model designed as patient specific hip resurfacing Implant.

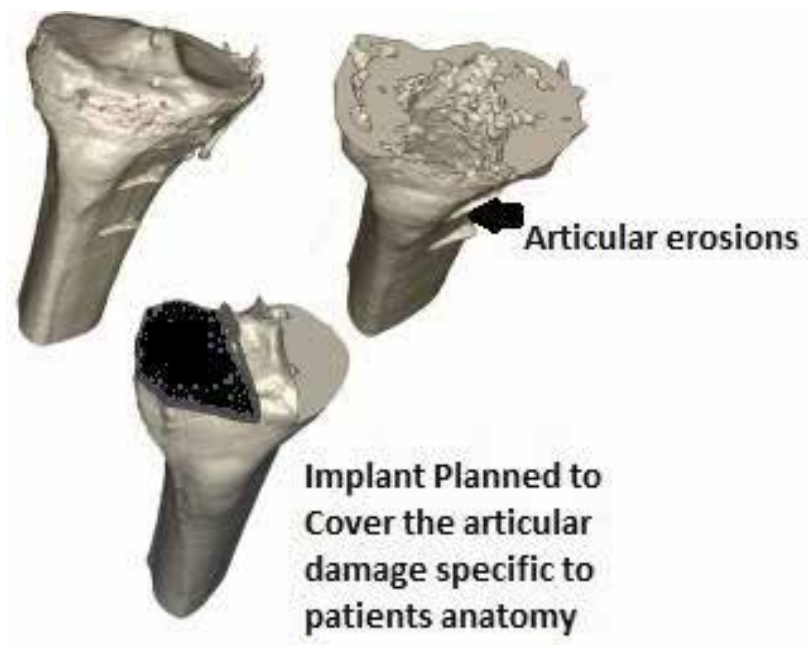

Fig. 22. Planning using CAD software to design patient specific knee Implants. 


\subsection{Illustrative case report: Designing Temporo-Mandibular Joint (TMJ)}

There is significant variation in the structure and shape of the human skull and hence it is difficult to replace a jaw joint successfully without customising it according to the patient's anatomy. In contrast, prostheses for knees and hips are designed for large number of population who fall within the normal range and vary little, except for the size of the patient. Case: A 12-year-old female presented to plastic and orthopaedic surgeon for management of a unilateral left Temporo-mandibular joint ankylosis. The ankylosis had probably been caused by trauma suffered 7 years back. The patient was significantly disabled secondary to difficulty with speech, mastication, and oral hygiene. There was a bony hard swelling around the left TMJ. Her inter-incisal opening distance was $3 \mathrm{~mm}$. 3D-CT scan suggested feature of bony ankylosis of left TMJ with minimal changes in right TMJ. There was no facial deformity. Occlusion was of class I. It was decided to replace the left TMJ with customized TMJ prosthesis. [Fig 23].

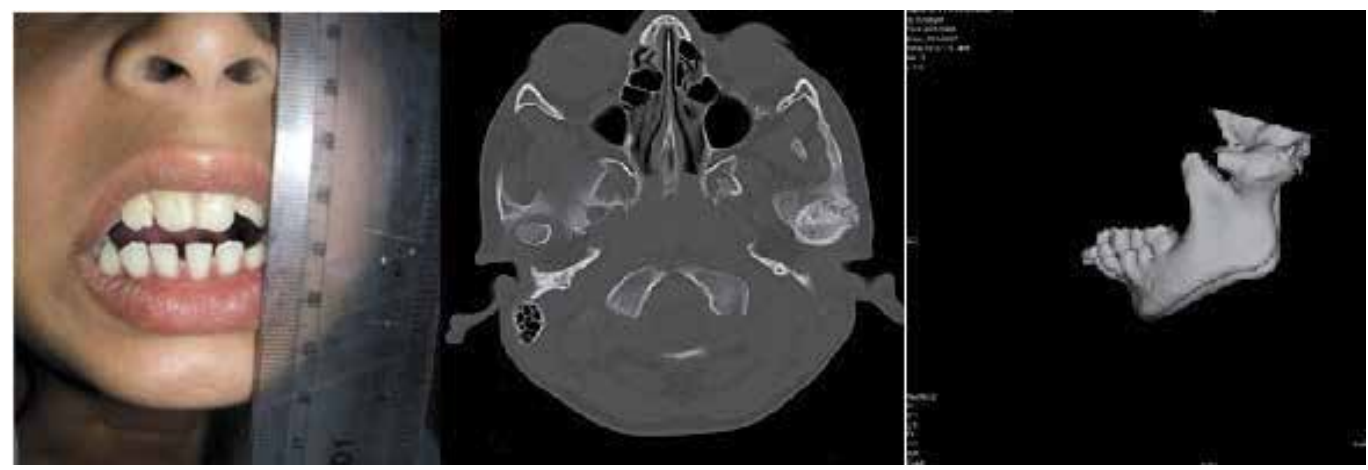

Fig. 23. Preoperative clinical, Ct images of a case of Temporomandibular (TMJ) joint ankylosis.

For the purpose, a preoperative computed tomography (CT) scan of the jaws and jaw joints was obtained using a specific protocol. Using the CT data in form of DIACOM images, a 3$\mathrm{D}$ ABS plastic model of the joint and associated structures was made using stereo lithographic technology and CAD (Fig 24). The mandible was spatially repositioned on the model to correct the functional and aesthetic misalignment problems. From these models the

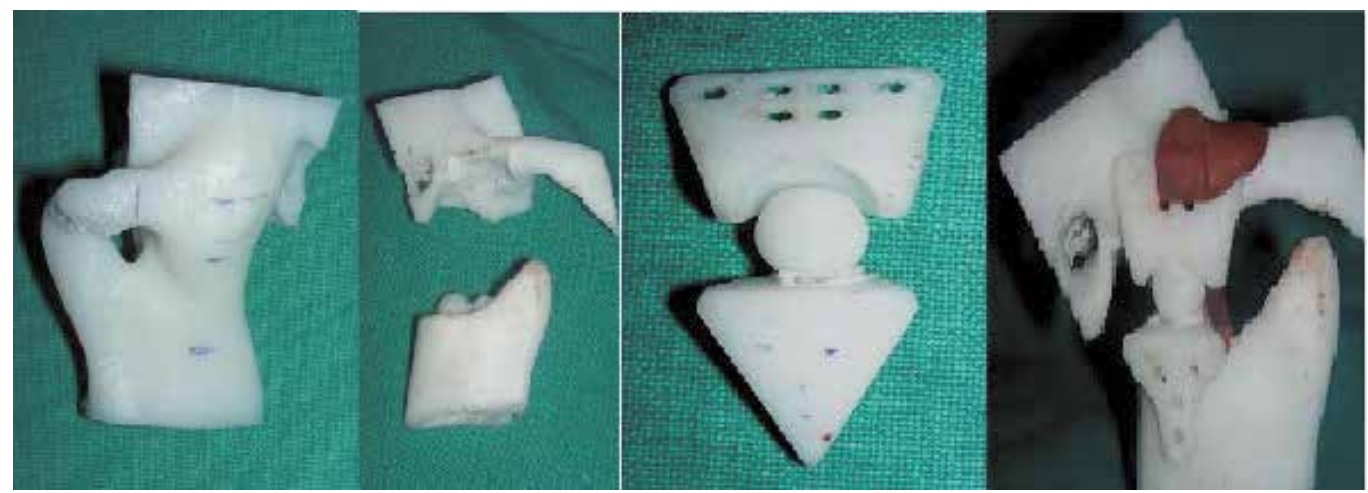

Fig. 24. Creation of RP model of TMJ joint and surgical planning. 
distance of gap arthroplasty was planned. Cad was then used to design the prosthesis conforming to the patient anatomy and as per the surgical planning (Fig 25, 26). The procedure was successfully completed and patient had an excellent mouth opening exceeding $5 \mathrm{~cm}$ post operatively and the results were same at the end of 3 years with good patient satisfaction.

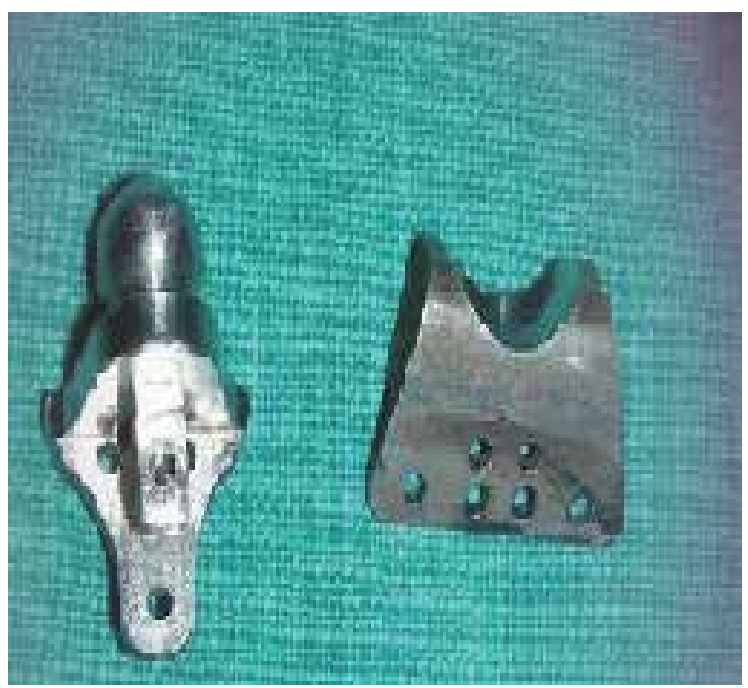

Fig. 25. Customised temporomandibular joint prepared based on RP model generated from patients CT data.

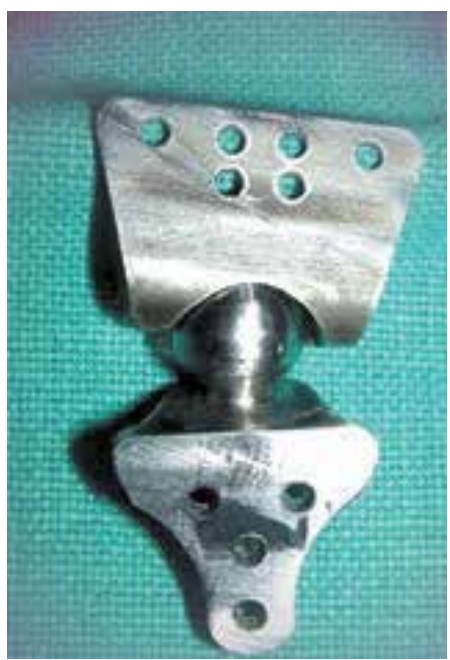

Fig. 26. Ball and socket mechanism employed in developing TMJ.

\subsection{Didactic models for teaching purposes}

As a vehicle for visualisation rapid prototyping models may also serve as important learning tools to young surgeons for practicing complex surgery which have a steep learning curve. The models of various conditions and fracture patterns may also provide 
students an opportunity to understand the pathology and classifications better. This would be in some way similar to the use of cadaveric dissection and bone models to teach normal anatomy. These models can be made in different colours to better illustrate various anatomical structures (Fig 27).

Werner et al in 2008 published an interesting article in Ultrasound in Obstetrics and Gynaecology on the use of rapid prototyping didactic models in study of foetal malformation. In their study of eight cases, MRI scan of foetus was done at 36 weeks. The protocol consisted of: T2-weighted sequence in the three planes of the foetal body (HASTE; repetition time, shortest; echo time, $140 \mathrm{~ms}$; field of view, 300-200 mm; $256 \times 256$ matrix; slice thickness, $4 \mathrm{~mm}$; acquisition time, $17 \mathrm{~s} ; 40$ slices). The entire examination time did not exceed $30 \mathrm{~min}$. After procuring DIACOM images, segmentation software (ScanIP version 2.0, Simpleware Ltd., Exeter, UK) was used to select the contours, allied to design and engineering software (Dassault Systèmes, SolidWorks Corp., and Autodesk Maya) used to create virtual model. Physical materialization was completed using thermoplastic acrylonitrile butadiene styrene. Santos J et al later enhanced and widened the horizon of this application in the field of obstetric by employing Ultrasound images to develop the rapid prototyping images. This is considered a significant step further in our understanding of feto maternal physiology and foetal anatomy and also picking up foetal anomalies at an early stage. Similar studies have also been reported from various fields but especially from orthopaedics, pulmonology and radiology department from across the world.

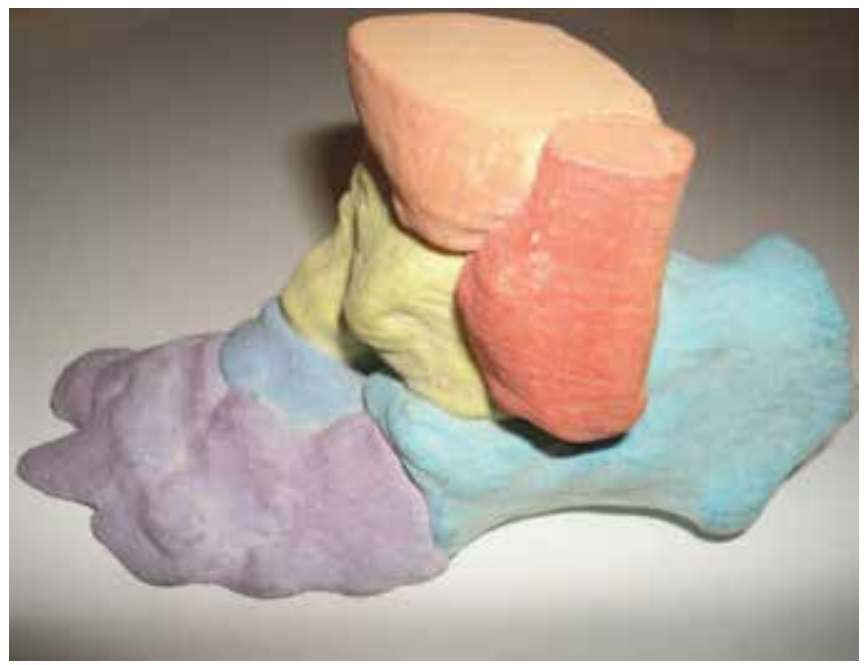

Fig. 27. Didactic model for teaching students anatomy and mechanics of the ankle joint. Differently coloured bone help easy identification and better understanding of the concepts

\subsection{Provide templates for designing bioactive and biocompatible material for tissue engineering}

There are limitations of existing clinical treatment in managing end stage organ damage. Patient specific biological substitutes may provide a viable alternative in managing these cases. The primary regenerative approach has been transplantation the toti-potent cells with regenerative capacity on to a scaffold. The scaffold attempts to mimic the function of the natural extracellular matrix, providing a temporary template for the growth of target 
tissues. In order to serve the purpose the scaffold must have suitable architecture and optimal strength. Rapid prototyping promises to overcome the limitations of conventional scaffolds which are mainly inability to replicate the microscopic pores and structures so commonly found in the body. It can help replicates the porous and hierarchical structures of natural tissues at an unprecedented level thus ensuring that complicated organs like liver, kidney and heart are one day made to order in a lab.

\section{References}

Bagaria V, Deshpande S, Rasalkar DD, Kuthe A, Paunipagar BK. Use of rapid prototyping and three-dimensional reconstruction modeling in the management of complex fractures. Eur J Radiol. 2011 Jan 20

Brown GA, Firoozbakhsh K, DeCoster TA, Reyna JR Jr, Moneim M. Rapid prototyping: the future of trauma surgery? J Bone Joint Surg Am 2003;85:49-55.

McGurk M, Amis AA, Potamianos P, Goodger NM. Rapid prototypingtechniques for anatomical modelling in medicine. Ann R Coll Surg Engl 1997;79:169-74.

Fortheine F, Ohnsorge JA, Schkommodau E, Radermacher K. CT-based planning and individual template navigation in TKA. In: Stiehl JB, Konermann WH, Haaker RG, eds. Navigation and Robotics in Total Joint and Spine Surgery. Berlin, Germany: Springer; 2004. p. 336-42.

Radermacher K, Portheine F, Anton M, Zimolong A, Kaspers G, Rau G, et al. Computer assisted orthopaedic surgery with image-based individual templates. Clin Orthop Relat Res 1998;354:28-38.

Harris J, Rimell J. Can rapid prototyping ever become a routine feature in general dental practice? Dent Update 2002;29:482-6.

Wagner JD, Baack B, Brown GA, Kelly J. Rapid 3-dimensional prototyping for surgical repair of maxillofacial fractures: a technical note. J Oral Maxillofac Surg 2004; 62:898-901.

Xia D, Gui L, Zhang Z, Lu C, Niu F, Jin J, et al. Fabrication of 3-dimensional skull model with rapid prototyping technique and its primary application in repairing one case of craniomaxillofacial trauma. Zhongguo Xiu Fu Chong Jian Wai Ke Za Zhi 2005;19:811-4.

Cheung LK, Wong MC, Wong LL. Refinement of facial reconstructive surgery by stereomodel planning. Ann R Australas Coll Dent Surg 2002;16:129-32.

Heissler E, Fischer FS, Bolouri S, Lehmann T, Mathar W, Gebhardt A, et al. Custom-made cast titanium implants produced with CAD/CAM for the reconstruction of cranium defects. Int J Oral Maxillofac Surg 1998;27:334-8.

Weigel T, Schinkel G, Lendlein A. Design and preparation of polymeric scaffolds for tissue engineering. Expert Rev Med Devices 2006;3:835-51.

Schantz JT, Hutmacher DW, Lam CX, Brinkmann M, Wong KM, Lim TC, et al. Repair of calvarial defects with customised tissue-engineered bone grafts II. Evaluation of cellular efficiency and efficacy in vivo. Tissue Eng 2003;9:S127-39.

Wiria FE, Leong KF, Chua CK, Liu Y. Poly-epsilon-caprolactone/hydroxyapatite for tissue engineering scaffold fabrication via selective laser sintering. Acta Biomater 2007;3:1-12.

Chaware SM, Bagaria V, Kuthe A. Application of the rapid prototyping technique to design a customized temporomandibular joint used to treat temporomandibular ankylosis. Indian J Plast Surg. 2009 Jan-Jun;42(1):85-93. 


\title{
The Use of Rapid Prototyping in Clinical Applications
}

\author{
Giovanni Biglino, Silvia Schievano and Andrew M. Taylor \\ Centre for Cardiovascular Imaging, UCL Institute of Cardiovascular Sciences, London, \\ United Kingdom
}

\section{Introduction}

This chapter will present a brief overview of the possible applications of rapid prototyping in the medical context. Different options of clinical inputs will be discussed as well as five detailed case studies which will demonstrate the flexibility and clinical usefulness of this technique.

Rapid prototyping broadly indicates the fabrication of a three-dimensional (3D) model from a computer-aided design (CAD), traditionally built layer by layer according to the 3D input (Laoui \& Shaik, 2003). Rapid prototyping has also been indicated as solid free-form, computer-automated or layer manufacturing (Rengier et al., 2008). The development of this technique in the clinical world has been rendered possible by the concomitant advances in all its three fundamental steps:

1. Medical imaging (data acquisition),

2. Image processing (image segmentation and reconstruction by means of appropriate software) and

3. Rapid prototyping itself (3D printing).

These steps are visually summarised in Figure 1.

In clinical terms, the possibility of observing, manipulating or manufacturing an anatomical model can serve a range of significant functions (Kim et al., 2008). For instance, it can address visualisation issues that virtual examination cannot always resolve. Also, it can be adopted as a simulation tool or a teaching device. Moreover, it allows medical practitioners and researchers to fully make use of the "patient-specific" concept, in terms of prosthesis design and implant fitting but also in terms of ad hoc simulations. Finally, it can facilitate the communication between the clinician and the patient.

The functions of rapid prototyping in the current clinical world are several (Adler \& Vickman, 1999):

- Pre-surgical planning: A 3D model not only can be useful in surgical practice (i.e. a better fitting, purposefully designed implant), but it can also help a surgical team in visually analysing the location, size and shape of the problem. In the event of a long operation, the model can also be used to plan and customise the surgery. This can be especially valuable when the surgery is performed on anatomical abnormalities.

- Mechanical replicas: A 3D model can be tailored to specific material properties, including non-homogenous variations within a region. Specifically, mechanically 
correct bone replicas are useful in evaluating the behaviour of the bone under different testing conditions.

- Teaching aids: Offering both visualisation of anatomical details and the possibility of practicing directly on a specimen without involving a patient, 3D models can be a valuable tool for training nurses and doctors.

- Customised implants: Instead of using a standard implant and adapting it to the implantation site during the surgical procedure, rapid prototyping enables the fabrication of patient-specific implants, ensuring better fitting and reduced operation time.

- Microelectromechanical systems (MEMS): These are micro-sized objects that are fabricated by the same technique as integrated circuits. MEMS can have different applications, including diagnostics (used in catheters, ultrasound intravascular diagnostics, angioplasty, ECG), pumping systems, drug delivery systems, monitoring, artificial organs, minimally invasive surgery.

- Forensics: Reconstruction of crime scene and wound are also benefiting from rapid prototyping. In particular, in the case of a surviving victim where a wound is of difficult access, e.g. the skull, a model can be used for detailed analysis.

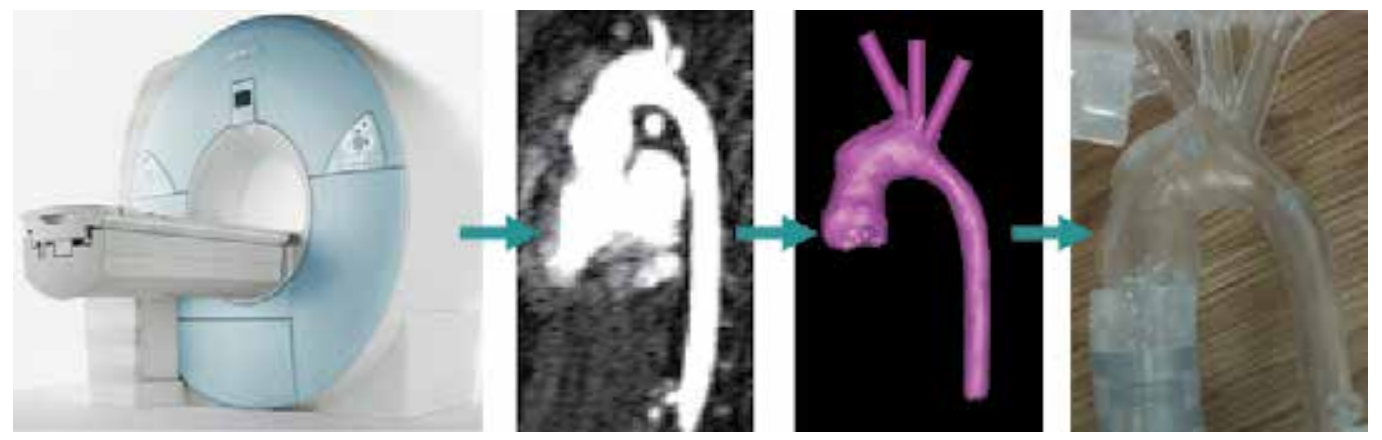

Fig. 1. Stages of rapid prototyping in a clinical setting. From left to right: data acquisition (in this case with magnetic resonance (MR) imaging), image processing, 3D volume reconstruction with appropriate software (in this case, Mimics ${ }^{\circledR}$, Materialise, Leuven, Belgium) and final 3D model printed in a transparent resin. The above example (aortic arch of a paediatric patient) is discussed further in paragraph 4.2.

Despite its clinical use to the present day is still somewhat limited, considering the potential and flexibility of this technique, it is likely that applications of rapid prototyping such as individual patient care and academic research will be increasingly utilised (Rengier et al., 2010).

\section{Anatomical data and image acquisition}

The clinical input for rapid prototyping is represented by all the information contained in imaging data. Most commonly, MR and computerized tomography (CT) imaging are used for this purpose. Other sources include laser surface digitizing, ultrasound and mammography. The output of the imaging acquisition process and input of the rapid prototyping following appropriate processing is a DICOM image (Digital Imaging and Communications in Medicine), which is the outcome of virtually all medical professions 
utilising images, including endoscopy, mammography, ophthalmology, orthopaedics, pathology and even veterinary imaging (Lim \& Zein, 2006).

\subsection{Magnetic resonance imaging}

MR imaging is an imaging technique based on detecting different tissue characteristics by varying the number and sequence of pulsed radio frequency fields, taking advantage of the magnetic relaxation properties of different tissues (Liu et al., 2006). MR imaging has the crucial advantage of not emitting X-ray radiations. Instead, the MR scanner provides a strong magnetic field, which causes protons to align parallel or anti-parallel to it. MR measures the density of a specific nucleus, normally hydrogen, which is magnetic and largely present in the human body, approximately 63\% (Hornak, 1996), except for bone structures. The speed at which protons lose their magnetic energy varies in different tissues allowing detailed representation of the region of interest. This measurement system is volumetric, producing isometric 3D images (i.e. the same resolution in all directions).

\subsection{Computerized tomography}

Hard tissues and bony structures, which are assessed less well by MR imaging, can be captured by means of CT. This is a radiographic technique that uses a narrow fan X-ray beam to scan a slice of tissue from multiple directions. The absorption of different tissues is calculated and displayed according to gray-scale values. The resolution of CT data can be increased by decreasing the slice thickness, producing more slices along the same scanned region. However, the resulting longer scanning time has to be weighed by the clinician against the consequence of increased radiation dose (Liu et al., 2006). The technology known as spiral CT allows for shorter scanning time and small slice intervals with respect to previous scanners. In this case the patient is translated continuously through the gantry while the X-ray tube and detector system are continuously rotating, the focus of the X-ray tube essentially describing a spiral.

\subsection{Other methods}

Laser surface digitizing is a technique that permits acquisition only of external data, while MR and CT comprise both internal and external data, thus reducing scanning time and file size (Liu et al., 2006). This technology is based on a laser probe emitting a diode-based laser beam which forms profiles on the surface of the anatomy being imaged. Each profile is collected as a polyline entity and the combination of profiles yields a 3D volume. Apart from the speed of acquisition, this method has the advantage of not emitting any radiation. An early proposed application of laser surface digitizing regarded the case study of an ear prosthesis model (Ching et al., 1998).

3D ultrasound has also been used as input for rapid prototyping applications, as in the case of foetal modelling (Werner et al., 2010)

\section{Model fabrication}

The methods used for manufacturing a physical model by rapid prototyping can be generally divided into two major categories: "additive" and "subtractive". Additive manufacturing indicates the fabrication of a part by adding materials to a substrate. On the other hand, a subtractive process involves machining using high-speed spindles and fairly 
machinable aluminium alloys in order to provide fast turnarounds for tooling and functional parts (Destefani, 2005). The choice between additive and subtractive rapid prototyping requires the evaluation of parameters such as speed of manufacturing, desired accuracy and budget (Mishek, 2009). In the clinical context, since subtractive techniques have the limitation of reduced ability in printing complex geometries and of requiring hard materials, additive techniques are more commonly employed.

- Stereolithography: A stereolithographic system includes a bath of photosensitive resin, a model-building platform and an ultraviolet laser for curing the resin (Winder \& Bibb, 2005). The input image is divided into slices and such data is fed to the stereolithography machine. Layers are cured in sequence, the laser guided onto the surface of the resin by means of a PC-controlled mirror. The support platform is lowered following the completion of each layer. Further curing occurs in an apposite cabinet once the model is removed from the resin bath. Support structures are added to the model in order to aid layers adhesion and then removed once the model is printed. It is regarded that stereolithography provides the most accurate 3D models with best surface finishing.

- Fused deposition modelling: Similarly to stereolithography, this is a layer-by-layer process, the main difference between the two being that the layers are deposited as a thermoplastic that is extruded from a fine moving tip (Laoui \& Shaik, 2003). As for stereolithography, support structures are necessary and are extruded with a second nozzle. The supporting elements are often printed in a different colour or using soluble material (Winder \& Bibb, 2005).

- Selective laser siltering: In this case an infrared laser is used to cure a thermoplastic powder. This technique does not require supporting structures, facilitating the cleaning process of the models (Berry et al., 1997).

- Laminated object manufacturing: Models produced with this technique are formed by layers of paper, cut using a laser and bonded by a heating process. By nature this is an inexpensive printing method, thus advantageous for large volumes. In clinical terms, however, hollow structures cannot be properly modelled by this technique, so its clinical application is limited. It has been used to produce bioceramic bone implants and prostheses for craniofacial defects (Laoui \& Shaik, 2003).

Alongside these additive-printing methods, a subtractive rapid prototyping technology can be employed for clinical applications:

- Computerised numerically controlled milling: In this case the printing process consists in removing a layer at a time from a block of material. Albeit the complexity of the surfaces and the detail of internal finishing are limited, this subtractive technology has been applied to medical modelling. One example is the construction of custom titanium implants for cranioplasty (Joffe et al., 1999).

\section{Clinical case studies}

The following case studies present a range of different, specific applications of rapid prototyping in the clinical context.

\subsection{Cardiac I: Refining the process of patient selection}

In the past two decades, great advances in transcatheter treatment of several cardiovascular disorders have been reported. In September 2000, Bonhoeffer et al. reported the first successful 
case of a minimally-invasive procedure known as percutaneous pulmonary valve implantation, or PPVI (Bonhoeffer et al., 2000). PPVI combines the replacement of a functional valve with relief of stenosis of the right ventricular outflow tract (RVOT) in patients with repaired congenital heart disease who require pulmonary valve replacement (Hijazi et al., 2006; Lurz et al., 2008). This technique potentially offers a major alternative to surgical valve replacement, but the success of PPVI is greatly dependent on patient selection based on assessment of implantation site morphology and dimensions (Schievano et al., 2007).

Rapid prototyping can be a valuable instrument in assessing patient-specific characteristics determining PPVI suitability, as demonstrated by a recent study (Schievano et al., 2007). A population of twelve patients was retrospectively investigated, including a range of different anatomical configurations. All patients had been referred for possible PPVI treatment. All patients also underwent MR examinations and the MR angiogram data was used as input for rapid prototyping development. Image processing was carried out using Mimics ${ }^{\circledR}$ software (Materialise, Leuven, Belgium). Imaging data was viewed in 2D (sagittal, coronal and transverse planes) and in 3D following segmentation. Segmentation masks were appropriately modified in order to highlight the area of interest, i.e. the RVOT. Following operations of thresholding and region-growing, a 3D volume was obtained by means of pattern recognition and interpolation algorithms. Such volume corresponds to the blood volume of the RVOT and, if further modifications are necessary, it is possible to operate on a pixel-by-pixel basis on the corresponding segmentation mask and render an "updated" 3D volume. The outer surface of the resulting volume essentially corresponds to the inner surface of the RVOT walls. The final step of RVOT model creation is hence to create an additional layer of fixed thickness (in this case $2 \mathrm{~mm}$ ) around the blood volume and delete the latter. The final volume is saved as a standard stereolithography solid-to-layer format (STL file) and is ready to be exported into a rapid prototyping machine. The 3D printer employed in this study was a drop-on-demand machine using thermoplastic resin (Stratasys Genisys, Eden Prairie, MN, USA). The printer operates by means of a nozzle driven by an xy stage to create outlines of each layer, whose thickness was $0.33 \mathrm{~mm}$. The software controlling the machine is able to determine optimal orientation for printing the object and the supports necessary during the printing phase. Total time for printing one model was 3-4 hours. The thin-layer finishing of the printer ensures fine definition of the physical model. All twelve anatomical models are shown in Figure 2.

For all patients, clinical decision regarding PPVI suitability was agreed by cardiologists, image experts and cardiac surgeons. The result was that four patients were judged as unsuitable for PPVI, while for the remaining eight cases, where PPVI was attempted, the procedure was successful only in four patients.

The utility of the 3D models was then evaluated retrospectively. 3D MR images alone or 3D physical models alone were given randomly to two cardiologists who were unaware of the clinical outcomes and who blindly re-evaluated each case solely on the base of the data provided. For the four cases previously clinically rejected for PPVI, both cardiologists confirmed that PPVI should have not been performed. Regarding the remaining eight patients, the two observers correctly determined PPVI suitability in four and two cases respectively based on MR images alone. However, when assessing the 3D models, PPVI assessment was correct in five cases each (Table 1).

In the present application, some advantages of rapid prototyping were clearly shown, such as facilitating clinical assessment, enabling measurements and providing a quick and instinctive appreciation of different morphologies. One limitation of the aforementioned 
RVOT models is represented by the rigidity of the surface and its lack of transparency. A compliant surface could mimic more closely, and to varying degrees of accuracy, the mechanical properties of blood vessels. In the case of a valved stent-graft positioned in the RVOT, such as PPVI procedure, this additional element would allow the model's wall to deform and accommodate the device, thus simulating the in vivo case more correctly. In addition, wall transparency could facilitate assessment of the position of the device and also render the model suitable for visualisation experiments. Both these points will be further discussed in the following paragraphs.

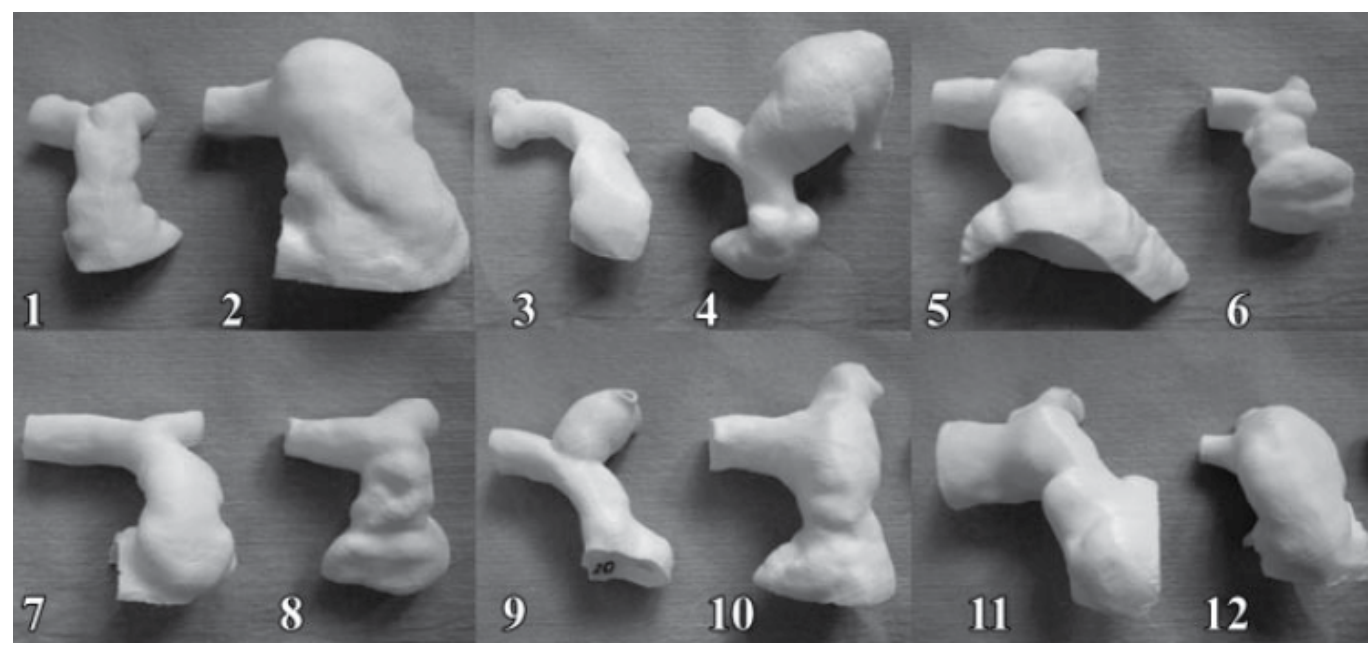

Fig. 2. Rapid prototyping 3D models of right-ventricular outflow tract, printed by means of stereolithography, for assessment of percutaneous pulmonary valve implantation in twelve patients. Note, these are 12 patients with the same congenital heart disease (tetralogy of Fallot), who have undergone the same surgical repair as neonates (complete repair), but present with a wide variety of anatomies 10-15 years later. This demonstrates the patientspecific nature and importance of understanding these individual anatomies. Image from Schievano et al., 2007.

\section{Diagnosis with MRI}

\section{Diagnosis with rapid prototyping}

\begin{tabular}{|c|c|c|c|c|c|c|c|c|}
\hline & \multicolumn{2}{|c|}{ Observer 1} & \multicolumn{2}{|c|}{ Observer 2} & \multicolumn{2}{|c|}{ Observer 1} & \multicolumn{2}{|c|}{ Observer 2} \\
\hline & Correct & Incorrect & Correct & Incorrect & Correct & Incorrect & Correct & Incorrect \\
\hline PPVI & 4 & 4 & 2 & 6 & 5 & 3 & 5 & 3 \\
\hline no PPVI & 4 & 0 & 4 & 0 & 4 & 0 & 4 & 0 \\
\hline $\begin{array}{l}\text { Correct } \\
\text { diagnosis }\end{array}$ & \multicolumn{4}{|c|}{$14 / 24$} & \multicolumn{4}{|c|}{$18 / 24$} \\
\hline
\end{tabular}

Table 1. Two operators evaluating patient suitability for percutaneous pulmonary valve implantation (PPVI): in comparison to assessment based on MR images alone, assessment based on rapid prototyping 3D models alone increased the number of cases evaluated correctly. 


\subsection{Cardiac II: Planning first-in-man device implantation}

Following directly from paragraph 4.1, the wide range of RVOT anatomies impinges on the suitability of PPVI in up to $85 \%$ of patients (Shievano et al., 2007). For this reason a secondgeneration device for PPVI was conceived in order to suit a larger proportion of patients. While the first-generation device (Melody ${ }^{\mathrm{TM}}$, Medtronic Inc., Minneapolis, MN, USA) is a cylindrical platinum-iridium stent, the new device is an hourglass-shaped nitinol covered stent (Schievano et al., 2010). At the time of first-in-man implantation, following bench and animal testing, rapid prototyping proved to be a precious tool for refining the procedure.

The patient-specific anatomy of RVOT, pulmonary trunk and proximal pulmonary arteries was reconstructed from 4D CT data. The model was printed in transparent rigid resin and the interventional cardiologists involved in this case of a novel PPVI device implantation could study access route and placement on the 3D phantom. As a result, the implanters could identify an optimal approach: guide wire in the left pulmonary artery, device deployment with the distal portion just within the left pulmonary artery, pullback of the device from the delivery system until correct positioning in the pulmonary trunk is achieved. This approach, together with the alternative and unsuccessful approach via the right pulmonary artery, is shown in Figure 3.

In this case, rapid prototyping enabled the interventional cardiologists with a visualisation tool that they cannot normally rely on, as opposed to a surgeon who has direct visual access to the area of interest. Testing correct positioning of the guide wire and practicing the implantation were important steps in ensuring procedural success.
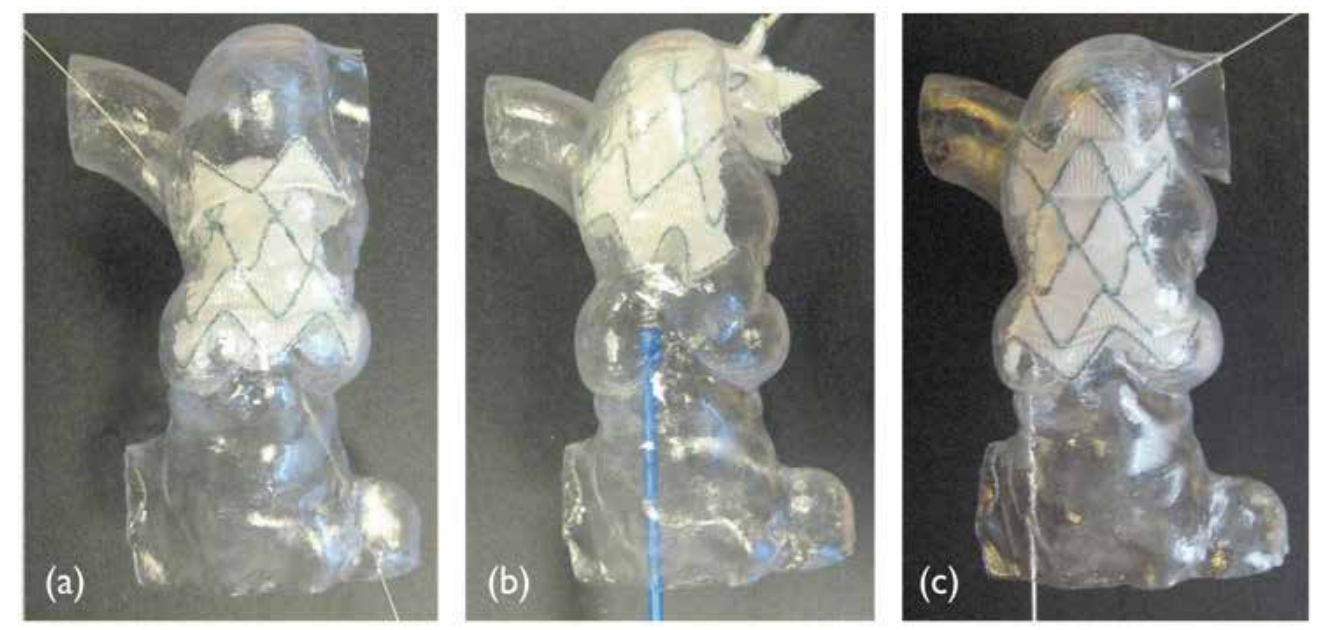

Fig. 3. Implantation of a new PPVI device into the same, patient-specific rapid prototyping model (a) via the right pulmonary artery (RPA) and (b \& c) the left pulmonary artery (LPA). It was impossible to place the device accurately via the RPA, but implantation into the LPA (b) with pullback into the pulmonary trunk (c) was successful. This trial implantation directed the implantation used in the actual first-in-man procedure, which was performed via the LPA.

\subsection{Cardiac III: Bench-top experiments to integrate clinical knowledge}

The first stage of Fontan palliation for neonates with hypoplastic left heart syndrome (HLHS), namely the Norwood procedure, aims to increase the flow of oxygenated blood to 
the systemic circulation while, simultaneously, provide a source of pulmonary blood flow in these single-ventricle patients (Norwood, 1991). This operation involves enlargement of the hypoplastic aorta by means of a patch, reconstruction of aortic coarctation and increase of pulmonary flow, the latter by means of an arterio-pulmonary (Norwood, 1991) or ventriculo-pulmonary (Sano et al., 2003) shunt or stenting of the ductus arteriosus (Galantowicz \& Cheatham, 2005). It is thus evident that Norwood patients present a very specific and complex arrangement of their circulatory system.

A computational model of the Norwood circulation has been already introduced (Migliavacca et al., 2001). On the experimental side, mock circulatory systems are acknowledged as a tool for addressing fluid mechanics questions in a systematic and rigorous way, allowing to isolate a variable of interest in a reproducible environment. Recent work from our group has shown the development of an in vitro setup suitable for studying features of the circulation following the Norwood procedure and focusing initially on the presence of aortic coarctation (Biglino et al., 2011). The setup is broadly based on the "multiscale" concept, as it includes an anatomically accurate 3D element (the region of interest, in this case the aortic arch) attached to a lumped parameter network (Quarteroni \& Veneziani, 2003). Rapid prototyping technology was thus employed to manufacture the 3D elements for this first - to our knowledge - Norwood mock circulatory system.

Initially, four distinct aortic arch geometries were selected: (a) "control" morphology, with straight unreconstructed arch, (b) enlarged arch, (c) aortic coarctation (coarctation index ${ }^{1}=$ 0.5 ) and (d) severe aortic coarctation (coarctation index $=0.3$ ). Retrospective $\mathrm{MR}$ angiographic data were used as input for the rapid prototyping process. Images were analysed in Mimics ${ }^{\circledR}$ (Materialise, Leuven, Belgium) as described in paragraph 4.1. Once a first volume rendering was available, each 3D model was modified considering the purpose of the study. In fact, since the aim was to comment on the effect of aortic coarctation in vitro, the brachiocephalic vessels were modified so that the variations in their dimensions from one case to the other would not influence flow distribution, thus rendering more difficult to discern the effect of varying arch geometry alone and nullifying one of the main benefits of bench experiments, i.e. the ability of varying one variable at a time. Instead, CAD cylindrical elements of equal, physiologically reasonable diameter and length were placed in the position of the brachiocephalic vessels. Also, another element was added on all models on one of the brachiocephalic branches (corresponding to the innominate artery) providing an attachment for an arterio-pulmonary (or modified Blalock-Taussig) shunt-equivalent conduit. Furthermore, conical elements were merged at all endings (shunt, upper body vessels, and descending aorta) in order to facilitate the insertion of the model into the mock circuit. Finally, in order to take pressure measurements at different locations, three small cylinders the size of a $4 \mathrm{~F}$ catheter were placed at different locations on the models (arch, just after the coarctation - if present - and descending aorta) in order to create three ports for pressure catheters insertion. All these volumes were merged in a unique volume, extruded with a thickness of $1.5 \mathrm{~mm}$ and exported as a STL file for printing.

Each model was printed twice, employing a rigid transparent resin and a compliant opaque composite, each offering different advantages. On the one hand, rigid models are suitable for visualisation experiments (such as particle image velocimetry) and, albeit non-

\footnotetext{
${ }^{1}$ The coarctation index $(\mathrm{CI})$ defines the severity of a coarctation as the ratio of the narrowest diameter at the isthmus $\left(\mathrm{D}_{1}\right)$ and the distal diameter in the descending thoracic aorta $\left(\mathrm{D}_{2}\right), \mathrm{CI}=\mathrm{D}_{1} / \mathrm{D}_{2}$ (Lemler et al., 2000).
} 
physiological, they allow observing flow distribution without the additional variable of compliant walls. On the other hand, flexible models are more physiological and can replicate the arterial Windkessel.

Both versions of the models were printed with a PolyJet machine (Object Geometries Inc, Billerica, MA, USA). The transparent resin is a commercially available material (Watershed ${ }^{\circledR}$ XC11122). Until recently, printing a compliant phantom proved to be more difficult, involving processes such as dripping or dipping and using rigid stereolithographic models as scaffolds (Armillotta et al., 2007). PolyJet technology, however, allows printing flexible models and the material of choice appears to be TangoPlus FullCure $930 \AA$ or $980 \AA$, the only difference between the two being that the first one is opaque while the latter has a black finishing. Preliminary work from our group reveals that this material can implement physiological compliance, depending on the wall thickness and the part of the vasculature being modelled (Biglino et al., 2011). Both rigid and compliant models could be printed within 24 hours$^{2}$. The four printed geometries are shown in Figure 4.

This study showed the usefulness of PolyJet technology in producing accurate patientspecific vascular models that can be inserted into mock circulatory systems.

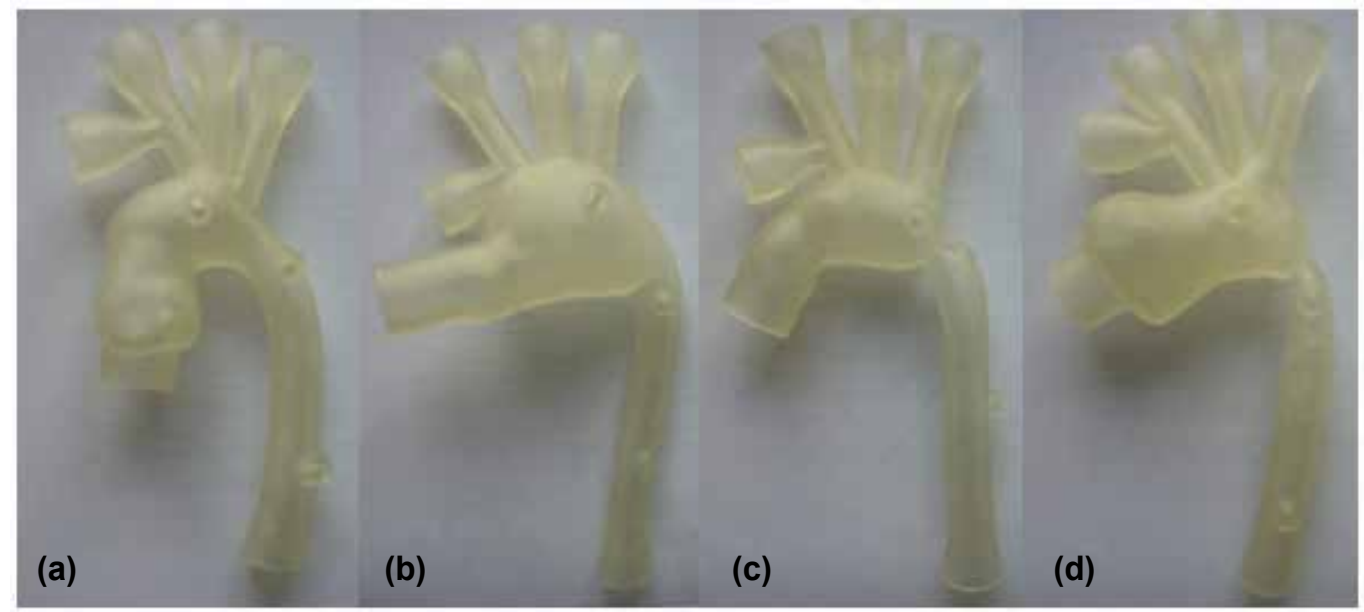

Fig. 4. Four neonate aortic arches, modified for in vitro experiments, printed in TangoPlus FullCure $930 ®$ material. Four patient-specific morphologies were selected: (a) control, (b) enlarged aortic arch, (c) aortic coarctation and (d) severe aortic coarctation.

\subsection{Dental: The use of stereolithography in maxillofacial surgery}

"Can rapid prototyping ever become a routine feature in general dental practice?" asked Harris \& Rimell less than ten years ago (Harris \& Rimell, 2002). Certainly, since they highlighted the potential of this technique in their study, experience has shown that rapid prototyping can play a role in this field. The importance of modelling in this context is further confirmed by publications of the late 1980s and early 1990s already exploring the potential of stereolithographic technology for maxillofacial surgery (Arvier et al., 1994; Bill et al., 1995; Karcher et al., 1992; Lambrecht et al., 1989).

${ }^{2}$ All models described in this paragraph were printed by Rapidform, Royal College of Art, London, UK. 
Robiony et al. recently showed an integrated process involving maxillofacial surgeons, radiologists and engineers for dental virtual surgical planning (Robiony et al., 2008). In this case, the input data for the printing process is represented by CT images. Once the images are imported in the dedicated software (Mimics $\left.{ }^{\circledR}\right)$, the anatomical region of interest is contoured by segmentation algorithms and the 3D structure is described by a triangle mesh which is exported as STL file for rapid prototyping. The printing process is a standard stereolithographic technique using liquid resin and polymerisation by a UV laser beam. While acknowledging the importance of the physical 3D model per se, this study also stressed the importance of being able to simulate a surgical procedure on the digital model. Manipulation of the STL file, rather than other formats such as IGES, appeared to be the best solution. Surgeons and engineers were thus able to import the skull model in the digital environment and replicate a surgical procedure. This study reports that 11 patients have been treated using this method: 3 cases of mandibular reconstruction, 5 cases of elongation of the vertical ramus and 3 cases of sagittal elongation of the mandible.

More specifically, one of the reported cases (surgical planning of emimandibular resection in oral cancer) shows how the rapid prototyping model can highlight cancerous tissues, enable the surgeon to make hypotheses of intervention for tumour resection and plan accurate postoperative reconstruction (Figure 5).

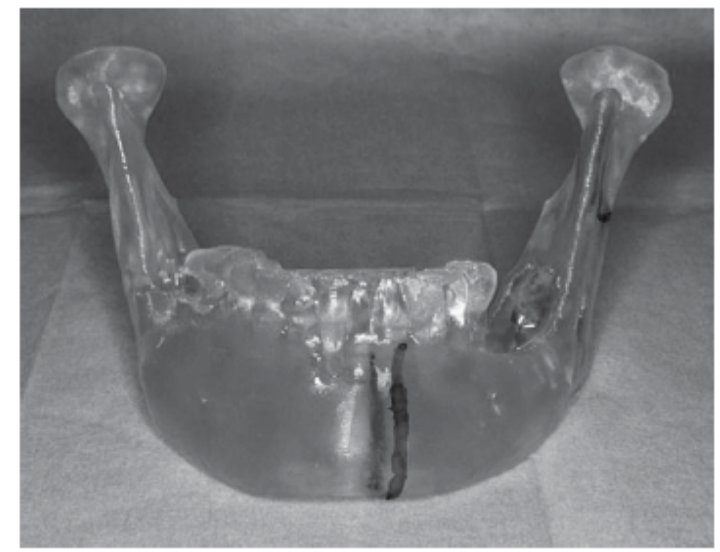

Fig. 5. Stereolithographic model of the mandible printed with rigid resin. Image from Robiony et al., 2008.

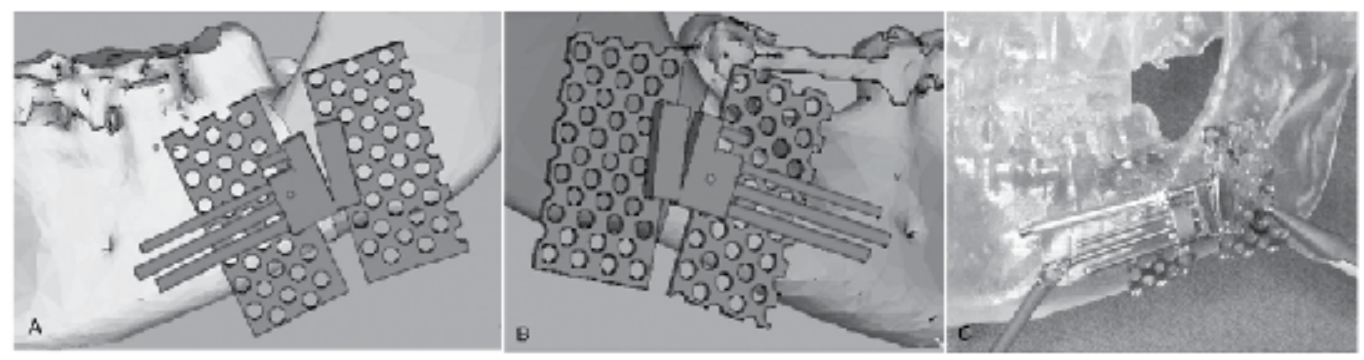

Fig. 6. Virtual simulation of mandibular elongation (A and B) and transfer of the surgical solution to the stereolithographic model (C). Image modified from Robiony et al., 2008. 
Another case reported in the same study (gradual elongation of the mandible by means of osteogenesis distraction) shows how correct mandibular elongation is planned virtually, by simulating its most desirable final position. In fact, the mandible is virtually cut performing bilateral sagittal osteotomy and moved in different ways (Figure 6A and 6B), the result being evaluated on the basis of aesthetics and functionality. Then, the surgical solution is first applied to the rapid prototyping patient-specific model (Figure 6C) and finally to the patient.

The advantages of employing rapid prototyping technology in maxillofacial surgery have been recognised as: providing an understandable clinical picture; allowing evaluation of different surgical options; shaping of implants (e.g. titanium plates) directly on the 3D model; more accurate evaluation of shape and dimensions of a bone graft avoiding unnecessary removal of bone; and consequently: reduced length of operation and improved exchange of ideas between experts of different backgrounds (D'Urso et al., 1999; Robiony et al., 2008).

\subsection{Orthopedic: Fabrication of patient-specific prostheses}

Orthopedics is probably one of the fields in which rapid prototyping technology has been employed more extensively. Different applications include for example: construction of an anatomical model in a case presenting a complex shoulder injury (Potamianos et al., 1998); generating patient-specific templates for total knee arthroplasty (Hafez et al., 2006); assessment, classification and surgical planning of acetabular fractures (Hurson et al., 2007); production of porous titanium scaffolds for orthopaedic implant design (Ryan et al., 2008); fabrication of a surgical guide for cup insertion in total hip arthroplasty (Hananouchi et al., 2009); understanding fracture configuration and management of complex fractures (Bagaria et al., 2011).

One recent application concerns tailored ankle-foot orthoses (Mavroidis et al., 2011; Schrank \& Stanhope, 2011). The ,Americans with Disabilities' report stated that in 2005 approximately 27 million people above the age of 15 had an ambulatory disability (Brault, 2008). Patients exhibiting weakness in the region of the ankle joint musculature as a consequence of motor neuron disorders or lower limb injuries are often treated with anklefoot orthoses, which support gait function (Pomeranz et al., 2006). The aforementioned studies used rapid prototyping technology aiming on the one hand to achieve better fitting patient-specific orthoses and on the other hand to facilitate the fabrication process of these devices, which currently is rather cumbersome.

Mavroidis et al. performed image acquisition of the ankle-foot complex using laser scanning (3D FaceCam 500 scanner, Technest Inc, Bethesda, MD, USA). Image reconstruction was carried out by means of commercially available software Rapidform ${ }^{\circledR}$ (Seoul, South Korea) which allowed operations such as removal of unwanted parts and remeshing. The model was extruded with a wall thickness of $3 \mathrm{~mm}$ and exported as an STL file for the rapid prototyping process. The physical models were printed using stereolithography (Viper Si2 machine, 3D Systems, Rock Hill, SC, US) curing liquid resin. An example is shown in Figure 7. Two ankle-foot orthoses were realised using two different materials (Accura 40 resin and Somos ${ }^{\circledR} 9120$ Epoxy photopolymer). Gait analysis was then conducted using a motion capture system and comparing four different scenarios: an healthy subject (a) walking without ankle-foot orthosis, (b) walking with a prefabricated ankle-foot orthosis, (c) walking with custom-made orthosis made in Accura 40 resin and (d) walking with custom-made orthosis made in Somos ${ }^{\circledR} 9120$ Epoxy. Gait 
analysis aimed to assess spatio-temporal parameters, kinematics (joint angles) and kinetics (joint moments and powers) of hip, knee and ankle.
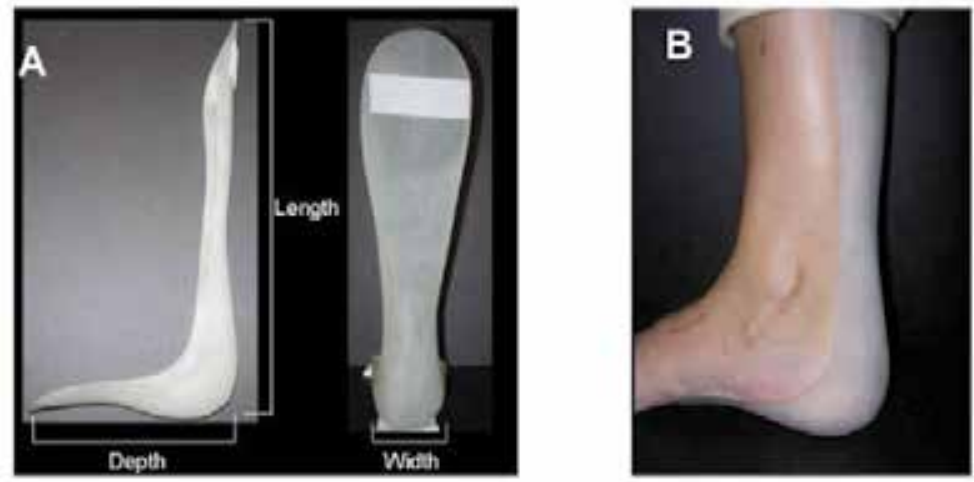

Fig. 7. Flexible ankle-foot orthosis fabricated by rapid prototyping (A) and fitting of the flexible orthosis on the leg of the tested subject (B). Image from Mavroidis et al., 2011.

On the other hand, Schrank \& Stanhope, after digital acquisition of the subject's shape and size characteristics, performed a virtual orthopaedic alignment process producing the custom-fit parameters for a fully parameterized CAD model. Without analysing in detail such parameterization procedure, aimed to the refinement of model fabrication, it is noted that in this case the authors opted for selective laser sintering printing technique (Vanguard HS SLS machine, 3D Systems, Rock Hill, SC, USA). Patient-specific orthoses were realised using data from two nondisabled subjects and assessed for dimensional accuracy and stiffness.

Both these studies show that rapid prototyping can be very valuable for the fabrication of ankle-foot orthoses. Results showed that the rapid prototyping prosthesis matched standards of prefabricated designs while increasing freedom with geometric features (Mavroidis et al., 2011) and that the accuracy of the orthoses fabricated by selective laser sintering was also within tolerance values from the literature (Schrank \& Stanhope, 2011). Moreover, both stressed the substantial reduction in prosthesis production time.

\subsection{Ethical predicament: Post mortem examinations}

Training of surgeons and pathologists has traditionally relied on dissections, but in 2007 the Royal Colleges and the Department of Health reported that a very substantial drop in the number of donor bodies was endangering medical training in the United Kingdom (www.bio-medicine.org, 2007). In order not to loose the important information provided by post mortem examinations, increasing interest has been focused on developing or exploring non-invasive techniques to perform these assessments. In particular, MR has been indicated as a viable alternative to invasive autopsies (Thayyil et al., 2009). At the same time, in the unfortunate case of neonatal or foetal autopsies, this sort of non-invasive examination is also more acceptable for parents, as it avoids dissection of the body (Brookes et al., 1996; Cohen et al., 2007; Griffiths et al., 2005).

A recent study from our group (Schievano et al., 2010) has highlighted the potential of rapid prototyping technology for foetal and infant anatomical reconstructions from post mortem MR images. A range of 11 cases was studied, with images using a 1.5 T MR scanner (Avanto, 
Siemens, Erlangen, Germany) or, for the case of one foetus under 20 weeks, a $9.4 \mathrm{~T}$ scanner (VNMRS, Varian, Palo Alto, CA, USA). The cases were selected purposefully to showcase a range of structural abnormalities or injuries, thus demonstrating the applicability of the technique to different organs. The images were analysed using suitable software (Mimics ${ }^{\circledR}$, Materialise, Leuven, Belgium) following the methodology described in paragraph 4.1 for a different application. Different volumes, including entire organs, were rendered in $3 \mathrm{D}$. In this case, two different machines were employed for printing the 3D models: a Z Corp printer (Z Corporation, Burlington, MA, USA) employing inkjet print heads depositing a binder into plaster powder was used for manufacturing opaque and coloured parts, while an iPro SLA machine (3D Systems Corporation, Rock Hill, SC, USA) employing an ultraviolet laser curing liquid resin was used for manufacturing transparent parts.

Visual examination of the 3D models allowed recognition of all structural anatomies, relationship between organs and relevant pathologies. The authors of this study stressed several advantageous applications of this technology, including:

- Improved understanding of complex congenital anatomies: the size of small foetuses renders studying congenital abnormalities intrinsically difficult. Rapid prototyping allows for a permanent physical record to be analysed at a later stage (e.g. for teaching purposes) but also allows for magnification/scaling of small features, as shown in Figure 8. In the presented example rapid prototyping facilitated the analysis of the specimen, since conventional autopsy was difficult due to autolysis and small dimensions.

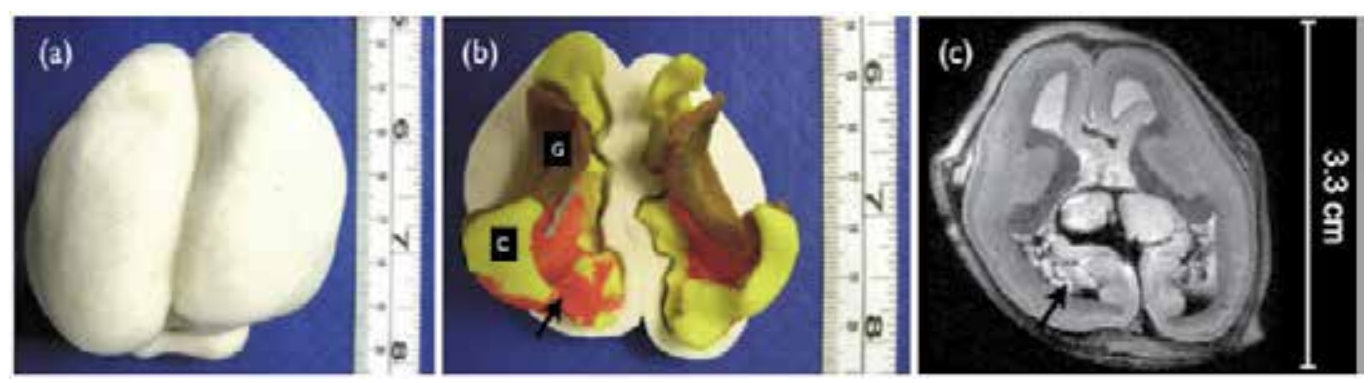

Fig. 8. Rapid prototyping of the brain of a 16 week foetus. (a) The outer surface, showing the absence of sulci and gyri. (b) Internal section in the axial plane, showing the germinal matrix $(\mathrm{G})$ and cerebrospinal fluid in the lateral ventricles (C) while the arrow indicates bleeding in the choroid plexus and ventricular cavity. (c) The 9.4 T MR image in the same axial plane.

Note that the rapid prototyping model is scaled to twice the original size. Image from Schievano et al., 2010.

- Medical training: rapid prototyping models facilitate storage and categorization of samples, which can be a precious tool for anatomy and pathology teaching.

- Parental counselling: in the event of identification of abnormalities and terminated pregnancy, traditional post mortem photographic images can be highly disturbing when shown to parents. A rapid prototyping model can instead facilitate communication with the parents, being less graphic and allowing the observer to be more detached from the object.

- Medico-legal demonstrations: court cases relating to injuries and deaths suffered by infants may require the use of post mortem images which, as in the case of parental 
counselling, may be regarded as inappropriate, as either gruesome or revealing features not strictly related to the case. Evidence of this nature is however crucial and rapid prototyping can provide a scientific way to show pathological findings to a jury. An example of this application is shown in Figure 9 and it is particularly interesting as it involves the use of MR images for identification of bleeding regions while CT data (SOMATOM Definition, Siemens, Forchheim, Germany) was used to reconstruct the fractured skull.

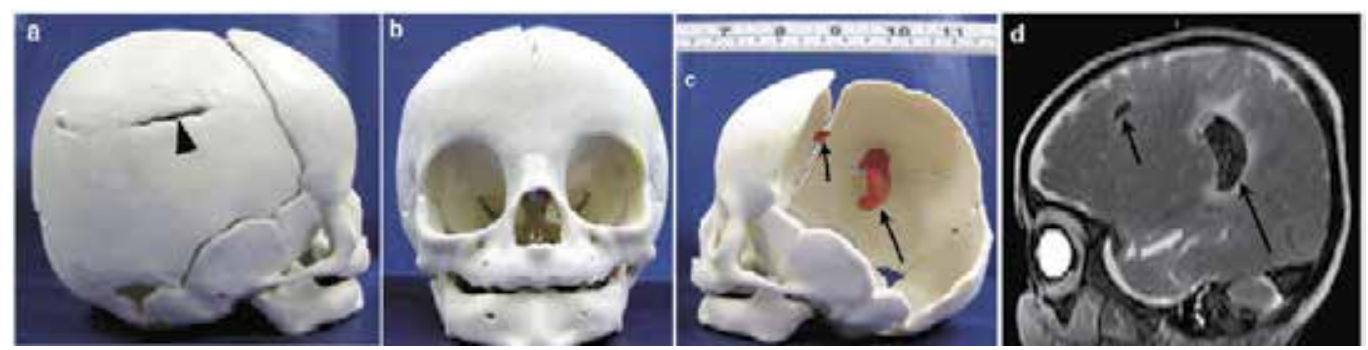

Fig. 9. The case of a fractured skull (a-c), showing parenchymal bleed in the brain of an infant. Post mortem MR images (d) indicated the position of the bleed. The model was built combining and registering CT images, providing the skull structure, and MR images, providing the bleed volume and position. Image modified from Schievano et al., 2010.

- In utero foetal diagnosis and management: it has been shown that 3D volume reconstruction of foetal organs can be achieved using rapid multi-slice snapshot MR sequences (Hayat et al., 2008). In utero foetal MR imaging can thus be used as input for rapid prototyping, which would be particularly useful for detecting the exact position of the internal organs in complex conditions such as foetal diaphragmatic hernia and conjoined twins (Christensen et al., 2004), identifying facial dysmorphism in some genetic conditions and diagnosis cases of skeletal dysplasia.

- $\quad$ Replacement for plastination: the use of human body parts for the process of plastination can "trivialis[e] the very important act of body donation" (http://news.bbc.co.uk, 2002), while rapid prototyping can preserve organs' appearance and size without the use of human tissues.

It is true that the abovementioned applications require the presence of specialised staff, either for image acquisition or image processing, and the need for proper printing equipment. On the other hand, Schievano et al. have reported in their comprehensive study that image processing required between 1 and 20 hours, rapid prototyping required between 1 and 12 hours and the cost of the models varied between $£ 10$ and $£ 240$ depending on the size, highlighting the effectiveness of the method (Schievano et al., 2010).

\section{Conclusion}

In broad terms, rapid prototyping is today extensively used in a variety of industries and fields, from more obvious applications (such as product design) to less intuitive ones (such as archaeology). It has been noted that "rapid prototyping is so pervasive that it would be unlikely any individual could go about his daily routine without using a product that has in some way benefited from rapid prototyping" (Grimm, 2004). Clinically, this technique represents a flexible tool, from diagnostics to prostheses design, as briefly outlined in this 
chapter. It is envisaged that, because of its relatively inexpensive nature and the wider range of available materials for printing, including compliant materials, rapid prototyping will be increasingly used in clinical practice.

\section{Acknowledgments}

The authors gratefully acknowledge the support of the Fondation Leducq (France), the Royal Academy of Engineering (UK), the Engineering and Physical Sciences Research Council (UK) and the National Institute for Health Research (UK).

\section{References}

Adler, D. \& Vickman, S. (1999). Medical applications of rapid prototyping at MSOE, Available from http://www.rpc.msoe.edu/medical.php

Armillotta, A.; Bonhoeffer, P.; Dubini, G.; Ferragina, S.; Migliavacca, F.; Sala, G. \& Schievano, S. (2007). Use of rapid prototyping models in the planning of percutaneous pulmonary valved stent implantation. Proceedings of the Institution of Mechanical Engineers, Part H, Journal of engineering in medicine, Vol.221, No.4, (May 2007), pp. 407-416, ISSN 0954-4119

Arvier, J.F.; Barker, T.M.; Yau, Y.Y.; D’Urso, P.S.; Atkinson, R.L. \& McDermant, G.R. (1994). Maxillofacial biomodelling. The British journal of oral and maxillofacial surgery, Vol.32, No.5, (October 1994), pp. 276-283, ISSN 0266-4356

Bagaria, V.; Deshpande, S.; Rasalkar, D.D.; Kuthe, A. \& Paunipagar, B.K. (2011). Use of rapid prototyping and three-dimensional reconstruction modelling in the management of complex fractures. European journal of radiology, Epub ahead of print, (January 2011), pp. 30-37, ISSN 0720-048X

Berry, E.; Brown, J.M.; Connell, M.; Craven, C.M.; Efford, N.D.; Radjenovic, A. \& Smith, M.A. (1997). Preliminary experience with medical applications of rapid prototyping by selective laser siltering. Medical engineering $\mathcal{E}$ physics, Vol.19, No.1, (January 1997), pp. 90-96, ISSN 1350-4533

Biglino, G.; Schievano, S.; Baker, C.; Giardini, A.; Figliola, R.; Taylor, A.M. \& Hsia, T.Y. (2011). A patient-specific paediatric mock circulatory system: investigating the circulation following the Norwood procedure. ASAIO journal - Abstracts of the $57^{\text {th }}$ annual conference, Vol.57, No.2, (March-April 2011), p.92, ISSN 1058-2916

Biglino, G.; Verschueren, P.; Zegels, R.; Taylor, A.M. \& Schievano, S. (2011). Quantification of TangoPlus FullCure ${ }^{\circledR} 930$ compliance for printing patient-specific vascular models. ASAIO journal - Abstracts of the 57th annual conference, Vol.57, No.2, (MarchApril 2011), p. 74, ISSN 1058-2916

Bill, J.S.; Reuther, J.F; Dittmann, W.; Kubler, N.; Meier, J.L.; Pistner, H. \& Wittenberg, G. (1995). Stereolithography in oral and maxillofacial operation planning. The international journal of oral and maxillofacial surgery, Vol.24, No.1 Pt2, (February 1995), pp. 98-103, ISSN 0901-5027

bio-medicine.org (March 2007). Shortage of human cadavers for medical training, 14.03.2007, Available from:

http:/ / www.bio-medicine.org/medicine-news/Shortage-of-Human-Cadavers-forMedical-Training-19041-1/ 
Bonhoeffer, P.; Boundjemeline, Y.; Saliba, Z.; Merckx, J.; Aggoun, Y.; Bonnet, D.; Acar, P.; Le Bidois, J.; Sidi, D. \& Kachaner, J. (2000). Percutaneous replacement of pulmonary valve in a right-ventricle to pulmonary-artery prosthetic conduit with valve dysfunction. The Lancet, Vol.356, No.9239, (October 2000), pp. 1403-1405, ISSN 0140-6736

Brault, M.W. (December 2008). Americans with disabilities: 2005, In: Census bureau, 12.2008, Available from http://www.census.gov/prod/2008pubs/p70-117.pdf

Brookes, J.A.; Hall-Craggs, M.A.; Sams, V.R. \& Lees, W.R. (1996). Non-invasive perinatal necropsy by magnetic resonance imaging. The Lancet, Vol.348, No.9035, (October 1996), pp. 1139-1141, ISSN 0140-6736

Ching, L.S.; Kai, C.C. \& Meng, C.S. (1998). A novel technique for fabricating facial prosthetic model, Proceedings of the 20 th annual International Conference of the IEEE Engineering in Medicine and Biology Society, pp. 2746-2749, ISBN 0-7803-5164-9, Hong Kong SAR, China, October 29-November 1, 1998

Christensen, A.M.; Humphries, S.M. \& Goh, K.Y.C. (2004). Advanced "tactile" medical imaging for separation surgeries of conjoined twins. Child's nervous system, Vol.20, No.8-9, (August 2010), pp. 547-553, ISSN 0256-7040

Cohen, M.C. \& Whitby, E. (2007). The use of magnetic resonance in the hospital and coronial pediatric post-mortem examination. Forensic science, medicine and pathology, Vol.3, No.4, (December 2007), pp. 289-296, ISSN 1547-769X

D’Urso, P.S.; Barker, T.M.; Earwaker, W.J.; Bruce, L.J.; Atkinson, R.L.; Lanigan, M.W.; Arvier, J.F. \& Effeney, D.J. (1999). Stereolithographic biomodelling in craniomaxillofcial surgery: a prospective trial. Journal of cranio-maxillo-facial surgery, Vol.27, No.1, (February 1999), pp. 30-37, ISSN 1010-5182

Destefani, J. (2005). Additive or subtractive? What rapid prototyping process is right for your job? Manufacturing engineering, Vol.134, No.4, (April 2005), pp. 2-5, ISSN 03610853

Galantowicz, M. \& Cheatham, J.P. (2005). Lessons learned from the development of a new hybrid strategy for the management of hypoplastic left heart syndrome. Pediatric cardiology, Vol.26, No.3, (March-April 2005), pp. 190-199, ISSN 0172-0643

Griffiths, P.D.; Paley, M.N. \& Whitby, E.H. (2005). Post-mortem MRI as an adjunct to fetal or neonatal autopsy. The Lancet, Vol.365, No.9466, (April 2005), pp. 1271-1273, ISSN 0140-6736

Grimm, T. (2004). User's guide to rapid prototyping, SME Society of manufacturing engineers, ISBN 0-87263-697-6, Dearborn, MI, USA

Hafez, M.A.; Chelule, K.L.; Seedhom, B.B. \& Sherman, K.P. (2006). Computer-assisted total knee arthroplasty using patient-specific templating. Clinical orthopaedics and related research, Vol.444 (March 2006), pp. 184-192, ISSN 0009-921X

Hananouchi, T.; Saito, M.; Koyama, T.; Hagio, K.; Murase, T.; Sugano, N. \& Yoshikawa, H. (2009). Tailor-made surgical guide based on rapid prototyping technique for cup insertion in total hip arthroplasty. The international journal of medical robotics and computer assisted surgery, Vol.5, No.2, (June 2009), pp. 164-169, ISSN 1478-5951 
Harris, J. \& Rimell, J. (2002). Can rapid prototyping become a routine feature in general dental practice? Dental update, Vol.29, No.10, (December 2002), pp. 482-486, ISSN 0305-5000

Hayat, T.; Jiang, S.; Allsop, J.; Hajnal, J. \& Rutherford, M. (2008). Quantification of human fetal brain development in utero using multislice snapshot MRI with 3D volume reconstruction. Ultrasound in obstetrics and gynecology - Abstracts of the $2^{\text {nd }}$ international conference on fetal MRI, Vol.31, (May 2008), p. 600, ISSN 1469-0705

Hijazi, Z.M.; Bonhoeffer, P.; Feldman, T. \& Ruiz, C.E. (2006). Transcatheter valve repair, Taylor \& Francis Group, ISBN 978-1-84184-472-5, Oxon, UK

Hornak, J.P. (1996). The basics of MRI, Available from http://www.cis.rit.edu/htbooks/mri, Rochester, NY, USA

Hurson, C.; Tansey, A.; O’Donnchadha, B.; Nicholson, P.; Rice, J. \& McElwain, J. (2007). Rapid prototyping in the assessment, classification and preoperative planning of acetabular fractures. Injury, Vol.38, No.10, (October 2007), pp. 1158-1162, ISSN 0020-1383

Joffe, J.; Harris, M.; Kahugu, F.; Nicoll, S.; Linney, A. \& Richards, R. (1999). A prospective study of computer-aided design and manufacture of titanium plate for cranioplasty and its clinical outcome. British journal of neurosurgery, Vol.13, No.6, (December 1999), pp. 576-580, ISSN 0268-8697

Karcher, H. (1992). Three-dimensional craniofacial surgery: transfer from a threedimensional model (Endoplan) to clinical surgery: a new technique (Graz). Journal of cranio-maxillo-facial surgery, Vol.20, No.3, (April 1992), pp. 125-131, ISSN 10105182

Kim, M.S.; Hansgen, A.R.; Wink, O.; Quaife, R.A. \& Carroll, J.D. (2008). Rapid prototyping: a new tool in understanding and treating structural heart disease. Circulation, Vol.117, No.18, (May 2008), pp. 2388-2394, ISSN 0009-7322

Lambrecht, J.T. \& Brix, F. (1989). Planning orthognathic surgery with three-dimensional models. The international journal of adult orthodontics and orthognathic surgery, Vol.4, No.3, (February 1989), pp. 141-144, ISSN 0742-1931

Laoui, T. \& Shaik, S.K. (2003). Rapid prototyping techniques used to produce medical models/implants, Proceedings of the $4^{\text {th }}$ national conference on rapid and virtual prototyping and applications, pp. 23-32, ISBN 1-86058-411-X, Centre for rapid design and manufacture, Buckinghamshire Chilterns University College, UK, June 20, 2003

Lemler, M.S.; Zellers, T.M.; Harris, K.A. \& Ramaciotti, C. (2000). Coarctation index: identification of recurrent coarctation in infants with hypoplastic left heart syndrome after the Norwood procedure. The American journal of cardiology, Vol.86, No.6, (September 2000), pp. 697-699, ISSN 0002-9149

Lim, J. \& Zein, R. (2006). The Digital Imaging and Communications in Medicine (DICOM): description, structure and applications, In: Rapid prototyping: theory and practice, A.K. Kamrani \& E.A. Nasr, (Ed.), 63-86, Springer, ISBN-10 0-387-23290-7, New York, NY, USA 
Liu, Q.; Leu, M.C. \& Schmitt, S.M. (2006). Rapid prototyping in dentistry: technology and application. The international journal of advanced manufacturing technology, Vol.29, No.3-4, (June 2006), pp. 317-335, ISSN 0268-3768

Lurz, P.; Coats, L.; Khambadkone, S.; Nordmeyer, J.; Boudjemline, Y.; Schievano, S.; Muthurangu, V.; Lee, T.Y.; Parenzan, G.; Derrick, G.; Cullen, S.; Walker, F.; Tsang, V.; Deanfield, J.; Taylor, A.M. \& Bonhoeffer, P. (2008). Percutaneous pulmonary valve implantation: impact of evolving technology and learning curve on clinical outcome. Circulation, Vol.117, No.15, (April 2008), pp. 1964-1972, ISSN 0009-7322

Mavroidis, C.; Ranky, R.G.; Sivak, M.L.; Patritti, B.L.; DiPisa, J.; Caddle, A.; Gilhooly, K.; Govoni, L.; Sivak, S.; Lancia, M.; Drillio, R. \& Bonato, P. (2011). Patient-specific ankle-foot orthoses using rapid prototyping. Journal of neuroengineering and rehabilitation, Vol.8, No.1, (January 2011), ISSN 1743-0003

Migliavacca, F.; Pennati, G.; Dubini, G.; Fumero, R.; Pietrabissa, R.; Urcelay, G.; Bove, E.L.; Hsia, T.Y. \& de Laval, M.R. (2001). Modeling of the Norwood circulation: effects of shunt size, vascular resistances and heart rate. American journal of physiology - Heart and circulatory physiology, Vol.280, No.5, (May 2007), pp. H2076-H2086, ISSN 03636135

Mishek, D. (2009). How and when to choose between additive and subtractive prototyping, In: Moldmaking technology, Available from

http:/ / www.vistatek.com/pdfs/Choosing-Between-Additive-and-Subtractive-

Prototyping-manufacturing.pdf

news.bbc.co.uk (June 2002). Body display 'may threaten donations', 11.06.2002, Available from: http://news.bbc.co.uk/1/hi/england/2038126.stm

Norwood, J. (1991). Hypoplastic left heart syndrome. The annals of thoracic surgery, Vol.52, No.3, (September 1991), pp. 688-695, ISSN 0003-4975

Pomeranz, B.; Adler, U.; Shenoy, N.; Macaluso, C. \& Parikh, S. (2006) Prosthetics and orthotics for the older adult with a physical disability. Clinics in geriatric medicine, Vol.2, No.2, (May 2006), pp.377-394, ISSN 0749-0690

Potamianos, P.; Amis, A.A.; Forester, A.J.; McGurk, M. \& Bircher, M. (1998). Rapid prototyping for orthopaedic surgery. Proceeding of the Institution of mechanical engineers, part $H$, journal of engineering in medicine, Vol.212, No.5, (1998), pp. 383-393, ISSN 0954-4119

Quarteroni, A. \& Veneziani, A. (2003). Analysis of a geometrical multiscale model based on the coupling of PDE's and ODE's for blood flow simulations. Multiscale modelling and simulations: a SIAM interdisciplinary journal, Vol.1, No.2, (2003), pp. 173-195, ISSN 1540-3459

Rengier, F.; Mehndiratta, A.; von Tengg-Kobligk, H.; Zechmann, C.M.; Unterhinninghofen, R.; Kauczor, H.U. \& Giesel, F.L. (2010). 3D printing based on imaging data: review of medical applications. International journal of computer assisted radiology and surgery, Vol.5, No.4, (July 2010), pp. 335-341, ISSN 1861-6410

Rengier, F.; von Tengg-Kobligk, H.; Zechmann, C.M.; Kauczor, H.U. \& Giesel, F.L. (2008). Beyond the eye - Medical applications of 3D rapid prototyping objects. European medical imaging review, Vol.1, (December 2008), pp. 76-80, ISSN 1759-7722 
Robiony, M.; Salvo, I.; Costa, F.; Zerman, N.; Bandera, C.; Filippi, S.; Felice, M. \& Politi, M. (2008). Accuracy of virtual reality and stereolithographic models in maxillo-facial surgical planning. The journal of craniofacial surgery, Vol.19, No.2, (March 2008), pp. 482-489, ISSN 1049-2275

Ryan, G.E.; Pandit, A.S. \& Apatsidis, D.P. (2008). Porous titanium scaffolds fabricated using a rapid prototyping and powder metallurgy technique. Biomaterials, Vol.29, No.27, (September 2008), pp. 3625-3635, ISSN 0142-9612

Sano, S.; Ishino, K.; Kawada, M.; Arai, S.; Kasahara, S.; Asai, T.; Masuda, Z.; Takeuchi, M. \& Ohtsuki, S. (2003). Right ventricle-pulmonary artery shunt in first stage palliation of hypoplastic left heart syndrome. The journal of thoracic and cardiovascular surgery, Vol.126, No.2, (August 2003), pp. 504-509, discussion pp. 509-510 ISSN 0022-5223

Schievano, S.; Coats, L.; Migliavacca, F.; Norman, W.; Frigiola, A.; Deanfield, J.; Bonhoeffer, P. \& Taylor, A.M. (2007). Variations in right ventricular outflow tract morphology following repair of congenital heart disease: implications for percutaneous pulmonary valve implantation. Journal of cardiovascular magnetic resonance, Vol.9, No.4, (February 2007), pp. 687-695, ISSN 1097-6647

Schievano, S.; Migliavacca, F.; Coats, L.; Khambadkone, S.; Carminati, M.; Wilson, N.; Deanfield, J.E.; Bonhoeffer, P. \& Taylor, A.M. (2007). Percutaneous pulmonary valve implantation based on rapid prototyping of right ventricular outflow tract and pulmonary trunk from MR data. Radiology, Vol.242, No.2, (February 2007), pp. 490-497, ISSN 0033-8419

Schievano, S.; Sebire, N.J.; Robertson, N.J.; Taylor, A.M. \& Thayyil, S. (2010). Reconstruction of fetal and infant anatomy using rapid prototyping of post-mortem MR images. Insights into imaging, Vol.1, No.4, (September 2010), pp. 281-286, ISSN 1869-4101

Schievano, S.; Taylor, A.M.; Capelli, C.; Coats, L.; Walker, F.; Lurz, P.; Nordmeyer, J.; Wright, S.; Khambadkone, S.; Tsang, V.; Carminati, M. \& Bonhoeffer, P. (2010). First-in-man implantation of a novel percutaneous valve: a new approach to medical device development. Eurointervention, Vol.5, No.6, (January 2010), pp. 745750, ISSN 1774-024X

Schrank, E.S. \& Stanhope, S.J. (2011). Dimensional accuracy of ankle-foot orthoses conducted by rapid customisation and manufacturing framework. Journal of rehabilitation research and development, Vol.48, No.1, (2011), pp. 31-42, ISSN 0748-7711

Thayyil, S.; Cleary, J.O.; Sebire, N.J.; Scott, R.J.; Chong, K.; Gunny, R.; Owens, C.M.; Olsen, O.E.; Offiah, A.C.; Parks, H.G.; Chitty, L.S.; Price, A.N.; Yousry, T.A.; Robertson, N.J.; Lythgoe, M.F. \& Taylor, A.M. (2009). Post-mortem examination of human fetuses: a comparison of whole-body high-field MRI at $9.4 \mathrm{~T}$ with conventional MRI and invasive autopsy. The Lancet, Vol.374, No.9688, (August 2009), pp. 467-475, ISSN 0140-6736

Werner, H.; Fontes, R.C.; Campbell, S. \& Santos, J.R.L. (2010). Rapid prototyping models of foetuses built from ultrasound $3 \mathrm{D}$ and magnetic resonance files, Innovative developments in design and manufacturing: advanced research in virtual and rapid 
prototyping - Proceedings of VR@P4, pp. 89-94, ISBN 978-0-415-87307-9, Leiria, Portugal, October 2009

Winder, J. \& Bibb, R. (2005). Medical rapid prototyping technologies: state of the art and current limitations for application in oral and maxillofacial surgery. Journal of oral and maxillofacial surgery, Vol.63, No.7, (July 2009), pp. 1006-1015, ISSN 0278-2391 


\title{
Circulation Type Blood Vessel Simulator Made by Microfabrication
}

\author{
Takuma Nakano and Fumihito Arai \\ Nagoya University \\ Japan
}

\section{Introduction}

Recently, Japanese have causes of the death. Figure 1.1 was reported by Ministry of Health, Labour and Welfare in 2008 in Japan and which shows the cause of the death. As you can see, the causes of death are cancer, cardiac disease, cerebrovascular disease and etc.. We can categorize cardiac disease and cerebrovascular disease as blood vessel disease. Blood vessel disease is the second highest cause of the death. To treat the cancer and blood vessel disease, many engineering approaches have been studied about tissue engineering, medical treatment tools, rehearsal systems and synthetic vascular prostheses. For treatment of cancer, there are researches of endoscope and abdominoscope for less-invasive surgical operation. For treatment of blood vessel disease, there are researches of endoscope which can use in blood vessels and synthetic vascular prostheses for replacement operation. These researches have the common identity that is improvement of quality of life (QOL). But, these lessinvasive operations are poor in extracting the organ size and controlling the posture of medical tools. To solve these problems, many researches from the other approaches are proposed. One of them is research about surgical simulators for rehearsal, practice and evaluation of real surgical operation. Generally, VR simulator and CFD analysis are famous as computer surgical simulator (Fig.1.2 (a) and (b)). These simulators enable to simulate surgical operation easily, and analyze fluid condition easily, however, these are poor in simulating pulsative system and not suitable to evaluate new treatment method using narrow vessels. Our surgical simulator (Endo Vascular Evaluator: EVE) has threedimensional (3D) blood vessel models fabricated in tailor-made (Fig.1.2 (c)) (Ikeda et al., 2005). And, EVE enables to simulate pulsative system and catheter operation easily, but, not suitable to evaluate new treatment method using narrow vessels. There are two famous methods using narrow vessels. One is a method of necrotizing cancer cells in the part of liver as shown in Fig.1.3 (a) (trancecatheter arterial chemoembolization: TACE, TAE). The other is a method of administering medicine as shown in Fig.1.3 (b) (drug delivery systems:DDS). TAE method protocol is to release anticancer drug and gelatin sponge in blood vessels for stack, then stop the blood flow and the supply nourishment. Stacking gelatin sponge has a possibility of effect to normal cells. Thus, gelatin sponge are needed to release from catheter, and it is very difficult to selectivity stack the only arteriole and capillary vessels which rink to cancer cells. However, blood fluidic condition is very complicated, and catheter's position, amount of anticancer drug and gelatin sponge, fluidic and pressure condition, environmental condition changed by catheter and effects to other 


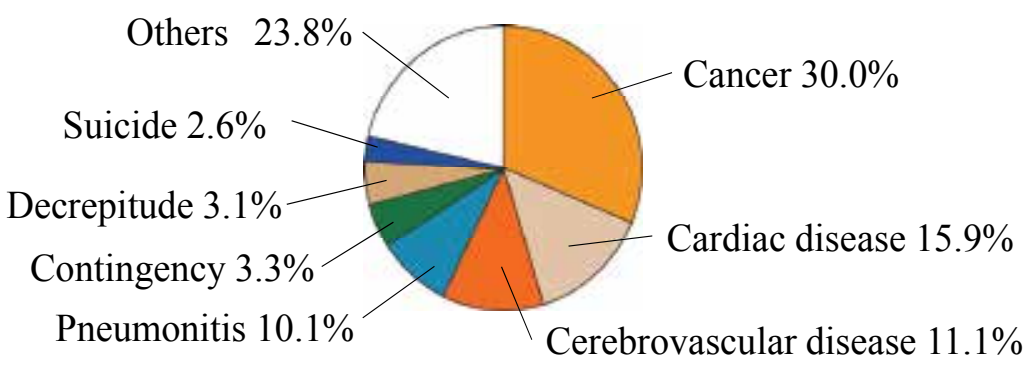

Fig. 1.1 The causes of the death in Japan in 2008.
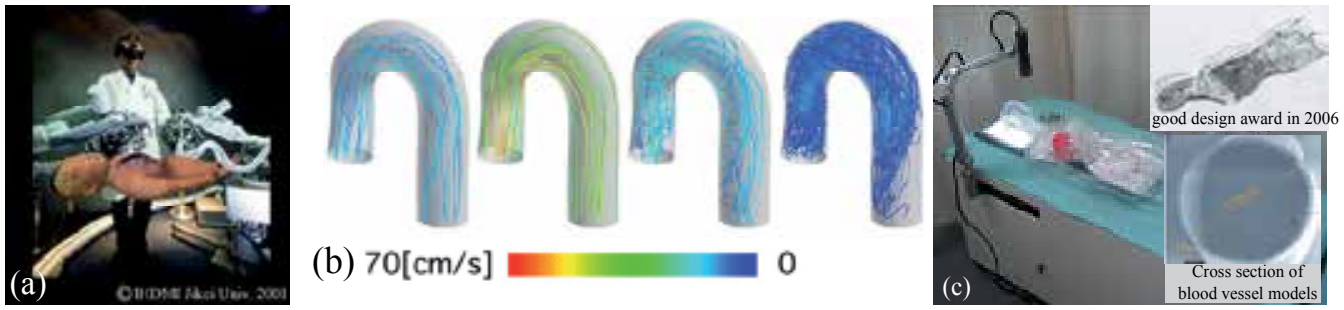

Fig. 1.2 Conventional surgical simulators. (a) VR surgical simulator (The Jikei Univ.). (b) Streamlines of blood flow by CFD analysis (Yamaguchi Lab. In Tohoku Univ.). (c) Our surgical simulator (Endo Vascular Evaluator: EVE).

tissue are unknown. DDS method protocol is similar to TAE, it is needed to discuss about optimizing applied dose, amount of diffusion of medicine and circulatory effect. On these accounts, the inspection based on detail plan and preparations experiment is necessary before performing real operations. And, the system for resolving these necessaries is required. For realizing a system of evaluating new treatment method using narrow vessels, we proposed circulation type blood vessel simulator (Fig.1.4). And, circulation type blood vessel simulator is needed to have artery models, narrow blood vessel models (arteriole and capillary vessel models) and vein models. In treatment of blood vessel diseases, medical doctors perform blood vessel shape maintenance with stent after a disease department was treated with a catheter for vascular disease treatment by manual skill. For practicing this manual skill, circulation type simulators having arteriole and capillary vessel models are required. Various studies are reported as surgical simulators and processing techniques of microchannels to arteriole and capillary vessel models. However, as for the conventional studies, narrow vessel models are not built into the systems, and a cross section of microchannels is near to a quadrangle. Therefore, they cannot realize human circulatory organ systems and blood vessels environments. In this chapter, realizing circulation type blood vessel simulators and producing arteriole and capillary vessel models having a circular cross section are aimed at.

Surgical simulators are used in practice and rehearsal for intravascular neurosurgery, and for development of new medical instruments such as catheters. Human carotid artery was made by combining processes of ink jet rapid prototyping, lost wax, dip coating, and selective dissolution. 3D configuration of carotid artery is developed by our 3D reconstructive method as described previously. These blood vessel models are made only for the artery, but reproduce the softness of a real blood vessel by controlling a film 

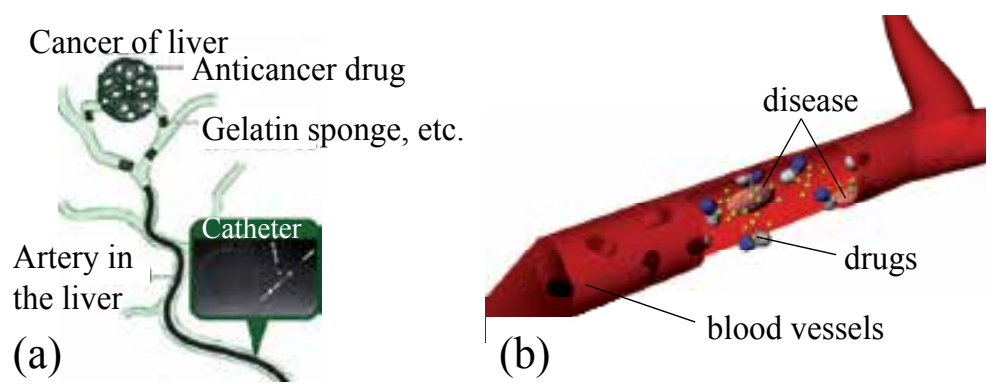

(b)

Fig. 1.3 Image of new treatment methods using narrow vessels. (a) Trancecatheter arterial chemoembolization (TACE, TAE). (The Japanese Society of Interventional Radiology, http://www.jsair.org/). (b) Drug delivery system (DDS).

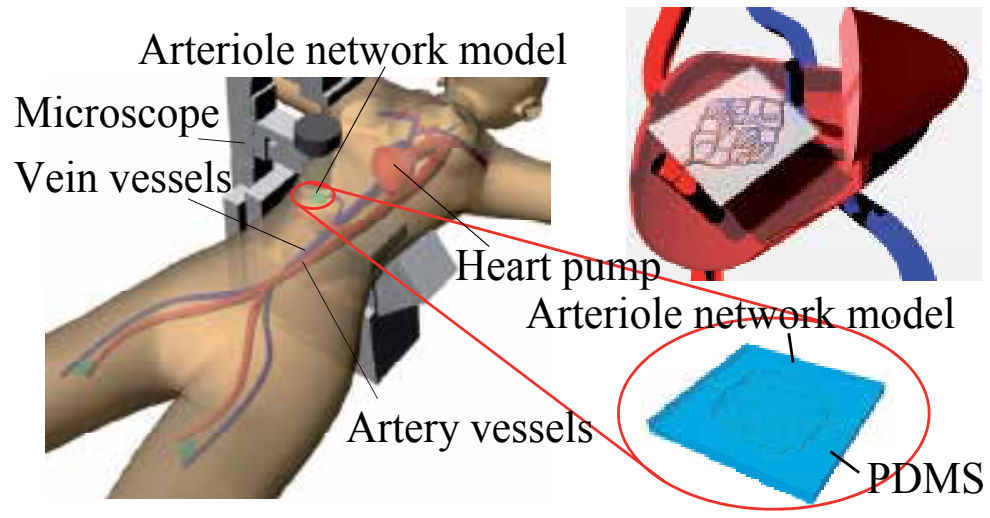

Fig. 1.4 Proposed circulation type blood vessel simulator.

thickness by dip lotion coating. Then, we developed a transparent surgical simulator by connecting the elastic tube models. Wax models are made by layer stacked modeling machine, however, the narrowest model's diameter is $500 \mathrm{um}$ due to its low resolution and the brittleness of the wax. Therefore, a simulator cannot make a blood vessel model of narrower than 500 um inside diameter. Diseases exist in blood vessels such as arteria basilaris that are narrower than $500 \mathrm{um}$. Narrower blood vessel models are needed to simulate a more realistic vessel environment as shown in Fig.1.4. Previous surgical simulators are not suitable for rehearsal and training for such diseases.

The latest treatments for cancer and blood vessel disease from blood vessels are researched, but the evaluations and rehearsals are demonstrated with animal bodies. These evaluations and rehearsals should be demonstrated with the blood vessel simulator which is mimicked real blood vessel structures of human body. Therefore, it is very important to make a blood vessel simulator mimicking the human body. Flexibility of our previous blood vessel models have no problem, but cannot know influence in an end blood vessel of treatment because a blood vessel model is larger than 500 um inside diameter. Therefore, the arteriole and capillary vessel models were needed as a simulator to know influence in an end blood vessel. This study is targeted to fabricate multiscale transparent 10-500 um diameter arteriole and capillary vessel models that enable easy simulation of blood circulations. In 
addition, fabricated microvessel models are needed to over the arteria pulmonalis's circularity which is $52.4 \%$, and the circularity of microchannels is calculated by the shortest axis divided by the longest axis (Attinger, 1964). For this purpose, fabricated 10-500 um microvessel models must be circular cross sections, and circularities must be higher than $52.4 \%$. In fact, a real blood vessel system has blood vessels of artery system and vein system. The targeted inside diameter of the blood vessel is 10-500 um (Hayashi, 1997). When microvessel models simulating real blood vessel are made, fabrication techniques should have high resolution processing about around 1 um (Noda et al., 2007; Itoga et al., 2004). Therefore, maicrofabrication processing technique is suitable for this requirement (Borenstein et al., 2002).

Many methods such as machining, stereolithography, ink jet rapid prototyping, and photolithography have been proposed for fabricating microchannels. Machining is suitable for a straight channel up to around $10 \mathrm{um}$ in diameter, but not for complicated capillary vessel networks. Stereolithography is applicable for fabricating a mold; however, it is difficult to dissolve it to create a hollow structure. Ink jet rapid prototyping is beneficial for thicker tube channels such as aortas, but not applicable for capillary vessel models. Photolithography is a fundamental technology for fabricating microchannels, and a high resolution around $1 \mathrm{um}$ is easily attained. We have chosen photolithography for fabricating microvessel models. In general, fabricating microchannels with a circular cross section is quite complex (Hanai et al., 2005; Eisner \& Schwider, 1996; Nicolas et al., 1998). Microchannels were fabricated by using diffused-light, reactive ion etch (RIE) lag, and light curable resin, but the cross sections of the fabricated microchannels were semicircular, large diameter and not fine surface (Futai et al., 2004). These processes are not suitable for fabricating fine and narrow diameter blood vessel models. Therefore, we proposed a new fabrication process for multiscale transparent arteriole and capillary vessel models. And, these models have circular cross section made by over exposure method, reflow method and grayscale lithography as photolithography process. The fabricated microvessel models were connected with conventional blood vessel models to realize a circulation simulator. For example, circulation models will allow simulation of animal testing and drug delivery systems by using microvessels. In this chapter, we report multiscale fabrication method of arteriole and capillary vessel models and prototyping results for the circulation models.

\section{Block type multi scale arteriole and capillary vessel models}

\subsection{Fabrication method}

Figure 2.1 shows multiscale fabrication methods of blood vessel models. We used layer stack molding machine and photolithography process such as over exposure method, reflow method, and grayscale lithography. Because of a limit of fabrication accuracy, it is necessary to choose an appropriate method to fabricate a model with targeted diameter. A 10-20 um diameter capillary vessel models are made by over exposure method, a 20-100 um diameter arteriole models are made by reflow method, and a 100-500 um diameter arteriole models are made by grayscale lithography. The fabricated patterns are transcribed onto poly(dimethylsiloxane) (PDMS) substrate (Wu et al., 2002; Whitesides et al., 2001). After these processes, each patterned surface of two patterned PDMS substrates are treated by plasma and heated (Fig.2.2). Then, arteriole and capillary vessel models with circular cross section are completed (Arai et al., 2007). 


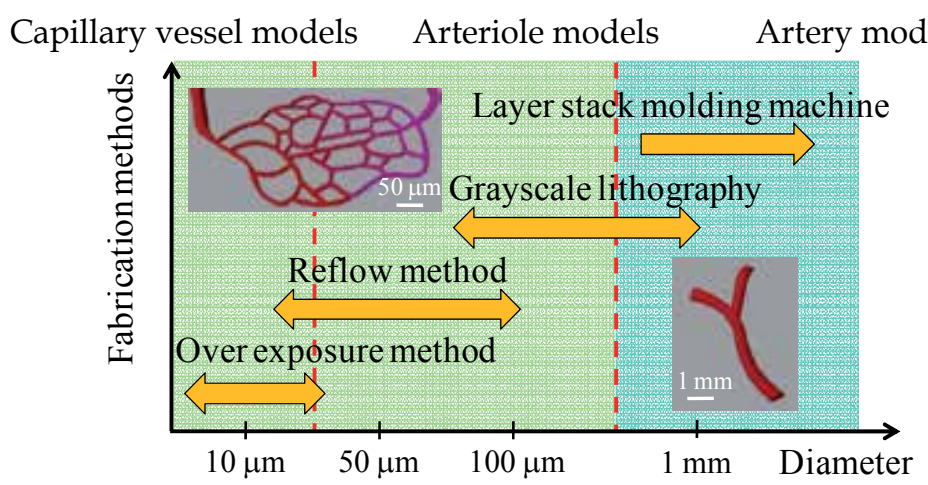

Fig. 2.1. Concept of fabrication methods for multi scale blood vessel models.

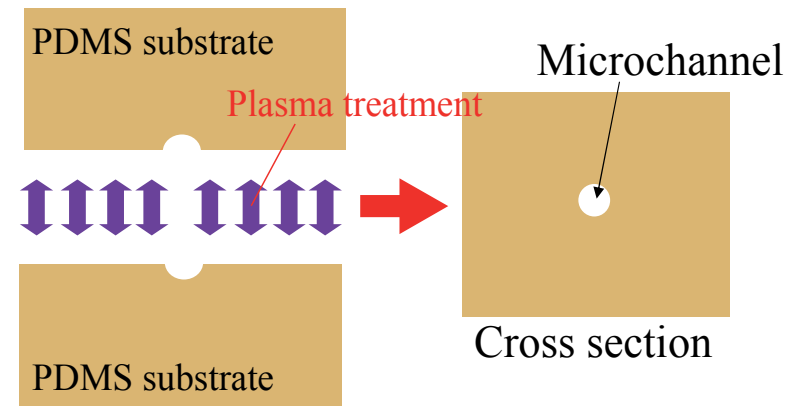

Fig. 2.2 Concept of making arteriole and capillary vessel model.

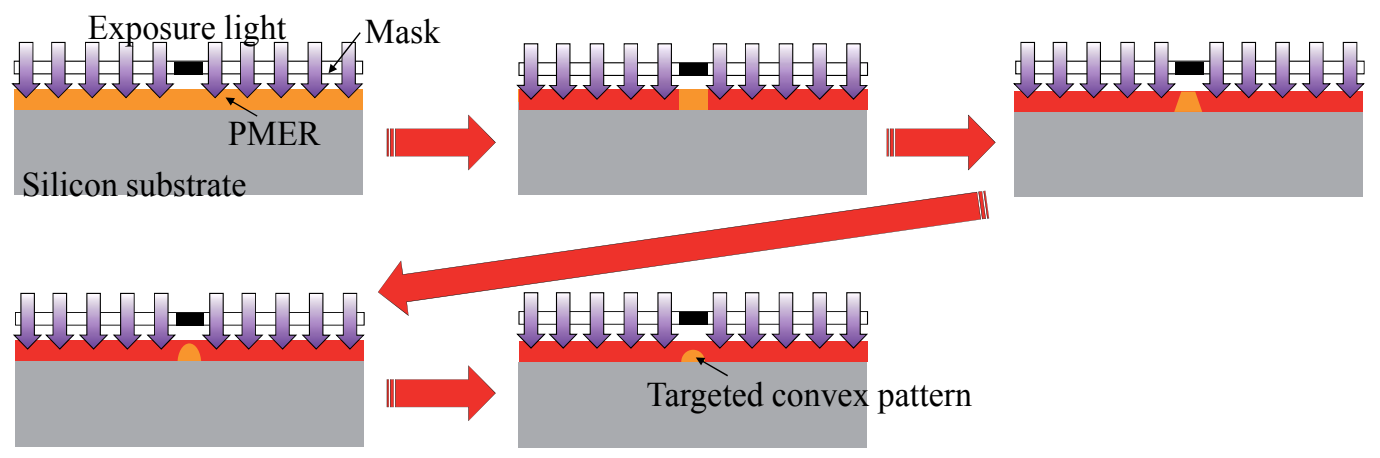

Fig. 2.3 The mechanism of over exposure method using diffracted light.

\subsubsection{Fabrication of $\mathbf{1 0 - 2 0}$ um capillary vessel models}

Fabrication method of capillary vessel models is over exposure method. In this exposure method, it is most important to expose at soft-contact using a mask and a resist surface. The soft-contact distance between the mask and a resist surface is 10-20 um. Exposure light is diffracted by the mask patterns. Exposed resist pattern can have the semicircular cross section by diffracted light. Figure 2.3 shows the mechanism of over exposure method for fabricating semicircular cross section patterns. In this process, a photoresist is PMER PLA900PM (recommended exposure amount: 800-1200 Doses) as posi-type photoresist. 

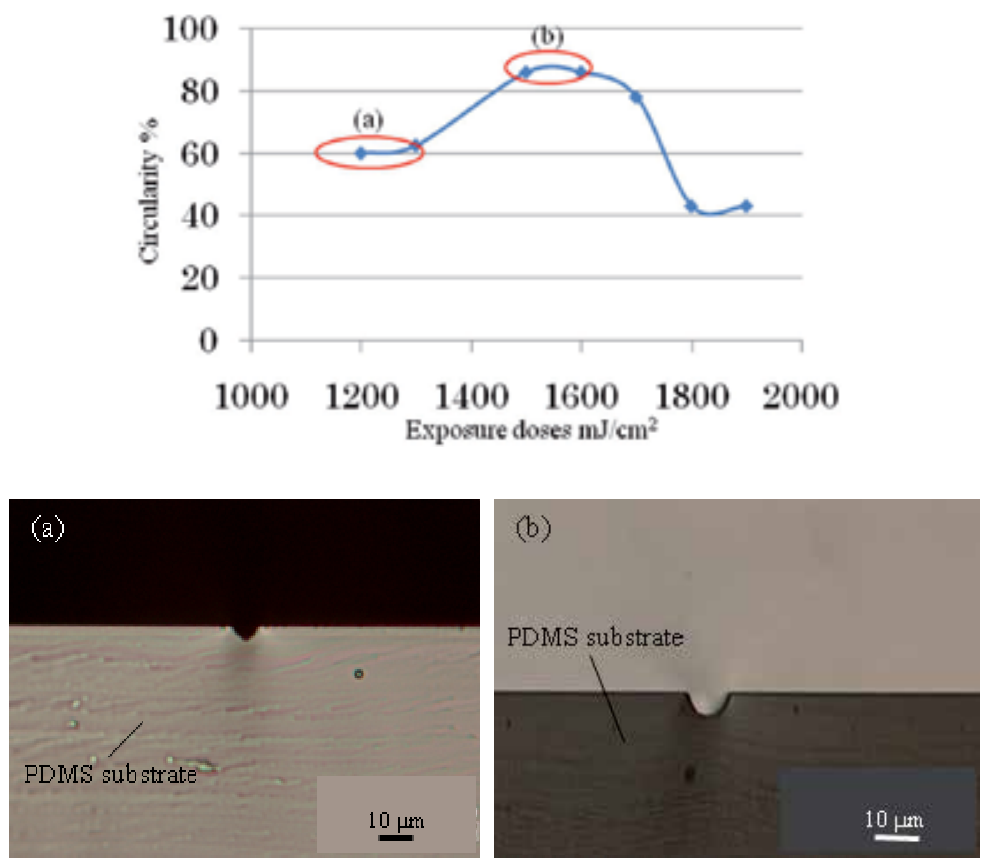

Fig. 2.4 Circularity result at each exposed dose. (a) shows the shape of microchannel in 12001400 doses. (b) shows the shape of microchannel in 1500-1700 doses.
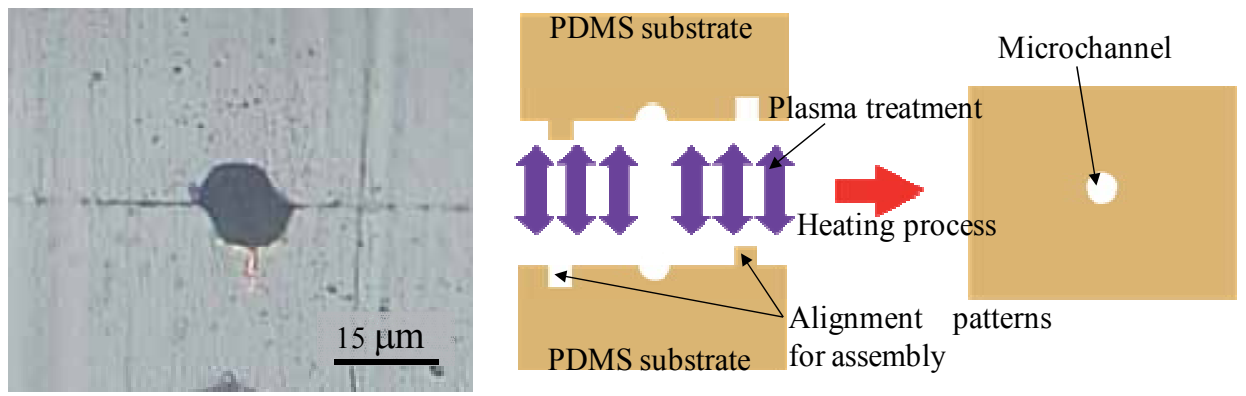

Fig. 2.5 Cross section of fabricated model, and new fabrication concept.

PMER is very sensitive resist for exposure light. Within the definite range of recommended exposure amount, the cross section of resist patterns is square. The cross section shape of resist patterns is varying trapezoid, triangular, semiround and oval by increasing the exposure light amount. Therefore, exposure light amount (exposure doses) should be controlled. And, we experimented and calculated relation between circularity and exposure doses. Figure 2.4 shows circularity result at each exposed dose. In 1200-1400 doses, the cross section of resist pattern shape is the trapezoid or triangular, and the circularity is low with about $60 \%$. In 1500-1700 doses, the cross section of resist pattern shape is the semicircular, and the circularity is high with about $80 \%$. In over 1700 doses, the resist pattern shape is the oval, and the circularity falls fast until about $40 \%$. (a) shows the peak at triangular shape, lowers afterwards the height part, and receives (b) that is the peak value at round shape in 
figure though are in the circularity two peak values of (a) and (b). The reason why the graph has increased and decreased is that the number of sampling to each light exposure was one time. Figure 2.5 shows cross section of fabricated capillary vessel models. When two PDMSsubstrates assemble, alignment is very difficult and patterns are out of alignment. To solve this problem, new patterns are needed for PDMS substrates assembly (Fig.2.5). And, new capillary vessel pattern and assembly pattern are fabricated by multi stage exposure photolithography. The fabrication process is following:

A. Fabrication of patterns for alignment

1. Coat a substrate with 8-um-thick photoresist PMER.

2. Expose at hard-contact using both a mask and a resist surface until 1000 Doses.

3. Develop for 7 minutes.

4. Hardbake at $145^{\circ} \mathrm{C}$ for $30 \mathrm{~min}$.

B. Fabrication of other patterns for alignment

1. After using plasma hydrophilic treatment, coat a substrate with 8-um-thick PMER.

2. Expose at hard-contact using both a mask and a resist surface until 1000 Doses.

3. Develop for 7 minutes.

4. Hardbake at $145^{\circ} \mathrm{C}$ for $30 \mathrm{~min}$.

C. Fabrication of pattern for microchannels

1. After using plasma hydrophilic treatment, coat a substrate with 8-um-thick PMER.

2. Expose at soft-contact using both a mask and a resist surface until 1500 Doses.

3. Develop for 5 minutes.

4. Transcribe resist patterns onto PDMS.

And, the capillary vessel model was completed by bonding two patterned PDMS substrates.

\subsubsection{Fabrication of $\mathbf{2 0 - 1 0 0 ~ u m ~ a r t e r i o l e ~ m o d e l s ~}$}

Fabrication method of 20-100 um arteriole models is reflow method. In this fabrication method, it is most important to control the resist pattern's height. Then, the evaluation of the resist patterns' height in a longer direction is needed. The resist patterns' height fabricated by reflow method was measured 10 times at random parts at $1 \mathrm{~mm}$ certain interval by using prove. Table 2.1 shows the evaluation results. The differences of resist patterns' height were less than $1.5 \mathrm{um}$. And, real blood vessels have a rough surface which is few micrometers, and reflow method is suitable for fabricating 20-100 um arteriole models. In reflow method, resist thickness must be changed by each targeted diameter. The 20-100 um arteriole models were fabricated by using this calculated resist thickness (Not shown). Next, the fabrication process based on reflow method was explained. The fabrication process is following:

1. Coating photoresist PMER on silicon substrate.

2. Exposure until 1000 Doses.

3. Development for 7 minutes.

4. Heating the resist patterns from bottom side by hot plate at $145^{\circ} \mathrm{C}$ for $30-60$ seconds.

5. Transcribing these resist patterns onto PDMS.

After the development, the resist pattern was heated from back side of silicon substrate. The patterned resists become liquid again in this process, and the semicircular resist pattern was fabricated by liquid surface tension. Then, the arteriole models were completed by bonding two patterned PDMS substrates. 


\begin{tabular}{|c|c|}
\hline Diameter [um] & Difference of patterns' height [um] \\
\hline 10 & 0.58 \\
\hline 20 & 0.18 \\
\hline 30 & 0.47 \\
\hline 40 & 1.0 \\
\hline 50 & 1.5 \\
\hline
\end{tabular}

Table 2.1 Profiling results of the resist patterns' height

\subsubsection{Fabrication of $100-500$ um arteriole models}

Fabrication method of $100-500$ um arteriole models is grayscale lithography. In this fabrication method, it is most important to control histogram of grayscale mask (O'Shea \& Rockward, 1995). In general, grayscale mask is very high cost. In this time, the grayscale emulsion mask is made by $1 / 20$ reduction machine with imagesetter film (3600 dpi) due tolow cost and simple process fabrications (Fig.2.6). Because fabricated grayscale mask has 256 shades of gray, the resists' height made by UV lamp power through the each gray tone has difference. Therefore, calibration of relation between curable depth (capable of fabricate resist patterns' height) and UV lamp power (gray level) is required. Figure 2.7 shows the evaluation result. Using this result, the grayscale emulsion mask was designed and fabricated. Next, the fabrication process based on grayscale lithography was explained. The fabrication process is following:

1. Coating naga-photoresist SU-8 on glass substrate.

2. Illuminating from the back side of substrate through grayscale emulsion mask.

3. Development to be 3D shape.

4. Transcribing these resist patterns onto PDMS.

And, the arteriole models were completed by bonding two patterned PDMS substrates.

\subsection{Results and evaluations}

We evaluated the alignment accuracy of the capillary vessel models. A model having no alignment pattern had an alignment error of $2.5 \mathrm{um}$, whereas, a model with alignment patterns had an alignment error of $0.6 \mathrm{um}$. The circularity of the capillary vessel model having no alignment pattern was $70 \%$ and for that with alignment patterns was $84 \%$. Thus, alignment patterns reduced the alignment error from 2.5 to $0.6 \mathrm{um}$ and improved the circularity from 70 to $84 \%$. Figure 2.8 shows the cross sections of fabricated arteriole and capillary vessel models. In addition, the circularity of $10 \mathrm{um}$ microchannel is $84.0 \%$, that of 50 um microchannel is $61.5 \%$, and that of 500 um microchannel is $82.3 \%$.

\section{Tube type arteriole models}

\subsection{Evaluation of wax + polyvinyl alcohol (PVA) mixture material}

We completed block type microvessel models by using photolithography in section 2 . However, block models cannot recreate moderate compliance which is similar to that of the real blood vessel. To solve this problem, arteriole and capillary vessel tube models are needed for surgical simulator. And, sacrificial models are needed to make tube models. But because of brittleness of wax, under 500 um diameter sacrificial models cannot be made by using layer stack molding machine. In this section, a novel fabrication method for transparent arteriole tube models is proposed. Improving the brittleness of wax by mixing 
wax with PVA was tried. After making sacrificial models made of mixture material, arteriole tube models were completed by dip coating.

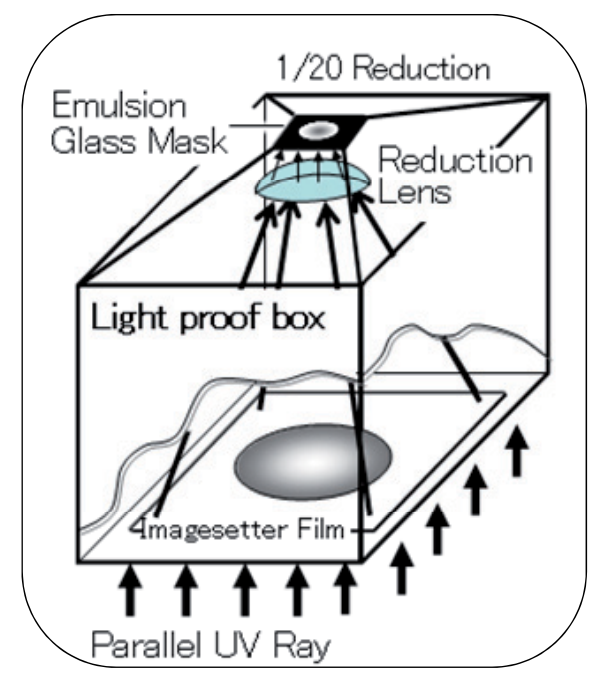

(a) Concept of 1/20 Reduction System.

\section{Grayscale emulsion mask}

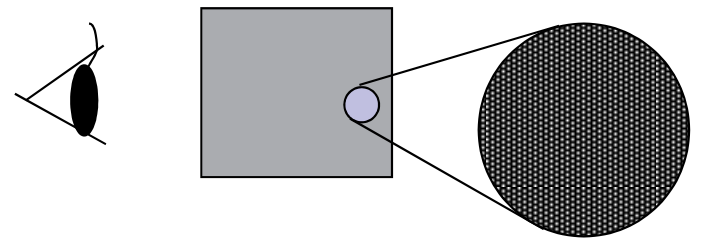

(b) Image of fabricated grayscale emulsion mask.

Fig. 2.6 Fabrication concept of grayscale emulsion mask.

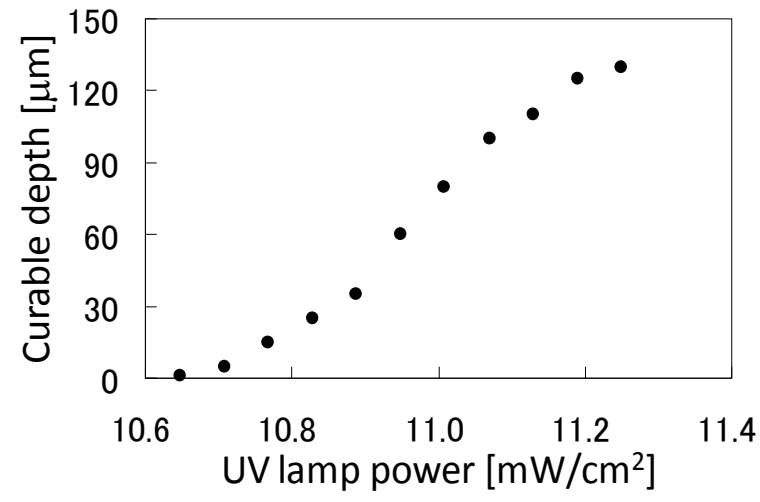

Fig. 2.7 Calibration of relation between curable depth (capable of fabricate resist patterns' height) and UV lamp power (gray level). 

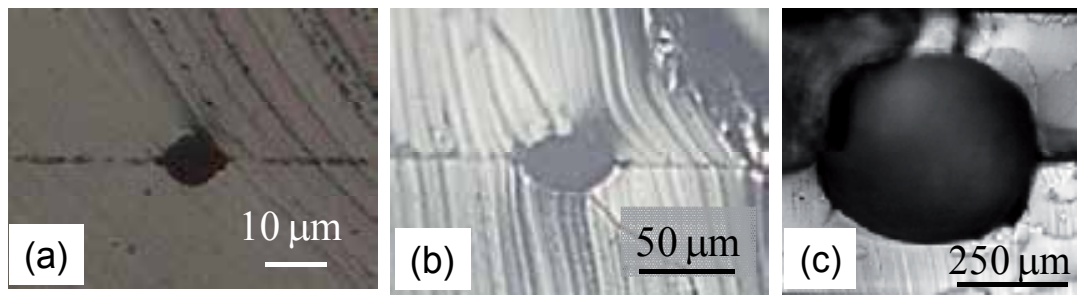

Fig. 2.8 Cross sections of fabricated models. (a) 10 um (b) 50 um (c) $500 \mathrm{um}$

In this fabrication process, wax and PVA mixture material is used to build sacrificial models. These models are very useful for fabricating tube models narrower than $500 \mathrm{um}$. If only wax is used for making sacrificial models, the temperature is needed to keep. And wax is not suitable to this fabrication process because of wax's fragility. If only PVA is used for making sacrificial models, PVA model is deformed easily by model's own weight when dip coating, since it has low bend strength. Therefore, the new method of fabricating sacrificial models was proposed with wax and PVA mixture materials. The mixture has the properties of both wax and PVA can be easily forecasted. The properties of mixture were evaluated by changing the mix ratio. Evaluated properties are Young's modulus as mechanical property and melting materials times in different liquid (solubility) as chemical property. Evaluated samples made by different mix ratio (mix ratio wax: PVA=1:0, 1:4, 2:3, 3:2, 4:1, 0:1).

Tensile tester is used for evaluation experiments of Young's modulus. Samples made by wax and PVA mixture materials are made $50 \mathrm{~mm} \times 10 \mathrm{~mm} \times 2 \mathrm{~mm}$ (long $\times$ wide $\times$ thick) in size. The tensile experiment was demonstrated three times for each wax and PVA mix ratio. Using these results, the Young's modulus was calculated. Young's modulus of each mix ratio is calculated by averaging the experimental results. Table 3.1 shows Young's modulus by each mix ratio. Young's modulus of PVA is $9.57 \mathrm{MPa}$. On the other hand, Young's modulus of wax is $20.6 \mathrm{MPa}$. As you can see, Young's modulus of mixture material is getting higher by increasing the ratio of wax. And also, Table 3.1 shows extension. Extension is expression of material's brittleness and ductibility. Extension of mixture material is getting higher by increasing the ration of PVA. Therefore, the mechanical property of wax and PVA mixture material can be controlled by changing mix ratio.

Next, melting time of each different ratio mixture materials was measured in different liquid by checking mass changes. The solubility of each mix ratio was defined from experiment. Using ultrasound bath was set at $50^{\circ} \mathrm{C}$ for solubility experiments. Deionized water (DI water), acetone, and DI water + acetone liquid mixture were provided for this test. DI water easily melts PVA. Acetone easily melts wax. Liquid mixture of 1:1 mix ratio was provided, since melting samples was wax and PVA mixture material. The liquid was used in the melting experiments. Figure 3.1 shows the experimental results about observing solubility of each sample in DI water, acetone, DI water and acetone liquid mixture. Because of DI water's feature, mixture material samples were melted easily. In this result, mass changes of the wax sample were observed. This is caused by heating in the process of ultrasound bath and etching effects. Acetone melted few mixture material was observed in this experiment, although acetone can melt wax easily. It happened since wax was coated with PVA, and acetone could not reach wax. On the other hand, mixture material was melted by liquid mixture. That was verified by experiment. Comparing these results, all mixture materials cloud be melted in DI water and acetone liquid mixture, however, dissolving rate was decreasing. PVA absorbed DI water and increased mass was observed when experiments 
started. These results suggested DI water and acetone liquid mixture is suitable for melting the wax and PVA mixture materials.

\begin{tabular}{|c|c|c|}
\hline PVA: wax ratio[\%] & Young's modulus [MPa] & Extension [\%] \\
\hline $1: 0$ & 9.57 & 75 \\
\hline $4: 1$ & 9.69 & 75 \\
\hline $3: 2$ & 8.78 & 75 \\
\hline $2: 3$ & 15.7 & 44 \\
\hline $1: 4$ & 22.8 & 4.0 \\
\hline $0: 1$ & 20.6 & 6.0 \\
\hline
\end{tabular}

Table 3.1. Experimental rigidity and extension
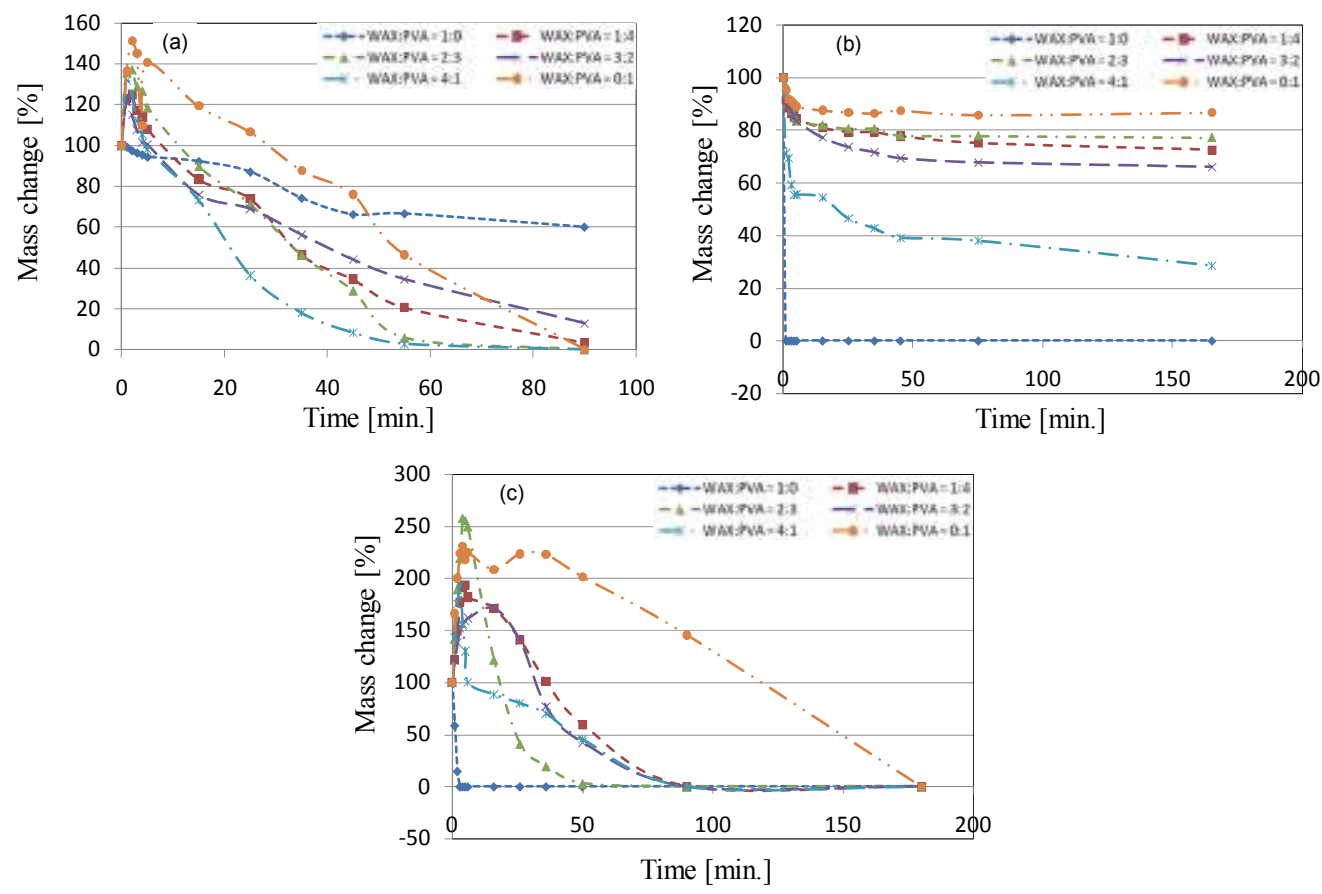

Fig. 3.1 Evaluation of chemical features. (a) DI water (b) Acetone (c) Acetone + DI water

\subsection{Fabrication of arteriole tube models}

For fabricating arteriole tube models, sacrificial models as molds for dip coating of PVA and silicone resin are needed. The sacrificial models with this wax and PVA mixture were fabricated. Patterned PDMS substrates for molding sacrificial models were made by explained method in section 2. Fabrication process flow for sacrificial models is following:

1. Coating wax and PVA mixture materials on patterned side of PDMS substrate.

2. Splitting and removing superfluous mixture.

3. Aligning two patterned PDMS substrates filled with mixture on patterned side.

4. After drying at room temperature, removing the PDMS substrates.

5. Removing mixture stacking on the sacrificial models. 
Figure 3.2 shows the sacrificial model before and after removing stacking mixture on model surface. The stacking mixture was cut and etched with DI water. And, Fig.3.2(b) shows the cleared and smoothed surface on sacrificial model. Then, we fabricated arteriole tube models by using the sacrificial models. This sacrificial model was coated with PVA for smoothing the surface and later coated with transparent silicone resin for tube structure by dip coating based on previous method. After dip coating, sacrificial model and PVA for smoothing were dissolved by DI water and acetone, and transparent arteriole tube models were completed. Fabrication process flow is following:
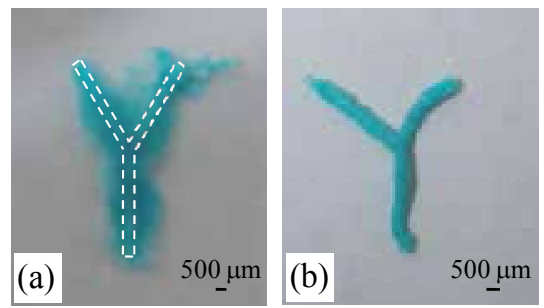

Fig. 3.2 Fabricated sacrificial models. (a) The before removing. (b) The after removing.
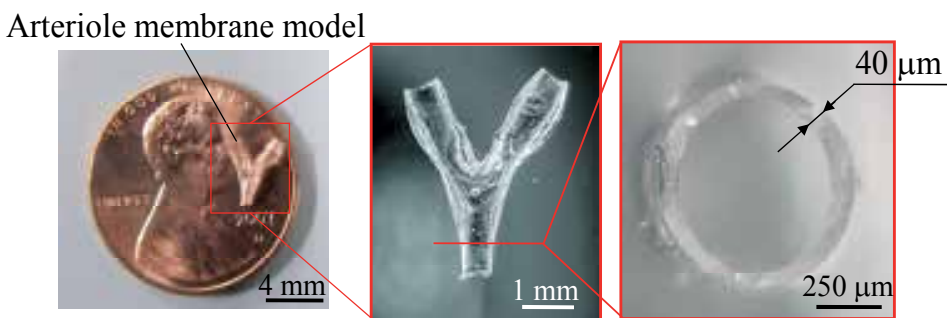

Fig. 3.3 Fabricated arteriole tube model. (a) Fabricated model. (b) Overall. (c) Cross section.

1. Dip coating the sacrificial models with $10 \mathrm{wt} \%$ PVA solution for smoothing the surface.

2. After drying, dip coating the PVA coated sacrificial models with silicone resin.

3. Dissolving sacrificial models and PVA layer by DI water and acetone liquid mixture.

4. Drying silicone tube structures and completing blood vessel tube models.

Figure 3.3 shows fabricated arteriole tube model. The tube and hollow structures were observed. And, fabricated arteriole tube model had circular cross section shown in Fig.3.3(b). The calculated circularity of this model was $90 \%$. As you can see, the thickness of fabricated model was not uniform. The thickest part is $350 \mathrm{um}$. Changing the ratio of base resin and curing agent consisted of silicone resin can decrease viscosity, then, coat uniform.

\section{Network type arteriole models}

\subsection{Design of network structure}

Our fabricated arteriole and capillary vessel models are transparent and $Y$ shape branched microchannels, but diameters are uniform around branched structure. These models cannot recreate the real blood vessel environments. To solve this problem, microvessel models based on real blood vessel branched rule is proposed and fabricated. This rule is based on the cube law between mother blood vessel's diameter and daughter blood vessels' diameters. Real blood vessel is branched structure based on the cube law shown in Fig.4.1. Therefore, the network branched microchannels were designed by using this rule. The 
microchannels are changed diameters when these are branched. The rule of changing diameter is shown in equation (1).

$$
\mathrm{R}_{0}{ }^{3}=\mathrm{R}_{1}^{3}+\mathrm{R}_{2}{ }^{3}
$$

In Eq. $1, R_{0}$ is mother blood vessel's diameter. $R_{1}$ and $R_{2}$ are daughter blood vessels' diameters (Sherman, 1981).

$$
\begin{aligned}
& \text { Mother branch's diameter: } \mathrm{R}_{0} \\
& \text { Daughter branch's diameter: } \mathrm{R}_{1}, \mathrm{R}_{2} \\
& \qquad \mathrm{R}_{0}{ }^{\mathrm{m}}=\mathrm{R}_{1}{ }^{\mathrm{m}}+\mathrm{R}_{2}{ }^{\mathrm{m}} \quad \cdots \text { (A) } \\
& \mathrm{m}=2.6 \sim 3.2 \text { in arterial system } \\
& \text { In general, we use } \mathrm{m}=3
\end{aligned}
$$

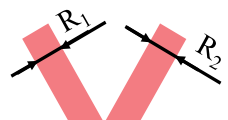

Fig. 4.1 The branched structure rule of real vascular.

\subsection{Fabrication and evaluation}

\subsubsection{0-500 um network type arteriole models}

Table 4.1 shows the parameters of blood vessel models' diameters using this rule. Using these parameters, grayscale mask was designed (Fig.4.2(a)). And, the 100-500 um arteriole network models were fabricated by grayscale lithography and PDMS bonding. The fabrication process is following.

\begin{tabular}{|c|c|c|}
\hline \multicolumn{2}{|c|}{ Daughter diameters } & Mother diameters \\
\hline $\mathrm{R}_{1}[\mathrm{um}]$ & $\mathrm{R}_{2}[\mathrm{um}]$ & $\mathrm{R}_{0}[\mathrm{um}]$ \\
\hline 100 & 100 & 126 \\
\hline 126 & 126 & 159 \\
\hline 159 & 159 & 200 \\
\hline 200 & 200 & 252 \\
\hline 252 & 252 & 317 \\
\hline 317 & 317 & 400 \\
\hline 400 & 400 & 504 \\
\hline
\end{tabular}

Table 4.1 Calculated the diameter parameter based on the rule (100-500 um).

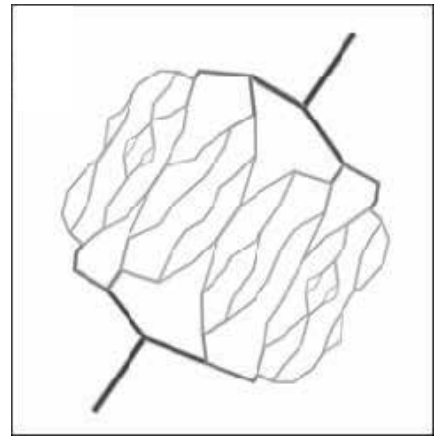

(a) Designed grayscale mask.

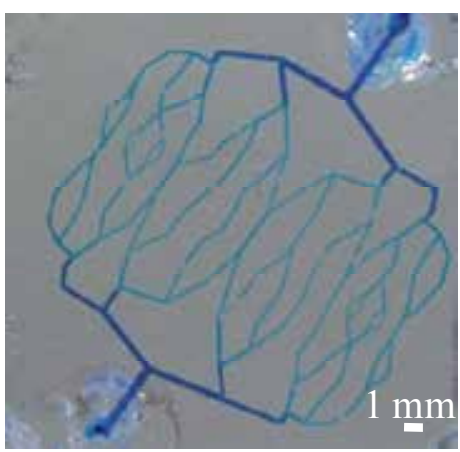

(b) Fabricated model.

Fig. 4.2 100-500 um diameter network type arteriole models. 

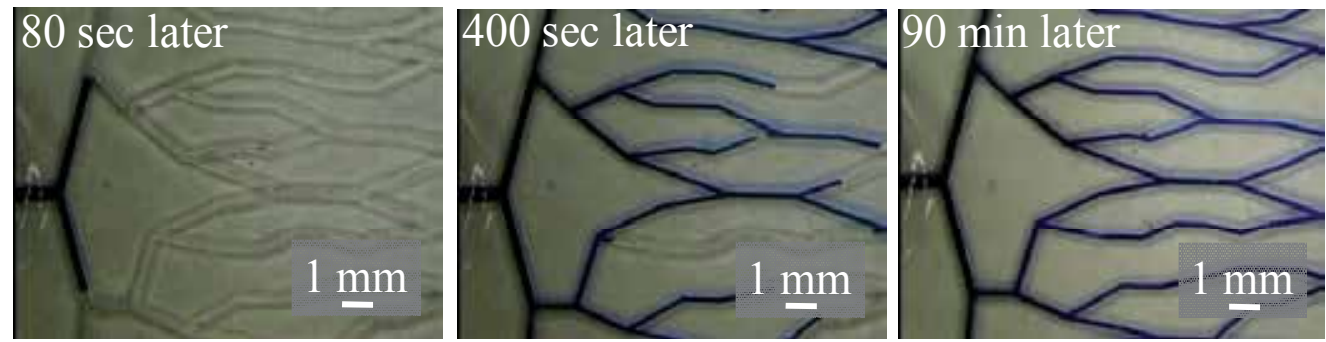

Fig. 4.3 The results of flow evaluation.

1. Coating nega-photoresist on glass substrate.

2. Exposing from the back side of substrate through grayscale mask.

3. Developing photoresist patterns to be 3D shape.

4. Transcribing these patterns onto PDMS substrate.

5. Bonding the two PDMS substrates by plasma treatment and heating.

Completed model was shown in Fig.4.2(b). Figure 4.3 shows the fluid evaluation using fabricated arteriole model which had 100-500 um diameter. A flow condition was $1 \mathrm{ul} / \mathrm{min}$. This fabricated model had no leakage, and was observed different flow speed in same diameter microchannel. It is because that branched angle may effect to flow speed and pressure.

\subsubsection{0-100 um network type arteriole models}

Using Eq. 1, diameter parameter was calculated for fabricating 20-100 um network type arteriole models. Table 4.2 shows the calculated parameters of arteriole network models based on the cube law. The mask for fabricating 20-100 um network type arteriole models was designed(Fig.4.4(a)). In reflow process, it is important to control the photoresist thickness. In this time, thickness of coated resist was $16 \mathrm{um}$, because reflowed resist tend to flow from narrow diameter to large diameter. Then, the 20-100 um arteriole network models were fabricated by reflow method and PDMS bonding. The fabrication process is following.

1. Coating photoresist PMER on silicon substrate.

2. Exposure until 1000 Doses.

3. Development for 7 minutes.

4. Heating the resist patterns from bottom side by hot plate at $145^{\circ} \mathrm{C}$ for $30-60$ seconds.

5. Transcribing these resist patterns onto PDMS.

\begin{tabular}{|c|c|c|}
\hline \multicolumn{2}{|c|}{ Daughter diameter } & Mother diameter \\
\hline $\mathrm{R}_{1}[\mathrm{um}]$ & $\mathrm{R}_{2}[\mathrm{um}]$ & $\mathrm{R}_{0}[\mathrm{um}]$ \\
\hline 19.8 & 19.8 & 25.0 \\
\hline 25.0 & 25.0 & 31.5 \\
\hline 31.5 & 31.5 & 39.7 \\
\hline 39.7 & 39.7 & 50.0 \\
\hline 50.0 & 50.0 & 63.0 \\
\hline 63,0 & 63.0 & 79.4 \\
\hline 79.4 & 79.4 & 100 \\
\hline
\end{tabular}

Table 4.2 Calculated the diameter parameter based on the rule (20-100 um). 


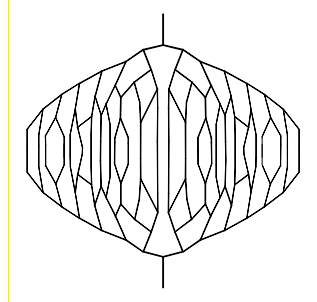

(a) Designed mask data (b) Fabricated model.

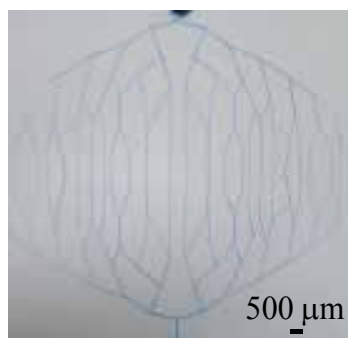

Fig. 4.4 20-100 um diameter network type arteriole models.

Fabricated network model was shown in Fig.4.4(b). It was confirmed fabricated microchannels had no leakage by flow test.

\section{Circulation type blood vessel simulators}

\subsection{Fabrication by seamless connecting}

Our artery and network arteriole models are made by the different processes, therefore, seamless connection is the key issue. Then, our concept of fabricating circulation models was proposed in Fig.5.1. In this method, the circulation model was fabricated by seamless connecting arteriole network models and artery models (whose smallest blood vessel model is $5.0 \mathrm{~mm}$ diameter) with connector models made of wax.

In seamless connecting method, wax connector models were used for connecting the $500 \mathrm{um}$ microchannel of the arteriole network and the $5.0 \mathrm{~mm}$ macrochannel. The designed wax connector model is shown in Fig.5.2 (a) and (b). This connector was fabricated by ink jet rapid prototyping, and had shafts for aligning the central axis of each channel. A 500 um shaft was needed to insert in the network model channel and a $0.8 \mathrm{~mm}$ shaft was needed to insert wax models in the artery model. The wax model of the artery had a $1.0 \mathrm{~mm}$ hole for insertion and alignment as shown in Fig.5.2 (c). The connection process flow is following:

A. Connection process for the network model (Fig.5.3)

1. Dip coating the wax connector with PVA to smoothen the surface.

2. Inserting the connector in the network model, and temporarily fixing with PDMS.

3. Completely fixing with PDMS.

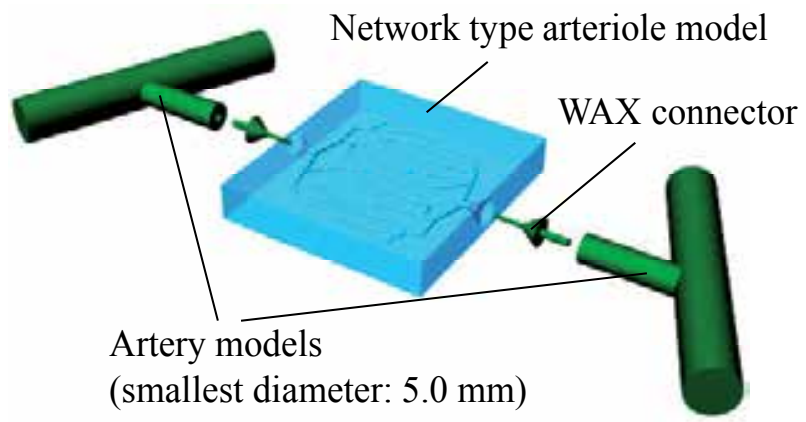

Fig. 5.1 Concept of fabricating circulation models for seamless connection. 


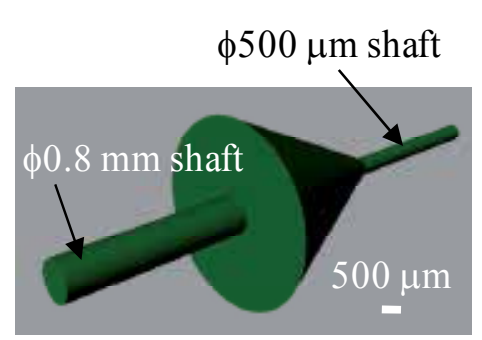

(a) Designed connector
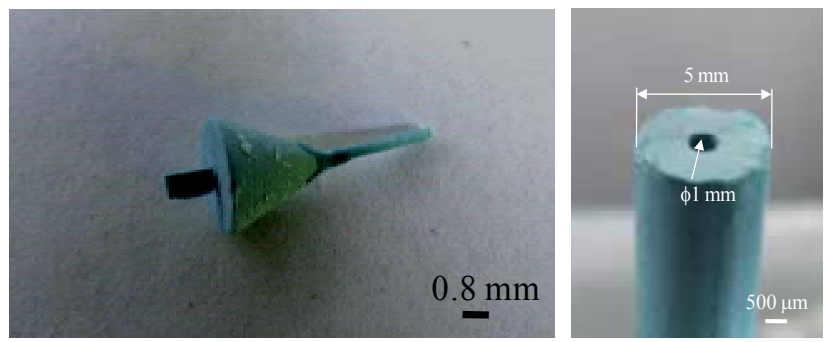

(b) Fabricated connector (c) artery wax model

Fig. 5.2 Wax connectors and connection hole of artery wax model.

1. Coating with PVA

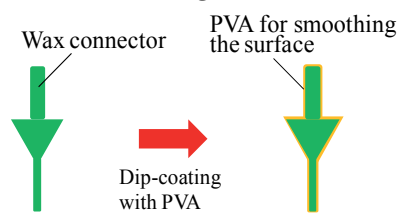

4. Removing superfluous parts

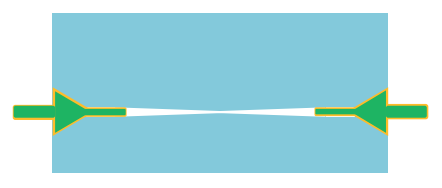

2. Inserting and fixing

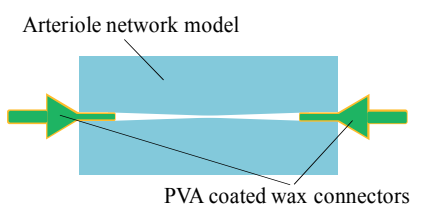

5. Dissolving wax and smoothing PVA

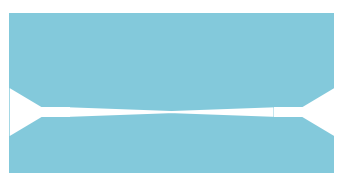

3. Fixing completely
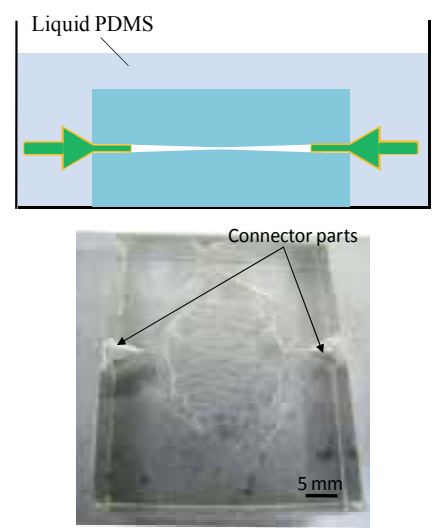

Fig. 5.3 Connection process for the network model.

4. Removing superfluous PDMS mold.

5. Dissolving the wax and smoothing PVA.

B. Connection process for an artery model (Fig.5.4)

1. Smoothing the surface of the wax artery model by dip coating with PVA.

2. Dip coating with silicone resin.

3. Removing the silicone resin and PVA from the alignment hole.

4. Inserting the $0.8 \mathrm{~mm}$ shaft of a PVA coated connector in the alignment hole.

5. Fixing the wax connector on the alignment hole with PVA.

These models were connected to make a circulation model. For fixing, the connector parts were coated with silicone resin, adjusted relative to each other, and heated at $55^{\circ} \mathrm{C}$ for an hour. This assembly process produced the circulation model. Figure 5.5 shows the fabrication process, and fabricated circulation blood vessel model.

\subsection{Evaluation with fabricated circulation model}

For evaluation of leakage check, we performed flow experiments with the fabricated circulation model using a methylene blue solution. The flow rate was $1 \mathrm{ul} / \mathrm{min}$, (This flow rate was $0.016 \mathrm{~mm} / \mathrm{s}$ in the $500 \mathrm{um}$ in diameter microchannel.) and the experiment demonstrated that these channels had no leakage. We also confirmed that the methylene blue solution flowed from the inlet to the outlet. The connected parts between the arteriole 
1. Coating with PVA

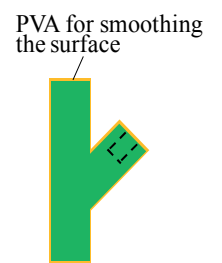

4. Inserting connector

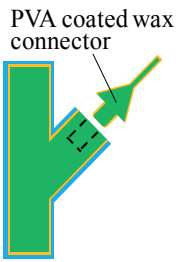

2. Coating with silicone resin

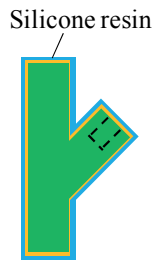

5. Fixing connector

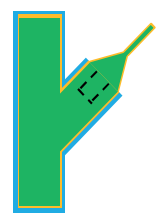

3. Cleaning alignment hole

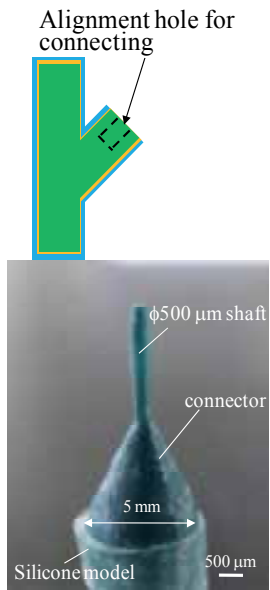

Fig. 5.4 Connection process for the artery model.

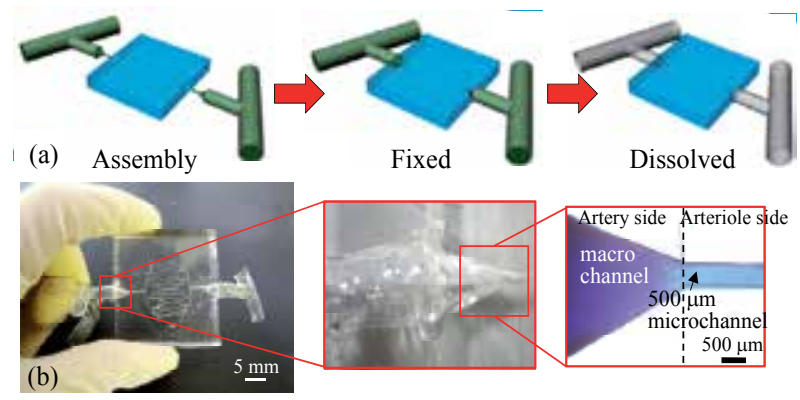

Fig. 5.5 Connection process for the artery model.
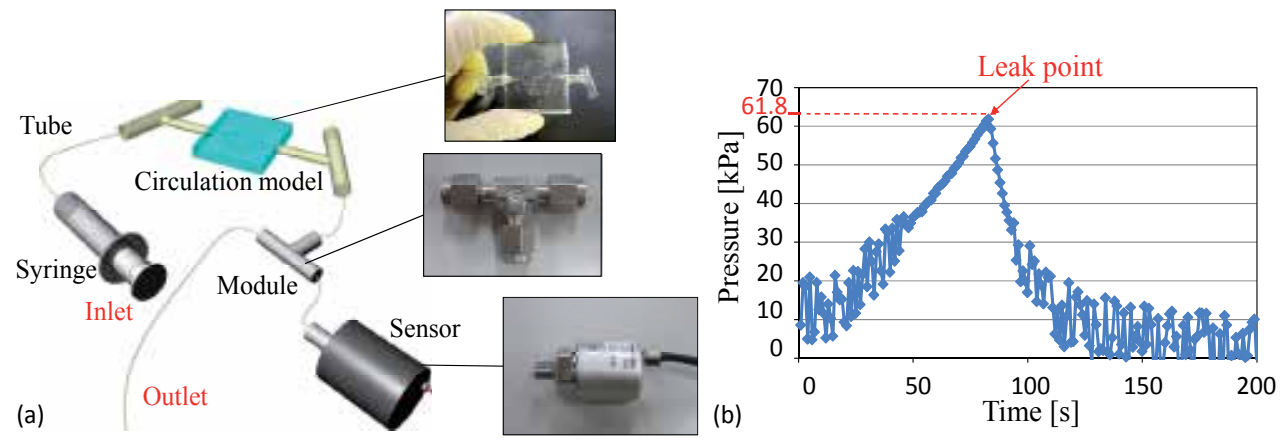

Fig. 5.6 Pressure test with fabricated model. (a)Setup for experiment. (b)Experimental result. network model and artery models exhibited no leakage. The proposed method was suitable for fabricating circulation models. Next, a pressure test was conducted using the fabricated circulation model. Figure 5.6(a) shows the pressure test setup. The prepared pressure sensor 
can measure the liquid pressure. First, the microchannels of the circulation model were filled with DI water. The outlet part was sealed off in order to increase the pressure inside the circulation model by the syringe pump. The experimental results revealed that the leak pressure was approximately $61.8 \mathrm{kPa}$ as shown in Fig.5.6(b). The leak point was located on the artery model. The pressure resistance of the fabricated circulation model was confirmed to be larger than the pressure of actual blood vessels. For example, the pressure in the artery is $16 \mathrm{kPa}$, and that in the arteriole is $13 \mathrm{kPa}$ (Whitmore, 1968). Therefore, the results of the pressure test using the fabricated circulation model show our model is capable of withstanding the pressures of real blood flow. Next, the pressure loss across the inlet and outlet of the fabricated circulation model was measured. This experiment required the use of two pressure sensors. One sensor was used to measure the pressure at the inlet side, and the other sensor was used to measure the pressure at the outlet side. The pressure loss was obtained by subtracting the pressure measured at the outlet side from the pressure measured at the inlet side. The pressure measured at the inlet side was $9.43 \mathrm{kPa}$, and that measured at the outlet side was $9.18 \mathrm{kPa}$. Thus, the pressure loss was $250 \mathrm{~Pa}$. This pressure loss may be caused by a high friction coefficient of silicone resin and PDMS and the length and structure of the channels. This pressure loss was sufficiently small.

\section{Conclusion}

This chapter reported four research topics that can be categorized into following two areas; (i) Various types of microvessel models for blood vessel simulator (Section 2, 3, 4), (ii) Seamless connecting for realizing circulation type blood vessel simulator (Section 5).

In section 1, we introduced the background of conventional surgical simulators, and explained the needs of arteriole and capillary vessel models as surgical simulator for higher precision simulating. For fabricating these models, photolithography process was selected in many maicrofabrications.

In section 2, multiscale fabrication method of 10-500 um arteriole and capillary vessel models was proposed and demonstrated. It is necessary to choose an appropriate exposure method. From the experimental results, it was confirmed proposed method is useful for making microvessel models. These models were fabricated by photolithography and plasma bonding with patterned PDMS substrates. In fabricating capillary vessel models, when two PDMS substrates assembled, alignment is very difficult and patterns are out of alignment. Then, we proposed a novel assembly method. The method is fabricating capillary vessel model pattern and assembly pattern by multi stage exposure. And, the alignment accuracy of the capillary vessel models was evaluated. Then, the circularity of fabricated microchannels was calculated. Fabricated microchannels were 10 um diameter capillary vessel model, $50 \mathrm{um}$ and $500 \mathrm{um}$ diameter arteriole models. The circularity of $10 \mathrm{um}$ microchannel is $84.0 \%$, that of $50 \mathrm{um}$ microchannel is $61.5 \%$, and that of $500 \mathrm{um}$ microchannel is $82.3 \%$. All cross sections of microchannels were circular or elliptical.

In section 3, new fabrication method of 100-500 um arteriole tube models for surgical simulator was proposed. And, fabrication method of arteriole tube model and evaluation of the molding material (wax and PVA mixture material) were reported. For making tube model having narrower than $500 \mathrm{um}$, it is very important to use wax and PVA mixture material. And, mechanical and chemical properties of wax and PVA mixture material were evaluated. From evolutional results, the brittleness of the previous sacrificial model was 
overcome by based on the proposed approach. And, we succeeded in making the tube and hollow structural arteriole model. This arteriole tube model had circular cross section inside the channel, and circularity of this channel was $90 \%$.

In section 4, 100-500 um transparent arteriole network model was successfully fabricated. And, fabricated microchannels had no leakage by the flow experiment. Therefore, using grayscale lithography and basing on real vessels' branched rule are useful to fabricate 100500 um transparent networked arteriole models. In addition, 20-100 um arteriole network model was successfully fabricated, too. The reflowed resist flowed from narrow diameter to large diameter. Therefore, comparing theoretical and experimental circularity, experimental circularity was dramatically improved. Then, reflow method is suitable for fabricating 20 100 um arteriole network models.

In section 5, circulation type blood vessel models were fabricated and demonstrated by using arteriole network models and artery models. A circulation model was fabricated using a wax connector for seamless connecting. The fabricated model had a seamless structure, and it was demonstrated by the flow experiments that this model had no leakage on the any parts. The proposed connection method was suitable for fabricating circulation models. And, fabricated circulation model can be used for blood vessel simulator from the result of pressure test. Therefore, the fabricated circulation model will be used to evaluate drug delivery systems, diacrisis, and medical treatments by ultrasound.

\section{Acknowledgements}

The present study was supported by a Grant-in-Aid for Scientific Research from the Ministry of Education, Culture, Sports, Science, and Technology of Japan and the Japan Society for the Promotion of Science (Grant Nos. 17076015, 18206027, and 214059).

\section{References}

Ikeda, S., Arai, F., Fukuda, T., Negoro, M., Irie, K. (2005). An In Vitro Patient-Tailored Biological Model of Cerebral Artery Reproduced with Membranous Configuration for Simulating Endovascular Intervention. Journal of Robotics and Mechatronics, Vol.17, No.3, June 2005, pp. 327-334, ISSN0915-3942 (print), 1883-8049 (online)

Attinger, E.O. (1964). Pulsatile Blood Flow., Mc.Graw-Hill Book Co., New York, USA

Hayashi, K. (April 1997). Biomechanical Engineering: A First Course, MARUZEN CO., LTD., ISBN 4-88898-081-0, Tokyo, Japan

Noda, D., Matsumoto, Y., Setomoto, M. (2007). Fabrication of Coil Lines with High Aspect Ratio for Electromagnetic Actuators. Proceedings of International Symposium on Micro-Nano Mechatronics and Human Science, pp.436-441, Nagoya, Japan, date, 2007

Itoga, K., Yamato, M., Kobayashi, J., Kikuchi, A., Okano, T. (2004). Cell micropatterning using photopolymerization with a liquid crystal device commercial projector. Biomaterials, Vol.25, No.11, May 2004, pp.2047-2053, ISSN 0142-9612

Borenstein, J., T., Terai, H., King, K., R., Weinberg, E., J., Kaazempur-Mofrad, M., R., Vacanti, J., P. (2002), Microfabrication Technology for Vascularized Tissue Engineering. Biomedical Microdevices, Vol.4, No.3, July 2002, pp.167-175, ISSN 1387-2176 (Print) 1572-8781 (Online) 
Hanai, K., Shimizu, S., Matsumoto, Y. (2005). Three Dimensional Structures of Negative-tone Photoresist by Binary Optics. IEEJ Trans. SM, Vol.125, No.10, January 2006, pp.424425, ISSN 1341-8939 (print), 1347-5525 (online)

Eisner, M., Schwider, J. (1996). Transferring resist microlenses into silicon by reactive ion etching. Opt. Eng., Vol.35, No.10, October 1996, pp.2979-2982, ISSN 0091-3286, 1560 2303 (eISSN)

Nicolas, S., Dufour-Gergam, E., Bosseboeuf, A., Bourouina, T., Gilles, J.-P., Grandchamp, J.P. (1998). Fabrication of a gray-tone mask and pattern transfer in thick photoresists. J. Micromech. Microeng., Vol.8, No.2, June 1998, pp.95-98, ISSN 0980-1317 (print), 1381-8439 (online)

Futai, N., Gu, W., Takayama, S. (2004). Rapid Prototyping of Microstructures with BellShaped Cross-Sections and Its Application to Deformation-Based Microfluidic Valves. Adv. Mater., Vol.16, No.15, August 2004, pp.1320-1323, ISSN 1521-4095

Wu, M-H., Park, C. \& Whitesides, G. M. (2002). Fabrication of Arrays of Microlenses with Controlled Profiles Using Gray-Scale Microlens Projection Photolithography. Langmuir, Vol.18, No.24, November 2002, pp.9312-9318, ISSN 0743-7463 (print), 1520-5827 (online)

Whitesides, G. M., Ostuni, E., Takayama, S., Jiang, X., Ingber, D. E. (2001). Soft Lithography in Biology and Biochemistry. Annu. Rev. Biomed. Eng., Vol.3, August 2001, pp.335373

Arai, F., Nakano, T., Tada, M., Lin, Y.-C., Ikeda, S., Uchida, T., Oura, H., Fukuda, T., Matsuda, T., Negoro, M. (2007). Fabrication of cell-adhesion surface and Arteriole Model by photolithography. Journal of Robotics and Mechatronics, Vol.19, No.5, October 2007, pp.535-543, ISSN 0915-3942 (print), 1883-8049 (online)

O'Shea, C. \& Rockward, W., S. (1995). Gray-scale masks for diffractive-optics fabrication: II. Spatially filtered halftone screens. Appl. Opt., Vol.34, No.32, November 1995, pp.7518-7526, ISSN 1559-128X, 2155-3165 (eISSN)

Sherman, T., F. (1981). On Connecting Large Vessels to Small. The Meaning of Murray's Law. J. Gen. Physiol., Vol.78, October 1981, pp.431-453, ISSN 0022-1295 (print), 15407748 (online)

Whitmore R. L. (1968). Rheology of the Circulation, Pergamon Press, ISBN 4-88898-081-0, Oxford, UK 


\title{
Rapid Prototyping for Training Purposes in Cardiovascular Surgery
}

\author{
Philippe Abdel-Sayed and Ludwig Karl von Segesser \\ Centre Hôspitalier Universitaire Vaudois, CHUV \\ Switzerland
}

\section{Introduction}

The emergence of new and rapidly growing therapies in cardiovascular surgery requires larger training opportunities and a precise identification of the heart structures and its disease. Nowadays, imaging techniques such as computed tomography, magnetic resonance imaging and others allow the virtual reconstruction of hearts in a three-dimensional conformation. Those techniques are being used for mainly for diagnostic purposes (Olson, Lange et al. 2007) and for operation planning (Gasparovic, Rybicki et al. 2005) as well as for educational training (Lermusiaux, Leroux et al. 2001). However, they do not give tangible practice in surgical simulation. This explains, why currently, animals are still widely used for training and are often involved in research and development. However, animal experiments are difficult to realize in large numbers for various reasons including ethical concerns and cost. Major efforts are made to replace the use of animals by artificial biomodels or by animal-cadaveric-models for surgical training purposes. Training on animal-cadaveric-models often proves to be complicated since it requires heavy material supports and standard slaughtering in abattoirs.

Artificial biomodeling appears to be a more and more promising alternative for training in cardiovascular surgery. Indeed, the realization in solid-replica of patient-specific and lifesize hearts contributes to the realism of the surgical procedure and facilitates recognition of the 3D structures, hence improving clinical performance. The procedure of heart biomodeling can be summarized as follows: (1) The acquisition of heart images, (2) the data processing and (3) the printing of the solid replica of the heart (Figure 1).

In this chapter we will present a literature review of last progresses of rapid prototyping in the field of cardiovascular surgery and cardiology. In particular, concerns regarding to the methodology and processing of modeling, limitations, applications and perspectives will be emphasized.

\section{Rapid prototyping methodology}

\subsection{Heart imaging}

In recent years, 3D imaging has rapidly entered the clinical world of cardiology and cardiovascular surgery. Indeed, several imaging modalities including magnetic resonance imaging (MRI), computed tomography (CT) and echocardiography have undergone significant advancements in imaging soft tissues, which allowed faithful volume renderings 


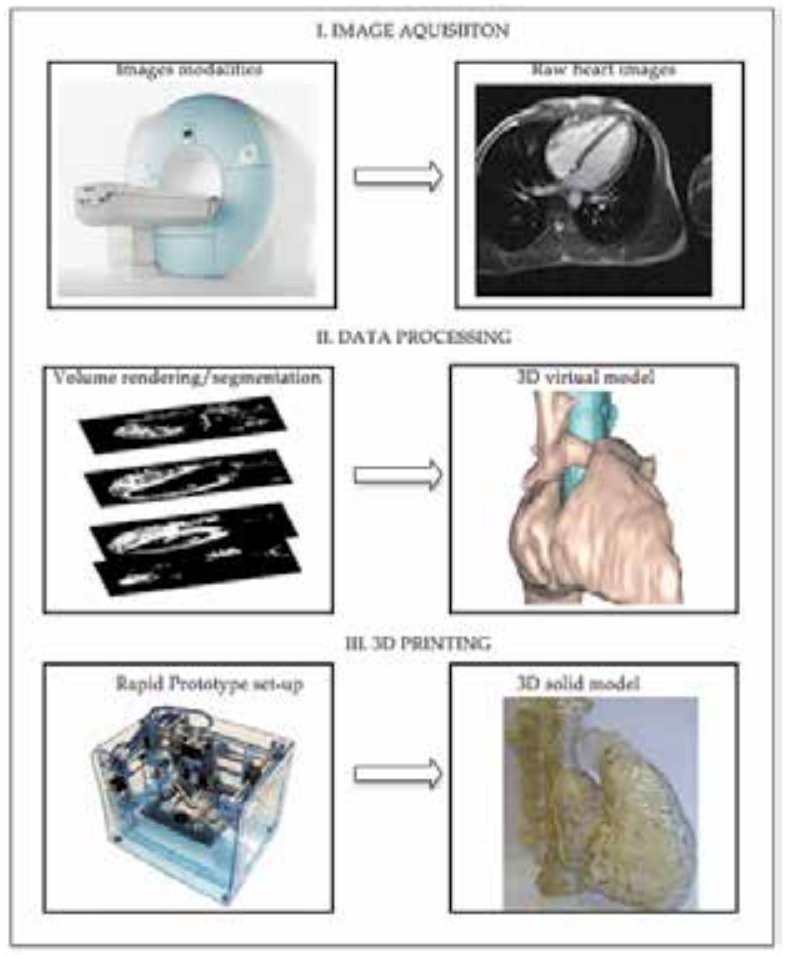

Fig. 1. The three major steps of heart prototyping

of heart structures through the stacking of multi-planar images acquired from those modalities (Figure 2). This trend from the 2D to the $3 \mathrm{D}$ is partly due to ambiguous interpretations that might be encountered in 2D images, which become crucial when considering options for surgical treatment (Riesenkampff, Rietdorf et al. 2009).

Virtual 3D reconstructions are used for diagnostic purposes, for operation planning as well as for education and training. They are particularly useful to visualize patient's heart with complex structural problems, such as congenital defects and aneurysms (Kellenberger, Yoo et al. 2007; Ou, Celermajer et al. 2007; Spevak, Johnson et al. 2008). However, incongruities between real anatomical structures and interpretation of virtual reconstructed threedimensional (3D) images still remain (Shiraishi, Yamagishi et al. 2010). This drives surgeons to realize solid replicas of hearts with structural defects, which would convey better and more tangible information about spatial relationships between heart structures.

Rapid prototyping (RP) is used to build up solid replicas of hearts. This process involves the conversion of medical images to a Stereolithography (STL) file format. This file format describes triangulated surfaces of the heart model in a 3D Cartesian coordinate system and is utilized in CAD manufacturing and by RP machines. The medical image files are in a Digital Imaging and Communications in Medicine (DICOM) format. This file format is standard for images of MRI, CT, echocardiography or other imaging modalities ${ }^{1}$. The 3D

${ }^{1}$ For the download and the exercise heart modeling, one can refer to the following online database of MRI/CT images, where various set of adult heart image files can be found (Casimage Database, Geneva University Hospital, http:/ / pubimage.hcuge.ch:8080/). 


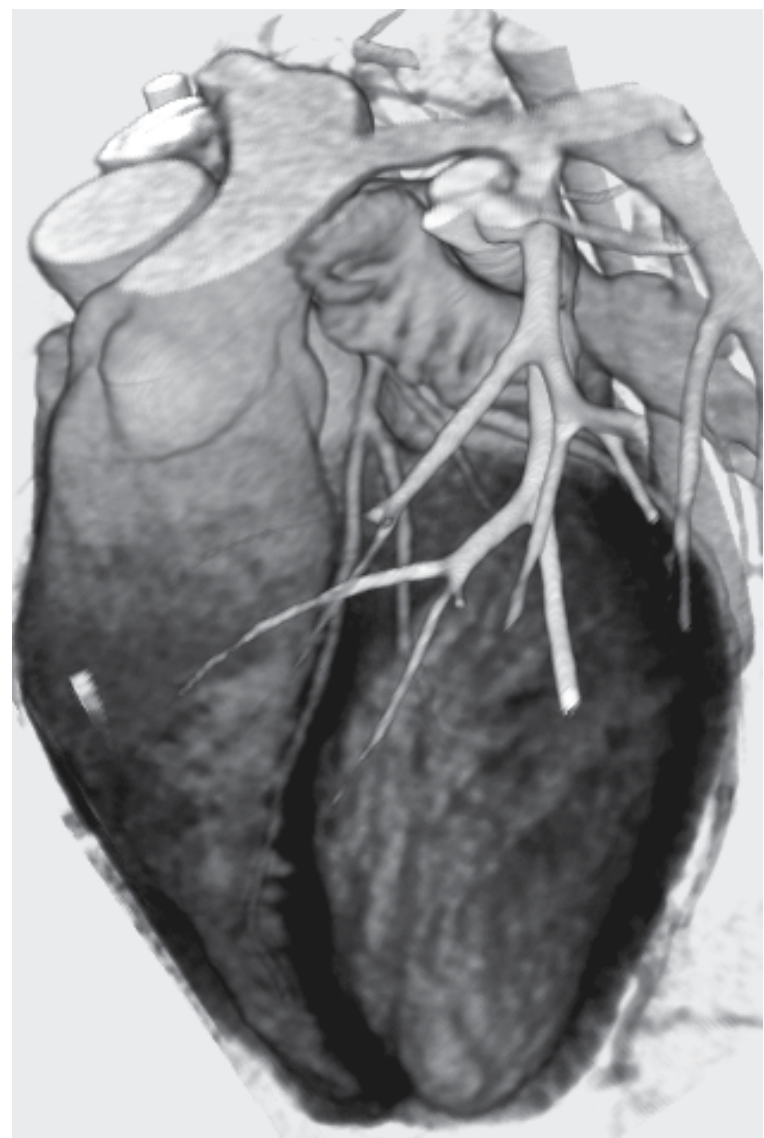

Fig. 2. Image of a volume rendering of a heart. The raw data come from a coronary angiogram recorded on a 64-detector CT scanner.

DICOM data must be of high resolution to make an accurate RP model. The following section aims to discuss the processing of DICOM data to isolate the structure of interest and turn it into an STL file, that will be upload to rapid prototyping machine to produce the solid 3D model.

\subsection{Data processing for heart modeling}

Several research groups have reported their utilization of user-friendly software packages for the processing of medical images, such as Jacobs et al who used Mimics software (Materialise NV, USA) to segment the medical images and to produce finished STL files (Jacobs, Grunert et al. 2008). Likewise Markert et al used MeVisLab software (MeVis Medical Solutions, Germany) to convert and finish the medical image file (Markert, Weber et al. 2007) and many others as well. However, in heart modeling literature, it is noticeable that a lack of information exists regarding to the methods of post-acquisition processing. Usually, the authors mention only the Computer-Aided Design (CAD) software they use for the processing of the images and their conversion to a STL. Hence, a significant missing part is the methodology of images segmentation. The dataset is a series of DICOM images, that when stacked together represent the heart with the surroundings tissues. The segmentation 
is the processing that allows isolating the structure of interest is the data files, i.e. it identifies voxels from a dataset corresponding to the desired tissue and removes the surrounding undesirable tissues.

\subsubsection{Automatic segmentation}

Automatic segmentation consists of applying pre-defined filters to the data set so that it isolates directly the desired heart structures. However this automatic process is defective, because it dependents on the quality of the data images, especially the artifacts introduced by the imaging modalities could induce errors in the segmentation. Most of the automatic segmentations are based on threshold techniques, which give satisfactory results only in isolating inner boundaries of the heart between myocardium and blood. However, they do not adequately locate the outer boundary of the heart, because the outer boundary is not well defined at some places.

As example of automatic segmentations, we would like to present here a preview of the methods existing in VolView software package (Figure 3) from the National Library of Medicine Insight Segmentation and Registration Toolkit (ITK). ITK is an open-source, crossplatform system that provides developers with an extensive suite of software tools for image analysis.

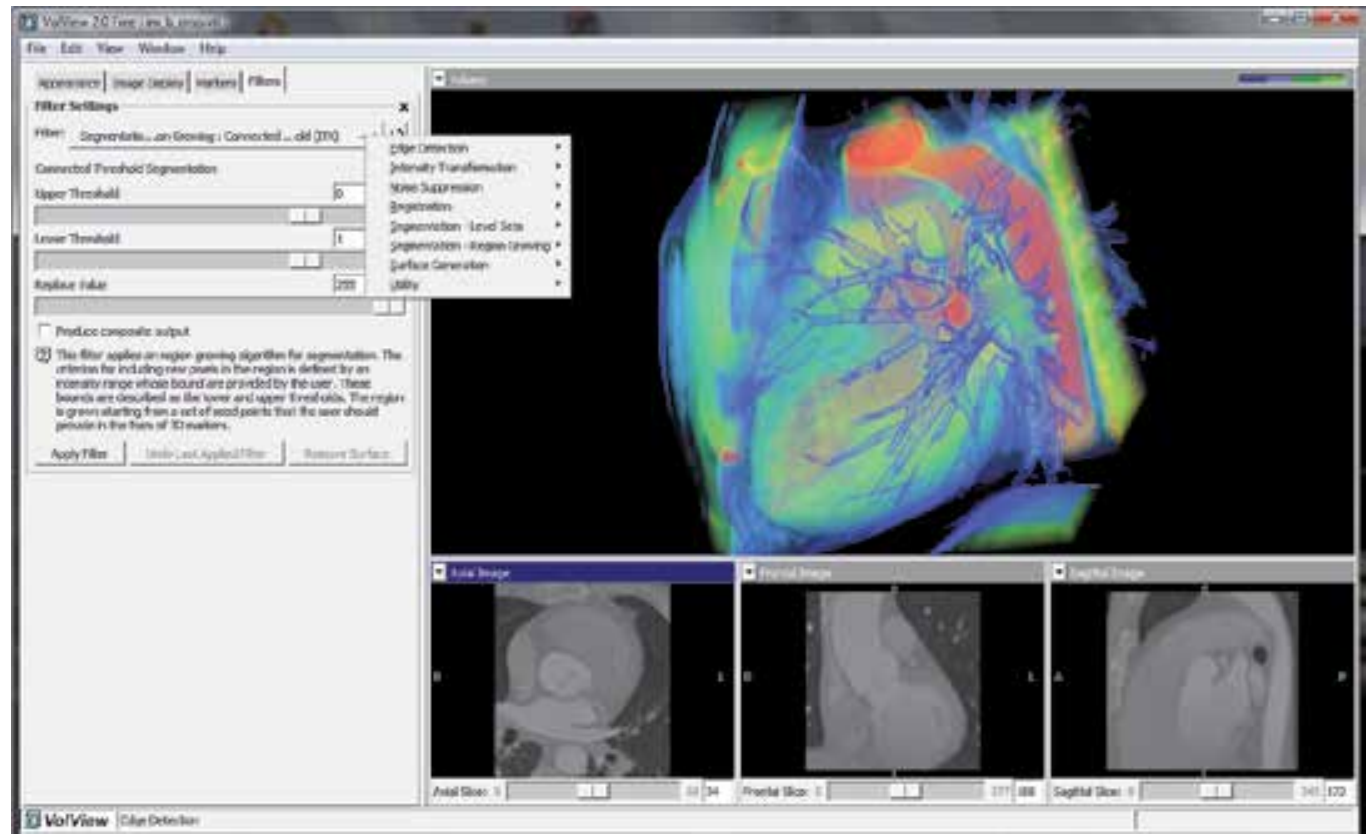

Fig. 3. View of the VolView panel. Several pre-programmed filters are available, among which some are defined for edge detection, noise suppression and segmentation of region growing.

Other than filters for noise suppression and edge detection, VolView software has two specific categories of segmentation filters. The first one has several algorithms based on the level sets concept and the second one has algorithms based on the region-growing concept. Briefly, statistical algorithms are applied to identify the voxels that might be admitted to the 
segmented region based on the distribution of voxel contrasts within defined regions. Those methods provide good results; the heart wall is clearly visible as well as major veins and arteries.

However, this automatic process has the disadvantage of detecting also surrounding structures, whose grey level is similar to cardiac muscle, including thus sometimes parts of ribs, spinal cord and the pulmonary branches within the lungs (Figure3). The algorithm also produces so-called false negatives within the heart muscle tissue, i.e. the algorithm removes parts of the heart that should not be removed from the volume of interest. The effort required to correct the errors produced by the algorithm remains high. This is why it would be equally efficient to perform a manual segmentation voxel by voxel.

\subsubsection{Manuel segmentation}

As example to illustrate a manual segmentation, we would like to present methods present in Amira (Visage Imaging $\mathrm{GmbH}$, Berlin, Germany), a software package proposed for processing biological images. Its Segmentation Editor has several tools available, including thresholding and laso tools that use the gradient of the image to detect boundaries. However, the more performing way to segment the heart remains to use the paintbrush, thanks to which one can trace specifically the boundaries of the heart in each slice (Figure 4). Nevertheless this technique is time consuming since it requires the processing one image at a time.

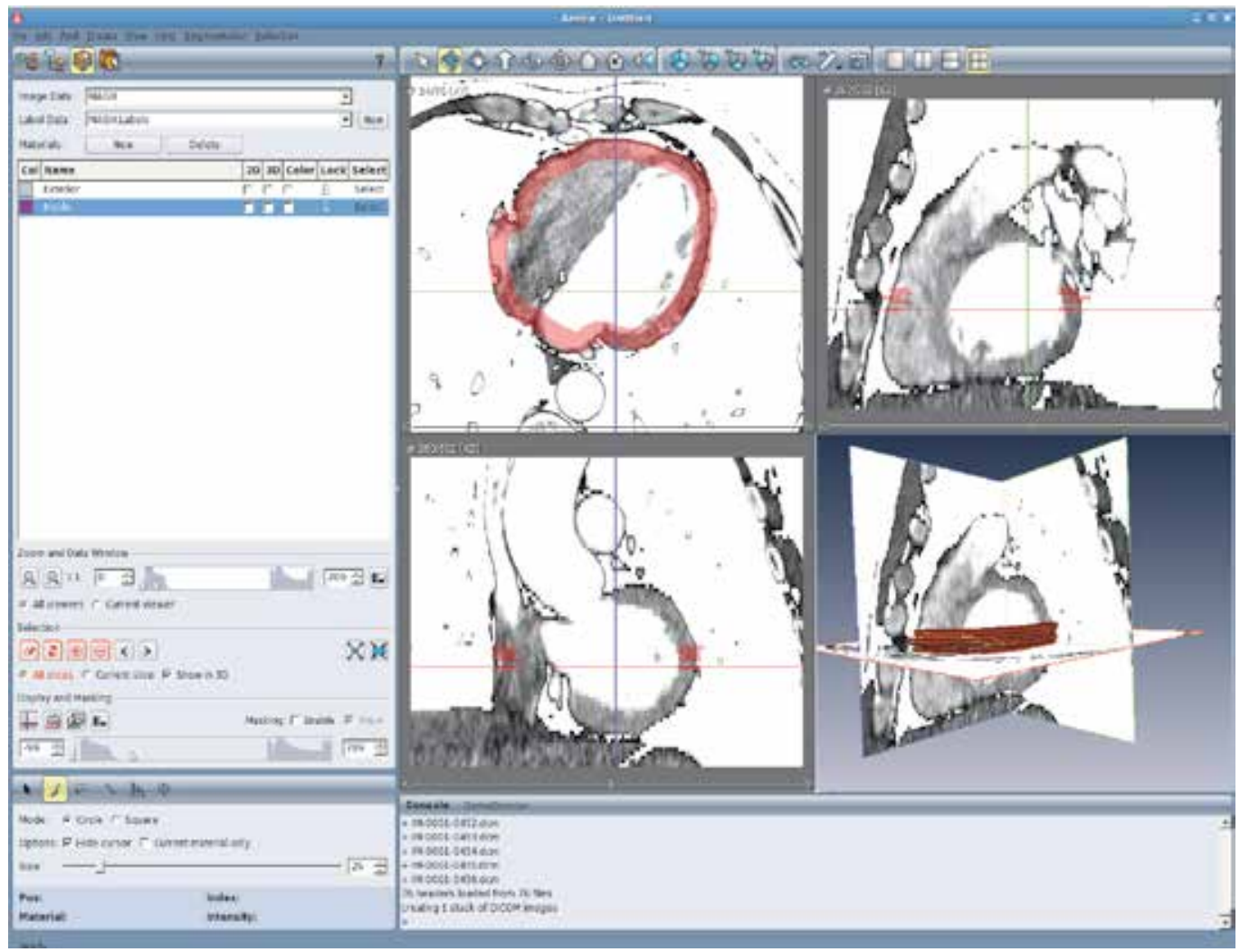

Fig. 4. A view of the manual segmentation. 
The current use of manual segmentation is only because it has not been developed yet a satisfactory automatic or semi- automatic methods. The fact that each set of images has its own specificities would explain why there is no unified protocol for heart segmentation. The final segmented heart is saved then in a STL file for the 3D printing.

\subsection{The model printing}

The reconstruction of the solid model is made from the STL file, which can be in principle read by any rapid prototyping machine, and is accomplished by the addition of material layer by layer according to the virtual design (Peltola, Melchels et al. 2008). Each layer, which corresponds to a virtual cross section from the CAD model, is solidified using different solidifiying agents, such as UV lasers or liquid binders. All the solidified layers joined together create the final shape. An advantage of this additive process is its ability to create complex shapes or particular geometric features. The concept of "rapid" is relative, because the additive process described above can typically produce models in few hours whereas reconstruction of models with contemporary methods can take days. But even for the additive process, the reconstruction time depends primarily on the size and complexity of the model and the RP printer type.

3D printing counts several manufacturing techniques among which some have already been used in cardiovascular surgery. Firstly comes the Stereolithography (SLA), which uses photopolymers that can be cured by UV laser. Lermusiaux et al. produced models on an SLA 250 stereolithography apparatus (3D Systems Corp., Valencia, CA). This prototyping device creates 3-D replicas of aortic aneurysm using epoxy resin. A low-powered but highly collimated laser beam is focused on the surface of a container filled with liquid resin. The laser draws a cross section of the model, converting the thin layer of the liquid resin to solid. The model was used for the development of new endovascular techniques for repair of abdominal aortic aneurysm (Lermusiaux, Leroux et al. 2001). Shiraishi et al. used ultraviolet laser beam to polymerize a selectively photosensitive polymeric liquid plastic solution to produce models used for simulative operations on congenital heart disease (Shiraishi, Yamagishi et al. 2010)

Other than Stereolithography there are other rapid prototyping systems such as Laser Sintering methods, which are based on small particles of metal, plastic or glass that are fused by a high power laser. As well, there is Fused Deposition methods that extrude small beads of fused thermoplastic materials that immediately attach to the below layer. Finally, Inkjet printing techniques, which use pistons to seed layers with fine powders; then an adhesive liquid dropped by another piston bonds the parts of these layers belonging to the 3D object. Depending on the manufacturing technique it is possible to combine materials of different elasticity or color in one model, which might help to create more realistic models in educational or training purposes (Rengier, Mehndiratta et al. 2010).

\subsection{Rapid prototyping benefits for surgical training}

Although RP application and benefit in craniofacial and maxillofacial surgery has been proven (Wagner, Baack et al. 2004), in cardiovascular surgery RP modeling is still in its early stage. However, its great potential to produce accurate models of heart and its structure of interest proves usefulness in cardiovascular surgery. Various studies using RP in adult and pediatric heart modeling have been already accomplished (Armillotta, Bonhoeffer et al. 2007; Jacobs, Grunert et al. 2008; Sodian, Weber et al. 2008; Shiraishi, Yamagishi et al. 2010). 
These studies showed that tangible 3D models (1) allow a better identification of structural abnormalities, (2) help to determine the best surgical option as treatment and (3) improve surgical skills of young surgeons by an intensive training simulating in vivo conditions without any risk of patient complications.

\section{Training of the trans-apical aprtic valve replacement}

\subsection{The 3D printing technology of the Fab@Home project}

As previously mentioned, several rapid prototyping machines can be used to make models from STL files. A particularly interesting machine is the open-source rapid prototyping system developed by the Fab@Home project (http://fabathome.orgy) and distributed by Koba Industries (Albuquerque, NM, USA). It consists of a 3D printer, which main component is a syringe used as a deposition tool and moved by several stepper motors (Figure 5).

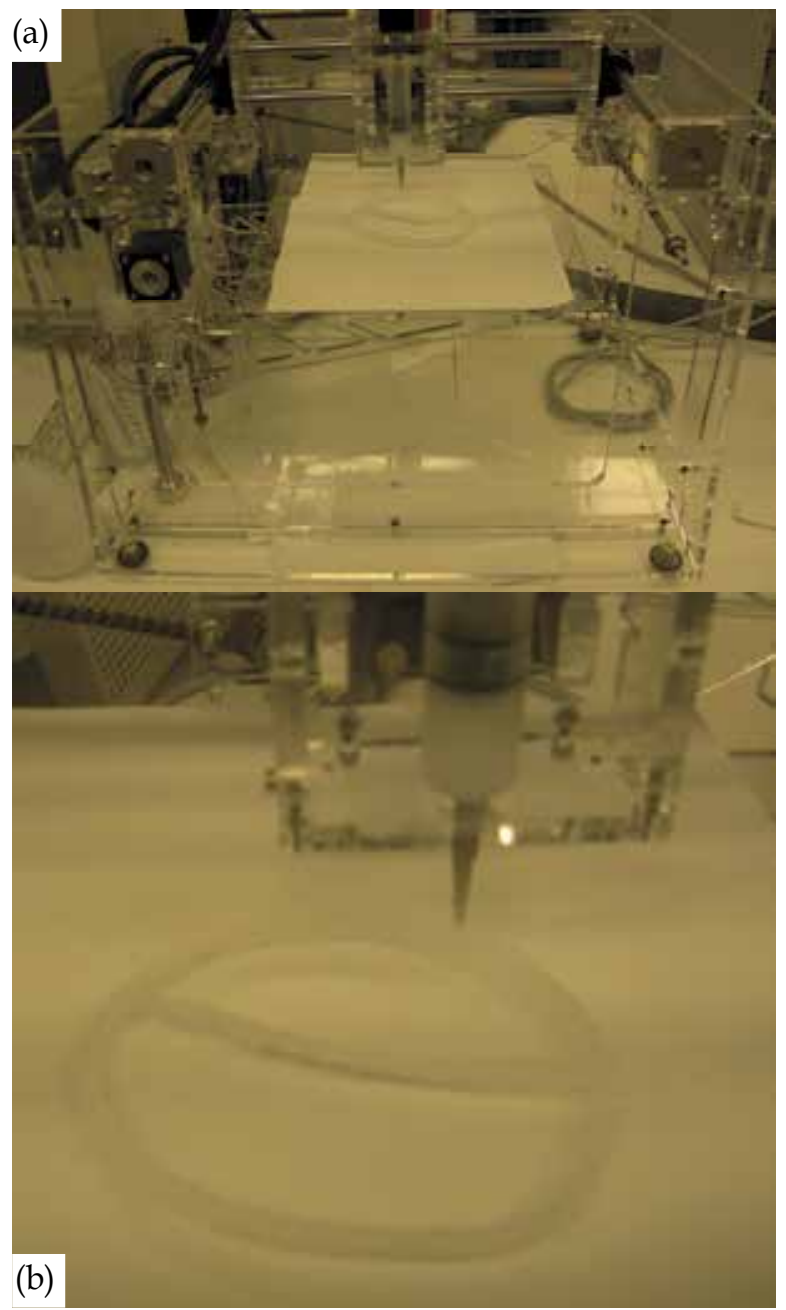

Fig. 5. (a) The Fab@Home 3D printer. (b) The syringe of the printer deposits silicone in building a heart model. 
This printer is the only 3D printer capable of using any viscous substance as a building material (Kalejs and von Segesser 2009), and for this reason it its particularly useful to build up from silicone a translucent model of a complete human heart. The property of translucency given by the silicone is important for the surgical training of trans-apical aortic valve replacement (AVR), because the translucency of the heart model is a way to circumvent the use of $X$ - rays to visualize the area of deployment of the stent during the trans-apical AVR (Abdel-Sayed, Kalejs et al. 2009).

The training of the trans-apical AVR is essential because far more than one thousand clinical trans-apical AVRs have been realized worldwide so far, and there is still little doubt that a high level of surgical skills is required for these procedures, which implies an unavoidable learning curve. In the past, animal experimentations were used for training, however, there are difficult to realize in large numbers for various reasons including ethical concerns and cost. Hence the importance to develop heart models that are life-size, compliant and translucent.

\subsection{Pseudo-volume rendering method for heart prototyping}

Unlike what has been presented before in this chapter for the modeling of the heart, a new pseudo-volume-rendering method has been established to create 3D geometries of the heart (Abdel-Sayed, Kalejs et al. 2009). This method uses SolidWorks 2007 CAD software (SolidWorks Corporation, Concord, MA, USA), and is more simple and less time consuming than conventional methods of segmentation and modeling. Basically, it requires the insertion into the graphical zone of SolidWorks only four representative CT-scan slices of a human heart. Heart contours are then extrapolate from those images thanks to spline curves, which consist of piecewise polynomial functions that allow the fitting complex shapes. Then the $3 \mathrm{D}$ wall of the heart is built up by a smoothing function that connects the spline curves (Figure 6).

Thereafter, the complete model geometry is saved in STL format and constructed using the printing set-up of Figure 5. As a material for 3D fabrication common house-hold (sanitary) silicone (Forbo international, Schoenenwerd, Switzerland) has been used. After printing, dip-coating of the entire heart model with dispersion silicone is performed two times so as to increase its mechanical strength, prior to its fitting within the heart trainer.

\subsection{The surgical procedure}

The realized heat model (Figure 6) has a straight path from the left ventricular apex towards the aortic annulus, the aortic root with a realistic sinus portion, and the ascending aorta suitable for trans-apical AVR. For this purpose, the heart model is then fitted in an artificial adult-chest (Figure 7) for trans- apical stent-valve replacement training.

\subsection{The surgical procedure}

The realized heat model (Figure 6) has a straight path from the left ventricular apex towards the aortic annulus, the aortic root with a realistic sinus portion, and the ascending aorta suitable for trans-apical AVR. For this purpose, the heart model is then fitted in an artificial adult-chest (Figure 7) for trans- apical stent-valve replacement training.

In the chest manikin used, the built-in thoracic incisions are placed anatomically correct, so that the apex of the heart model can be easily accessed from the antero-lateral left thoracotomy. The superior midline sternal splitting incision is used to visualize the implant 


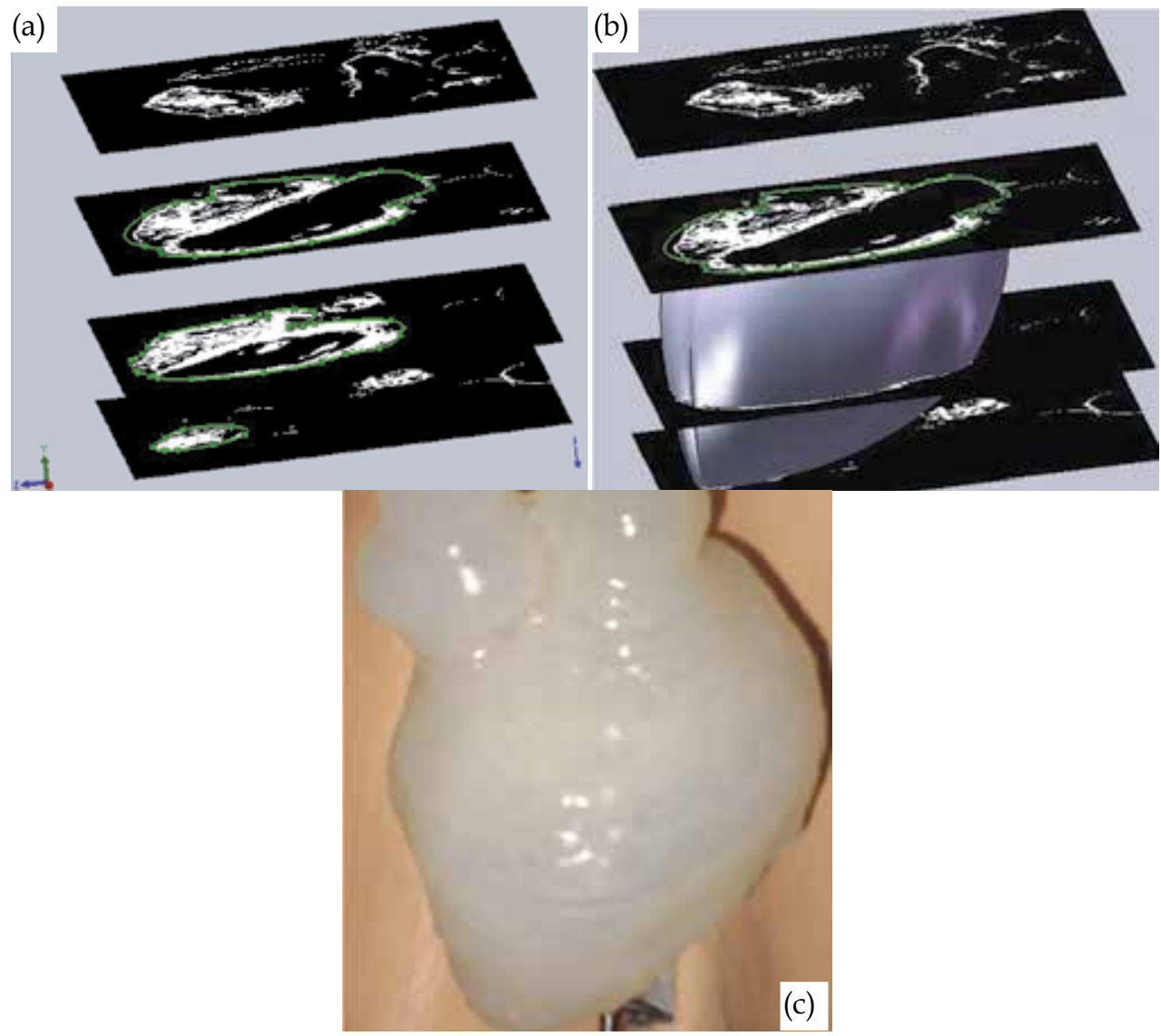

Fig. 6. Overview of the pseudo-volume rendering method. (a) Graphical zone of

SolidWorks, that includes the CT-scan images, the spline curves in green. (b) The smoothed surface of the heart wall from the spline curves. (c) finalized heart model with its aortic roots. Reproduced from Abdel-Sayed P, Kalejs M, von Segesser LK. A new training set-up for trans-apical aortic valve replacement. Interac CardioVasc Thorac Surg 2009; 8: 599-601, with permission of the European Association of Cardio-Thoracic Surgery.

procedure. A light source is positioned behind the heart model in order to see by translucency the area of interest, during catheterization, positioning of the introducer that bears the valved-stent and valve deployment (Figure 8).

Practically, the trans-apical AVR procedure is realized exactly like in the clinical setting. Through the left antero-lateral mini-thoracotomy, the apex is identified and punctured with a hollow needle. A soft J-type guide-wire is brought into the left ventricle, through the aortic annulus into the ascending aorta, all of this under visual control through the small superior sternotomy and the translucent aortic root. For implantation of a catheter mounted aortic valve prosthesis using the anterograde route, the guide wire has to be exchanged for a stiffer wire using a (pigtail-) catheter. Another pigtail catheter can be inserted in retrograde fashion for identification of the valve level. A balloon is then inserted in anterograde fashion and inflated for sizing. With the stiff wire in place, the balloon is exchanged for the large introducer allowing for insertion of the catheter, which carries the compressed valve. The 


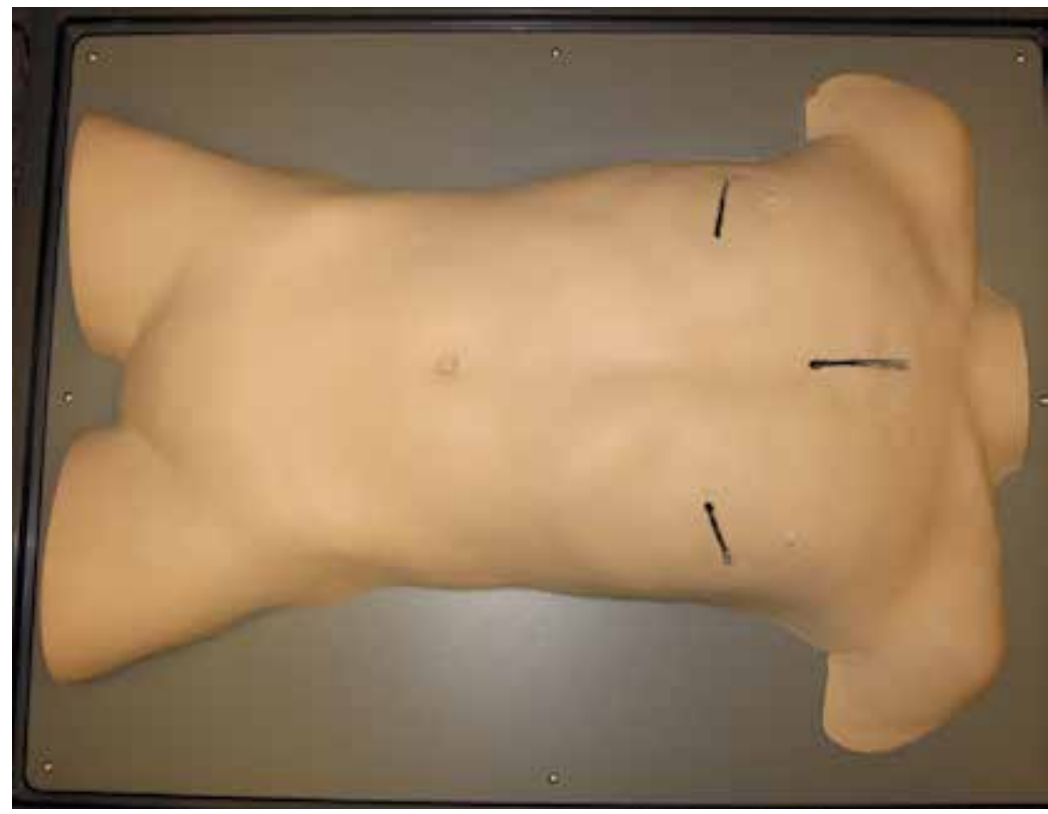

Fig. 7. Artificial adult-chest used for the training of trans-apical AVR.

latter can be either balloon expandable or self-expandable (Symetis Ltd, Lausanne, Switzerland) like demonstrated here.

The same heart model can also be used for training on pulmonary valve replacement. Whereas we perform this procedure in the clinical setting through a small epigastric incision, the cover of the phantom used here is exchanged for a cover with a full median sternotomy, in order to access the translucent heart through an inferior median sternotomy, and to visualize the anterograde pulmonary valve replacement within the infundibulum of the right ventricle and the pulmonary artery through a superior median sternotomy (Figure 9).

\section{Current limitations of heart biomodels}

The limitations of artifical heart models come first from the resolution and accuracy of the images from which the heart model is reconstructed. To make the better biomodels of the cardiovascular systems, image acquisition done for instance by multi-slice CT technique provides highly contrasted images compared with other techniques such as MRI and echocardiography. However, the major concern of multi-slice CT is exposure of patients to ionising radiation. So the improvements of artificial heart model are dependant on the development of images modalities and the balance between good images and the safeness of the acquisition procedure.

Nevertheless, the most important limitations of heart prototyping lay within the artifacts occurring during the segmentation procedure. Moreover the segmentation of soft tissues such as heart is difficult and time consuming. Those limitations can be reduced with a pseudo-volume rendering method presented in this chapter, however in this case the models would lost in accuracy and specificity to the real heart. 


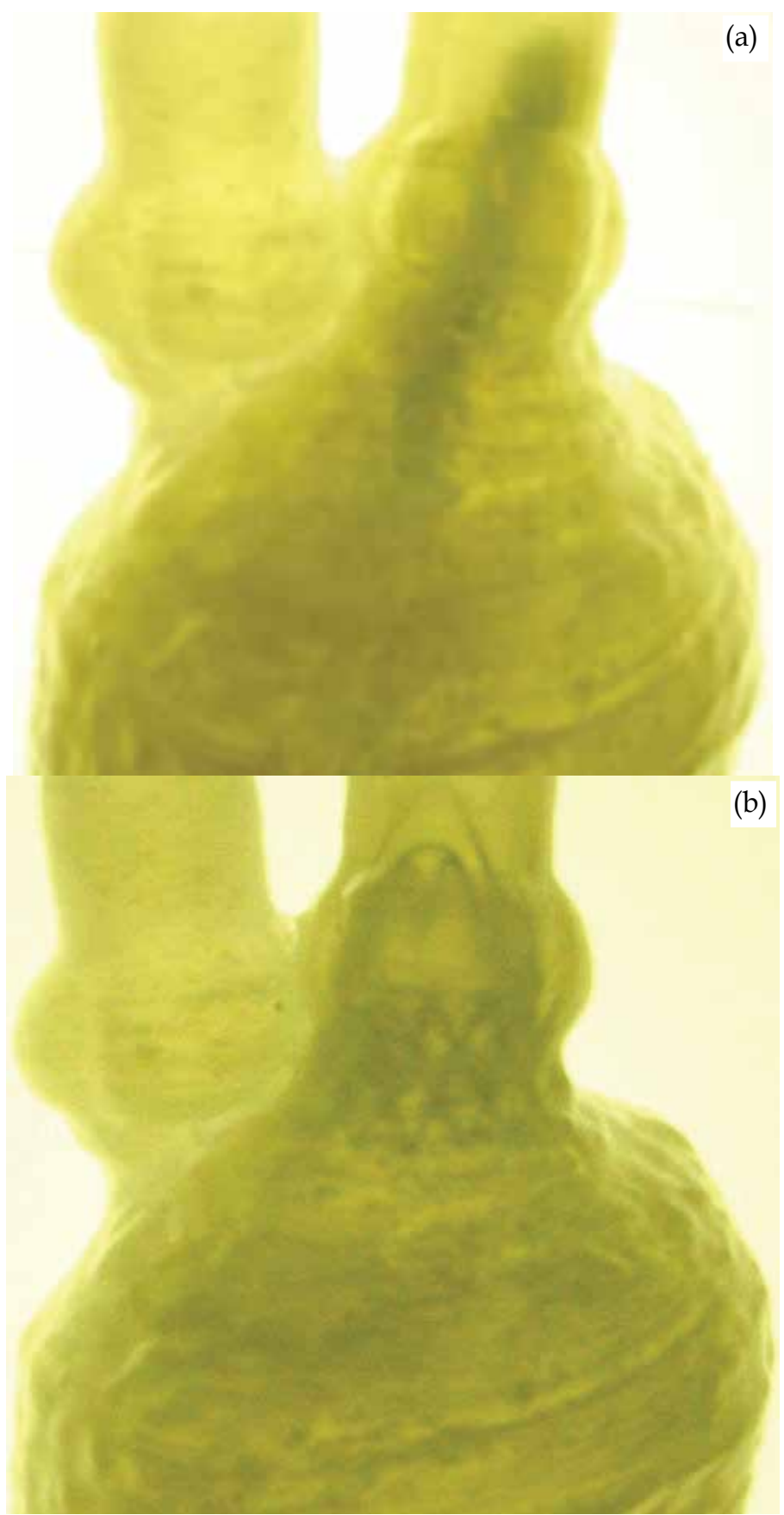

Fig. 8. (a) Catheter bearing the valved- stent seen by translucency and backlight; (b) selfexpanding valved-stent released at the level of the annulus. Reproduced from Abdel-Sayed $\mathrm{P}$, Kalejs M, von Segesser LK. A new training set-up for trans-apical aortic valve replacement. Interac CardioVasc Thorac Surg 2009; 8: 599-601, with permission of the European Association of Cardio-Thoracic Surgery. 


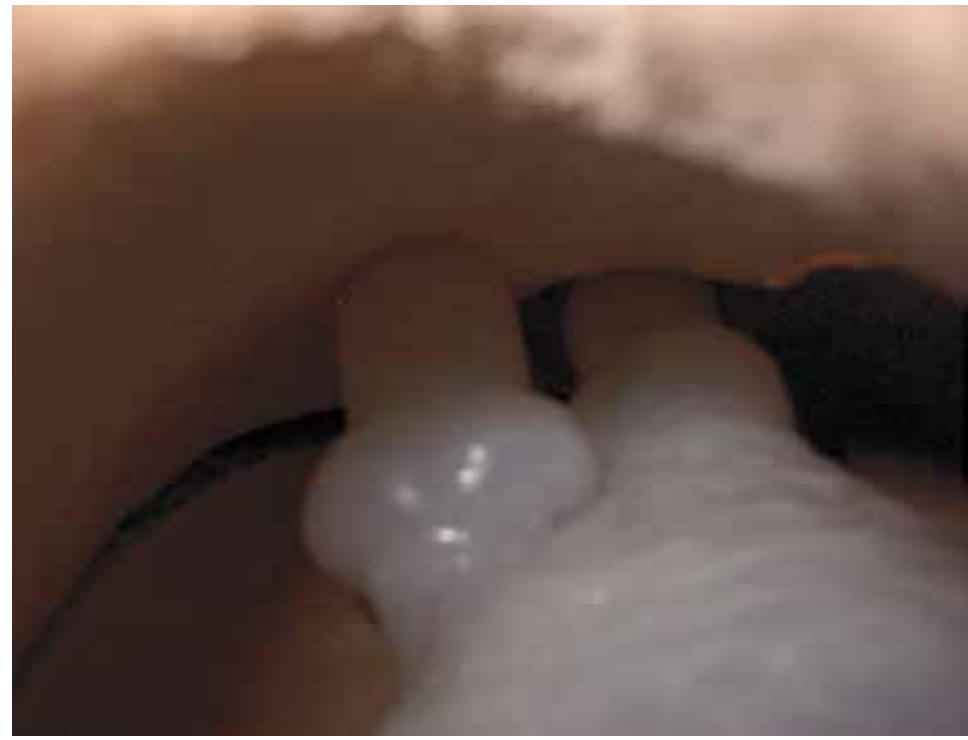

Fig. 9. View of the model valves through the manikin sternotomy.

Another major limitation of rapid prototyping comes from the costs for the generation of 3D hearts. Up to now, a use of rapid prototyping at large scale for surgical planning or training does not seem to be justified since standard planning procedures are in most of the cases satisfactory, though in some complex cases, additional costs of RP may be compensated by reduced operating times and higher success rate of the surgical procedure. The time needed for producing a patient-specific solid heart also limits its use in surgery to specific cases and is not appropriate for emergency cases.

Finally, the conviction of surgeons by the utility of these models in cardiovascular surgery still remains weak for some of them, because those models cannot mimic critical aspects such as bleeding and complications that surgeons might encounter during operation. Also, sometimes the flexibility, durability and texture of materials are not satisfactory to mimic heart and great vessels.

\section{Other applications and perspectives of rapid prototyping in cardiology}

Artificial heart models are not only useful for surgical training but they are also serving in the preparation of patient-specific cardiac implants (e.g. Titanium merged by laser for mitral valves rings). This customization of implant might be sometimes necessary for patients whose disease involves special requirements, or need implant size that is out of the standard range. Indeed, only $15 \%$ percent of the cases requiring percutaneous pulmonary valves implantations were apt with current devices, because of the wide variety of site morphology, size and dynamics (Capelli, Taylor et al. 2010). This percentage of patients, who could benefit from minimally invasive procedure such as percutaneous pulmonary valves implantations, can be improved with new-valved stent graft that would be designed on biomodels. Improved surgical outcome because of adequate match with individual anatomical needs emphasize the evolution towards a more patient's specific implant design that can be performed on biomodels reconstructed from images taken from the patient himself. 
Furthermore, heart biomodeling might be useful for the design of preformed 3D patches that would be used in cardiomyoplasty to correct complex malformations (Tsuchikane, Taketani et al. 2008). Also, for endovascular procedures more and more common (Althoff, Knebel et al. 2008), measurements taken on prototypes corresponding to patients could open new perspectives. For the planning procedure of these interventions, the assessment of collisions problems as well as the adequacy of customed implants might be performed on heart models (Harrison, Estefan-Ventura et al. 2007). Finally, issues related to growth that could be simulated with computational fluid dynamics and validated with in vitro tests performed solid prototypes.

Maybe the most promising medical perspective for rapid prototyping is the so-called "bioprinting" of heart structures. Bioprinting combine the deposition of biomaterials with cells into spatial orientations and complexities that physiologically emulate the organ geometries. This has been already initiated to bioprint vessel-like constructs using hyaluronan hydrogels crosslinked with polyethylene glycol tetracrylates (Skardal, Zhang et al. 2010). The overarching goal of this application of rapid prototyping in creating scaffolds for cellular growth and tissue engineering would be to generate a whole functional and living organ adapted to the individual patient anatomy and needs. Organ engineering would overcome the human organ deficiency for transplantation, and would allow building complex in-vitro tissue models promoting drug discovery (Boland, $\mathrm{Xu}$ et al. 2006; Mironov, Kasyanov et al. 2008). However, further research and development are needed until functional and viable tissues or organs can be created.

\section{Conclusions}

Rapid prototyping is a promising technique with numerous applications in cardiovascular surgery ranging from surgical training to designing of implants. Currently, research is in going to improve the limitations of this technique, which does not allow yet a common use in clinical practice. Nevertheless, the vast potential of this technique promises a growing use and development, not only in cardiovascular surgery, but also in other biomedical fields and academic activities.

\section{References}

Abdel-Sayed, P., M. Kalejs, et al. (2009). "A new training set-up for trans-apical aortic valve replacement." Interact Cardiovasc Thorac Surg 8(6): 599-601.

Althoff, T. F., F. Knebel, et al. (2008). "Long-term follow-up of a fenestrated Amplatzer atrial septal occluder in pulmonary arterial hypertension." Chest 133(1): 283-285.

Armillotta, A., P. Bonhoeffer, et al. (2007). "Use of rapid prototyping models in the planning of percutaneous pulmonary valved stent implantation." Proceedings of the Institution of Mechanical Engineers Part H-Journal of Engineering in Medicine 221(H4): 407-416.

Boland, T., T. Xu, et al. (2006). "Application of inkjet printing to tissue engineering." Biotechnol J 1(9): 910-917.

Capelli, C., A. M. Taylor, et al. (2010). "Patient-specific reconstructed anatomies and computer simulations are fundamental for selecting medical device treatment: application to a new percutaneous pulmonary valve." Philos Transact A Math Phys Eng Sci 368(1921): 3027-3038. 
Gasparovic, H., F. J. Rybicki, et al. (2005). "Three dimensional computed tomographic imaging in planning the surgical approach for redo cardiac surgery after coronary revascularization." European Journal of Cardio-Thoracic Surgery 28(2): 244-249.

Harrison, M. R., D. Estefan-Ventura, et al. (2007). "Magnetic Mini-Mover Procedure for pectus excavatum I. Development, design, and simulations for feasibility and safety." Journal of Pediatric Surgery 42(1): 81-86.

Jacobs, S., R. Grunert, et al. (2008). "3D-Imaging of cardiac structures using 3D heart models for planning in heart surgery: a preliminary study." Interact Cardiovasc Thorac Surg 7(1): 6-9.

Kalejs, M. and L. K. von Segesser (2009). "Rapid prototyping of compliant human aortic roots for assessment of valved stents." Interact Cardiovasc Thorac Surg 8(2): 182186.

Kellenberger, C. J., S. J. Yoo, et al. (2007). "Cardiovascular MR imaging in neonates and infants with congenital heart disease." Radiographics 27(1): 5-U1.

Lermusiaux, P., C. Leroux, et al. (2001). "Aortic aneurysm: Construction of a life-size model by rapid prototyping." Annals of Vascular Surgery 15(2): 131-135.

Markert, M., S. Weber, et al. (2007). "A beating heart model 3D printed from specific patient data." Conf Proc IEEE Eng Med Biol Soc 2007: 4472-4475.

Mironov, V., V. Kasyanov, et al. (2008). "Organ printing: promises and challenges." Regen Med 3(1): 93-103.

Olson, C. W., D. Lange, et al. (2007). "3D Heart: a new visual training method for electrocardiographic analysis." J Electrocardiol 40(5): 457 e451-457.

Ou, P., D. S. Celermajer, et al. (2007). "Three-dimensional CT scanning: a new diagnostic modality in congenital heart disease." Heart 93(8): 908-913.

Peltola, S. M., F. P. Melchels, et al. (2008). "A review of rapid prototyping techniques for tissue engineering purposes." Ann Med 40(4): 268-280.

Rengier, F., A. Mehndiratta, et al. (2010). "3D printing based on imaging data: review of medical applications." Int J Comput Assist Radiol Surg 5(4): 335-341.

Riesenkampff, E., U. Rietdorf, et al. (2009). "The practical clinical value of three-dimensional models of complex congenitally malformed hearts." Journal of Thoracic and Cardiovascular Surgery 138(3): 571-580.

Shiraishi, I., M. Yamagishi, et al. (2010). "Simulative operation on congenital heart disease using rubber-like urethane stereolithographic biomodels based on 3D datasets of multislice computed tomography." European Journal of Cardio-Thoracic Surgery 37(2): 302-306.

Skardal, A., J. X. Zhang, et al. (2010). "Bioprinting vessel-like constructs using hyaluronan hydrogels crosslinked with tetrahedral polyethylene glycol tetracrylates." Biomaterials 31(24): 6173-6181.

Sodian, R., S. Weber, et al. (2008). "Pediatric cardiac transplantation: Three-dimensional printing of anatomic models for surgical planning of heart transplantation in patients with univentricular heart." Journal of Thoracic and Cardiovascular Surgery 136(4): 1098-1099.

Spevak, P. J., P. T. Johnson, et al. (2008). "Surgically corrected congenital heart disease: Utility of 64-MDCT." American Journal of Roentgenology 191(3): 854-861.

Tsuchikane, E., S. Taketani, et al. (2008). "A novel catheter system for percutaneous intracoronary artery cardiomyoplasty." J Invasive Cardiol 20(7): 357-360.

Wagner, J. D., B. Baack, et al. (2004). "Rapid 3-dimensional prototyping for surgical repair of maxillofacial fractures: A technical note." Journal of Oral and Maxillofacial Surgery 62(7): 898-901. 


\title{
Rapid Prototyping in Biomedical Engineering
}

\author{
Kentaro Iwami and Norihiro Umeda \\ Tokyo University of Agriculture and Technology \\ Japan
}

\section{Introduction}

Tissue engineering has attracted great attention as a key technology for realizing regenerative medicine (Lysaght and Reyes, 2001, Ringe, et al., 2002). In particular, tissue and organ repair are a possible applications owing to recent advances such as the development of induced pluripotent stem (iPS) cells (Takahashi and Yamanaka, 2006). Cell culture on a 3dimensional extracellular matrix (3D ECM) is required to study differentiation of stem cells into various types and achieve in vitro tissue repair (Cukierman, et al., 2001, Stevens and George, 2005). Therefore, 2D and 3D patterning technologies for developing scaffolds are increasingly required in tissue engineering.

In this context, the role of rapid prototyping $(\mathrm{RP})$ in medicine and biomedical engineering is greatly expanding. Highly precise and fine scaffold patterning has been achieved not only by photolithography (Leclerc, et al., 2004) but also by several RP methods including 3D printing (Kim, et al., 2008, Landers, et al., 2002, Nakamura, et al., 2005, Roth, et al., 2004). In most of these technologies, 2D or 3D scaffold patterns are batchwise or sequentially fabricated, cells are subsequently cultured on the scaffold pattern, and cell viability in the mixture of patterns is evaluated. To investigate the process of tissue repair, interactions between various types of cells on 3D structures have been studied. The pattern complexity of these structures has been found to be increasing.

In biomedical engineering, not only tissue engineering but also the developments of implantable and other prostheses are important applications of RP technology. In biomedical engineering. Commencing with inlays, many implantable prostheses such as total joint prostheses and dental prostheses have been realized based on RP technology. Furthermore, bone regeneration is one of the principal applications of tissue engineering. RP of hydroxyapatite (HAp) is expected to play a significant role in this field. As described above, the application of RP in biomedical engineering is greatly expanding. In this chapter, some applications of RP in medicine and biomedical engineering fields and a systematic collection of the latest achievements in these areas have been described.

\section{Materials used in RP techniques for biomedical applications}

This section provides a review of the materials used in RP techniques for biomedical applications. For example, hydrogel is one of the important materials used for cell culture. Some advanced biomaterials such as thermoreversible hydrogels have also been discussed below. Ceramics are also used for orthopedic implants. Especially, porous composites such as HAp are expected to be used for bone tissue regeneration. Furthermore, cells themselves are directly printed or patterned through RP techniques. 


\subsection{Hydrogels}

Hydrogels are a water-containing networks of water-insoluble polymer chains. They are also referred as aquagels and can absorb a huge amount of water. Of note, a hydrogel can contain more than $99 \%$ water by weight. By modifying the polymer network, various functionalities of a hydrogel such as biocompatibility and stimulus responsibility can be realized. These hydrogels are often referred as "Smart gels" (Chaterji, et al., 2007) or "Intelligent gels"(Osada and Ross-Murphy, 1993). A hydrogel is widely used for embedding biological samples and/or pharmaceutical agents ecause it is a soft and wet material.

One of the most important applications of hydrogels in tissue engineering is their use as a scaffold for cell culture (Drury and Mooney, 2003, Nowak, et al., 2002). Many natural materials such as agarose, alginate and gelatine are used to produce bioactive materials (Awad, et al., 2004, Ladet, et al., 2008, Raghunath, et al., 2007). Natural materials as well as synthetic polymers are used as biocompatible hydrogels. Fabrication and shaping of the hydrogel scaffold is strongly demanded in tissue engineering (Landers, et al., 2002).

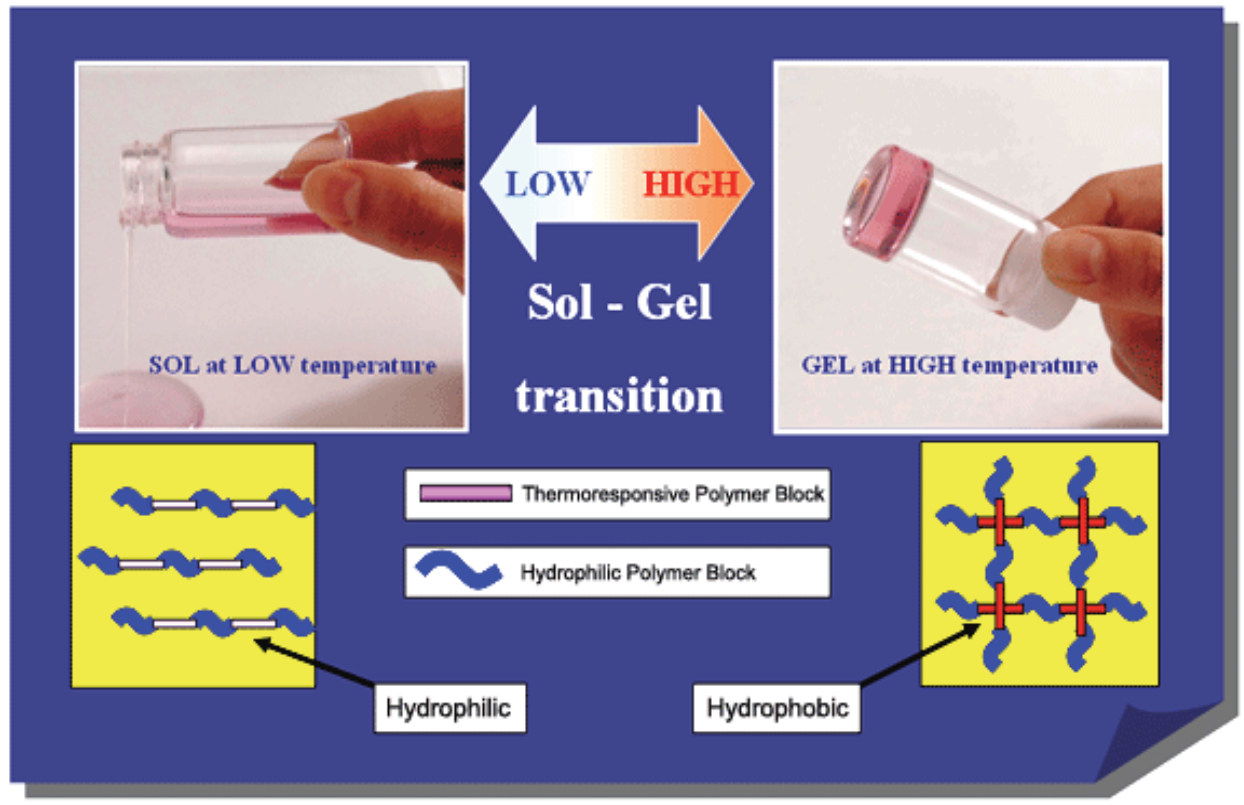

Fig. 1. Mebiol Gel

\subsubsection{Thermoreversible hydrogels}

Some natural hydrogels such as agarose and gelatin are in a gel state at low temperature, and their transition to the sol state occurs with heating. This property is often unsuitable for tissue engineering because cultured tissues or organs may be damaged during peeling or recovery. To solve this problem, a thermoreversible hydrogel, Mebiol ${ }^{\circledR}$ gel (Mebiol Inc., Kanagawa Japan) has been used (Yoshioka, et al., 1998). The Mebiol® gel MB-10 is composed of $\mathrm{N}$-isopropylamide and polyoxyethylene.

Fig. 1 shows MB-10 in sol and gel states. The sol-gel transition temperature of MB-10 is $22^{\circ} \mathrm{C}$. The sol state temperature of the gel is below $15^{\circ} \mathrm{C}$. Its viscosity slightly increases with temperature. The sol-gel transition of MB-10 has very little thermal hysteresis. MB-10 is 
supplied as a sterile freeze-dried powder ( $1 \mathrm{~g}$ in a glass vial), which is dissolved in $10 \mathrm{ml}$ of sterile water or culture medium.

\subsection{Ceramics}

Ceramics have been widely used for biomedical applications for a long time. The most common application of ceramics is a mold for fabrication of metal alloy implants. RP has played an important role in this application. A 3-D printed ceramic mold and cast metal alloy used for knee prosthesis are typical examples (Curodeau, et al., 2000).

In addition to molds, ceramics have recently been attracting great interest for use in clinical implants because of increased awareness of the problems associated with metal implants such as corrosion, ion elution, and fatigue cracking (Clarke, 1992). In contrast, ceramics have superior characteristics of chemical stability, bioinertia (alumina and zirconia), bioactivity (HAp), and porosity for tissue ingrowth (HAp and alumina) (Hench, 1991).

Both hard and porous ceramics are used as implants. The most representative examples of the former are alumina and zirconia. Materials containing these compounds such as zirconia-toughened alumina (ZTA) or yttria-stabilized zirconia are also used (He, et al., 2008, Manicone, et al., 2007). Because of their extreme hardness and scratch resistance, alumina and zirconia are often used as loaded implants such as total joint prosthesis and dental prosthesis prosthesesas described in Section 4.1. These hard ceramics are used in bulk, beads, powders, or as coatings (De Aza, et al., 2002, Dorlot, et al., 1989, Oonishi, et al., 2002). Alumina powder is used to form porous structures by sintering packed a powder with proper binder such as silica (Maca, et al., 2001).

\subsubsection{Porous ceramics and composites}

Because alumina ceramic itself is not bioactive, bone ingrowth is difficult to achieve and implant anchoring becomes challenging. Porous structures and bioactive coatings are often adopted to solve this problem. HAp is one of the most commonly used bioactive coating materials because of its porous structure, and a HAp coating provides favorable sites for cell attachment (Bose, et al., 2002).

\section{RP methods}

In this section, RP techniques used for biomedical applications have been described. Selective laser sintering (SLS) is used in the development of metal or ceramic implants. Stereolithography (STL) is used to develop 3D structures through both top-down and bottom-up approaches. Many materials are available for fused deposition modeling (FDM) and 3D printing. Some advanced methods such as tissue engineering assisted by laser (TEAL), inkjet-based 3D printing and a combination of extrusion/aspiration/refilling have also been described below.

\subsection{Selective laser sintering}

Selective laser sintering (SLS) is a method used to sinter thin layers of powdered polymeric or ceramic materials to form solid $3 \mathrm{D}$ objects by laser irradiation. $\mathrm{CO}_{2}$ or YAG lasers are often used. In this method, the object is fabricated layer-by-layer from slices of 3D CAD data files. During single layer fabrication, the laser beam is selectively scanned over the powder surface, following following the designing of cross-sectional profiles by the CAD slice. When the powder is irradiated by the laser, the powder temperature increases and reaches 
its melting point. The particles are then fused together and this layer is defined. Subsequent layers are fabricated on the top of previous layers.

The type of material fabricated by SLS ranges from polymers to ceramics. Calcium phosphates including HAp are the best example of the former (Duan and Wang, 2010, Tan, et al., 2003). Other polymers such as polyetheretherketones (PEEK), polylactides and polycaprolactones are also fabricated by SLS (Bukharova, et al., 2010, Williams, et al., 2005). Alumina is an important target for SLS (Maca, et al., 2001). Recently, composites of these materials have been widely studied because they can combine the characteristic advantages of existing materials (Chua, et al., 2004, Eosoly, et al., 2010).

\subsection{Stereolithography}

Stereolithography is a method used to produce a 3D structure of a UV- or photocurable resin. In this method, similar to SLS, the object is fabricated layer-by-layer. In general, this method has higher accuracy than that of SLS (Melchels, et al., 2010). The range of materials that can be used in this method is not limited to resin. Ceramic 3D structures can be formed by dispersing ceramic particles in resins (Doreau, et al., 2000). This process also offers the ability to form $3 \mathrm{D}$ ceramic structures.

\subsection{Fused deposition modeling and 3D printing}

Fused deposition modelling (FDM) is an RP method based on extrusion of filament through a nozzle. A 3D structure is produced by traversing the nozzle over a substrate. This method is often applied in the manufacturing of scaffolds used in tissue engineering (Zein, et al., 2002). Fig. 3 shows a schematic illustration of scaffold manufacturing by FDM.

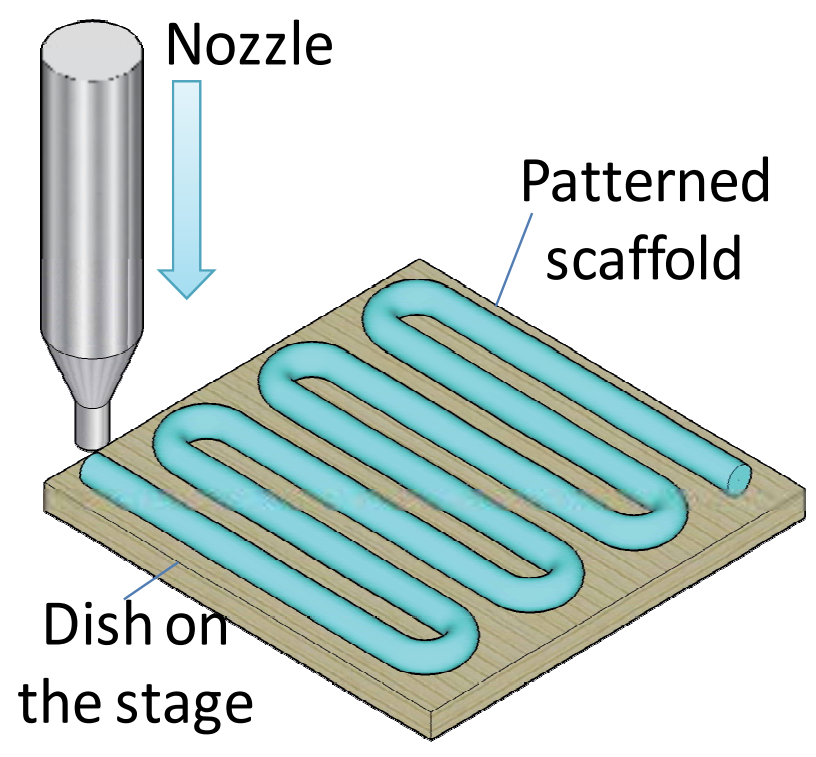

Fig. 3. Schematic of scaffold patterning based on FDM

Polymers are often used for FDM in bioengineering (Hutmacher, et al., 2001). This method can also be used to form polymer-ceramic composites (Kalita, et al., 2003). 


\subsection{Advanced printing methods}

Printing technology is widely used in biomedical engineering. Novel applications of printers in this field include printing images of cells and organs. Conventional inkjet technologies are now applied to cell printing, and patterning and seeding of viable cell have been realized (Mironov, et al., 2003, Nakamura, et al., 2005, Roth, et al., 2004). In these methods, not only $2 \mathrm{D}$ but also 3D printing of cell images has been realized using the layer-by-layer scheme. Laser printing is also applied to create cell patterns with higher throughput using pulsed IR laser (Guillemot, et al., 2010).

Another important application of printing technology in this field is 3D printing of hard materials. Porous ceramic scaffolds manufactured by 3D printing are expected to have applications in bone replacement (Seitz, et al., 2005). This method is also applied to fabricate molds for orthopedic implants (Curodeau, et al., 2000).

\section{Advanced FDM by extruding/aspirating/refilling of thermoreversible hydrogels}

As long as injection- or extrusion-based technologies are used in conventional FDM using hydrogels, it is difficult to locally remove or modify cultured cells. Furthermore, generation of cell patterns with a large-area is difficult because of the low throughput of extrusion patterning. A combination of extrusion and aspiration could provide a solution to this problem. The patterning throughput for a large-area cell is increased by adopting aspiration technology. Furthermore, local modification of the pattern is possible by refilling the previously aspirated groove with a cell-scaffold mixture. This system could have a wide range of applications in tissue engineering. However, such technology has not yet been reported.

To solve this problem, a novel cell-tissue RP method has been developed using a cellscaffold mixture (Iwami, et al., 2010). This method offers both extrusion of the mixture and aspiration on the basis of Bernoulli suction; extrusion and aspiration can be easily interchanged. This system has broad applications in cell patterning. For example, cell patterns can be filled into another cell matrix. Fig. 4 shows a conceptual drawing of the system.
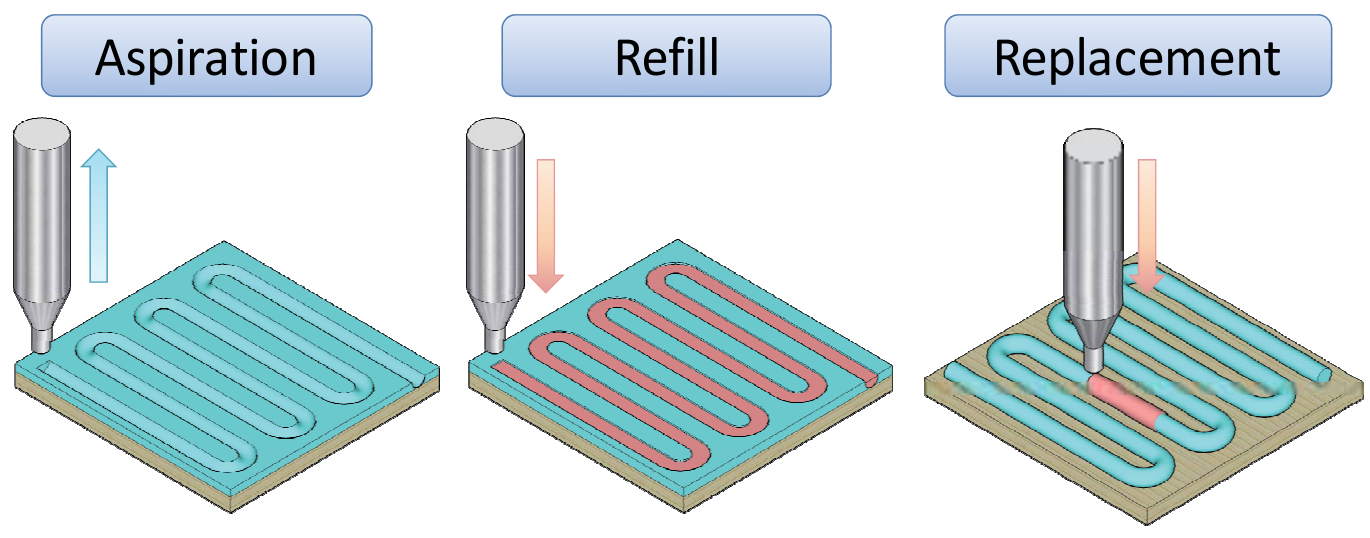

Fig. 4. Conceptual drawing of FDM based on extruding/aspirating/refilling of a thermoreversible hydrogel 
Here we show scaffold patterning by combining extrusion and aspiration of a thermoreversible hydrogel, and a culture of Sf-9 insect cells mixed in the patterned gel is illustrated.

\subsection{Hydrogel extruding/aspirating/refilling system}

The apparatus for the gel patterning system is shown in Fig. 5. The system is based on RP. The nozzle and substrate are placed in an incubator box with controlled internal temperature and humidity. Substrate temperature on the 3D stage can be controlled between room temperature and $90^{\circ} \mathrm{C}$.

MB-10 solution is dispensed from a metal nozzle. The solution in the nozzle is retained in the sol state by maintaining the temperature of the nozzle below $10^{\circ} \mathrm{C}$ using an integrated cooler, which consists of a heat sink, a heat pipe, and a Peltier device (UT3030CE-M, 27W; VICS Co., Tokyo, Japan). The Peltier controller (VPE-10, VICS Co.) maintains the temperature of the device between $-10^{\circ} \mathrm{C}$ and $50^{\circ} \mathrm{C}$. In this system, other materials can be used by replacing the nozzle. Because the temperature of this Peltier device ranges from $-40^{\circ} \mathrm{C}$ to $150^{\circ} \mathrm{C}$, if the Peltier controller is replaced, other materials that require a higher solgel transition temperature (i.e., agar) can be used.

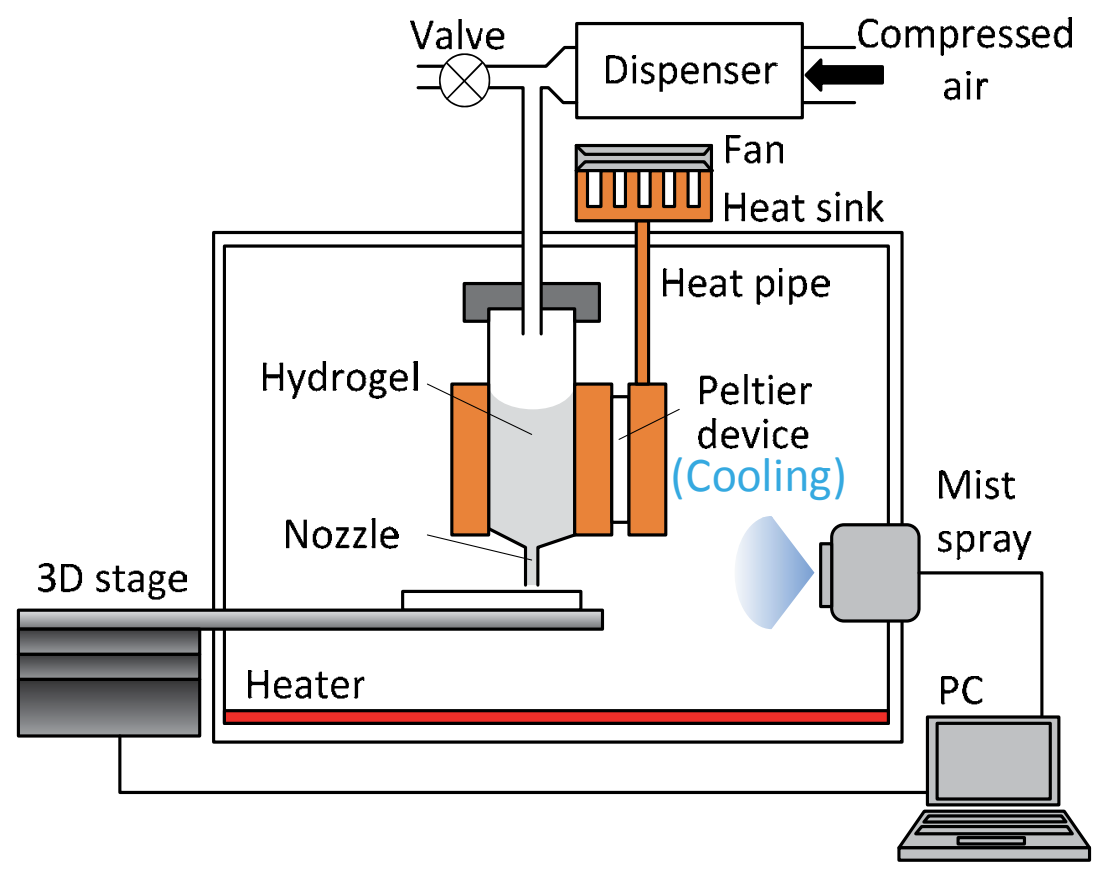

Fig. 5. Experimental setup for the FDM system based on extruding/aspirating/refilling

The bottom of the nozzle is connected to the dispenser and valve. The dispenser regulates the pressure of the compressed air, controlling the air flow. A 0.01- $\mu$ m air filter (NHM-T8, Kitz Microfilter Co., Tokyo Japan) is placed before the dispenser.

This system operates in 3 modes: extrusion, aspiration, and refilling. These modes are toggled by closing or opening the valve. When the valve is closed, compressed air is supplied to the nozzle, and the MB-10 solution is extruded to the substrate or refilled into 
the aspirated groove. When the valve is opened, the compressed air is directed away through the valve, and the MB-10solution is aspirated by Bernoulli suction. The substrate is placed below the nozzle, and the required gel patterns are drawn on the substrate using a computer-controlled 3D stage.

In this system, the medium on the substrate is maintained in the gel state by keeping the temperature in the box higher than the sol-gel transition temperature. The temperature control enables immediate sol-gel transition of the solution extruded from the nozzle. In contrast, when the system is operated in the aspiration mode, the medium in the gel state is cooled by the nozzle and reverted to the sol state, which can then be aspirated. In this system, the gel must be prevented from desiccating to maintain cell viability. This is achieved using a mist spray. And a supersaturated atmosphere is maintained inside the box.

A Labview program was developed to control the stage and dispenser. The control procedure for this program is similar to that used for numerical control (NC) machining. The program toggles the dispenser and moves the stage into the given coordinate with the given feed speed. Therefore, the design of the scaffold is given by a table consisting of stage coordinates, feed speeds, and toggle flags. The width of the gel or groove can be controlled by the feed speed of the stage.

\subsection{Experimental}

In the extrusion mode, a metal nozzle with 100- $\mu \mathrm{m}$ diameter (SHN-0.1N, Musashi Engineering Inc., Tokyo Japan) was used. Because this nozzle has a large reservoir diameter and a short tapered tip, the temperature of the nozzle tip can be effectively lowered. The distance between the nozzle and substrate was $100 \mu \mathrm{m}$. The temperature in the incubator box was maintained at $30^{\circ} \mathrm{C}$, and the nozzle was cooled to $12^{\circ} \mathrm{C}$. Straight gel lines with a length of $10 \mathrm{~mm}$ were formed by varying the pressure applied to the nozzle and the feed speed of the stage. A single line was drawn under each condition. The width of each line was measured by laser microscopy. Patterning of planar gel meshes and a square column were demonstrated.

In the aspiration mode, metal nozzles with diameters of 100, 200, and $500 \mu \mathrm{m}$ (SHN series, Musashi Engineering Inc.) and a pulled micropipette with a 50- $\mu \mathrm{m}$ diameter were used. The sample covered with an MG layer was prepared by spin-coating. The coating speed and time were $1000 \mathrm{rpm}$ and $20 \mathrm{~s}$, respectively, and the thickness of the MG layer was $17 \mu \mathrm{m}$. Time variation of aspirated gel weight was measured to evaluate the aspiration speed. Straight grooves of $10 \mathrm{~mm}$ length were formed by aspiration, thereby varying the feed speed. A single groove was drawn under each condition, and the width of the grooves was measured by laser microscopy. Cross-line patterning was demonstrated.

In the refilling mode, a $5 \mathrm{~mm}$ square groove pattern was formed on the MG layer with a thickness of $17 \mu \mathrm{m}$. Then, $10-\mu \mathrm{m}$ diameter glass beads were mixed with MG, and used as a pattern indicator. Beads mixed with MG were refilled into the groove by extruding and tracing the trajectory of aspiration patterning. Refilling of the aspirated grooves with the bead-mixed gel was demonstrated in this mode.

Patterning of the cell-mixed gel was demonstrated in the extrusion mode. A $100-\mu \mathrm{m}$ diameter pulled micropipette was used as the nozzle. An acrylic plate was used as the substrate. The gel pattern was overlaid with $3 \mathrm{ml}$ of the culture medium (Sf-900 III SFM), and its temperature was maintained at $30^{\circ} \mathrm{C}$. The patterned cells were cultured in the incubator. Cell viability in the pattern was evaluated by the trypan blue method. 


\subsection{Results and discussion}

The extruded gel line is shown in Fig. 6. The widths of straight lines formed by extrusion mode was obtained by laser microscopy and is shown in Fig. 7. When the feed speed of the stage was higher than $0.5 \mathrm{~mm} / \mathrm{s}$ with an applied pressure of $30 \mathrm{kPa}$, the lines were patterned intermittently. Except under these conditions, continuous line patterns were formed. As the feed speed of the stage increased, the line width decreased, except under the condition of $50 \mathrm{kPa}$ and $1.67 \mathrm{~mm} / \mathrm{s}$. The line width could be controlled from $114 \pm 15$ to 300 $\pm 25 \mu \mathrm{m}$ by changing the extrusion conditions. The extruded gel was swollen because a 100$\mu \mathrm{m}$ diameter nozzle was used. The die swell ratio for a circular cross-section nozzle is usually expressed as De/D where De and D are the diameters of the extrudate and nozzle, respectively (Liang, 2004). Table 1 shows the relationship between the applied pressure and swell ratio. The die swell ratio increased linearly with increasing applied pressure.

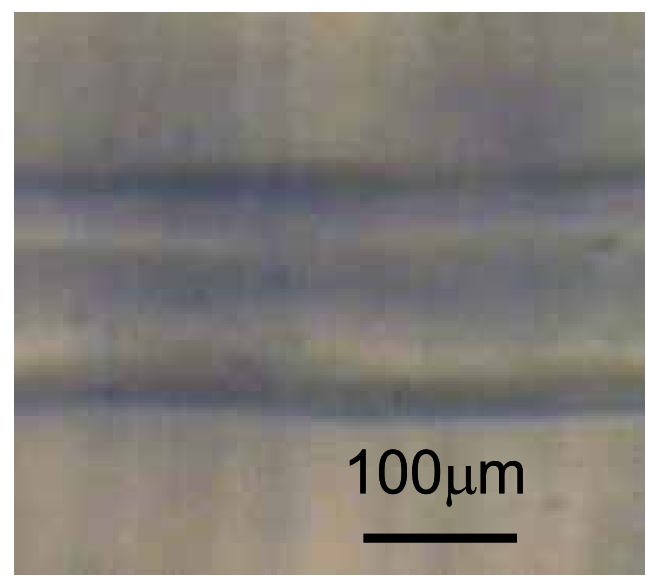

Fig. 6. A gel line extruded through a metal nozzle with $100 \mu$ m diameter.

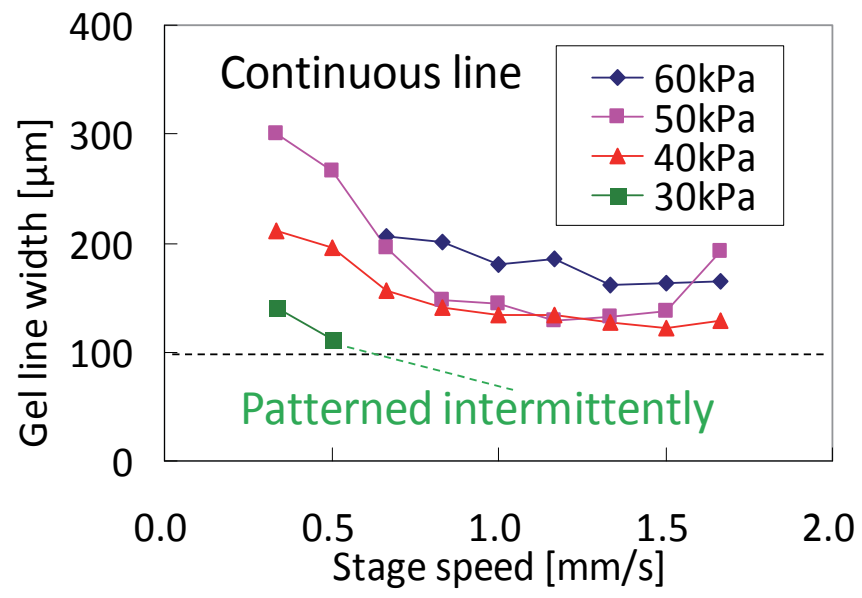

Fig. 7. Width of the extruded gel at different stage speeds and applied pressures (gauge pressure). Error bars indicate standard deviations (S. D.) for each data point $(n=10)$. 


\begin{tabular}{|c|c|c|}
\hline $\begin{array}{c}\text { Applied presssure } \\
(\mathrm{kPa})\end{array}$ & Swell ratio & S. D. \\
\hline 30 & 1.27 & 0.21 \\
\hline 40 & 1.50 & 0.32 \\
\hline 50 & 1.83 & 0.62 \\
\hline 60 & 1.80 & 0.18 \\
\hline
\end{tabular}

Table 1. Relationship between applied pressure and the swell ratio

Mesh patterning was demonstrated. In this experiment, the feed speed and applied pressure were $0.83 \mathrm{~mm} / \mathrm{s}$ and $40 \mathrm{kPa}$, respectively. Under this condition, the linewidth estimated from the straight-line result was $141 \pm 9 \mu \mathrm{m}$. Fig. 8 shows the designed trajectory of the nozzle (a) and the extruded mesh pattern of the gel with a period of $300 \mu \mathrm{m}(\mathrm{b})$. As shown in Fig. 8, gel patterns were well defined. However, the line widths broadened at crossing points. This was considered to be caused by pattern stacking.
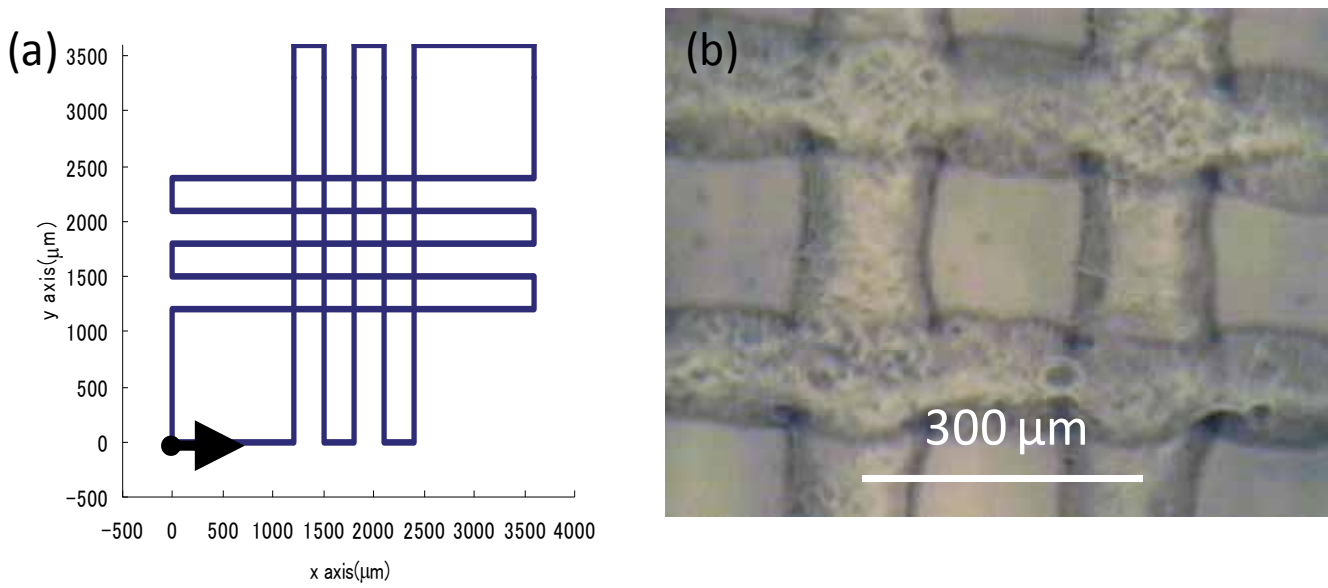

Fig. 8. Designed trajectory of the nozzle (a) and the extruded mesh pattern of the gel with a period of $300 \mu \mathrm{m}(\mathrm{b})$

In the aspiration mode, the output pressure of the dispenser was fixed at $0.2 \mathrm{MPa}$. Time variation of aspirated gel weight with the $500-\mu \mathrm{m}$ diameter nozzle is shown in Table 2 . A constant aspiration speed of $7 \mathrm{mg} / \mathrm{s}$ was obtained. However, with diameters of 50, 100 and $200 \mu \mathrm{m}$, the measured weights were less than $10 \mathrm{mg}$ after 30 minutes of aspiration. Therefore, the $500-\mu \mathrm{m}$ diameter nozzle was chosen for the following aspiration patterning. Fig. 9 shows the trajectory of the nozzle (a) and a close-up photograph of the cross-line aspirated pattern (b).

Fig. 10 shows photographs of refilled patterns of gel-bead mixtures $(a-d)$ and their position in the aspirated 5-mm square groove (e). Glass beads obtained in the pattern indicate successful refilling. The height of the refilled surface is $11 \mu \mathrm{m}$ lower than that of the original gel surface.

Table 3 shows the patterning condition of Sf-9 insect cells produced by extrusion using a $100-\mu \mathrm{m}$ nozzle diameter. A sinusoidal line pattern with a period of $1 \mathrm{~mm}$ was obtained by controlling the stage. Fig. 11 shows the patterned Sf-9 insect cells obtained by extrusion. The 
cell pattern had a width of $300 \mu \mathrm{m}$. The pattern retained its structure while the temperature was maintained above $30^{\circ} \mathrm{C}$. When the substrate was cooled to $10^{\circ} \mathrm{C}$, the gel melted and the cell pattern was dispersed in the culture medium.

\begin{tabular}{|c|c|}
\hline Time (min.) & $\begin{array}{c}\text { Aspirated gel } \\
\text { weight }(\mathrm{mg})\end{array}$ \\
\hline 0 & 0 \\
\hline 5 & 21 \\
\hline 10 & 71 \\
\hline 15 & 97 \\
\hline 20 & 146 \\
\hline 25 & 184 \\
\hline 30 & 233 \\
\hline 35 & 258 \\
\hline 40 & 296 \\
\hline 45 & 308 \\
\hline 50 & 309 \\
\hline
\end{tabular}

Table 2. Time variation of aspirated gel weight
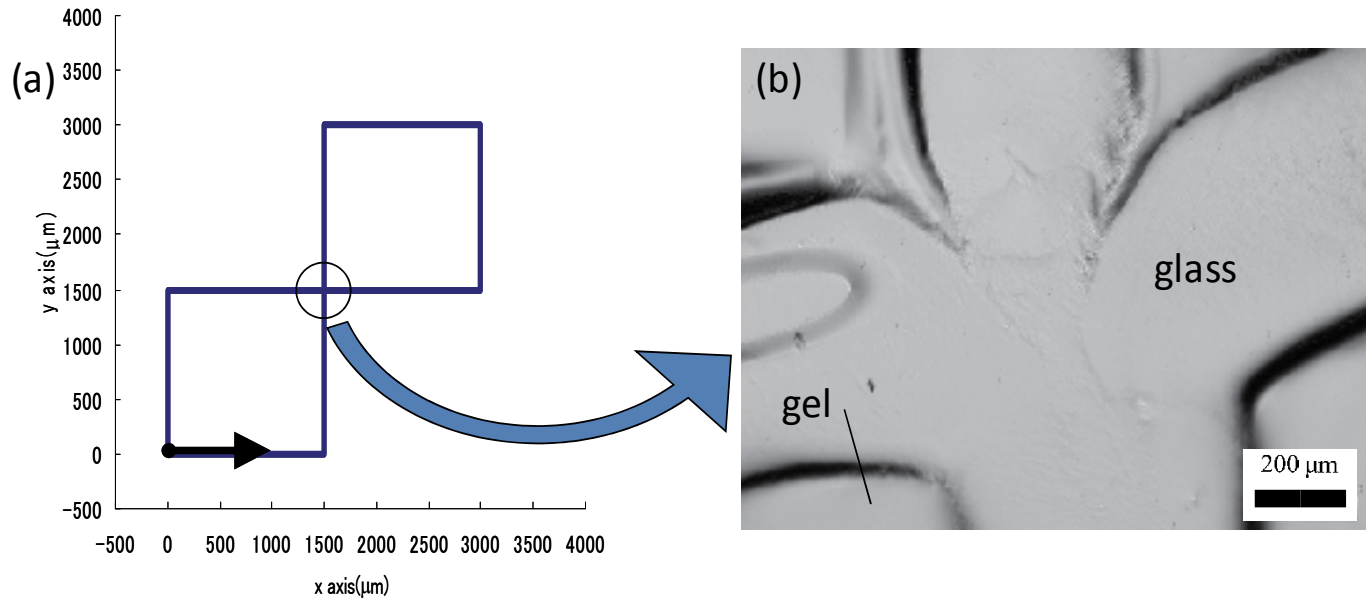

Fig. 9. Designed trajectory of a nozzle (a) and an aspirated cross pattern of a gel developed using a 500- $\mu \mathrm{m}$ diameter nozzle (b).

\subsection{Section conclusion}

An RP system based on extrusion, aspiration, and refilling was developed and proposed. Cell patterning using this system was demonstrated by extrusion of a mixture of cells and thermoreversible hydrogel. In the extrusion mode, the width of the pattern ranged from $114 \pm$ 15 to $300 \pm 25 \mu \mathrm{m}$ using a nozzle of $100 \mu \mathrm{m}$ diameter. In the aspiration mode, grooves with a 
width of $355 \pm 10$ and $636 \pm 21 \mu \mathrm{m}$ were obtained using a nozzle with a diameter of $500 \mu \mathrm{m}$. Refilling of the aspirated groove with gel was also demonstrated. Cell patterning using this system in the extrusion mode was successfully performed. This system offers a novel procedure for the field of tissue engineering. To enhance this system so that it becomes possible to inject/aspirate/refill multiple materials automatically, further challenges, such as construction of a rotary nozzle changing system, need to be addressed.
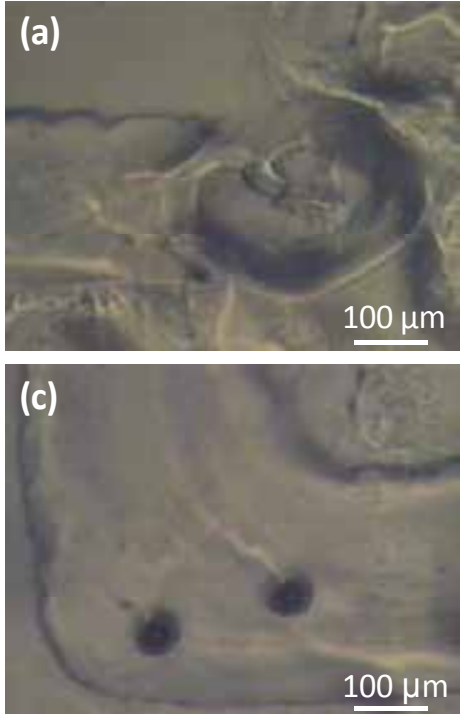

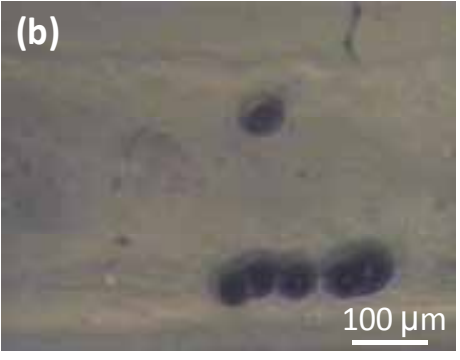

(d)

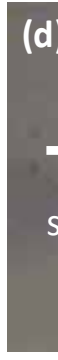

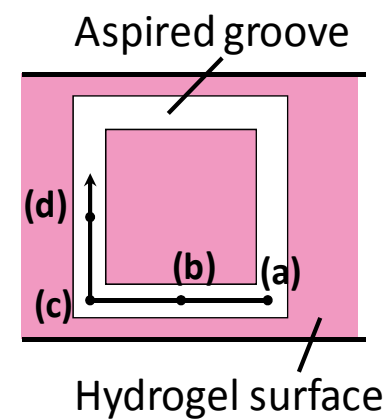

Hydrogel surface

Fig. 10. Photographs of a gel-bead mixture refilled pattern (a-d) and their position in the aspirated groove (e)

\begin{tabular}{|c|c|}
\hline Cell & Sf-9 insect cell \\
\hline Nozzle diameter & $100 \mu \mathrm{m}$ \\
\hline Gel & $\begin{array}{c}\text { Mebiol } \AA, 1 \mathrm{~g} / \mathrm{ml} \\
\text { vial }\end{array}$ \\
\hline Adhesive & $0.1 \%$ gum arabic \\
\hline Binding agent & $\begin{array}{c}0.5 \% \\
\text { methylcellulose }\end{array}$ \\
\hline Spray & $75 \%$ DPBS \\
\hline Medium & SF-900 SFM \\
\hline
\end{tabular}

Table 3. Conditions of Sf-9 insect cell patterning 


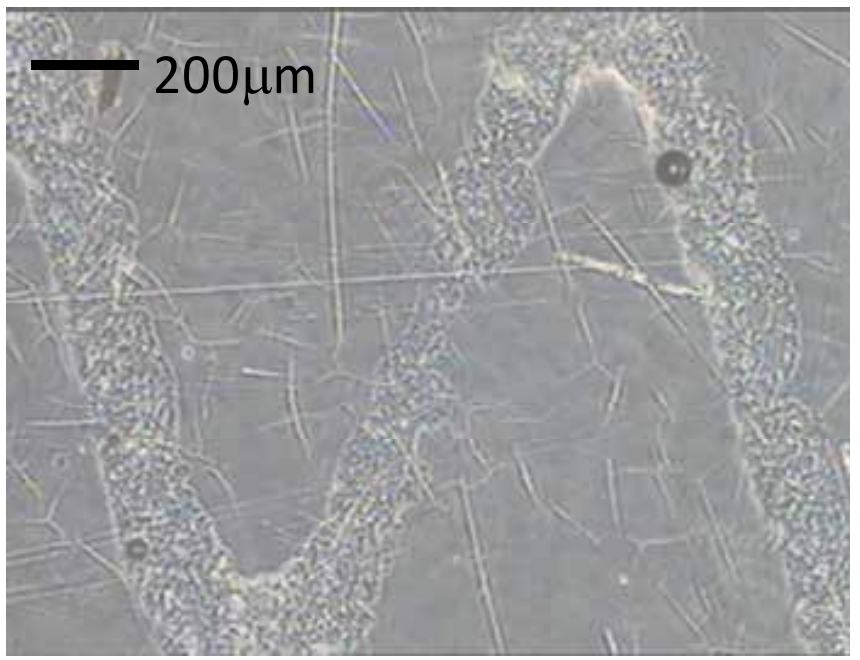

Fig. 11. Sinusoidally patterned Sf-9 insect cells obtained by extrusion

\section{Applications}

Commencing with inlays, alloys are used for implantable prostheses. Recently, in addition to ceramics, these prostheses as bone replacement materials are fabricated through RP technologies. Furthermore, bone regeneration through RP fabrication of Hap scaffolds will be one of the biggest applications of RP in biomedical engineering. Tissue engineering is another important application of RP. Fabrication of 3D scaffolds mimicking the ECM will make great contributions to the fields of regenerative medicine and in vitro organ regeneration.

\section{Conclusion}

$\mathrm{RP}$ in biomedical engineering is attracting great interest. Although this field had been important for a long time, recent advantages and breakthroughs are opening up research frontiers. Because the range of materials has broadened from hard and dry to soft and wet, proper methods should be designed for the application of each type of material.

\section{Acknowledgment}

This work was supported by a Grant-in-Aid for Scientific Research (B) from Japan Society for the Promotion of Science (JSPS).

\section{References}

Awad, H. A., Quinn Wickham, M., Leddy, H. A., Gimble, J. M. \& Guilak, F., (2004). Chondrogenic differentiation of adipose-derived adult stem cells in agarose, alginate, and gelatin scaffolds. Biomaterials, Vol.25, No.16, pp. 3211-3222. 
Bose, S., Darsell, J., Hosick, H. L., Yang, L., Sarkar, D. K. \& Bandyopadhyay, A., (2002). Processing and characterization of porous alumina scaffolds. Journal of Materials Science-Materials in Medicine, Vol.13, No.1, pp. 23-28.

Bukharova, T. B., Antonov, E. N., Popov, V. K., Fatkhudinov, T. K., Popova, A. V., Volkov, A. V., Bochkova, S. A., Bagratashvili, V. N. \& Gol'dshtein, D. V., (2010). Biocompatibility of Tissue Engineering Constructions from Porous Polylactide Carriers Obtained by the Method of Selective Laser Sintering and Bone MarrowDerived Multipotent Stromal Cells. Bulletin of Experimental Biology and Medicine, Vol.149, No.1, pp. 148-153.

Chaterji, S., Kwon, I. K. \& Park, K., (2007). Smart polymeric gels: Redefining the limits of biomedical devices. Progress in Polymer Science, Vol.32, No.8-9, pp. 1083-1122.

Chua, C. K., Leong, K. F., Tan, K. H., Wiria, F. E. \& Cheah, C. M., (2004). Development of tissue scaffolds using selective laser sintering of polyvinyl alcohol/hydroxyapatite biocomposite for craniofacial and joint defects. Journal of Materials Science-Materials in Medicine, Vol.15, No.10, pp. 1113-1121.

Clarke, I. C., (1992). ROLE OF CERAMIC IMPLANTS - DESIGN AND CLINICAL SUCCESS WITH TOTAL HIP PROSTHETIC CERAMIC-TO-CERAMIC BEARINGS. Clinical Orthopaedics and Related Research, No.282, pp. 19-30.

Cukierman, E., Pankov, R., Stevens, D. R. \& Yamada, K. M., (2001). Taking Cell-Matrix Adhesions to the Third Dimension. Science, Vol.294, No.5547, pp. 1708-1712.

Curodeau, A., Sachs, E. \& Caldarise, S., (2000). Design and fabrication of cast orthopedic implants with freeform surface textures from 3-D printed ceramic shell. Journal of Biomedical Materials Research, Vol.53, No.5, pp. 525-535.

De Aza, A. H., Chevalier, J., Fantozzi, G., Schehl, M. \& Torrecillas, R., (2002). Crack growth resistance of alumina, zirconia and zirconia toughened alumina ceramics for joint prostheses. Biomaterials, Vol.23, No.3, pp. 937-945.

Doreau, F., Chaput, C. \& Chartier, T., (2000). Stereolithography for manufacturing ceramic parts. Advanced Engineering Materials, Vol.2, No.8, pp. 493-496.

Dorlot, J. M., Christel, P. \& Meunier, A., (1989). Wear analysis of retrieved alumina heads and sockets of hip prostheses. Journal of Biomedical Materials Research, Vol.23, No.S14, pp. 299-310.

Drury, J. L. \& Mooney, D. J., (2003). Hydrogels for tissue engineering: scaffold design variables and applications. Biomaterials, Vol.24, No.24, pp. 4337-4351.

Duan, B. \& Wang, M., (2010). Customized Ca-P/PHBV nanocomposite scaffolds for bone tissue engineering: design, fabrication, surface modification and sustained release of growth factor. Journal of the Royal Society Interface, Vol.7, pp. S615-S629.

Eosoly, S., Brabazon, D., Lohfeld, S. \& Looney, L., (2010). Selective laser sintering of hydroxyapatite/poly-epsilon-caprolactone scaffolds. Acta Biomaterialia, Vol.6, No.7, pp. 2511-2517.

Guillemot, F., Souquet, A., Catros, S., Guillotin, B., Lopez, J., Faucon, M., Pippenger, B., Bareille, R., Remy, M., Bellance, S., Chabassier, P., Fricain, J. C. \& Amedee, J., (2010). High-throughput laser printing of cells and biomaterials for tissue engineering. Acta Biomaterialia, Vol.6, No.7, pp. 2494-2500. 
He, X., Zhang, Y., Mansell, J. \& Su, B., (2008). Zirconia toughened alumina ceramic foams for potential bone graft applications: fabrication, bioactivation, and cellular responses. Journal of Materials Science: Materials in Medicine, Vol.19, No.7, pp. 2743-2749.

Hench, L. L., (1991). Bioceramics: from concept to clinic. Journal of the American Ceramic Society, Vol.74, No.7, pp. 1487-1510.

Hutmacher, D. W., Schantz, T., Zein, I., Ng, K. W., Teoh, S. H. \& Tan, K. C., (2001). Mechanical properties and cell cultural response of polycaprolactone scaffolds designed and fabricated via fused deposition modeling. Journal of Biomedical Materials Research, Vol.55, No.2, pp. 203-216.

Iwami, K., Noda, T., Ishida, K., Morishima, K., Nakamura, M. \& Umeda, N., (2010). Bio rapid prototyping by extruding/aspirating/refilling thermoreversible hydrogel. Biofabrication, Vol.2, No.1, pp.

Kalita, S. J., Bose, S., Hosick, H. L. \& Bandyopadhyay, A., (2003). Development of controlled porosity polymer-ceramic composite scaffolds via fused deposition modeling. Materials Science \& Engineering C-Biomimetic and Supramolecular Systems, Vol.23, No.5, pp. 611-620.

Kim, G., Son, J., Park, S. \& Kim, W., (2008). Hybrid Process for Fabricating 3D Hierarchical Scaffolds Combining Rapid Prototyping and Electrospinning. Macromolecular Rapid Communications, Vol.29, No.19, pp. 1577-1581.

Ladet, S., David, L. \& Domard, A., (2008). Multi-membrane hydrogels. Nature, Vol.452, No.7183, pp. 76-79.

Landers, R., Hubner, U., Schmelzeisen, R. \& Mulhaupt, R., (2002). Rapid prototyping of scaffolds derived from thermoreversible hydrogels and tailored for applications in tissue engineering. Biomaterials, Vol.23, No.23, pp. 4437-4447.

Leclerc, E., Furukawa, K. S., Miyata, F., Sakai, Y., Ushida, T. \& Fujii, T., (2004). Fabrication of microstructures in photosensitive biodegradable polymers for tissue engineering applications. Biomaterials, Vol.25, No.19, pp. 4683-4690.

Liang, J.-Z., (2004). A relationship between extrudate swell ratio and entry stored elastic strain energy during die flow of tyre compounds. Polymer Testing, Vol.23, No.4, pp. 441-446.

Lysaght, M. J. \& Reyes, J., (2001). The growth of tissue engineering. Tissue Engineering, Vol.7, No.5, pp. 485-493.

Maca, K., Dobsak, P. \& Boccaccini, A. R., (2001). Fabrication of graded porous ceramics using alumina-carbon powder mixtures. Ceramics International, Vol.27, No.5, pp. 577-584.

Manicone, P. F., Rossi Iommetti, P. \& Raffaelli, L., (2007). An overview of zirconia ceramics: Basic properties and clinical applications. Journal of dentistry, Vol.35, No.11, pp. 819-826.

Melchels, F. P. W., Feijen, J. \& Grijpma, D. W., (2010). A review on stereolithography and its applications in biomedical engineering. Biomaterials, Vol.31, No.24, pp. 6121-6130. 
Mironov, V., Boland, T., Trusk, T., Forgacs, G. \& Markwald, R. R., (2003). Organ printing: computer-aided jet-based 3D tissue engineering. Trends in Biotechnology, Vol.21, No.4, pp. 157-161.

Nakamura, M., Kobayashi, A., Takagi, F., Watanabe, A., Hiruma, Y., Ohuchi, K., Iwasaki, Y., Horie, M., Morita, I. \& Takatani, S., (2005). Biocompatible inkjet printing technique for designed seeding of individual living cells. Tissue Engineering, Vol.11, No.11-12, pp. 1658-1666.

Nowak, A. P., Breedveld, V., Pakstis, L., Ozbas, B., Pine, D. J., Pochan, D. \& Deming, T. J., (2002). Rapidly recovering hydrogel scaffolds from self-assembling diblock copolypeptide amphiphiles. Nature, Vol.417, No.6887, pp. 424-428.

Oonishi, H., Clarke, I. C., Good, V., Amino, H., Ueno, M., Masuda, S., Oomamiuda, K., Ishimaru, H., Yamamoto, M. \& Tsuji, E., (2002). Needs of bioceramics to longevity of total joint arthroplasty. Key Engineering Materials, Vol.240, pp. 735-754.

Osada, Y. \& Ross-Murphy, S. B., (1993). Intelligent gels. Scientific American, Vol.268, No.5, pp. 42-7.

Raghunath, J., Rollo, J., Sales, K. M., Butler, P. E. \& Seifalian, A. M., (2007). Biomaterials and scaffold design: key to tissue ]engineering cartilage. Biotechnology and applied biochemistry, Vol.46, No.2, pp. 73-84.

Ringe, J., Kaps, C., Burmester, G.-R. \& Sittinger, M., (2002). Stem cells for regenerative medicine: advances in the engineering of tissues and organs. Naturwissenschaften, Vol.89, No.8, pp. 338-351.

Roth, E. A., Xu, T., Das, M., Gregory, C., Hickman, J. J. \& Boland, T., (2004). Inkjet printing for high-throughput cell patterning. Biomaterials, Vol.25, No.17, pp. 3707-3715.

Seitz, H., Rieder, W., Irsen, S., Leukers, B. \& Tille, C., (2005). Three-dimensional printing of porous ceramic scaffolds for bone tissue engineering. Journal of Biomedical Materials Research Part B-Applied Biomaterials, Vol.74B, No.2, pp. 782-788.

Stevens, M. M. \& George, J. H., (2005). Exploring and Engineering the Cell Surface Interface. Science, Vol.310, No.5751, pp. 1135-1138.

Takahashi, K. \& Yamanaka, S., (2006). Induction of Pluripotent Stem Cells from Mouse Embryonic and Adult Fibroblast Cultures by Defined Factors. Cell, Vol.126, No.4, pp. 663-676.

Tan, K. H., Chua, C. K., Leong, K. F., Cheah, C. M., Cheang, P., Abu Bakar, M. S. \& Cha, S. W., (2003). Scaffold development using selective laser sintering of polyetheretherketone-hydroxyapatite biocomposite blends. Biomaterials, Vol.24, No.18, pp. 3115-3123.

Williams, J. M., Adewunmi, A., Schek, R. M., Flanagan, C. L., Krebsbach, P. H., Feinberg, S. E., Hollister, S. J. \& Das, S., (2005). Bone tissue engineering using polycaprolactone scaffolds fabricated via selective laser sintering. Biomaterials, Vol.26, No.23, pp. 4817-4827.

Yoshioka, H., Mori, Y., Tsukikawa, S. \& Kubota, S., (1998). Thermoreversible gelation on cooling and on heating of an aqueous gelatin-poly ( $\mathrm{N}$ ]isopropylacrylamide) conjugate. Polymers for Advanced Technologies, Vol.9, No.2, pp. 155-158. 
Zein, I., Hutmacher, D. W., Tan, K. C. \& Teoh, S. H., (2002). Fused deposition modeling of novel scaffold architectures for tissue engineering applications. Biomaterials, Vol.23, No.4, pp. 1169-1185. 


\title{
Usage of Rapid Prototyping Technique in Customized Craniomaxillofacial Bone Tissue Engineering Scaffold
}

\author{
Dong Han ${ }^{1}$, Jiasheng Dong1 ${ }^{*}$, De Jun $\mathrm{Cao}^{1}$, Zhe-Yuan Yu1 ${ }^{1}$, Hua Xu1, \\ Gang Chai ${ }^{1}$, Shen Guo-Xiong ${ }^{1}$ and Song-Tao $\mathrm{Ai}^{2}$ \\ ${ }^{1}$ Department of Plastic and Reconstructive Surgery, Ninth People's Hospital, Medical \\ School of Shanghai Jiao Tong University, Shanghai \\ ${ }^{2}$ Department of Radiology, Ninth People's Hospital, Medical School of Shanghai Jiao Tong \\ University, Shanghai, \\ People's Republic of China
}

\section{Introduction}

Congenital defect, posttraumatic asymmetries, and depressive deformities in craniofacial skeleton are common in the craniomaxillofacial surgical practice. In these patients, the injury or hypoplasty zone and precise shape of the implant that will fill the bone defect must be determined preoperatively, and an appropriate estimate of the quantity of bone needed must be made before plastic surgery. A combination of digital medical technology and tissue engineering has shown great promise for repairing these defects.

Computer-aided design (CAD) and computer-aided manufacturing (CAM) systems can manipulate three-dimensional (3D) computed tomographic (CT) images of bone, using a virtual reality force feedback device. Rapid prototyping (RP) is an exceptional innovation in engineering that has been applied to medicine to build models that provide both visual and tactile information. CAD/CAM and RP techniques allow the generation of scaffolds for cell delivery that are custom-made to fit into given bone defects. Craniomaxillofacial bone is irregular and has a subtle 3D structure, and individualized repair of bone defects is very important. CAD, CAM, laser scanning and RP technologies have therefore been applied in craniomaxillofacial surgery.

The scaffolds employed must fit into the anatomical defect and must have sufficient mechanical integrity as well as a controllable degradation rate. The synthetic biodegradable polymers poly (lactic acid) (PLA), poly (glycolic acid) (PGA) and poly (lactic-co-glycolic acid) (PLGA) have attracted much attention in tissue engineering because of their excellent biocompatibility, ease of fabrication into desired shapes, uniform quality and controllable degradation timescales compared to natural macromolecules.

The cells for bone tissue engineering must be available in large numbers and must be able to express the cartilage and bone phenotypes. Bone marrow stem cells (BMSCs) can differentiate into fibroblastic, osteogenic and adipogenic cells. The method for harvesting these cells is well established, and BMSC lines can be readily propagated for long periods without losing their potency. Many BMSCs can be cultured, enabling fabrication of 
transplantable constructs that are composed of appropriate scaffolds with successful BMSC ingrowth.

Based our previous research work, we developed a novel digital medical support system that allows us to manipulate 3D CT images and develop the prototype of a custom-made scaffold for surgical repair of a craniomaxillofacial bone defect in an animal model. In this chapter, we will describe our workflow of the construction of customized craniomaxillofacial bone tissue engineering scaffold with an example of dog's mandibular condyle.

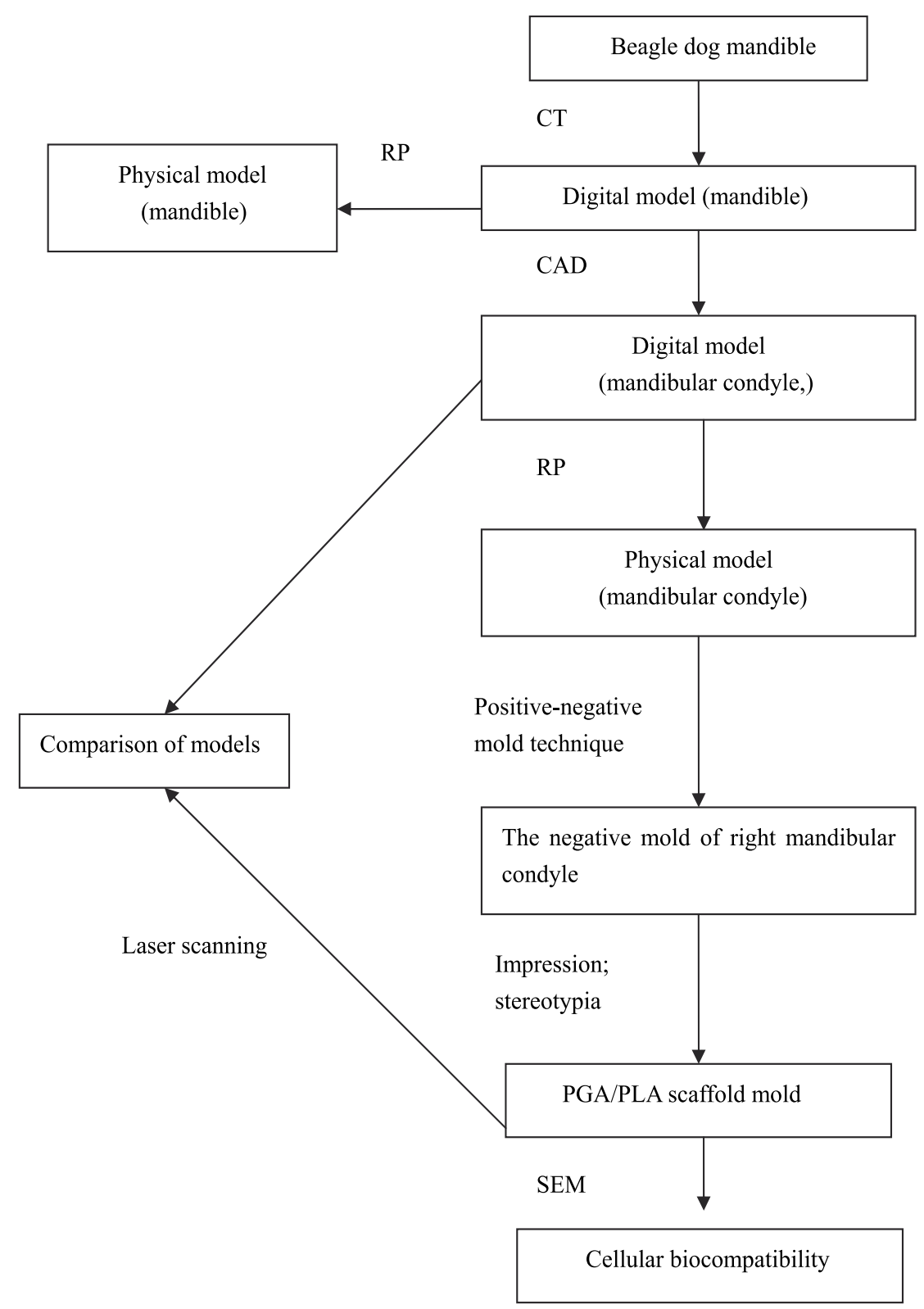

Fig. 1. Flow chart describing the protocol 
In the pilot study, 12-week-old beagle dogs (male, weighing 10-15 kg) were selected. An institutional review committee of Shanghai Jiao Tong University School of Medicine approved all animal study protocols. The animals were examined using 3D CT. For each dog, a model of the mandibular condyle was produced by RP to guide construction of a fitted bone substitute scaffold made of PGA/PLA, which was then seeded with BMSCs. The workflow could be divided into 5 major steps as described in figure 1 .

\section{STEP 1: CAD and RP of the dog mandible}

The mandibles of beagle dogs were examined using a multi-slice CT scanner (GE Medical Systems, Light-Speed16, New York, USA). CT scans were carried out with a slice thickness of $0.625 \mathrm{~mm}(120 \mathrm{kV}, 70 \mathrm{~mA})$. The raw data were exported to Digital Imaging and Communications in Medicine (DICOM) 3.0 format files. With the help of SimMed (PAMI of Shanghai Jiaotong University, Shanghai, China), we imported the formatted images and selected the bone tissue scale (CT threshold, 251-3072) for every slice. A 3D digital model of the mandible (without soft tissue and cartilage) was then reconstructed slice by slice and compiled into a solid geometric model in STL format (Figure 2).

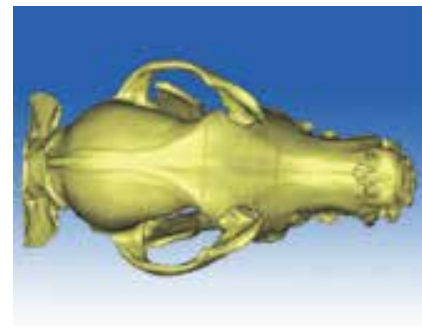

(a)

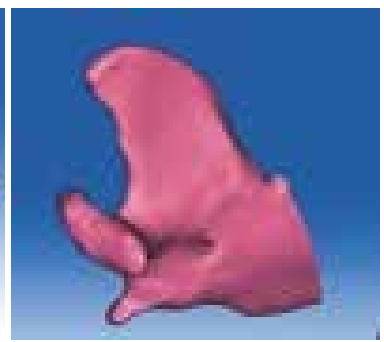

(b)

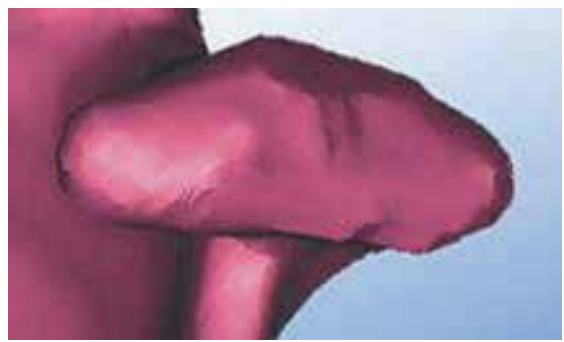

(c)

Fig. 2. Virtual model: (a) 3D reconstruction of the skull; (b) mandibular ramus; and (c) mandibular condyle

The STL files were then imported into Z-Printer 510 (Z Corp Inc., MA, USA). This system enables 3D printing (3D-P) using resin powder. The process consists of a printer-like computer system that directs the deposition of layers of sealant alternating with layers of resin powder (each $0.1 \mathrm{~mm}$ thick), resulting in the fabrication from CAD data of 3D models of the left mandibular condyle, using layer-by-layer manufacturing (Figure 3). Actually, the detail method of 3D outputting was not important. The keynote procedure here was to have the first positive solid model that was same as which we had reconstructed in cyber world.

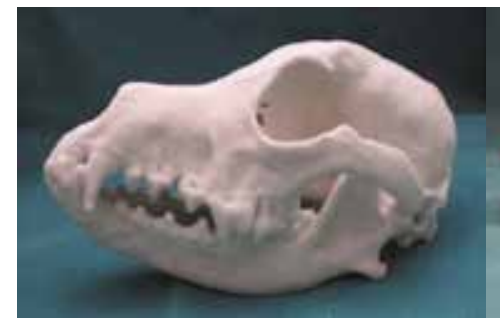

(a)

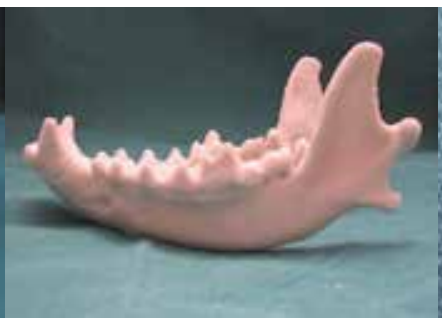

(b)

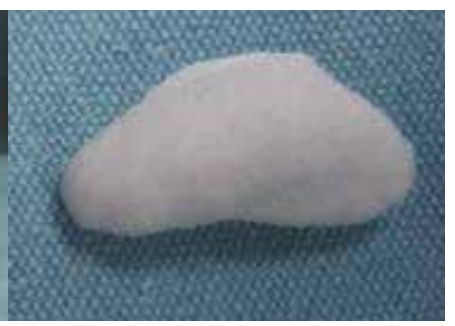

(c)

Fig. 3. (a) Completed RP model: lateral view of the skull. (b) Completed RP model: lateral view of the mandible. (c) Completed RP model: posterior view of the left mandibular condyle 


\section{STEP 2: Fabrication of a negative mould of the mandibular condyle}

The RP model of the mandibular condyle was used as the positive model and was impressed into semisolid-state gypsum or silicone fibrosum, creating a negative mould, which was dried at room temperature. The mould was clamshelled by sectioning two sides and leaving the bottom intact to allow for easy removal of the positive model (Figure 4).

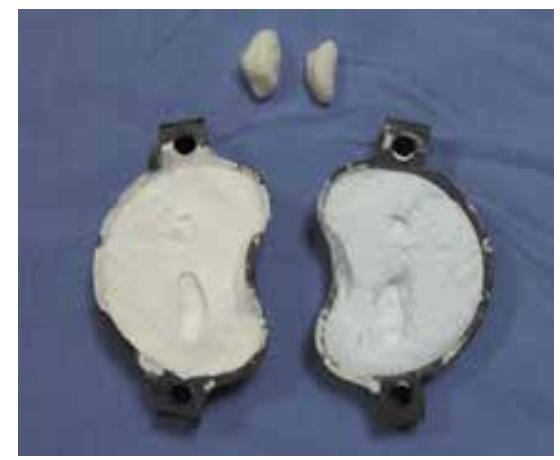

Fig. 4. Negative mould of the left mandibular condyle. gypsum fibrosum mould, open. Rapid prototyped PGA/PLA scaffolds in mandibular condyle bone defect reconstruction

\section{STEP 3: Preparation of the PGA/PLA scaffold}

Unwoven PGA fibres (60 mg; Albany International Research, Albany, NY, USA) were layered into the negative mould and $0.6 \mathrm{ml}$ 1.5\% PLA (Sigma-Aldrich, St. Louis, MO, USA) was diluted in dichloromethane solvent and added to maintain the scaffold shape. The scaffold was then removed from the mould carefully (Figure 5). Then, the geometry accuracy of the scaffold model was needed to be checked.

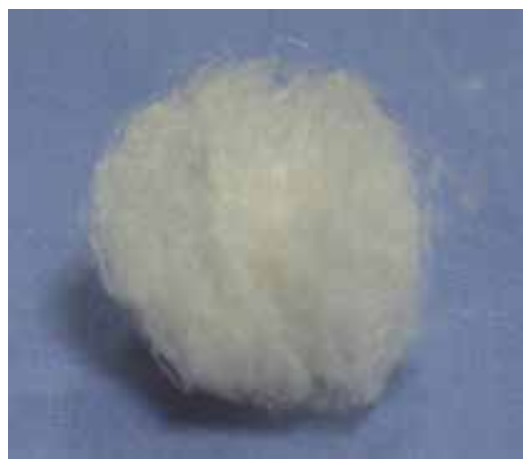

(a)

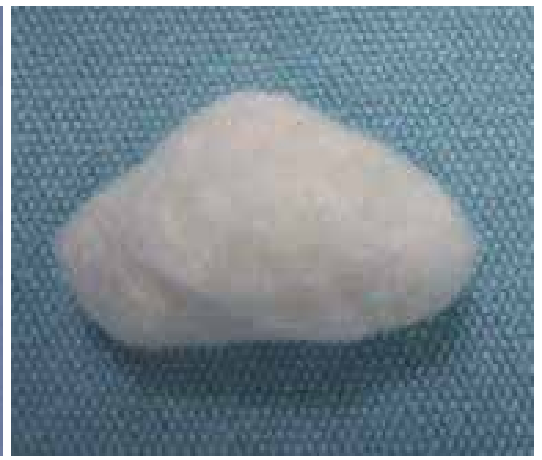

(b)

Fig. 5. (a) Unwoven fibres of PGA. (b) PGA/PLA model scaffold of the left mandibular condyle

\section{STEP 4: Laser scanning of the scaffold model}

A 3D laser surface scanning system (VIVID910 3D, Konica Minolta, Tokyo, Japan) with a Polygon Editing Tool v. 1.03 (Konica Minolta, Tokyo, Japan) was used to acquire the 3D geometric surfaces of the regenerated left mandibular condyle. The scan was performed with a $25 \mathrm{~mm}$ focus camera at a scan distance of $750 \mathrm{~mm}$. Every model was scanned from 
five perpendicular angles, and the scanned images from each model were noise-filtered, smoothed, registered and merged into a single, equidimensional-scale, digital, stereoscopic model in STL format. The left condyle of the CT-reconstructed STL model was put aside as the comparison object. Afterwards, the scan result was finely registered to the CT model using Rapidform2006 (ISUS Ltd, Korea) to match up certain reference points on the geometric surface, ensuring that the two models had the same coordinates. Then, we compared the divergence of the two sample datasets, using the Whole Inspection Model tool in Rapidform2006, which automatically measures the distance ( $\mathrm{mm}$ ) between each corresponding triangle surface on two registered surfaces and displays the measured distances as a coloured chart (Figure 6).

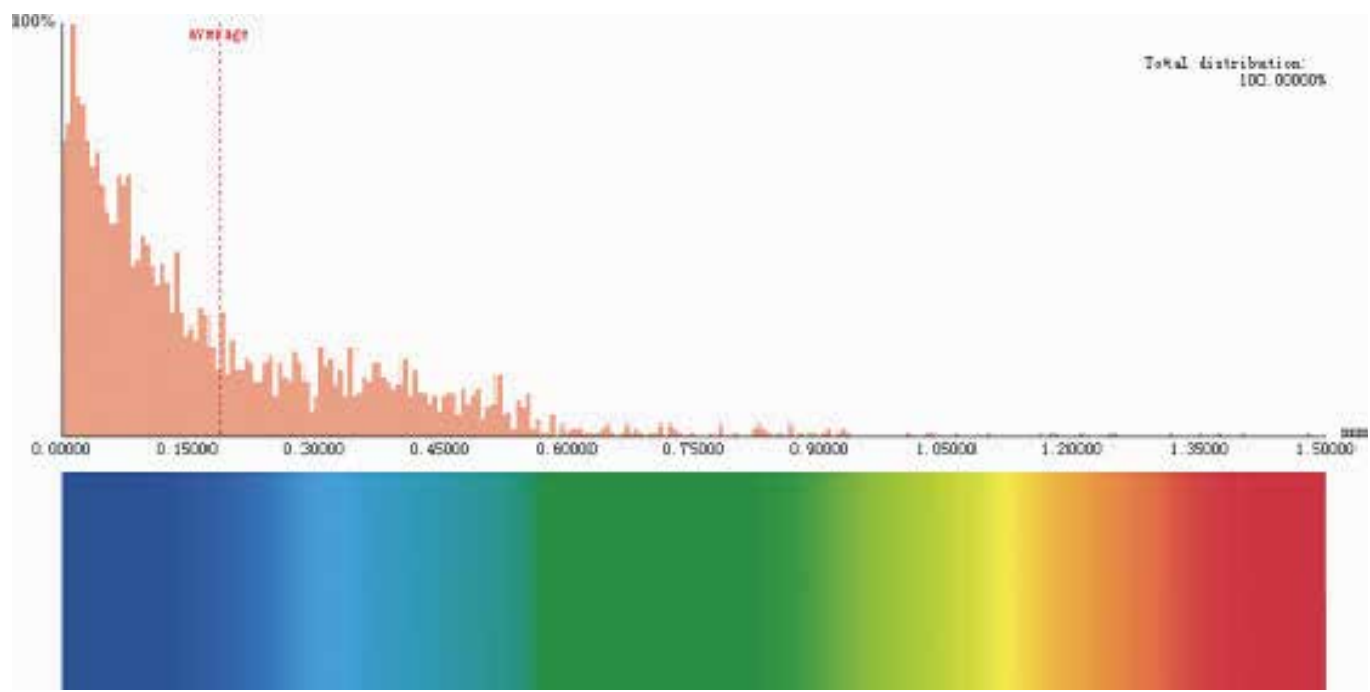

(a)

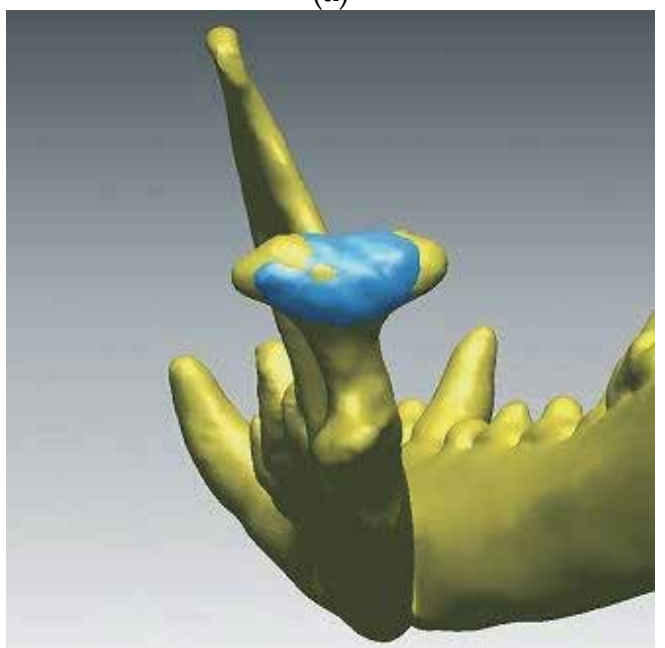

(b)

Fig. 6. (a) Total distribution of scan data. (b) Comparison of the three acquisitions: point clouds obtained by CT and from the laser scanner 


\section{STEP 5: Cellular biocompatibility with the PGA/PLA scaffold in vitro}

The model scaffold was sterilized by soaking in 75\% alcohol and washing three times with phosphate-buffered saline (PBS). This was followed by two washes with Dulbecco's modified Eagle's medium (DMEM; Gibco, USA). Bone marrow aspirates (5 ml) were taken from the iliac crests of beagle dogs. The aspirates were resuspended in $20 \mathrm{ml}$ DMEM with $0.5 \mathrm{ml}$ heparin sodium. The mixture was centrifuged at $1000 \mathrm{rpm}$ for 5 minutes and the supernatant removed. The cell pellets were resuspended, loaded onto Ficoll-Paque PLUS and centrifuged at $1500 \mathrm{rpm}$ for $10 \mathrm{~min}$. Fractions containing low-density mononuclear cells were harvested with a pipette and washed twice with PBS before culturing. The cells were cultured in DMEM supplemented with $10 \%$ fetal bovine serum. The cultures were maintained at $37^{\circ} \mathrm{C}$ in an atmosphere of $5 \% \mathrm{CO}$. BMSCs at passage $2(2.5 \times 107$ cells in 0.5 $\mathrm{ml})$ were evenly dropped onto PGA/PLA scaffolds to form cell/scaffold constructs. These constructs were then cultured in DMEM supplemented with $10 \% \mathrm{FBS}$ at $37^{\circ} \mathrm{C}$ in a $5 \% \mathrm{CO} 2$ atmosphere. After 7 days of incubation, cell attachment and matrix production on the polymer were observed via scanning electron microscopy (SEM; JEOL JSM-6360LV, Japan).

Post-processed 3D geometric models from laser scanning demonstrated that the mean error between the two samples was $<0.3 \mathrm{~mm}$; moreover, at error $<1 \mathrm{~mm}$, confidence remained at $\geq 95 \%$. For each tolerance level, the degree of deviation was shown as a percentage (Table 1 ). The results indicate that the amount of overlap between the two datasets, expressed as percentages for tolerance levels $0.1,0.3,0.5,0.8$ and $1.00 \mathrm{~mm}$, were $54.01 \%, 77.88 \%, 90.26 \%$, $94.83 \%$ and $95.65 \%$, respectively. SEM showed that BMSCs were able to adhere to the scaffold surface and spread and grow along its fibres. Most of these cells had synthesized and organized abundant extracellular matrices, with which they had created bridges between one another.

\begin{tabular}{cccccc}
\hline $\begin{array}{c}\text { Subjects } \\
(\mathrm{n}=8)\end{array}$ & $\begin{array}{c}1.0 \mathrm{~mm} \\
(\%)\end{array}$ & $\begin{array}{c}0.8 \mathrm{~mm} \\
(\%)\end{array}$ & $\begin{array}{c}0.5 \mathrm{~mm} \\
(\%)\end{array}$ & $\begin{array}{c}0.3 \mathrm{~mm} \\
(\%)\end{array}$ & $\begin{array}{c}0.1 \mathrm{~mm} \\
(\%)\end{array}$ \\
\hline Mean & 95.65 & 94.83 & 90.26 & 77.88 & 54.01 \\
SD & 1.47 & 2.13 & 2.78 & 3.44 & 3.31 \\
Maximum & 97.98 & 97.08 & 93.77 & 82.66 & 59.63 \\
Minimum & 93.45 & 90.63 & 85.81 & 71.28 & 49.27 \\
\hline
\end{tabular}

Table 1. Degree of deviation (\%) at $0.1,0.3,0.5,0.8$ and $1.00 \mathrm{~mm}$

In craniomaxillofacial surgery, morphological characteristics of the bone and relevant mechanisms of injury dictating the complexity and variety of the methods are currently used to repair the bony defect, of which the most important components are restoration of tissue volume and reconstruction of the 3D contour and shape.

New technology such as RP has been widely used in craniomaxillofacial reconstructive surgeries. These technologies have facilitated the transformation of reconstructive surgical practice from complicated, general, and experience based to simple, accurate, and digitalized. Because the structure of the craniofacial skeleton is comparatively delicate and the bone surface is irregular, artificial implant materials cannot be easily molded during operation. When RP is applied for the preoperative design of the implant, the operation time will be effectively shortened and the result will be substantially improved. Moreover, a personalized preoperative design shall make the implant an accurate match for the defect. Aesthetic needs are both met when the defect is corrected. Rapid prototyping is the digitalized technology grounded on theories of discrete and accumulative forming that 
produces the substance layer by layer or point by point. Based on the CT scan data and the CAD model of the substance, the production for the prototype is accurately conducted. With the rapid development of the computer software and hardware and further introduction of mathematics, the CAD/CAM as the importance technology can be applied more widely.

Three-dimensional CT reconstruction was first applied to craniomaxillofacial research and treatment in 1986. Craniofacial skeleton conditions can be found out clearly through threedimensional CT scan. Virtual digital data of CT scan are transferred into a solid threedimensional reconstruction model. Therefore, replication of the deformed state can be interpreted directly, and craniofacial anatomy and periphery relationship can be described in detail. The accuracy of the model, which has been tested by both domestic and international scholars, helps physicians to find out the degree and the specificity of the defect easily, thoroughly, and directly. Thus, the preoperative simulation and the treatment plan are carried out to reduce both the difficulty and the duration of the surgery. With the effective communication between the physician and the patient, consensus is reached and postoperative satisfaction is met. Overall, the three-dimensional reconstruction model is an irreplaceable tool in the contemporary craniomaxillofacial surgery.

Reconstruction of 3D images from CT data has been used for many years to enhance interpretation of two-dimensional (2D) CT slices. Construction of CAD/CAM scaffolds derived from 3D computer-generated images has found various uses in craniofacial and plastic surgery, and has been particularly valuable when planning complex reconstructive procedures, such as repairs of large traumatic and complex deformities of the temporomandibular joint. Collaborations between engineers, surgeons and prosthetic designers have resulted in efficient exploitation of the available instruments and technologies .

In a previous study, the unit of craniofacial surgery in our department had applied this combined technique in the produce of $\mathrm{EH}$ (hydroxyapatite granules mixed with an epoxide acrylate maleic medical resin molding agent) compound artificial bone implants. Between January 2005 and October 2008, 39 patients with craniomaxillofacial deformities were treated. All the 39 patients were successfully operated on according to the preoperative plans. The results after surgery were satisfactory. One patient with temple augmentation needed secondary surgery to smooth the periphery of the implanted material because of the prominent edge of the implant that can be felt by the patient. Subcutaneous effusion in 2 patients was treated with suction and compressive bandaging. The recovery was satisfying. Implants in the 2 patients operated on through intraoral incisions had to be eventually removed 2 months after the surgeries owing to severe local symptoms of infection such as swelling and pain. All the other patients showed no complications during uneventful postoperative follow-ups.

With the application of CAD/CAM and RP, the implant material is perfectly matched for the skeleton defect. In the discussed case group, the combination of RP and CAD/CAM is applied in the craniomaxillofacial treatment. It has been proved after surgery that customized bone implants adhere to the defected area perfectly, and no migration has been noticed. The adhesion is tight, and the appearance is comparatively symmetrical. As for defect or depression that is close to the midline of the face, there is no mirror image for reference. Proper adjustments for the implant production are made during computerized simulation in accordance with the continuity of the geometric curve of the skeleton, although the patients' own opinions are properly taken at the same time. The postsurgical results were mostly well accepted by the patients. In the EH operation group, 2 patients 

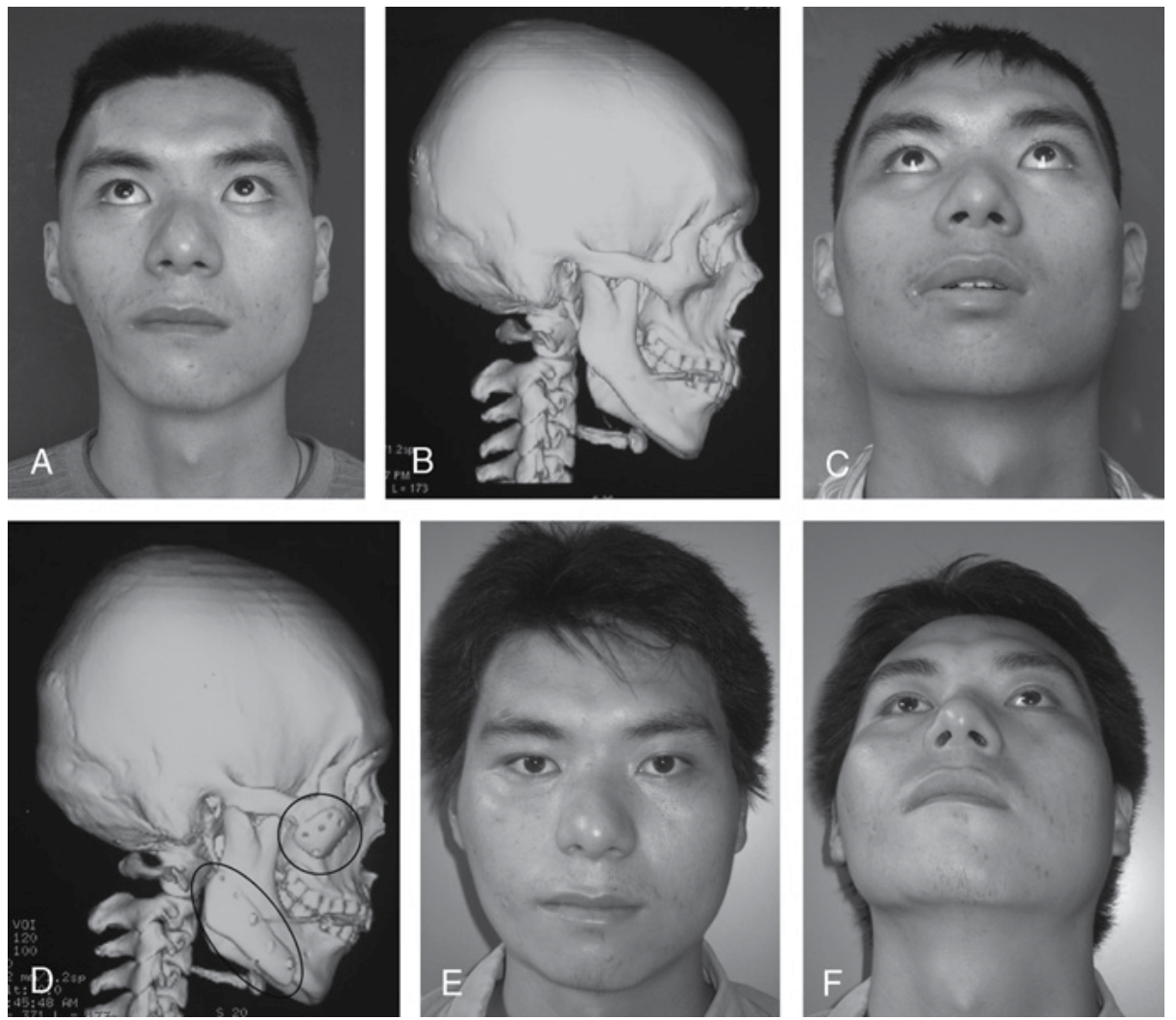

Fiq. 8 A, Preoperative inferior view for patient 1. B, Preoperative three-dimensional CT. C, One-week postoperative inferior view. D, Postoperative three-dimensional CT. E, Anteroposterior view of the same patient after 1 year. F, Inferior view after 1 year.
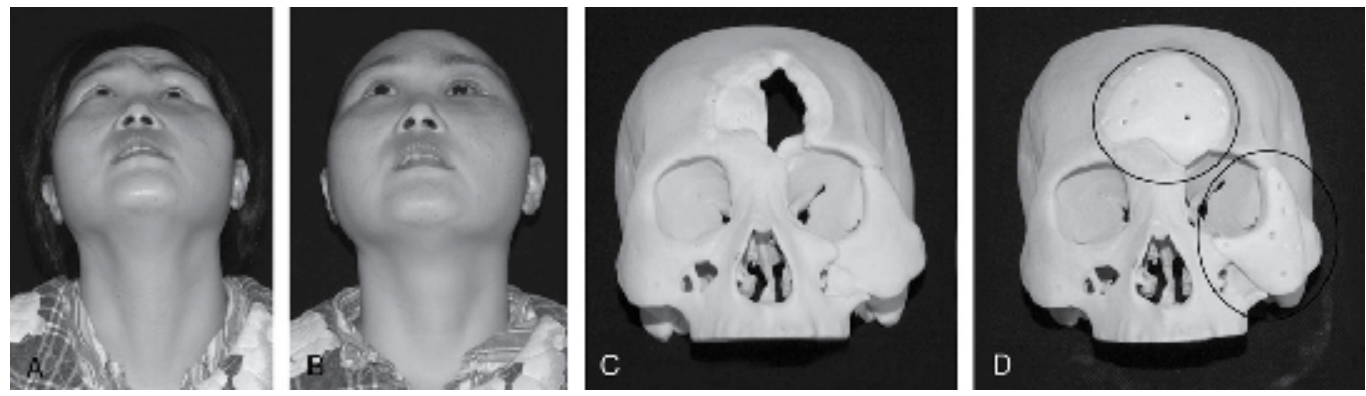

Fig. 9. A, Preoperative inferior view of patient 2. B, Postoperative inferior view (3 mo). C, Three-dimensional skull model. D, Preoperatively made EH compound artificial bone implant.

with temple augmentation were operated on. Usually, a lipoinjection or a Medpor implant is chosen for the reconstructive surgery. However, after sufficient communication with the patients, computerized reconstruction was applied for the design of the $\mathrm{EH}$ compound artificial bone implants to meet the patients' demands. The surgical procedures were easy 
and safe. The implants were placed subperiosteumly. No foreign body rejection or extrusion was noticed during the follow-up.

While studies have investigated the suitability of various materials in the construction of CAD/CAM scaffolds, histological studies focusing on BMSC seeding in scaffolds have demonstrated that PGA/PLA is one of the best materials available for the regeneration of new bone and cartilage. However, sculpting the external volume of the scaffold and generating strategies for its RP using PGA/PLA are significant challenges.

We have described a new method to tailor-make PGA/PLA mandibular condylar scaffolds for bone and cartilage regeneration that ensures excellent morphological alteration and accuracy. A physical replica of the mandible was generated from 3D CT data using $\mathrm{CAD} / \mathrm{CAM}$ and $\mathrm{RP}$, and was used as the positive model in the negative mould technique to prefabricate a PGA/PLA scaffold in the shape of the mandibular condyle. To evaluate the accuracy of the copy thus obtained, a laser scanning system was used. In general, if the difference could be seen in $<90 \%$ of the face after simulated implant placement, this was doomed to be a faithful reproduction; in addition, when aligned, maps of the merged scans showed that, on average, $90 \%$ of the created composite facial scans correlated to the original with an error up to $0.85 \mathrm{~mm}$, which was considered to be clinically acceptable. As for bone reconstruction of craniomaxillofacial features, the tolerance levels reached in our results are more acceptable. A comparison between the copy and the original morphology confirmed the efficacy of this method for accurate reconstruction of craniomaxillofacial features.

Synthetic absorbable implants made of materials such as elastic PGA/PLA can be readily adapted to the shape of a given defect. Because of their resilience, these materials may not sufficiently resist static or dynamic stresses. While each material has its advantages and disadvantages, it must at least accomplish its specified objectives. PGA and PLA are degradable, and their degradation product is weakly acidic. The acid-base neutralization reaction that occurs locally during degradation can prevent aseptic inflammation, maintain acid-base balance and promote osteogenesis. Zhou demonstrated that physiological repair of defects in articular cartilage and the corresponding subchondral bone was achievable using autologous BMSCs and PGA/PLA polymers. This study shows that BMSCs dispersed throughout a PGA/PLA scaffold in in vitro culture, proving that PGA/PLA have excellent cellular compatibility. BMSCs also have multi-lineage differentiation potential, particularly for osteogenic and chondrogenic differentiation, making them ideal seeding cells for tissue engineering.

In summary, our digital medical system enabled the fabrication of a customized PGA/PLA scaffold that very accurately recreated the original anatomical form and also had excellent cellular compatibility. Furthermore, the cost of equipment for this procedure was not high, since RP machines and laser scanners are available at low cost. This digital medical support system may be useful for craniomaxillofacial surgeons when reconstructing complex bony injuries and abnormalities.

\section{References}

[1] Ciocca L, De Crescenzio F, Fantini M, et al. CAD/CAM and rapid prototyped scaffold construction for bone regenerative medicine and surgical transfer of virtual planning: a pilot study. Comput Med Imaging Graph 2009; 33(1): 58-62. 
[2] Klein M, Glatzer C. Individual CAD/CAM fabricated glass-bioceramic implants in reconstructive surgery of the bony orbital floor. Plast Reconstr Surg 2006; 117(2): 565-570.

[3] Yeong WY, Chua CK, Leong KF, et al. Rapid prototyping in tissue engineering: challenges and potential. Trends Biotechnol 2004;22(12): 643-652.

[4] Dean D, Min KJ, Bond A. Computer aided design of large-format prefabricated cranial plates. J Craniofac Surg 2003; 14(6): 819-832.

[5] Peltola SM, Melchels FP, Grijpma DW, et al. A review of rapid prototyping techniques for tissue engineering purposes. AnnMed 2008; 40(4): 268-280.

[6] Ciocca L, Mingucci R, Gassino G, et al. CAD/CAM ear model and virtual construction of the mold. J Prosthet Dent 2007; 98(5):339-343.

[7] Zhou G, Liu W, Cui L, et al. Repair of porcine articular osteochondral defects in nonweightbearing areas with autologous bone marrow stromal cells. Tissue Eng 2006; 12(11):3209-3221.

[8] Xu H, Han D, Dong JS, Shen GX, Chai G, Yu ZY, Lang WJ, Ai ST. Rapid prototyped PGA/PLA scaffolds in the reconstruction of mandibular condyle bone defects. Int J Med Robot. 2010; 6(1):66-72.

[9] Rosen JM, Long SA, McGrath DM, et al. Simulation in plastic surgery training and education: the path forward. Plast Reconstr Surg 2009; 123(2): 729-738; discussion, 739-740.

[10] Hollister SJ. Porous scaffold design for tissue engineering. Nat Mater 2005; 4(7): 518524.

[11] Hutmacher DW, Sittinger M, Risbud MV. Scaffold-based tissue engineering: rationale for computer-aided design and solid free-form fabrication systems. Trends Biotechnol 2004; 22(7): 354-362 [review].

[12] Moroni L, de Wijn JR, van Blitterswijk CA. Integrating novel technologies to fabricate smart scaffolds. J Biomater Sci Polym Ed 2008; 19(5): 543-572.

[13] Wang L, Detamore MS. Tissue engineering the mandibular condyle. Tissue Eng 2007; 13(8): 1955-1971.

[14] Hutmacher DW. Scaffolds in tissue engineering bone and cartilage. Biomaterials 2000; 21(24): 2529-2543.

[15] Kau CH, Richmond S, Zhurov AI, et al. Reliability of measuring facial morphology with a three-dimensional laser scanning system. Am J Orthod Dentofacial Orthop 2005; 128(4): 424-430.

[16] $\mathrm{Yu} \mathrm{D}, \mathrm{Li} \mathrm{Q}, \mathrm{Mu}$ X, et al. Bone regeneration of critical calvarial defect in goat model by PLGA/TCP/rhBMP-2 scaffolds prepared by low-temperature rapid-prototyping technology. Int J Oral Maxillofac Surg 2008; 37(10): 929-934.

[17] Cao W, Miyamoto Y. Direct slicing from AutoCad solid models for rapid prototyping. Int J Adv Manuf Technol 2003;21:739-742

[18] Muller A, Krishnan KG, Uhl E, et al. The application of rapid prototyping techniques in cranial reconstruction and preoperative planning in neurosurgery. J Craniofac Surg 2003;14:899-914.

[19] Mavili ME, Canter HI, Saglam-Aydinatay B, et al. Use of three-dimensional medical modeling methods for precise planning of orthognathic surgery. J Neurosurg Sci 2008;52:113-116 
[20] Cho YR, Gosain AK. Biomaterials in craniofacial reconstruction. Clin Plast Surg 2004;31:377-385

[21] Baker SB, Weinzweig J, Kirschner RE, et al. Applications of a new carbonated calcium phosphate bone cement: early experience in pediatric and adult craniofacial reconstruction. Plast Reconstr Surg 2002;109:1789-1796

[22] Muhitdin E, Mustafa S, Murat T, et al. Contour restoration of the secondary deformities of zygomaticoorbital fractures with porous polyethylene implant. J Craniofac Surg 2007;18:520-525

[23] Menderes A, Baytekin C, Topcu A, et al. Craniofacial reconstruction with high-density porous polyethylene implants. J Craniofac Surg 2004;15:719-724

[24] Mohamed ED, Tompach PC, Morstad AT, et al. Long-term follow-up of the use of nonporous hydroxyapatite for augmentation of the alveolar ridge. J Oral Maxillofacial Surg 1991;49:257-261

[25] Habal MB, Reddi AH. Bone grafts and bone induction substitutes. Clin Plast Surg 1994;21:525-529

[26] Hashem FK, AI Homsi M, Mahasim ZZ, et al. Laryngotracheoplasty using the Medpor implant: an animal model. J Otolaryngol 2001;30: 334-339

[27] Gosain AK, Song L, Riordan P, et al. A 1-year study of osteoinduction in hydroxyapatite-derived biomaterials in an adult sheep model: part I. Plast Reconstr Surg 2002;109:619-630

[28] Gosain AK, Song L, Riordan P, et al. A 1-year study of osteoinduction in hydroxyapatite-derived biomaterials in an adult sheep model: part II. Bioengineering implants to optimize bone replacement in reconstruction of cranial defects. Plast Reconstr Surg 2004;114:1155-1163

[29] Gosain AK, Song L, Riordan P, et al. A 1-year study of hydroxyapatite-derived biomaterials in an adult sheep model: III. Comparison with autogenous bone graft for facial augmentation. Plast Reconstr Surg 2005;116:1044-1052

[30] Schliephake H, Neukam FW. Bone replacement with porous hydroxyapatite blocks and titanium screw implants: an experimental study. J Oral Maxillofacial Surg 1991;49:151-156

[31] Durham S, McComb J, Levy M. Correction of large cranial defects with reinforced hydroxyapatite cement: technique and complications. Neurosurgery 2003;52:842845

[32] Xu Y, Dong T, Xu X, et al. Design and fabrication of EH composite material and evaluation of its biomechanical properties. Acta Acad Med Zuzou 1995;15:17-18

[33] Fan X, Zhang D, Feng S, et al. Late reconstruction and repositioning of enophthalmos of orbital blow-out fracture. China J Ophthalmol 2002;38:644-647

[34] $\mathrm{Mu} \mathrm{X}$, Dong J, Wang W, et al. Epoxide acrylate maleic resin and hydroxyapatite composite material as a hone graft substitute in surgical correction of orbital reconstruction. China J Ophthalmol 1995;31: 447-449

[35] Zins JE, Moreira-Gonzalez A, Papay FA. Use of calcium-based bone cements in the repair of large, full-thickness cranial defects: a caution. Plast Reconstr Surg 2007;120:1332-1342

[36] Matic DB, Manson PN. Biomechanical analysis of hydroxyapatite cement cranioplasty. J Craniofac Surg 2004;15:415 
[37] Bianchi SD, Ramieri G, De Gioanni PP, et al. The validation of stereolithographic anatomical replicas: the authors' own experience and a review of the literature [in Italian]. Radiol Med 1997;94:503-510

[38] Choi JY, Choi JH, Kim NK, et al. Analysis of errors in medical rapid prototyping models. Int J Oral Maxillofac Surg 2002;31:23-32

[39] Matic D, Phillips JH. A contraindication for the use of hydroxyapatite cement in the pediatric population. Plast Reconstr Surg 2002;110:1-5 


\title{
Use of Rapid Prototyping and 3D Reconstruction in Veterinary Medicine
}

\author{
Elisângela Perez de Freitas ${ }^{1}$, Pedro Yoshito Noritomi ${ }^{2}$ \\ and Jorge Vicente Lopes da Silva ${ }^{2}$ \\ ${ }^{1}$ Department of Veterinary Surgery and Anesthesiology, \\ College of Veterinary Medicine and Animal Science \\ - São Paulo State University (UNESP) \\ ${ }^{2}$ Tridimensional Technologies Division, \\ Renato Archer Information Technology Center (CTI) \\ Brazil
}

\section{Introduction}

\subsection{Medical image processing}

In veterinary medicine, as well as in medicine, many noninvasive image techniques have been used for pathology diagnosis. The most oftenly used methods are computed tomography (CT) and magnetic resonance imaging (MRI) (Lim \& Zein, 2006). In general, these equipments generate a sequence of $2 \mathrm{D}$ images which make it difficult to display the spatial relationship of the important structures (Qiu et al., 2004). The 2D images are digitally stored in the international standard DICOM (Digital Imaging and Communications in Medicine) file format simplifying the interoperability among different medical systems with no extra costs for data conversion. This file format contains the necessary data for threedimensional (3D) reconstruction and patient information. Specifically developed reconstruction software, based on these 2D DICOM files, can generate the correspondent 3D models of the region of interest. By means of the software, internal and external anatomy can be clearly shown, measured, highlighted, and segmented either simultaneously or separately.

\subsection{Rapid prototyping}

Rapid prototyping is an additive process based on a computer controlled deposition of layers of materials. It was first available a little more than two decades ago, to speed up and facilitate solutions in automobile and aeronautical product development. In a few years it started to be used in the medical field, for reconstructive surgery, helping surgeons with a perfect replica of the anatomy of the patient. Nowadays, rapid prototyping is also known as additive manufacturing and is widespread in many areas of knowledge.

A virtual model is electronically sliced and sent to the machine that deposits each layer at a time. In the process to build a physical model from a virtual one many different processes are present using polymeric materials in different forms such as liquids, 
powders, sheets, and filaments. Other materials such as metals, ceramics, and paper can also be used. To glue each layer on top of the other there are processes based on lasers, binders, extrusion, etc.

The advantage of RP technologies is their ability to produce complex 3D physical models from a three-dimensional computer model (Geng et al., 2005). The main processes of rapid prototyping are: Selective Laser Sintering (SLS) based on the melting of a fine polymeric powder by means of heating lasers; Estereolitography (SLA) that is based on the polymerization of a liquid resin by means of specific wavelength lasers; Fused Deposition Modeling (FDM) where a filament is extruded and deposited continuously in thin threads of polymeric materials; and Tridimensional Printing (3DP) where each layer of powder is glued to the upper by means of ink jetting a binder. There are available today about thirty commercial processes including the above and its variations.

\section{Veterinary clinical cases}

\subsection{Dogs: 3D model of different type of dog's skull}

Dogs are categorized as dolichocephalic (Doberman, Collie, Setter, Whippet), mesaticephalic (Rottweiler, Poodle, Cocker spaniel) and brachycephalic (Boxer, Bulldog, Pug). The mandibular structure can be different according to the type of dog's skull. This mandibular structure may vary in three ways: on the cortical bone's width of the mandibular body, mandibular size in relation to the maxilla or the type of dental occlusion in these animals. It is really important that the surgeon is knowledgeable about the specifics of each kind of $\mathrm{dog}$, in order for him to choose the best surgical technique for the mandible fracture or orthognathic corrections, then to re-habilitate occlusion and masticatory function, to make them the closest to normal function as possible. This study has the goal to offer mandibular images in a normal pattern of Doberman, Rottweiler, Poodle and Boxer so that they can help as comparison tools between normal or affected structures. The animals were anesthetized and physical examination indicated no abnormalities in the mandible. Sequential transverse images of each dog's head were acquired on a helical CT Scanner (Shimadzu SCT-7800CT). The data obtained was saved in DICOM file format. 3D reconstructed images of the mandible were made by software programs commercially available. The virtual models were used to perform the measurements of length, height and width of the mandible and measurements of the cortical bone of the mandible body. The results are described in Table 1 and Figure 1, 2.

\begin{tabular}{ccccc}
\hline Dog breed & Cortical bone $(\mathbf{m m})$ & Length $(\mathbf{m m})$ & Height $(\mathbf{m m})$ & Width $(\mathbf{m m})$ \\
\hline Doberman & 4,3 & 185,8 & 28,3 & 11,8 \\
Rottweiler & 5,2 & 162,8 & 27,2 & 19,5 \\
Poodle & 2,7 & 77,0 & 10,2 & 7,9 \\
Boxer & 4,7 & 137,7 & 20,8 & 11,6 \\
\hline
\end{tabular}

Table 1. Measurements of the mandible for the different dog breeds.

This study has the goal to build the 3D reconstructed model of the mandible in a normal pattern of Doberman, Rottweiler, Poodle and Boxer so that they can help as comparison tools between normal and affected structures. 

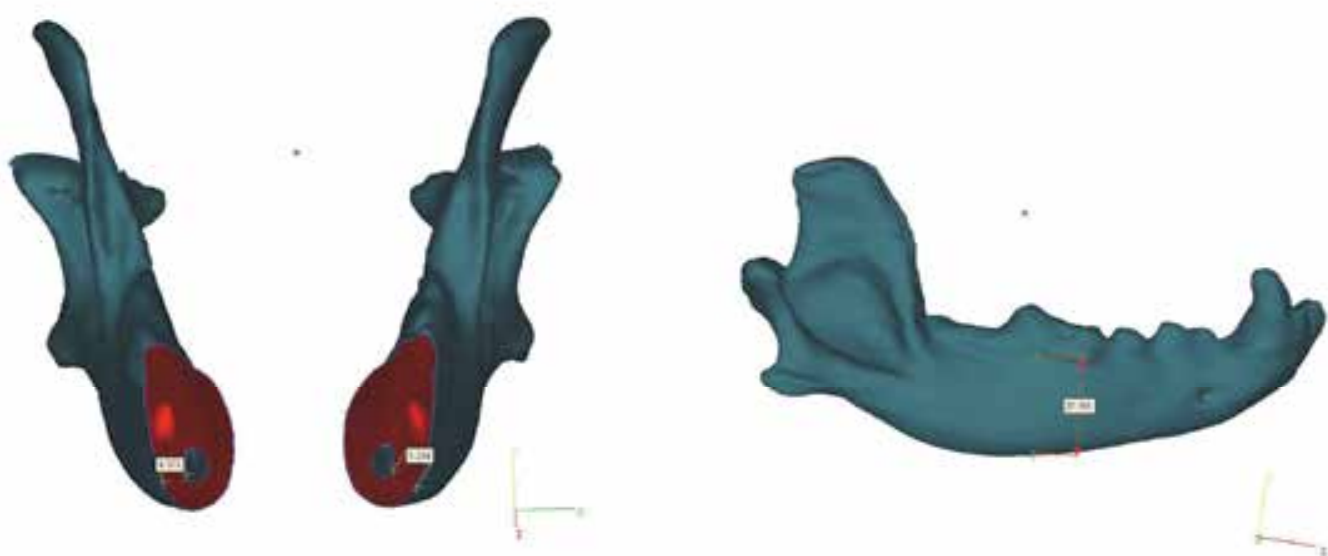

Fig. 1. Measurements of the cortical bone and height of the Rottweiler's mandible.
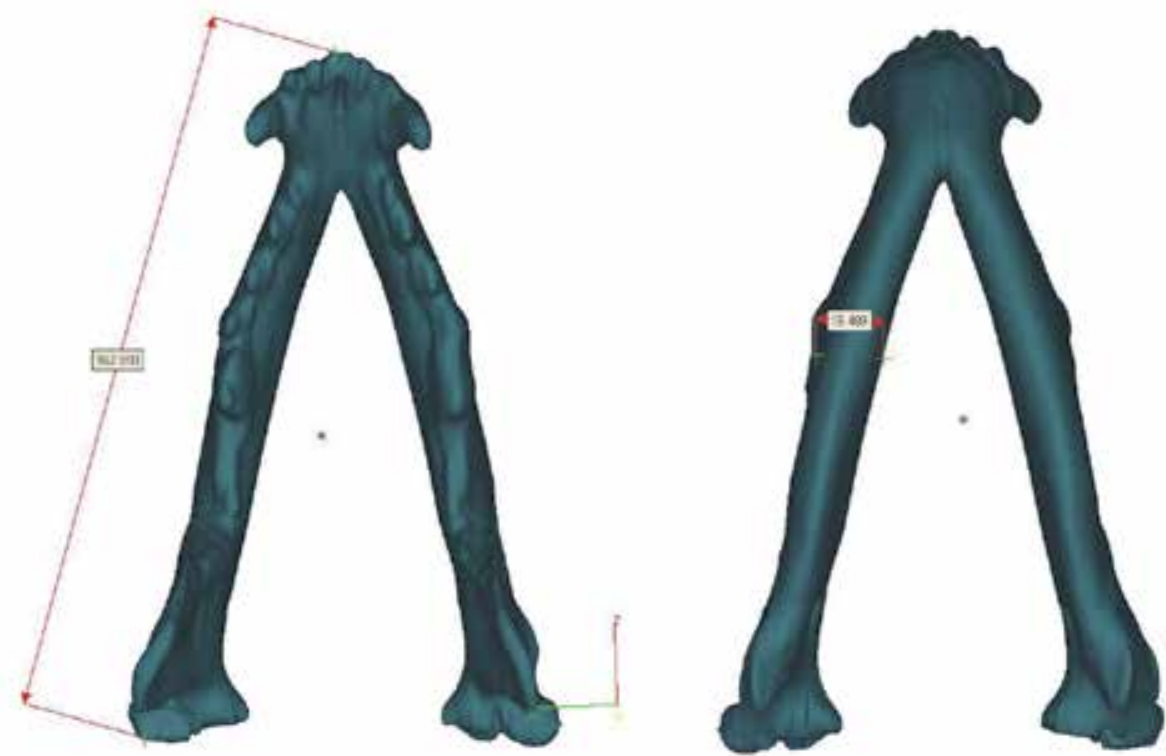

Fig. 2. Measurements of the length and width of the Rottweiler's mandible.

\subsection{Fox: orthopedic treatment of the maxillofacial malformation}

A fox (Pseudalopex vetulus), 9-month-old free-ranging, $3.7 \mathrm{~kg}$ male was presented to the Veterinary Hospital with Class II malocclusion - posterior cross-bite - on the right side (Freitas et al., 2010a). The animal was anesthetized and oral examination revealed the right 
mandibular canine tooth distal to the right maxillary canine tooth and tipped in a buccal direction. Because of this, the left mandibular canine caused a lesion on the palate between the left maxillary $3^{\text {rd }}$ incisor and canine teeth. Sequential transverse images of the head were acquired on a helical CT Scanner (Shimadzu SCT-7800CT) with the fox placed in dorsal recumbency. The InVesalius software was also used to edit and measure the original 3D virtual model obtained directly from the CT scan, and the Magics X SP2 was used to generate the final model for the prototype machine. The virtual 3D model of the head showed facial and mandibular asymmetry (Figure 3 ). The physical prototype constructed by the 3DP (Z-Cortp) technique enabled the preoperative planning (Figure 4 and 5). The inclined plane technique was used to correct crossbite and avoid palate injury. The fox adapted well to the treatment showing signs of discomfort only in the first $48 \mathrm{~h}$. The inclined plane was removed after three months and a new CT exam was performed. Visualization of $3 \mathrm{D}$ images in combination with rapid prototyping allowed the surgeon to perform 3 sets of geometric measurements in the virtual model on the first and second examination. Comparison of the prototypes and oral examination confirmed less maxillofacial discrepancy. Furthermore, the palate injury healed because the left mandibular canine tooth was not making contact with the palatal tissues. This orthopedic treatment showed that the Plana's technique has satisfactory results while modifying the skeletal structure stimulating the development of the stomatognatic system. In this case, the visualization and segmentation of CT images and 3D reconstruction of skull aided the surgical planning. The comparison of the prototype before and after treatment allowed a qualitative improvement of the occlusion.

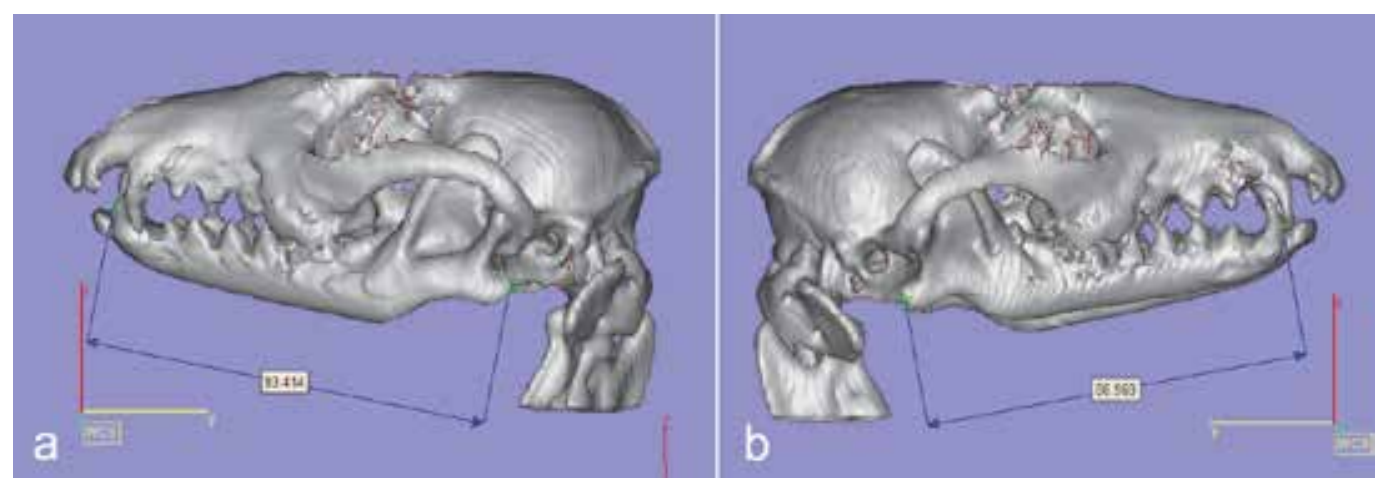

Fig. 3. Virtual 3D model (Magics X SP2) of the head based on CT image data showing the left mandible (a) is bigger than the right mandible (b).

\subsection{Rabbit: 3D finite element analysis}

The finite element analysis (FEA) is one of the most promising tools in the study of functional morphology of the craniofacial skeleton in humans and other species (Richmond et al., 2005). It is a computationally intensive engineering technique that estimates how objects of complex design resist loads (Wang et al., 2006). CT imaging is a useful method to gather three-dimensional physiological data such as bone geometry and density in vivo (Keyak et al., 1990). The results of von Mises' stress distribution in a human model of normal mandible showed that the model was reliable (Vollmer et al., 2000). Despite advances in commercially available meshing programs for creating subject-specific models 


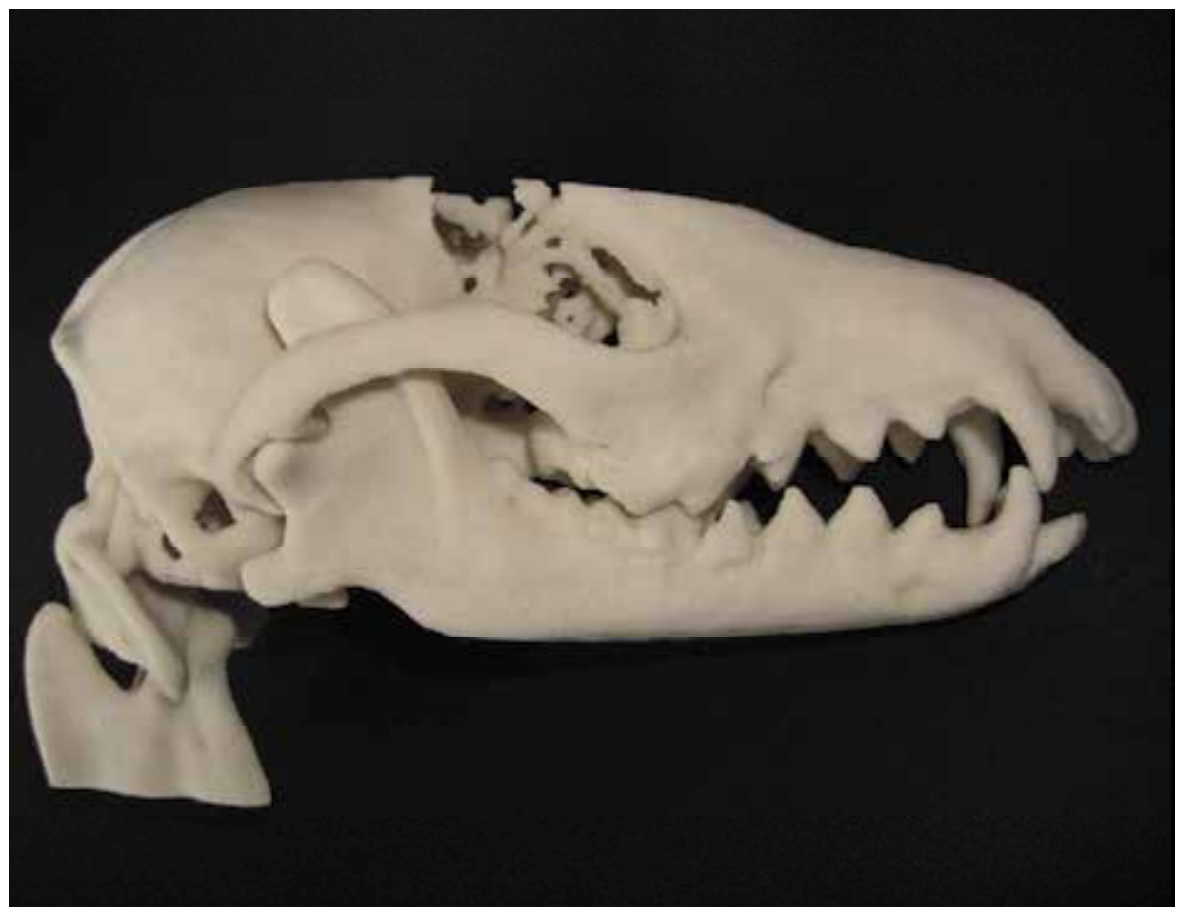

Fig. 4. Photograph of the prototype of the fox's skull.

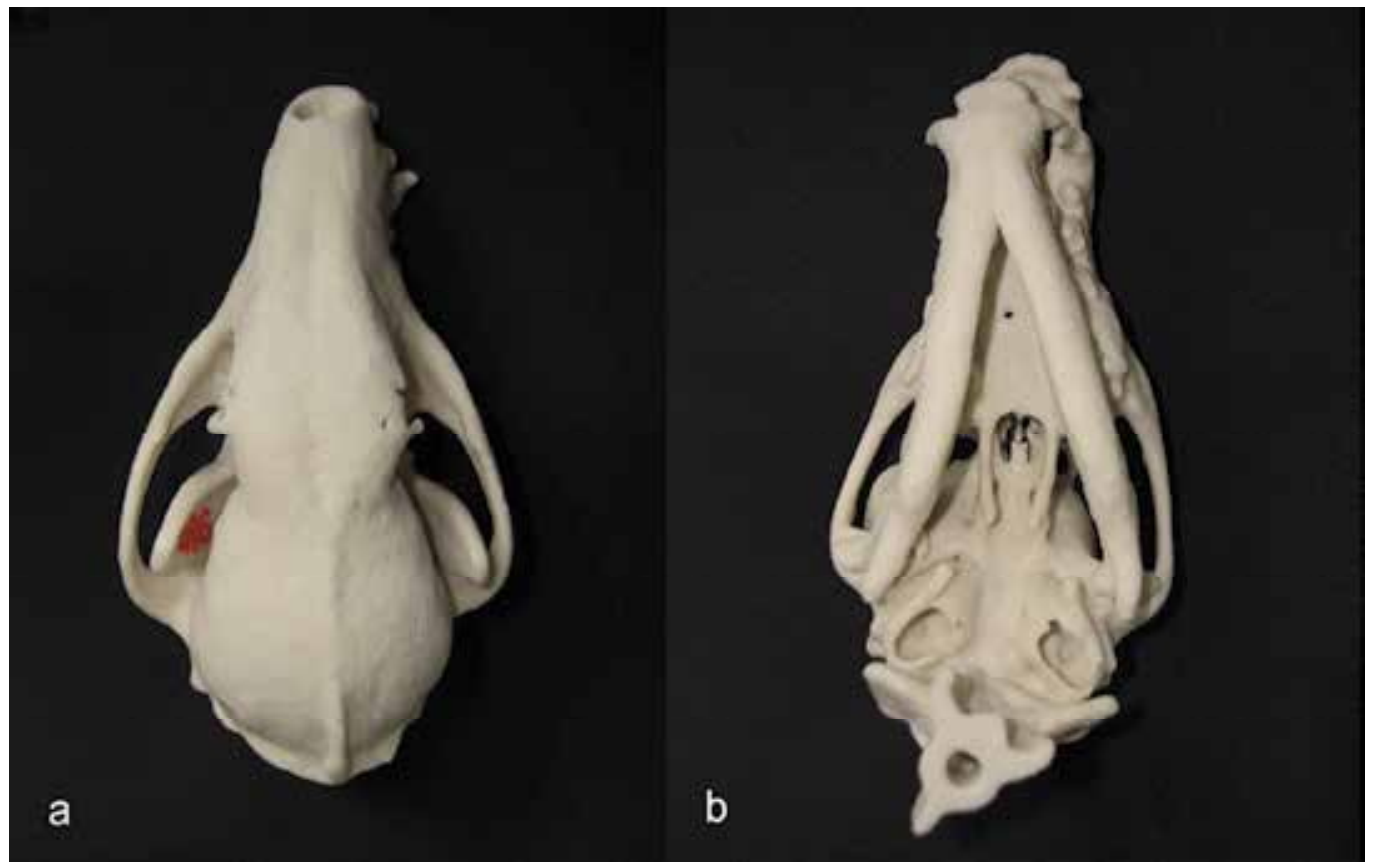

Fig. 5. Photography showing dorsal (a) and ventral (b) view of the prototype. Note the mandibular asymmetry. 
from image data, the process is still expensive, in terms of time, and usually requires high level of user intervention (Wilcox, 2007). In this study, three-dimensional (3D) geometrical and finite element analysis (FEA) of the mandible of a rabbit were built in order to evaluate the distribution of stresses on mandible of the rabbit applying a masticatory force of 10 Newtons $(\mathrm{N})$.

A computerized tomography (CT) examination was performed on the head of the Norfolk rabbit, female, 2 months of age. In order to perform CT studies the animal was submitted to general anesthesia. Sequential transverse images of the head were acquired on a helical scanner (Shimadzu SCT-7800CT) with the rabbit placed in a dorsal recumbency. The scanning parameters were $120 \mathrm{kVp}, 170 \mathrm{~mA}, 1.0 \mathrm{~mm}$ slice thickness, $1.0 \mathrm{~mm}$ interval, pitch of 2.0, and $1 \mathrm{~s} /$ rotation (Figure 6). A virtual 3D model of the head was generated based on CT image data, in DICOM (Figure 7). The 3D model was saved in STL format and it was prototyped (Figure 8). The STL model was imported into the software Rhinoceros ${ }^{\circledR}$ in order to create the BioCAD representation of the mandible prototype of the head was created. The BioCAD was used as a reference to create a more complex 3D model, demanded by the finite element analysis. The complete CAD models were exported to the NEiNastran ${ }^{\circledR}$, finite elements analysis program, using IGES file format. The finite elements analysis was performed by using a tetrahedral elements mesh (Figure 9). Isotropic, linear and homogeneous material properties were assumed for the bone. The study of total translation distribution on mandible and opposite action of the masseter muscle had to be made by NEiNastran ${ }^{\circledR}$ (Figure 10). The masticatory forces of the $10 \mathrm{~N}$ were applied on mandible in order to simulate a typical rabbit bite.

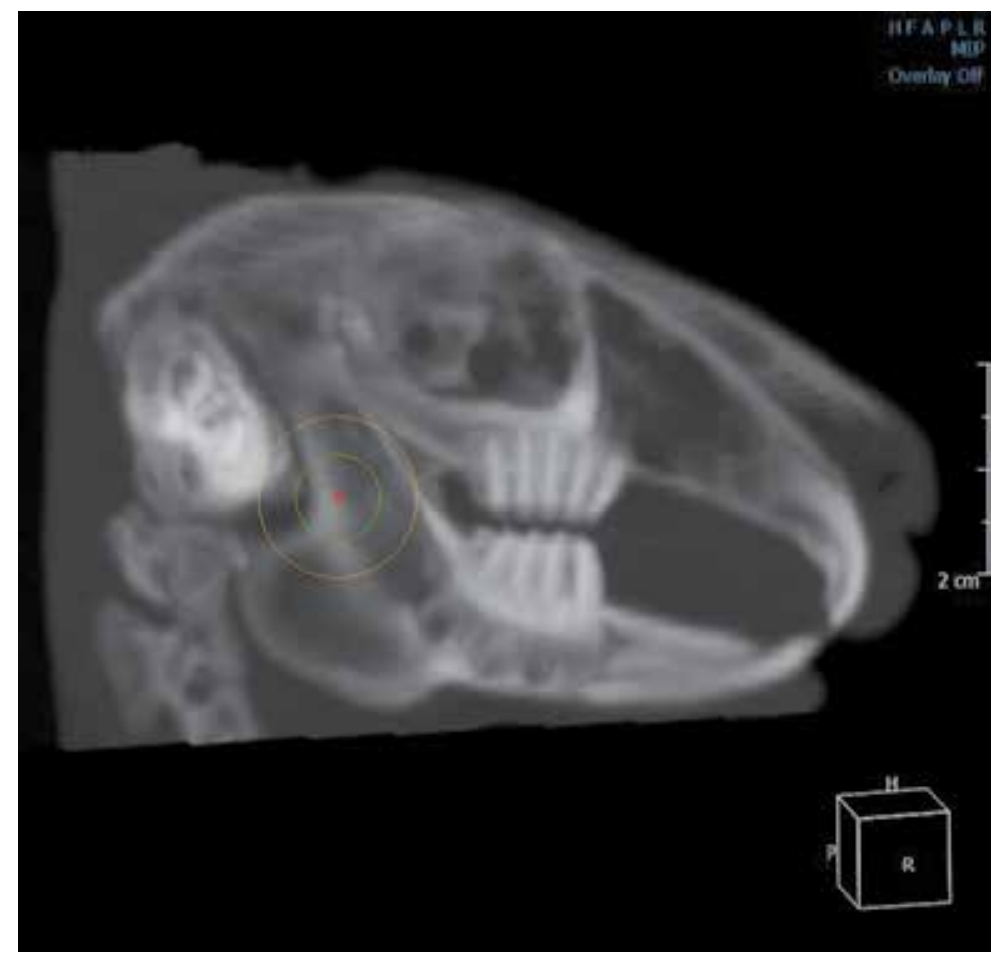

Fig. 6. Image showing the reconstruction of the rabbit's head. 


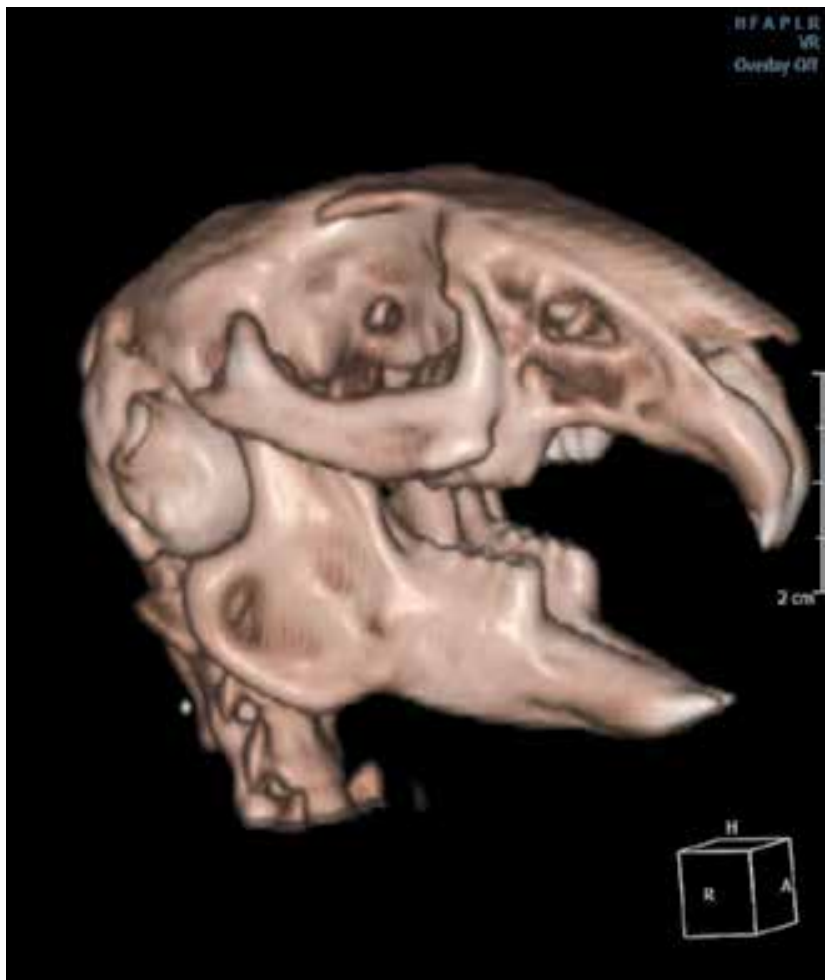

Fig. 7. Image of the 3D virtual model of the rabbit's skull.

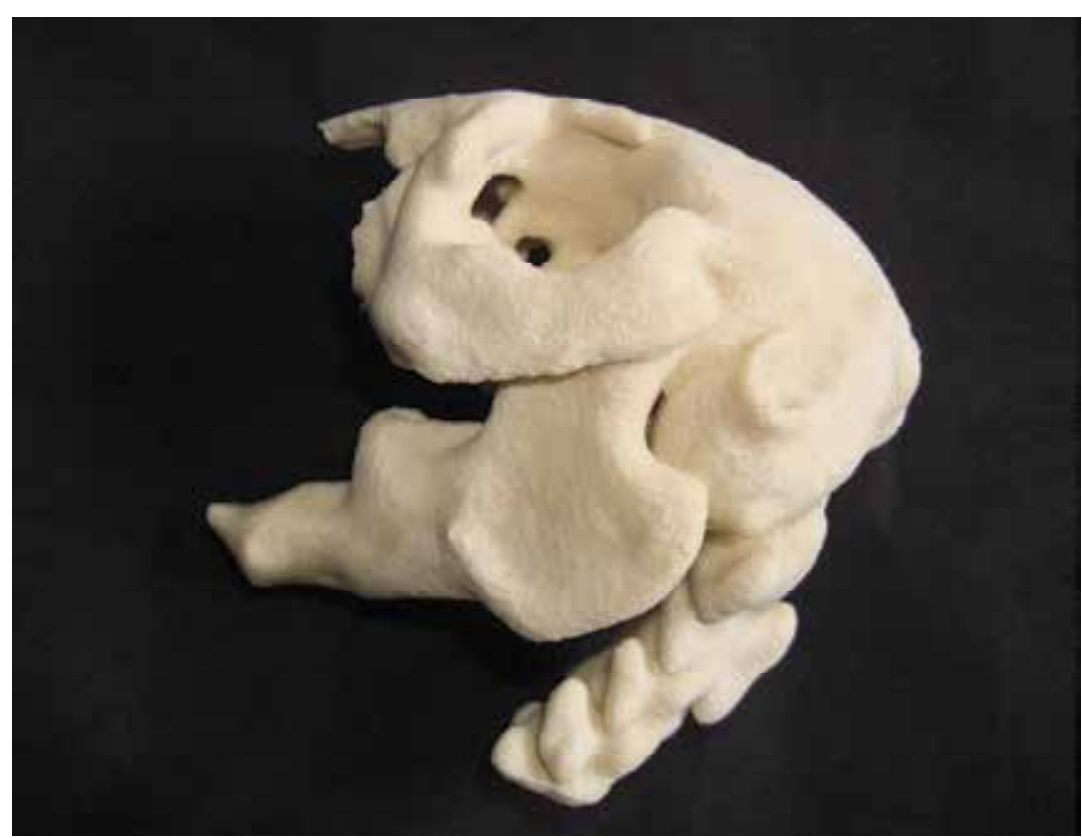

Fig. 8. Photography of the rabbit skull prototype. 


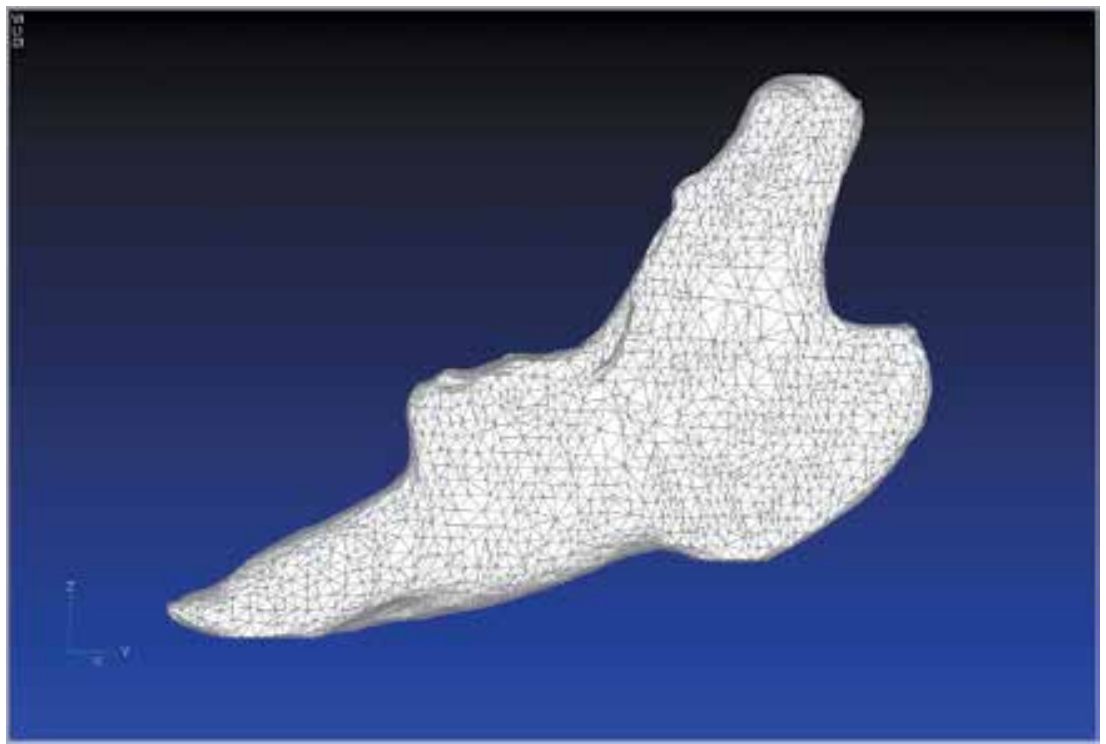

Fig. 9. Finite element mesh on the rabbit's mandible.

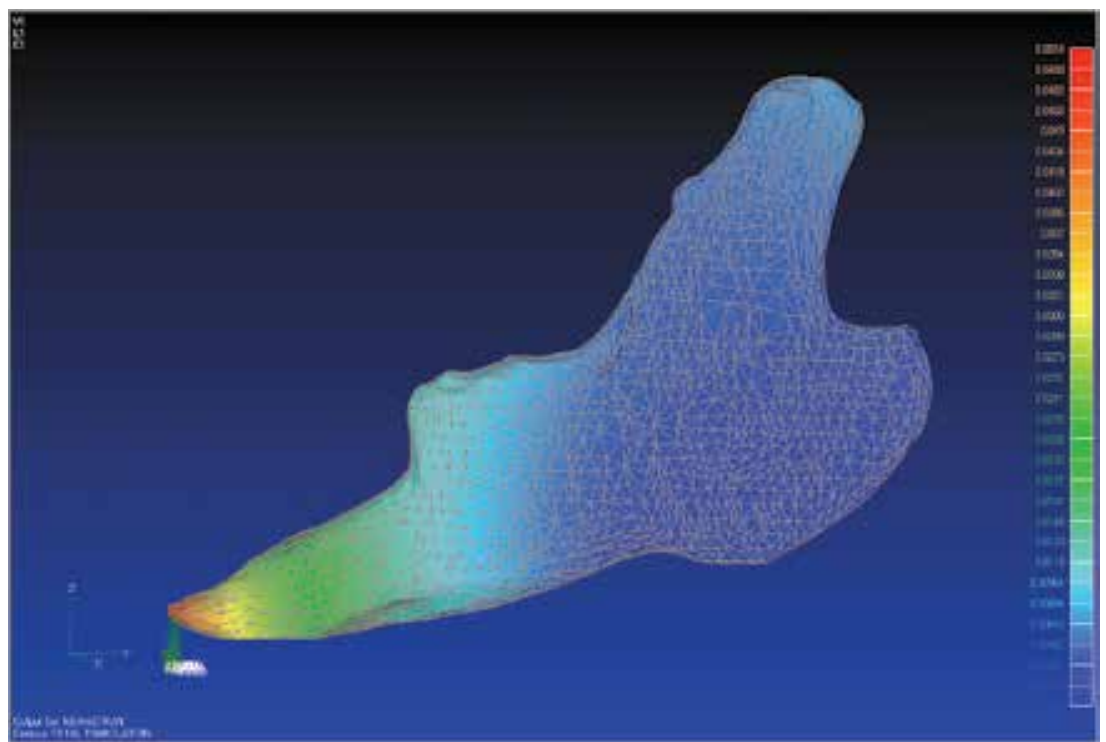

Fig. 10. Total translation distribution on the rabbit's mandible.

The FEA has show von Mises' stress concentration for the rabbit's mandible. There was a major von Mises' stress concentration on the cranial region of the mandible (Figure 11), with compressive stress propagation on the ventral side of the mandibular canal, with a tractive stress on the dorsal side of the same mandibular canal, on the third middle of the mandibular body. Both, the compressive and tractive stress fields follow through the mandibular body, drawing a stress triangle with the vertical ramus of the mandible on retro-molar region. 
Previous studies have reported that the distribution of von Mises' stresses on the human normal mandible occurred on the posterior coronoid process, mandibular angle and distal alveolar ridge in the molar area (Tie et al., 2006). In another hand, in this study with rabbit, the von Mises' stress concentration occurred on the cranial region of the mandible. Both the compressive stress and tractive stress distribution occurred through the mandibular body of the rabbit, drawing a stress triangle with the vertical ramus of the mandible on retro-molar region. Analyzing von Mises' stress concentration for normal mandible, it was important because the stress distribution has a great effect on biomechanical properties of reconstructed mandibles. If stress distribution is too concentrated, the bone will not regenerate but will be absorbed (Song \& Xue, 1999).

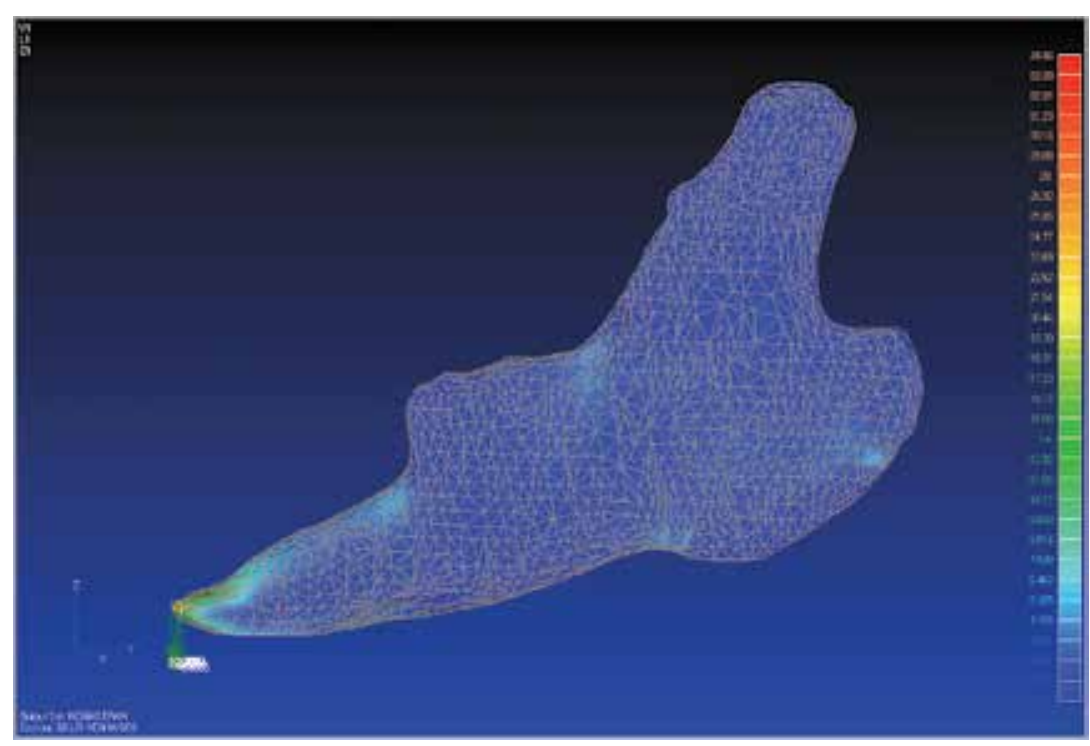

Fig. 11. Rostral area of the rabbit mandible showing a maximum von Mises' stresses.

\subsection{Dog: bone plate for mandibular fracture}

The present study aimed to develop a plate for treating oblique fractures of the mandibular body in dogs and to validate the project by using finite elements and biomechanical essays from the prototype of the mandible (Figure 12).

A computerized tomography (CT) examination was performed on the head. The obtained 3D virtual model was used as reference to develop the plate and the monocortical screws. The prototypes were used as samples for the mechanical evaluations. The same head utilized for the CT scan was used to perform the surgical procedure. The dog's head was positioned in left lateral recumbency, and the right mandible area was clipped, prepared, and draped using a sterile technique. A longitudinal skin incision was performed to the ventral midline of the right mandible from the level of the third molar tooth to the level of the fourth lower right premolar tooth. The subcutaneous fascia, and platysma and digastric muscles were incised and retracted to expose the vestibular face of the mandible. An oblique ventrorostral fracture was induced between the second and the first lower right molar using a gig saw. Then, fracture reduction and osteosynthesis were performed using titanium screws and a plate, the same procedure as in the prototype (Figure 13). 


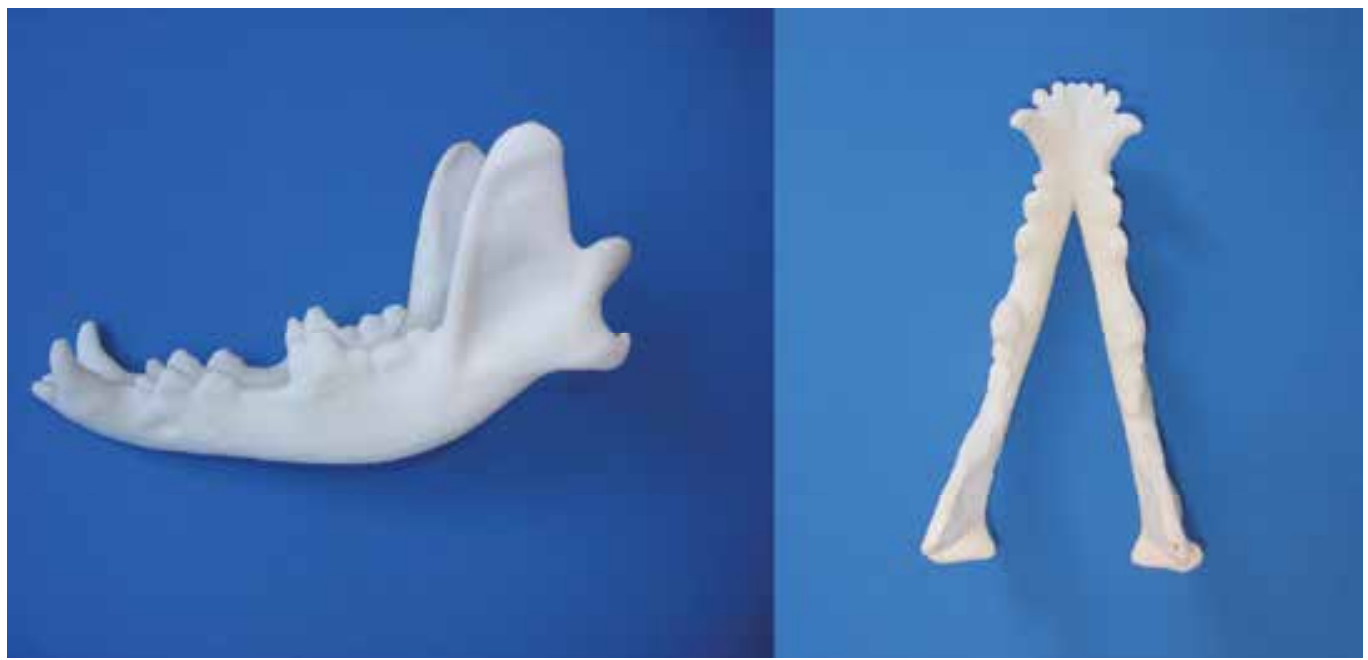

Fig. 12. Prototype of the mandible.

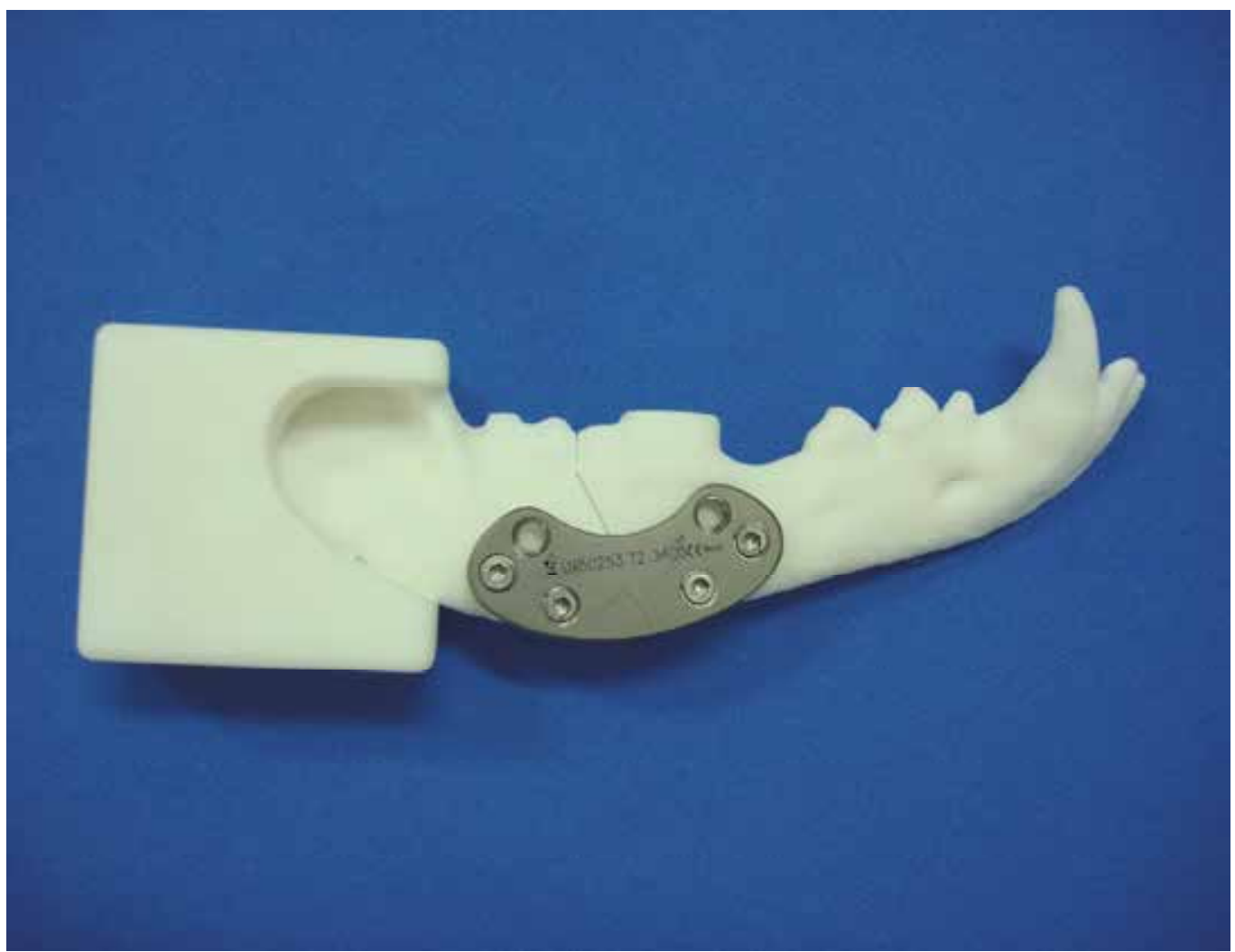

Fig. 13. Prototype of the mandible with plate and screws. 
Two screws were placed at each end of the fracture, since the mechanical tests had shown this to be a satisfactory system for this type of fracture. The elevated muscles were sutured to the fascia on the surface mandible using a simple continuous pattern, and the skin incision closed using simple interrupted pattern. Nylon monofilament 4-0 was the suture material used for all tissue layers. Another CT scan was performed to observe the placement of screws (Figure 14).

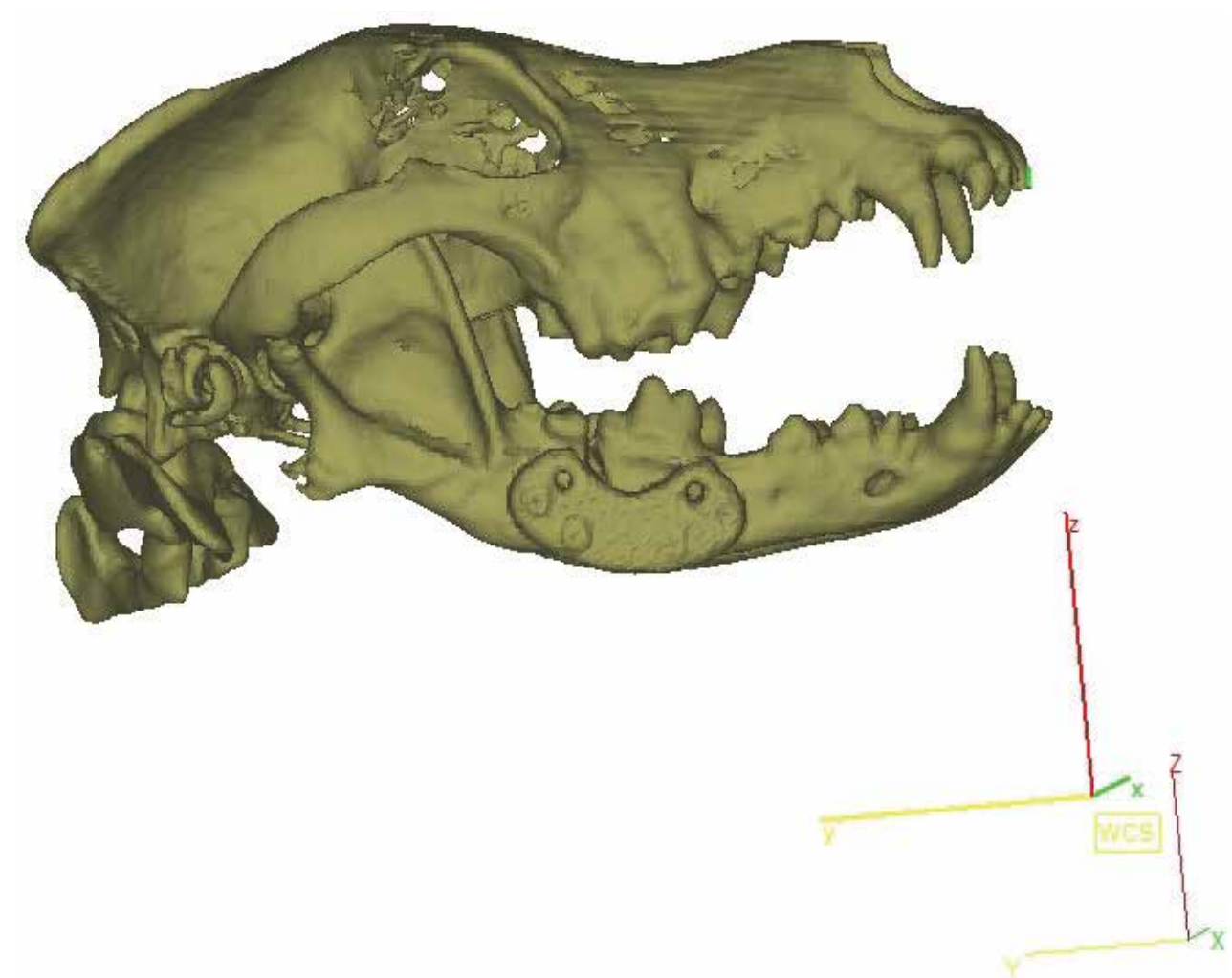

Fig. 14. 3D virtual model of the dog head with plate and screws.

The bone plate developed in the present experiment achieved proper mechanical resistance as proven by mechanical tests and finite element studies. Furthermore, its positioning next to the alveolar surface of the mandible bone, considered the site of maximum tension stress (Freitas et al., 2010b), as well as its design and thickness, render it better for application. In addition, the stabilization of the bone plate using blocked monocortical screws avoided the damage to dental roots and neurovascular structures that was observed in CT studies. In the SPS Free-Block system the screw is blocked due to the presence of a lock ring positioned inside the plate hole. Besides, this lock ring allows the screw be positioned at different angles. The guidelines adopted during the development of implants and the validation tests used will be discussed.

The mandible bone has a thick cortex and high mechanical resistance (Ashman et al., 1985). Because of this the cortical bone tends to behave as an isotropic material (Ashman et al., 1985; O'Mahony et al., 2000). The materials used to produce the prototypes and the system of plates and screws, nylon 6 and titanium, respectively, are also considered isotropic, 
linear, homogeneous and elastic materials. However, in order to establish the mechanical properties of the sintered nylon 6, traction tests were performed on rectangular specimens. This test enabled the establishment of the elasticity module and Poisson coefficient (Anusavice, 2005), both necessary to perform the finite element analysis. The mechanical test performed in the prototype showed the resistance difference between the fracture types. The maximum force to reach the plastic phase, when the material lost its original properties, was four times higher for favorable than for unfavorable fracture (Freitas et. al, 2010b). Unfavorable fracture tends toward dorsal distraction of the caudal fractured segment and ventral displacement of the cranial fractured segment (Harvey \& Emily, 1993; Wiggs \& Lobprise, 1997; Hall \& Wiggs, 2005). Due to these results and high cost of the prototypes, a force of $40 \mathrm{~N}$ was established to perform the tests. This force is within the range of elastic deformation for the fracture with less resistance. Since the prototypes maintained their initial properties, the tests were considered valid.

The results of the flexile tests performed in favorable and unfavorable fracture prototypes showed that the greater the number of screws used for stabilization of the bone plate, the higher the relative rigidity of the system, and the smaller the deflection. However, comparing both fracture types, the relative rigidity values of the favorable fracture were approximately two times those of the unfavorable fracture. These findings suggest that not only the number of screws used, but also the fracture type, had significant influence on plate fixation.

On the other hand, comparing these findings with the finite element data, it can be observed that the principal maximum stress for favorable fractures displayed an unexpected behavior. Based on the number of screws removed there was better stress distribution in the bone, with a decrease of principal maximum stress value from $20.60 \mathrm{MPa}$ to $15.34 \mathrm{MPa}$ (Freitas et al, 2010b).

The stress distribution has significant influence on biomechanical properties of surgically reconstructed mandible; if concentration of stress distribution occurs the bone will be reabsorbed instead of being regenerated (Tie et al., 2006).

Both von Mises' stress and force transmission showed that in a favorable fracture based on screw removal there was higher stress concentration on the lower screw positioned in the cranial fractured segment. Probably this is associated with the positioning of this screw in the direction of the load application. On the other hand, in the unfavorable fracture the von Mises' stress was higher in the lower screw positioned in the caudal fractured segment, suggesting that the bone plate probably supports and rotates around this screw. Since this type of fracture does not offer support to cranial fractured segment by means of the caudal fractured segment, the stress distribution was concentrated in the ventral part of the bone plate that suffered constant traction during load application. Since the caudal fractured segment tends to dorsal displacement due to the muscular pull and the caudal fractured segment does not offer resistance, only the plate was able to maintain the stability in this fracture type.

In conclusion, the double-arch geometry plate fixed with blocked monocortical screws presents sufficient resistance to stabilize oblique fractures of the mandibular body, without compromising the dental or neurovascular structures.

\subsection{Bird: orthosis produced by rapid prototyping}

A Brazilian bird of prey called southern caracará (C. plancus) adult was found in wild life and brought to the veterinary hospital with a thin thread constricting its posterior left 
member. The clinical examination showed necrosis in the tibiotarsal joint. The patient was submitted to general anesthesia to perform amputation of the member. After surgery the bird presented difficulties in to adaptating to the captive environment, specially concerning to locomotion and standing.

In order to improve the bird's quality of life an orthosis was specially designed using the concepts of the paralympic runner Oscar Pistorius. Using the information of a CT acquisition of the contralateral healthy member and mirroring the data the perfect shape and dimensions were obtained. The information was used to design an optimized fitting orthosis that was later produced directly by means of the SLS rapid prototyping technique (Figure 15). The internal region that should be in contact with the animal's skin was covered by a layer of dentistry addition silicone to avoid injury in the area (Figure 16).

The final result showed a difficulty of the animal to adapt with the osthosis and after some hours it was taken out (Figure 17). The same process was repeated for three days and at the end the animal presented a trauma on the skin, having tried to release it with its beak (Figure 18). Unfortunately the animal did not adapt to the orthosis and further studies have to be developed for successful adaptation.

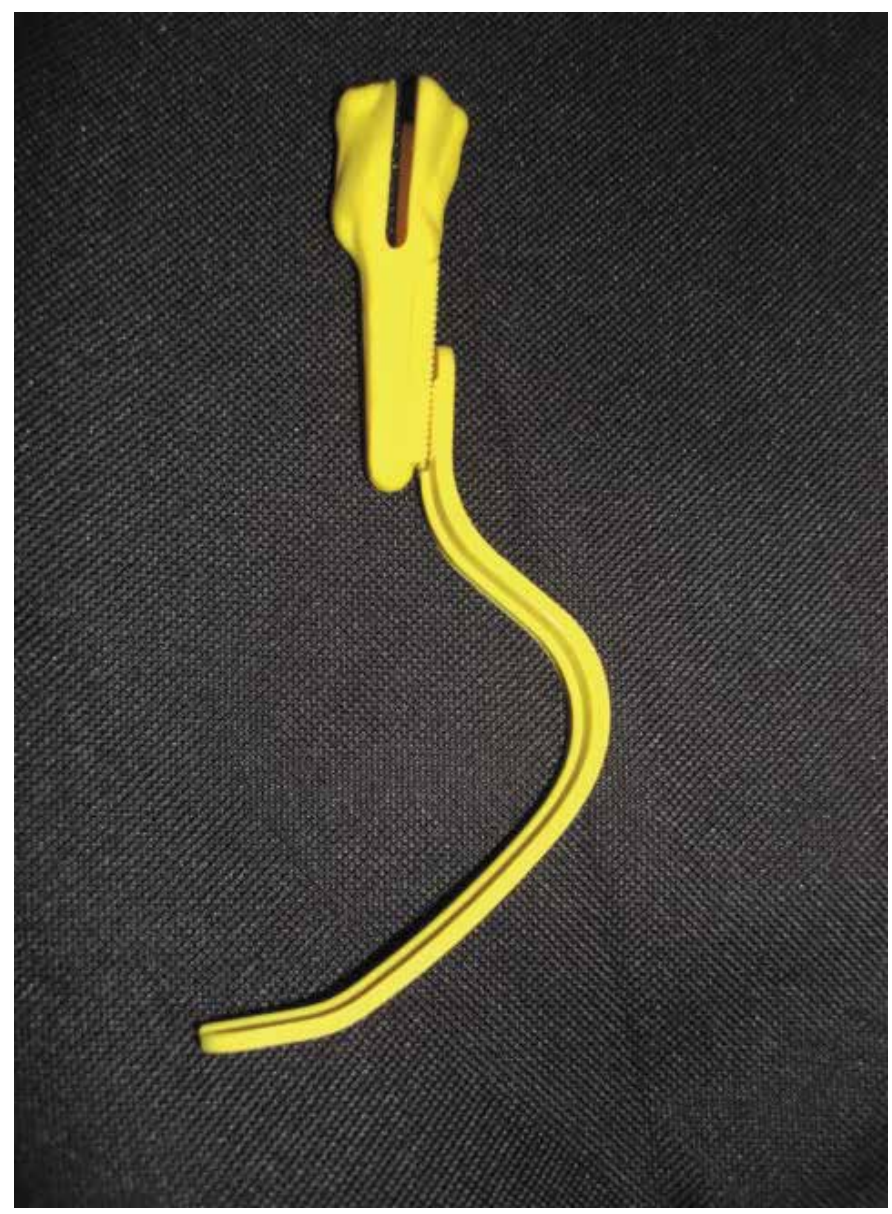

Fig. 15. Orthosis produced by means of the SLS rapid prototyping technique. 


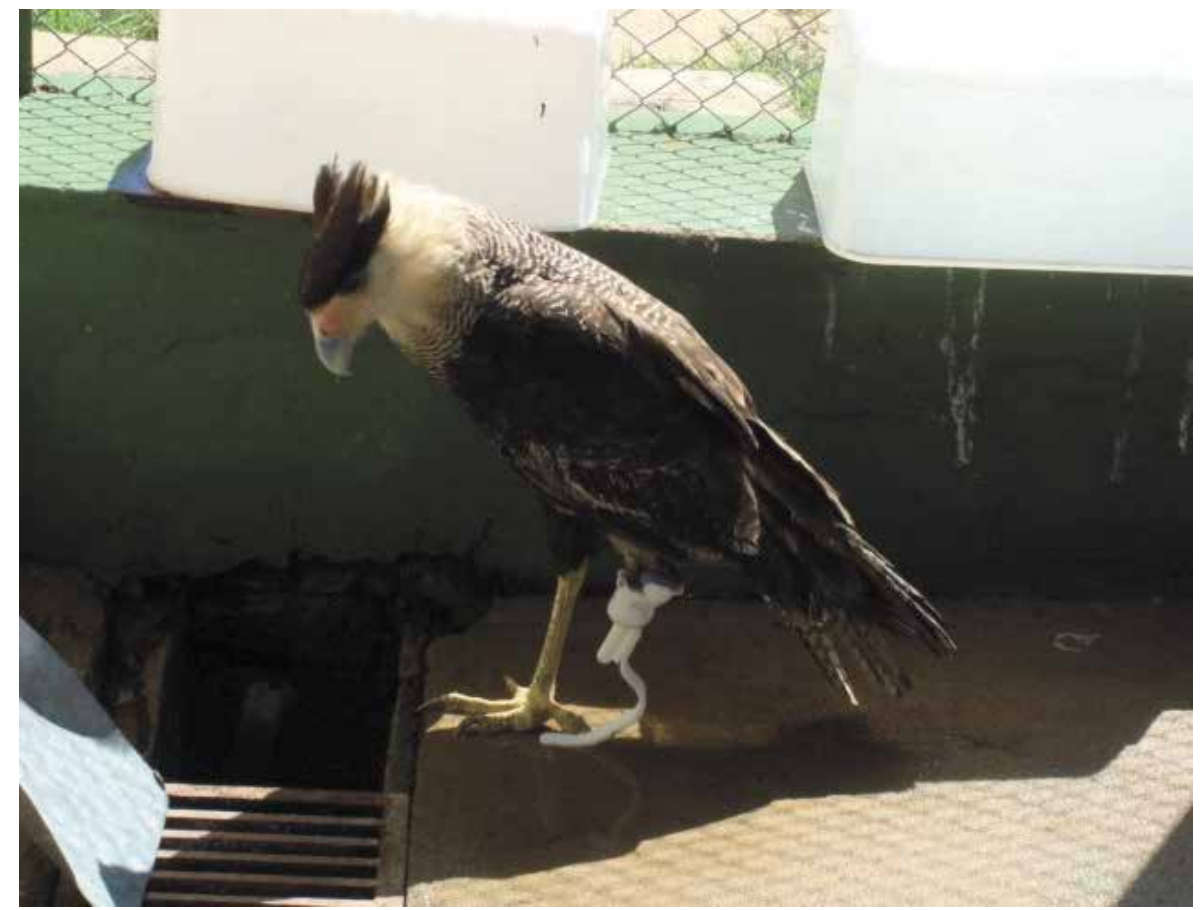

Fig. 16. Southern Caracará with orthosis on the posterior left member.

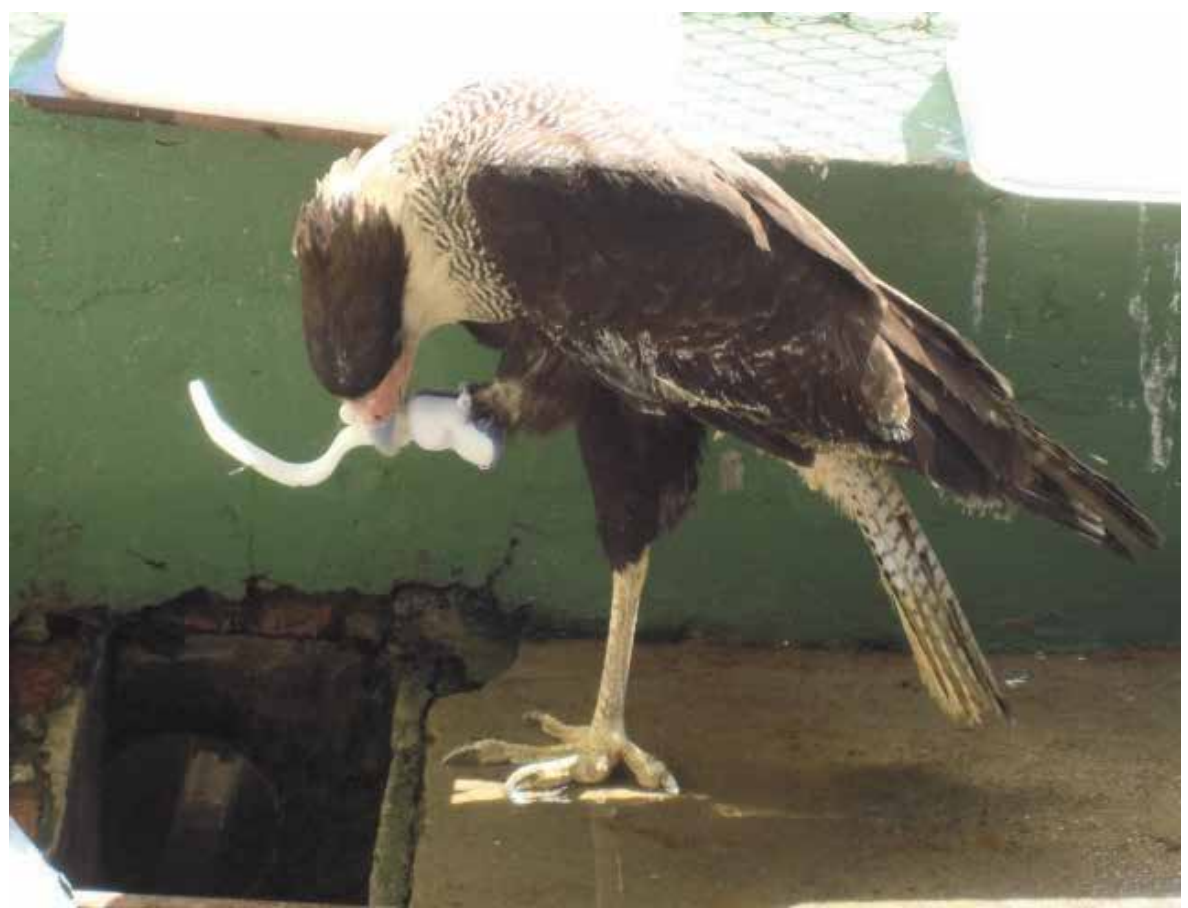

Fig. 17. Southern Caracará trying to release the member with beak. 


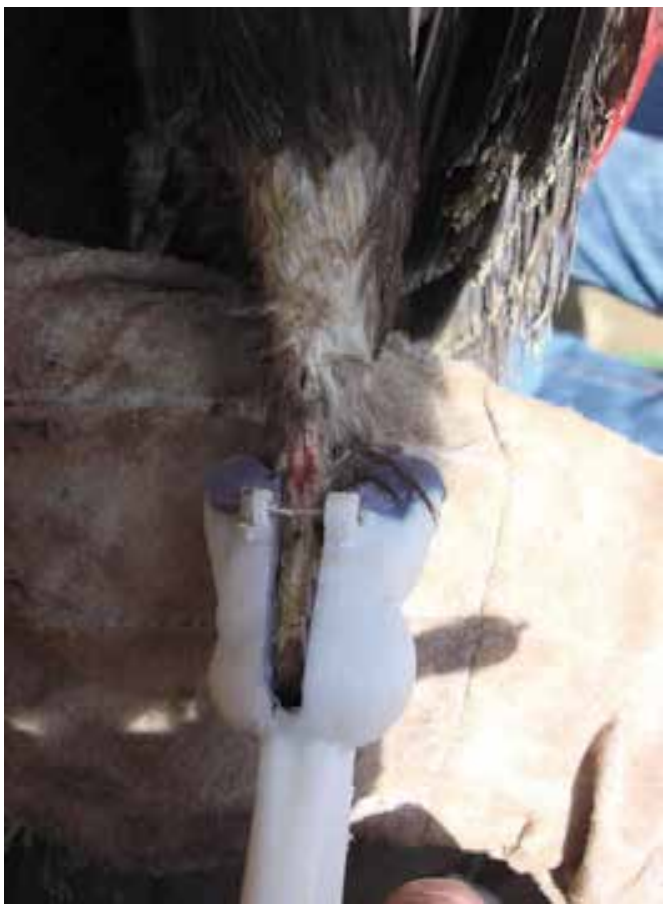

Fig. 18. Injury on the skin of the Caracará.

\section{Conclusions}

3D medical models and rapid prototyping opened up new possibilities for the surgical planning and treatment of wild and domestic animals, allowing for anatomy studying and to support diagnosis in order to minimize the anesthetic period during surgery. It can be a good opportunity to increase the welfare of some rare animals or of the ones considered to be in danger of extinction. In this situation these animals can even have a better reproduction.

\section{Acknowledgements}

The authors are grateful to the professionals of Tridimensional Technologies Division of Renato Archer Information Technology Center - CTI.

\section{References}

Anusavice, K.J. (2005). Phillips materiais dentários, 11ed, Elsevier, ISBN 85-352-1532-8, Rio de Janeiro.

Ashman, R.B.; Rosinia, G.; Cowin, S.C.; Fontenot, M.G. \& Rice, J.C. (1985). The bone tissue of the canine mandible is elastically isotropic, Journal of Biomechanics, 18, (1985), 717721, ISSN 0021-9290.

Freitas, E.P.; Rahal, S.C.; Teixeira, C.R.; Silva, J.V.L.; Noritomi, P.Y.; Villela, C.H.S.; Yamashita, S. (2010a). Rapid prototyping and inclined plane technique in the 
treatment of maxillofacial malformations in a fox, Canadian Veterinary Journal, 51 (March 2005), 267-270, ISSN 0008-5286.

Freitas, E.P.; Rahal, S.C.; Gioso, M.A.; Vulcano, L.C.; Shimano, A.C.; Silva, J.V.L.; Noritomi, P.Y. \& Warrak, A.O.E. (2010b). Finite element modeling for development and optimization of a bone plate for mandibular fracture in dogs, Journal of Veterinary Dentistry, 27, 4 (December 2010), 212-221, ISSN 0898-7564.

Geng, L.; Feng, W.; Hutmacher, D.W.; Wong, Y.S.; Loh, H.T. \& Fuh, J.Y.H. (2005). Direct writing of chitosan scaffolds using a robotic system, Rapid Prototyping Journal, 11, 2, (2005), 90-97, ISSN 1355-2546.

Hall, B.P. \& Wiggs, R.B. (2005). Acrylic splint and circumferential mandibular wire for mandibular fracture repair in a dog, Journal of Veterinary Dentistry, 22, 3, (September 2005), 170-175, ISSN 0898-7564.

Harvey, C.E.; Emily, P.P. (1993). Small animal dentistry, Mosby, St. Louis.

Keyak, J.H.; Meagher, J.M.; Skinner, H.B. \& Mote, Jr. C.D. (1990). Automated threedimensional finite element modeling of bone: a new method, Journal of Biomedical Engineering, 12, 5, (September 1990), 389-397, ISSN 0141-5425.

Lim, J.; Zein, R. (2006). The digital imaging and communications in medicine (DICOM): description, structure and applications, In: Rapid Prototyping - Theory and Practice, Kamrani, A. \& Nasr, E.A. (Ed.), 63-86, Springer, ISBN-13 9780387232904, New York.

O'Mahony, A.M.; Williams, J.L.; Katz, J.O. \& Spencer, P. (2000). Anisotropic elastic properties of cancellous bone from a human edentulous mandible, Clinical Oral Implants Research, 11, (2000), 415-21, ISSN 0905-7161.

Qiu, M.G.; Zhang, S.X.; Liu, Z.J.; Tan, L.W.; Wang, Y.S.; Deng, J.H. \& Tang, Z.S. (2004). Three-dimensional computational reconstruction of lateral skull base with plastinated slices, The Anatomical Record Part A, 278, A, (2004), 437-442, ISSN 15524892.

Richmond, B.G.; Wright, B.; Grosse, I.; Dechow, P.C.; Ross, C.F.; Spencer, M.A. \& Strait, D.S. (2005). Finite element analysis in functional morphology, Anatomical Record, 283, A, (March 2005), 259-274, ISSN 0003-276X.

Song, G. \& Xue, M. (1999). Stress-adaptation of bone, Journal of Biomedical Engineering, 16, 4 (December 1999), 502-505, ISSN 0141-5425.

Tie, Y.; Wang, D.M.; Ji, T., Wang, C.T. \& Zhang, C.P. (2006). Three-dimensional finiteelement analysis investigating the biomechanical effects of human mandibular reconstruction with autogenous bone grafts, Journal of Cranio-maxillofacial Surgery, 34, 5, (July 2006), 290-298, ISSN 1010-5182.

Vollmer, D.; Meyer, U \& Joos, D. (2000). Experimental and finite element study of a human mandible, Journal of Cranio-maxillofacial Surgery, 28 (April 2000), 91-96, ISSN 10105182.

Wang, Q.; Strait, D.S. \& Dechow, P.C. (2006). A comparison of cortical elastic properties in the craniofacial skeletons of three primate species and its relevance to the study of human evolution, Journal of Human Evolution, 51, 4, (October 2006), 375-382, ISSN 0047-2484.

Wiggs, R.B. \& Lobprise, H.B. (1997). Veterinary Dentistry: principles and practice, LippincottRaven, Philadelphia.

Wilcox, R.K. (2007). The influence of material property and morphological parameters on specimen-specific finite element models of porcine vertebral bodies. Journal of Biomechanics, 40, 3 (2007), 669-673, ISSN 0021-9290. 


\title{
Rapid Prototyping in Correction of Craniofacial Skeletal Deformities
}

\author{
Libin Zhou and Yanpu Liu \\ Department of Oral and Maxillofacial Surgery, School of Stomatology, \\ Fourth Military Medical University
}

China

\section{Introduction}

The skull of a human being, comprising two parts: the cranium and the maxillofacial bone, is a bony structure in the head that forms a cavity for the brain and supports the structures of the face. The functions of the skull include protection of the brain, fixing the position of the eyeballs and ears, compositing the oral cavity and airway. Bone deformities in the craniofacial area, including acquired deformities (such as defects result from oncological resection and trauma, and micrognathia syndrome after mandibular condyle injuries), and congenital deformities (various craniofacial syndromes, such as hemifacial microsomia) will create tremendous cosmetic and functional handicaps (Mehta \& Deschler, 2004).

Such deformities heavily affect the patients' quality of life and pose a vast challenge to craniofacial surgeons. Various methods, including autogenous bone grafting (free bone grafting and vascularised grafting of compound tissue flaps), and alloplastic and allogeneic materials, have met with limited success (Boyne, 1973; Tideman et al., 1998; Samman et al., 1999). To achieve satisfactory esthetic and functional reconstruction using the traditional methods remains extremely challenging.

At present most of the mandible restoration implants are fabricated by hand forming technique (Boyne, 1973; Tideman et al., 1998; Samman et al., 1999; Eufinger et al., 1997; Stojadinovic et al., 1999). The method involves pre-bending the reconstruction plate on the stereolithography model to cover the defect. Bone graft also was traditionally adapted on the sterilized stereolithography model during the operation to reconstruct the facial defect. The precision of fit and durability of implant mainly relies on the abilities of the technicians and surgeons.

Rapid prototyping refers to a group of techniques that are used to produce stereolithographical models based on digital images, including reverse engineering (RE), computer-aided design (CAD), rapid prototyping (RP), etc. RE is a viable approach to create a 3-dimensional virtual model of an existing physical part by measuring an object using 3dimensional scanning technologies such as laser scanners, structured light digitizers, computed tomography (CT) or magnetic resonance (MR). CAD takes advantage of computer technology to aid in the design and, especially, the drafting (technical and engineering drawing) of a part or product. It is both a visual (or drawing) and symbol-based method of communication whose conventions are particular to a specific technical field. RP takes CAD files and transforms them into thin, virtual, horizontal cross-sections. 
Stereoscopic lithography carries out layer by layer according to the virtual structure to build up the physical model. This process allows the production of geometrically complex shaped models that otherwise would be impossible to produce.

Use of the rapid prototyping in the craniofacial area has been extensive. It has been used primarily as a means of evaluation of the craniofacial deformity, preoperative planning, surgical simulation, guiding surgical procedures, postoperative evaluation, and longitudinal follow-up for outcome assessment, using tactile models derived from patient computerized tomography (CT) data.

The following flowchart (fig.1) reveals the conventional approach to manufacture a custom implant for reconstruction of a mandibular defect. It starts from the CT scanning of the patient's skull. The CT images are imported into the CAD software and modified as a surface. The surface is then converted to an STL file, which converts the surface to triangles (triangulation). The surface can then be fed to the RP machine. A mandible implant is then produced by manually shaping the titanium plate on the surface of the 3D stereolithography model. The resulting shaped titanium plate is then directly used to bridge the residual mandibular blocks (Tideman et al., 1998; Samman et al., 1999; Eufinger et al., 1997; Stojadinovic et al., 1999).

However, the potential to intimately control the microstructure and the overall macroscopic shape of the implants makes RP an ideal process for fabricating individual implant which is more important and promising in offering simpler and more rapid surgical implementations.

A new approach has been developed to design the mandible reconstruction implant from Computer Aided Design (CAD) (fig.2). The proposed approach includes serial steps: CT data acquisition, medical image processing - 3D reconstruction, design of the custom titanium implant, Rapid Prototyping, rehearsal of surgery and implant fitting evaluation and production of titanium implant (Singare et al., 2004; Zhou et al., 2011; Zhou et al., 2010).

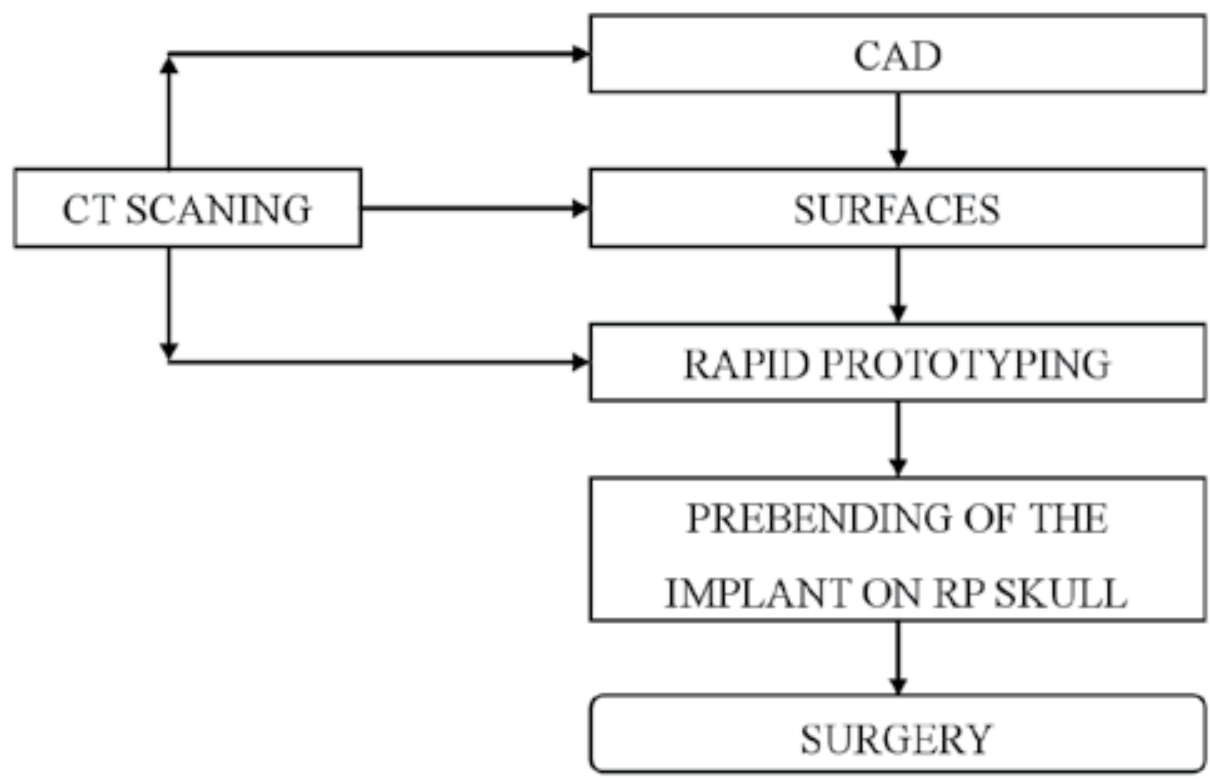

Fig. 1. The approach to manufacture a custom implant for a mandibular defect patient 


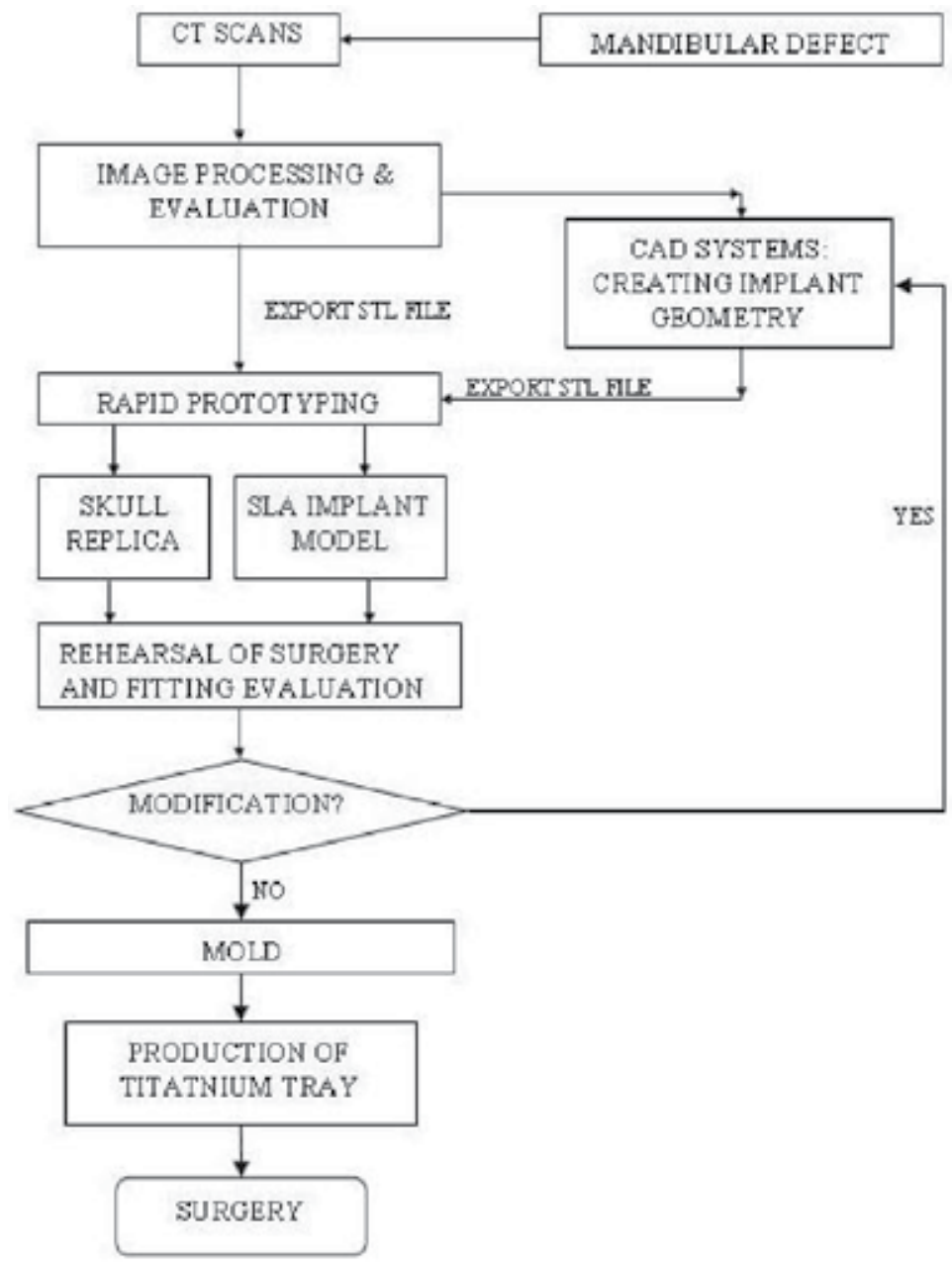

Fig. 2. Computer-aided geometric modeling for the manufacturing of custom implants

\subsection{CT data acquisition}

CT data acquisition was performed by the spiral volumetric technique (Picker MX8000). Suitable CT parameters for data acquisition were as follows: zero degree gantry, a resolution of $512 \times 512$ pixel reconstruction matrix image, $1.3 \mathrm{~mm}$ slice thickness and a slice reconstruction interval of $0.6 \mathrm{~mm}$.

The scan data are recorded according to the DICOM Norm (a standard of data formatting and of communication used in medical imagery).

\subsection{Medical image processing-segmentation and 3D reconstruction}

The 2D image slices from the CT scans were imported into the Materialise's Interactive Medical Imaging Control System (Mimics). A thresholding and region growing technique were used to extract the contour of the skeleton from the CT data. After removing the soft tissue, a 3-D region-growing technique is then used to isolate the skeletal part of the head from the CT dataset. 
A 3D image is reconstructed and visualized. The skull data was converted to a mesh based surface representation (STL) format and was then download to an RP machine to fabricate the skull replica.

\subsection{Design of the custom titanium implant}

Customized bone grafting trays were designed using Geomagics studio, version 6.0 (Raindrop Geomagic, Research Triangle Park, NC). Different techniques are applied for individual cases, including mirroring the non-defect side, implant design from other skull CT data and geometry modeling. The designs of different implant are to be elaborated in each case report.

\subsection{Rapid prototyping}

The CAD model of the skeleton structure and the bone grafting tray were then transferred in a stereolithography (STL) format, and input to a laser stereolithographic rapid prototyping system, LPS 600, to manufacture the skull models and customized implants. The model was sliced into $0.1 \mathrm{~mm}$ layer thickness, and then processed through a layer by layer building process. A physical resin model was thus obtained.

\subsection{Rehearsal of surgery and implant fitting evaluation}

The RP model makes a clear view about the defect and allow for a surgeon to gain operative experience and get a clear view of the specific demands required for such an operation.

Preoperative rehearsal of surgery via fitting the physical model of the custom tray with the patient's skull replica facilitates the optimal placement for the prosthesis onto the residual mandible; thereby evaluate the quality of the custom tray. This could reduce the operation time, and allow for modification of the surgical plans and the implants.

\subsection{Production of titanium implant}

To obtain a biocompatible titanium tray, the prototyped resin model of the tray was embedded with a high temperature resistant phosphate investment material. After successive drying and dipping, the resin model was burn out in an oven, at a temperature of $300-600^{\circ} \mathrm{C}$. This led to a casting mold and a titanium tray was cast using this model. The titanium tray was then subject to post processing: trimming, sandblasting and drilling, among others.

\section{Clinical applications}

\subsection{Case study: Unilateral mandible defect (fig. 3)}

A 24-year-old man with an adamantoma on the left mandibular angle and ramus was admitted. The surgical plan was to make a block resection treatment to cure the tumor, and to repair the defect with a rapid prototyped tray.

The CT data of the patient's skull was acquired and the computer assisted design of the tray was based on the mirror imaging technique.

Since the defect only involved the left side of the mandible. It was decided to mirror the undamaged right side onto the left side. To mirror the non-affected side image, a reference plan is needed. Usually, the center-plane can be established by the landmark of the anatomy structure, such as maxillary and mandibular adjacent point of central incisors, nasion, nasal 
septum, the central point of the sella turcica, etc (Zhou et al., 2010). It can also be established based on the midpoints of the symmetric landmarks, such as the orbital cavity, the condyles, the temporomandibular joint glenoid fossa, opposite teeth, etc. Generally, cranium and maxillas and zygomas are more stable than madible, since the latter will displace easily after unilateral bone resection or overgrowth in one side. Sometimes, to identify
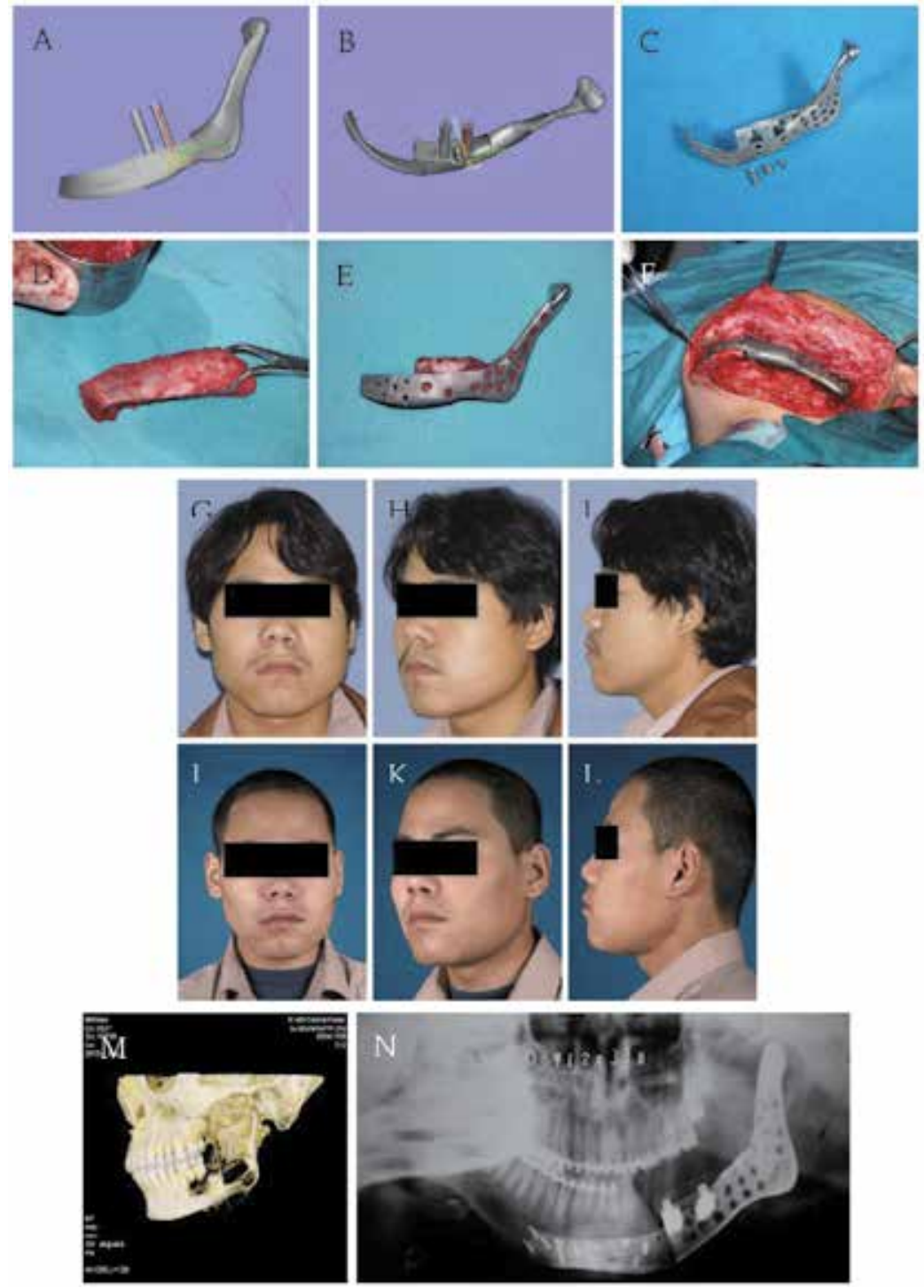

Fig. 3. (A\&B) the design of the tray. (C) the titanium tray. (D-F) intraoperative photos, D, shaping the iliac bone graft, E, marrow-cancellous bone grafts packed in the tray and bone blocks covered the tray, $\mathrm{F}$, the tray-bone graft complex was fixed onto the mandible to restore the defect. (G-L) pre- and post-operative facial appearance of the patient. (M) preoperative $3 \mathrm{D}$ view of the skull, tumor on the left mandible. $(\mathrm{N})$ postoperative X-ray view of the reconstructed mandible. 
the mirror plane is a great challenge. For the deformity only affect a part of the facial or cranial bone, the unaffected normal part can be used to establish and adjust for the mirror plane.

A suitable mirror plane could be obtained by trial and error. A reference plane that allows maximum overlap between the mirrored image and the native image of the normal part of the skull after mirroring can be considered to be the mirror plane.

After mirroring, the mirrored symmetric structure is considered to be the target contour to be restored. Then the mirror image was used to design the implant geometry. And footplates were designed based on the residual mandible ends.

The implant was manufactured by RP process. The SLA model was used to cast the titanium tray. And the implant was sterilized and prepared for the surgery.

The reconstructive surgery was performed via an extraoral approach. The residual mandible end was exposed and the bone bed was prepared, the tray was fitted onto the mandible and highly accurate match was observed. Autologous ilium was harvested from the anterior iliac crest. Crushed bone marrow-cancellous bone particles were densely packed into the tray and a cortical-cancellous bone block was placed on the top to cover entire tray. The cortical bone was drilled and secured on the top of the tray by two dental fixture implants. The tray-bone graft complex was then fixed onto the mandible with titanium screws. The wound was then closed. Satisfactory facial appearance and normal occlusion were restored. Over denture was made to rehabilitate the occlusion.

\subsection{Case study: Unilateral cranium defect (fig. 4)}

A patient with a huge unilateral cranium defect, involving the left parietal, temporal, frontal and sphenoid bone, due to traffic accident trauma was admitted for reconstruction.

Using the same mirroring technique, cranium prosthesis was designed and manufactured, to protect the intracranial contents. An implantation surgery was performed, with taking good care of the brain. Using the prototyped prosthesis, symmetric cranium was restored, via a straight approach. The general appearance and radiologic picture demonstrated the symmetry.

In conclusion, the computer assisted design and rapid prototyping technique facilitate the reconstructive surgery. By applying the mirroring method, excellent symmetry can be restored for the asymmetric skeletal defect.

\subsection{Case study: Mandibular retraction (fig. 5)}

A 28-year-old woman with a mandibular retraction needed chin augmentation. A chin augmentation of $6 \mathrm{~mm}$ was predicted by cephalometric analysis. And an individual prosthesis was designed and manufactured.

By using the same technology in case study one, a 3D reconstructed CT data was generated (MIMICS). The defect couldn't be reconstructed by mirror imaging technique. The patient's 3D mandible CT data was measured and these measurements data were used to select a similar mandible. A skull model of a healthy woman with normal mandible contour was selected and used to design the implant geometry.

The CAD design of the implant was based on the normal mandible data used as a template to create an anatomically correct mandibular contour. The inner surface of the implant was based on the anatomic structure of the chin surface, which allow for an easy placement of the implant onto the chin. 


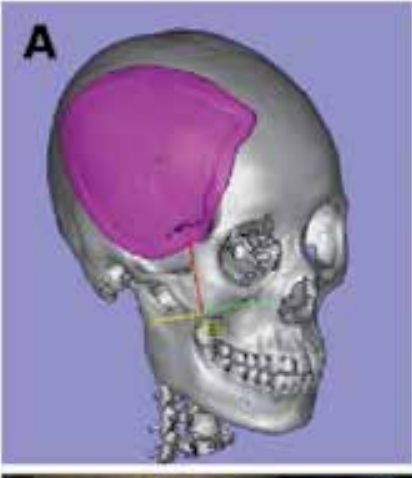

\section{B}
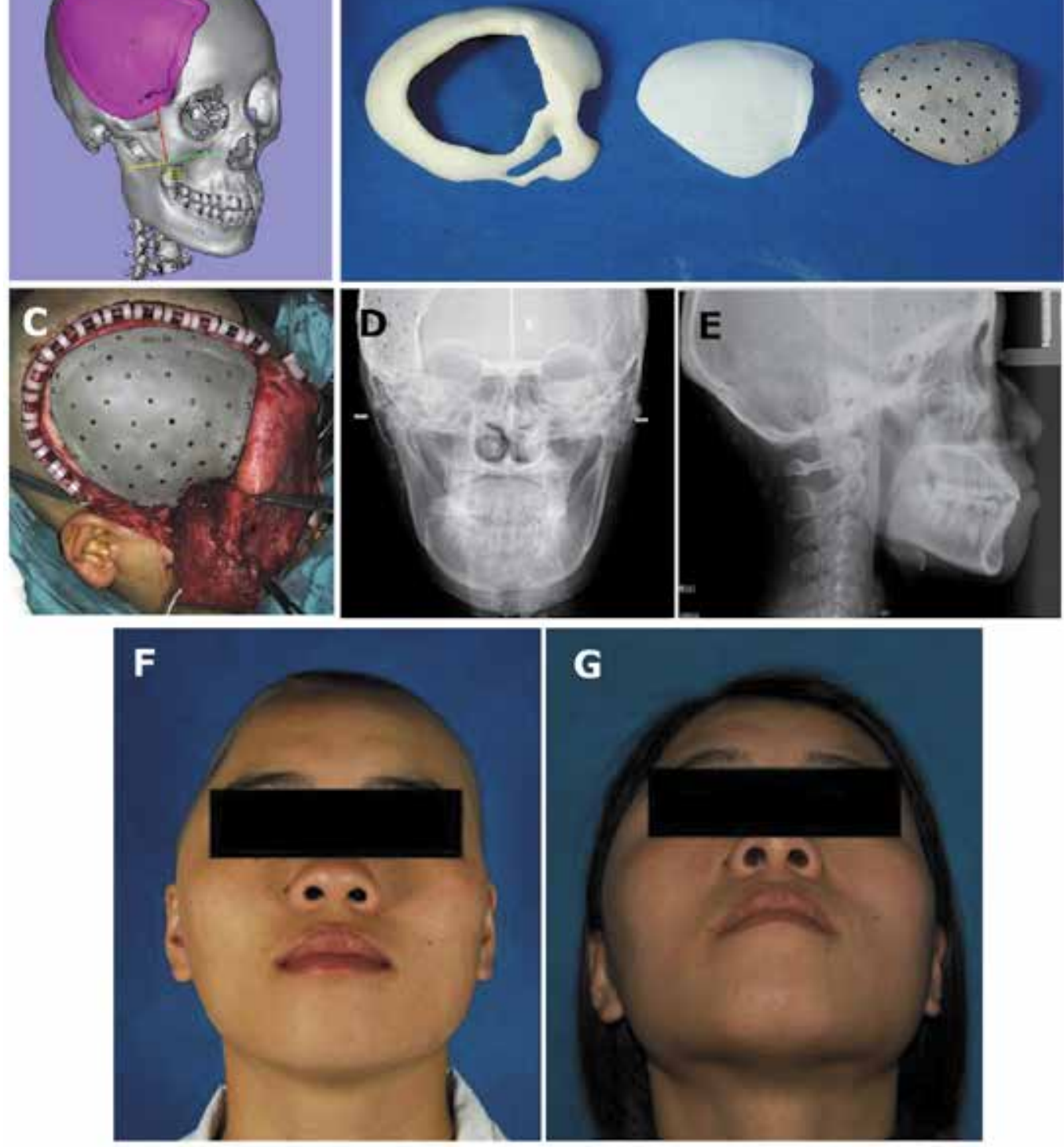

Fig. 4. (A) the design of the cranium prosthesis. (B) the resin model of the cranium and prosthesis, and the titanium prosthesis. $(\mathrm{C})$ intraoperative photo: the fixation of the prosthesis to the cranium to repair the defect. (D\&E) postoperative X-ray view of the skull., $\mathrm{F}$, expose the chin, $\mathrm{G}$, place the prosthesis. $(\mathrm{F} \& \mathrm{G})$ pre- and post-operative facial appearance of the patient.

A three-dimensional model was manufactured by using a rapid prototyping machine and the prototype was used to cast the titanium implant. The prosthesis manufactured using rapid prototyping technology resulted in simple surgical implantation and better facial 
contour. This technique can also be used to reconstruct segmental defect in the chin region and to cure hemifacial mocrosomia.
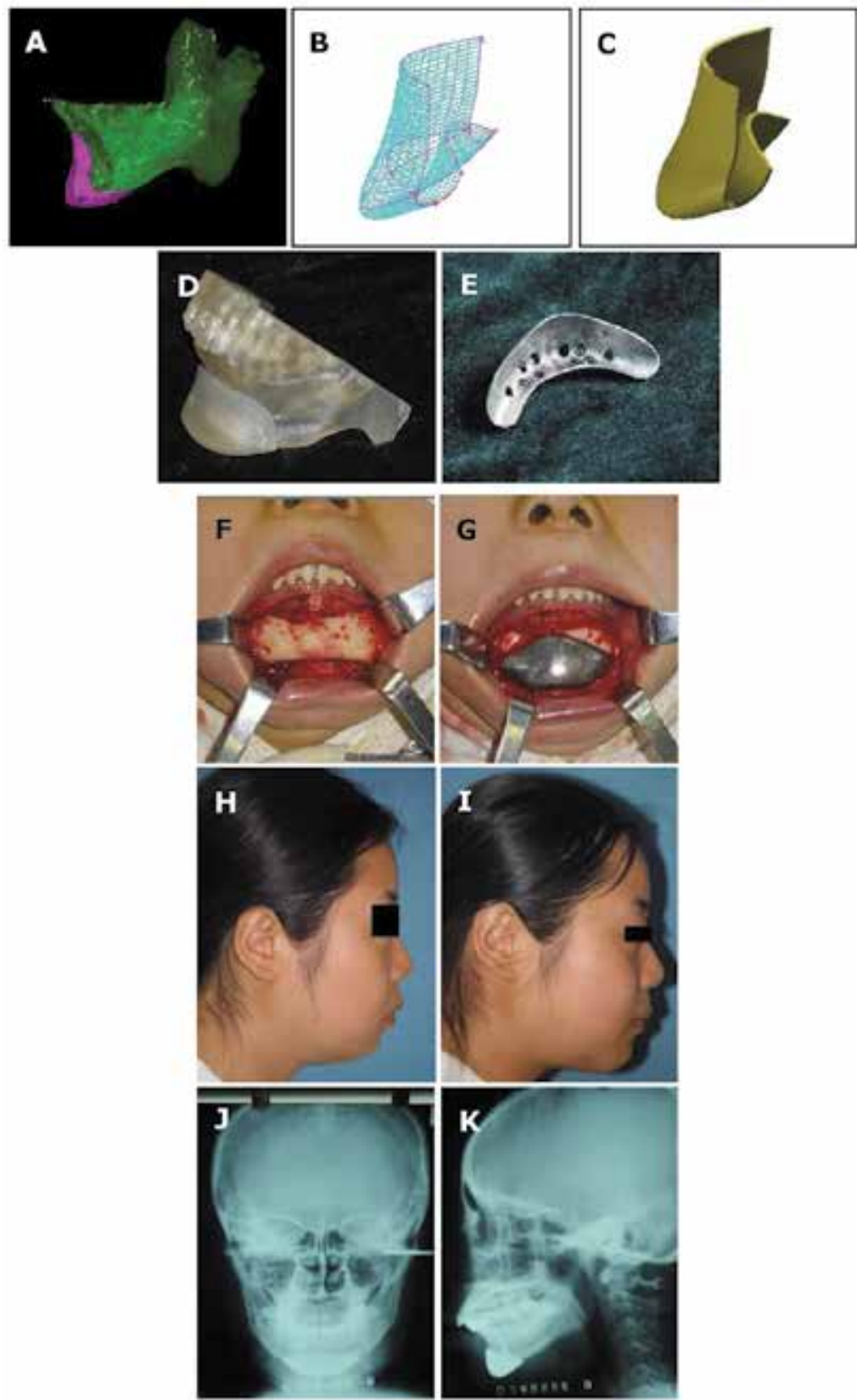

Fig. 5. (A-C) the design of the chin prosthesis. (D) resin model of the prosthesis. (E) the titanium chin prosthesis. (F\&G) intraoperative photos, F, expose the chin, $G$, place the prosthesis. (H\&I) pre- and post-operative facial appearance of the patient. (J\&K) postoperative X-ray view of the reconstructed mandible. 


\section{Results}

We compared the skull model and the CT scan data and found that the physical model's dimension was in agreement with the CT scan data and the error was less than $0.3 \%$.

The prototyped models, the skull and the implant, were used to evaluate the design and the surgical planning. It was found that the physical tray made from virtual data, fitted perfectly with the exact replica of the patient's skull anatomy. Furthermore, an adequate symmetry of the jaw was obtained. The surgical planning was accurate and was facilitated by the RP model.

The custom titanium implants were well fitted in patients. In all cases, the implants were just inserted and fixed by screws, so that the duration of the surgery was reduced with the aid of the customized implants.

No complications were observed except that the cancellous bone packed in the grafting tray was absorbed after a period of time as shows in fig. $3 \mathrm{~N}$.

\section{Conclusions}

We introduced the technology of manufacturing individual reconstructive prosthesis for craniofacial bone defects. This technology involves implant shape design in CAD environment from CT data, fabrication of the physical model by rapid prototyping process, creating the mold from the prototype, and then cast of the titanium implant. Clinical studies demonstrated that this new method can create accurate implant for bone various defects. We conclude that, with the development of the relative techniques of RP, perfect individual implants can be manufactured. Also the RP technique facilitates the reconstruction surgery and makes it more controllable and accurate. Satisfactory aesthetics and functional rehabilitation of craniofacial deformities can be achieved, that otherwise would remain difficult.

\section{Acknowledgment}

We appreciate the assistance by the Institute of Advanced Manufacturing Technology, Xi'an Jiaotong University, in designing and manufacturing the individual implants.

\section{References}

Mehta RP. \& Deschler DG. (2004). Mandibular reconstruction in 2004: an analysis of different techniques. Current Opinion in Otolaryngology \& Head and Neck Surgery, Vol 12, No.4, (August 2004), pp. 288-2893, ISSN 1068-9508

Boyne PJ. (1973). Methods of osseous reconstruction of the mandible following surgical resection. Journal of Biomedical Materials Research, Vol 7, No.195, (1973), pp. 195-204, ISSN 1549-3296

Tideman H., Samman N. \& Cheung LK. (1998). Functional reconstruction of the mandible: a modified titanium mesh system. International Journal of Oral and Maxillofacial Surgery, Vol.27, No.5, (October 1998), pp. 339-345, ISSN 1399-0020

Samman N., Luk W., Chow T., Cheung L., Tideman H. \& Clark R. (1999). Custom-made titanium mandibular reconstruction tray. Australian Dental Journal, Vol.44, No.3, (September 1999), pp. 195-199, ISSN 0045-0421 
Eufinger H., Wehmöller M. \& Machtens E. (1997). Individual prostheses and resection templates for mandibular resection and reconstruction. British Journal of Oral and Maxillofacial Surgery, Vol.35, No.6, (December 1997), pp. 413-418, ISSN 0266-4356

Stojadinovic S., Eufinger H., Wehmöller M. \& Machtens E. (1999). One-step resection and reconstruction of the mandible using computer-aided techniques--experimental and clinical results. Mund Kiefer Gesichtschir, Vol.3, (May 1999), pp. S151-153, ISSN 1432-9417

Singare S., Dichen L., Bingheng L., Yanpu L., Zhenyu G. \& Yaxiong L. (1997). Design and fabrication of custom mandible titanium tray based on rapid prototyping. Medical Engineering \& Physics, Vol.26, No.8, (October 2004), pp. 671-676, ISSN 1350-4533

Zhou L., Zhao J., Shang H., Liu W., Feng Z., Liu G., Wang J. \& Liu Y. (2011). Reconstruction of Mandibular Defects Using a Custom Made Titanium Tray in Combination with Autologous Cancellous Bone. Journal of Oral and Maxillofacial Surgery, Vol.69, No.5, (May 2011), pp. 1508-1518, ISSN 0278-2391

Zhou L., Shang H., He L., Bo B., Liu G., Liu Y., \& Zhao J. (2010). Accurate reconstruction of discontinuous mandible using a reverse engineering/computer-aided design/rapid prototyping technique: a preliminary clinical study. Journal of Oral and Maxillofacial Surgery, Vol.68, No.9, (September 2010), pp. 2115-2121, ISSN 0278-2391 


\title{
Application of a Novel Patient - Specific Rapid Prototyping Template in Orthopedics Surgery
}

\author{
Sheng Lu, Yong-qing $\mathrm{Xu}$ and Yuan-zhi Zhang \\ Department of Orthopedics, Kunming general hospital, \\ Chengdu military district, PLA, Kunming,
}

China

\begin{abstract}
1. Introduction
Conventional surgical handwork requires competences such as dexterity or fine motor skills, which are complemented by visual and tactile feedback. Computer-assisted orthopaedic surgery aims at improving the perception that a surgeon has of the surgical field and the operative manipulation. Bony manipulation such as drilling, chiseling, or sawing can be performed more accurately and implants can be placed more exactly. This reduces the risk of harming the patient intra-operatively by damaging sensitive structures.

CT scans are very suitable for surgical navigation, especially in orthopaedics. The bones can be easily distinguished from any other tissue, and can be easily segmented out. The bones are also the least deformable parts of the body, and therefore the most stable references for navigation, making it possible for different phases of surgical planning and execution to be performed well after the patient imaging. Pre-operative planning is typically done in three orthogonal cross-sectional views made through the CT scan volume.
\end{abstract}

\section{Application of a novel patient - specific rapid prototyping template in orthopedics surgery}

The rapid prototyping template first apply in the hip and knee athroplasty and then apply in the spine surgery. But the limitation of template design and produce technique, the authors introduced and validated a novel rapid prototyping templates in the clinical setting. Report on their experience with spinal pedicle screw placement, [1,2] placement of C2 laminar screws, [3] accurate prosthesis implantation in hip resurfacing arthroplasty, etc using a novel computer- assisted drill guide template.

\subsection{A novel computer- assisted rapid prototyping drill guide template for spinal pedicle screw placement}

Spinal Pedicle screw fixation systems provide three-dimensional (3D) fixation in the spine. Compared with conventional hook instrumentation, the clinical advantages of such systems include enhanced correction and stabilization of various deformities, shorter fusion length, more solid and reliable fixation, and no encroachment into the spinal canal. Therefore, pedicle screw fixation systems have gained popularity for internal fixation of fractures, tumors, and deformities of the spine . In spinal pedicle screw insertion, it is important both 
to select the correct size of screw and to place it properly within the pedicle to ensure good anchoring. Manual placement has a high associated rate of unplanned perforation, which is the major specific complication of pedicle screw placement and causes a high risk of bone weakening or lesions of the spinal cord, nerve roots, or blood vessels.

Successful placement of pedicle screws in the cervical spine requires a thorough threedimensional understanding of the pedicle morphology in order to accurately identify the ideal screw axis. Several methods have been explored for precise cervical pedicle screw placement including anatomic studies, image-guided techniques, computer-assisted surgery system, and drill templates. These techniques can be broadly classified into five types: (1) techniques relying on anatomical landmarks and averaged angular dimensions; (2) techniques with direct exposure of the pedicle, e.g. by laminaminotomy; (3) CT-based computer assisted surgery (CAS), and (4) fluoroscopy-based CAS techniques. (5) Drill template techniques.

The principle of image guidance is to register the patient's pre-operative computed tomography (CT) scans, thus permitting the surgeon to navigate simultaneously within the patient and the CT scan volume. Such navigation systems have shown good clinical results. There are, however, several disadvantages associated with navigation systems. In cases where screws are to be placed in more than one vertebra, it is necessary to perform a separate registration step for each vertebra. Intraoperative registration of bone structures takes up to several minutes, and thus the time taken for the overall procedure is increased compared with a conventional approach. The navigation equipment often requires additional personnel to be present during surgery, and this, together with the increased operating time, leads to a higher risk of intraoperative infection. The navigation equipment is cumbersome and occupies a lot of space in the operating room. Finally, only a few hospitals can bear the costs of sensor or robot-based systems. One way to overcome these drawbacks is the production of personalized templates. These are designed using preoperative CT to fit in a unique position on the individual's bone, and they have carefully designed holes to guide the drill through a pre-planned trajectory.

\subsubsection{A novel patient-specific navigational template for cervical screw placement}

Successful placement of pedicle screws in the cervical spine requires a thorough threedimensional understanding of the pedicle morphology in order to accurately identify the ideal screw axis. The accuracy of computer-assisted screw insertion has been demonstrated recently. The rate of pedicle perforations was $8.6 \%$ in the conventional group and $3.0 \%$ in the computerassisted surgery group in 52 consecutive patients who received posterior cervical or cervicothoracic instrumentations using pedicle screws. [4] Another group has also reported similar results, in which the rate of pedicle wall perforation was found to be significantly lower in the computer-assisted group (1.2\%) than in the conventional group (6.7\%).[5] However, despite advances in instrumentation techniques and intra-operative imaging, successful implementation of posterior cervical instrumentation still remains a challenge.

Considering these difficulties, this study introduces an ingenious, custom-fit navigational template for the placement of pedicle screws in the cervical spine and further validate it in the clinical settings. Based on this technique, the trajectory of the cervical pedicle screws were first identified based on the preoperative CT scan model. The drill template was then patient-specifically designed so that it can keep in close contact with the postural surface of the cervical vertebra in order to provide the best stability for drilling. 
25 patients (14 male, 11 female, age 17-53 years) with cervical spinal pathology included 10 patients with destabilizing cervical spine injuries, 4 patients with cervical spondylotic myelopathy, and 11 patients with basilar invagination requiring instrumentation underwent cervical pedicle screw placement using a novel, patient-specific navigational template technique. According to this technique, a spiral three-dimensional (3-D) CT scan (LightSpeed VCT, GE, USA) was performed preoperatively on the cervical spine of each patient with a $0.625-\mathrm{mm}$ slice thickness and $0.35-\mathrm{mm}$ in-plane resolution. The images were stored in DICOM format, and transferred to a workstation running MIMICS 10.01 software (Materialise, Belgium) to generate a 3-D reconstruction model of the desired cervical vertebra (Fig. 1).

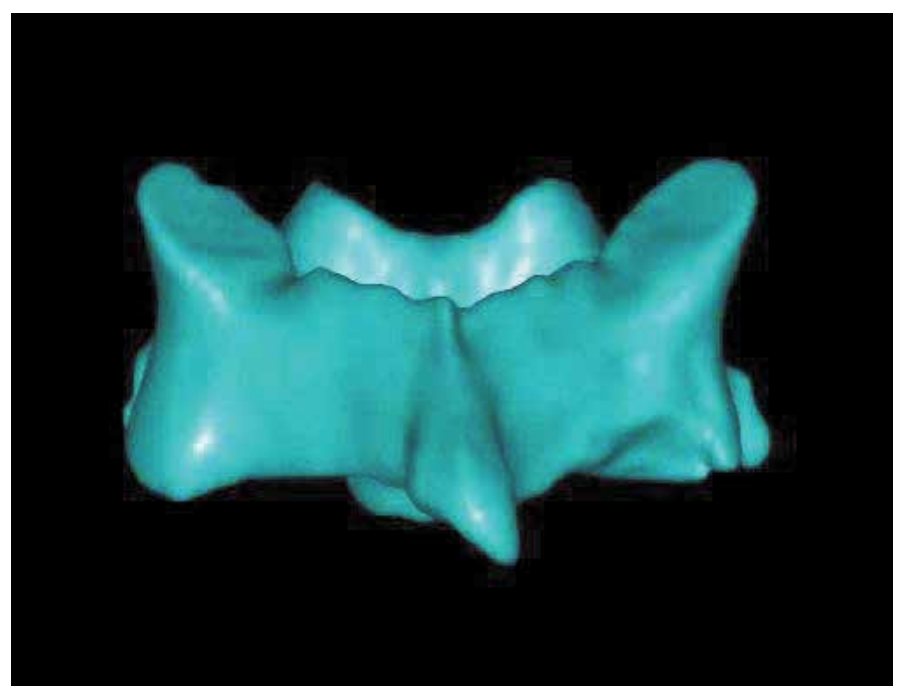

(a)

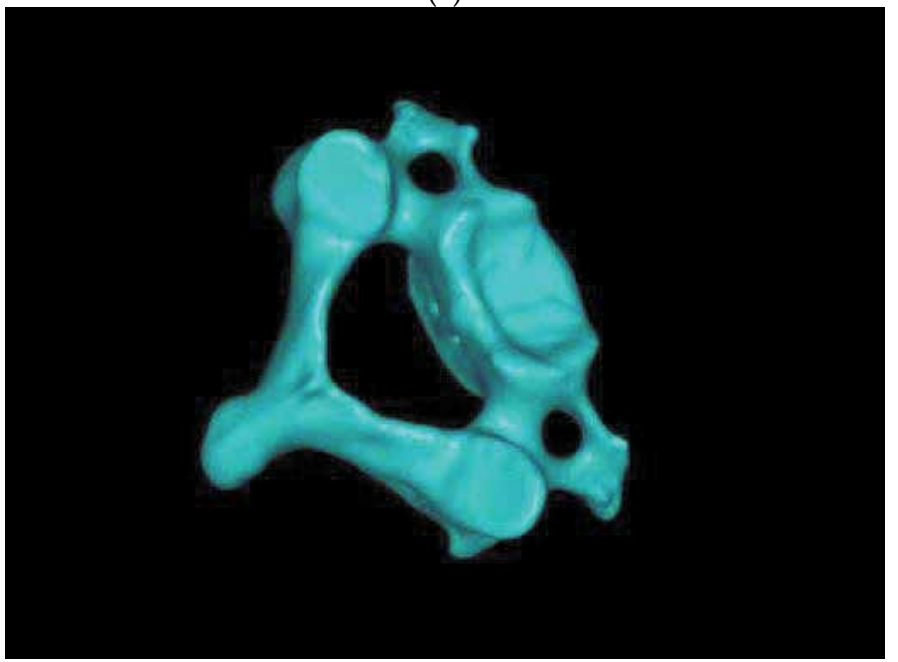

(b)

Fig. 1. 3-D model of the cervical vertebra (C3).

a: posterior view ; b: lateral view 
The 3-D cervical vertebral model was then exported in STL format to a workstation running Reverse Engineer software -UG imageware12.0 (EDS, US), for determining the optimal screw size and orientation. Using the UG Imageware software, the pedicles (left and right pedicle) were projected towards the vertebra and lamina (Fig.2a). As the thickness and cross section of the pedicle vary along its length, the smaller diameter of the elliptical inner boundary of the pedicle's projection was used in determining the maximum allowable dimension for screw diameter (Fig.2b). This diameter was further used to draw a circle and projected between the vertebra and the lamina to obtain the optimal pedicle screw trajectory (Fig.2c,). A 3D vertebral model was reconstructed with a virtual screw placed on both sides(Fig.2d).
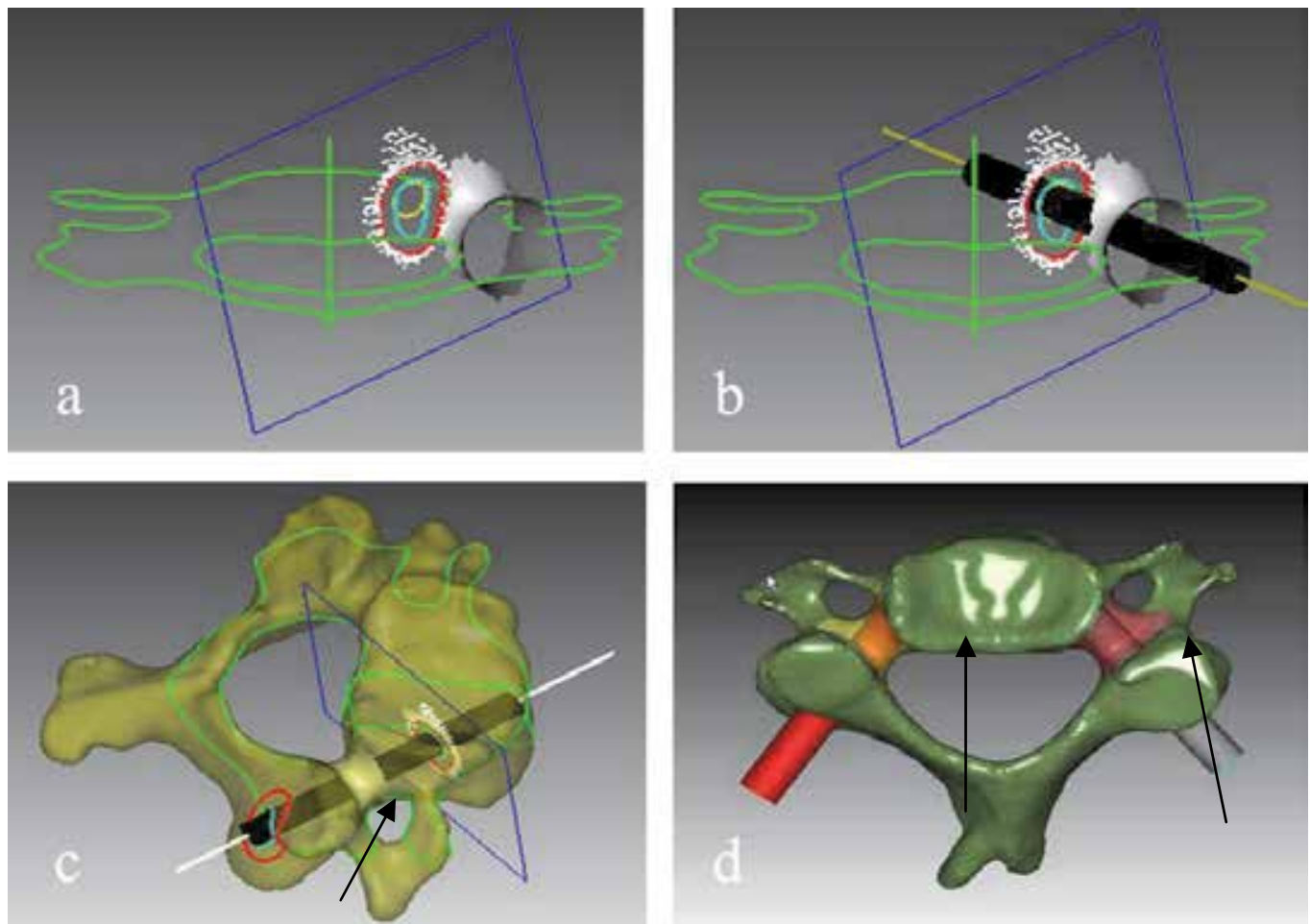

Fig. 2. Analysis of cervical pedicle screw trajectory by the Reverse Engineering software a: Pedicle and its positive projection; b: the best trajectory of pedicle screw projection;c: Pedicle screw channel. (arrow) d: Planned screw trajectory (arrows) 
Following the determination of the optimal pedicle screw trajectory, a navigational template was constructed with a drill guide on either side. The template surface was created as the inverse of the vertebral posterior surface, thus potentially enabling a nearperfect fit. It was also made sure that there was no overlapping of the template onto adjacent segments (Fig.3).

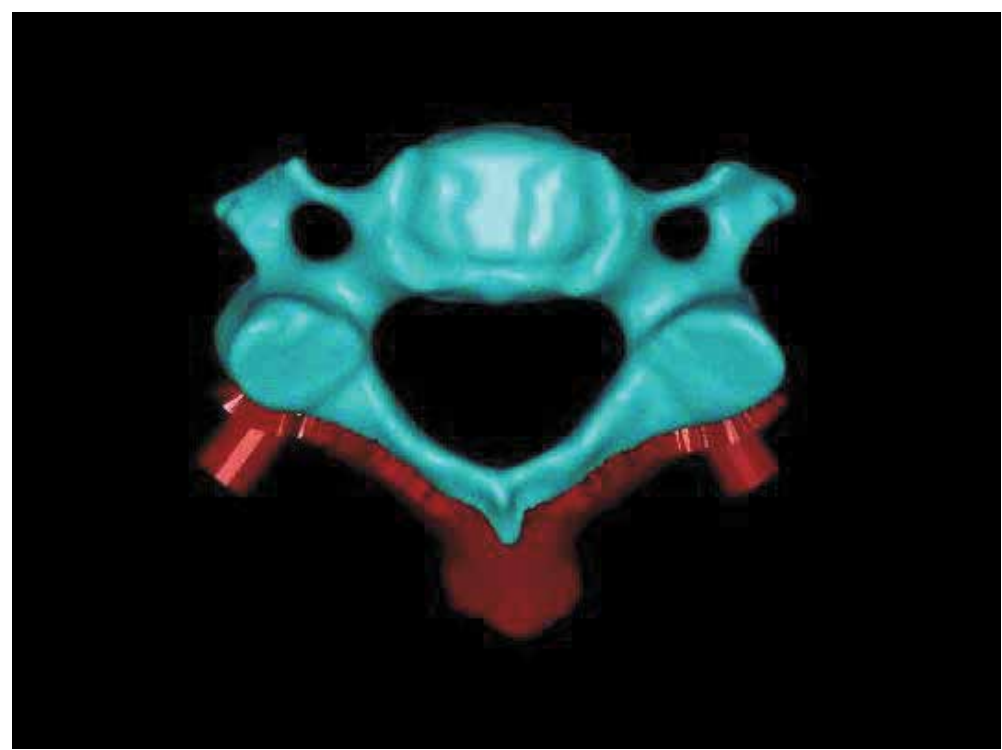

(a)

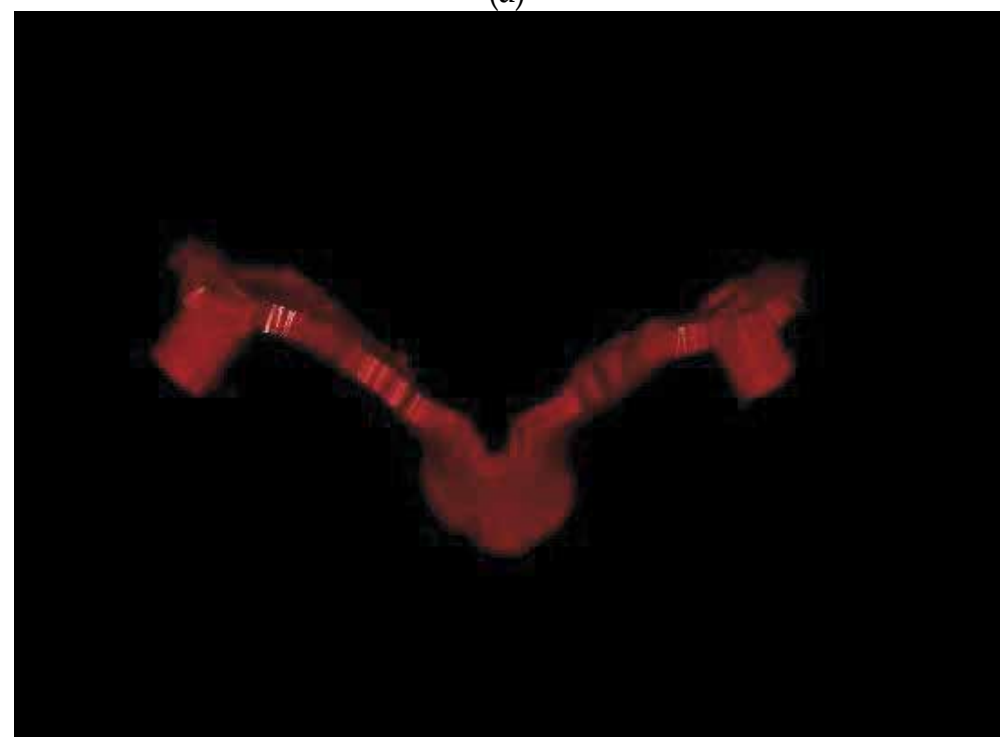

(b)

Fig. 3. Design of the navigational template a: Navigational template fits with the vertebra perfectly; b: The 3-D computer model of navigational template 
The biomodel of the desired vertbera as well as its corresponding navigational template were produced in acrylate resin (Somos 14120, DSM Desotech Inc, USA) using stereolithography - a rapid prototyping (RP) technique (Hen Tong company, China). The accuracy of the navigational template was examined by visual inspection before surgery. The biomodel of the vertebra and its corresponding template were placed together, and a standard electric power-drill was used to drill the screw trajectory into the biomodel of the vertebra through the template navigation holes. Visual inspection was performed for identifying any violation (Fig.4)

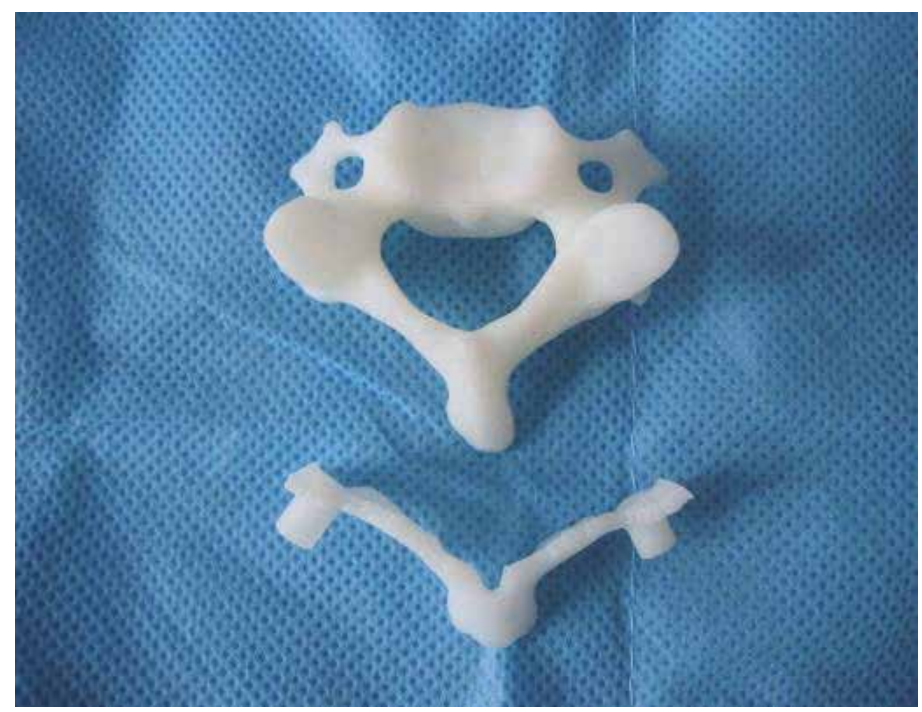

(a)

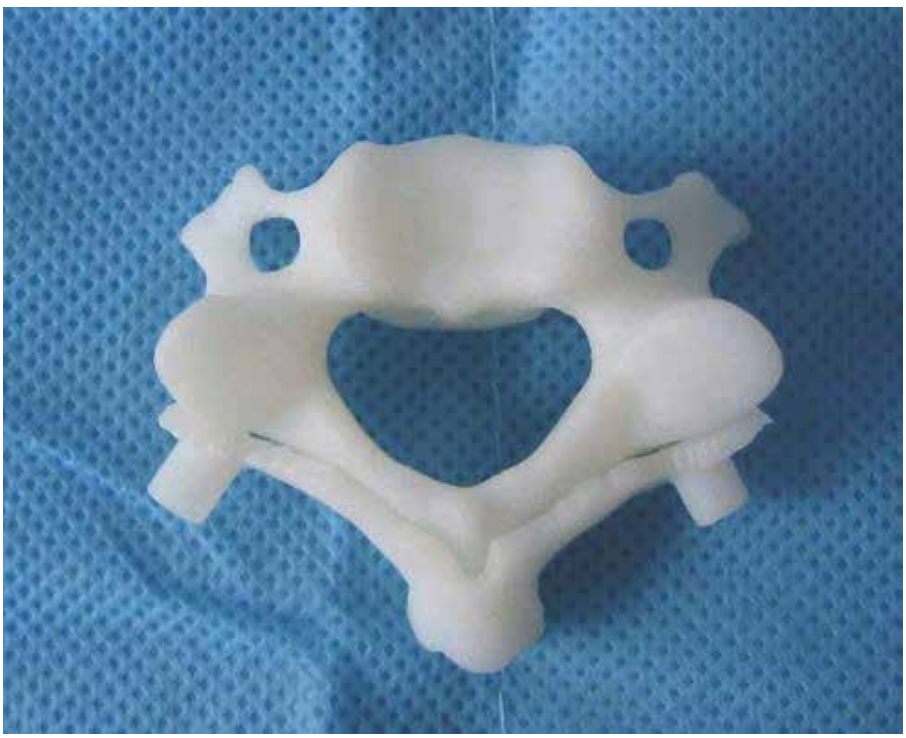

(b) 


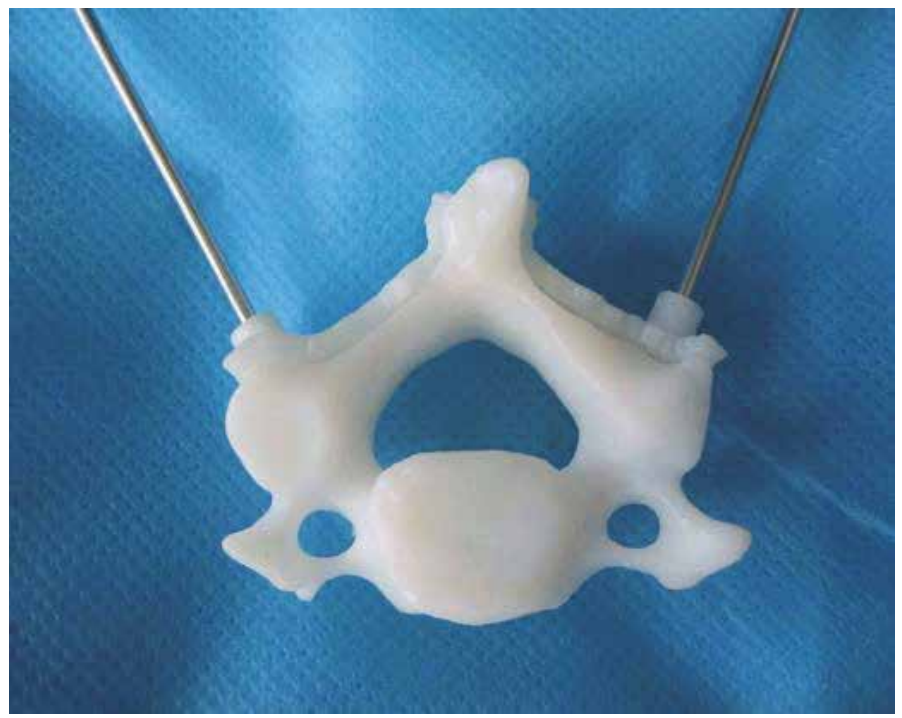

(c)

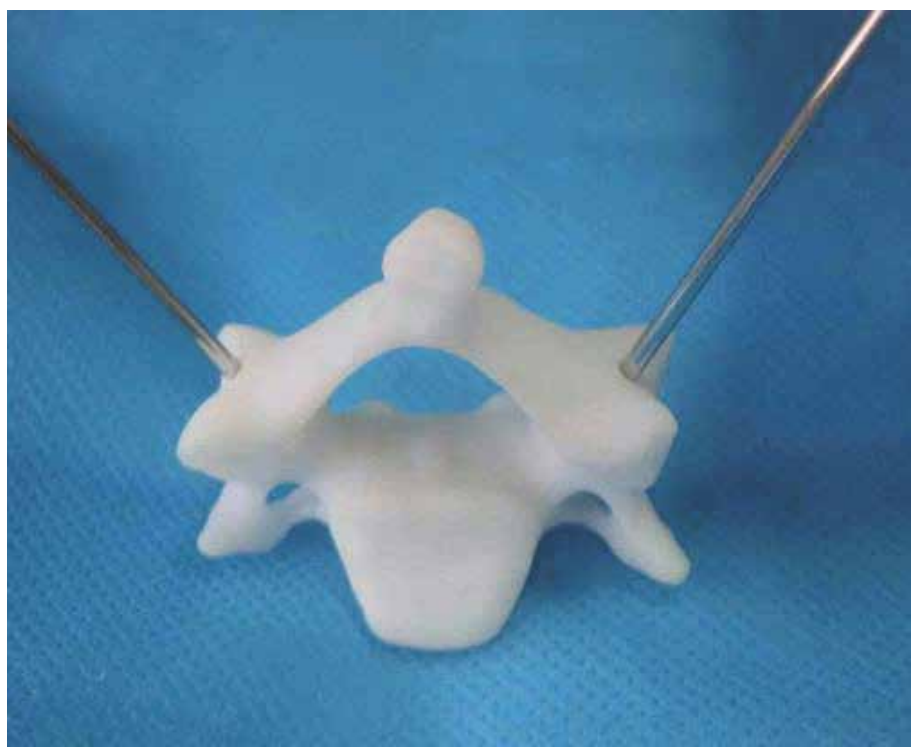

(d)

Fig. 4. The accuracy of the navigational template was examined by visual inspection a: RP model of vertebra and navigational template; b: navigational template fits RP model of vertebra perfectly; $\mathrm{c}$ K wires inserted through navigational template into the pedicles; $\mathrm{d}$ : accuracy of the navigational template examined by visual inspection.

The template was sterilized and used intraoperatively for navigation and for confirming anatomic relationships. For safety reasons, fluoroscopy was performed intraoperatively during drilling and insertion of the pedicle screw on the first 3 patients. For the remaining cases, fluoroscopy was performed only after the insertion of all the pedicle screws, thus 
considerably reducing the exposure time to radiation. After surgery, the positions of the pedicle screws were evaluated using X-ray and CT scan. An axial image, including the whole length of each screw, was obtained, and the medial and lateral deviation of the screw was classified into 4 grades 6 . Grade 0, no deviation; the screw was contained in the pedicle. Grade 1, deviation less than $2 \mathrm{~mm}$ or less than half of the screw diameter. Grade 2, deviation more than $2 \mathrm{~mm}$ and less than $4 \mathrm{~mm}$, or half to one screw diameter. Grade 3, deviation more than $4 \mathrm{~mm}$, or complete deviation.

The accuracy of the navigational template was examined before operation by drilling the screw trajectories into the vertebral biomodels. Each navigational template was found to be fitting to its corresponding vertebral biomodel appropriately without any free movement, and the $\mathrm{K}$ wires were found to be inserted through the drill hole through the pedicle and into the desired vertebra without any violation as found by visual inspection.

During the operation, it was easy to find the best fit for positioning the template manually, as there was no significant free motion of the template when it was placed in position and pressed slightly against the vertebral body. As such, the navigational template fulfilled its purpose for use as in situ drill guide.

A total of 88 screws were inserted into levels C2-C7 with 2-6 screws on each patient. Of these pedicle screws, 71 were in Grade-0, 14 in Grade-1, 3 in Grade-2, and no screw was in Grade-3. None of the cases had complications caused by pedicle perforation and especially there were no injury to the vertebral artery or to the spinal cord, nor was there a need for revision of pedicle perforation in any of the cases.

In this study, cervical pedicle abnormality existed in five patients. The pedicles (four C2 and one C7) of these patients were very narrow with a minimum diameter of $3.5 \mathrm{~mm}$. Screws of relatively smaller diameter (3- $\mathrm{mm}$ ) were chosen for these patients accordingly, and were placed inside pedicles accurately using navigational templates. (Fig.5)

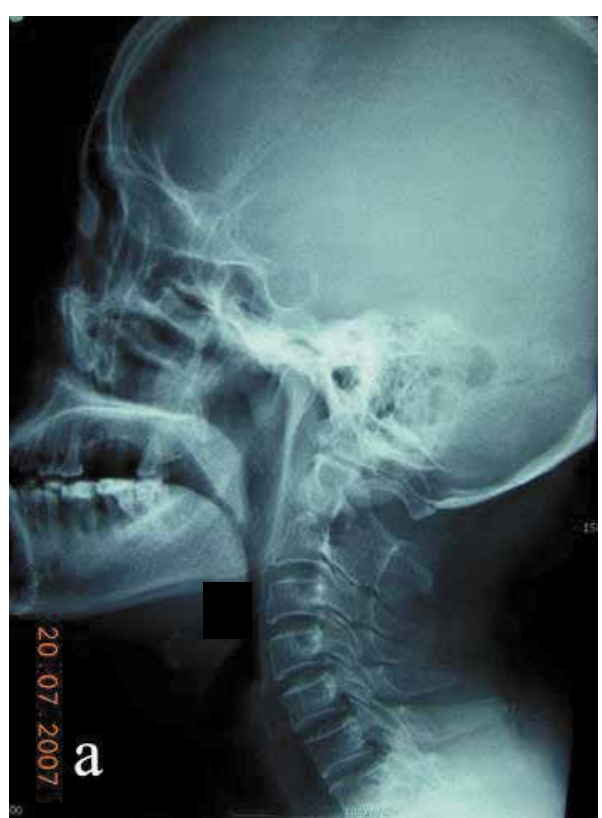

(a)

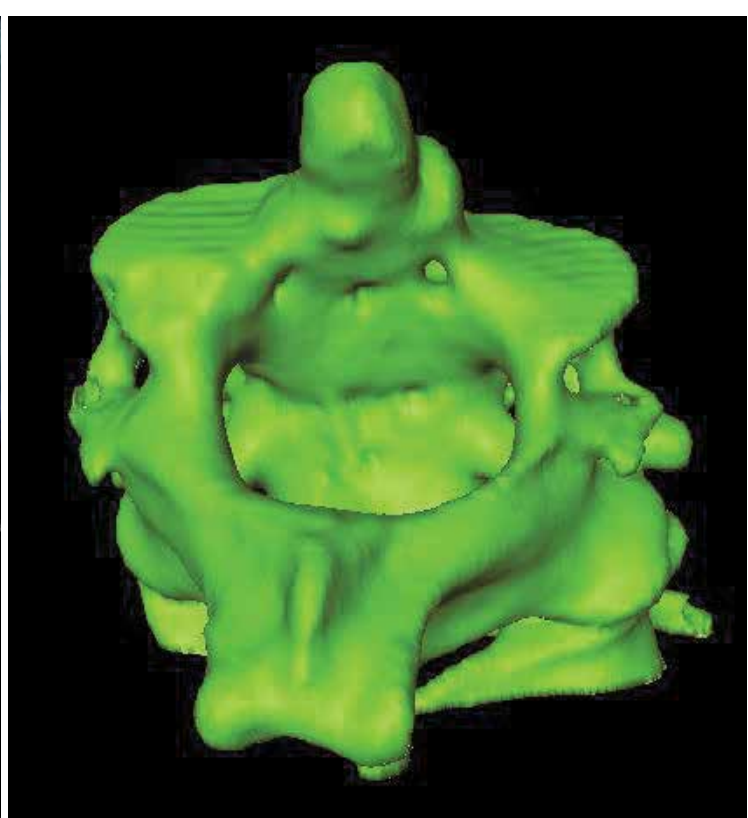

(b) 


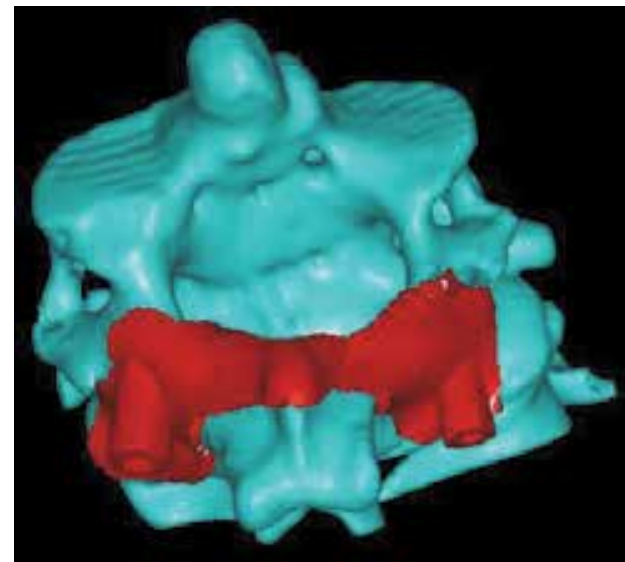

(c)

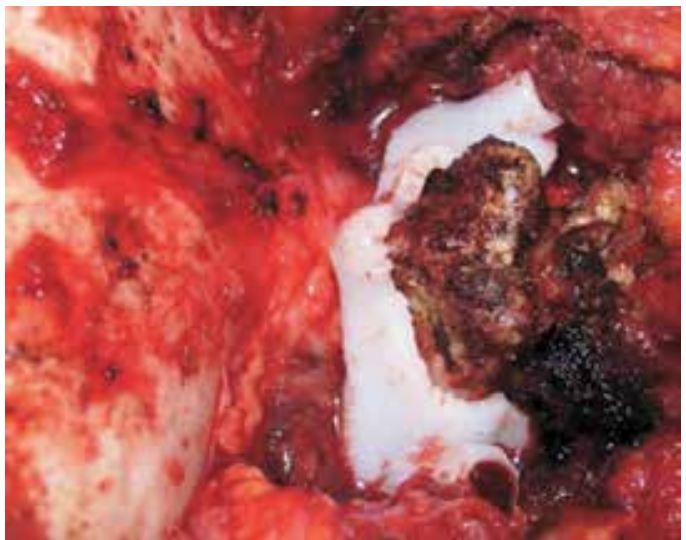

(e)

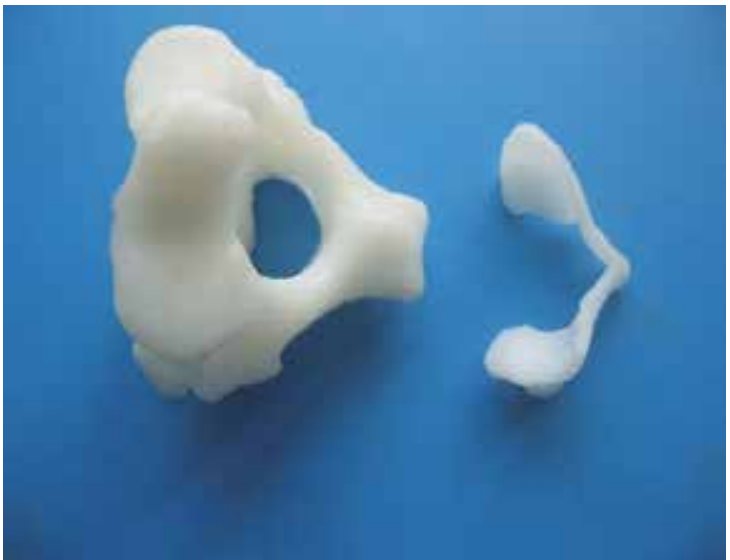

(d)

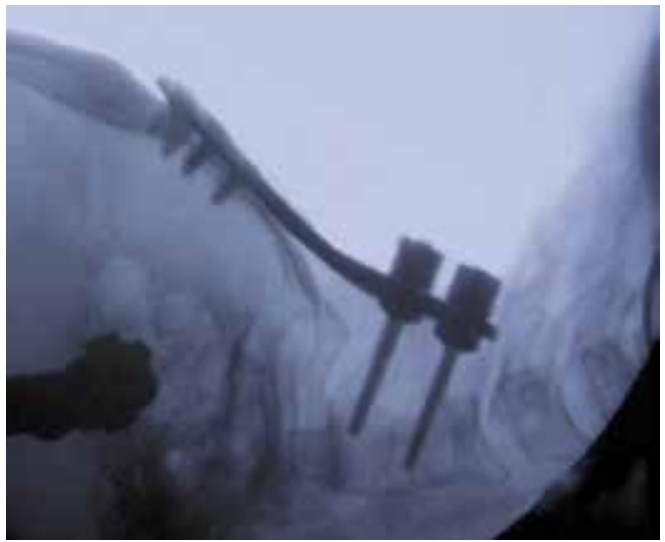

(f)

Fig. 5. A female patient was diagnosised of basilar invagination, trans-C2 Pedicle screw Occipto-Cervical Fusion was done; In this case the C2,3 fusion was observed and the diameter of left $\mathrm{C} 2$ pedicle was only $3.5 \mathrm{~mm}$, the $\mathrm{C} 2$ pedicle screw was inserted using the navigational template;

a: the X-ray shows atlantoaxial dislocation; d: 3-D model of C2,3; b,c: Pedicle screw trajectory and design of the C2 navigational template; $\mathrm{d}$ : RP model of C2,3 and navigational template, e: the navigational template fit the posterior part of $\mathrm{C} 2$ perfectly; f: fluoroscopy show good positioning of pedicle screw;

In another case, the pedicle was extremely narrow in level C2 with a minimum diameter of only $1.5-\mathrm{mm}$ and therefore the $\mathrm{C} 2$ cervical fixation was not performed. The $\mathrm{CT}$ data showed congenital fusion between C2 - C3 and therefore pedicle screw fixation was successfully performed on level C3 using the drill template.

By using this novel, custom-fit navigational template, the operation time has been considerably reduced. On an average, each vertebral pedicle screw insertion took about 80 seconds. Fluoroscopy was required only once after the insertion of the entire pedicle screws, which has considerably reduced the duration of radiation exposure to the members of the surgical team. Currently the production time for RP model is about 2 days and the cost is 
about $\$ 50$ per vertebral level. The production time can be brought down to 1 day and the cost can be reduced to $\$ 20$ if the RP model of the vertebra is not needed.

\subsubsection{A novel patient-specific navigational template for thoracic pedicle placement}

Most studies have shown that the rates of misplacement for the free-hand technique are usually between $28 \%$ and $43 \%$, while only a few studies have shown rates of less than $5 \%$. Hence, the screw breach rate may be dangerously high when the anatomy is altered as in scoliosis. Lonner et al. [6] suggested that there should be a considerable learning curve for using the pedicle screws in scoliosis surgery to avoid complications. The need for improved accuracy and consistency in the placement of thoracic pedicle screws has led to investigations on the application of computer-navigated spine surgery. Computer-assisted pedicle screw installation allows for an increased accuracy in using screws, thus decreasing the incidence of misplaced screws. Considering these difficulties, surgeons must use whatever techniques they find helpful to create a safe environment when placing thoracic pedicle screws into the deformed pediatric spine.

16 patients (12 females, 4 males, age 5-18 years) with scoliosis (14 adolescent idiopathic scoliosis, 2 congenital scoliosis) undergoing spinal deformity correction surgeries using posterior pedicle screw instrumentation of the thoracic spine formed the study group. Before the operation, a spiral three-dimensional (3-D) CT scan (LightSpeed VCT, GE, USA) was performed on the thoracic spine of each patient with $0.625 \mathrm{~mm}$ slice thickness and 0.35 $\mathrm{mm}$ in-plane resolution. The images were stored in DICOM format and transferred to a workstation running MIMICS 10.01 software (Materialise company, Belgium) to generate a 3-D reconstruction model for the desired thoracic vertebra (Fig. 6a). The 3-D vertebral model

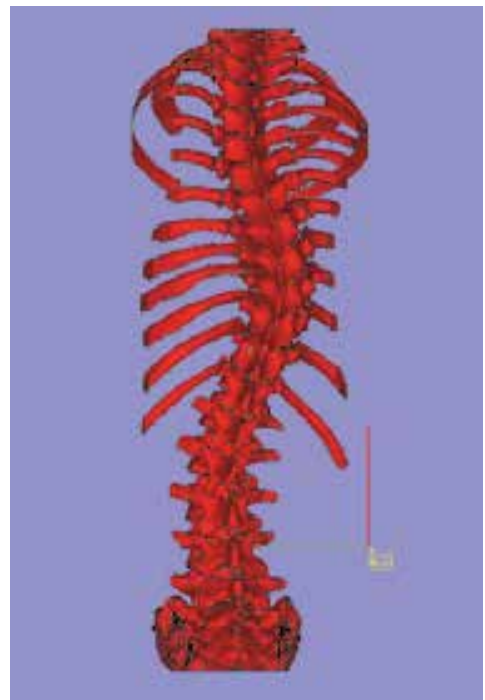

(a)

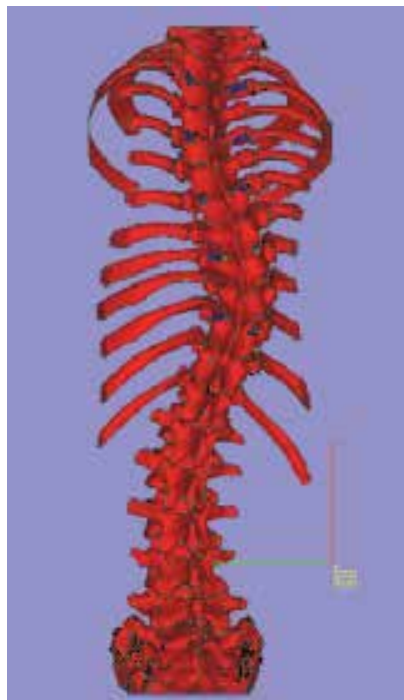

(b)

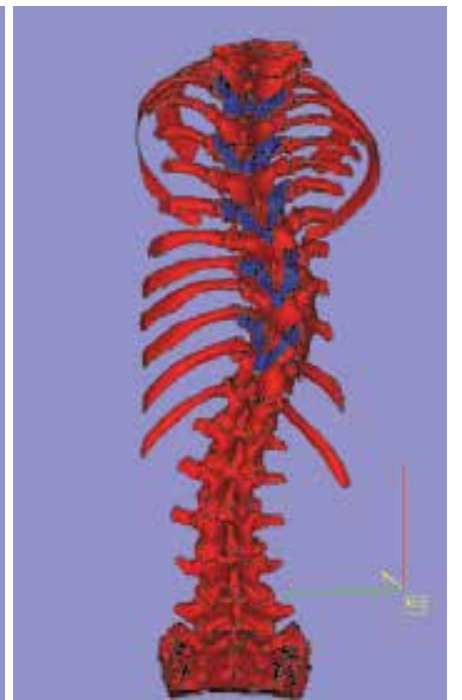

(c)

Fig. 6. Trajectory of the pedicle screw and design of the navigational template a: Three-dimensional model of the scoliosis vertebra; b: Planned screw trajectory (blue). c: The navigational template was created as the inverse of the posterior vertebral surface fitting with the vertebra perfectly. 
was then exported in STL format and opened in a workstation running Reverse Engineering (RE) software UG imageware12.0 (EDS, USA) to determine the optimal screw size and orientation. A screw with a diameter of $5 \mathrm{~mm}$ was placed virtually into the 3-D spinal model on both sides. The virtual screw's entry point and the trajectory were placed at the center of the pedicle without violating the cortex.

Pre-operative Planning

According to the type of scoliosis, the fusion level is determined, and the instrumentational vertebra is chosen. The design and development of the drill template for each vertebra are also made according to the instrumentational vertebra. The vertebral rotation, axes, length, and diameter of the pedicle were measured from the pre-operative CT scan. Thus, the length and diameter of every pedicle screw were decided upon before operation. The concave periapical (T5-8) pedicles are often deformed and are considered to be the most difficult area to work on during pedicle screw placement [3,27-29]. If the pedicle is very narrow, an inout-in technique can be chosen. (Fig 7).

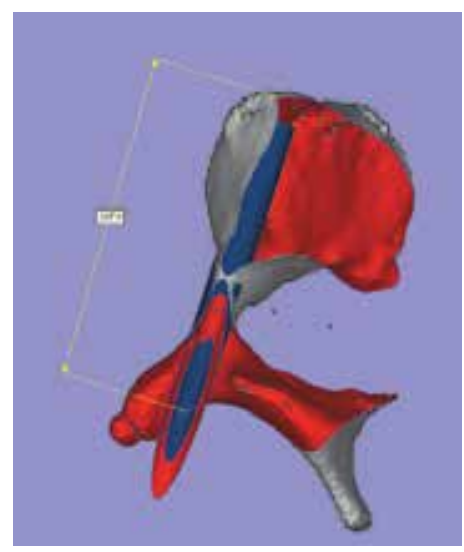

(a)

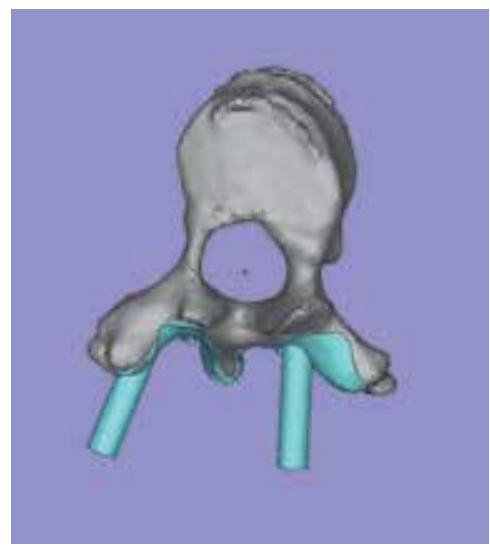

(c)

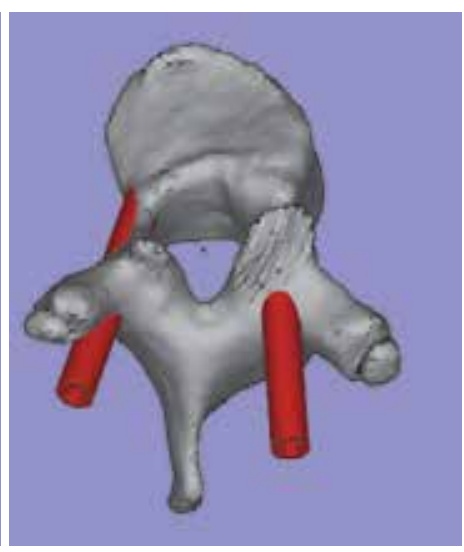

(b)

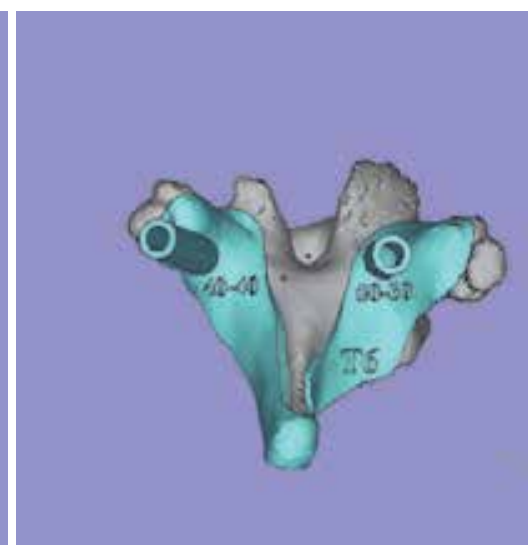

(d) 


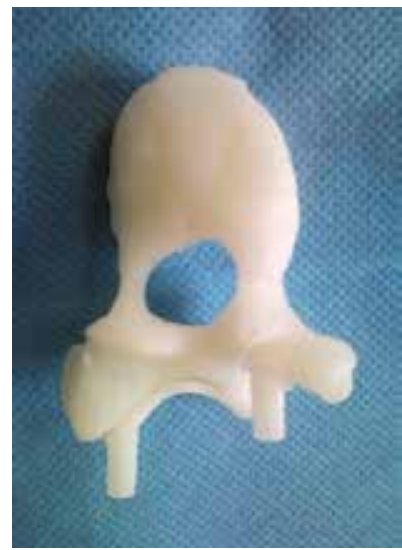

(e)

Fig. 7. 3D reconstruction of a single vertebra and the biomodel of the drill template a: The best trajectory of the pedicle screw and measurement of the length of the pedicle; $b$ : In-out-in technique in the concave side; c: The navigational template fits the vertebra perfectly; $d$ : The template can show the location, diameter, and length of the pedicle screw; e: The navigational template fits the RP model of the vertebra very well.

\section{Operation procedure}

The spine was exposed subperiosteally on both sides, up to the tips of the transverse processes. For the thoracic spine, the soft tissues on the facet joints were thoroughly cleaned off to ensure better visualization of the bony landmarks. The drill template was then placed on the spinous, lamina, and transverse processes. The drill template and the corresponding spinous process were fitted well. A high-speed drill was used along the navigational channel to drill the trajectory of each pedicle screw. Using a hand drill, the trajectory of the pedicle screw was carefully drilled to a depth in accordance with the pre-operation plan. The pedicle screw, the diameter and length of which had been chosen pre-operatively, was carefully inserted along the same trajectory. After screw placement and correction of deformity, all exposed laminar surfaces were decorticated, and the autologous iliac crest bone was grafted.

A total of 168 screws were placed from T2 to T12 in the 16 cases, and post-operative CT scans were obtained in all 16 patients. About 157 screws were considered intrapedicular, while 11 screws were considered to have a $0-2 \mathrm{~mm}$ breach (1 medial, 10 lateral in which 8 belonged to the planned in-out-in screws). No pedicle screw breached more than $2 \mathrm{~mm}$, and the overall screw accuracy ( $<2 \mathrm{~mm}$ breach is safe) was $100 \%$. No screws penetrated the inferior or superior cortex in the sagittal plane.

\subsubsection{A Novel Patient-specific Navigational Template for laminar Screw Placement}

Instability of the occipitocervical junction requiring surgical stabilization may be treated with a variety of techniques. The objective is to obtain solid fusion of the involved segments, which is best achieved by minimizing motion between them. Older methods such as the Brooks-Jenkins or modified Gallie wiring techniques, are simpler procedures, have been known for a long time and are associated with failure rates of fusion up to $25 \%$, primarily in cases with rotational instability. Newer techniques have been described that effectively limit 


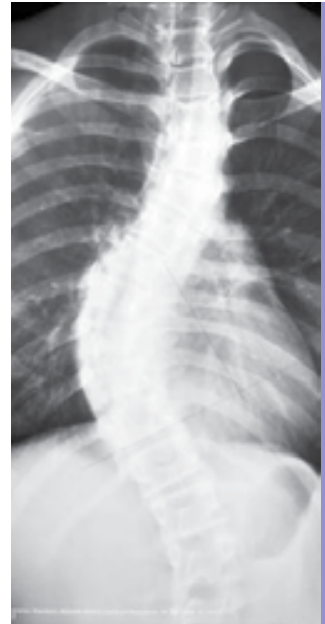

(a)

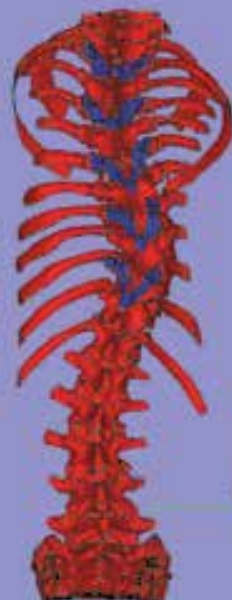

(b)

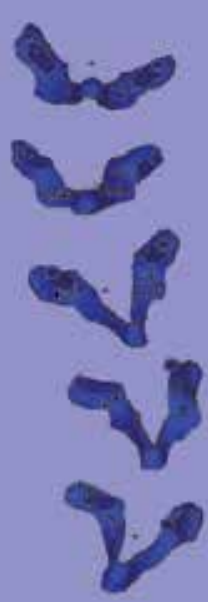

(c)

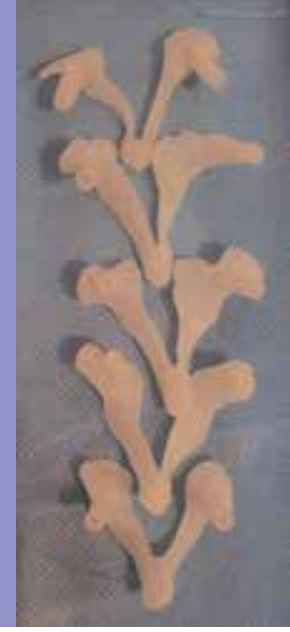

(d)

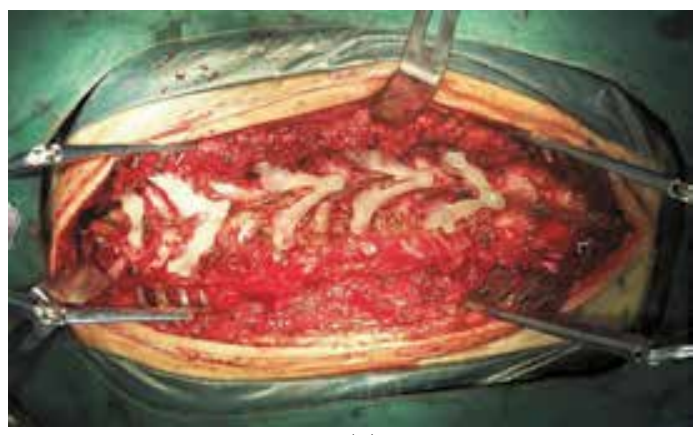

(e)

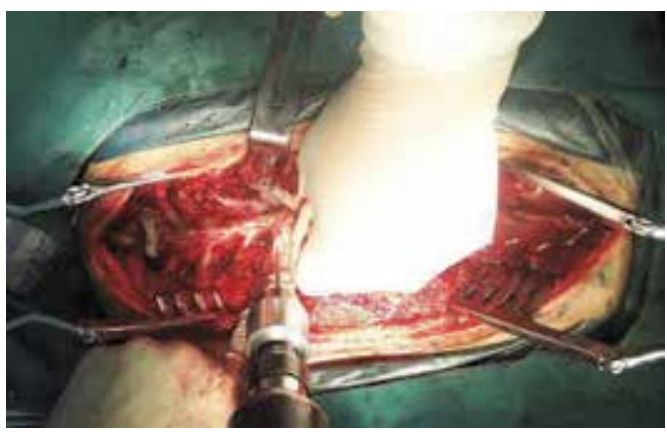

(f)

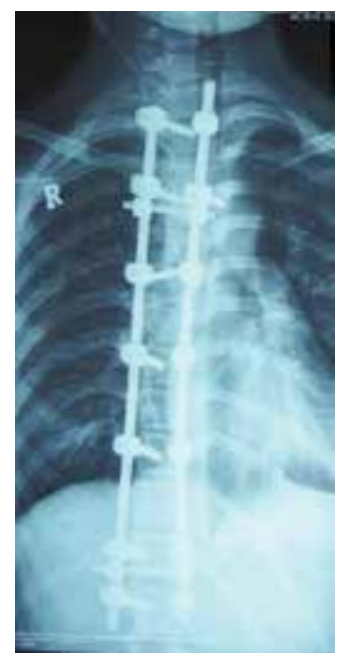

(g)

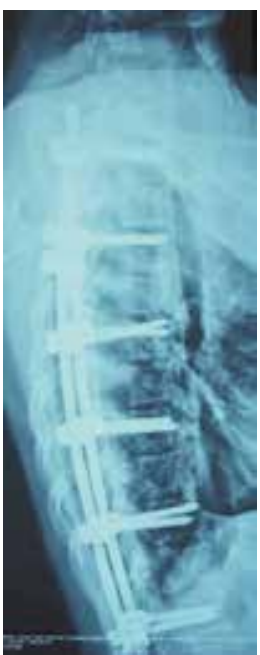

(h) 


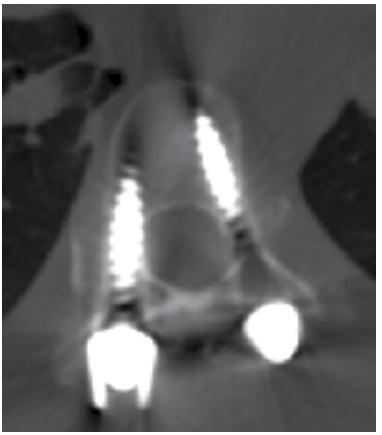

(i)

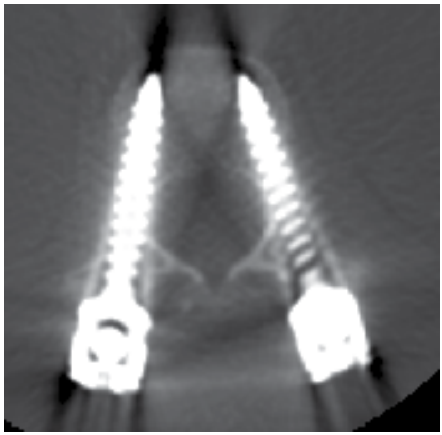

(j)

Fig. 8. A 12 -year-old male with a $62^{\circ}$ pre-operative curve; thoracic pedicle screw was inserted using the drill template;

a: AP X-ray; b: 3-D model of the spine; c: Pedicle screw trajectory and design of the navigational template; $\mathrm{d}$ : RP model of the navigational template, e: The navigational template fits the posterior part of the thoracic perfectly in the operation; $\mathrm{f}$ : Drill the trajectory by a power drill. $\mathrm{g}, \mathrm{h}, \mathrm{I}, \mathrm{j}$ : $\mathrm{X}$ ray and $\mathrm{CT}$ scan show that the screws were fully contained within the pedicle and body; corrected to $11^{\circ}$ one year after surgery, giving $82.3 \%$ correction of the major curve.

motion along all axes. The addition of transarticular screw fixation, according to Magerl and Seemann, offers a better biomechanical stability. But, Magerl screw fixation must be very precise point of placement to make screw fixation in the lateral mass of atlas, this can result in a significant risk to the vertebral artery. The size of the $\mathrm{C} 2$ pedicle can limit the ability to safely place these screws, particularly taking into account the anomalous position of the vertebral artery in relation to the isthmus of C 2 in up to $20 \%$ of the population. Leonard and Wright [7] described a new technique of C2 laminar screw for rigid screw fixation of the axis and incorporation into atlantoaxial fixation or subaxial cervical constructs, and subsequent cases have shown good clinical results with this technique. C2 laminar screws are appealing due to the reduced risk of injury to the vertebral artery and biomechanical stability. The present method of C2 laminar screw placement relies on anatomical landmarks for screw placement. Placement of C2 laminar screws using drill template has not been described in the literature. A novel computer- assisted drill guide template for placement of C2 laminar screws, Which designed to simplify and shorten the surgical act and the same time further enhance the accuracy of screw positions in the C2 laminar.

Before the operation, a spiral three-dimensional (3-D) CT scan (LightSpeed VCT, GE, USA) was performed on cervical spine of each patient with $0.625-\mathrm{mm}$ slice thickness and $0.35-\mathrm{mm}$ in-plane resolution. The images were stored in DICOM format, and transferred to a workstation running MIMICS 10.01 software (Materialise company, Belgium) to generate a 3D reconstruction model for the desired C2 vertebrae (Fig. 9a). The 3-D vertebral model was then exported in STL format, and opened in a workstation running Reverse Engineering (RE) software UG imageware12.0 (EDS, USA) for determining the optimal screw size and orientation. A screw with a diameter of $4 \mathrm{~mm}$ was placed virtually into the 3-D spinal model on both sides. The virtual screw's entry point and the trajectory was placed centered on the lamina without violating the cortex and two screws will not interfere with each other. (Fig. 9b). The optimal screw size was determined according to the size of laminea as well. Afterwards, a navigational template was constructed with one drill guide on either side. The template 
surface was created to be the inverse of C2 spinous process and laminar, thus potentially enabling a fit in a lock-and-key fashion similar to a physical casting of the vertebral surface, and specifically avoided overlap onto adjacent segments (Fig. 9c). The inner diameter of the hollow cylinder was created to accommodate the preplanned trajectory for drilling. Once these had been done, a drill template was constructed with a surface designed to be the inverse of the vertebral surface (Fig. 9d).

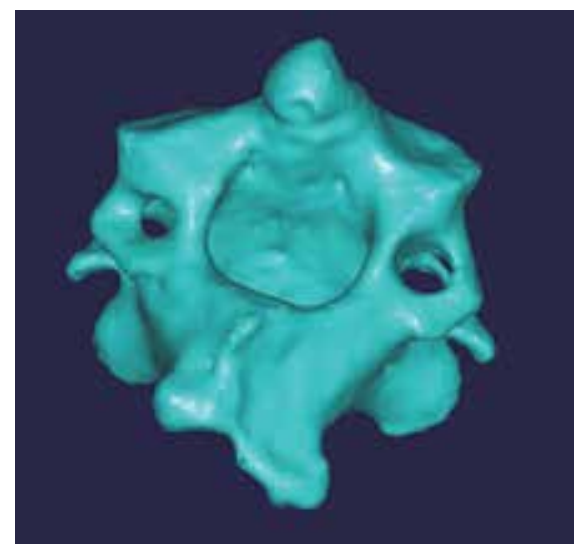

(a)

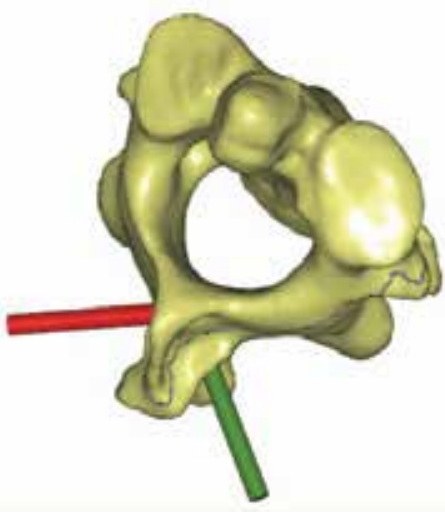

(b)

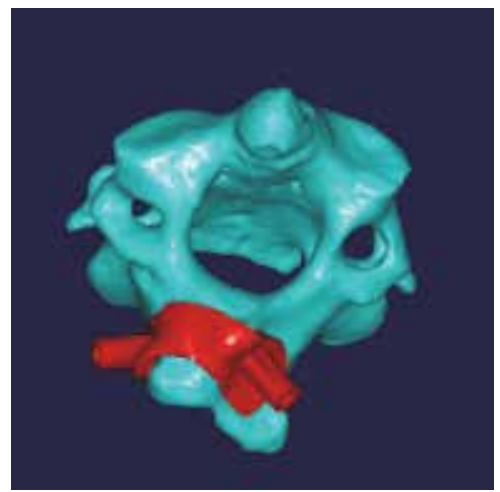

(c)

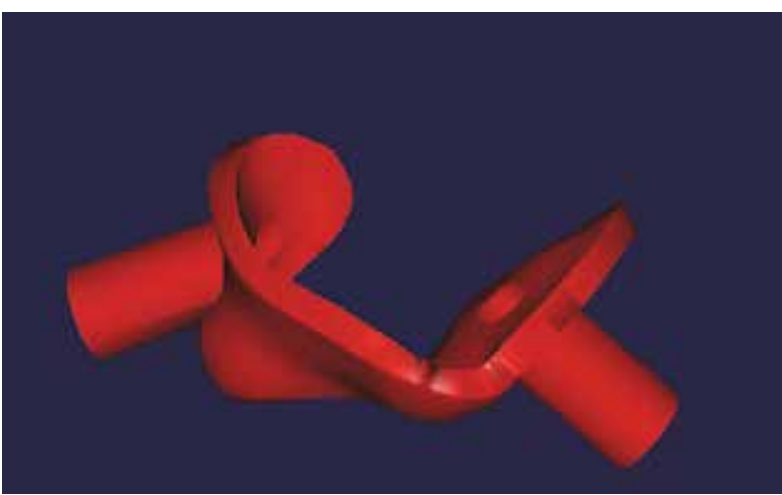

(d)

Fig. 9. The design of the virtual navigational template

a: 3-D model of C2 vertebra; b: virtual display of the planned laminar screw trajectory; c: navigational template fit the vertebral perfectly; $\mathrm{d}$ : The virtual 3-D model of navigational template

The computer model was then exported in STL format. The biomodel of the C2 vertebra as well as its corresponding navigational template were both produced by acrylate resin (Somos 14120, DSM Desotech Inc, USA) using stereolithography rapid prototyping (RP) technique. The accuracy of the navigational template was before surgery examined by visual inspection. The biomodel and its coresponding template were placed together, and a standard electric power drill was used to drill screw trajectory into the biomodel at the predefined placement, and visual inspection was taken for any violation of $C 2$ laminar (Fig. 10). 


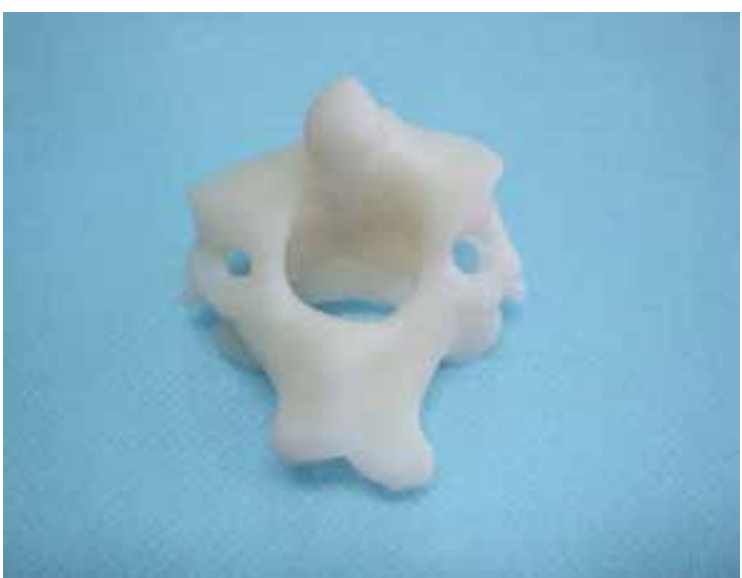

(a)

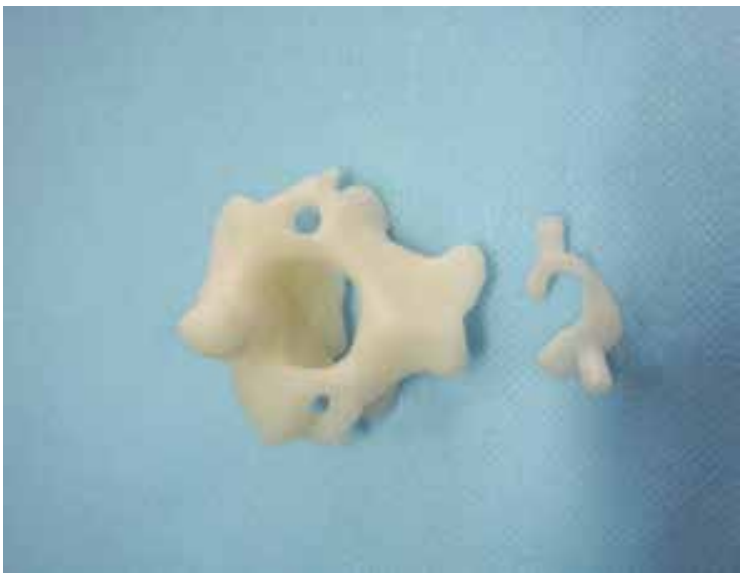

(b)

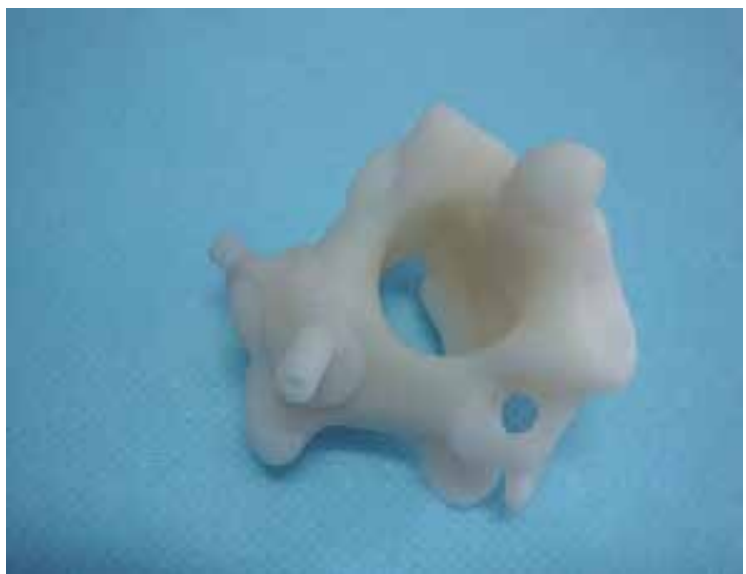

(c) 


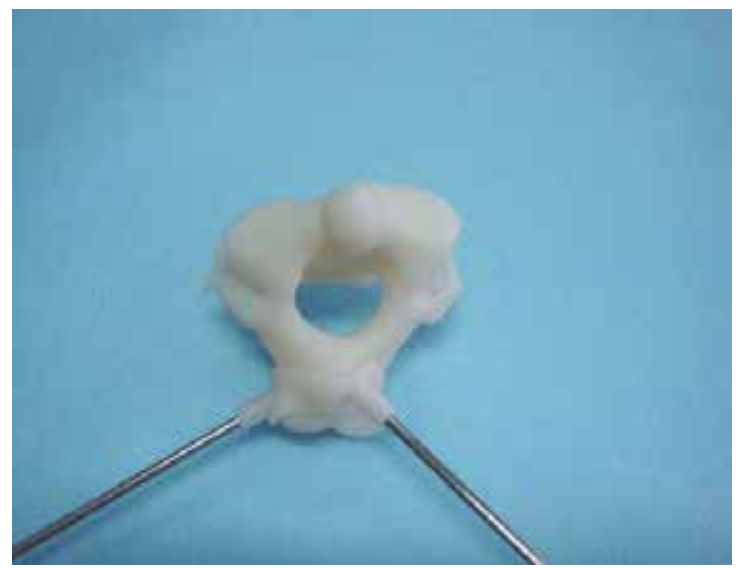

(d)

Fig. 10. The accuracy of the navigational template was examined by visual inspection a: RP model of C2 vertebra; $b$ : RP model of C2 vertebra and navigational template; $c$ : navigational template fit RP model of vertebra perfectly; $d$ : insert the K wire by navigational template; the accuracy of the navigational template was examined by visual inspection.

From June 2006 to Septempber 2008, 9 patients (four males, five females, age 17-53 years) with basilar invagination requiring the posterior instrumentation were performed occipitocervical fusion surgery by $\mathrm{C} 2$ laminar screw fixation. There are eight cases with occipitalization of the atlas, six cases accompanied by $\mathrm{C} 2-\mathrm{C} 3$ vertebral fusion. In three patients a transoral surgery was performed firstly, followed by posterior surgery. The anatomy of $\mathrm{C} 2$ pedicle was observed preoperative $\mathrm{X}$ ray and CT scan, if the pedicle is very narrow, thin, or vertebral artery high riding, it would be inappropriate placement of pedicle screw, the laminea screw fixation can be choosed.

Tracheal intubation using general anesthesia, patients are placed in the prone position with the head and cervical spine maintained in the neutral position using the Mayfield head holder. The spinous process, laminae and lateral masses of of C2 are then exposed as needed. Then the drill template was placed on the spinous process and laminae of C2. Template and the corresponding spinous process were fitted well. The high-speed drill is used along the navigational channel to drill the trajectory of laminar screw. Using a hand drill, the trajectory of laminar screw is carefully drilled to a depth of preoperation plan. A $4.0 \mathrm{~mm}$ screw is carefully inserted along the same trajectory. Using the same technique as above, a $4.0 \mathrm{~mm}$ screw is placed into the other lamina. After screw placement, all exposed laminar surfaces are decorticated with the high-speed drill. Autologous iliac crest bone grafts are wedged under the rods between the occipital bone and the spinous process and lamina of $\mathrm{C} 2$.

Using the virtual 3-D model, the optimal entry point for the bore can be choosed, thus determining the entry point and direction for the $\mathrm{C} 2$ laminar screw. The drill template was created to fit the postural surface of $\mathrm{C} 2$ spinous process very well. The accuracy of the drill template was before operation examined by drilling $\mathrm{K}$ wire trajectory into the vertebra biomodel. Each navigational template fits its corresponding vertebral biomodel perfectly, and $\mathrm{K}$ wire was found to be inserted through the drill hole into the $\mathrm{C} 2$ lamina, no violation was found by visual inspection.

Nine patients with basilar invagination were performed occipitocervical fusion surgery, the bilateral cross laminar screw fixation was preformed in eight patients, unilateral laminar 
screw fixation and other side of pedicle screw fixation was preformed in a patient. Average follow-up was 9 months (range 4-13 months). Preoperative and postoperative functional comparisons were made using the ASIA grading scale for all cases. Seven Of the patients $(77.8 \%)$ improved at least one ASIA grade. Two patients have not improve. No patient suffered neurological deterioration as a result of the procedure.

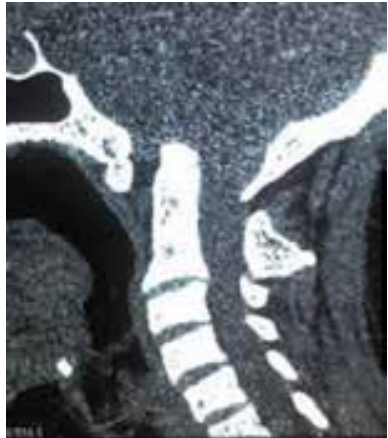

(a)

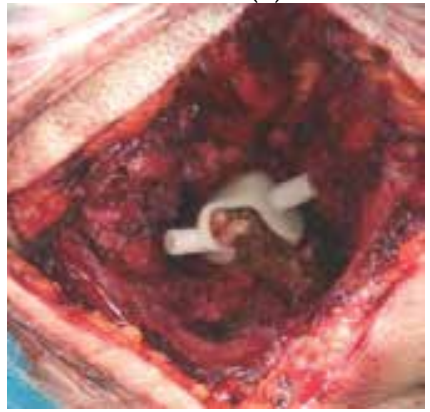

(d)

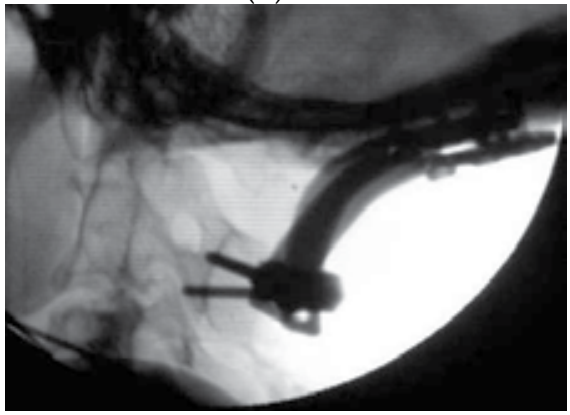

(g)

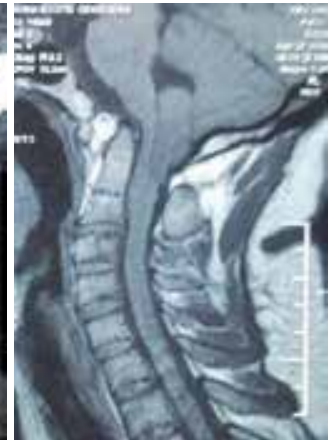

(b)

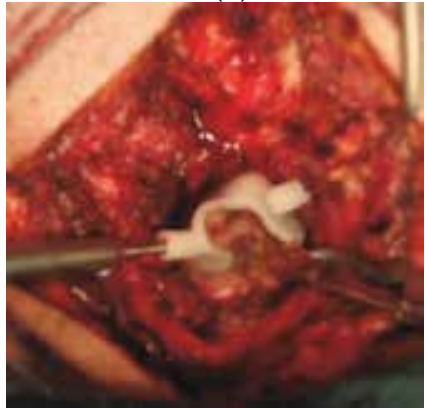

(e)

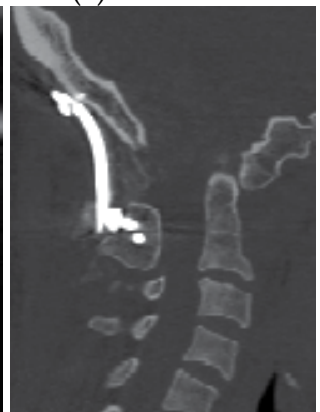

(h)

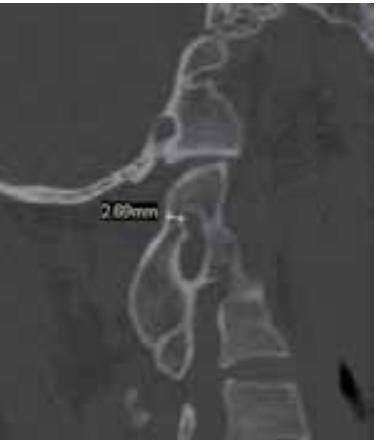

(c)

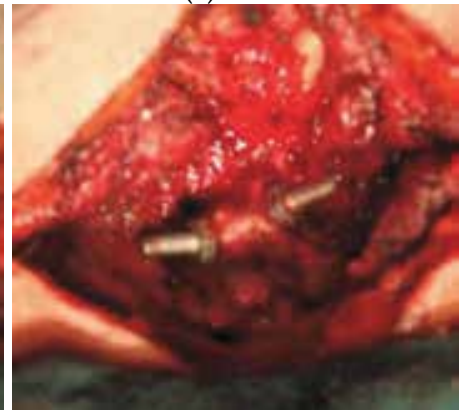

(f)

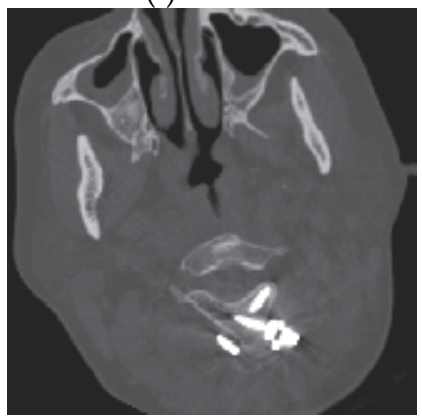

(i)

Fig. 11. The navigational template applied in the operation of a 42- year- old women with basilar invagination, occipitalization of the atlas and C2- C3 vertebral fusion.

$\mathrm{a}, \mathrm{b}$ : Pre-operative CT and T1 sagittal MRI displaying ventral brainstem compression; c: Preoperation CT show the pedicle of C2 is narrow and can not accommodate the $3.5 \mathrm{~mm}$ pedicle screw; d: Navigational template fit the spinous process and lamina of C2 perfectly; e: The high-speed drill is used along the navigational channel to drill the trajectory of laminar screw; $\mathrm{f}$ : Insert the cervical pedicle screw by navigational template; $\mathrm{g}, \mathrm{h}, \mathrm{i}$ : Postoperative CT showing accurate placement of C2 laminar screws 
Seventeen C2 laminar screws were inserted using drill emplate. No screw inserted complications such as spinal cord, nerves, vertebral artery injury appear in this group. The mean operative time between fixation of the template to the lamina and placement of the screw was 1 to 2 minutes. Operation time reduced through use of the navigational template. No additional computer assistance was needed during surgery, and fluoroscopy was used only once, after all the C2 laminar screws had been inserted. The method thus significantly reduced radiation exposure for the members of the surgical team.

It takes about 16 hours to manufacture the RP model, and the price of each RP model of the vertebra and navigational template is about $\$ 20$. Postoperative CT scans showed that the individual template has a higher precision. No laminar screw misplacement occurred using the individual template. (Fig. 11)

\subsection{Design and primary application of computer-assisted, patient-specificnavigational templates in metal-on-metal hip resurfacing arthroplasty}

Total hip resurfacing arthroplasty, viewed by many as representing an evolution from the mold arthroplasty procedure of Smith-Petersen, has been considered an alternative to total hip arthroplasty for adult patients with osteoarthritis of the hip or congenital hip dysplasia. It has been performed with a variety of materials, designs, surgical approaches, techniques, and fixation methods [1]. Many advantages of hip resurfacing arthroplasty have been suggested, including bone conservation [2-4], improved function as a consequence of retention of the femoral head and neck and more precise biomechanical restoration [5], decreased morbidity for revision arthroplasty [6], reduced dislocation rates [7,8], and normal femoral loading and reduced stress shielding [9]. In hip resurfacing arthroplasty, prosthesis location is key to postoperative joint stability. In conventional hip arthroplasty, the neck-shaft angle is determined by the design of the prosthesis itself, whereas in hip resurfacing, it is determined by valgus or varus placement of the femoral prosthesis. When the prosthesis is inserted by conventional positioning, some positioning devices are needed; however, accuracy cannot always be achieved with current devices. Successful positioning thus depends largely on how experienced the surgeon is. With the development of computer-aided design and computer-aided engineering, the trend in medical technology has been toward individualization. We conducted a study in which we introduced and validated novel locating navigation templates in the clinical setting. The templates' designs are based on reverse engineering. The patient-specific design allows close contact with acetabular and femoral features to provide better stability and function than is possible with conventional positioning devices.

Three-dimensional (3-D) computed tomography (CT) pelvic scan image data were obtained from 10 healthy volunteers who underwent a spiral 3-D CT scan (Light- Speed VCT; GE, Fairfield, CT) using a $0.625-\mathrm{mm}$ slice thickness and $0.35-\mathrm{mm}$ in-plane resolution. Data were transferred via a digital imaging and communications in medicine network into a computer workstation. Three dimensional models of the hips were reconstructed using Amira software (version 3.1; TGS, San Diego, CA) and saved in stereolithography format. The 3-D models were then imported into Imageware software (version 12.0; EDS, Palo Alto, CA). First, data for the transaction planes (1-mm thickness) of the acetabular and femoral head surfaces were extracted; and the correctly fitting globe was produced. The globe center was considered to be the rotation center of both the acetabulum and the femoral head. Second, we presumed an ideal acetabular location of about $45^{\circ}$ abduction and $18^{\circ}$ anteversion and 
an optimum location for the femoral head implant of $140^{\circ}$ abduction along the axis of the femoral neck. Finally, we designed specific navigation templates according to the anatomical features of the acetabular contour and the femoral head (Figs. 12 and 13).
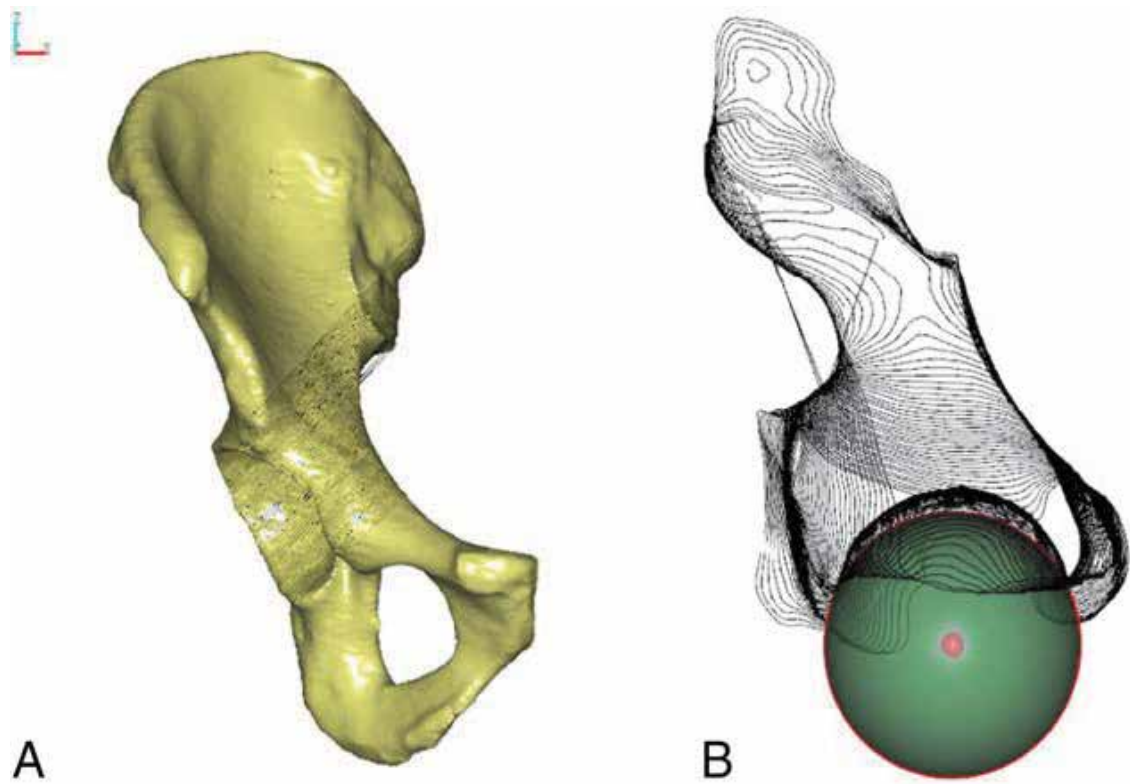

C
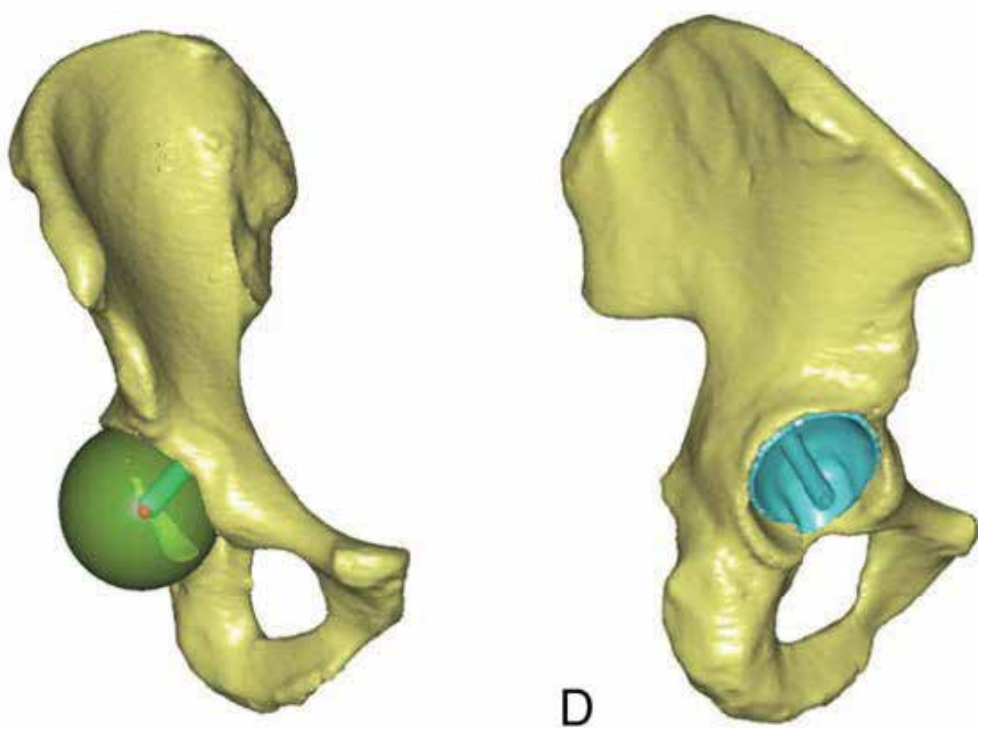

Fig. 12. Determination of the rotation center and the design process for a navigation template for the acetabulum: (A) preparing a 3-D reconstruction of the hip and dissection of acetabular surface, (B) constructing the rotation center of the acetabulum (small ball), (C) determining the navigation channel, and (D) producing the navigation template. 

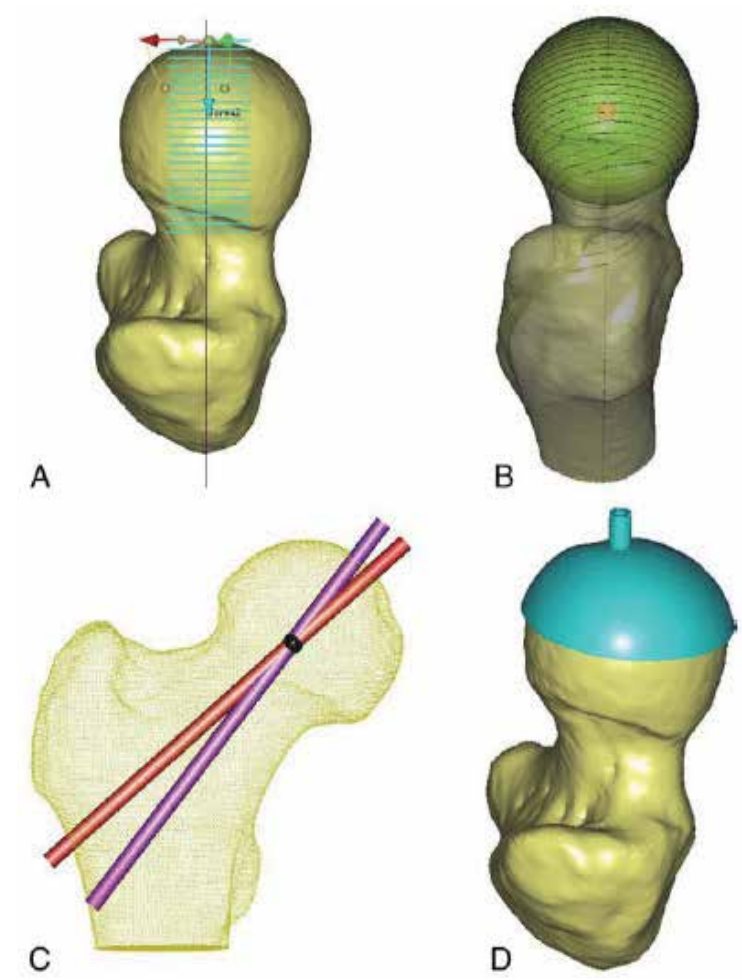

Fig. 13. The design process for a femoral head navigation template: (A) determining the axial ray on the femoral neck and dissecting the femoral head, (B) constructing the center of femoral head (small ball), (C) determining the navigation channel, and (D) producing the navigation template.

20 patients (11 men and 9 women) with pathologic changes in one hip who were 24 to 37 years old were scheduled for total hip resurfacing arthroplasty. They were randomly assigned to undergo either conventional implantation of a prosthesis (control group) or implantation of a prosthesis guided by navigation templates (NT group). The navigation templates were made as described in the preceding section. Navigation template models were exported in stereolithography format and formed from acrylate resin (Somos 14120;DSMDesotech Inc, Stanley, NC) using the stereolithography rapid prototyping (RP) technique (Fig. 14).
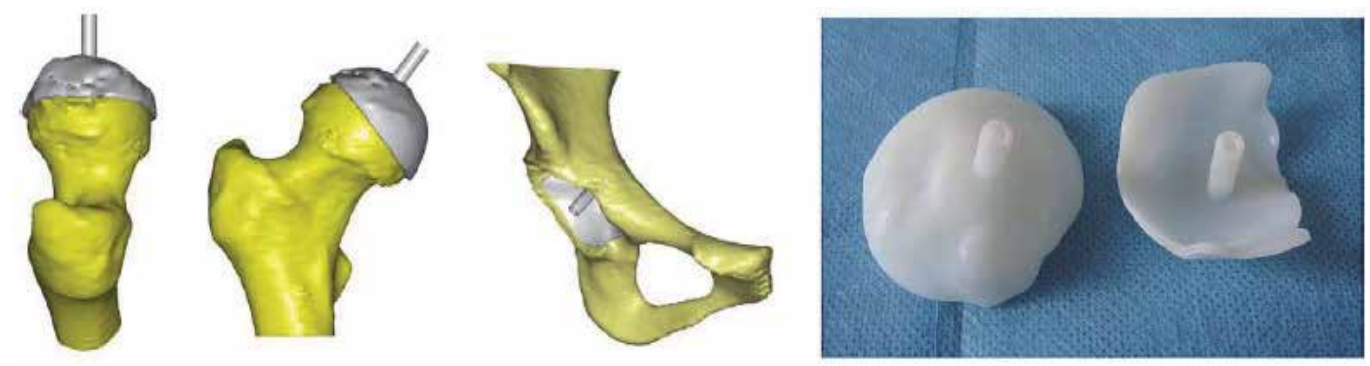

Fig. 14. Template models of the acetabulum and femoral head. 
Duration of surgery and intraoperative blood loss for the 2 groups were compared. Deviation between the ideal abduction angles and the actual angles of the implanted acetabular cup was calculated, as was deviation of cup anteversion angles. The deviation between the neck-shaft angle and the actual implanted short stem-shaft angle (SSA) and that between the anteversion angle of the femoral neck and the anteversion angle of the actual implanted short stem were measured. Angle deviations in the NT group were compared with those in the control group. In the NT group, after dislocation of the hip and insertion of the template into the acetabulum, a 3.2-mm-diameter guide pin was placed in the template hole. We followed the guide pin with the acetabular reamer to drill and shape the prosthetic socket at $45^{\circ}$ abduction and $18^{\circ}$ anteversion.
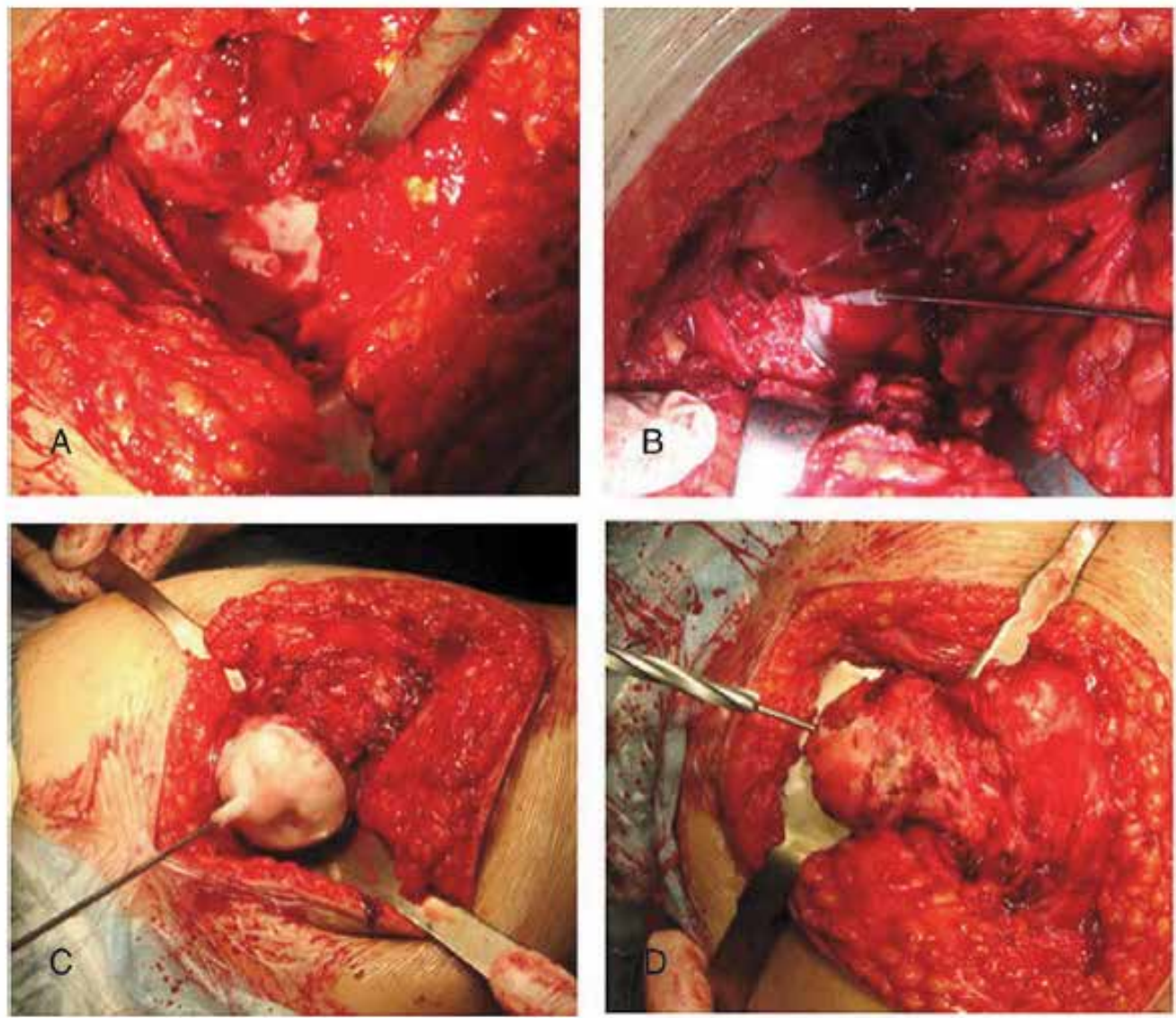

Fig. 15. Surgical procedure in the navigation template group: (A) locating the acetabulum, (B) inserting a guide pin, $(\mathrm{C})$ matching the navigation template and inserting a Kirschner wire, and (D) enlargement canals.

When the femoral head was dislocated and correctly exposed, we matched the locating template to the surface of the femoral head as well as possible. We then inserted a Kirschner wire into the femoral neck according to the template. We evaluated the axial location of the femoral component (shown by the Kirschner wire) with anteroposterior (AP) and lateral position photographs obtained during surgery by a $\mathrm{C}$ arm machine (Fig. 15). We obtained 
radiographs for all patients at some point between 7 and 10 days after surgery. Radiographic analysis included an AP view of the pelvis and AP and lateral views of the hip. On AP radiographs, the SSA was measured between the axial line of the femur and the extension line of the component stem with a measuring tool from Adobe Photoshop software (version 7.0; Adobe, San Jose, CA).

The navigation templates were found to be highly accurate (Fig. 16). The average duration of surgery for the NT group was 118.6 minutes vs 140.2 minutes for the control group (P b .05). The average intraoperative blood loss in NT group was $410.9 \mathrm{~mL}$ vs $480.6 \mathrm{~mL}$ for the control group ( $\mathrm{P} \mathrm{b} .05)$. The average deviation of the cup abduction angle $\left(1.2^{\circ} \pm 0.9^{\circ}\right)$ was significantly less in the NT group than in the control group $\left(5.4^{\circ} \pm 3.2^{\circ}, \mathrm{P} \mathrm{b} .05\right)$, and the average deviation of the cup anteversion angle $\left(2.1^{\circ} \pm 1.2^{\circ}\right)$ was significantly less in the NT group than in the control group $\left(4.1^{\circ} \pm 2.8^{\circ}, \mathrm{P} \mathrm{b} .05\right)$. In addition, the average deviation of the femoral SSA $\left(1.3^{\circ} \pm 1.0^{\circ}\right)$ was significantly less in the NT group than in the control group $\left(10.2^{\circ} \pm 1.5^{\circ}, \mathrm{P}\right.$ b .05).
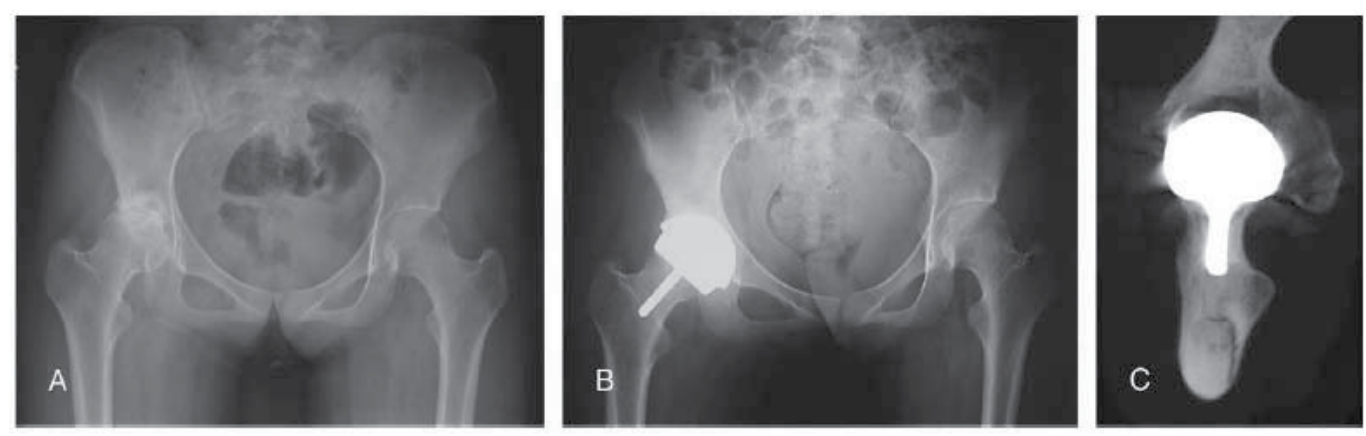

Fig. 16. (A) Anteroposterior radiograph of a patient from the navigation template group shows hip pathology before surgery. (B, C) Anteroposterior radiograph and computed tomography image from the same patient show prosthesis position after surgery.

\section{Conclusion}

The authors have developed a novel patient-specific navigational template for spinal pedicle screw placement, placement of C2 laminar screws, and accurate prosthesis implantation in hip resurfacing arthroplasty with good applicability and high accuracy. The potential use of navigational templates in orthopedics surgery is promising. Our methodology appears to provide an accurate technique and trajectory for orthopedics surgery.

\section{Acknowledgment}

This work was supported by Yunnan Natural Science Foundation (2008CD210 and 2010ZC183).

\section{References}

[1] Lu S, Xu YQ, Zhang YZ, Li YB, Xie L, Shi JH, Guo H, Chen GP, Chen YB.(2009) A novel computer-assisted drill guide template for lumbar pedicle screw placement: a cadaveric and clinical study. Int J Med Robotics Comput Assist Surg, 5(2): 184-191 
[2] Sheng Lu, Yong Q. Xu, William W. Lu, Guo X. Ni, Yan B. Li, Ji H. Shi, Dong P. Li, Guo P. Chen, Yu B. Chen and Yuan Z. Zhang. (2009) A Novel Patient-Specific Navigational Template for Cervical Pedicle Screw Placement. SPINE, 34(26):E959-E964.

[3] Sheng Lu, Yong Q. Xu, Yuan Z. Zhang, Le Xie, Hai Guo, Dong P. Li. (2009) A novel computer-assisted drill guide template for placement of C2 laminar screws. Eur Spine J, 18(9):1379-1385.

[4] Richter M, Cakir B, Schmidt R.(2005) Cervical pedicle screws: conventional versus computerassisted placement of cannulated screws. Spine, 30: 2280-2287.

[5] Kotani Y, Abumi K, Ito M, Minami A. (2003) Improved accuracy of computer-assisted cervical pedicle screw insertion. J Neurosurg, 99:257-263.

[6] Lonner BS, Auerbach JD, Estreicher MB, et al. (2009) Thoracic pedicle screw instrumentation: the learning curve and evolution in technique in the treatment of adolescent idiopathic scoliosis. Spine, 34:2158-64

[7] Leonard JR, Wright NM (2006) Pediatric atlantoaxial fixation with bilateral, crossing C-2 translaminar screws. Technical note. J Neurosurg, 104:59-63 


\title{
Rapid Prototyping Applied to Maxillofacial Surgery
}

\author{
Marcos Vinícius Marques Anchieta1, \\ Marcelo Marques Quaresma ${ }^{2}$ and Frederico Assis de Salles ${ }^{3}$ \\ ${ }^{1}$ Private Practice, Brasilia, \\ ${ }^{2}$ Computer Science, Brasilia, \\ ${ }^{3}$ Former Visiting Professor at School of Medicine, University of Brasilia, Brasilia, \\ ${ }^{3}$ Former Head of the Maxillofacial Surgery Department, \\ Hospital do Aparelho Locomotor-Sarah, Brasilia, \\ Brazil
}

\section{Introduction}

What is rapid prototyping $(\mathrm{RP})$ ?

The word prototyping was first used in engineering to describe the act of producing a prototype, a unique product, the first product, or a reference model.

In the past, prototypes were handmade by sculpting or casting, and their fabrication demanded a long time. Any and every prototype should undergo evaluation, correction of defects and approval before the beginning of its mass or large scale production. Prototypes may also be used for specific or restricted purposes, in which case they are usually called a pre-series model.

With the development of information technology, three-dimensional models can be devised and built based on virtual prototypes. Computers can now be used to create accurately detailed projects that can be assessed from different perspectives in a process known as computer-aided design (CAD). To materialize virtual objects using $C A D$, a computer-aided manufactory (CAM) process has been developed. To transform a virtual file into a real object, CAM operates using a machine connected to a computer, similar to a printer or peripheral device. ${ }^{13}$

In 1987, Brix and Lambrecht used, for the first time, a prototype in health care. It was a three-dimensional model manufactured using a computer numerical control (CNC) device, a type of machine that was the predecessor of rapid prototyping. ${ }^{6}$

Some rapid prototyping machines had already been in experimental use in the 1970s, and computed tomography (CT) was invented in the 1960s by Godfrey N Hounsfield, an electronic engineer, in collaboration with Allan McLeod Cormack, a physicist. However, it was only in the 1990s that an actual three-dimensional model was built to reproduce the anatomy of a patient based on CT images obtained during that patient's examination, thanks to advances in CT scanner quality and the development of specific software for this purpose. In 1991, human anatomy models produced with a technology called stereolithography were first used in a maxillofacial surgery clinic in Viena. ${ }^{16}$

Prototyping was developed to respond to the need to test functionality, ergonomics, shape, adaptation and design in production engineering, an area in which the term prototyping is 
widely known. ${ }^{13}$ This term has been adopted by health care, although models manufactured according to CT images are not exactly prototypes, but rather replicas, because they are not created by a designer or planner, but replicated according to images scanned by, in this case, a CT unit. The model for use in health care is the materialization of a three-dimensional image provided by the CT scanner.

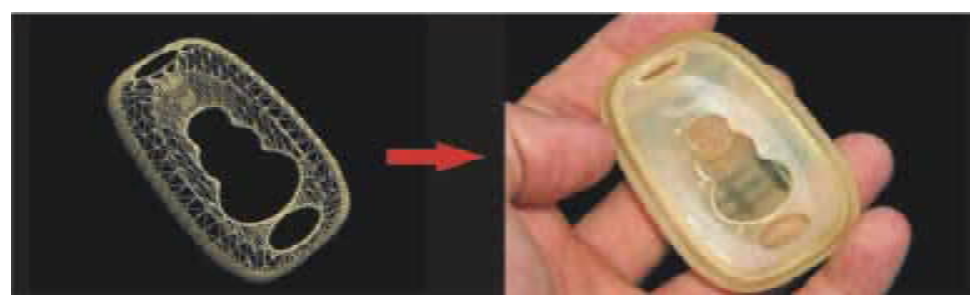

Fig. 1. Virtual file and corresponding prototype built using rapid prototyping.

The graph below lists some economic sectors that use rapid prototyping.

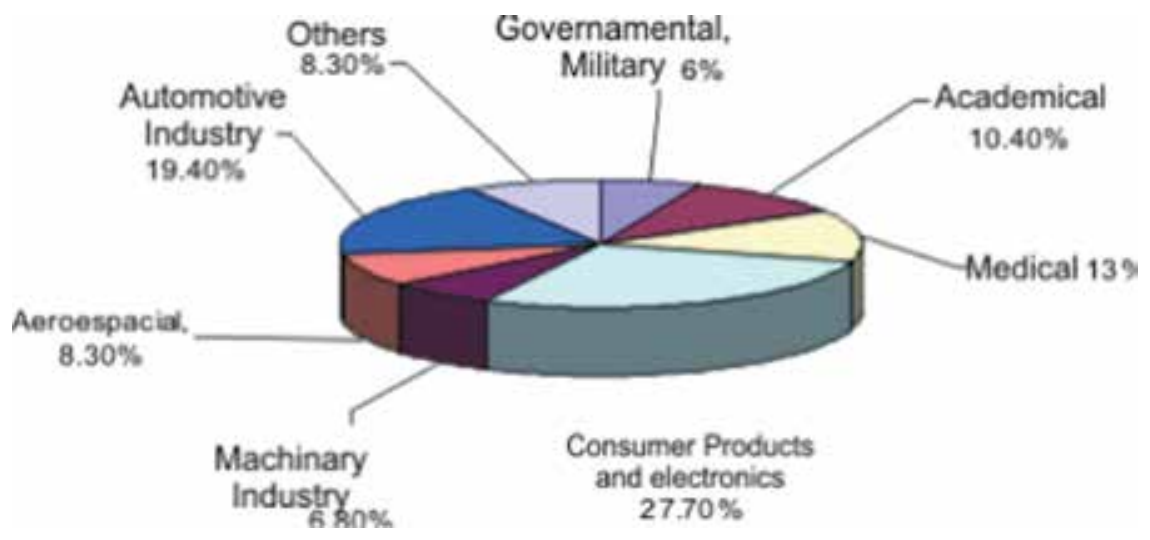

\section{Prototype quality in health care}

The quality of prototypes in the areas of dental and medical care has been the focus of some discussions. The accuracy assigned to prototypes is variable, and, according to the literature, prototype distortion reaches $0.6 \%$ when compared with CT scans. ${ }^{19}$

To understand the several factors that affect the quality of a prototype used in healthcare, it is important to understand how the anatomic model to be reproduced is created.

Quality and final precision of a prototype to be used in health care depend on four factors, summarized in the four steps below:

- Patient preparation

- CT scanning

- Image manipulation

- Prototyping technology

\subsection{Patient preparation}

To obtain good quality images, the patient should be evaluated, receive the necessary information and be prepared before undergoing CT scanning. These procedures should 
minimize the harmful effects of artifacts on CT images and ensure accurate image reproduction when the prototype is manufactured. The critical area of facial CT scanning is tooth occlusion, or the area of contact between mandibular and maxillary teeth when the mouth closes. Therefore, the crowns, restorations and metal cores should be removed before scanning. The mouth should be kept open during scanning to avoid that artifacts produce fusion between maxillary and mandibular teeth; if there is fusion, the jaws will not separate after the model is complete.

In cases CT scanning is planned to occur after orthodontic appliance placement, the orthodontist should choose brackets without nickel or ceramic brackets, because they produce fewer artifacts.

$\mathrm{Ct}$ scanning can produce very relevant additional information when radiopaque markers are used, but they should be carefully manufactured and fixed to be faithfully reproduced. Figure 2 shows a prosthesis with duplicate teeth and a radiopaque marker (barium sulfate). The model was built to establish the tooth-bone relation; however, the prosthesis, which should be over the alveolar ridge, was displaced from the desired position. Image reconstruction revealed that the undesired displacement resulted form the improper use of a luer syringe plunger to keep the mouth open. This example stresses the importance of the information that should be given to the patient. CT scanning had to be repeated and the patient was exposed to another radiation dose.

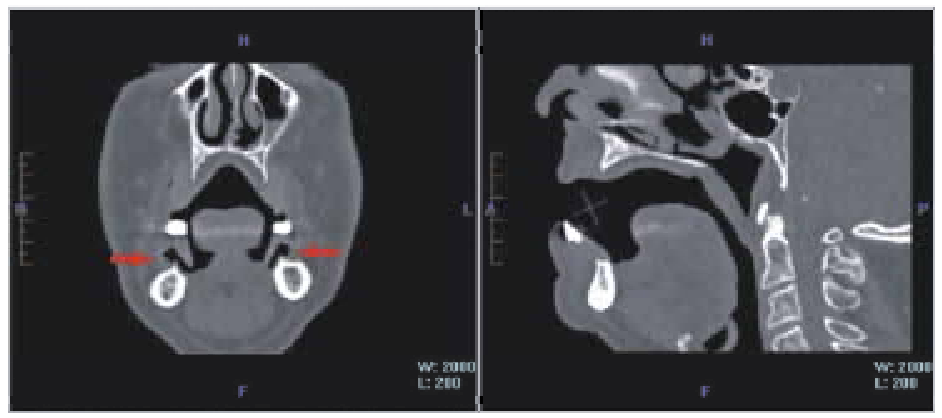

Fig. 2. Arrows indicate prosthesis displacement on both sides due to improper advice to bite on a syringe plunger to keep mouth open during CT scanning of mandible.

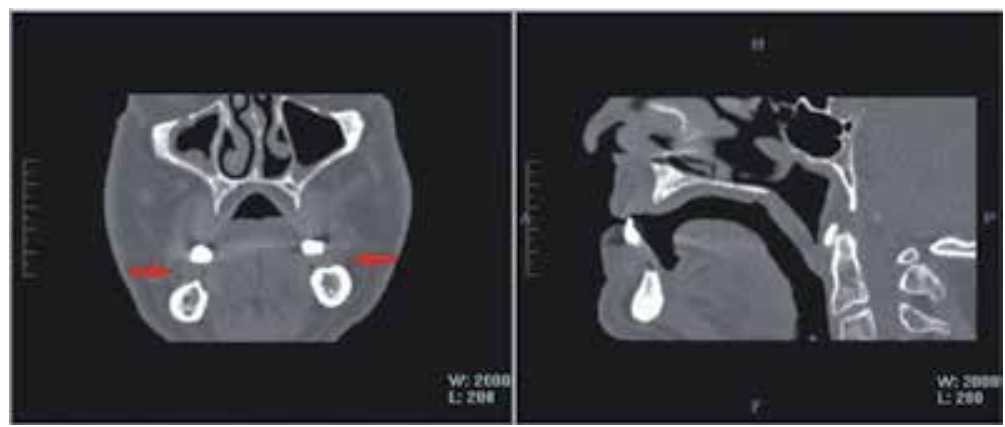

Fig. 3. Arrows point to correction of position of prosthesis in Figure 2 in patient's second CT scanning. Models produced from these images reproduced a perfect bone-teeth relation and ensured correct implant placement. 
While radiographic contrast media are often used to examine the vascular and digestive systems in Medicine, in Dentistry radiopaque markers are frequently used to reproduce missing teeth and guide the surgeon during planning procedures that involve bones. To reproduce missing teeth on $\mathrm{CT}$ images, several radiopaque markers can be used, such as metal balls, gutta-percha points, metal rings, stored teeth, or a mixture of autopolymerizing acrylic resin and barium sulfate.

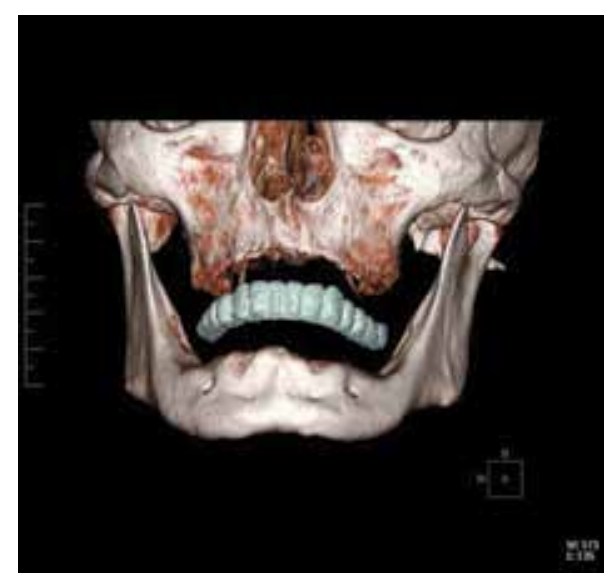

Fig. 4. Image of a maxillary total prosthesis with teeth duplicated in radiopaque material to determine tooth-bone relation and define most adequate points to insert implants.

\subsection{Computed tomography (CT)}

Of the advances in CT equipment, two deserve special attention due to their importance in the production of models to be used in health care: the reduction in CT slice capture time, which results in faster and more accurate evaluations and avoids distortions in threedimensional image reconstruction; and image manipulation, which significantly improves its high-definition quality.

The principle of CT image production, similarly to conventional radiology, is the partial absorption of X-rays by the human body. While fat and air, for example, are easily passed through, bone and metal are not.

Basically, a CT scan indicates the amount of radiation attenuated at each portion of the section under analysis and translates variations into a grey scale that produces an image.

As a tissue's capacity to absorb X-rays is closely associated with its density, zones with different densities will have different colors, which will enable their differentiation.

Each image pixel corresponds to the mean attenuation of tissues in a certain zone, and attenuation is expressed in Hounsfield units in honor of the creator of the first CT machine.

- Hounsfield scale (HU)

- $\quad$ water: zero HU

- $\quad$ air: $-1000 \mathrm{HU}$

- bone: 300 to $350 \mathrm{HU}$

- $\quad$ fat: -120 to $-80 \mathrm{HU}$

- muscle: 50 to $55 \mathrm{HU}$

For CT scanning, the patient is placed on a table that moves into an aperture of about $70 \mathrm{~cm}$ in diameter. Around this aperture there is an X-ray tube in a circular gantry. At 180 degrees, 
that is, opposite the X-ray tube, there is an X-ray detector that captures radiation and sends information to the computer to which it is connected.

\subsubsection{Terms}

Images are stored in Digital Imaging and Communications in Medicine (DICOM) files.

Threshold - selection of density according to anatomic structure of interest;

Matrix size - number of lines and columns that form CT images, usually 512 x 512;

FOV - field of view, or area captured during scanning;

Shades of gray:

- $\quad$ 8-bit scanner $-2^{8}=256$ shades of gray

- $\quad$ 12-bit scanner - $212=4,096$ shades of gray

- $\quad$ 16-bit scanner $-216=65,536$ shades of gray

- Humarl eye - detects about 10 to 60 shades of gray.

\subsubsection{Types of CT scanners}

Conventional: during examination using conventional machines, the gantry moves a full rotation around the patient, and the $X$-ray tube emits radiation that, after passing through the patient's body, is captured in the opposite side by the X-ray detector. Data are then processed in a computer, which analyzes the attenuation variations along the section under examination; these data are used to reconstruct an image as a shape. The table moves a little further then, and the process is repeated for a new image a few centimeters from the first site.

Helical, Multislice, Ultrafast: more recent machines, called helical CT scanners, move as a helix around the body of the patient instead of in full circles. Therefore, if the slice is to be 10 $\mathrm{cm}$ thick, the gantry will advance $10 \mathrm{~cm}$ during each gantry rotation. Intermediate slices at, for example, each $2 \mathrm{~cm}$ may be obtained simply by digital reconstruction because the area under examination was scanned during one helical movement. Therefore, the patient receives lower radiation doses during scanning. Multislice units scan multiple simultaneous slices during each gantry rotation because they have several radiation emission and reception units. Ultrafast machines are used in CT scanning of the heart because they can produce slices at 50 to 100 millisecond intervals.

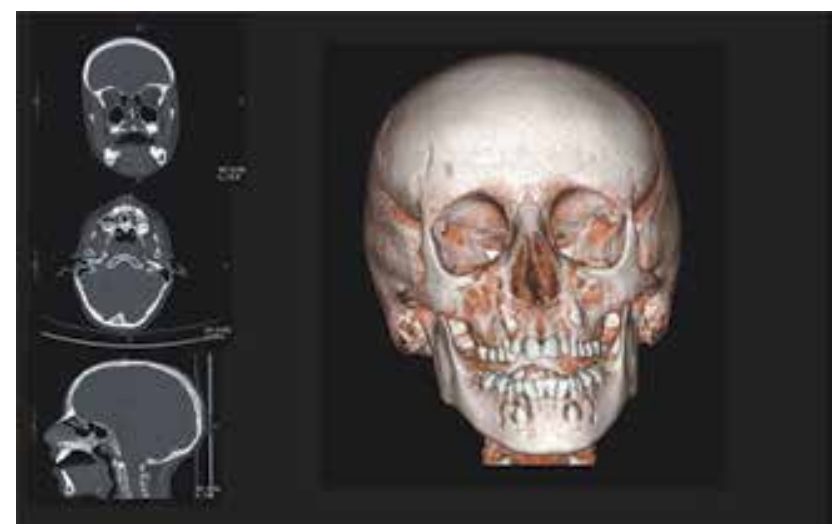

Fig. 5. Three-dimensional image formatted according to scan obtained using a multislice unit. 
Cone-Beam CT: Cone-beam CT scanners do not build images slice by slice. Instead, they capture all the area to be examined with a few exposures, and image formatting and slicing is performed by software developed for this specific purpose. This type of CT unit has the advantage of exposing the patient to a lower radiation dose than the other scanners. However, due to the lower number of exposures, the most peripheral areas of the image are much sharper, whereas the most central fields, which receive a lower radiation dose, lose part of their sharpness. Because of that, the radiologist should receive information about the region of interest, so that the center point or fulcrum of acquisition does not overlap with structures of interest in this region. Currently, three brands of cone-beam CT scanners for dentistry are available in the market: NewTom, Accuitomo and I-Cat.
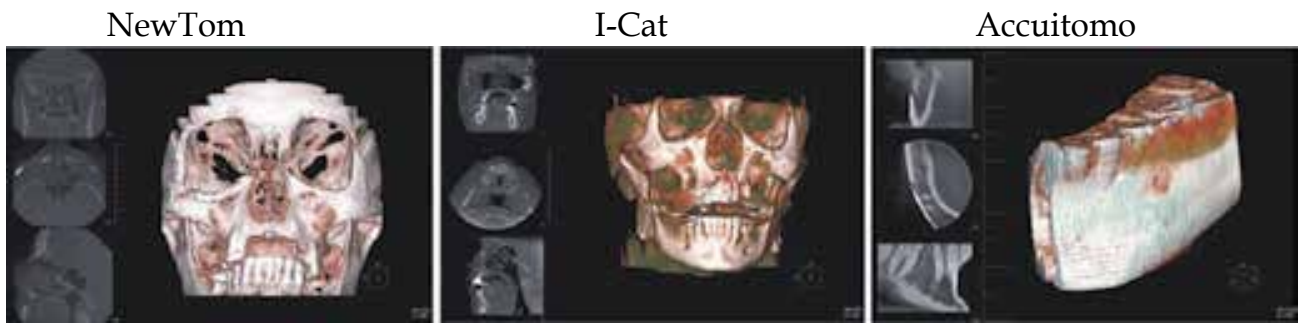

Fig. 6. Images captured with the three types of cone-beam CT scanners available in the market.

\subsection{Image manipulation}

Images captured in the DICOM format are processed using specific three-dimensional reconstruction software. Good-quality software provides several auxiliary tools to process images and convert them to STL format, which is the standard file format used by prototyping machines. A 3D STL file consists of a mesh of triangles, and the greater the number of triangles used in producing the image, the better the definition, precision and fidelity in the reproduction of anatomic details of the area of interest. ${ }^{24}$

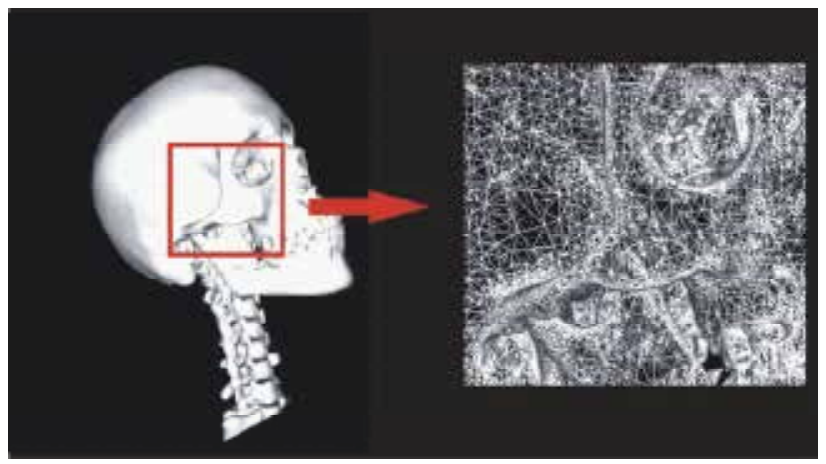

Fig. 7. On the left, 3D reconstruction. On the right, example of triangle mesh in a STL file.

The quality of a STL file depends on the software used for the conversion of CT images into a three-dimensional format. After the STL file is prepared, the prototyping technology that will be used to convert it into a model should be chosen. Images will then be sliced at thicknesses compatible with the technology chosen and sent to the prototyping unit to build 
the model. Therefore, the same STL file may generate prototypes with great differences in quality and accuracy because these features depend on the technology chosen.

Images should be manipulated by specialized personnel to avoid distortions that may affect the accurate reproduction of anatomy. The threshold should also be carefully chosen, because differences may result in more or less bone being detected and may compromise the quality of the prototype.

The region of interest for the surgeon should be made very clear so that it is fully scanned during CT to produce images that will later be used to fabricate the prototype. The CT scan shown on the right in Figure 8 was captured using a large FOV that included the whole face. The region of interest, however, was only the maxilla, and only part of the image would be used. The correct procedure in this case would be the restriction of FOV to the maxilla because the whole scanned image would then be used, and the resulting quality would be substantially better.
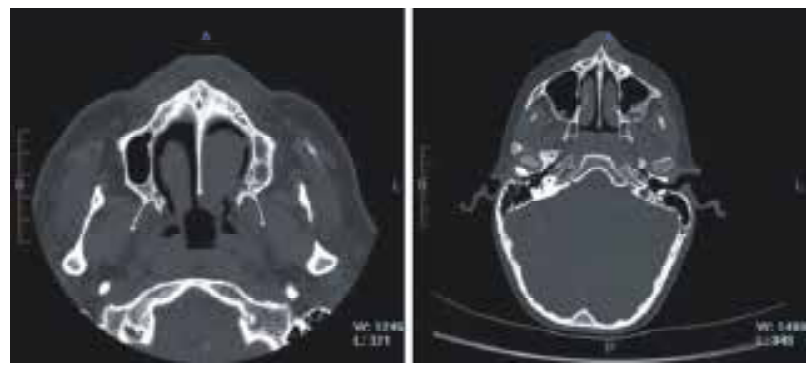

Fig. 8. On the left, CT scan in which FOV was restricted to the maxilla, which produced a more detailed image. On the right, FOV included a large area and reduced details of the maxillary area, the area of interest in this case.

Spatial Relation Retainers (SRR), which may be ordered from the prototyping laboratory, are very useful aids in establishing the tooth-alveolar bone relation. They preserve the position of the teeth used as radiopaque markers in relation to bone structures by means of retainers created virtually and reproduced together with the prototype. The patient's prosthesis is placed over this model to reproduce the thickness of the mucosa. SRR may also be used to retain the spatial relation of bone fragments in complex fractures.

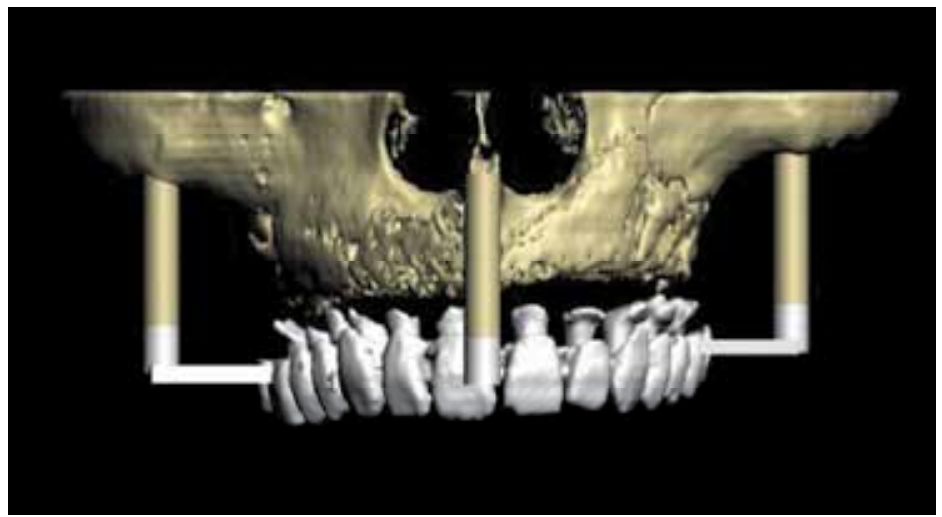

Fig. 9. Spatial relation retainers (SRR) 
The first software developed to establish the interaction between CT and RP was Mimics, produced by the Belgian company Materialise. Several other packages have been developed since then, each one with advantages as well as limitations. Currently, some software packages allow surgeons to interact and perform virtual planning, which can then be saved directly in a STL file.

\subsection{Rapid prototyping technology}

Todd Grim seems to have found the best definition of rapid prototyping. According to him, rapid prototyping may be understood as "a collection of technologies that are driven by CAD data to produce physical models and parts through an additive process".4,13 Based on this concept, all rapid prototype technologies create their three-dimensional models by means of addition of layers of a material that will fuse to give shape to the object previously planned. The main technologies currently available in the market are:

\subsubsection{Stereolithography (STL)}

Stereolithography (STL) is the oldest and most important rapid prototyping technology and the one best known all over the world. The term STL is sometimes mistakenly used interchangeably with "rapid prototyping". STL uses a wide range of different materials and may be used for several types of production.

In its process, a liquid resin (acrylic, epoxy or vinyl) is photosensitized using an ultraviolet (UV) laser beam. The STL unit has a vat that is filled with resin and a platform inside it that moves downwards. The computer sends information to the platform about each layer of the virtual model to be polymerized. Machine number control positions the platform on the surface of the layer of resin, and the laser beam literally draws the first layer. After the completion of each layer, the platform moves downward and dips the previously solidified layer into the liquid resin so that a new layer can be polymerized and stacked on the top of the previous layer, and successively so until all the model is complete. At the end of the process, the model will be immersed in resin and should, therefore, be rinsed in an isopropyl alcohol bath to remove all the non-polymerized material. ${ }^{13}$

The thickness of the layers that generate prototypes using STL is $0.025 \mathrm{~mm}$, which results in a surface of good quality.

\subsubsection{Fused Deposition Modeling (FDM)}

Fused deposition modeling (FDM) is based on the superposition, on a platform, of successive layers formed by the deposition of filaments of a plastic material for the production of the model. At the same time, two types of material are deposited: a plastic material used in building the body of the object, and a brittle material that fills ups empty spaces and gives support to the object. Later, this brittle material is removed to obtain a clean prototype.

The material used for the model is ABS, and the material used for the support structures is a mixture of ABS and lime. The FDM machine has a platform that moves vertically along the $\mathrm{Z}$ axis and a head with two extrusion tips that extrude heated filaments of build material: one to feed the model layers and the other to deposit the layers of support structures. For each layer, coordinates, or "roads" are generated along which the extrusion tip deposits the molten filament material. At the end of each layer, the platform moves down and the head begins the deposition of more material for the other layer, repeating the operation until the model is complete. ${ }^{13}$ 
The thickness of the layers that generate FDM prototypes is $0.178 \mathrm{~mm}$, which generates a rough surface of poor quality. As the model is made of a plastic material, it cannot be cut because melted plastic will stick to the tool.

\subsubsection{D printing}

Three-dimensional (3D) printing is a technology that is similar to inkjet printing in computers. However, instead of ink, the heads spread a binder composed of an aqueous solution and a glue. The machine has a reservoir for one type of powder, which may contain mixtures of material, such as plaster and starch, a platform that moves horizontally and down while the powder solidifies, a roller to distribute and evenly spread the powder to the fused, and a print head that is filled with the binder.

The building process is described below: the roller moves over the build tray and evenly spreads a uniform layer of powder that resembles a rug; the print head moves in the $X$ and $\mathrm{Y}$ axes and releases a jet of binder onto the powder; and the binder fuses the powder; the platform then moves down and another layer of powder is deposited and receives a binder jet. This second layer binds and adheres to the previous layer, and the process is repeated.

When completed, the remaining loose powder is aspirated from the surface of the model. This process does not confer great resistance to the model. Therefore, after completion, the models have to receive infiltrating materials to improve their resistance. This finishing process may change some anatomic details in the prototype surface. ${ }^{13}$

The thickness of the layers generated by powder binding is $0.1 \mathrm{~mm}$, which gives the prototype a slightly irregular surface, low resistance and low accuracy due to the nature of the material used in its fabrication.

\subsubsection{Inkjet - PolyJet}

Launched by the Objet company in 1999 and introduced in Brazil in 2004 by the ARTIS Prototyping Company, the major characteristic of this technology is the construction of highly-accurate prototypes using liquid acrylic resin.

The production begins with the deposition of successive layers of acrylic resin that are stacked up to create the prototypes.

During production, two types of material are used: acrylic resin, which will form the body of the object, and a gelatinous material to fill empty spaces during production and serve as a support structure for the prototype. The gelatinous material is easily removed later using a jet of water.

The thickness of build layers in this technology is $0.016 \mathrm{~mm}$, which ensures excellent surface finish and the reproduction of small details.

In health care, a translucent amber resin is used to provide visualization of canals, ducts and sinuses. The model can be cut and fixed with screws because its resistance is similar to that of bone.

\subsubsection{Selective laser sintering}

Selective laser sintering (SLS) is a versatile technology that can be used to build prototypes with several materials, such as nylon, metal, elastomers and plastic.

This technology uses a laser beam that fuses powder particles of the material to be used. After a layer is laser-sintered, a new powder layer is added to continue the production of the prototype. 
The thickness of the layers in this technology is $0.1 \mathrm{~mm}$, and the objects have a slightly irregular, highly porous surface. ${ }^{13}$ Because of the large variety of materials used, it is well known in engineering, although its cost is high. ${ }^{13}$

\subsubsection{Comparison of layer thickness using rapid prototyping technology}

Figure 10 shows a comparison between the number of layers to build a section measuring 1 $\mathrm{mm}$ high and $1 \mathrm{~mm}$ thick using the different rapid prototyping technologies. Each technology has a minimum material deposition thickness to form a build layer. The thinner this layer, the better the surface finishing and the smoother the prototype surface.

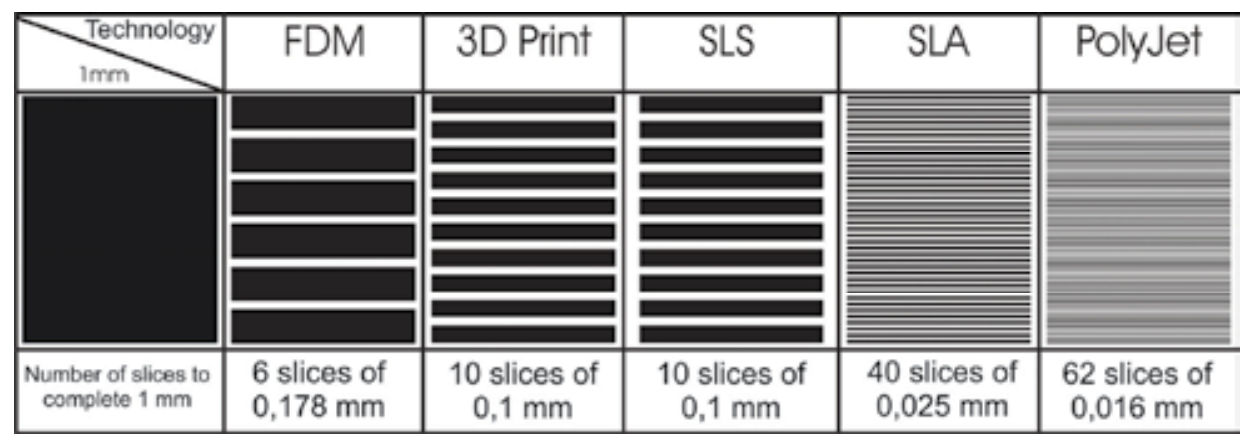

\subsubsection{Adequate prototyping technology}

The choice of an adequate prototyping technology is fundamental for the final quality of a prototype because, as seen before, each technology uses different types of material and has different characteristics.

In some cases, prototype translucency may be important for the evaluation of drill direction and depth. In other situations, it might be more important to have an opaque material to evaluate asymmetries, for example. Still in other cases, the specialist may choose a technology that uses more resistant material, such as nylon, to reproduce fine, delicate structures, although it is difficult to work with drills in this material because friction and heat may melt it and block the advancement of the drill. There are also wax models, cast models, or even models made directly using metal.

The choice, therefore, depends on the purpose of the prototype. The characteristics of the several technologies, described in Table 1, should, therefore, be well known to make the right choice.

\begin{tabular}{|l|l|l|l|l|l|}
\hline & FDM & 3D Printing & SLS & STL & PolyJet \\
\hline Translucency & No & No & No & Yes & Yes \\
\hline Resistance & High & Low & High & High & High \\
\hline Precision & Low & Low & High & High & High \\
\hline Material & Plastic & Plaster & Nylon & Resin & Resin \\
\hline
\end{tabular}

Table 1. Characteristics of the prototypes built using the major rapid prototyping technologies

These characteristics should be analyzed also according to prototype application so that the results of their use are satisfactory. 


\begin{tabular}{|l|l|l|l|l|l|}
\hline & $\begin{array}{l}\text { Graft } \\
\text { planning }\end{array}$ & $\begin{array}{l}\text { Implant } \\
\text { planning }\end{array}$ & $\begin{array}{l}\text { Surgery } \\
\text { simulation }\end{array}$ & $\begin{array}{l}\text { Guide } \\
\text { fabrication }\end{array}$ & $\begin{array}{l}\text { Visual } \\
\text { assessment }\end{array}$ \\
\hline Translucency & No & Yes & Yes & Yes & No \\
\hline Resistance & Low & High & High & High & Low \\
\hline Precision & Low & High & High & High & Low \\
\hline Material & $\begin{array}{l}\text { Resin, } \\
\text { plaster, } \\
\text { plastic, } \\
\text { nylon }\end{array}$ & Resin & Resin & Resin & $\begin{array}{l}\text { Resin, plaster, } \\
\text { plastic, nylon }\end{array}$ \\
\hline
\end{tabular}

Table 2. Necessary characteristics of prototypes according to their clinical use

Previous studies about CT quality indicated that a good CT examination currently provides axial images with a thickness of about $1 \mathrm{~mm}$ and distance between slices never greater than $1 \mathrm{~mm}$. Therefore, RP technologies have greater precision than current CT scanners. In the case of PolyJet, each build layer is about 62 times thinner than the thickness of a good quality CT slice.

\section{Rapid prototyping protocol in health care}

The quality of a prototype depends, first, on the quality of the images, as in the case of a digital camera.

The good quality of a 3D reconstruction depends on the use of all the axial sequential slices, because reconstruction is produced by stacking up the different axial slices. For example: a scan with 200 1-mm thick slices will produce a 3D image of $200 \mathrm{~mm}$, or $20 \mathrm{~cm}$.

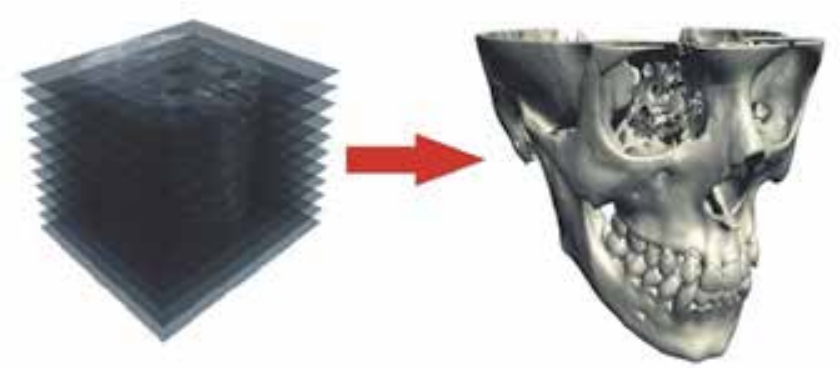

Fig. 11. Principle of superposition of axial CT slices to create a three-dimensional model.

The region of interest and its boundaries should be clearly stated in writing in the exam request, together with the observation that the protocol should be followed.

The protocol to guide radiologist to acquire good-resolution CT scans is: ${ }^{29}$

- CT scanning should acquire axial slices $1 \mathrm{~mm}$ thick or thinner;

- CT scanning should keep a distance of $1 \mathrm{~mm}$ or less between slices;

- $\quad$ Gantry should not be tilted (gantry tilt $=0$ );

- $\quad$ Only the sequence of 2D axial images in DICOM format should be recorded in the CD;

- FOV should be limited, but all the region of interest described in the request should be included;

- When the region of interest is the face, the patient's mouth should be kept open during CT scanning to ensure the separation between mandible and maxilla; 
- $\quad$ Voxel ratio should be 1:1 (pitch = 1:1);

- The window should be standard, in the original format of the CT scanner.

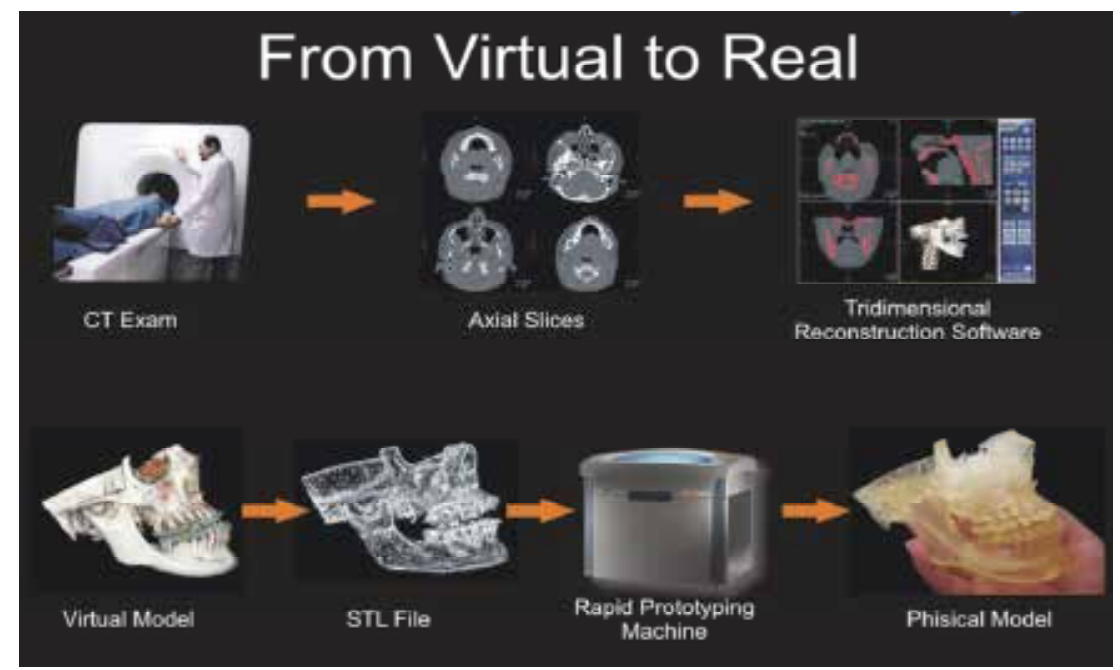

Fig. 12. Step by step sequence to fabricate a prototype of a human anatomic structure

* Attention: to fabricate the prototype, images should be stored in DICOM format and recorded in a CD-ROM or sent via the Internet to the prototyping bureau; the $\mathrm{CT}$ scan films are not necessary.

\section{Uses of prototypes in health care}

In December 1998, the Phidias Project was established in the European Community to explore and evaluate the applications of rapid prototyping in medical and dental care. The project brought together over 40 health care organizations in $11 \mathrm{EU}$ countries and lasted four years. It included 253 surgeries that used prototypes in several specialties. ${ }^{31}$

This Project findings showed that most prototypes were used in implant surgery planning $(29 \%)$, tumor evaluation $(22 \%)$, trauma treatment $(17 \%)$, and treatment of congenital anomalies, in $13 \%$ of the cases. ${ }^{31}$

Some results of the Phidias Project are analyzed in the tables below:

$\begin{array}{lll}\text { Indications for medical model } & & \\ \text { Diagnosis } & \mathrm{n} & \text { Percentage (\%) } \\ \text { Tumor } & 56 & 22 \\ \text { Trauma: } & 43 & 17 \\ \text { Congenital anomalies } & 32 & 13 \\ \text { Orthognathic surgery } & 18 & 7 \\ \text { Skull/implant } & 15 & 6 \\ \text { Tooth implant } & 74 & 29 \\ \text { Orthopedic surgery } & 11 & 4 \\ \text { Other diagnoses } & 4 & 2 \\ \text { TOTAL } & 253 & 100\end{array}$

Table 3. Phidias Project data about indication of prototypes in health care 
Reasons to use model in planning

$\begin{array}{ll}\text { Increase diagnostic quality } & 109\end{array}$

$\begin{array}{ll}\text { Produce preoperative guide implant } & 108\end{array}$

$\begin{array}{ll}\text { Plan surgery } & 183\end{array}$

Prepare resection guide $\quad 31$

Obtain patient approval $\quad 99$

Use as guide during intervention 123

Simulate intervention preoperatively 105

Other reasons 36

Table 4. Phidias Project data about reasons to use prototype during surgery planning.

\section{How preoperative planning affected the intervention}

Planning skin incision $\quad 34$

Whether to operate or not $\quad 66$

The general surgical concept 131

Details of surgical concept 167

$\begin{array}{ll}\text { Composition of surgical team } & 77\end{array}$

Positioning patient on surgical table $\quad 22$

Selection of material for osteosynthesis 94

Selection of instruments and resources 115

Site of osteosynthesis material implant $\quad 108$

Sequence of intervention $\quad 121$

Table 5. Phidias Project data according to effect of rapid prototyping on planning

How model planning

affected the result of surgery in comparison with other imaging modalities

Precision and quality of bone transplant

Precision and quality of osteotomy

Communication with other health workers

Communication with patient

Little

1

$3 \quad 2 \quad 94$

"Confidence" during intervention

$8 \quad 11 \quad 134$

$17 \quad 6 \quad 164$

$17 \quad 19 \quad 156$

Table 6. Phidias Project data according to comparisons between rapid prototyping and other diagnostic imaging modalities.

There are several applications for 3D models, and new purposes constantly arise in different areas of health care. The use of prototypes in immediate trauma is not common, but it is rather frequent in trauma sequelae. Prototypes are very useful in planning surgeries ${ }^{8}$ for facial reconstruction and are essential to reestablish symmetry for the patient. ${ }^{6}$

Models can be used for rehearsal and more accurate planning and bring reductions in surgical time.6,8,23 When surgery is performed in a hospital, time reduction implies lower costs with anesthesia and hospitalization because the patient is usually discharged earlier than expected. 14

Prototypes can and should be used in several situations, such as:

- Evaluation of asymmetrical features 
- $\quad$ Reconstruction of symmetrical structures using mirroring

- Fracture assessment

- Modeling rigid internal fixation plates and screw selection

- Modeling osteogenic distractors

- Calculation and adaptation of bone grafts

- Tumor assessment

- Fabrication of surgical guides

\subsection{Evaluation of asymmetries}

The use of prototypes provides the exact degree of asymmetry and defines the skeletal contribution to deformities. Models provide accurate measures that facilitate planning and performance of corrective surgeries. $6,8,23$ A complete face model provides access to all the patient's anatomic structures bilaterally and defines what symmetry relations have to be restored. During surgery it is practically impossible to accurately establish the asymmetrical relations because only one side is exposed.

\subsection{Reconstruction of symmetry using mirroring}

When trauma or asymmetry is limited to one of the sides of the face and does not go beyond the middle line, what is necessary to reestablish symmetry with the unaffected side can be evaluated.

Three-dimensional software resources may be used to mirror the unaffected hemifacial structure and the superposition of this structure over the affected side. This procedure provides information to perform a Boolean process, the subtraction of the affected structure, leaving only what would be necessary to reconstruct it. In cases of bone loss, the volume and outline of the missing area can be reproduced accurately using data from the opposite side. The same process may be used in reverse to define how much should be removed in cases of unilateral bone excess.

This virtual mathematical process opens several possibilities: the prototype may be produced only for the area that needs reconstruction (result of the Boolean process) and then this specimen can be duplicated in biocompatible material and implanted in the patient. Also, the prototype of the reconstruction site, together with the affected anatomic structure, may be used to determine the best site for plates and fixation screws, or they may suggest not to use any of these, but, rather, to use a negative structure (impression) virtually created over the structure for reconstruction, thus producing a prototype that would be a cast for the reconstruction site. ${ }^{6}$

This process is not limited to bone, and may be used even for the reconstruction of soft tissues, such as in the cases an ear is lost, in which the ear in the opposite side would be mirrored to reproduce the missing ear.

When loss affects the middle region, a CT database of other patients may be used to search for a similar bone fragment to reconstruct the missing anatomic structure virtually or to use $\mathrm{CAD}$ software and literally redesign the region.

\subsection{Fracture assessment}

To interpret fractures using radiographs has always been a problem in maxillofacial surgery. No matter how skilled the surgeon, the radiologist, or both, difficulties arise due to the superposition of images of different anatomic structures. CT has reduced this 
problem, but has not eliminated it. Anatomic image slicing has provided a more complete evaluation and reduced interferences, but the real dimension of the injury still depends on the imagination and experience of the surgeon despite the latest resources. Virtual 3D reconstructions on the computer screen, for example, are not palpable, and, no matter how real they seem to the observer's eyes, they are always, after all, flat images displayed on a screen or printed on paper. ${ }^{19}$

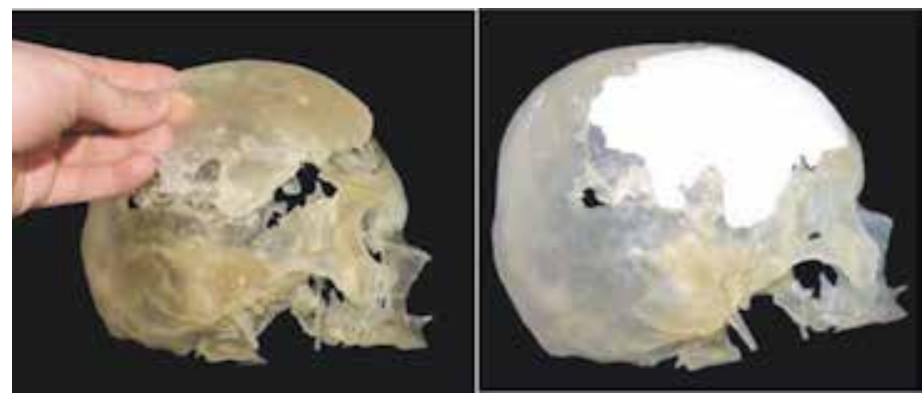

Fig. 13. Reconstruction of skull anatomy using mirroring. The prototype of the area to be reconstructed was built in duplicate in surgical acrylic to be implanted in the patient.

This problem has been finally solved with the advent of prototypes, which enlarge the capacity of the surgeon to understand the real extension of injuries, as if handling the fragments in an open surgical field. ${ }^{16}$

Surgical planning became simple because these new technologies determine the most appropriate point for access, plate casting and screw size. ${ }^{16}$ Moreover, prototypes can be used for a more detailed case documentation and to facilitate communication with the patient, who can visualize and better understand the extension of the problem.

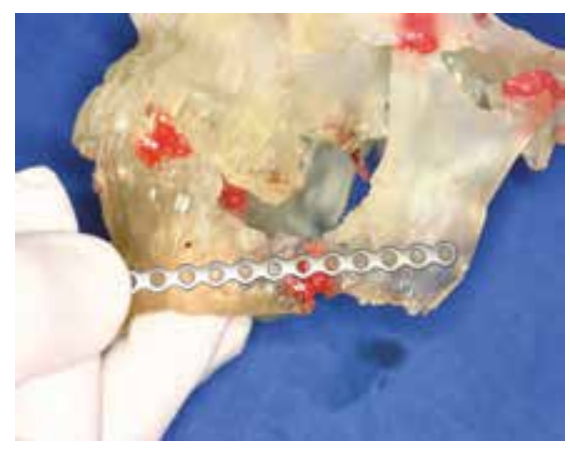

Fig. 14. Preoperative reconstruction plate preparation according to prototype

\subsection{Modeling rigid internal fixation plates and screw selection}

Reconstruction plate preparation for operations reduces surgical time dramatically. As seen before, the plate can be modeled previously, be used as a surgical guide, and ensure an accurate and efficient adaptation. ${ }^{17}$ Patients that undergo partial resection of the mandible have major displacements of the bone stumps due to the traction of the masticatory muscles. In such cases, prototypes can be used to reposition the displaced fragments and move the condyles and the middle line back to their previous positions. 
The previous selection of mini-plates and screws has several advantages in orthognathic surgeries. Surgical simulations using plate models and the calculation of screw size, as well as the choice of the best place for their fixation, greatly reduce surgical time.16,17 However, the most important factor is accurate planning, and modeled plates will serve as guides to reposition bone segments.
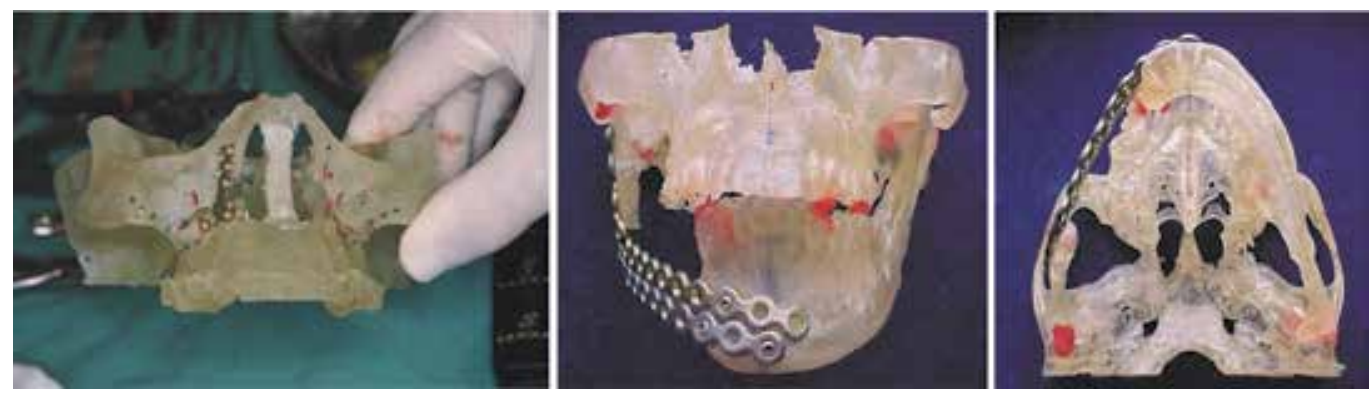

Fig. 15. Modeling rigid internal fixation plates for orthognathic surgery (left) and fabrication of double personalized mandible reconstruction plate by soldering (center and right).

\subsection{Modeling osteogenic distractors}

Modeling osteogenic distractors directly on the bone at the time of surgery poses a great problem due to the difficulty in evaluating distractor vector, because the distal fragment cannot be moved far enough to evaluate its direction. Prototypes not only predict distraction direction, but also evaluate all the process. All patient movements can be assessed until the final fragment position is reached. ${ }^{12}$ Planning the path that the fragment will follow may indicate possible complications, such as limitations in distractor excursion in convergent osteotomies.
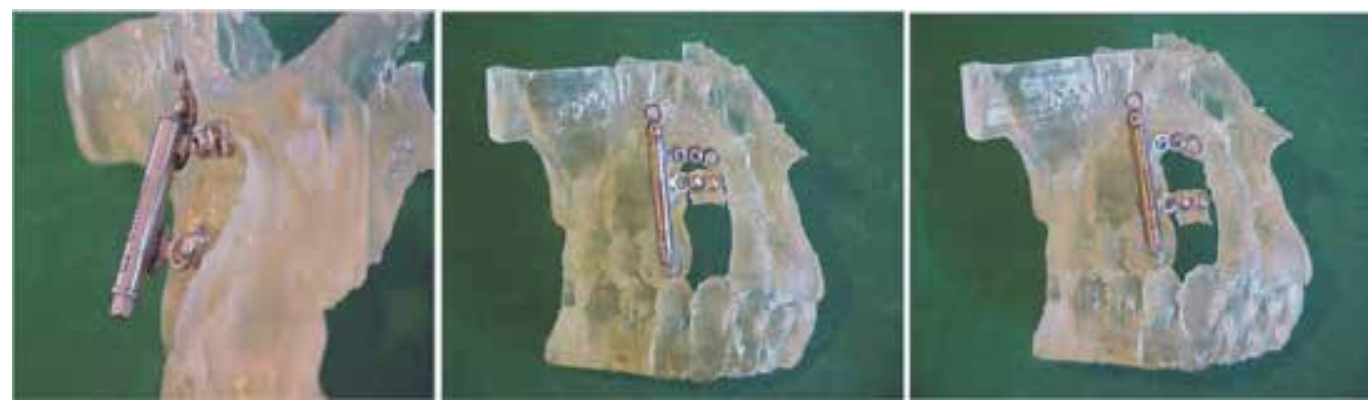

Fig. 16. Modeling and evaluation of distraction vector in a case of osteogenic distraction.

\subsection{Calculation and adaptation of bone grafts}

Prototypes are equally important in planning bone graft reposition, either to repair loss or correct a defect. Graft size and shape are calculated by sculpting the graft, adapting it to the previously sterilized model, and taking it to the surgical field. ${ }^{11}$ This calculation is easily made when any modeling material is used, such as wax and acrylic resin. The volume guides the surgeon to decide about the amount of bone that should be removed from the donor site or selected from bone segments obtained from bone banks. 

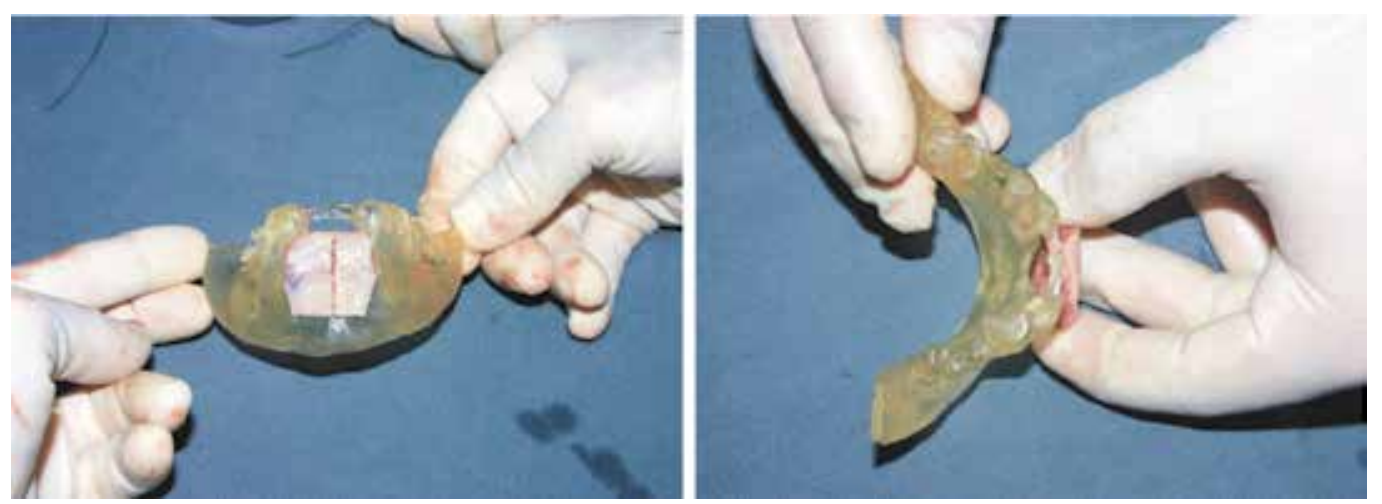

Fig. 17. Adaptation of bone graft on the prototype.

\subsection{Tumor assessment}

The assessment of tumors by using radiographs may not answer questions about the real extension of the lesion. In addition to providing a more careful evaluation of extension, prototyping also facilitates the conversation with the patient about the real situation and severity of the case, and serves, therefore, not only as a diagnostic instrument, but also as a valuable tool for the communication between the specialist and the patient.9,14,17,23

Prototypes also enable previous planning and rehearsing, which ensures the detailed analysis of each case. 8,17

Figure 18 shows a mandibular keratocyst extending from the ascending ramus to the apex of the first premolar. The prototype played an extremely important role in explaining the need for a surgery to the patient, and was also used to calculate the amount of PRP necessary to fill the bone defect completely.

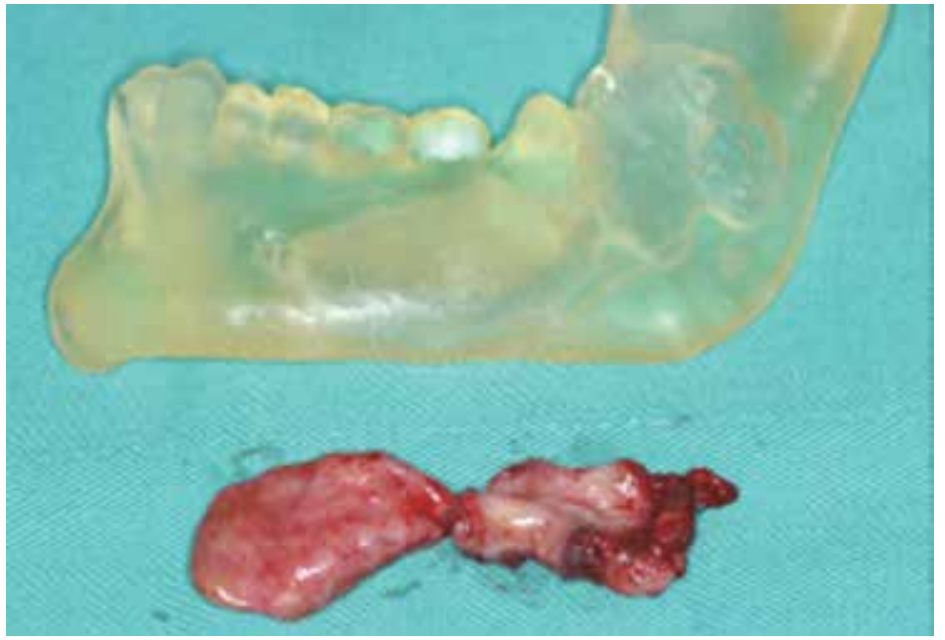

Fig. 18. Prototype of a mandible segment demonstrates odontogenic keratocyst.

The large ameloblastoma seen in the figure 19 had compromised all right mandibular body, the ascending ramus and coronoide process. Only the rapid prototyping is able to reproduce the reality with such accuracy. 


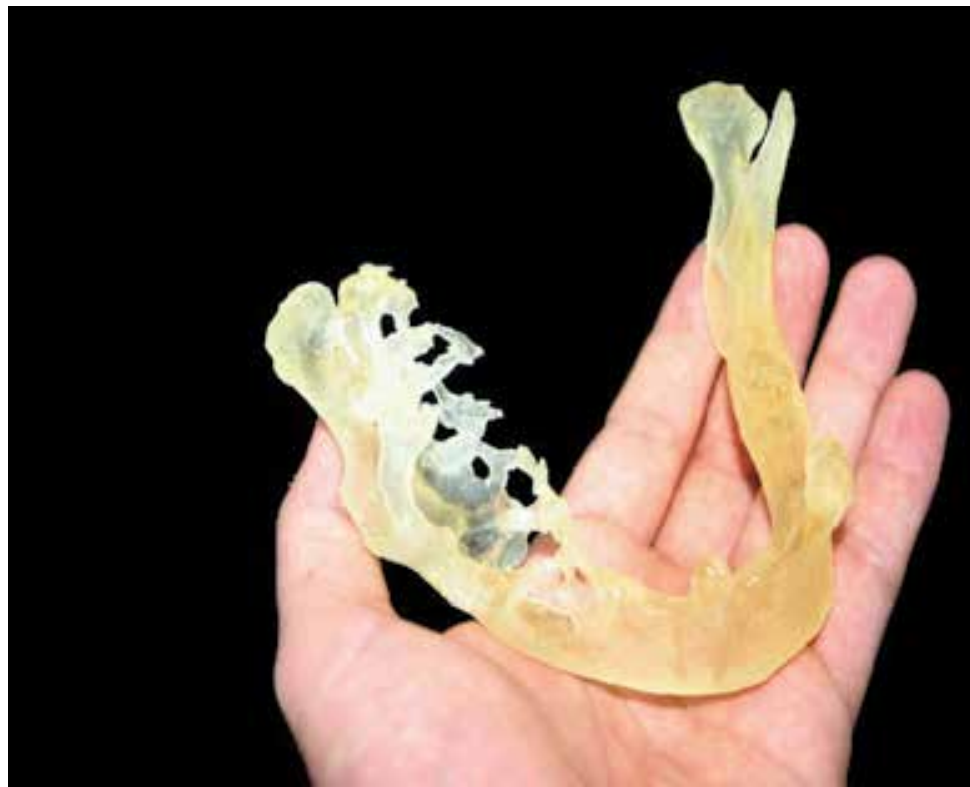

Fig. 19. Evaluation of a multilocular ameloblastoma of mandible.

\subsection{The creation of surgical guides}

The prototype offers a unique opportunity to work and the cost-benefit becomes positive only when using this feature for something beyond mere illustration ${ }^{19}$.

The production of surgical guides, which are common in implants can also be of great valuable in Maxillofacial Surgery.

The possibility of the producing a guide that can be taken to the surgical field and adapted on the patient's bone to guide various types of procedures, can significantly expedite a surgery and it can increase its probability of success ${ }^{23}$.

\section{References}

[1] Almong DM, Onufrak JM, Hebel K, Meitner SW. Comparison between planned prosthetic trajectory and residual bone trajectory using surgical guides and tomography - a pilot study. J Oral Implantol. 1995; 21(4): 275-80.

[2] Almong DV, Sanchez R. Correlation between planned prosthetic and residual bone trajectories in dental implants. J Prosthet Dent. 1999; 81(5): 562-7.

[3] Asher ES, Evans JH, Wright RF, Wazen JJ. Fabrication and use of a surgical template for placing implants to retain an auricular prothesis. J Prosthet Dent. 1999; 81: 228-33.

[4] Barry E, Brown JM, Connell M, Cravens CM, Efford ND, Radjenovic A, et al. Preliminary experience with medical applications of rapid prototyping by selective laser sintering. Med Eng Phys. 1997; 19:90-6.

[5] Becker CM, Kaiser DA. Surgical guide for dental implant placement. J Prosthet Dent. 2000; 83: 248-51.

[6] Bill JS, Reuther JF, Dittmann W, Kübler N, Meier JL, Pistner H, et al. Stereolithography in oral and maxillofacial operation planning. Int J Oral Maxillofac Sur. 1995; 24:98-103. 
[7] Dixon DR, Morgan R, Hollender LG, Roberts FA, O`Neal B. Clinical application of spiral tomography in anterior implant placement. J Periodontol. 2002; 73:1202-9.

[8] Erickson DM, Chance D, Schmitt S, Mathis J. An opinion survey of reported benefits from the use of stereolithographic models. J Oral Maxillofac Surg. 1999; 57:1040-3.

[9] Faber J, Berto PM, Quaresma MM. Rapid prototyping as a tool for diagnosis and treatment planning for maxillary canine impaction. Am J Orthod Dentofacial Orthop. 2006;129 (4):583-9.

[10] Fortin T, Coudert JL, Champleboux G. Computer-assisted dental implant surgery using computed tomography. J Image Guid Surg. 1995; 1: 53-8.

[11] Gaggl A, Schultes G, Santler G, Kärcher H. Treatment planning for sinus lift augmentations through use of 3-dimensional milled models deriver from computed tomography scans. Oral Surg Oral Med Oral Pathol Oral Radiol Oral Endod. 1998; 86:388-92.

[12] Gateno J, Allen ME, Teichgraeber, JF Messersmith M. An in vitro study of the accuracy of a new protocol for planning distraction osteogenesis of the mandible. J Craniomaxillofac Surg. 200; 58: 985-90.

[13] Grimm T. User's guide to rapid prototyping. 1st ed. Michigan: Society Of manufacturing Engineers: 2004.

[14] James WJ, Slabbekoorn MA, Edgin WA, Hardin CK. Correction of congenital malar hypoplasia using stereolithography for presurgical planning. J Oral Maxillofac Surg. 1998; 56:517-21.

[15] Kennedy BD, Collins Jr. TA, Kline PCW. Simplified guide for precise implant placement: a technical note. Int J Oral Maxillofac Implants. 1998; 13: 684-8.

[16] Kermer C, Lindner A, Friede I, Wagner A, Millesi W. Preoperative stereolithographic model planning for primary reconstructions in craniomaxillofacial trauma surgery. J Craniomaxillofac Surg. 1998; 26:136-9.

[17] Poulsen M, Lindsay C, Sullivan T, D'Urso P. Stereolithographic modeling as an aid to orbital brachytherapy. Int J Rad Biol Phys. 1999; 44:731-5.

[18] Quinlan P, Richardson CR, Hall EE. A multipurpose template for implant placement. Implant Dent. 1998; 7(2): 113-21.

[19] Sailer HF, Haers PE, Zollikofer CPE, Warnke T, Calrs FR, Stucki P. The value of stereolithographic models for preoperative diagnosis of craniofacial deformities and planning of surgical corrections. Int J Oral Maxillofac Surg. 1998; 27: 327-33.

[20] Salles FA, Anchieta MVM, Carvalho GP. Esteriolitografia auxiliando o planejamento cirúrgico em enfermidades orais. Rev Bras Patol Oral [periódico na Internet]. 2002 Out [acesso em 27/06/2005]; [aproximadamente 5 pag]. Disponível em: http://www.patologiaoral.com.br/texto10.asp.

[21] Shahrasbi AH, Hansen CA. Surgical oral radiographic guide with a removal component for implant placement. J Prosthet Dent. 2002; 87: 330-2.

[22] Tan KBC. The use of multiplanar reformatted computerised tomography in the surgical-prosthodontic planning of implant placement. Ann Acad Med Singapore. 1995; 24: 68-75.

[23] Urso PS, Barker TM, Earwaker J, Bruce LJ, Atkinson L, Lanigan MW, et al. Stereolithographic biomodeling in cranio-maxillofacial surgery: a prospective trial. J Craniomaxillofac Surg. 1999; 27:30-7. 
[24] Vaneziz P, Vanezis M, McCombe G, Niblett T. Facial reconstruction using 3-D computer graphics. Forensic Sci Int. 2000; 108: 81-95.

[25] Weinberg LA, Kruger B. Three-dimensional guidance system for implant insertion: part I. Implant Dent. 1999; 7(2): 81-93.

[26] Weinberg LA, Kruger B. Three-dimensional guidance system for implant insertion: part II. Dual axes table - problem solving. Implant Dent. 1999; 8(3): 255-63.

[27] Whitman DH, Connaughton B. Model surgery for mandible midline distraction osteogenesis. Int J Oral Maxillofac Surg. 1999; 28:421-3.

[28] Wohlers, T. Wohlers Report 2003: Rapid Prototyping and Tooling State of the Industry Annual Worldwide Progress Report. Fort Collins, CO:Wohlers Associates, Inc. 2003.

[29] ARTIS. Protocolo para exame tomográfico [documento na Internet]. 2007 Out [acesso em 02/05/2007]; Disponível em: http://www.artis.com.br/artis/Images/prototipagem/protocolo.pdf .

[30] Anchieta, M.; Salles, F. 44 years of temporomandibular ankylosis. Proceedings of 16th International Congress and Exhibition, Paris: France, p. 1120, Jun. 2002.

Erben Ch., Vitt KD, Wulf J. The Phidias Validation Study of Stereolithographic Models. Phidias Rapid Prototyping in Medicine. 2002; 8:15-16. 


\title{
Clinical Applications of Rapid Prototyping Models in Cranio-Maxillofacial Surgery
}

\author{
Olszewski Raphael and Reychler Hervé \\ Université catholique de Louvain \\ Belgium
}

\section{Introduction}

Medical models or bio-models represent portions of human anatomy at a scale of 1:1 obtained from three-dimensional (3D) medical imaging (CT scan, MRI). The procedure for the fabrication of medical models consists of multiple steps: 1) the acquisition of highquality volumetric 3D image data of the anatomical structure to be modelled, 2) 3D image processing to extract the region of interest from surrounding tissues, 3) mathematical surface modelling of the anatomic surfaces, 4) formatting of data for rapid prototyping (RP) (this includes the creation of model support structures that support the model during building, which are subsequently manually removed), 5) model building, and 6) quality assurance of the model and its dimensional accuracy. These steps require significant expertise and knowledge of medical imaging, 3D medical image processing, computerassisted design, and software manufacturing and engineering processes. The production of reliable, high-quality models requires a team of specialists that may include medical imaging specialists, engineers, and surgeons (Winder \& Bibb, 2005). Rapid prototyping was introduced in the 1980's to define new techniques for the manufacturing of physical models based on CAD-CAM (computer-aided design, computer-aided manufacturing). RP technology allows the building of a medical model layer by layer, reproducing almost every form of the external and internal anatomic structure. Other categories of RP technologies are solid freeform fabrication, layer additive manufacturing, and 3D printing. RP techniques are different from physical models obtained by milling. RP medical modelling in craniomaxillofacial (CMF) surgery has mainly been developed over the last ten years (Phidias European network), and concerns the following range of applications: 1) aiding in the production of surgical implants, 2) improving surgical planning, 3) acting as an orientation aid during surgery, 4) enhancing diagnostic quality, 5) using in preoperative simulation, 6) achieving a patient's consent prior to surgery, and 7) preparing a template for resection for surgeons (Winder \& Bibb, 2005).

\section{Review of current RP techniques in CMF surgery}

\subsection{Introduction}

Almost all of the RP techniques that have been described were developed to construct 3D medical models for CMF surgery, and these will be presented in the following literature review. These techniques include stereolithography (SL), selective laser sintering (SLS), fused deposition modelling (FDM), 3D printing (3DP), and polyjet modelling. 


\subsection{Materials and method}

A systematic review of the literature was conducted on PubMed (Medline). A search strategy employed was based on title-abstract sifting by one observer. Our exclusion criteria consisted of general dentistry, prosthodontics, orthodontics, forensic medicine, orthopaedics, biomechanics, finite element analysis, and virtual imaging. The inclusion criteria consisted of medical rapid prototyping, three-dimensional models, stereolithography, selective laser sintering, fused deposition modelling, 3D printing, polyjet, maxillofacial, craniofacial, cranioplasty, implantology. The search strategy was based on eight search equations that combined free terms and MeSh terms: 1) ((RP[All Fields] AND models[All Fields]) AND maxillofacial[All Fields] AND ("humans"[MeSH Terms] AND English[lang])) (search on 15.03.11), with 10 articles found, and 10 articles selected; 2) ((Medical[All Fields] AND rapid[All Fields] AND prototyping[All Fields]) AND (maxillofacial[All Fields] OR craniofacial[All Fields]) AND ("humans"[MeSH Terms] AND English[lang])) (search on 17.03.11), with 19 articles found, and 19 articles selected; 3) ((three-dimensional[All Fields] AND models[All Fields]) AND (maxillofacial[All Fields] OR craniofacial[All Fields]) AND ("humans"[MeSH Terms] AND English[lang])) (search on 17.03.11 to 19.03.11), with 450 articles found, 350 articles excluded, and 100 articles selected; 4) ((stereolithography[All Fields] AND maxillofacial[All Fields] AND ("humans"[MeSH Terms] AND English[lang])) (search on 15.03.11), with 37 articles found, 6 articles excluded, and 31 articles selected; 5) ((Selective laser sintering [All Fields] AND maxillofacial[All Fields] AND ("humans"[MeSH Terms] AND English[lang])) (search on17.03.11), with 9 articles found, 1 article excluded, and 8 articles selected; 6) (fused deposition modelling [All Fields] AND maxillofacial[All Fields] AND ("humans"[MeSH Terms] AND English[lang])) (search on 17.03.11), with 1 article found, and 1 article selected; 7) ((three[All Fields] AND dimensional[All Fields]) AND (printer[All Fields] OR ("printing"[MeSH Terms] OR "printing"[All Fields])) AND maxillofacial[All Fields] AND ("humans"[MeSH Terms] AND English[lang]) (search on 15.03.11), with 7 articles found, 1 article excluded, and 6 articles selected; and 8) (polyjet[All Fields] AND (maxillofacial[All Fields] OR craniofacial[All Fields]) AND ("humans"[MeSH Terms] AND English[lang]) (search on 17.03.11), with 1 article found, and 1 article selected. The limits were human studies and English language. There were no limits for the time of publication. The total number of articles found was 534, with 365 articles excluded, and 169 articles selected. The number of duplicate article found among the eight search equations was 42 .

After title-abstract sifting, 127 articles were retained. There were 25 articles excluded after reading of the articles. There were also 3 redundant publications. Finally, we selected 99 articles for review.

\subsection{Discussion}

\subsubsection{Stereolithography}

SL has been the most used RP technique in CMF surgery since it was first applied in grafting a skull defect in 1994 (Mankovich et al., 1994).

\subsubsection{Technique}

An SL RP system consists of a bath of photosensitive resin, a model-building platform, and an ultraviolet (UV) laser for curing the resin. A mirror is used to guide the laser's focus onto the surface of the resin, where the resin becomes cured when exposed to UV radiation. The mirror is computer- controlled, with its movement being guided to cure the resin on a slice- 
by-slice basis. A model is initially designed with CAD software in a suitable file format (commonly STL) and transferred to the SL machine for building. The CAD data file is converted into individual slices of known dimensions. These slice data are then fed into the RP machine, which guides the exposure path of the UV laser onto the surface of the resin. The layers are cured sequentially and bond together to form a solid object beginning from the bottom of the model and building upward. As the resin is exposed to the UV light, a thin, well-defined layer becomes hardened. After a layer of resin is exposed, the resin platform is lowered within the bath by a small known distance. A new layer of resin is wiped across the surface of the previous layer using a wiper blade, and this second layer is subsequently exposed and cured. The process of curing and lowering the platform into the resin bath is repeated until the full model is complete. The self-adhesive property of the material causes the layers to bond to each other and eventually form a complete, robust, 3D object. The model is then removed from the bath and cured for an additional period of time in a UV cabinet. The built portion may contain layers, that significantly overhang layers below. If this is the case, then a network of support structures, made of the same material, is added beneath the overhanging layers at the design stage to add support during the curing process. These support structures, analogous to a scaffold, are removed by hand after the model is fully cured, which is a labour-intensive and time-consuming process. Generally, SL is considered to provide the greatest accuracy and best surface finish of any RP technology. The model material is robust, slightly brittle, and relatively light, although it is hydroscopic and may physically warp over time (a few months) if exposed to high humidity (Ono et al., 1994; Choi et al, 2002; Winder \& Bibb, 2005).

\subsubsection{Clinical applications}

SL models have been used as preoperative planning tools in CMF traumlatology, CMF surgery for craniofacial syndromes and the correction of asymmetric faces, orthognathic surgery, distraction osteogenesis, CMF post-tumoral reconstructive surgery, temporomandibular joint surgery, skull defects reconstruction and cranioplasty, and implantology. This technique can also be used for ear or orbital reconstruction and could be potentially applied in anthropological studies or in the study of facial aging (Papadopoulos et al., 2002). Selectively colored SL models have also been used for the diagnosis and planning of treatments related to supernumerary teeth extraction in cleidocranial dysplasia (Sato et al., 1998), in planning a complex maxillofacial tumour surgery (Kermer et al., 1998), and for the visualisation of rapports between the disc and mandibular condyle in the tempormandibular joint (Undt et al., 2000).

SL models may assist in the diagnosis of and preoperative planning for facial fractures, especially in late primary repair, when open reduction and internal fixation have to wait for a decrease in facial swelling or cerebral oedema. SL models facilitate anatomical reduction, minimise surgical approaches, save operating time, and lead to improvement of postoperative results, which may reduce the number of secondary corrections required for post-traumatic deformities (Kermer et al., 1998; Powers et al., 1998). Post-traumatic deformities after fractures of the zygomatic complex are seen less often in the age of routine open reduction and rigid fixation; nevertheless, they can occur because of diagnostic and therapeutic failures. In the case of delayed reconstruction, the surgeon has to cope with the patients' functional symptoms (diplopia, hypoesthesia, and reduced mouth opening), as well as aesthetic challenges. The repositioning of the entire zygomatic complex is a method that promises the complete reversal of all symptoms. However, this method represents the 
most difficult part of the surgery because of the limited access to the region. As a consequence of remodelling processes, no obvious edges of a fracture remain that could serve as landmarks to determine correct positioning (Klug et al., 2006). An SL model allows for analysis of the actual displacement of bone in all 3 dimensions. Additionally, SL models can be employed to plan a surgery and move the zygoma to its final ideal position. Using these models, osteosynthesis plates can be individually pre-bent before actual surgery, thereby shortening operating time. To transfer the preoperative plan to the operating theatre, a 3D- CT-based navigation system can be associated with SL models to transfer the exact positions of the screws from the SL model to the patient (Klug et al., 2006). However, SL models have proven to be less useful in cases of consolidated fractures of the periorbital and naso-ethmoidal complex, except where there is major dislocation, because of the limited representation of detailed structures (sutures) present in this region (Sailer et al., 1998). A surgery can be simulated in SL models prior to operation on malformative craniofacial syndromes, in which the visualisation of complex anatomy may considerably modify the surgical approach applied, as well as avoiding unnecessary complications (Pelo et al., 2006; Sinn et al., 2006). SL models have provided additional relevant anatomical information for surgeons related to hypertelorism, severe asymmetries of the neuro- and viscerocranium (Wong et al., 2005), complex cranial synostoses and large skull defects. For hemifacial microsomia (unilateral hypoplasia of the craniofacial skeleton and its overlying soft tissue), Zhou et al. (Zhou et al., 2009) developed a customised mandibular implant model that was designed in a computer-assisted manner by projecting a mirror image of the healthy mandible onto the affected side in a three-dimensional CT model. A resin SL model of the implant was then made using a RP machine. Finally, a polymeric biomaterial was sculpted according to the SL model and implanted into the affected side of the mandible to restore the patient's facial symmetry (Chang et al., 1999; Wong et al., 2005; Zhou et al., 2009). The value of these models as realistic "duplicates" of complex or rare dysmorphic craniofacial pathologies for the purpose of creating a didactic collection should also be emphasised (Sailer et al., 1998). However, SL models representing only bone do not reveal the spatial relationships between soft tissue and bone in complicated craniofacial deformities. Therefore, a mixed SL model has been developed showing both soft and bony tissue by first using CT values, resulting in a model in which soft tissue is solid and bone is replaced by empty space (Nakajima et al., 1995). The space is then filled with plaster to represent the skeleton. This model also can provide baseline data for evaluating facial growth after surgical repair of clefts (Nakajima et al., 1995; Al-Ani et al., 2008).

The aim of orthognathic surgery is to treat sagittal, vertical or transverse skeletal congenital or post-traumatic dysmorphoses, based on different types of osteotomies of the maxilla/and or mandible, and to modify the relative position of the maxilla to the mandible, and the absolute position of both the maxilla and mandible to the skull base. Orthognathic surgery is almost associated with pre- and post- surgery orthodontic treatement, as the stable occlusion between the jaws is one of the main goals of the treatment. In orthognathic surgery, SL models replicate the facial skeleton with precise internal anatomy, which can facilitate the design of the osteotomy and the preparation for osteosynthesis. Each sectioned segment of the maxilla and mandible can be accurately repositioned by transferring the positional relationships of multiple reference points on the SL model to the bone surface using pre-bent titanium plates (Hibi et al., 1997). Efforts have been made to replace CTimages, which are often affected by artefacts (due to metallic dental amalgams), thus resulting in poor representation of the tooth area in SL models, as occlusion plays a major 
role in orthogntahic surgery in terms of aestehics, and in avoiding postoperative relapse. Therefore, hybrid SL models based on scanning of plaster casts and on skull CTs have been obtained to allow for more accurate planning of orthognathic surgeries (Hoffmann et al., 2002). The SL technique allows the generation of digital templates that are used during surgery to assist the surgeon in repositioning the maxilla and/or the mandible in relation to each other (Gateno et al., 2007).

Distraction osteogenesis is a surgical process used to reconstruct skeletal deformities and lengthen long bones of the body. A corticotomy is used to fracture the bone into two segments, and the two ends of the bone are gradually drawn (with a distraction device) apart during the distraction phase, allowing new bone to form in the gap. When the desired or possible length is reached, a consolidation phase follows, in which the bone is allowed to continue healing. Distraction osteogenesis has the benefit of simultaneously increasing bone length and the volume of surrounding soft tissues. Its application to the craniofacial skeleton allowed for better corrections of multiple complex maxillo-mandibular craniofacial deformities to be achieved. SL models have been used preopeatively to evaluate various surgical solutions (Whitman \& Connaughton, 1999; Robiony et al., 2007), simulate osteotomies, simulate the positioning of the distractor device (Poukens et al., 2003), prebend plates or inserts of the distraction device (Minami et al., 2007; Varol \& Basa, 2009), define the vector of distraction (the direction of the movement of the elongated bone), simulate final results (Yamaji et al., 2004; Robiony, 2010), and to develop a surgical guide to transfer a surgical plan (osteotomy lines, and the positions of inserts on both sides of the distracted area) to the operating theatre (Poukens et al., 2003). If the distractor is to be prepared for mandibular elongation, the position of the screws (inserts) can be determined preoperatively according to growth trends and the location of the tooth buds and inferior alveolar nerve (Feiyun et al., 2010). In correcting mandibular micrognathia and tempormandibular joint (TMJ) ankylosis in particular, 3D SL models have several advantages: 1) the range of bilateral TMJ ankylosis and the position of the osteotomy line can be easily determined; 2) the transport disc can be designed at the posterior edge of the mandibular ramus, with individual, tailored ramus distractors being made; 3 ) the precise distraction length of the bilateral mandible body can be determined for later orthodontic therapy and orthognathic surgery; 4) the position of the osteotomy line in the mandible body can be determined, with an individual tailored distractor being made; 5) for immobilisation, the attachment plate of the distractor can be adjusted and attached to the surface of the mandible; and 6) surgical procedures can be explained clearly to patients using the 3D model. Thus, 3D CMF model have great potential in therapy for bilateral TMJ ankylosis accompanied by mandibular micrognathia (Feiyun et al., 2010). However, surgery using an STL model cannot not be repeated, because RP is expensive, and models often become useless after primary model surgery (Varol \& Basa, 2009). SL models can be used for preoperative planning of maxillary resection due to oral cancer (Lethaus et al., 2010) and for the planning of maxillary reconstruction with osseo-cutaneous microvascularised free-flaps. A 3D SL model can serve to 1) visualise the extent of a tumour (Eisele et al., 1994); 2) evaluate the anatomy of a defect, and define the residual anchor bone for integration with free-flap segments (He et al., 2009); 3) design osteotomies based on free-flaps and the direction of segment replacement to simulate symmetric maxilla reconstruction (He et al., 2009); 4) fit a graft exactly, with or without reduced reshaping (He et al., 2009); 5) preadapt plates based on a SL model (Al-Sukhun et al., 2008); 6) manufacture a surgical guide for 
tumoral resection (Ekstrand \& Hirsch, 2008); 7) shorten the surgical time before a free-flap is reanastomosed and reduce the risk of microsurgery (He et al., 2009); 8) predict the outcome of surgery (He et al., 2009); and 9) provide a permanent record for future needs or reconstructions (Eisele et al., 1994).

SL models have also been used for the preoperative planning of mandibular resection and reconstruction (Matsuo et al., 2010). Mandibular reconstruction is often needed after partial resection and due to continuity defects (Cohen et al., 2009). The aims for the reconstruction are maintaining the proper aesthetics and symmetry of the face and achieving of a good functional result, thus preserving the form and the strength of the jaw and allowing future dental rehabilitation (Cohen et al., 2009). Reconstruction poses a challenge for the maxillofacial surgeon for a number of reasons, such as the complicated geometry of the mandible, the muscles attached to the mandible, which act in different directions, the shape and position of the condyles in the glenoid fossa, and occlusion (Cohen et al., 2009). Reconstruction of the mandible can be achieved using a temporary bridging titanium locking bone plate until bony reconstruction of the gap is accomplished (Cohen et al., 2009). The use of the reconstruction plate is also advocated when predicted life expectancy is low and when medical conditions preclude prolonged general anaesthesia (Cohen et al., 2009). Further rehabilitation of the mandible can be performed using autogenous bone grafting (iliac crest, fibula free-flap), which is a reliable standard procedure (Cohen et al., 2009). Incorporation of the bone graft into the mandible provides the continuity and strength necessary for its proper functioning, with the possibility of dental implant rehabilitation (Cohen et al, 2009). Bone tissue can be harvested during the first surgical procedure or at a later stage (Cohen et al., 2009).

SL 3D models of the mandible are used to assist in developing a presurgical plan, including consideration of the length of the resection (Kernan \& Wimsatt, 2000). On the SL model, the mandibular and mental foramina are marked, the course of the mental nerve is demarcated, and the boundaries of the mandibular resection are chosen. The reconstruction plate is premolded to the planned neomandible SL model. Intraoperative time is not expended moulding the plate imprecisely. Instead, the plate can be bent as exactly as possible before the operation without the pressure of time. This method serves as a valuable learning tool for junior surgeons. Patients can also gain a significantly better understanding of the problem and the challenges of reconstruction by using such models, which results in a better alignment of hopes and expectations between patients and surgeons. Some potential drawbacks of these techniques include the cost of SL models and the difficulty in adapting them to situations in which the surgical plan changes intraoperatively (ie, tumour-positive bone margins demanding a larger bone resection) (Hirsch et al., 2009). Intraoperative navigation could be associated with the use of SL models to ensure that the locations of mandibular osteotomies coincide with the planning phase (Ewers \& Schicho, 2009; Juergens et al., 2009). Plates (Kernan \& Wimsatt, 2000), trays (Matsuo et al., 2010), or titanium mesh cages for iliac bone (Yamashita et al., 2008), can be easily bent and adapted to fit a mandibular SL models (Zhou et al., 2010; Kernan \& Wimsatt, 2000). SL model also enable the surgeon to determine the required length of a plate, and the length and number of screws (Kernan \& Wimsatt, 2000). As a result, before resection, there is an accurately fitted and contoured reconstruction plate ready for placement. Decreased exposure time to general anaesthesia, decreased blood loss, and lessened wound exposure time are all significant patient benefits from reduced operating times (Kernan \& Wimsatt, 2000). The ability to complete nonsurgical aspects of a patient's treatment in the laboratory also allows 
for precision that is often not achievable during the operative procedure (Kernan \& Wimsatt, 2000). A method to transfer a reconstructive plate from an SL model to a patient has been proposed: Two acrylic guides were built ( 1 for each side) with prints of the occlusal surfaces of the remaining teeth and the titanium plate, allowing the accurate replication of the guide position in the mandibular remnant. This procedure allows for very accurate replication of the $3 \mathrm{D}$ position of the plate, without the need for partial resection of the tumour before fixing the plate (Fariña et al., 2009).

Reconstruction of major surgical defects in the oral cavity after oncological resections requires the use of a free flap. Vascularised free fibular flaps are considered the most suitable choice for mandible reconstruction because of their favourable aesthetics and their functional outcomes. Fibular bone allows the planning of osteotomies in relation to the orientation of the bone and to its vascular pedicle. Thick cortical bone readily accepts plates and screws for secure interosseous fixation, and osteointegrated implants may be placed in this bone safely. The length of bone that can be removed is up to $25 \mathrm{~cm}$; the bone may be osteotomised in 2 to 4 fragments, and retains its vitality. Other tissue structures such as the skin, fascia, and muscle, are removed with the bone. A fibula free-flap graft has to be contoured to fit the mandibular defect, so preoperative planning is required. Shaping of the fibular graft can be performed using computer-aided design and computer-aided modelling procedures for evaluation of the presurgical anatomy, whereby 3D SL models of the fibula graft are obtained (Liu et al., 2009). The 3D SL models of the fibula graft allow for selecting the best titanium plates for each case and bending the plates preoperatively, which reduces the time spent in the operating theatre. While the application of computer-assisted maxillofacial surgery is becoming increasingly popular, translation from virtual models and surgical plans to the operating theatre remains a major challenge. Methods have been described to translate virtual plans to surgical applications using an RP guide based on a 3D SL model for resection of the fibula and for its insertion into the resected defect in the mandible (Hirsch et al., 2009). Additioanlly, osteotomies could be translated into surgical situations through an RP guide (Hirsch et al., 2009), and the length of the resected bone, the mandibular curvature, and the width of the basal bone could be transferred to the fibula flap with template modelling (Hirsch et al., 2009).

Finally, SL models have also been used for preoperative planning and to guide the bending of titanium plates to be used in the resection of benign tumours with mandibular bone involvement such as ameloblastoma (Mainenti et al., 2009).

Temporomandibular joint (TMJ) surgery is mainly performed to treat mandibular condyle fractures, degenerative osteoarthritic diseases, congenital aplasia, tempormandibular ankylosis, and tumours. SL models based on CT imaging (Zhang et al., 2011) or MRI (Undt et al., 2000) can help in the visualisation of bony structures and the shape of the articular disc in relation to bony structures (Undt et al., 2000).

SL models can also serve in constructing a custom-made, total TMJ prosthesis that is adapted surgically to a patient's unique anatomy (Worrall \& Christensen, 2006; Zizelmann et al., 2010).

Additionally, SL models have been used for patients with skull bony defects requiring corrective cranioplasty after the resection of osseous tumours, with congenital and posttraumatic craniofacial deformities, requiring reconstructive cranioplasty, and requiring planning of difficult skull base approaches (Müller et al., 2003). SL models for corrective cranioplasty allowed for the simulation of osteotomies for advancement plasty and craniofacial reassembly in the model before surgery, thus reducing operating time and 
intraoperative errors. The usefulness of SL models in congenital craniofacial deformities depended directly on the size and configuration of the cranial defect. The indications for the manufacture of individual 3D SL models could be cases of craniofacial dysmorphism that require meticulous preoperative planning and skull base surgery with difficult anatomical and reconstructive problems. The SL models provide 1) a better understanding of the anatomy, 2) presurgical simulation, 3) intraoperative accuracy in the localisation of lesions, 4) accurate fabrication of implants, and 5) improved education of trainees (Müller et al., 2003; Wong et al., 2002). A titanium plate can be customised based on an SL model for ideal adaptation to convex (Dattilo \& Bursick D, 1994; Arnaud et al, 1997), and/or concave skull defects. The reconstruction of unilateral bony defects was also based on the use of virtual mirror imaging of the side controlateral to the side with the defect. An STL mirror model was then produced that served as a template (Lo et al., 2004) for a cranioplasty implant (Bill et al., 1995). Finally, implants from diverse sources, such as artificial bone (Cao et al., 2010), bone allotransfers (Kübler et al., 1995), and titanium mesh (Wu et al., 2008), were manufactured to fit into cranial defects.

An approach combining computer-aided design, SL models and surgical navigation could help manage complex lesions in the skull base and craniofacial area requiring rigid reconstruction (Wu et al., 2008).

Finally, selectively coloured SL models have been used to construct surgical guides for dental implant placement. The colour allowed for the identification of internal structures, such as the inferior alveolar nerve canal inside the mandible or maxillary sinuses inside the maxilla. It is of major importance when using these RP models to build on surgical guides for implantology (Cillo et al., 2010). SL models have also been used to build surgical guides for zygoma and pterygoid implants in severely atrophied maxillae (Vrielincket al., 2003) and to fix an obturator prosthesis after a large maxillary malignant tumour (Ekstrand \& Hirsch, 2008).

\subsubsection{Stereolithography accuracy}

SL models can provide a highly exact reproduction of the skull in children with craniofacial malformations (Frühwald et al., 2008). However, Chang et al. (Chang et al., 2003) found that the mean differences in the overall dimensions between SL models and skull specimens were $1.5 \mathrm{~mm}$ (range: 0-5.5 $\mathrm{mm}$ ) for craniofacial measures, $1.2 \mathrm{~mm}$ (range: 0-4.8 mm) for skull base measures, $1.6 \mathrm{~mm}$ (range: 0-5.8 $\mathrm{mm}$ ) for midface measures, $1.9 \mathrm{~mm}$ (range: 0-7.9 $\mathrm{mm}$ ) for maxilla measures, and $1.5 \mathrm{~mm}$ (range: 0-5.7 $\mathrm{mm}$ ) for orbital measures. The mean differences in defect dimensions were found to be $1.9 \mathrm{~mm}$ (range: $0.1-5.7 \mathrm{~mm}$ ) for unilateral maxillectomy, $0.8 \mathrm{~mm}$ (range: $0.2-1.5 \mathrm{~mm}$ ) for bilateral maxillectomy, and $2.5 \mathrm{~mm}$ (range: 0.2-7.0 mm) for orbitomaxillectomy defects. Midface SL models may be more prone to error than those for other craniofacial regions because of the presence of thin walls and small projections. Thus, one should consider designing midface bone replacements that are larger in their critical dimensions than those predicted by preoperative modelling. Choi et al. (Choi et al., 2002) found that the absolute mean deviation between an original dry skull and an SL RP model over 16 linear measurements was $0.62+/-0.35 \mathrm{~mm}(0.56+/-0.39 \%)$. These errors were mainly due to the volume-averaging effect, threshold value, and difficulty in the exact replication of landmark locations. Schicho et al. (Schicho et al., 2006) compared the accuracy of CT and SL models. The accuracy for SL models expressed as the arithmetic mean of the relative deviations ranged from $0.8 \%$ to $5.4 \%$, with an overall mean deviation of $2.2 \%$. The mean deviations of the investigated anatomical structures ranged from $0.8 \mathrm{~mm}$ to $3.2 \mathrm{~mm}$. An overall mean of deviations (comprising all structures) of $2.5 \mathrm{~mm}$ was found. 
Kragskov et al. (Kragskov et al., 1996) also compared the accuracy of CT and SL models and found that the mean difference over all of the investigated cases was $1.98 \mathrm{~mm}(3.59 \%)$. It should also be noted that the limiting factor in SL model accuracy is the imaging technique, rather than the RP technology used. In general, CT and MRI imaging methods acquire image slices that have a slice thickness on the order of 1.0 to $3.0 \mathrm{~mm}$, which is much greater than the limiting build resolution of any of the RP technologies (Winder \& Bibb, 2005). In performing a prospective study on the clinical use of SL models, D'Urso et al. (D'Urso et al., 1999) concluded that SL models significantly improved operative planning and diagnosis. SL models were found to improve measurement accuracy significantly (image measurement error $44.14 \%$, biomodel measurement error $7.91 \%, \mathrm{P}<.05$ ). Surgeons estimated that the use of SL models reduced operating time by average of $17.63 \%$ and were cost effective with a mean price of $\$ 1,031$ AUS. Patients found SL models to be helpful in the informed consent process. SL modelling is an intuitive, user-friendly technology that has facilitated diagnosis and operative planning. SL models have allowed surgeons to rehearse procedures readily and improved communication between colleagues and patients. (D'Urso et al., 1999).

\subsubsection{Stereolithography artefacts}

The CT scanning step is important because the quality of the original CT images directly influences the accuracy of a 3D SL model (Choi et al., 2002). The volumetric or 3D image data required for RP models has to follow isotropic multislice CT scanning protocols with a pixel size on the order of $0.5 \mathrm{~mm}$ and a slice thickness as low as $1.0 \mathrm{~mm}$ (Winder \& Bibb, 2005). The CT scanning step can introduce errors in numerous ways, including with respect to section thickness, pitch, gantry tilt, tube current and voltage, patient movement, metal artefacts of intraoral prostheses, and the slice image construction algorithm itself (Choi et al., 2002). Due to the nature of the voxel dimension, the reconstruction of 3D models from CT images involves the interpolation of slices to convert the image data volume into an isotropic dataset set for mathematical modelling (Winder \& Bibb, 2005). An inherent problem in this computation is that it smoothes out sharp corners or edges between two slices, which is referred to as the partial volume averaging effect or inter-slice-averaging effect. This effect makes it very difficult to replicate a 3D volume precisely, and because many landmarks are located on sharp vertices or acute edges, the effect may greatly affect the accuracy of 3D models (Choi et al., 2002). The next step consists of the identification and separation of the anatomical structure of interest (segmentation) for modelling from its surrounding structures, which can be performed by image thresholding, manual editing, or autocontouring to extract volumes of interest (Winder \& Bibb, 2005). Final delineation of the anatomical structure of interest may require $2 \mathrm{D}$ or $3 \mathrm{D}$ image editing to remove any unwanted details. A number of software packages are available for data conditioning and image processing for medical RP, including Analyze (Lenexa, KS, www.AnalyzeDirect.com), Mimics by Materialise (Leuven, Belgium, www.materialise.com), and Anatomics (Brisbane, Australia, www.anatomics.net). There remains a need for seamless and inexpensive software that provides a comprehensive range of data interpretation, image processing, and model-building techniques to interface with RP technology (Winder \& Bibb, 2005). The size of 3D models depends on the threshold value, which is a specific density in a slice image that separates the organ of interest and other regions. When the threshold value is specified in a slice, it defines contour lines representing the boundary of the organ of interest. The boundaries obtained from every slice form an isosurface with the same density. Therefore, it is important to select the proper threshold value 
(Choi et al., 2002). The first SL models created were for bone, which was easily segmented in CT image data. Bone has a CT number range from approximately 200 to 2,000. This range is unique to bone within the human body, as it does not numerically overlap with any other tissues (Winder \& Bibb, 2005). All soft tissues outside the threshold range were deleted, leaving only bone structures. Thresholding required the user to determine the CT number value that represented the edge of bone where it interfaces with soft tissue. The choice of threshold may cause a loss of information in areas where only thin bone is present (Winder $\& \mathrm{Bibb}, 2005)$. If the bone was particularly thin or the threshold inappropriately measured, a continuous surface was unachievable, leaving the model with a hole where the surface was not closed. In some cases, large areas of bone were removed completely, especially at the back of the orbit and around the malar region (partial volume effect) (Winder \& Bibb, 2005). In many circumstances, the volume of the body that is scanned is much larger than that actually required for model construction. To reduce the model size and, therefore the cost, 3D image editing procedures may be employed. The most useful tool for this procedure was a mouse-driven 3D volume editor that enabled the operator to delete or cut out sections from the volume of data. The editing function deleted sections to the full depth of the data volume along the line of sight of the operator. Image editing reduced the overall model size, which also reduced RP building time. Clearer and less complex models may be generated, making structures of interest more clearly visible. Other image processing functions, such as smoothing, volume data mirroring, image addition, and subtraction should be available for the production of models (Winder \& Bibb, 2005). When importing data, the key characteristics that determine the size and scale of the data are the pixel size and the slice thickness (Winder \& Bibb, 2005). The pixel size is calculated by dividing the field of view by the number of pixels. The field of view is a variable set by the radiographer at the time of scanning (Winder \& Bibb, 2005). The number of pixels in the $\mathrm{x}$ axis and the $\mathrm{y}$ axis is typically $512 \times 512$ or $1,024 \times 1,024$. If there is a numerical error in any of these parameters while data are being translated from one data format to another, the model may be inadvertently scaled to an incorrect size (Winder \& Bibb, 2005). The slice thickness (Choi et al., 2002) and any interslice gap must be known (although the interslice gap is not applicable in CT imaging, in which images are reconstructed contiguously or overlapping) (Winder \& Bibb, 2005). Numerical error in the slice thickness dimension will lead to inadvertent incorrect scaling in the third dimension. This distance is typically on the order of $1.5 \mathrm{~mm}$ but may be as small as $0.5 \mathrm{~mm}$ or as great as $5 \mathrm{~mm}$. Smaller scan distances result in higher quality of the 3D reconstruction. The use of the internationally recognised DICOM (Digital Image Communications in Medicine) standard for the format of medical images has largely eliminated these errors (Winder \& Bibb, 2005). Additional sources of error in 3D model reconstruction include topological defects, such as tessellation, triangle edge, and closure errors, the decimation ratio for surface smoothing, and the methods of interpolation used. The RP manufacturers that provide 3D reconstruction software are concerned with the ability to deal with topological incompleteness and surface smoothness. Errors can arise during the actual production and curing of RP models, including errors associated with the residual polymerisation and transformation of RP materials, the creation and removal of support structures (to avoid unsupported or weakly supported structures), laser diameter, laser path, thickness of layers, and finishing (Choi et al., 2002). Model stair-step artefacts represent the stepped effect seen in medical models. One contribution to these artefacts comes from the discrete layer thickness at which the model is built, which is a characteristic of the particular RP process and material being used. Typically, these thickness range from 
$0.1 \mathrm{~mm}$ to $0.3 \mathrm{~mm}$. This effect can be minimised by selecting processes and parameters that minimise the build layer thickness. However, thinner layers result in longer build- times and increased costs, and an economic compromise is typically found for each RP process. As the layer thickness is typically an order of magnitude smaller than the slice thickness of the CT images, it does not have an overriding effect on the quality of the model. The second effect arises from the slice thickness of the acquired CT or MR images and any potential gap between them (Winder \& Bibb, 2005). Both SL and fusion deposing modelling (FDM) required support structures during the build process. These are subsequently cleaned from the model manually, although they generally leave a rough surface, which does not affect the overall accuracy of the model but contributes to a degradation of its aesthetic appearance. It is unlikely that these structures will have a detrimental effect on surgical planning or implant design (Winder \& Bibb, 2005). The mathematical modelling of a surface will introduce its own surface effects. The smoothness (governed by the size of the triangle mesh) of the model surface becomes poorer as the surface mesh becomes larger. A larger mesh results in a lower number of triangles, reduced computer file size, and faster rendering. A smaller mesh results in a much better surface representation, much greater computer file size, and slower rendering (Winder \& Bibb, 2005).

\subsubsection{Conclusions}

Complex surgical procedures, especially those related to craniofacial structures, appear to benefit significantly from the preplanning and implant construction allowed by physical prototypes. It has been hypothesised that the costs of RP are offset by associated reductions in the number of inaccurate and incomplete complex surgical procedures. The costs of revision procedures and for the replacement of ill-fitting custom implants (which can cost up to $\$ 3,000$ ) are significant compared with the cost of applying a centralised rapid medical prototyping service. Many advantages of SL models have been identified: 1) the quality of preoperative planning is greatly improved by allowing a better understanding of the anatomy, and the extent of the disease; 2) the best approach to an osteotomy, and, to the associated surgical site can be assessed, and a more realistic simulation of the surgical steps can be conducted; 3) SL medical models provide an excellent reference when discussing surgical procedures with patients, thus enhancing the validity of informed consent, as the patients gain a greater understanding of the technical difficulties and limitations of the proposed surgery; 4) medical training and surgical education can be undertaken, away from already overcrowded surgical suites, and communication between different specialties allows for a more comprehensive multidisciplinary approach (Cheung et al., 2002); 5) the predicting of results improves with more accurate custom implant manufacturing, preplanned screw placement, and osteotomy design, which also reduces operative time (Arvier et al., 1994; Korves et al., 1995; Murray et al., 2008). SL models can also be sterilised and used directly in the operating theatre. The disadvantages of this technique are mainly those inherent in MRI and CT imaging. Additionally, only one model per simulation can be used, and storage areas will be needed with intense use of these biomodels (Lo \& Chen, 2003). Furthermore, the necessary equipment for producing these models is quite costly, and the cost of the fabrication of a composite skull model is presently and is likely to remain very expensive. Although the use of SL models in routine cases is quite rare, they are already in use at various universities and institutions with very satisfactory results, especially in severe cases of maxillofacial deformities (Papadopoulos et al., 2002). Finally, the limitations of the SL modelling technique include a lengthy production time which 
renders it unsuitable for emergency cases, and radiation exposure of the patient. With wider use and further technological development, these drawbacks will be minimised. In the future, 3D SL biomodels may become an adjunct not only to maxillofacial surgery but also to other medical specialties (Yau et al., 1995).

\subsubsection{Stereolithography Selective Laser sintering (SLS)}

\subsubsection{SLS technique}

The SLS technique uses a $\mathrm{CO}_{2}$ laser beam to selectively fabricate models in consecutive layers. First, the laser beam scans over a thin layer of powder previously deposited on the build tray and levelled with a roller. The laser beam heats the powder particles and fuses them to form a solid layer, and then moves along the $X$ and $Y$ axes to design the structures according to computer-aided design (CAD) data. After the first layer fuses, the build tray moves downward, and a new layer of powder is deposited and sintered, and the process is repeated until the object is completed. When the manufacturing process is complete, the prototype is removed from the tray, and the surrounding unsintered powder is dusted off. The prototype surface is finished by sandblasting. The SLS prototype is opaque, and its surface is abrasive and porous. Prototype fabrication time is $15 \mathrm{~h}$, and its approximate cost is 600 USD (Sannomiya et al., 2008; Silva et al., 2008). SLS models do not require support structures and are, therefore, relatively easily cleaned, thus saving labour costs (Winder \& Bibb, 2005). This technique has been used in the field of craniofacial surgery, reconstructive surgery, orthognathic surgery, pre-prosthetic surgery, tumour surgery, and dental implants.

\subsubsection{SLS clinical applications}

SLS model has been used in the presurgical planning for a tumour surgery (ameloblastoma) to assist with mandibular reconstruction using fibular grafts after ameloblastoma resection. Before surgery, the surgeon used the initial SLS biomodel with the tumour to mark the areas where osteotomies should be performed and to determine the shape and magnitude of an autogenous graft implant. An SLS model could also be used during surgery as a guide for the surgeon to mark the bone graft taken from the fibula and transfer the position of osteotomies from the SLS preoperative model to the operating theatre (Sannomiya et al., 2008). A custom-made SLS model has also been developed that can be fitted at any site of a microvascular fibula flap, taking into account the vascular anatomy (Leiggener et al., 2009). This procedure enhanced the visualisation of points to be remodelled in an autogenous fibular graft to reproduce a new mandible (Sannomiya et al., 2008). The accuracy of SLS model is relatively high with standard errors of a maximum of 0.1 to $0.6 \mathrm{~mm}$. This accuracy depends on the thickness of the CT scans used, which should be as thin as possible (1 to 2 $\mathrm{mm}$ is a good compromise for a skull study); the field of view should have a resolution of $512 \times 512$ and not generate tilting during image acquisition (Leiggener et al., 2009).

Relying on the accuracy of the guide, osteotomies and plating can be safely and swiftly performed with the osseous flap in place, which reduces the ischaemic time. Having access to a virtual plan preoperatively allows a surgical team to discuss a procedure in detail, and surgeons can improve or refine treatment plans and produce custom-made devices in advance. Such virtual plans allow for the movement of bony segments to find the best positions with regard to function, aesthetics and blood supply (the vascular anatomy can be visualised), which means that the optimal donor location on the fibula can be determined. Using RP model to manufacture a guide directly from a dataset obtained from the virtual 
plan eliminates the intermediate steps of model construction, from which different types of guides are produced (Leiggener et al., 2009).

A 3D SLS skull model has been found to be able to accurately reproduce and reconstruct a fracture model (Aung et al, 1999) and fully reveal the anatomical structure of the craniomaxillary bone and its relationship to surrounding tissues. It has been used to mimic surgeries for repairing craniomaxillofacial trauma, to determine the validity of a surgical design, to predict surgical outcomes, to weigh various approaches to determine an intraoperative guiding template, and to shorten operation time and minimise surgical risks (Li et al., 2009). The advantage of SLS technology over SL is that it produces models with higher accuracy. This accuracy is especially important in reproducing thin osseous structures of fractured orbital floors for the purpose of manufacturing new custom-made titanium orbital floors (Williams \& Revington, 2010). An SLS polyamide model has been used for repairing large skull defects by constructing custom-made cranial plates. Custommade cranioplasty implants are associated with the advantages of reduced operative time, less invasive surgery, improved cosmetic results, faster recuperation, and reduced costs due to short operative times (Rotaru et al., 2006). SLS models have also allowed for the analysis of abnormalities regarding calvaria morphology, nasal bones and maxilla, improving the criteria for diagnosis and the surgical plan in a case of craniofacial dysostosis (Apert syndrome) (da Rosa et al., 2004; Sannomiya et al., 2006).

\subsubsection{SLS accuracy}

The accuracy of the SLS model is relatively high, with maximum standard errors of 0.1 to 0.6 $\mathrm{mm}$. This accuracy depends on the thickness of the CT scans used, which should be as thin as possible ( 1 to $2 \mathrm{~mm}$ is a good compromise for a skull study); the field of view should have a resolution of $512 \times 512$ and not generate tilting during image acquisition (Sannomiya et al., 2008). Silva et al. (Silva et al., 2008) and Ibrahim et al. (Ibrahim et al., 2009) found a dimensional error of $2.10 \%$ for the SLS prototype in comparison with the dry skull. The authors found an inverse correlation between the external and internal dimensions that may be explained by the dumb-bell effect described by Choi et al. (Choi et al., 2002), in which an increase in external dimensions and a simultaneous decrease in internal dimensions indicated that the prototypes had larger dimensions than the original skull and that the selected threshold may have been too low. Therefore, accuracy is dependent primarily on the choice of scanning protocol, on data segmentation and, especially, on the determination of the threshold. One factor that may partially explain the smaller dimensions of SLS prototypes is the superficial wear caused by sandblasting (Silva et al., 2008). The unused powder that surrounds the prototype in the SLS system cannot be reused. Because of the high cost of the material, several parts are fabricated simultaneously. The long fabrication time for the SLS technique $(16 \mathrm{~h})$ is very close to the time required for fabrication with the SL system (Silva et al., 2008).

\subsubsection{Fused deposition modelling (FDM)}

\subsubsection{FDM technique}

Fused deposition modelling (FDM) uses a similar principle as SL in that it builds models on a layer-by-layer basis. The main difference is that the layers are deposited as a thermoplastic that is extruded from a fine nozzle. A commonly used material for this procedure is acrylonitrile butadiene styrene (ABS). The physical properties of ABS are that it is rigid, has 
dimensional stability, exhibits thermoplastic properties, and is inexpensive. The 3D model is constructed by extruding the heated thermoplastic material onto a foam surface in a path guided by the model data. Once a layer has been deposited, the nozzle is raised between 0.178 to $0.356 \mathrm{~mm}$, and the next layer is deposited on top of the previous layer. This process is repeated until the model is complete. As with SL, support structures are required for FDM models, as it takes time for the thermoplastic to harden and the layers to bond together. The supports are added to the model at the design stage and are built using a different thermoplastic material, which is extruded through a second nozzle. The support material is of different colour than the building material and does not adhere to it, which enables the easy identification and subsequent removal of the supports by hand after the model is completed. A recent development related to this technique is the availability of a soluble support material, which enables support structures to be dissolved from the model in an agitated water bath (Winder \& Bibb, 2005). In this systematic review, we found that only a surgical template for dental implant drilling had thus far been developed based on this technique (Sohmura et al., 2009).

\subsubsection{D Printing (3DP)}

\subsubsection{3DP technique}

The 3D printing (3DP) system uses print heads to selectively disperse a binder onto powder layers. This technology has a lower cost than similar techniques. First, a thin layer of powder is spread over a tray using a roller similar to the one used in the SLS system. The print head scans the powder tray and delivers continuous jets of a solution that binds the powder particles (gypsum powder with a lateral resolution of $200 \mu \mathrm{m}$ or less) as it touches them. No support structures are required while the prototype is fabricated because the surrounding powder supports the unconnected parts. When the process is complete, the surrounding powder is aspirated. In the finishing process, the prototype surfaces are infiltrated with a cyanoacrylate-based material to harden the structure (Silva et al., 2008). The printing technique enables the formation of complex geometrical structures, e.g., hanging partitions inside cavities, without artificial support structures. After the CT scan the rendering of the DICOM data and transformation into STL takes a maximum of a half an hour, and the printing process and infiltration takes approximately $4-6 \mathrm{~h}$. The material costs for the construction of each model are $€$ 150-200 each (Silva et al., 2008; Klammert et al., 2009). The $3 \mathrm{D}$ printers used in this process are relatively inexpensive $(£ 25,000)$, have fast build times ( $4 \mathrm{~h}$ for a full skull), and are easy to maintain. Additionally, 3D printers are cost effective $\left(£ 1 / \mathrm{cm}^{3}\right)$, associated with low waste, accurate $( \pm 0.1 \mathrm{~mm}$ in the $Z$ plane, $\pm 0.2 \mathrm{~mm}$ in the $X, Y$ planes), have small dimensions and can make hard, soft or flexible models. These printers can also be used to identify different types of body tissue depending on the predefined threshold setting selected (Aleid et al., 2010).

\subsubsection{3DP clinical applications}

The reduction of fractures in 3DP models of the maxillofacial region before surgery provides a number of advantages. The most important of these is the global perspective of the reconstruction that the $3 \mathrm{D}$ model provides. The widths of the maxillary and mandibular arches and the symmetry of the reconstruction can be easily evaluated in 3 dimensions using this technique. Additionally, planning for certain types of surgery, particularly the repair of discontinuity defects of the mandible, is greatly improved by the ability to evaluate the 
alignment of mandibular segments based on mirror imaging of the contralateral side, which greatly enhances the accuracy of the reconstruction. Of course, there is an effort investment required in preparing the model. The task of converting CT images using interactive segmentation software and the generation of the model can be managed by technical staff. However, the surgery on the RP model and the reduction of fractures both require the skills of a surgeon (Aleid et al., 2010). It is important to emphasise the fact that reconstruction plates contoured before surgery act as templates to ensure the accuracy of the reconstruction. When plates contoured on 3D models before surgery are used, they bring the segments into alignment when they are fixed to the bone with screws. Therefore, the contoured plates not only serve to reconstruct the fracture but also act as templates to establish the final phase of alignment with precision (Wagner et al., 2004). A 3DP model has also been used for repairing orbital floor fractures using pre-shaped titanium mesh implants formed based on anatomical 3DP models of the orbit (Kozakiewicz et al., 2009). The unaffected orbit was mirrored onto the contralateral side, i.e., the injured orbit. This model contained numerous artefacts, which is typical of CT studies because of the very thin bone structures of the orbit. To create a rigid physical model that will be strong enough to be used as a template, all of the empty spaces (air) surrounding the mirrored orbit in the virtual model were filled in, which resulted in a virtual model of the orbit that was surrounded only by hard tissue. Next, the virtual model data were converted to an STL format, and solid physical models were created from acrylic resin using a 3D printer. The resulting physical models were stronger and more rigid than if they had been built containing hollow structures i.e., maxillary and ethmoid sinuses (Kozakiewicz et al., 2009). The use of 3DP models in orbital floor reconstruction has numerous advantages, such as the following: 1) increasing the understanding of orbital disruption; 2) shortening the operation time; 3 ) decreasing the number of attempts at positioning an implant in the orbit and verifying the shape and fit; 4) serving as a guide for the surgeon during an operation; 5) being relatively inexpensive. However, this method is also associated with some disadvantages, such as the following: 1) the length of time required to build model; 2) the cooperation required between a number of people in different locations; and 3) the use of this method in panfacial fractures is challenging because it is difficult to find any stable orbital margins for virtual planning of the model and to establish an accurate position for the preshaped plates (Kozakiewicz et al., 2009).

Preoperative orthognathic surgery plans can be tested using 3DP models. The relationship between proximal and distal mandibular segments after bilateral sagittal split osteotomies has been evaluated on models preoperatively. Studying the planned movements of osteotomised bone segments preoperatively and observing the relationships of osteotomised segments of the mandibula and maxilla in orthognathic surgery increased the intraoperative accuracy (Mavili et al., 2007). A 3DP multi-position model was also used to prebend titanium plates and produce a surgical guide for transferring osteotomies from the 3DP model to the operating theatre in genioplasty (Olszewski et al., 2010).

Additionally, 3DP models have been used for planning distraction osteogenesis related to complex craniofacial malformations (osteotomies, vector of distraction). The customised fixation plates of a distractor primarily prepared based on the 3DP model can be easily adapted during surgery to predicted positions due to their high accuracy of fit, and they enable the parallel alignment of both connecting pins, which ensures the proper transmission of distraction forces to the mobilised segment (Klammert et al., 2009).

Furthermore, 3DP models have been used in mandibular resection (Ortakoglu et al., 2007) and reconstruction using a reconstructive plate. The plate was precontoured according to 
the 3DP model. Precise adaptation of the plate and excellent symmetry were achieved within a relatively short operation time. Plate handling in the operating theatre was minimal, thus preserving its strength. Other benefits of using 3DP models include decreased exposure time to general anaesthesia, decreased blood loss and shorter wound exposure time. The advantages of 3DP model techniques include the special understanding of bone morphology that is provided, accurate and easier planning of preoperative plate bending, and much more accurate bone harvesting due to using the negative imprint of the gap to be reconstructed. Thus, 3DP technology is a reliable method for assisting in precise mandibular reconstruction using bone plates and bone grafts. Compared with other $3 \mathrm{D}$ methods, this method can be performed more quickly and easily and is more cost effective. Furthermore, it is superior in printing smaller and more complex structures (Cohen et al., 2009).

\subsubsection{3DP accuracy}

Silva et al. (Silva et al, 2008) reported a mean dimensional error of $2.67 \%$ in prototypes produced using 3DP technologies in comparison with a dry human skull (criterion standard). In the 3DP system, the printing mechanism, the type and quality of the materials used in the fabrication of the prototypes, and the absorption properties of the powder when in contact with the binder and infiltration material are parameters that should be controlled to obtain a reliable final product. It is possible that the 3DP prototypes were larger than the dry skulls because of cyanoacrylate infiltration. The powder remaining in the 3DP system may be reused, and the parts may be fabricated individually, which substantially reduces prototype fabrication time $(4 \mathrm{~h})$. Therefore, the 3DP technique has a lower final cost than the SLS technique, which, in turn, has a lower cost than the SL technique (Silva et al., 2008). Advantages of 3DP over SLS include a faster printing time and lower costs (Cohen et al., 2009). However, SLS prototypes have a better dimensional precision and reproduce anatomical details of the craniomaxillary region more accurately than 3DP prototypes (Silva et al., 2008). Ibrahim et al. obtained a dimensional error for 3DP of $1.07 \mathrm{~mm}$ (2.67\%) when comparing SLS (0.89 mm and 2.10\%) and 3DP models and dry skulls (Ibrahim et al., 2009).

\subsubsection{Polyjet modelling}

Polyjet modelling is performed by jetting state-of-the-art photopolymer materials in ultrathin layers $(16 \mu \mathrm{m})$ onto a build tray layer by layer until the model is completed. Each photopolymer layer is cured by UV light immediately after it is jetted, producing fully cured models that can be handled and used immediately, without post-curing. The gel-like support material used, which is specially designed to support complicated geometries, is easily removed by hand and water jetting. At present, this technique is too time- consuming and, therefore, too expensive to be used in CMF surgery clinical applications. Ibrahim et al. (Ibrahim et al., 2009) reported a dimensional error of $2.14 \%$ in reproducing a dry mandible when using this technique.

\section{Clinical examples of applications of use of RP models in CMF surgery}

\subsection{Three-dimensional rapid prototyping model, modeling clay, surgical guide, and pre-bent titanium mesh in reconstruction of the posttraumatic orbital floor 3.1.1 Introduction}

Different methods, surgical approaches, and materials (Schon et al., 2006; Kozakiewicz et al., 2009) were proposed for reconstruction of the posttraumatic orbital floor. Recently, the use 
of three-dimensional (3D) pre-bent titanium implants in a 3D rapid prototyping (3D RP) model was introduced (Kozakiewicz et al., 2009). However, even if the pre-bent titanium mesh fits perfectly on the 3D RP model, transfer of the pre-bent mesh from the 3D RP model to the operating room while maintaining exact an position remains challenging. Therefore, we present a method that involves the use of a rapid prototyping model-based prefabricated surgical drill guide to improve the pre-bent titanium mesh positioning.

\subsubsection{Case report}

A 38-year-old male patient presented to our Department of Oral and Maxillofacial Surgery three weeks after facial trauma incurred during his weekly boxing course. Anamnesis revealed a period of extensive left periorbital swelling immediately following the injury. However, no medical consultation was performed at that time. The clinical examination revealed a left eye enophtalmos and an upgaze diplopia. The patient also presented a hypoesthesia of the left infra-orbital nerve. The patient's main concern was esthetic, related to the accentuated palpebral fold on the left side. A low-dose CT scan was performed (Olszewski et al., 2008). The patient presented with a combined maxillofacial fracture of the left orbital floor, the left anterior maxillary sinus wall and the nasal bones (Fig 1A, C).

\subsubsection{Method}

A 3D RP model (Z Corp, Burlington, USA) was created based on low-dose CT data (DICOM files, STL format) (Olszewski et al., 2008). We used modeling clay (Décor fin, Royal Talens, Holland) to fill in all the holes of the orbital floor on the 3D RP model. The modeling clay also served to reconstruct the left orbital floor such that it was symmetric to the right side. Then, a sheet of paper was cut to fit in the left orbital floor. A titanium mesh $(0.4-\mathrm{mm}$ in width) was then cut from a $100 \times 100-\mathrm{mm}$ titanium mesh plate (Synthes, Oberdorf, Switzerland) with a sheet of paper as a guide. The titanium mesh was then applied and prebent on the 3D RP model. The holes for the screws were marked with a pencil on the anterior orbital rim of the 3D RP model (Fig 2 A). The acrylic guide for positioning the screws was prepared according to the shape of the left orbital rim. Aluminum cylinders were inserted into the acrylic surgical guide to guide the $1.8-\mathrm{mm}$ diameter drill. The aluminum cylinders were inserted perpendicular to the underlying bone surface (Fig 2 B). The pre-bent titanium mesh and the acrylic guide were sterilized using a standard procedure. The fracture site was exposed with the patient under general anesthesia, via a subciliary approach to the left orbital floor. The herniated fat and muscle tissue were moved up to avoid further necrosis and to increase the intra-orbital volume. The prefabricated surgical acrylic guide was inserted in the inferior left orbital rim (Fig $2 \mathrm{C}$ ). Four holes were drilled in the left orbital rim, through the guide, using an 8 -mm drill. The pre-bent titanium mesh was then positioned in the orbit and fixed to the inferior orbital rim by means of three 4 -mm screws and one 6 -mm screw. The diameter of each screw was 1.8 -mm (Fig $2 \mathrm{D}$ ). Clinical postoperative follow-ups at one week and one month showed no diplopia and correction of the palpebral fold. Radiological follow-up revealed a restitutio ad integrum of the left orbital floor (Fig. 1 B, D).

\subsubsection{Discussion}

The recently presented use of pre-bent titanium mesh in 3D RP models (Kozakiewicz et al., 2009; Scolozzi et al., 2009) allows for accurate repositioning of a de novo reconstructed 
orbital floor. However, there are multiple alternative positions for insertion and positioning of the pre-bent titanium mesh inside the orbit. This is especially true for medio-lateral positioning of the titanium mesh, due to relative lack of precise anatomical landmarks on the inferior orbital rim. The acrylic surgical guide allows for transfer of holes for screws from the position appropriate for 3D RP planning to that used in the operating theater (Olszewski et al., 2010). Therefore, there exists only one three-dimensional position for the pre-bent titanium mesh inside the orbit. This cost-effective method could also be an alternative to most cost- and time- consuming navigation-based methods (Ewers et al., 2005; Bell \& Markiewicz, 2009). To pre-bend the intra-orbital part of the titanium mesh a 3D virtual model of the orbit was described and constructed as a 3D RP model (Kozakiewicz et al., 2009). The 3D virtual model required two steps: 1) a mirroring of the right side of the orbital floor on the left side and, 2) a virtual filling in all the empty virtual spaces present because of partial volume effect and of true spaces, that are anatomically present. All these steps were time-consuming and necessitated an experienced engineering team (Kozakiewicz et al., 2009). Use of the modeling clay directly on the 3D RP model precludes the need for time-consuming, complex computer-assisted manipulations, knowledge of advanced software, or an engineering team. Finally, the use of a sheet of paper allows economical use of $100 \times 100-\mathrm{mm}$ mesh titanium plates.

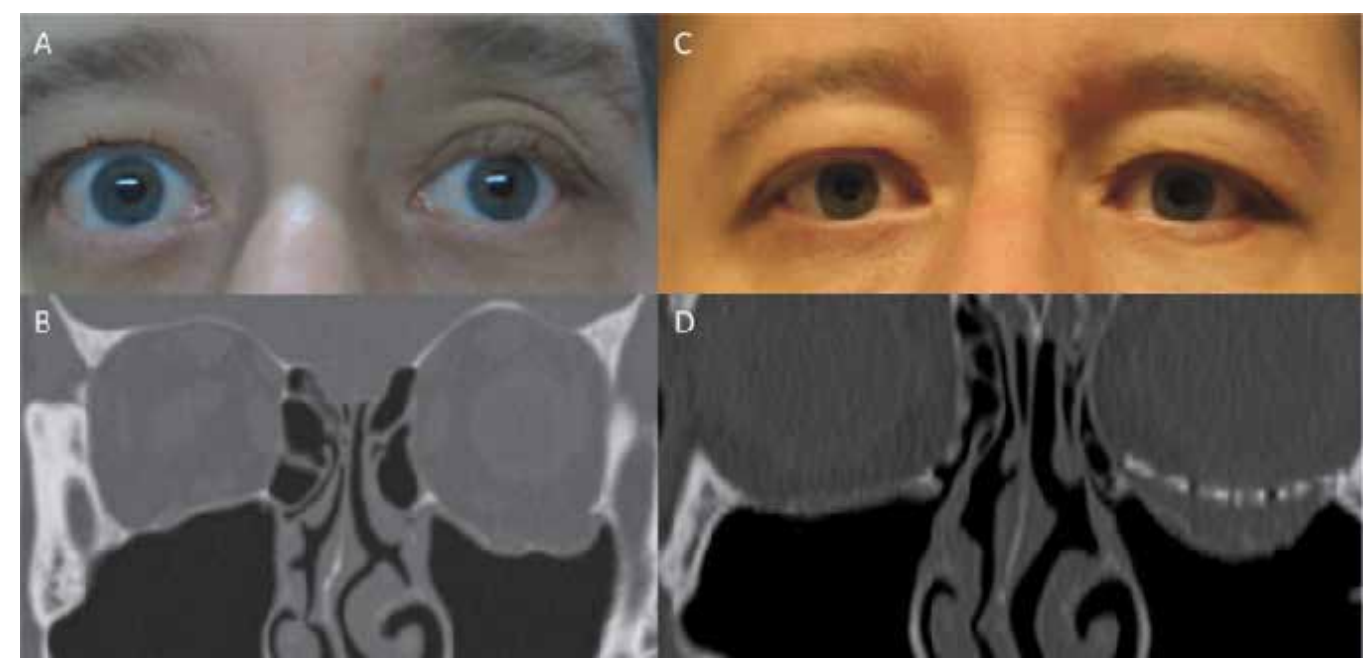

Fig. 1. (A) Pre-operative appearance of the face, accentuated palpebral fold on the left side; (B) Preoperative low-dose CT scan, coronal view; (C) Postoperative appearance of the face, correction of the upper left palpebral fold; (D) Postoperative low-dose CT scan, coronal view, restoratio ad integrum of the inferior orbital wall with the preshaped titanium mesh. 


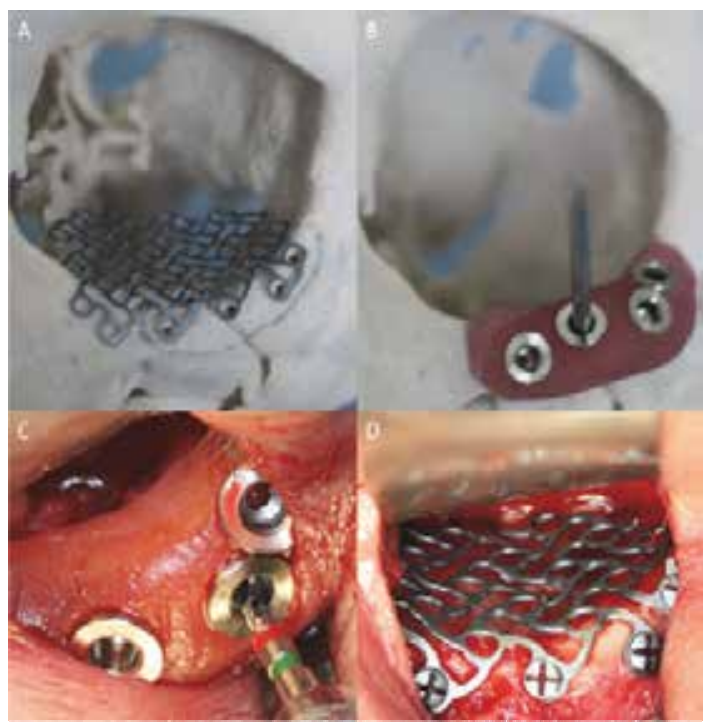

Fig. 2. (A) Pre-bent titanium mesh on the three-dimensional rapid prototyping (3D RP) model, with holes for screws and size of the guide marked with black pencil on the 3D RP model; (B) Acrylic surgical guide for positioning the holes for screws on the 3D RP model, positioning of the drill at $90^{\circ}$ in relation to the bone surface; (C) Intra-operative drilling of holes through the surgical guide; (D) Intra-operative view of the positioning of the pre-bent titanium mesh on the left orbital floor.

\subsection{Innovative procedure for computer-assisted genioplasty: three-dimensional cephalometry, rapid prototyping model and surgical splint.}

\subsubsection{Introduction}

Genioplasty plays an important role in harmonizing facial proportions and profiles. However, planning a genioplasty remains a difficult task because of limited means of diagnosis, planning and information transfer to the operating room (OR). Specifically, the theoretical virtual anteroposterior and vertical positions of the chin are reduced to the landmarks "menton", "B point", and "pogonion" on two-dimensional cephalograms (Ayoub et al., 1994). We propose and describe the combined use of three-dimensional (3D) cephalometry, a 3D rapid prototyping model, and pre-bent titanium plates as a new means of computer-assisted genioplasty.

\subsubsection{Method}

A young adult patient presented in our clinics after an orthodontic treatment was completed elsewhere. At clinical examination, the patient still presented a retrusive profile and refused any orthognathic treatment for the occlusion (Figure 3A). Therefore, we proposed an advancement genioplasty. We received approval from the local ethics committee (B40320084307) for the clinical application of the 3D cephalometric analysis, and the subject gave informed consent for the study. A low-dose CT scan of the head was performed (Olszewski et al., 2008) from which we determined the anterior, posterior, and inferior limits of the chin with the newly-developed and validated 3D cephalometric planar analysis (ACRO 3D) (Figure 3B) (Olszewski, 2007, 2008). The osteotomy lines were 
planned and visualized with Mimics software (Materialize, Leuven, Belgium). We positioned the upper osteotomy line at a distance of at least $5 \mathrm{~mm}$ from both mental foramina. The amount of movement was virtually planned with Mimics software in relation to the reference planes from the ACRO 3D analysis (Figure 3B). We then built a $3 \mathrm{D}$ rapid-prototyping model (RPM) from the low-dose CT scan with a 3D printer (Z-Corp, Burlington, USA) (Silva et al., 2008). The 3D RPM was presented as a multi-position 3D model (Figure 4) with initial, intermediary, and final positions of the bony slices of the chin. We used the final position of the 3D RPM to pre-bend the titanium plates and to indicate the positions of the holes for screws corresponding to the pre-bent plates. The 3D RPM model was then repositioned to its initial position and an acrylic surgical guide was made. The role of the surgical guide was to transfer the position of the holes for the screws (in their final position) and the osteotomy lines. The surgical guide was sterilized at $120^{\circ}$ Celcius for 20 minutes in an autoclave. The pre-bent titanium plates were also sterilized (Kozakiewicz et al., 2009). During the surgery and before the osteotomy, the acrylic guide was positioned on the osseous chin of the patient. First, we drilled the holes for the screws through the surgical guide (Figure 5). Then, with a sterilized pencil (Husami et al., 1988) we drew the osteotomy lines based upon the surgical guide (Figure 5). Osteotomy cuts were performed following the pencil tracings. Finally, after complete separation of the bony fragments, we positioned and screwed the pre-bent plates (Figure 6). A low-dose CT scan showed a good result in the patient's profile (Figure 3, C).

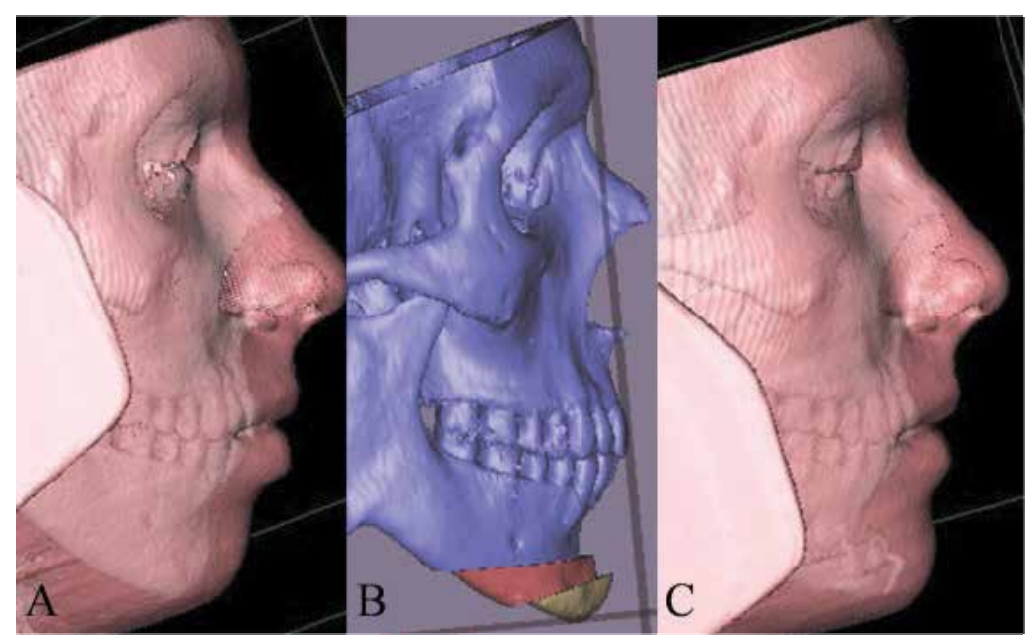

Fig. 3. A. Pre-operative profile; B. Virtual planning, double advancement of the chin; C. Post-operative profile.

\subsubsection{Discussion}

Computer-assisted genioplasty seems to be the next step in the evolution of computerassisted orthognathic surgery (Xia et al., 2000). The combination of different threedimensional methods for the diagnosis (3D cephalometric analysis), virtual planning (Mimics software), and transfer (3D RPM, surgical guide, and pre-bent plates) allowed for a complete 3D treatment of this case. The 3D cephalometry plays an important role in planning a genioplasty. For the first time, the chin region was evaluated with more than a 


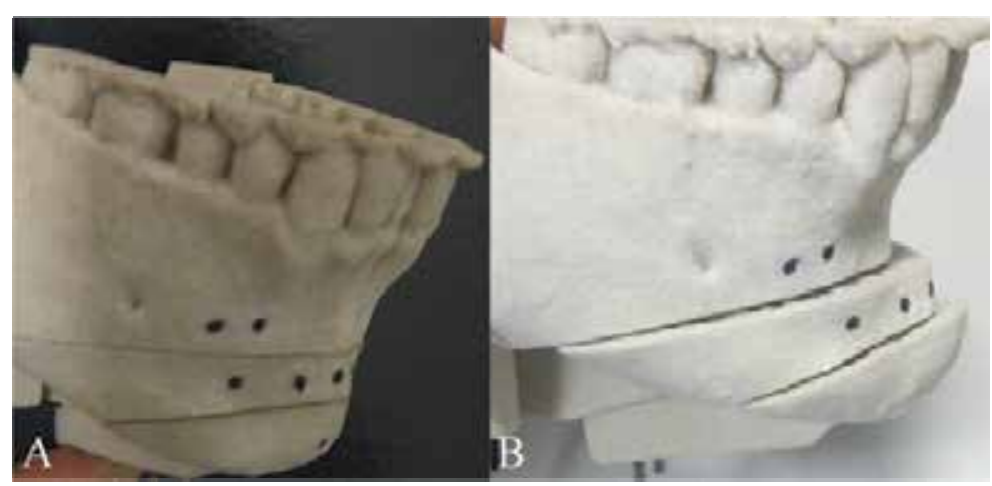

Fig. 4. A. Pre-operative initial position. B. Postoperative final position. Black dots indicate the position of holes for the screws.

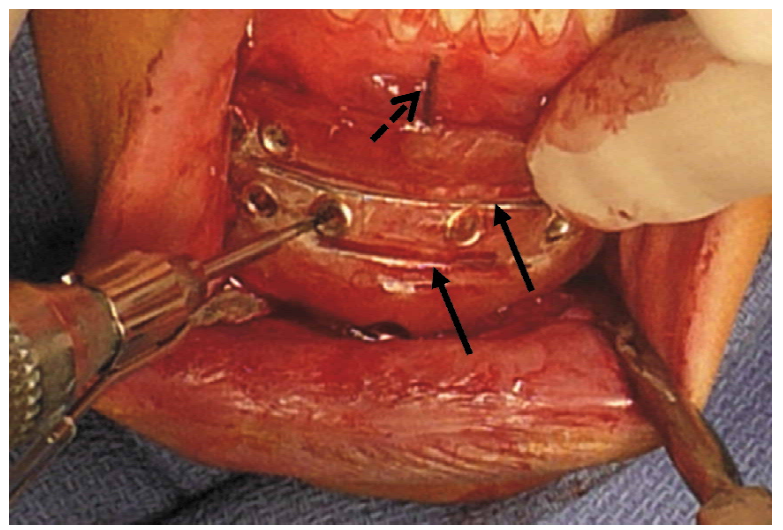

Fig. 5. Acrylic guide positioned on the osseous chin of the patient. Holes for the screws drilled through the surgical guide. Plain arrows showing transfer lines for osteotmy paths. Discontinoous arrow shows the midline indicator of the acrylic guide.

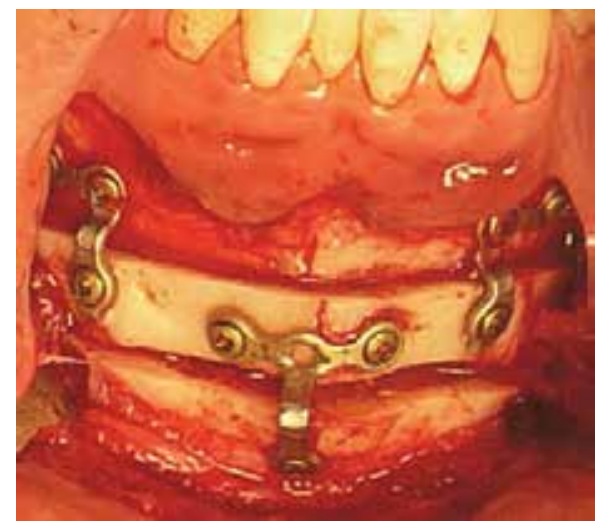

Fig. 6. Positioning and screwing of the pre-bent plates. 
single landmark ("menton", or "pogonion") as is normally the case with 2D cephalometrics (Ayoub et al., 1994). We have limited the presentation of the 3D cephalometry in Figure 3 to the region of interest, which was the evaluation of the chin. The concept, software, and experimental validation of the 3D cephalometric planar analysis have been previously published (Olszewski et al., 2007). Three planes (anterior, posterior and inferior) determine the theoretically ideal and individual position for the osseous chin volume in the 3D space. However, the final decision for the position of the chin must also involve consideration of the soft tissue in the patient's profile. The position of the chin is dependent on the desires of the patient and on the clinical judgment of the surgeon. Therefore, there can be a discrepancy between the conclusions of the theoretical 3D cephalometry and the clinical experience. In this case, the final amount of bone movement in the anteroposterior direction was inferior to that proposed by the 3D cephalometric analysis (anterior plane). It must be stressed that the visualization of soft tissues in figures $3 \mathrm{~A}$ (preoperative) and $3 \mathrm{C}$ (postoperative) represents the true soft-tissue profile of the patient pre- and postoperatively. Virtual planning showed that there was only a place for one screw on the lower part of the chin osteotomy. With the virtual planning we decided preoperatively on the best positioning for that screw. The stability of the lower part of chin osteotomy was also achieved with a pre-bent plate formed on the 3D rapid model. Virtual planning allows for the visualization of the roots and for better positioning of the screws in relation to the roots. For that reason, the chance of root damage is very low in comparison to techniques without a computer-generated surgical splint. The 3D RP model allowed for a transfer of parallel osteotomy lines to the OR. We also were able to propose a sandwich technique, which allowed for the following: 1) a bigger advancement compared to one-piece movement, and 2) improved stability and healing of the displaced bony slices. The use of the sterilized computer-generated surgical guide and pencil was also cost-effective compared to currently available navigation systems (Ewers et al., 2005). We modified the use of the 3D RPM from a diagnosis-only purpose (Santler et al., 1998) to making a transfer of virtual information to the OR. It should be stressed that building a 3D RPM with 3D printers is now more affordable (less than 300 Euros for a 3D RP model) than stereolithographic models (Kozakiewicz et al., 2009). Also, the time spent on the osteotomy and on bending plates was decreased during surgery. The possible chin drop related to increased muscle detachment in the sandwich technique was avoided due to mental muscle reattachment at the end of the surgery. The postoperative results of the new technique are promising. The technique is fast and is easy-to-use due to its computer-generated surgical splint and pre-bent plates. More patients are needed for definitive clinical validation of this procedure. Of note, the same approach of combining 3D cephalometric analysis, 3D multi-position RPMs, surgical costeffective computer-generated surgical guides and pre-bent plates may be of interest in other orthognathic surgery.

\subsection{New three-dimensional (3D) surgical guide for frontal-nasal-ethmoid-vomer osteotomy}

3.3.1 Introduction

Lefort III surgery is a classical surgery performed to correct craniofacial craniosynostoses (Epker \&Wolford, 1980). The majority of the osteotomy lines are performed through via open sky access or with the tactile contact (pterygopalatine disjunction with an Obwegeser osteotome). However, frontal-nasal-ethmoid-vomer osteotomy is performed in a blind manner based only on the experience of the surgeon. The main risks during this type of 
osteotomy are linked to the initial wrong three-dimensional orientation of the osteotome in relation to the patient's anatomy. The anatomy of a craniosynostotic syndromic patient could also be misleading for initial orientation of the osteotom (Sannomiya et al., 2006). An osteotomy performed in too anterior and/or too lateral a direction could result in a bad split of the midline during the down-fracture with Rowe forceps. If performed in too posterior a direction, the ethmoid body could be entered, resulting in intense bleeding and olfactive nerve damage. Finally the depth of insertion of the osteotome in relation to the frontonasal suture is also an issue: too short an insertion will result in incomplete midfacial disjunction and uncontrolled midfacial fracture during the down-fracture with Rowe forceps. Therefore, we propose a new technique based on a three-dimensional (3D) surgical guide for frontalnasal-ethmoid-vomer osteotomy based on a 3D rapid prototyping model. The method was applied to a 7- year- old Apert craniosynostosis patient. Lefort III osteotomies were associated with internal distraction devices for the midface advancement (Nout et al;; 2008).

\subsubsection{Material and methods}

A three-dimensional (3D) computed tomography (CT) of the skull was acquired in a standard head position with a previously validated low- dose CT protocol (Olszewski et al., 2008) (Brilliance 64, Philips, Eindhoven, the Netherlands). The protocol dictates a $1 \mathrm{~mm}$ slice, with $512 \times 512$ matrix, $210 \mathrm{~mm}$ field of view, $120 \mathrm{kV}$ and $42 \mathrm{~mA}$. The native data were saved on a CD (DICOM format). The 3D CT reconstruction was performed by Mimics software (Materialize, Leuven, Belgium) and saved under STL format. A rapid prototyping (RP) model of the skull was obtained with a 3D printer (Z Corp, Burlington, USA). We drilled a groove in the nasal bones until the frontal-nasal suture was reached. A osteotome of $5 \mathrm{~mm}$ width was positioned inside the nasal fossa, with anterior-posterior orientation, from the nasal-frontal suture toward the level of posterior nasal spine. Following this, PMMA resin (Palacos, Heraeus Medical, Germany) was moulded around the osteotome and around the nasal bones (Figures 7,8).

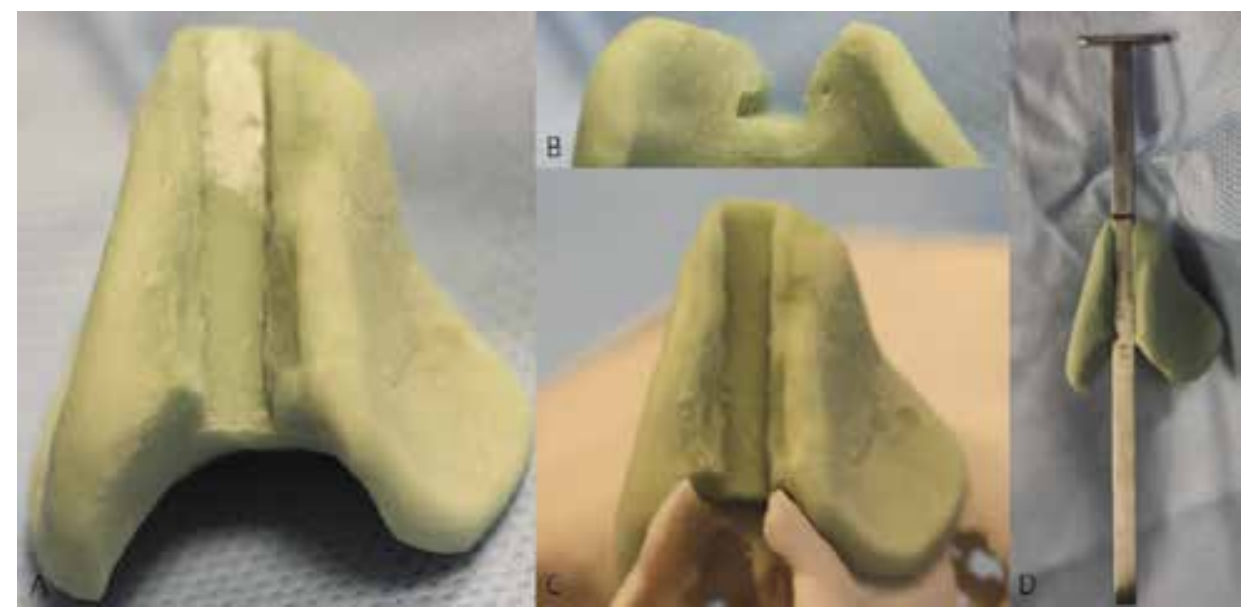

Fig. 7. A. Anterior view of customized 3D osteotomy guide in PMMA resin . B. Inferior view of the 3D guide: the groove for osteotome. C. Anterior view: positioning of the 3D guide on the 3D RP model. D. Anterior view: checking the sliding movement of the osteotome through the 3D guide. The final depth to insert the osteotome is indicated with alcohol pen. 
After the thermo-reaction ended, the 3D guide was polished with dental burrs and sterilised in an autoclave under standard conditions $\left(135^{\circ} \mathrm{C}, 20\right.$ minutes). The distance from the top of the $3 \mathrm{D}$ guide and the posterior nasal spine was also measured on the 3D RP model $(8.3 \mathrm{~cm})$.

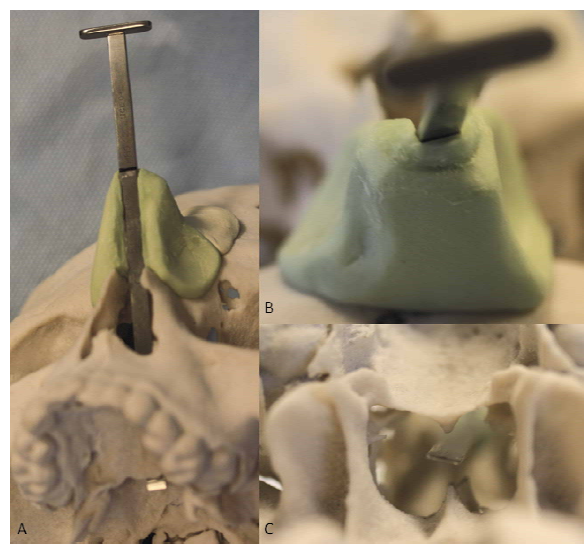

Fig. 8. A. Positioning of the 3D guide, and osteotome on the patient's 3D RP skull model. B. Superior view: checking the insertion of the osteotome inside the 3D guide. C. Inferior view: checking the posterior limit of the osteotomy at the level of posterior nasal spine.

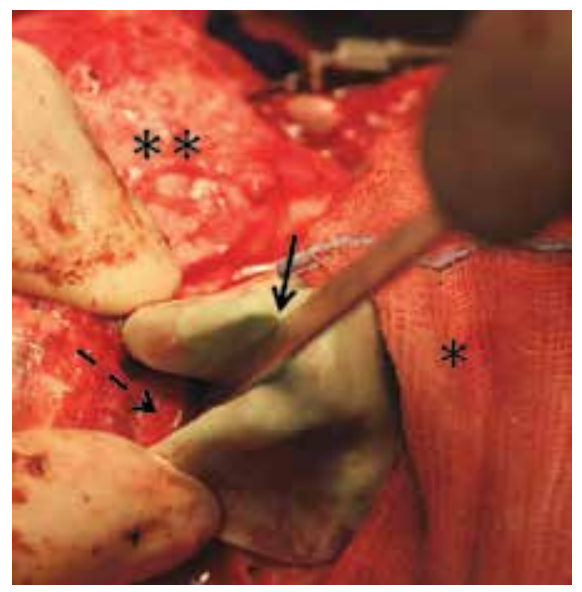

Fig. 9. A. Intra-operative view. Insertion of the osteotome with the 3D guide trough the nasal bones (dashed arrow) until reaching the predicted depth (arrow shows a mark on osteotom). On the right, orbitofrontal bandeau is deposed at that time of surgery, and compresses cover the brain $\left({ }^{*}\right)$. On the left, bicoronal flap raised up at the beginning of the surgery $\left({ }^{* *}\right)$.

\subsubsection{Results}

The surgery was then performed classically with bi-coronal and intraoral accesses. All Lefort III osteotomies were performed classically with drills and osteotomes. The 3D guide was positioned on the top of the frontal-nasal sutures. No cranial pressure was induced with the $3 \mathrm{D}$ guide at any time during the procedure. We used an osteotome of $5 \mathrm{~mm}$ width. We 
marked a reported distance from the 3D RP model (Figure 8.A) on the osteotome with an alcohol pen (Figure 9, arrow). The osteotome was then positioned in the flat groove inside the $3 \mathrm{D}$ guide and inserted into the bone with the orientation provided by the 3D guide until it reached the marked depth (at the level of posterior nasal spine) (Figure 9, arrow).

\subsubsection{Discussion}

Performing frontal-nasal-ethmoid-vomer osteotomy seems to be one of critical issues in Lefort III surgery (Gosain et al., 2002; Matsumoto et al., 2003). Therefore, every technique facilitating this procedure could immediately help the surgeon and protect the patient from major complications. We presented a technical approach based on the optimisation of the use of 3D RP models. 3D RP models are mainly used for diagnostic purposes (Santler et al., 1998). However, 3D RP models allow also for the transfer of 3D data from planning to the operating theatre (Kozakiewicz et al., 2009). We used a 3D printer technique (Z Corp, Burlington, USA) to build a 3D RP model of the skull (Kozakiewicz et al., 2009). The 3D printed models have an economical advantage over stereolithography and retain the accuracy required for medical modelling (Ibrahim et al., 2009). The PMMA resin (Palacos, Heraeus Medical, Germany) was used to create the 3D guide. This material is easy to use, cost -effective, and allows for fast 3D moulding of the frontal-nasal area. The positioning of the 3D guide on the patient does not require a supplementary task by the surgeon, such as registration and tracking in intra-operative navigation (Jeelani et al., 2009). The individualized 3D guide allows three main pieces of information to be transferred from the 3D RP model: impact point for the osteotome, orientation in the 3D space, and the depth for the insertion of the osteotome. This technique allows a critical osteotomy path in Lefort III surgery to be transferred in a secure, fast, and cost-effective manner from the 3D RP model to the operating room. Further study will consists in verification of the accuracy of using customized 3D guides for subcranial separation of the face at the nasofrontal region on cadavers.

\section{Conclusions and future directions}

Three-dimensional RP models are used in association with a variety of applications in CMF surgery. However, there is still room for innovation in these models and for new uses related to additional indications. Increasing the accuracy of RP techniques is still required but without supplementary irradiation of the patient. For this reason, attention should focus on the implementation of low-dose CT scans and cone beam CT scan protocols for data acquisition. Additionally, an effort should be made to develop 3D RP models from alternative image sources, such as MRI, ultrasounds, and laser scan imaging. More costeffective methods are required for the broad application of these modeling technique beyond the most developed countries. For this reason, 3DP currently appears be more realistic for current clinical use than SL or SLS techniques. Other cost-effective RP techniques, such as 3D paper printing (http://www.mcortechnologies.com), should also be investigated in terms of accuracy and applicability and, to increase the availability of 3D RP technology to CMF surgeons and to improve patient care. Such 3D RP models should be used not only for diagnostic purposes but also mainly for transferring virtual plans to the operating theatre. In this process, 3D RP models will be competitive against computerassisted navigation techniques, which are accurate but still very expensive and timeconsuming. Another important factor related to the use of these models is the time required 
for their fabrication, which should be shortened to allow the use 3D RP techniques directly in emergency rooms to enlarge the field of potential indications they may be used to adress. There is still a need for the development of guidelines for 3D RP models related to their clinical use in CMF surgery for the purpose of determining which technique (with which protocol) is best for addressing each specific indication. Finally, CMF surgery will certainly profit from the most advanced and emerging 3D printing techniques, such as organ printing (Song et al., 2010). Organ printing is a biomedically relevant variant of rapid prototyping technology, which is based on tissue fluidity. Computer-assisted deposition (printing) of natural materials (cells or matrices) is performed one layer at a time until a particular 3D form is achieved (Jakab et al., 2010). However, recent attempts using rapid prototyping technologies to design solid synthetic scaffolds (Song et al., 2010) suffered from an inability to precisely place cells or cell aggregates into a printed scaffold. Thus, organ-printing technology will become increasingly more secondum naturam. Mironov et al., (Mironov et al., 2003, 2011) defined organ printing as a rapid prototyping computer-aided 3D printing technology based on using the layer-by-layer deposition of cells and/or cell aggregates into a 3D gel, with the subsequent maturation of the printed construct in perfused and vascularised living tissues or organs. This definition of organ printing includes the many different printer designs and components associated with the deposition process that are currently available, such as jet-based cell printers, cell dispensers or bioplotters, different types of 3D hydrogels and varying cell types. Such computer-assisted tissue engineering using 3D live RP technology will certainly open a new era for reconstructive CMF surgery.

\section{References}

Al-Ani SA, Locke MB, Rees M \& de Chalain TM. (2008). Our experiences managing a rare cranio-orbital cleft. J Craniofac Surg, 19, 3, (May 2008), pp. (819-822) ISSN 1049-2275

Aleid W, Watson J, Sidebottom AJ \& Hollows P. (2010). Development of in-house rapid manufacturing of three-dimensional models in maxillofacial surgery. Br J Oral Maxillofac Surg, 48, 6, (September 2010), pp. (479-481)

Al-Sukhun J, Törnwall J, Lindqvist C, Kontio R \& Penttila H. (2008). One-stage zygomaticomandibular approach for improved access to the hemimaxilla and the middle base of the skull. J Craniofac Surg, 19, 2, (March 2008), pp. (528-533)

Arnaud E, Marchas D \& Renier D. (1997). Aplasia of the vertex without scalp defect. J Craniofac Surg, 8, 2, (March 1997), pp. (146-150)

Arvier JF, Barker TM, Yau YY, D'Urso PS, Atkinson RL \& McDermant GR. (1994). Maxillofacial biomodelling. Br J Oral Maxillofac Surg, 32, 5, (October 1994), pp. (276283) ISSN 0266-4356

Aung SC, Tan BK, Foo CL \& Lee ST. (1999). Selective laser sintering: application of a rapid prototyping method in craniomaxillofacial reconstructive surgery. Ann Acad Med Singapore, 28, 5, (September 1999), pp. (739-743) ISSN 0304-4502

Ayoub AF, Stirrups DR \& Moos KF. (1994). Assessment of chin surgery by a coordinate free method. Int J Oral Maxillofac Surg, 23, 1, (February 1994), pp. (6-10) ISSN 0901-5027

Bell RB \& Markiewicz MR. (2009). Computer-assisted planning, stereolithographic modeling, and intraoperative navigation for complex orbital reconstruction: a 
descriptive study in a preliminary cohort. J Oral Maxillofac Surg, 67, 12, (December 2009), pp. (2559-2570)

Bill JS, Reuther JF, Dittmann W, Kübler N, Meier JL, Pistner H \& Wittenberg G. (1995). Stereolithography in oral and maxillofacial operation planning. Int J Oral Maxillofac Surg, 24, 1 Pt 2, (February 1995), pp. (98-103) ISSN 0901-5027

Cao D, Yu Z, Chai G, Liu J \& Mu X. (2010). Application of EH compound artificial bone material combined with computerized three-dimensional reconstruction in craniomaxillofacial surgery. J Craniofac Surg, 21, 2, (March 2010), pp. (440-443)

Chang PS, Parker TH, Patrick CW Jr \& Miller MJ. (2003). The accuracy of stereolithography in planning craniofacial bone replacement. J Craniofac Surg, 14, 2, (March 2003), pp. (164-170)

Chang SC, Liao YF, Hung LM, Tseng CS, Hsu JH \& Chen JK. (1999). Prefabricated implants or grafts with reverse models of three-dimensional mirror-image templates for reconstruction of craniofacial abnormalities. Plast Reconstr Surg, 104, 5, (October 1999), pp. (1413-1418)

Cheung LK, Wong MC \& Wong LL. (2002). Refinement of facial reconstructive surgery by stereo-model planning. Ann R Australas Coll Dent Surg, 16, (October 2002), pp. (129132)

Choi JY, Choi JH, Kim NK, Kim Y, Lee JK, Kim MK, Lee JH, Kim MJ. (2002). Analysis of errors in medical rapid prototyping models. Int J Oral Maxillofac Surg, 31, 1, (February 2002), pp. (23-32) ISSN 0901-5027

Cillo JE Jr, Theodotou N, Samuels M \& Krajekian J. (2010). The tent pole splint: a bonesupported stereolithographic surgical splint for the soft tissue matrix expansion graft procedure. J Oral Maxillofac Surg, 68, 6, (June 2010), pp. (1365-1370)

Cohen A, Laviv A, Berman P, Nashef R \& Abu-Tair J. (2009). Mandibular reconstruction using stereolithographic 3-dimensional printing modeling technology. Oral Surg Oral Med Oral Pathol Oral Radiol Endod, 108, 5, (November 2009), pp. (661-666)

da Rosa EL, Oleskovicz CF \& Aragão BN. (2004). Rapid prototyping in maxillofacial surgery and traumatology: case report. Braz Dent J, 15, 3, (March 2004), pp. (243-247)

Dattilo DJ \& Bursick D. (1994). Management of traumatic cranial vault deformities using three-dimensional computer-generated models. J Trauma, 36, 5, (May 1994), pp. (691-694)

D'Urso PS, Barker TM, Earwaker WJ, Bruce LJ, Atkinson RL, Lanigan MW, Arvier JF \& Effeney DJ. (1999). Stereolithographic biomodelling in cranio-maxillofacial surgery: a prospective trial. J Craniomaxillofac Surg , 27, 1, (February 1999), pp. (30-37) ISSN 1010-5182

Eisele DW, Richtsmeier WJ, Graybeal JC, Koch WM \& Zinreich SJ. (1994). Three-dimensional models for head and neck tumor treatment planning. Laryngoscope, 104, 4, (April 1994), pp. (433-439) ISSN 0023-852X

Ekstrand K \& Hirsch JM. (2008). Malignant tumors of the maxilla: virtual planning and realtime rehabilitation with custom-made R-zygoma fixtures and carbon-graphite fiber-reinforced polymer prosthesis. Clin Implant Dent Relat Res, 10, 1, (March 2008), pp. (23-29) ISSN 1523-0899

Epker BN, \& Wolford LM. (1980). Dentofacial deformities. Surgical-orthodontic correction CV Mosby, ISBN 0801616069, St Louis, Toronto, London 
Ewers R \& Schicho K. (2009). Augmented reality telenavigation in cranio maxillofacial oral surgery. Stud Health Technol Inform, 150, pp. (24-25)

Ewers R, Schicho K, Undt G, Wanschitz F, Truppe M, Seemann R \& Wagner A. (2005). Basic research and 12 years of clinical experience in computer-assisted navigation technology: a review. Int J Oral Maxillofac Surg, 34, 1, (January 2005), pp. (1-8)

Fariña R, Plaza C \& Martinovic G. (2009). New transference technique of position of mandibular reconstructing plates using stereolithographic models. J Oral Maxillofac Surg, 67, 11, (November 2009), pp. (2544-2548)

Feiyun P, Wei L, Jun C, Xin X, Zhuojin S \& Fengguo Y. (2010). Simultaneous correction of bilateral temporomandibular joint ankylosis with mandibular micrognathia using internal distraction osteogenesis and 3-dimensional craniomaxillofacial models. J Oral Maxillofac Surg, 68, 3, (March 2010), pp. (571-577)

Frühwald J, Schicho KA, Figl M, Benesch T, Watzinger F \& Kainberger F. (2008). Accuracy of craniofacial measurements: computed tomography and three-dimensional computed tomography compared with stereolithographic models. J Craniofac Surg, 19, 1, (January 2008), pp. (22-26) ISSN 1049-2275

Gateno J, Xia JJ, Teichgraeber JF, Christensen AM, Lemoine JJ, Liebschner MA, Gliddon MJ \& Briggs ME. (2007). Clinical feasibility of computer-aided surgical simulation (CASS) in the treatment of complex cranio-maxillofacial deformities. J Oral Maxillofac Surg, 65, 4, (April 2007), pp. (728-734)

Gosain AK, Santoro TD, Havlik RJ, Cohen SR \& Holmes RE. (2002). Midface distraction following Le Fort III and monobloc osteotomies: problems and solutions. Plast Reconstr Surg, 109, 6, (May 2002), pp. (1797-1808) ISSN 0032-1052

He Y, Zhu HG, Zhang ZY, He J \& Sader R. (2009). Three-dimensional model simulation and reconstruction of composite total maxillectomy defects with fibula osteomyocutaneous flap flow-through from radial forearm flap. Oral Surg Oral Med Oral Pathol Oral Radiol Endod, 108, 6, (December 2009), pp. (e6-12)

Hibi H, Sawaki Y \& Ueda M. (1997). Three-dimensional model simulation in orthognathic surgery. Int J Adult Orthodon Orthognath Surg, 12, 3, pp. (226-232) ISSN 0742-1931

Hirsch DL, Garfein ES, Christensen AM, Weimer KA, Saddeh PB \& Levine JP. (2009). Use of computer-aided design and computer-aided manufacturing to produce orthognathically ideal surgical outcomes: a paradigm shift in head and neck reconstruction. J Oral Maxillofac Surg, 67, 10, (October 2009), pp. (2115-2122)

Hoffmann J, Schwaderer E \& Dammann F. (2002). The use of hybrid stereolithographic models for the planning of complex craniofacial procedures. Biomed Tech (Berl), 47, Suppl 1 Pt 1, pp. (278-281)

Husami T, Leffler K, Churnik R \& Lehman JA Jr. (1988). Sterilization of the bone pencil. Plast Reconstruct Surg, 82, 6, (December 1988), p. (1100)

Ibrahim D, Broilo TL, Heitz C, de Oliveira MG, de Oliveira HW, Nobre SM, Dos Santos Filho JH \& Silva DN. (2009). Dimensional error of selective laser sintering, threedimensional printing and PolyJet models in the reproduction of mandibular anatomy. J Craniomaxillofac Surg, 37, 3, (April 2009), pp. (167-173)

Jakab K, Norotte C, Marga F, Murphy K, Vunjak-Novakovic G \& Forgacs G. (2010). Tissue engineering by self-assembly and bio-printing of living cells. Biofabrication, 2, 2 (June 2010), 022001. doi: 10.1088/1758-5082/2/2/022001 
Jeelani NU, Khan MA, Fitzgerald O'Connor EJ, Dunaway D \& Hayward R. (2009). Frontofacial monobloc distraction using the StealthStation intraoperative navigation system: the ability to see where you are cutting. J Craniofac Surg, 20, 3, (May 2009), pp. (892-894)

Juergens P, Krol Z, Zeilhofer HF, Beinemann J, Schicho K, Ewers R \& Klug C. (2009). Computer simulation and rapid prototyping for the reconstruction of the mandible. J Oral Maxillofac Surg, 67, 10, (October 2009), pp. (2167-2170)

Kermer C, Lindner A, Friede I, Wagner A \& Millesi W. (1998). Preoperative stereolithographic model planning for primary reconstruction in craniomaxillofacial trauma surgery. J Craniomaxillofac Surg, 26, 3, (June 1998), pp. (136-139) ISSN 1010-5182

Kermer C, Rasse M, Lagogiannis G, Undt G, Wagner A \& Millesi W. (1998). Colour stereolithography for planning complex maxillofacial tumour surgery. J Craniomaxillofac Surg, 26, 6, (December 1998), pp. (360-362) ISSN 1010-5182

Kernan BT \& Wimsatt JA 3rd. (2000). Use of a stereolithography model for accurate, preoperative adaptation of a reconstruction plate. J Oral Maxillofac Surg, 58, 3, (March 2000), pp. (349-351) ISSN 0278-2391

Klammert U, Böhm H, Schweitzer T, Würzler K, Gbureck U, Reuther J \& Kübler A. (2009). Multi-directional Le Fort III midfacial distraction using an individual prefabricated device. J Craniomaxillofac Surg, 37, 4, (June 2009), pp. (210-215)

Klug C, Schicho K, Ploder O, Yerit K, Watzinger F, Ewers R, Baumann A \& Wagner A. (2006). Point-to-point computer-assisted navigation for precise transfer of planned zygoma osteotomies from the stereolithographic model into reality. J Oral Maxillofac Surg, 64, 3, (March 2006), pp. (550-559)

Korves B, Klimek L, Klein HM \& Mösges R. (1995). Image- and model-based surgical planning in otolaryngology. J Otolaryngol 24, 5, (October 1995), pp. (265-270) ISSN 0381-6605

Kozakiewicz M, Elgalal M, Loba P, Komunski P, Arkuszewski P, Broniarczyk-Loba A \& Stefanczyk L. (2009). Clinical application of 3D pre-bent titanium implants for orbital floor fractures. J Craniomaxillofac Surg, 37, 4, (June 2009), pp. (229-234)

Kragskov J, Sindet-Pedersen S, Gyldensted C \& Jensen KL. (1996). A comparison of threedimensional computed tomography scans and stereolithographic models for evaluation of craniofacial anomalies. J Oral Maxillofac Surg, 54, 4, (April 1996), pp. (402-411, discussion 411-412) ISSN 0278-2391

Kübler N, Michel C, Zöller J, Bill J, Mühling J \& Reuther J. (1995). Repair of human skull defects using osteoinductive bone alloimplants. J Craniomaxillofac Surg, 23, pp. (337346) ISSN 1010-5182

Leiggener C, Messo E, Thor A, Zeilhofer HF \& Hirsch JM. (2009). A selective laser sintering guide for transferring a virtual plan to real time surgery in composite mandibular reconstruction with free fibula osseous flaps. Int J Oral Maxillofac Surg, 38, 2, (February 2009), pp. (187-192)

Lethaus B, Kessler P, Boeckman R, Poort LJ \& Tolba R. (2010). Reconstruction of a maxillary defect with a fibula graft and titanium mesh using CAD/CAM techniques. Head Face Med, 19, 6, (July 2010), 16

Li WZ, Zhang MC, Li SP, Zhang LT \& Huang Y. (2009). Application of computer-aided three-dimensional skull model with rapid prototyping technique in repair of 
zygomatico-orbito-maxillary complex fracture. Int J Med Robot, 5, 2, (June 2009), pp. (158-163)

Liu XJ, Gui L, Mao C, Peng X \& Yu GY. (2009). Applying computer techniques in maxillofacial reconstruction using a fibula flap: a messenger and an evaluation method. J Craniofac Surg, 20, 2, (March 2009), pp. (372-377)

Lo LJ \& Chen YR. (2003). Three-dimensional computed tomography imaging in craniofacial surgery: morphological study and clinical applications. Chang Gung Med J, 26, 1, (January 2003), pp. (1-11) ISSN 0255-8270

Lo LJ, Chen YR, Tseng CS \& Lee MY. (2004). Computer-aided reconstruction of traumatic fronto-orbital osseous defects: aesthetic considerations. Chang Gung Med J, 27, 4, (April 2004), pp. (283-291) ISSN 0255-8270

Mainenti P, Oliveira GS, Valério JB, Daroda LS, Daroda RF, Brandão G \& Rosa LE. (2009). Ameloblastic fibro-odontosarcoma: a case report. Int J Oral Maxillofac Surg, 38, 3, (March 2009), pp. (289-292)

Mankovich NJ, Samson D, Pratt W, Lew D \& Beumer J 3rd. (1994). Surgical planning using three-dimensional imaging and computer modeling. Otolaryngol Clin North Am, 27, 5, (October 1994), pp. (875-889) ISSN 0030-6665

Matsumoto K, Nakanishi H, Seike T, Koizumi Y \& Hirabayashi S. (2003). Intracranial hemorrhage resulting from skull base fracture as a complication of Le Fort III osteotomy. J Craniofac Surg, 14, 4, (July 2003), pp. (545-548) ISSN 1049-2275

Matsuo A, Chiba H, Takahashi H, Toyoda J \& Abukawa H. (2010). Clinical application of a custom-made bioresorbable raw particulate hydroxyapatite/poly-L-lactide mesh tray for mandibular reconstruction. Odontology, 98, 1, (February 2010), pp. $(85-88)$

Mavili ME, Canter HI, Saglam-Aydinatay B, Kamaci S \& Kocadereli I. (2007). Use of threedimensional medical modeling methods for precise planning of orthognathic surgery. J Craniofac Surg, 18, 4, (July 2007), pp. (740-747) ISSN 1049-2275

Minami K, Mori Y, Tae-Geon K, Shimizu H, Ohtani M \& Yura Y. (2007). Maxillary distraction osteogenesis in cleft lip and palate patients with skeletal anchorage. Cleft Palate Craniofac J, 44, 2, (March 2007), pp. (137-141) ISSN 1055-6656

Mironov V, Kasyanov V \& Markwald RR. (2011). Organ printing: from bioprinter to organ biofabrication line. Curr Opin Biotechnol, 16, (March 2011)

Mironov V, Boland T, Trusk T, Forgacs G \& Markwald RR. (2003). Organ printing: computer-aided jet-based 3D tissue engineering. Trends Biotechnol, 21, 4, (April 2003), pp. (157-161)

Müller A, Krishnan KG, Uhl E \& Mast G. (2003). The application of rapid prototyping techniques in cranial reconstruction and preoperative planning in neurosurgery. $J$ Craniofac Surg, 14, 6, (November 2003), pp. (899-914) ISSN 1049-2275

Murray DJ, Edwards G, Mainprize JG \& Antonyshyn O. (2008). Optimizing craniofacial osteotomies: applications of haptic and rapid prototyping technology. J Oral Maxillofac Surg, 66, 8, (August 2008), pp. (1766-1772)

Nakajima T, Yoshimura Y, Nakanishi Y, Koga S \& Katada K. (1995). Integrated life-sized solid model of bone and soft tissue: application for cleft lip and palate infants. Plast Reconstr Surg, 96, 5, (October 1995), pp. (1020-1025) ISSN 0032-1052

Nout E, Cesteleyn LL, van der Wal KG, van Adrichem LN, Mathijssen IM \& Wolvius EB. (2008). Advancement of the midface, from conventional Le Fort III osteotomy to Le 
Fort III distraction: review of the literature. Int J Oral Maxillofac Surg, 37, 9, (September 2008), pp. (781-789)

Olszewski R, Tranduy K \& Reychler H. (2010). Innovative procedure for computer-assisted genioplasty: three-dimensional cephalometry, rapid prototyping model and surgical splint. Int J Oral maxillofac Surg, 39, 7, (July 2010), pp. (721-724)

Olszewski R, Tran Duy K, Raucent B, Hebda A \& Reychler H.(2008). Communicating a clinical problem to the engineers: towards a common methodology. Int J Oral Maxillofac Surg, 37, 3, (March 2008), pp. (269-274)

Olszewski R, Reychler H, Cosnard G, Denis JM, Vynckier S \& Zech F. (2008). Accuracy of three-dimensional (3D) craniofacial cephalometric landmarks on a low-dose 3D computed tomograph. Dentomaxillofac Radiol, 37, 5, (July 2008), pp. (261-267)

Olszewski R, Zech F, Cosnard G, Nicolas V, Macq B \& Reychler H. (2007). 3D CT cephalometric craniofacial analysis: Experimental validation in vitro. Int $J$ Oral Maxillofac Surg, 36, 9, (September 2007), pp. (828-833)

Ono I, Gunji H, Suda K \& Kaneko F. 1(994). Method for preparing an exact-size model using helical volume scan computed tomography. Plast Reconstr Surg, 93, 7, (June 1994), pp. (1363-1371) ISSN 0032-1052

Ortakoglu K, Akcam T, Sencimen M, Karakoc O, Ozyigit HA \& Bengi O. (2007). Osteochondroma of the mandible causing severe facial asymmetry: a case report. Oral Surg Oral Med Oral Pathol Oral Radiol Endod, 103, 5, (May 2007), pp. (e21-e28)

Papadopoulos MA, Christou PK, Christou PK, Athanasiou AE, Boettcher P, Zeilhofer HF, Sader R \& Papadopulos NA. (2002). Three-dimensional craniofacial reconstruction imaging. Oral Surg Oral Med Oral Pathol Oral Radiol Endod, 93, 4, (April 2002), pp. (382-393)

Pelo S, Tassiello S, Boniello R, Gasparini G \& Longobardi G. (2006). A new method for assessment of craniofacial malformations. J Craniofac Surg, 17, 6, (November 2006), pp. (1035-1039) ISSN 1049-2275

Poukens J, Haex J \& Riediger D. (2003). The use of rapid prototyping in the preoperative planning of distraction osteogenesis of the cranio-maxillofacial skeleton. Comput Aided Surg, 8, 3, pp. (146-154) ISSN 1092-9088

Powers DB, Edgin WA \& Tabatchnick L. (1998). Stereolithography: a historical review and indications for use in the management of trauma. J Craniomaxillofac Trauma, 4, 3, (Fall 1998), pp. (16-23)

Robiony M. (2010). Distraction osteogenesis: a method to improve facial balance in asymmetric patients. J Craniofac Surg, 21, 2, (March 2010), pp. (508-512)

Robiony M, Salvo I, Costa F, Zerman N, Bazzocchi M, Toso F, Bandera C, Filippi S, Felice M \& Politi M. (2007). Virtual reality surgical planning for maxillofacial distraction osteogenesis: the role of reverse engineering rapid prototyping and cooperative work. J Oral Maxillofac Surg, 65, 6, (June 2007), pp. (1198-1208)

Rotaru H, Baciut M, Stan H, Bran S, Chezan H, Iosif A, Tomescu M, Kim SG, Rotaru A \& Baciut G. (2006). Silicone rubber mould cast polyethylmethacrylate-hydroxyapatite plate used for repairing a large skull defect. J Craniomaxillofac Surg, 34, 4, (June 2006), pp. (242-246)

Sailer HF, Haers PE, Zollikofer CP, Warnke T, Carls FR \& Stucki P. (1998). The value of stereolithographic models for preoperative diagnosis of craniofacial deformities 
and planning of surgical corrections. Int J Oral Maxillofac Surg, 27, 5, (October 1998), pp. (327-333)

Sannomiya EK, Reis SA, Asaumi J, Silva JV, Barbara AS \& Kishi K. (2006). Clinical and radiographic presentation and preparation of the prototyping model for presurgical planning in Apert's syndrome. Dentomaxillofac Radiol, 35, 2, (March 2006), pp. (119-124)

Sannomiya EK, Silva JV, Brito AA, Saez DM, Angelieri F \& Dalben Gda S. (2008). Surgical planning for resection of an ameloblastoma and reconstruction of the mandible using a selective laser sintering 3D biomodel. Oral Surg Oral Med Oral Pathol Oral Radiol Endod, 106, 1, (July 2008), pp. (e36-40)

Santler G, Karcher H \& Ruda C. (1998). Indications and limitations of three-dimensional models in cranio-maxillofacial surgery. J Craniomaxillofac Surg, 26, 1, (February 1998), pp. (11-16) ISSN 1010-5182

Sato K, Sugawara J, Mitani H \& Kawamura H. (1998). Use of selectively colored stereolithography for diagnosis of impacted supernumerary teeth for a patient with cleidocranial dysplasia. Int J Adult Orthodon Orthognath Surg, 13, 2, pp. (163-167)

Schicho K, Figl M, Seemann R, Ewers R, Lambrecht JT, Wagner A, Watzinger F, Baumann A, Kainberger F, Fruehwald J \& Klug C. (2006). Accuracy of treatment planning based on stereolithography in computer assisted surgery. Med Phys, 33, 9, (September 2006), pp. (3408-3459) ISSN 0094-2405

Schon R, Metzger MC, Zizelmann C, Weyer N \& Schmelzeisen R. (2006). Individually preformed titanium mesh implants for a true-to-original repair of orbital fractures. Int J Oral Maxillofac Surg, 35, 11, (November 2006), pp. (990-995)

Silva DN, Gerhardt de Oliveira M, Meurer E, Meurer MI, Lopes da silva JE \& Santa-Barbara A. (2008). Dimensional error in selective laser sintering and 3D-printing of models for craniomaxillary anatomy reconstruction. J Craniomaxillofac Surg, 36, 8, (December 2008), pp. (443-449)

Sinn DP, Cillo JE Jr \& Miles BA. (2006). Stereolithography for craniofacial surgery. J Craniofac Surg, 17, 5, (September 2006), pp. (869-875) ISSN 1049-2275

Sohmura T, Kusumoto N, Otani T, Yamada S, Wakabayashi K \& Yatani H. (2009). CAD/CAM fabrication and clinical application of surgical template and bone model in oral implant surgery. Clin Oral Implants Res, 20, 1, (January 2009), pp. (87-93)

Song SJ, Choi J, Park YD, Lee JJ, Hong SY \& Sun K. (2010). A three-dimensional bioprinting system for use with a hydrogel-based biomaterial and printing parameter characterization. Artif Organs;34, 11 (November 2010), pp. (1044-1048)

Scolozzi P, Momjian A, Heuberger J, Andersen E, Broome M, Terzic A \& Jaques B. (2009). Accuracy and predictability in use of AO three-dimensionally preformed titanium mesh plates for posttraumatic orbital reconstruction: a pilot study. J Craniofac Surg, 20, 4, (July 2009), pp. (1108-1113)

Undt G, Wild K, Reuther G \& Ewers R. (2000). MRI-based stereolithographic models of the temporomandibular joint: technical innovation. J Craniomaxillofac Surg, 28, 5, (October 2000), pp. (258-263) ISSN 1010-5182

Varol A \& Basa S. (2009). The role of computer-aided 3D surgery and stereolithographic modelling for vector orientation in premaxillary and trans-sinusoidal maxillary distraction osteogenesis. Int J Med Robot, 5, 2, (June 2009), pp. (198-206) 
Vrielinck L, Politis C, Schepers S, Pauwels M \& Naert I. (2003). Image-based planning and clinical validation of zygoma and pterygoid implant placement in patients with severe bone atrophy using customized drill guides. Preliminary results from a prospective clinical follow-up study. Int J Oral Maxillofac Surg, 32, 1, (February 2003), pp. (7-14) ISSN 0901-5027

Wagner JD, Baack B, Brown GA \& Kelly J. (2004). Rapid 3-dimensional prototyping for surgical repair of maxillofacial fractures: a technical note. J Oral Maxillofac Surg, 62, 7, (July 2004), pp. (898-901)

Whitman DH \& Connaughton B. (1999). Model surgery prediction for mandibular midline distraction osteogenesis. Int J Oral Maxillofac Surg, 28, 6, (December 1999), pp. (421-423)

Williams JV \& Revington PJ. (2010). Novel use of an aerospace selective laser sintering machine for rapid prototyping of an orbital blowout fracture. Int J Oral Maxillofac Surg, 39, 2, (February 2010), pp. (182-184)

Winder J \& Bibb R. (2005). Medical rapid prototyping technologies: state of the art and current limitations for application in oral and maxillofacial surgery. J Oral Maxillofac Surg, 63, 7, (July 2005), pp. (1006-1015)

Wong TY, Fang JJ, Chung CH \& Huang JS. (2002). Restoration of the temporal defect using laser stereolithography technique. J Oral Maxillofac Surg, 60, 11, (November 2002), pp. (1374-1376) ISSN 0278-2391

Wong TY, Fang JJ, Chung CH, Huang JS \& Lee JW. (2005). Comparison of 2 methods of making surgical models for correction of facial asymmetry. J Oral Maxillofac Surg, 63, 2, (February 2005), pp. (200-208)

Worrall SF \& Christensen RW. Alloplastic reconstruction of the temporomandibular joint in treatment of craniofacial developmental or congenital anomalies: a surgical case report. Surg Technol Int, 15, pp. (291-304) ISSN 1090-3941

Wu CT, Lee ST, Chen JF, Lin KL \& Yen SH. (2008). Computer-aided design for threedimensional titanium mesh used for repairing skull base bone defect in pediatric neurofibromatosis type 1 . A novel approach combining biomodeling and neuronavigation. Pediatr Neurosurg, 44, 2, (January 2008), pp. (133-139) ISSN 1016-2291

Xia J, Ip HH, Samman N, Wang D, Kot CS, Yeung RW \& Tideman H. (2000). Computerassisted three-dimensional surgical planning and simulation: 3D virtual osteotomy. Int J Oral Maxillofac Surg 29, 1, (February 2000), pp. (11-17) ISSN 0901-5027

Yamaji KE, Gateno J, Xia JJ \& Teichgraeber JF. (2004). New internal Le Fort I distractor for the treatment of midface hypoplasia. J Craniofac Surg, 15, 1, (January 2004), 124-127 ISSN 1049-2275

Yamashita Y, Yamaguchi Y, Tsuji M, Shigematsu M \& Goto M. (2008). Mandibular reconstruction using autologous iliac bone and titanium mesh reinforced by laser welding for implant placement. Int J Oral Maxillofac Implants, 23, 6, (NovemberDecember 2008), pp. (1143-1146)

Yau YY, Arvier JF \& Barker TM. (1995). Technical note: maxillofacial biomodelling-preliminary result. Br J Radiol, 68, 809, (May 1995), pp. (519-523) ISSN 0007-1285

Zhang S, Liu X, Xu Y, Yang C, Undt G, Chen M, Haddad MS \& Yun B. (2011). Application of rapid prototyping for temporomandibular joint reconstruction. J Oral Maxillofac Surg, 69, 2, (February 2011), pp. (432-438) 
Zhou L, He L, Shang H, Liu G, Zhao J \& Liu Y. (2009). Correction of hemifacial microsomia with the help of mirror imaging and a rapid prototyping technique: case report. $\mathrm{Br} \mathrm{J}$ Oral Maxillofac Surg, 47, 6, (September 2009), pp. (486-488)

Zhou LB, Shang HT, He LS, Bo B, Liu GC, Liu YP \& Zhao JL. (2010). Accurate reconstruction of discontinuous mandible using a reverse engineering/computer-aided design/rapid prototyping technique: a preliminary clinical study. J Oral Maxillofac Surg, 68, 9, (September 2010), pp. (2115-2121)

Zizelmann C, Bucher P, Rohner D, Gellrich NC, Kokemueller H \& Hammer B. (2010). Virtual restoration of anatomic jaw relationship to obtain a precise 3D model for total joint prosthesis construction for treatment of TMJ ankylosis with open bite. Int J Oral Maxillofac Surg, 39, 10, (October 2010), pp. (1012-1015) 


\title{
A Wafer-Scale Rapid Electronic Systems Prototyping Platform
}

\author{
Walder André1, Yves Blaquière ${ }^{2}$ and Yvon Savaria ${ }^{1}$ \\ ${ }^{1}$ École Polytechnique de Montréal (EPM) \\ 2Université du Québec à Montréal (UQAM) \\ Canada
}

\section{Introduction}

Aggressive time to market requirements are key drivers of electronic system design methods. The need of rapid prototyping platforms for electronic systems is present in both academia and industry. Accordingly, several platforms have been proposed such the Zebu XXL in (Zebu) and the NanoBoard ${ }^{\mathrm{TM}}$ in (ALT, 2009). While the verification platform Zebu XXL plays the role of a system emulator capable of testing Field Programmable Gate Arrays (FPGAs) or Application Specific Integrated Circuits (ASICs) the NanoBoard platform can be considered as one of the first rapid prototyping platforms of digital systems available in the market (Eve Corp, 2011). This chapter provides an overview of that rapid prototyping platform. The technology and the wafer scale device at its core are presented, describing its key components and techniques used to supply power to the wafer, as well as challenges regarding tools required to operate this prototyping platform. The novel rapid prototyping platform for electronic systems is developed as part of a large project involving more than 40 participants from several universities and organizations. The core concept was proposed by Norman (Norman, 2006). Further information on the project with related patents and papers are provided at www.DreamWafer.com (DreamWafer, 2011).

Several technologies such as FPGAs and Field Programmable Interconnect Chips (FPICs) have been proposed (Mohsen, 1995) to support rapid prototyping of complex electronic systems and to reduce the time-to-market. With typical FPGA based rapid prototyping platforms, digital circuits to be prototyped are described using VHDL (VHSIC Hardware Description Language) or Verilog; a flow is then used to place, route and download the design into the FPGA (Ricci, 2002; Dollas, 1994). With FPICs, the area of a printed circuit board is shared between user components and the programmable interconnect chips (Mohsen, 1995). Space constraints impose stringent limits on the number of pins that may be dedicated to debugging electronic system prototypes. The novel rapid prototyping platform (Norman et al., 2008; Norman, 2006) presented in this chapter can significantly reduce the time-to-market by allowing hardware, from simple electronics to high-density electronics, micro-nano systems or system-on-chip application specific integrated circuits (ICs) to be prototyped in minutes instead of months.

The novel prototyping platform is depicted in figure 1 (a). As illustrated in figure 1(b), user's IC packages are placed on the wafer scale active surface of the WaferICTM and then the cover is closed. The package type and pins are detected, recognized and interconnected using a 
configurable routing network embedded in the active wafer. The prototype is now ready to be brought up and run. This platform can considerably reduce the time to market when developing electronic micro-nano systems. Such systems may include ICs such as memories, field programmable gate arrays (FPGAs), sensors, and processors. The proposed platform can speed up the prototyping of a wide range of electronic systems, which may be the new technology under development or simply a support to that new technology providing power, ground and communications between components specified by a system developer and the external world. The main advantage of the proposed WaferIC is not to achieve greater computational power, as proposed in some previous work on Wafer Scale Integration (WSI) (Brewer, 1989; Jalowiecky, 1990; Minges, 1989). Indeed, the main goal of the WaferIC is to provide a smart active interconnect area that can be configured in a short period of time, and that is large enough to implement a densely interconnected system, possibly composed of multiple ICs having more than 2,000 pins each.

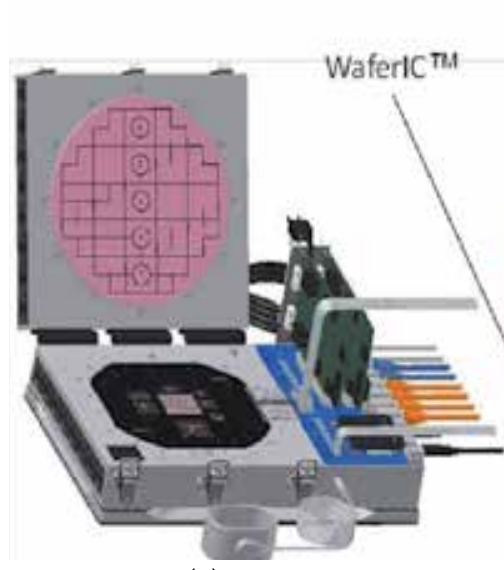

(a)

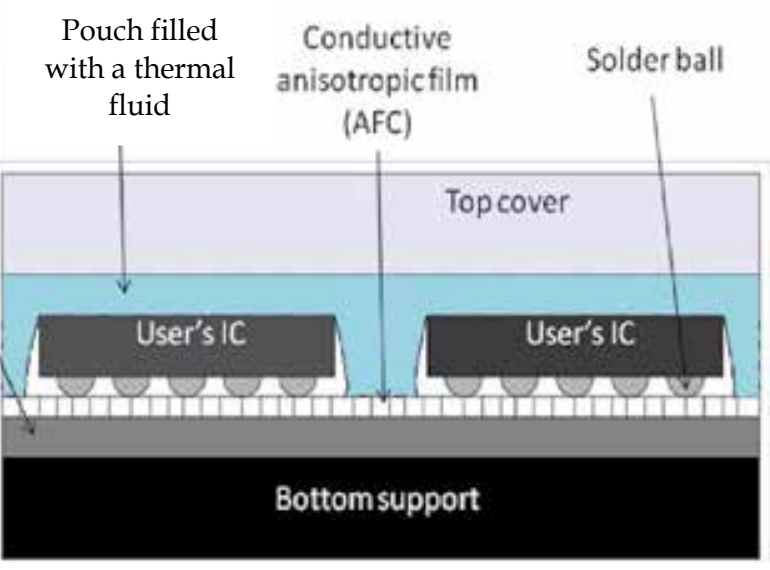

(b)

Fig. 1. WaferBoard ${ }^{\mathrm{TM}}$, a rapid prototyping platform for electronic systems.

Wafer-scale integration feasibility has been demonstrated and several design rules that contribute to make it feasible have been defined as well (Landis, 1990; Boulori, 1991; Anderson, 1992; Koren, 1998; Sharifi, 2007). Moreover fault-tolerance and yield enhancement of WSI have been addressed in (Lea, 1988; Chen, 1994; Moore, 1985) as well as fundamental design methodologies for wafer scale integration in (Hedge, 1991). In addition to wafer scale integration of electronic circuits, this concept has been extended to MEMs WSI (Shimooka, 2008; Braun, 2010).

\section{A Wafer-scale reconfigurable platform}

This section briefly summarizes the so-called WaferBoard technology. It covers (1) the physical aspects of the WaferIC, (2) its core interconnection network, (3) the power distribution strategy, (4) the proposed development software workflow and (5) the fabrication of WaferBoard prototypes.

\subsection{Physical aspects of the WaferIC}

The electronic system prototyping platform depicted in figure 1 is composed of four main building-blocks (Fig. 2): (1) the WaferIC, (2) the top PCB providing external interfaces, 
including those to a host computer, (3) the power supply, and (4) the bottom PCB to support power distribution.

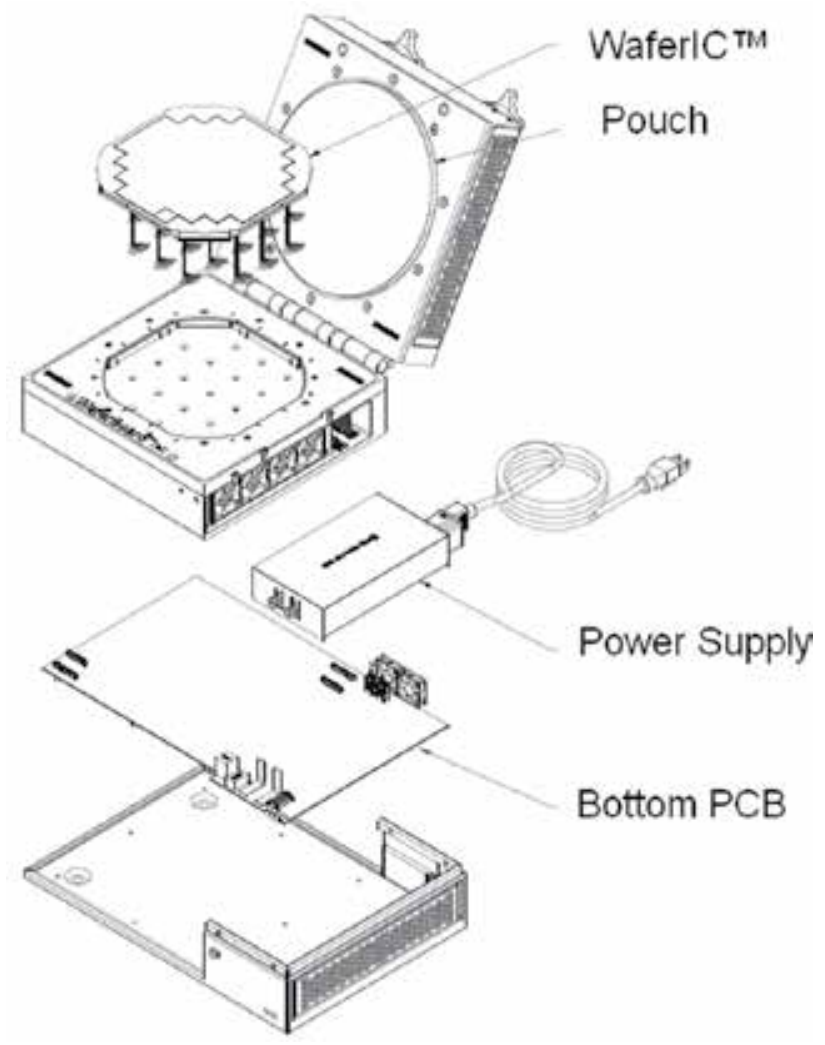

Fig. 2. WaferBoard basic building-blocks.

This platform is made possible by leveraging a full $200 \mathrm{~mm}$ diameter wafer-scale integrated circuit. Its active surface is covered with a very dense array of very fine conductive pads called NanoPads (Fig. 3). As depicted in figure 1(b), the top surface of the active wafer is covered by an anisotropic conductive film (ACF). This anisotropic Z-axis film comprises as many as 80 million conductive fibers (BtechCorp, 2011, Diop, 2010). It establishes electrical contact between the NanoPads and the balls of ICs deposited on the surface by the user. The active surface of the wafer has 1,245,184 micro-sized NanoPads. Due to the high density of NanoPads, each user's IC (uIC) solder ball will make electrical contact with several NanoPads. Each NanoPad embeds sensors that can detect electrical contact established between neighbor NanoPads through uIC balls. From these uIC contacts, a map is built and then the wafer's internal digital network structure is dynamically configured to establish connection between uIC pins according to a user specified netlist.

This platform requires innovative CAD tools. A machine learning algorithm analyzes detected UIC pins in order to recognize IC packages. The user specified netlist may be used for that task. A fast routing algorithm is used to configure on the fly the wafer-scale defecttolerant interconnect network (WaferNet ${ }^{\mathrm{TM}}$ ). Connections are established as a function of user specified constraints. As a wafer scale device normally contains a number of defects 
(one every $5 \mathrm{~cm}^{2}$ on average is assumed as a general design guideline), the router works around pre-diagnosed defects. Based on routing results, and considering the user specified netlist and constraints, the tools may propose a better IC placement. Another remarkable feature of this prototyping platform is the possibility, as part of the system debugging and validation process, to add control and observation test points at will. A workflow linking the envisioned tool set was proposed (Lepercq et al., 2009).

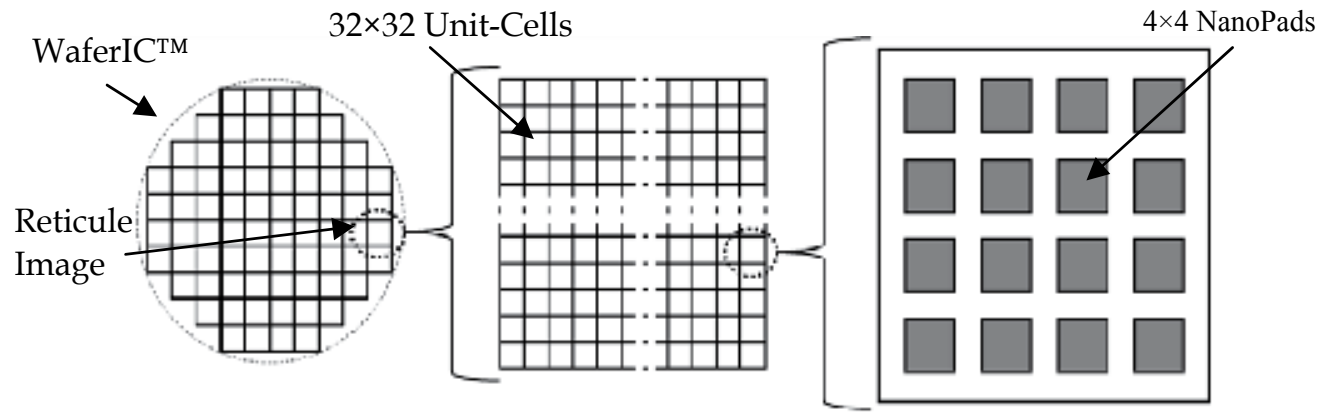

Fig. 3. The cell-based architecture of the WaferIC.

Supported uIC packages include BGA, QFP, and TSOP, to name a few. Each NanoPad can be configured as floating, digital input/output, controlled-voltage power supply or ground, according to the type of uIC pin in contact with it. An array of $4 \times 4$ NanoPads is grouped into a defect tolerant Unit-cell (Fig. 3), which can manage eventual detected and localized defects.

A regular array of $32 \times 32$ Unit-Cells is assembled to obtain a part of the integrated structure. This $32 \times 32$ Unit-Cells array fits into a single reticle image. That chip-size part of the system is photo-repeated 76 times to completely cover a $20 \mathrm{~cm}$ wafer. Using a process step called interreticle stitching, connections can be established between neighbor reticle images, as depicted in figure 3. Several metal layers can be stitched as needed. The system under development takes advantage of multiple metal layers in mature $0.18 \mu \mathrm{m}$ CMOS technologies, combined with stitching, to fabricate a wafer-wide defect-tolerant interconnection network, capable of implementing a large number of interconnections between any NanoPad combination specified by the user's netlist. The implemented WaferIC has 1,477,120 millimeter-scale programmable interconnection segments, 1,710,848 sub-millimeter programmable interconnect segments, which are configured using 22,750,000 bits of static memory.

\subsection{Unit-cell architecture of the WaferIC}

The functional architecture of the Unit-Cell is depicted in figure 4. Each Unit-Cell has complex logic and analog circuits to ensure communication between the uICs and the $4 \times 4$ array of NanoPads. From a network point of view, a Unit-Cell is an interconnection node which can route signals from any direction to any destination. Once the signal has reached the destination cell, its internal $N \times M$ crossbar can be configured in a mode that injects the signal into a NanoPad on the surface of the wafer.

To ensure fast propagation of digital signals between uICs, an interconnect chain implemented as depicted in figure 5 is used. This interconnect chain starts from the input/output (IO) stage and continues through interconnect stages composed of active repeaters and crossbars. The crossbars can steer signals in any direction possible from one 


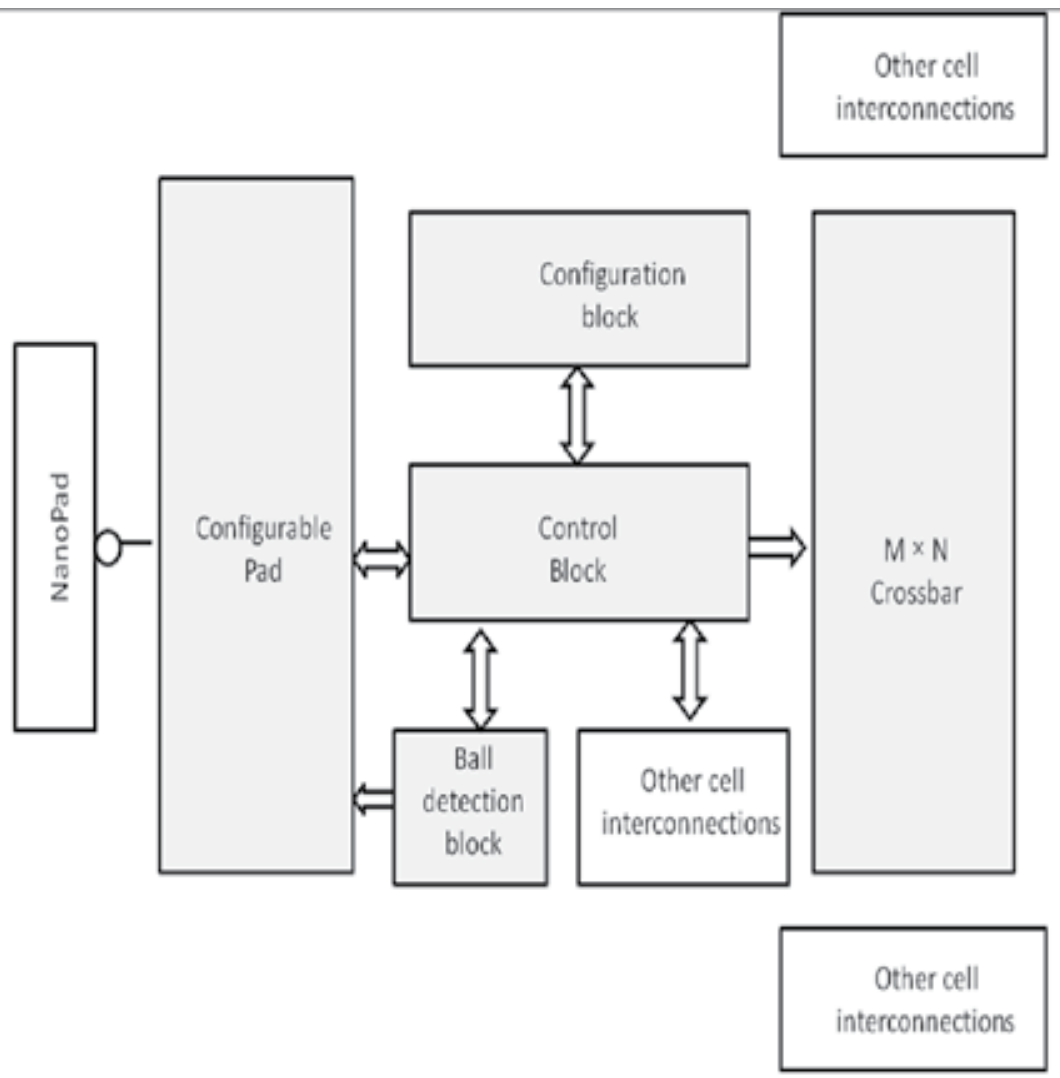

Fig. 4. Architecture of a Unit-Cell.

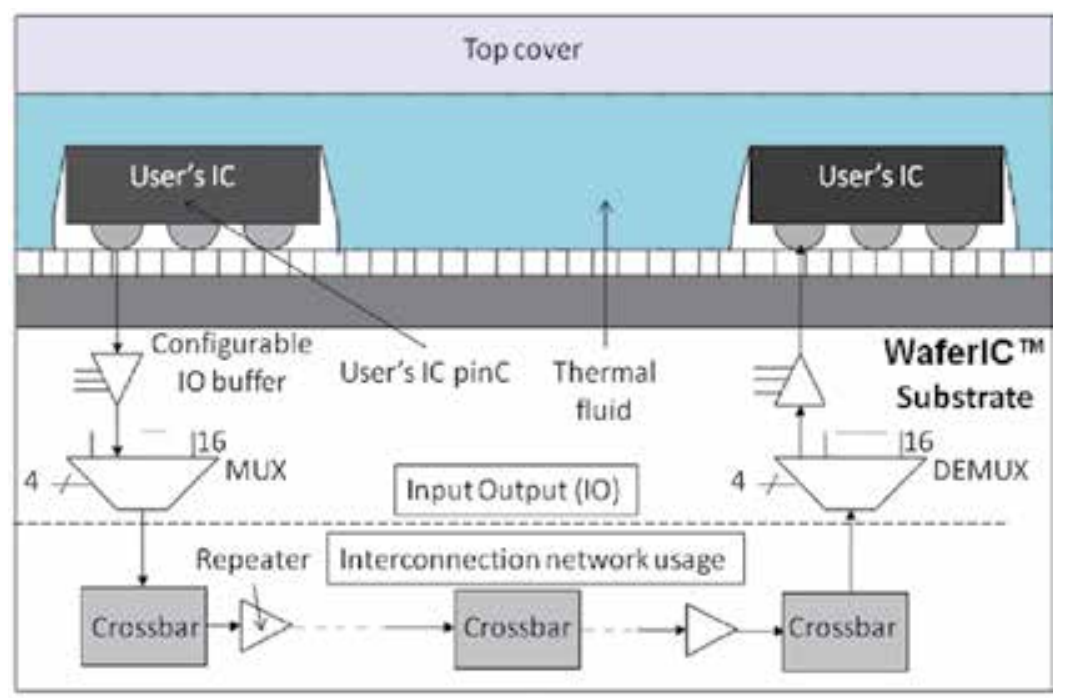

Fig. 5. Overview of an interconnect chain embedded in the WaferIC. 
NanoPad to another. Figure 5 presents an example of one possible route through the interconnect chain. The interconnect chain is made of three main elements. The first element is the input/output (IO). It is made of configurable IO buffers set up at each NanoPad, which allow the WaferIC to supply a variety of voltage levels in order to support different types of uICs. To maintain signal integrity, an interconnect chain is implemented with the second element, a set of repeaters inserted at regular interval. The third element is the crossbar, used to route the signal in different directions as needed in the WaferNet. More details about the crossbar architecture are given in section 2.3.

\subsection{WaferNet, a defect tolerant interconnection network}

The WaferNet was designed to support most standard uICs, including processors, FPGAs, and memories regardless of the packages' pin-count and density. The large interconnect density offered by the multiple metal layers available in mature CMOS technologies enables the WaferNet to support point-to-point, point-to-multipoint and busses. The WaferNet is a scalable multi-dimensional mesh network, which can route a large number of connections without conflict as required by dense PCBs.

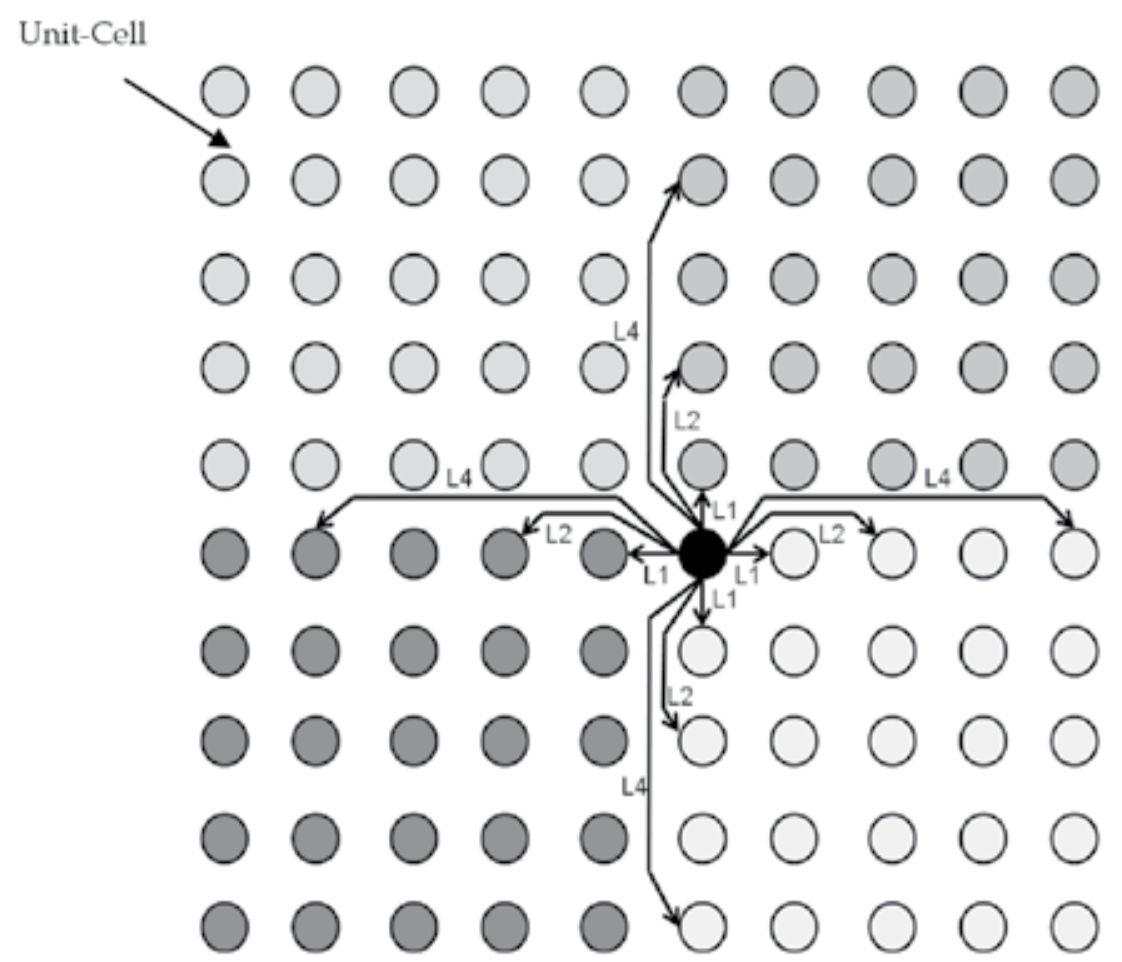

Fig. 6. Example of interconnections with other cells for the Unit-Cell colored in black where $\mathrm{L}$ stands for the length of links measured as a distance in Unit-Cells.

Contacts between adjacent interconnections can be achieved using a two dimensional neighbor-to-neighbor mesh, but this approach becomes inefficient in the case of distant interconnections. Thus, in WaferNet, each network node is linked to $K$ others in each physical direction (N-S-E-W). Figure 6 shows the progressive increase in length of $K$ links in 
a given direction. Indeed, the length of links grows according to a geometric series. As depicted in figure 6 , each node follows the same pattern of connection; when $K=3$, for example, the node depicted in black is connected to the 1st, 2nd and 4th neighboring nodes in each direction (N-S-E-W). The value of $K$ is a key design parameter that influences complexity, interconnect density and defect tolerance. Indeed, increasing $K$ contributes to improve defect tolerance as each crossbar supports more links than the minimum required. Moreover a dense NanoPad array in which each component ball intersects a plurality of NanoPads contributes to defect tolerance.

The WaferNet has a regular architecture based on a Unit-Cell elementary tile in order to meet the wafer-scale integration constraints. The $N \times M$ crossbar that is part of each cell can route its $4 \times K$ ( 4 directions) inward signals to its $4 \times K$ outward signals. Each Unit-Cell is designed to handle up to B uIC balls. A regular uIC ball implies one or more crossbar input or output. By contrast, each bi-directional uIC ball consumes two crossbar outputs. One of them is used to control the signal direction. The size of the crossbar $(N \times M)$ is therefore related to the number of uIC balls supported by a cell and its neighbor (as needed to support defect tolerance), where $N \geq 4 \times K+B$ and $M \geq 4 \times K+2 B$. In general, increasing $M$ or $N$ makes the network more robust to faults or defects.

\subsection{Crossbar implementations}

The crossbar required by the network architecture utilizes a large part of the Unit-Cell logic. To illustrate that complexity, the internal architecture of a crossbar for a given variable $K$, $B=2$ is shown in figure 7 . Three approaches were considered for crossbar implementation: (1) crosspoint-based crossbar, (2) tri-state based crossbar, and (3) switch-based crossbar. All

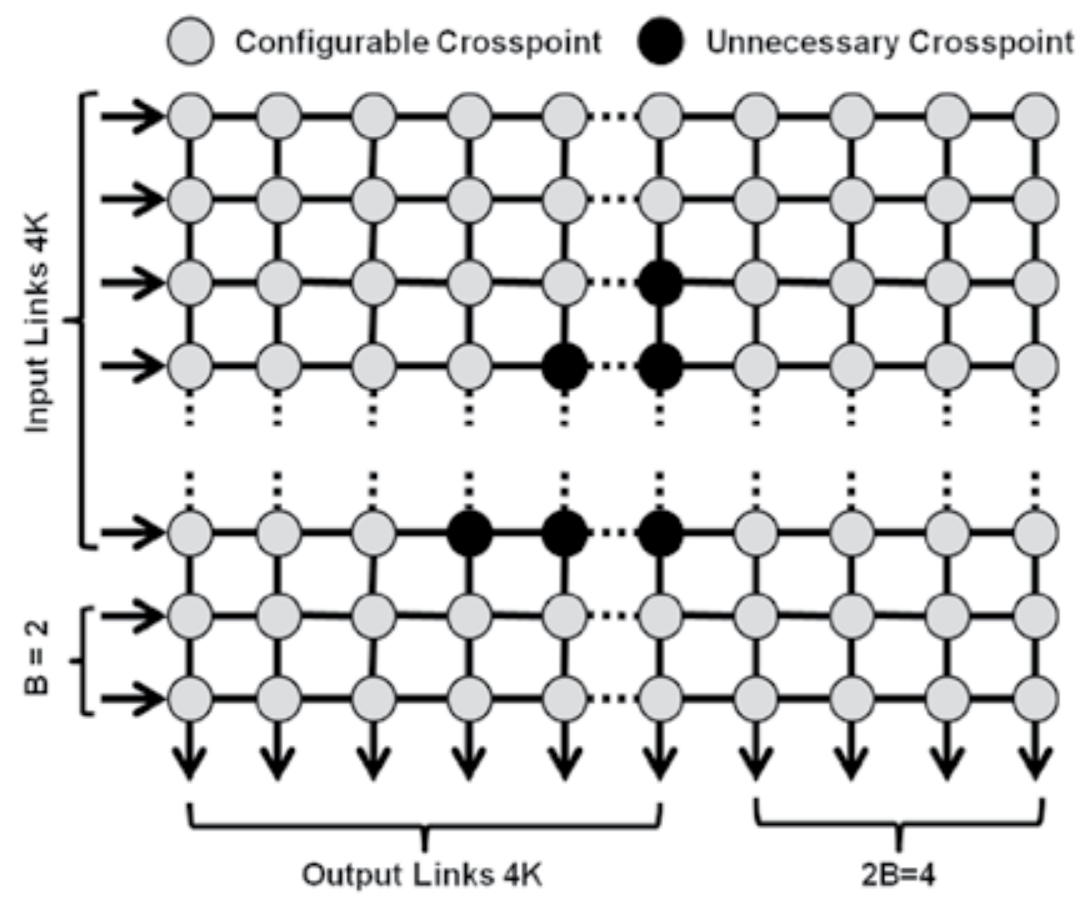

Fig. 7. Crossbar implementation using crosspoints. 
three techniques were implemented at the circuit level and these implementations were compared in order to find the best solution for the WaferNet.

The first solution implements crossbars with crosspoints. This crosspoint-based crossbar (Fig. 7) uses a single memory element per crosspoint that is responsible for propagating or not propagating some signals to any given column. In figure 7, several crosspoints are labeled as unnecessary from a functional standpoint. However, some implementations may keep these crosspoints for layout regularity. Another solution is to use pass-transistor switches. Such switches (see Fig. 8 (b)) are notably used to implement FPGA crosspoints. While pass transistor switches are bi-directional, their implementation requires special care due to the threshold voltage losses they induce. A solution to alleviate threshold voltage losses is to use transmission gate switches at the cost of more silicon area and parasitic capacitances. The advantage of using pass transistors or transmission gates to implement switch based-crosspoints may be offset by the relatively large resistivity that accumulates as a signal passes through several layers of switches. A common means to combat this effect is to regenerate the signals by inserting buffers at regular intervals. To avoid unintentional shorts on column lines, the crosspoints and their related configuration sequence must be carefully designed. Shorts could generate high peak currents that would stress the components. This could reduce significantly the reliability and product life of the WaferBoard, and increase its power consumption.

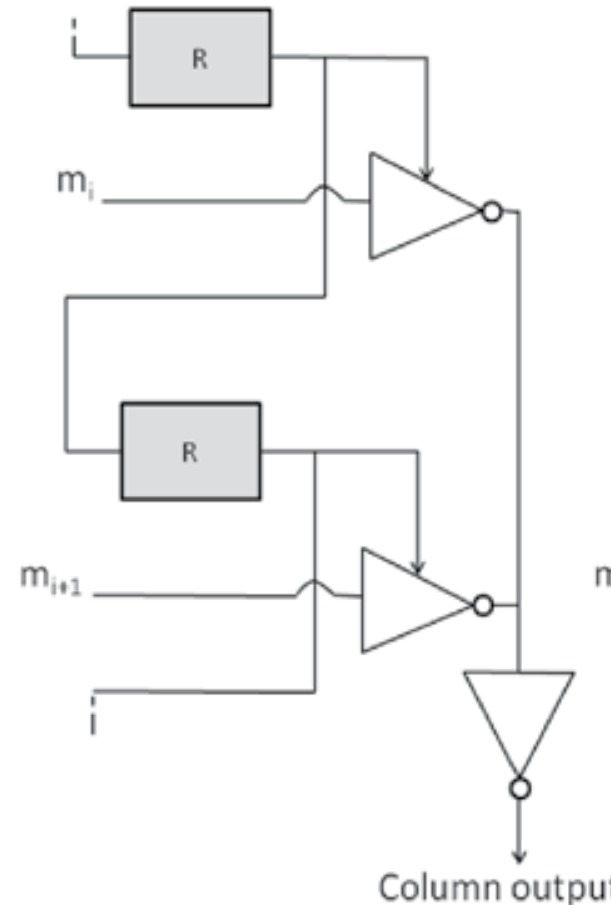

(a)

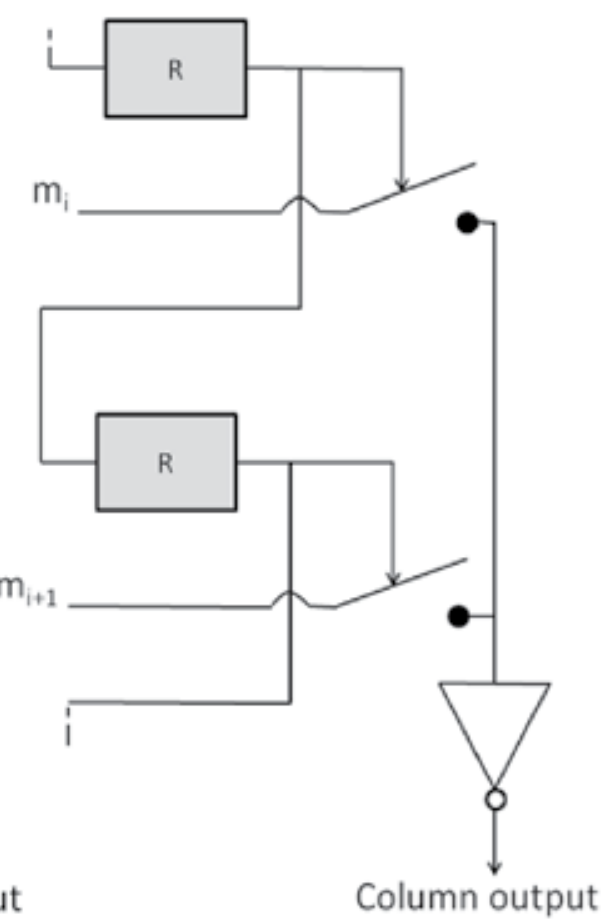

(b)

Fig. 8. Examples of crossbar implementations (a) Tri-state based crosspoints, (b) switchbased crosspoints. 
Another approach for implementing crossbars is to use multiplexers. To implement a full $N \times M$ crossbar, $M N$-input multiplexers (one per column) are needed. A total of $N \log _{2} \mathrm{M}$ configuration memory elements are required while $\log _{2} \mathrm{M}$ memory elements are required for each column multiplexer. An advantage of using multiplexers to implement the crossbar is the manner in which short-circuits are prevented compared to crosspoint-based crossbars. Moreover, the number of memory elements, especially for large $K$, is considerably reduced with this approach.

Several crossbars were designed according to these various styles for $K=7$, and the resulting implementations were compared. Table 1 summarizes the logic area extracted from RTL synthesis for four implementations. Some results are also reported for partial pruned crossbars (see figure 7). In figure 9 that shows internal details of a cell , the incoming links in the N-E-S-W physical directions are called CI0,[0..6], CI1,[0..6], CI2,[0..6], and CI3,[0..6] respectively.

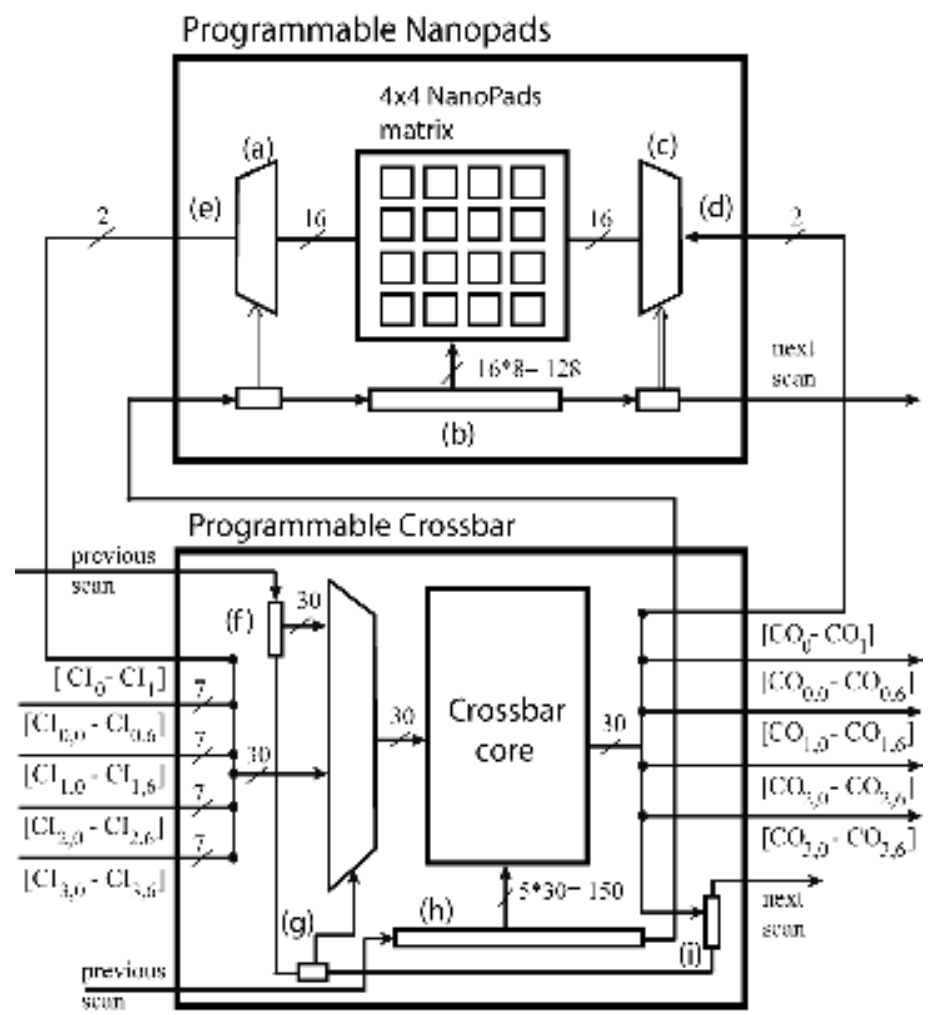

Fig. 9. Unit-Cell structure with its two internal scan chains : configuration scan ((h) and (b)), test and diagnosis scan ((f), (g) and (i)). (Basile-Bellavance, Blaquière \& Savaria, 2009).

Our results, reported in Table 1, demonstrate that the multiplexer based crossbar occupies less area than the tri-state based crossbar. This is due to the fact that the crossbar complexity grows as $O\left(N \log _{2} M\right)$, where $N$ is the number of inputs and $M$ is the number of outputs. This is to be compared with a linear growth $O(N \times M)$ in total area with crosspoint-based crossbars. 


\begin{tabular}{|c|c|c|c|}
\hline Crossbar type & Total area $\left(\mu \mathrm{m}^{2}\right)$ & Memory area $\left(\mu \mathrm{m}^{2}\right)$ & $\begin{array}{c}\text { Memory proportion } \\
(\%)\end{array}$ \\
\hline Full Tri-state based & 125400 & 54240 & 43.3 \\
\hline Partial tri-state based & 91521 & 41764 & 45.6 \\
\hline Full mux based & 45050 & 9040 & 20.2 \\
\hline Partial mux based & 43538 & 9040 & 20.8 \\
\hline
\end{tabular}

Table 1. Synthesis results of crossbar implementations for $K=7$.

Based on these results, the first full wafer prototype that was produced use $K=6$ to fully take advantage of the interconnect density that multi-metal-layer lithography provides with the adopted standard $0.18 \mu \mathrm{m}$ CMOS process (logic and interconnect complexities were too high to fit in the available area when $K=7$ ). Thus the implemented crossbar includes 6 incoming 6 links and outgoing links in each direction (Fig. 9). The Unit-Cell includes also internal scan chains that are used to configure the crossbar and to get access to crossbar I/Os using a protocol similar to that found in the IEEE standard 1149.1 (referred as JTAG here) (Parker, 1998). The Unit-Cell internal scan chains can be daisy chained and accessed through the standard four JTAG ports, one set per reticle image (TDI, TDO, TCK and TMS).

\subsection{WaferBoard power distribution}

The first implemented WaferIC has 4,864 regularly distributed Through Silicon Vias (TSVs). The TSV technology (Motoyoshi, 2009; Rimskog, 2008) is a mature technology that allows integration of 3D IC or 3D packaging (Papanikolaou, 2011; Lau, 2009; Mitsumasa, 2009). Power and ground must be distributed to uICs through these TSVs with embedded programmable regulators within the NanoPads to ensure proper power supply integrity. Effectively, decoupling capacitors cannot be placed on the top side of the WaferIC and integration of sufficient capacitance is impossible due to silicon area constraints imposed by the WaferIC. Consequently, the chosen architecture needs to rapidly deliver a regulated

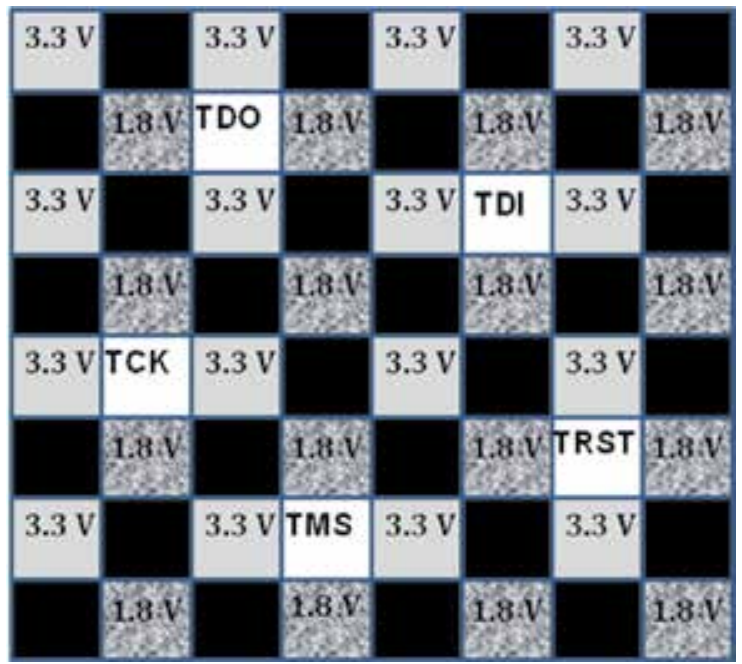

Fig. 10. TSV distribution over the reticle image. 
voltage without the benefit of adding capacitors. The voltage regulator in each NanoPad is designed to provide a range of standard VDD levels such as 1.0, 1.5, 1.8, 2 and $2.5 \mathrm{~V}$. Each reticle image has an array of $8 \times 8$ TSVs as depicted in Figure 10, which are used to supply ground (27 TSVs), and two levels of power $1.8 \mathrm{~V}$ and $3.3 \mathrm{~V}$ (16 TSVs each). A set of 5 TSVs is reserved for JTAG signals to configure the device. Each NanoPad can deliver up to $100 \mathrm{~mA}$ to a uIC ball load. The power delivered to the wafer through TSVs comes from an array of independent power sources that can supply $15 \mathrm{~A}$ each, and a total of $315 \mathrm{~A}$ to the WaferIC. The WaferIC is made of analog and digital parts. The analog part comprises I/O buffers, one per NanoPad, and distributed power regulators, responsible to supply power to uICs. The digital portion consists of the embedded programmable interconnect network and of the defect tolerant scan chains used for configuration purposes.

Figure 11 presents the WaferIC power-supply tree structure with a single power-source at its root and a distributed set of regulators that constitute slave stage embedded in the NanoPads at its leaves. These regulators, very close to uIC pins, are designed to respond rapidly to uICs power demands. The WaferIC receives power through modules called PowerBlocks, each of which feeds several reticle images from the back side through TSVs. Discrete regulators providing ground, $1.8 \mathrm{~V}$, and $3.3 \mathrm{~V}$ are embedded in each PowerBlock.

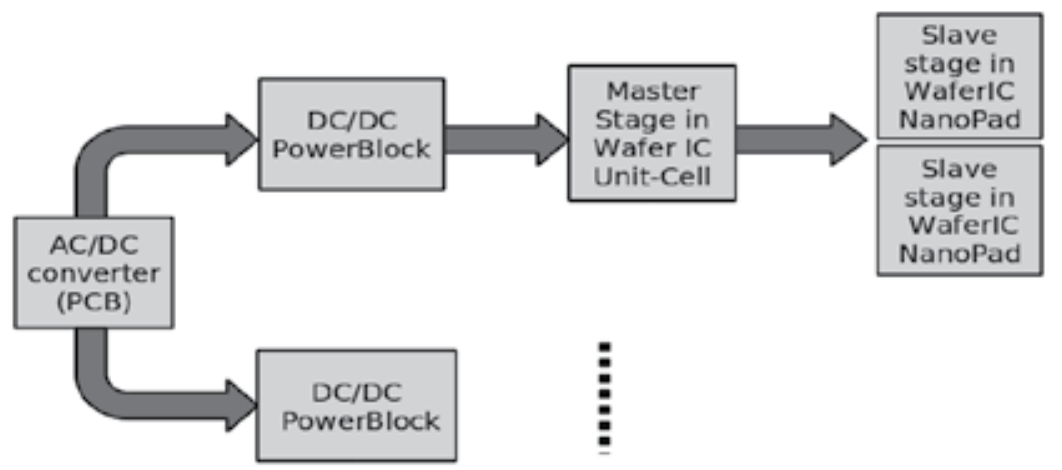

Fig. 11. WaferIC power-supply tree structure.

Each voltage reference circuit embedded in the NanoPads is structured as depicted in figure 12. These regulators could have a substantial quiescent current. In this case, the total quiescent current consumed by the large number of voltage references embedded in the WaferIC could significantly contribute to the power consumption of the wafer-scale circuit. The use of a master-slave architecture helps in reducing the power consumption by a factor of 16 . For example, the WaferIC contains $~ 1.3$ million NanoPads; if each of them consumed 100 $\mu \mathrm{A}$, this would result in a contribution to total current of $130 \mathrm{~A}$, which is not acceptable. The proposed solution is to share low-power circuitries in the master stage in a Unit-Cell. This solution considerably reduces the power consumption of the whole wafer-scale system.

The topology of the embedded regulators in the WaferIC is such that each Unit-Cell contains one master stage and 16 slave stages (Fig. 12). There is only one VSET reference voltage node for the $4 \times 4$ NanoPads within the same Unit-Cell. The main function of the master stage is to set a stable control signal VSET for all the slave stages. A programmable voltage reference is followed by an Operational Transconductance Amplifier (OTA) in its feedback loop, which controls the output of a buffer, followed by a fast load regulation module. The Slave stage is controlled by VSET and provides a stable output to drive the Nanopads. The 


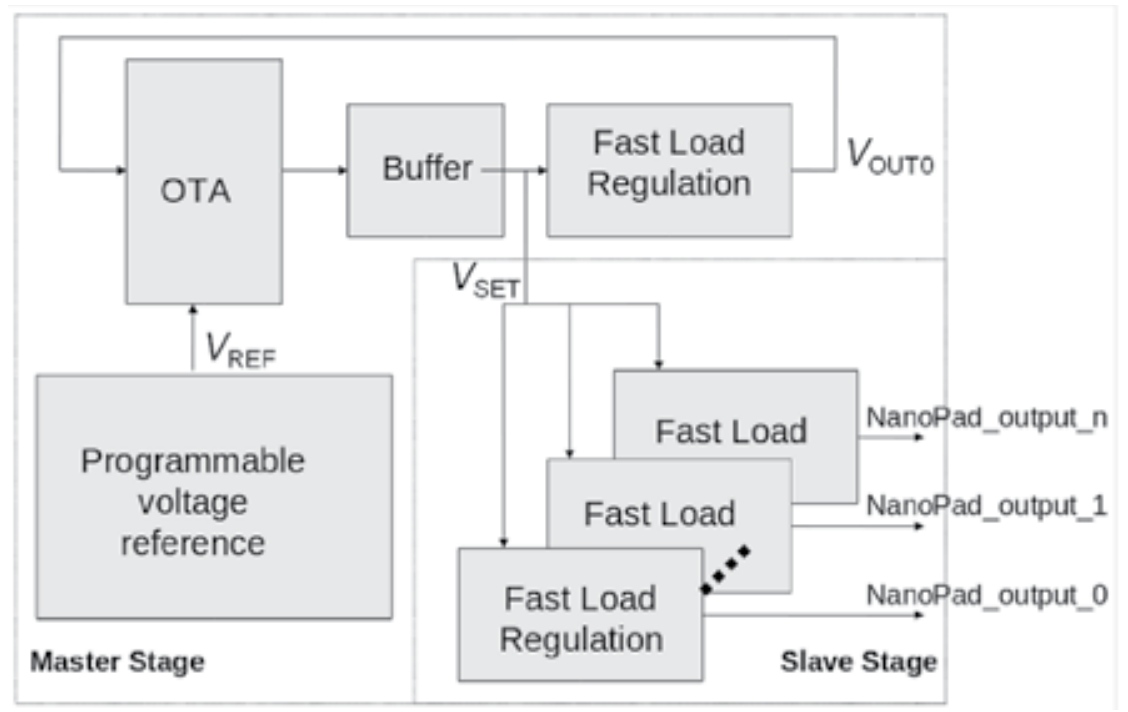

Fig. 12. Hierarchical topology of the embedded regulators in the WaferIC Unit-Cell [Laflamme-Mayer et al., 2010].

in-situ distributed part of the regulator has a low power quiescent mode necessary due to the fact that a full wafer device contains many copies of this circuit, most of which are normally unused.

\subsection{WaferConnect, a software tool for the WaferBoard}

The rapid prototyping platform is supported by a suite of software tools called WaferConnect. This toolbox supports a workflow defined as proposed by the Workflow Management Coalition (WfMC, 1999). This model has been extensively used for building general workflows (Geogakopoulos \& Hornick, 1995) as well as computer-aided design tools (Huang \& Liao, 2007; Trappey et al., 2007). The proposed workflow has a total of nine steps (see figure 13). In step 1, the user puts the required ICs on the active WaferIC surface. Step (2) is the boot-up and diagnosis process step. At this stage the wafer is automatically powered-up and the whole wafer is scanned to extract a defect map. That information is forwarded to other tools designed to ensure that the system will not make use of these defective resources. In step (3), a map of connected NanoPads (NanoPads that are in contact with a uIC package balls) is extracted. About one million contact sensors embedded into the WaferIC are used to extract the contact map based on shorts between adjacent NanoPads created by a uIC solder ball.

This contact map is then used by the UIC package pin/netlist recognition process (4). The user provides a netlist and constraints in step (5). This netlist contains information that defines interconnections required between uICs deposited on the surface of the WaferIC. It can be manually defined or preferably read from a standard netlist file (e.g. EDIF, GRB, Protel). The netlist and its specified constraints are used in step (6) to compute feasible routes for each net. The constraints supported by the proposed system are somewhat similar to those supported by PCB routers. However, in our prototyping system, routes must be assigned to predefined wire segments of a defect-tolerant multi-dimensional mesh interconnection network described in (Lepercq, 2008). For instance, bus latency (timing constraints), skew, and bandwidth are 
other routing constraints. In step 7 the network is configured using suitable drivers. The user then debugs his systems as part of step (8) with the support of a set of debugging tools that will be defined as part of our future research. Finally, in step (9) of the workflow, reports are generated to confirm compliance with the specification.

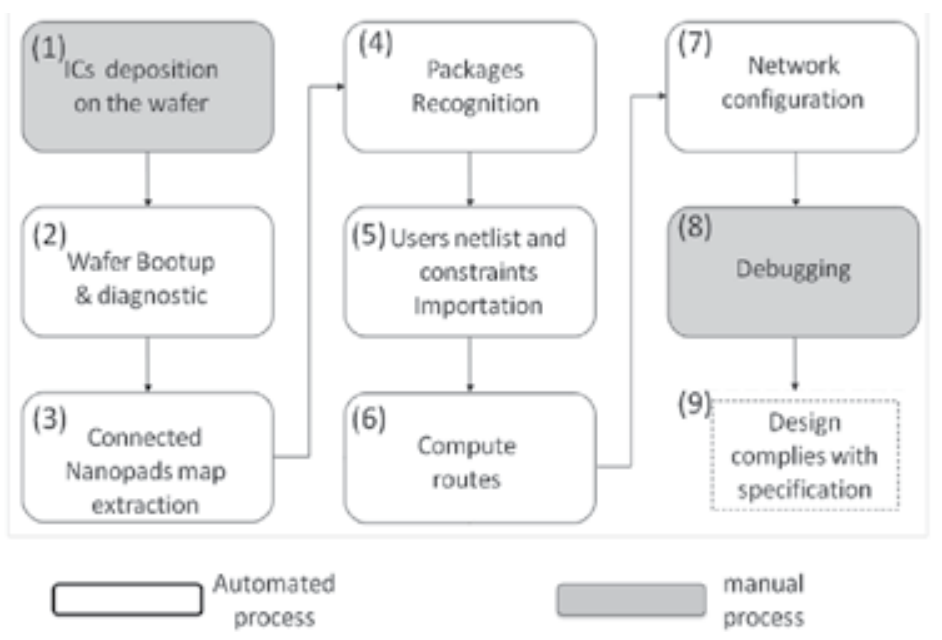

Fig. 13. The WaferBoard workflow: from WaferIC characterization up to a working electronic system prototype.

Only two critical tools of the work flow are described here. The first is the package recognition tool and the second is the routing tool for one type of constraints. Prior to package recognition of the user's IC deposited on the WaferIC surface, all ball positions of the user's IC are estimated. The scale-space theory (Babaud, 1986) is used in the package recognition algorithm. The position and size of each ball are estimated from the set of NanoPads that are in contact with that ball. This problem is similar to the geometric problem of finding the smallest bounding circle for $\mathrm{n}$ points in a two dimensional space (Arvo, 1991). The package recognition is possible whenever all balls are connected to at least two NanoPads. After estimating the balls position, a package orientation is extracted based on two IC characteristics. Finally, the package recognition is completed by searching in a library of known IC packages. The implemented algorithm is based on (Tuytelaars \& Mikolajezyk, 2008).

The second critical tool is the routing algorithm. In order to find the shortest path between connected NanoPads, the interconnection network is modeled as a dense graph $G(V, E)$ with $\# E>>\# V$, where $E$ is the set of WaferNet segments, $V$ its set of cells and \#S is the cardinality of respective set $S$. Moreover two heuristic approaches are proposed to manage conflicts. The first heuristic approach is called In-Order, which routes each net of the user netlist incrementally, while the second heuristic approach computes a route independently for each net, with the assumption of an ideal and fully functional WaferNet. A defective interconnect resource is treated as a resource that was previously assigned to another net.

\subsection{Prototypes of the WaferBoard ${ }^{\mathrm{TM}}$ : three implemented test chips}

A first test chip has been implemented as a proof of concept. This test chip embeds an array of $3 \times 3$ Unit-Cells of the WaferIC (Fig. 14). It is a miniature version of the WaferIC, where most of the functionality of the WaferIC was validated without the need to have an 
expensive prototype including an entire silicon wafer. Each cell contains $4 \times 4$ NanoPads, giving a checkerboard of $12 \times 12$ NanoPads. Among the 144 NanoPads, only 22 were accessible for testing. The others were not accessible as they were not bounded to the output pins of the chip. Five control signals allow a JTAG scan chain to program that test chip, and two voltage levels $(3.3 \mathrm{~V}$ and $1.8 \mathrm{~V})$ were needed to provide power to the user's integrated circuit. The analog block section and programmable drivers section of each NanoPad were validated. The digital part of the integrated circuit was implemented with standard cells.

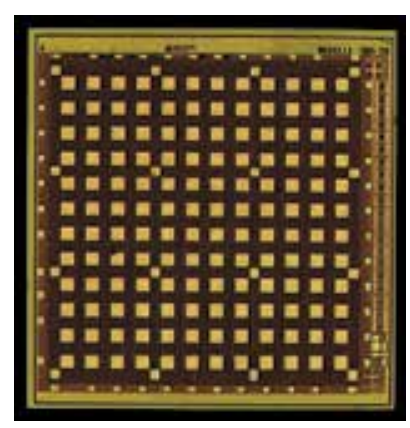

Fig. 14. First test chip silicon die.

A test and measurement protocol has been developed: all signals transmitted to the circuit under test were recorded by a logic analyzer that provides all the information necessary to diagnose failures. The digital tests were performed by applying test vectors and by measuring the output response. To validate the behavior of the WaferNet, signals were injected into NanoPads accessible from the pins of the circuit under test. The signals injected into NanoPads were generated by programmable waveform generators. These signals entering and leaving the WaferNet were observed using digital oscilloscopes. Figure 15 shows the results of this test on the oscilloscope. The current passing through the power pins (VDD) and that passing through the ground (GND) were measured, as well as the current passing through the NanoPad VDD3.3V connection.

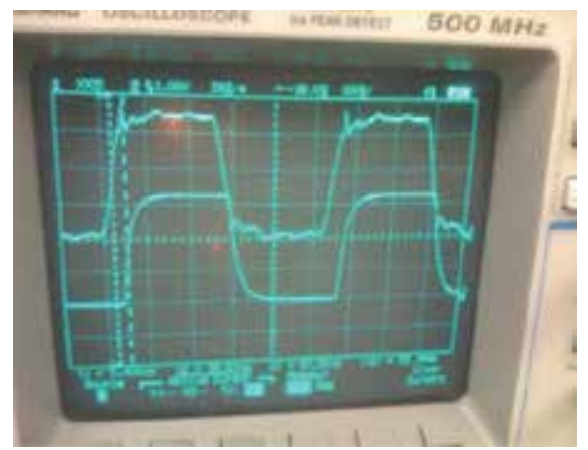

Fig. 15. Signal propagation in the WaferNet of the first test chip.

The results obtained with the first test Chip were very significant for the WaferBoard proof of concept. The positive results obtained demonstrated clearly that it is possible to create a circuit embedding the WaferNet configurable network. 
A second test chip (Fig. 16) was also fabricated using the standard CMOS technology in which a novel architecture was implemented to support fast differential signaling. This is particularly useful when uICs have some fast differential IOs. According to post layout simulations, the proposed architecture supports a data rate of $2.5 \mathrm{Gbps}$ with $200 \mathrm{mV}$ of voltage swing. More details on the internal architecture can be found in (Valorge, Blaquière \& Savaria, 2010).

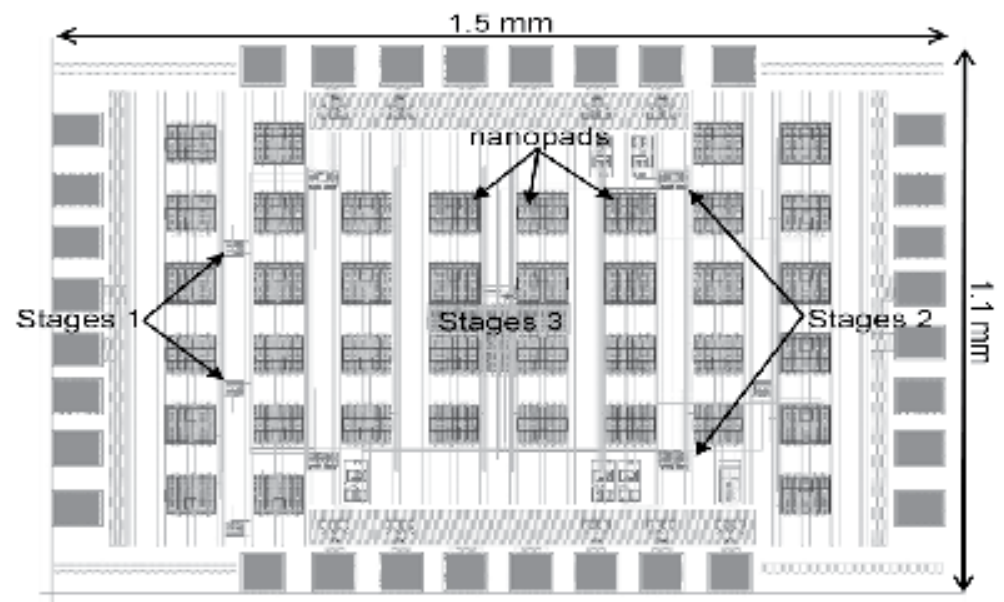

Fig. 16. Second test chip silicon die layout (Valorge, Blaquière \& Savaria, 2010).

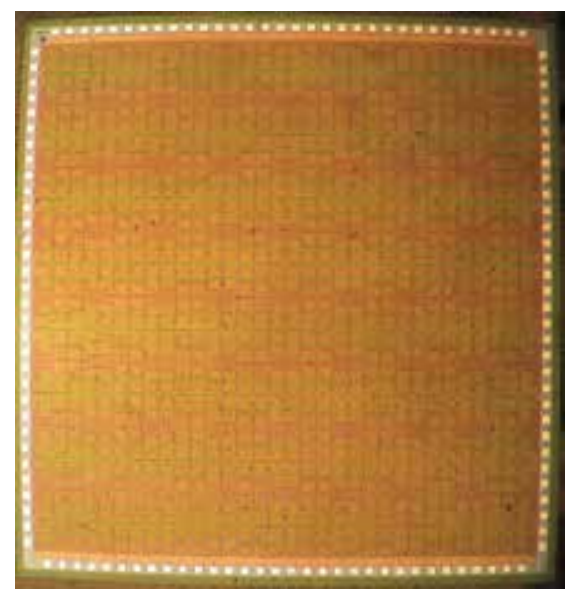

Fig. 17. Third test chip silicon die.

As part of this project, a third test chip was fabricated. It has an area of approximately $1 / 10,000$ th that of a full $200 \mathrm{~mm}$ WaferIC. It was also fabricated with a standard 6-metal layer $0.18 \mu \mathrm{m}$ CMOS technology to prove the proposed concepts. This third test chip (Fig. 17) was created to further test a more elaborate version of the programmable pad embedded inside each NanoPad. A beta-multiplier architecture was used in the proposed programmable voltage reference circuit to provide a reference current IREF that ideally depends only on transistor parameters. This current is duplicated into a Programmable Reference Array (PRA). The test results show that the NanoPad can be configured to one the following standard levels: $1.0 \mathrm{~V}, 1.5 \mathrm{~V}, 1.8 \mathrm{~V}, 2.0 \mathrm{~V}, 2.5 \mathrm{~V}$ 
or $3.3 \mathrm{~V}$. The programmable voltage reference, when it is in a sleep mode, showed an ultra-low quiescent power consumption of $0.66 \mathrm{nW}$ from a $3.3 \mathrm{~V}$ supply.

\section{Conclusion}

In this chapter, we presented an innovative rapid prototyping platform, developed to facilitate and accelerate the development of a wide range of electronic systems made of several integrated circuits. We summarized the main building blocks that comprise the proposed platform. Finally, we briefly exposed the challenge regarding CAD tools needed to make this platform functional and user-friendly. The goal of this project is to demonstrate an easy to use system that allows rapid configuration of functional systems from handplaced packaged components deposited over an active surface that embeds a high capacity configurable routing network, as well as means to test, diagnose and control the system, and to supply power to user ICs at the needed voltage, level of current, and integrity.

The first set of full functionality stitched wafers have been fabricated at a CMOS fab and are now processed to create the required through silicon vias. On-going research activities should lead to a functional prototype system in 2011-2012. Its successful completion leverages leading edge technology and skills of four different companies (Tower Semiconductor, Allvia, Sound Design Technologies and Btech Corp) providing unique and compatible technologies.

\section{Acknowledgements}

The authors would like to acknowledge Gestion TechnoCap Inc., DreamWafer Division, which provided technical support and contributed Intellectual Property. Tower Semiconductor is acknowledged for providing wafer fabrication and Allvia for TSV processing. Special thanks to Gestion TechnoCap Inc., DreamWafer Division, NSERC, Prompt, Precarn Inc., MITACS, FQRNT, and CMC are also acknowledged for their financial support. The DreamWafer ${ }^{\mathrm{TM}}$ concept originates from Richard Norman. More than 40 persons have participated to the project. The authors would like to recognize Richard Prytula for making this project possible, Olivier Valorge for his influential contribution and Philippe Dauphin for creating the very detailed 3D system models illustrating the system under development.

\section{References}

Altium, (April 29). Available from http://www.altium.com/products/the-nanoboard/en/the-nanoboard_home.cfm

Anderson, A. H.; Raffel, J. I.; \& Wyatt, P. W. (1992). Wafer Scale Integration Using Restructurable VLSI, In Journal Computer - Special Issue on Wafer Scale Integration, Vol. 25, Issue 4, Apr. 1992

Arvo, J. (1991). An Easy Bounding Circle, Jon Rokne, Graphics Gems II, Academic Press, Boston, U.S.A., 1991

Babaud, J.; Witkin, A. P.; Baudin M.; \& Duda, R. O. (1986). Uniqueness of the Gaussian Kernel for Scale-Sace Filtering, in IEEE Trans. Pattern Anal. Mach. Intel.; pp. 26-33, 1986

Basile-Bellavance, Y.; Blaquière, Y. \& Savaria, Y. (2009). Fault Diagnostic Methodology for the WaferNet Interconnection Network, IEEE/NEWCAS International Conference, Toulouse, France, June $28^{\text {th }}$-July $1^{\text {st }}, 2009$

Boulori, H. (1991). A Structured Approach to Architectural Yield Optimization for WSI, In IEE Colloquium on Wafer Scale Integration, 1991, pp. 6/1 -6/3, London, UK, 28 May 1991 
Braun, S. (2010). Wafer Level Heterogeneous Integration of MEMS Actuators, ISBN 978-917415-493-1, ISSN 1653-5146, TRITA-EE 2010:002

Brewer, J. E. (1989). Promises and Pitfalss of WSI, In Wafer Scale Integration, Kluwer, pp.1-29, 1989

BtechCorp (April 2011). Available from http:/ / www.btechcorp.com

Chen, Y. Y. \& Upadhyaya, S. J. (1994). Modeling the Reliability of a Class of Fault-Tolerant VLSI/WSI Systems Based on Multiple Level Redundancy, In IEEE Transactions on Computers, Vol. 43, Issues 6, Jun 1994

Diop, M. D.; Radji, M.; André, W.; Blaquière, Y.; Izquierdo, R. \& Hamoui, A. A. (2010). Electrical Characterization of Annular Through Silicon Via for a Reconfigurable Wafer Sized Circuit Board, IEEE 19th Conference on Electrical Performance of Electronic Packaging and Systems (EPEPS), pp. 1-17, Austin, TX, USA, 25-27 Oct. 2010

Dollas, A.; Ward, B.; \& Bancock, J. D. S. (1994). FPGA based Low cost Reusable Module for the Rapid Prototyping of Subsystems, In Lecture Notes in Computer Science, 1994, Vol. 849/1994, pp. 259-270, DOI: 10.1007/3-540-58419-6_96

DreamWafer, (April 2011). Available from http://www.dreamwafer.com/Research.html http:/ / www.dreamwafer.com/Patents.pdf

Eve Corp, (April 2011). Available from http://www.eve-team.com/products/products.html

Georgakopoulos, D. \& Hornick, M. (1995). An Overview of Workflow Management: From process Modeling to Workflow Automation Infrastructure, in Special issue on software support for work flow management, vol. 3, issue 2, pp. 119-153, 1995

Huang, C. J. \& Liao L.M. (2007). An Intelligent Agent-base Collaborative Workflow for Enterprise PCB Product Design," IEEE International Conference on Industrial Engineering and Engineering Management, pp. 189-193, Dec. 2007

Huang, C. J.; Trappey; C. V. \& Ku, C. C. (2007)., The Design of a JADE-based Autonomous Workflow Management System for Collaborative IC Design, in the 11 International Conference on CSCW in Design, Melbourne, Australia, Apr. 26 - 28, 2007

Jalowiecki, I. P. \& Hedge, S. J. (1990). The WASP2 WSI Massively Parallel Processor Demonstratiors, IEEE Custom Integrated Circuis Conference, 1990

Jalowiecki, I. P.; Hedge, S. J.; \& Lea, R. M. (1991). A Practical WSI Experimental Programme, In IEE Colloquium on Wafer Scale Integration, London, UK, pp. 7/1-7/3, 28 may 1991

Landis, D. L. (1990). A Self-test Methodology for Restructurable WSI, International Conference on Wafer Scale Integration, 1990, pp. 258-264, San Francisco, CA, 23-25 Jan 1990

Lea, R. M \& Boulori, H. S. (1988) Fault Tolerance: Step Towards WSI, In IEE Proc. Computers and Digital Techniques, 1988, Vol. 135, Issue 6, pp. 289-297, Nov. 1988

Koren, I \& Koren, Z. (1998). Defect Tolerance in VLSI Circuits : Techniques and Yield Analysis, IEEE, 86(9): 1819-1833, 1998

Laflamme-Mayer, N.; Valorge, O.; Blaquière, Y. \& Sawan, M. (2010). A Low-Power, SamallArea Voltage Reference Area for a Wafer-Scale Prototyping Platform, NEWCAS Conference, $8^{\text {th }}$ IEEE International Conference, Montreal, QC, pp. 189-192, June 2010

Lau, J. H., Yue, T. G. (2009). Thermal Management of 3D IC Integration with TSV (through silicon via), In ECTC 2009. 59th Electronic Components and Technology Conference, 2009, San Diego, CA, pp. 635-640m 12 Jun. 2009

Lepercq, É.; Blaquière, Y.; Norman, R. \& Savaria, Y. (2009). Workflow for an Electronic Configurable Computing System, ISCAS Conference 2009, International Symposium on Circuits and Systems, pp. 2005-2008, ISBN 978-1-4244-3827-3, Tapei, China, May 24-25, 2009

Minges, M. L. (1989). Electronic Materials Handbook: Packaging, Electronic Materials Handbook, Vol. 1, ESBN 9780871702852 
Mitsumasa, K.; Takafumi, F.; \& Tetsu, T. (2009). High-Density Through Silicon Vias for 3-D LSIs: Silicon stacked chips that perform highly-parallel data transfer have been successfully fabricated for image processing, artificial retinas, and for microprocessor and memory testing, Proc. of the IEEE 2009, Vol. 97, No. 1, pp. 49-59

Mohsen, A.M. (1995). Field Programmable Printed Circuit Board, USA Patent 5661409, 537124, 1995

Moore W. R. \& Mahat, R. (1985). Fault Tolerant Communication for Wafer-Scale Integration of Processor Area, In Microelectronic and Reliability, Vol. 25, Issue 2, pp. 291-294, 1985

Motoyoshi, M. (2009). Through-Silicon Via (TSV), In Proc. Of the IEEE, 2009, Vol. 97, Issue 1, pp 43-48, 27 Feb 2009

Norman, R. (2006). Reprogrammable Circuit Board with Alignment-Insensitive Support for Multiple Component Contact Types US patent application 11/611,263, filed Dec. 2006

Norman, R.; Valorge, O.; Blaquière, Y.; Lepercq, É.; Basile-Bellavance, Y.; El-Alaoui, Y.; Prytula, R. \& Savaria, Y. (2008). An Active Reconfigurable Circuit Board, NEWCAS-TAISA Conference 2008, 6th International IEEE Northeast Workshop on Circuits and Systems, pp. 351-354, ISBN 978-1-4244-2331-6, Montreal, Quebec, Canada, June 22-25, 2008

Norman, R.; Valorge, O.; Blaquière, Y.; Lepercq, É.; Basile-Bellavance, Y.; El-Alaoui, Y.; Prytula, R. \& Savaria, Y. (2008). An Interconnection Network for a Novel Reconfigurable Circuit Board, MNRC Conference 2009, 2nd Microsystems and Nanoelectronics Research Conference, pp. 53-56, ISBN 978-1-4244-4751-0, Ottawa, Ontario, Canada, October 13-14, 2009

Papanikolaou, A.; Soudris, D.; \& Radojcic, R. (2011). Three Dimensional System Integration : IC Stacking Process and Design, (Eds 2011) ESBN 978-1-4419-0961-9

Parker, P. K., (1998). The Boundary-scan Handbook: Analog and Digital, $2^{\text {nd }}$ ed. Kluwer Academic Publisher, Boston/Dordrecht/London, ch. 1, 4, 1998

Ricci, F \& Hoang, L. H. (2002) An FPGA-based Rapid Prototyping Platform for Variablespeed Drives, In IEEE 2002 28th Annual Conference on Industrial Electronics Society IECON 02, Vol. 2, pp. 1156-1161, 20 March 2003

Rimskog, M.\& Bauer, T. (2008) High Density Throug Silicon Via (TSV), DTIP 2008, Nice, French Riveria, France, 9-11 April 2008

Sharifi, H.; Choi, T. Y.; \& Mahommadi, S. (2007). IEEE Transaction on Advanced Packaging, Vol.. 30, No. 1, Feb. 2007

Shimooka, Y.; Inoue, M. E.; Obata, S.; Miyagi, T. ; Mori, I.. \& Shibata, Y. (2008). Robust Hermetic Wafer Level Thin Film Encapsulation technology for Stacked MEMS/IC Package, In Proc. ECTC, 2008, pp. 824-828.

Tuytelaars, T. \& Mikolajezyk, K. (2008). Local Invariant Feature Detectors: a survey, Foundations and Trends in Computer Graphics and Vision, Col. 3, Issue 3, pp. 177-280, 2008

Valorge, O.; Blaquière, Y. \& Savaria, Y. (2010). A Spatially Reconfigurable Fast Differential Interface for a Wafer Scale Reconfigurable Platform, ECECS, 2010, 17th IEEE International Conference on Electronic, Circuits, and Systems, Athens, pp. 1176-1179, 12-15 Dec. 2010

WfMC, (1999). Workflow Management Coalition Terminology and Glossary, document number, WfMC-tc-1011, document status - issue 3.0, 1999. http:/ / www.wfmc.org

Zebu XXL, (April 29). Available from

http://www.eve-team.com/products/products.html 


\title{
Rapid Prototyping for Mobile Robots Embedded Control Systems
}

\author{
Leonimer Flavio de Melo, Jose Fernando Mangili Junior \\ and Jose Augusto Coeve Florino \\ State University of Londrina \\ Brazil
}

\section{Introduction}

One of the main motivations of this work is to propitiate a virtual environment that facilitates development of archetypes of embedded systems, emphasizing implementation of tools that allow the simulation of kinematic, dynamic and control conditions, with real time monitoring of all important points of the system. In this way, the proposal of a virtual simulator of mobile robotic systems is presented together with techniques of rapid prototyping.

The use of the rapid prototyping technique in mobile robotic systems differs from the traditional target used in mechanics engineering and enters in a new field of research and development for projects of mobile robots mechatronics systems. In this way, the rapid prototyping of these systems is associated not only with the project of the physical system, but mainly with the experimental implementations in the fields of hardware and software of the robotic system.

In the context of this work, the rapid prototyping is the technology that allows, in conjunction with a simulation virtual environment, the development of a mobile robots controller project. After tested and validated in the simulator, the control system is programmed in the control onboard memory of the mobile robot. In this way, a economy of time and material are obtained, validating first all the model virtually for later operating the physical implementation of the system.

It is fundamental that the architecture of hardware of the considered system be opened and flexible in the way of effecting the necessary modifications for system optimization. A proposal of open architecture system was presented in Melo et al. (2005) and summarized in this work. The software of the embedded control system of the mobile robot, in the context of the rapid prototyping, can be elaborated in simulators and have all the parameters tested for adjustments that makes necessary in accordance with the physical system to be implemented, the hardware architecture, the actuators and the sensors. In this way, in the context of this work, the rapid prototyping is then the methodology that allows the creation of a virtual environment of simulation for the project of a controller for mobile robots. After being tested and validated in the simulator, the control system is programmed in the control board memory of the mobile robot. In this way, an economy of time and material are obtained, firstly validating all the model virtually and later operating the physical implementation of the system (Dudek \& Jekin, 2000).

The choice of DSP TMS320C6474 multicore digital signal processor as main onboard manager device falls into two main factors. First, its great information and instructions processing 
capacity, operating at $1,2 \mathrm{GHz}$ clock frequency and executing up to 10 billion information per seconds in its maximum performance, makes the system sufficiently efficient for implementations of complex software techniques required in the robotic navigation systems. Second because it has one specific MatLab tool box that can be used for its real time programming with hardware-in-the-Loop (HIL) techniques, that it is one of the simulation techniques utilized in the rapid prototyping systems for embedded mobile robotic controllers. This new technique of simulation, HIL (previously only available in the aerospace and aeronautical industry), can be used for the development and establishment of parameters of embedded mobile robotic controllers (Ledin, 2001).

The development of control systems for independent mobile robots has appeared as a great challenge for researchers until current days. Different platforms for project of control system for independent mobile robots have being used in diverse research areas. For many years the researchers have been designing control systems that present an intelligent behavior in controlled environments, with ideal situations, but that normally does not keep the same performance in real world. Innumerable systems of control exist to be used in real world, but generally these systems are limited and they do not present an independent or intelligent behavior.

For mobile robotics systems, so many possible applications exist, for example in transport, monitoring, inspection, cleanness of houses, space exploration, aid physical deficient, among others (Braunl, 2008). However, independent mobile robots had not yet caused much impact in domestic or industrial applications, mainly had the lack of a system with robust, trustworthy and flexible control that it would allow these robots operated in dynamic environments, less structuralized, and inhabited by human beings. The development of a mobile robotic model system with open architecture and flexible control, with robust control system, that incorporates the most modern embedded hardware technology and that makes possible the operation of a mobile robotic systems in a real world environment is one of the motivations of this work.

The locomotion planning, under some types of restrictions, is a very vast field of research in area of mobile robotics (Graf, 2001). The basic planning of trajectory for mobile robots imply the determination of a way in space-C (configuration space) between an initial configuration of robot and a final configuration, in such a way that robot does not collide with no obstacle in the environment, and that the planned movement is consistent with kinematic restrictions of the vehicle (Beeson et al., 2007). In this context, one of the boarded points in this work was development of a trajectory calculator for mobile robots.

\section{The mobile robot platform}

Platforms for knowledge consolidation could be used in several educational and research areas, such as modeling, control, automation, power systems, sensors, transmission of data, embedded electronics and software engineering. In fact, the use of mobile robots as a basis for knowledge consolidation has been successfully adopted in many educational and research institutions mainly because they appear to be a quite attractive low cost solution that allow integration of several important areas of knowledge (Braunl, 2008). Mobile robots also become a better solution for practical problems in modern society. These appointments show a large applicability of mobile robots and a crescent request in modern world (Beeson et al., 2007). One of the proposals of this project is developing a generic open system for control a mobile robot and supplies this need.

The system emphasizes control structure, supervision and transfer of information. In a context of educational and research aims the project aspects and integration solution compose the 
desired know-how acquired during development of system, which certainly would not to be approached if a commercial mobile robot was acquired.

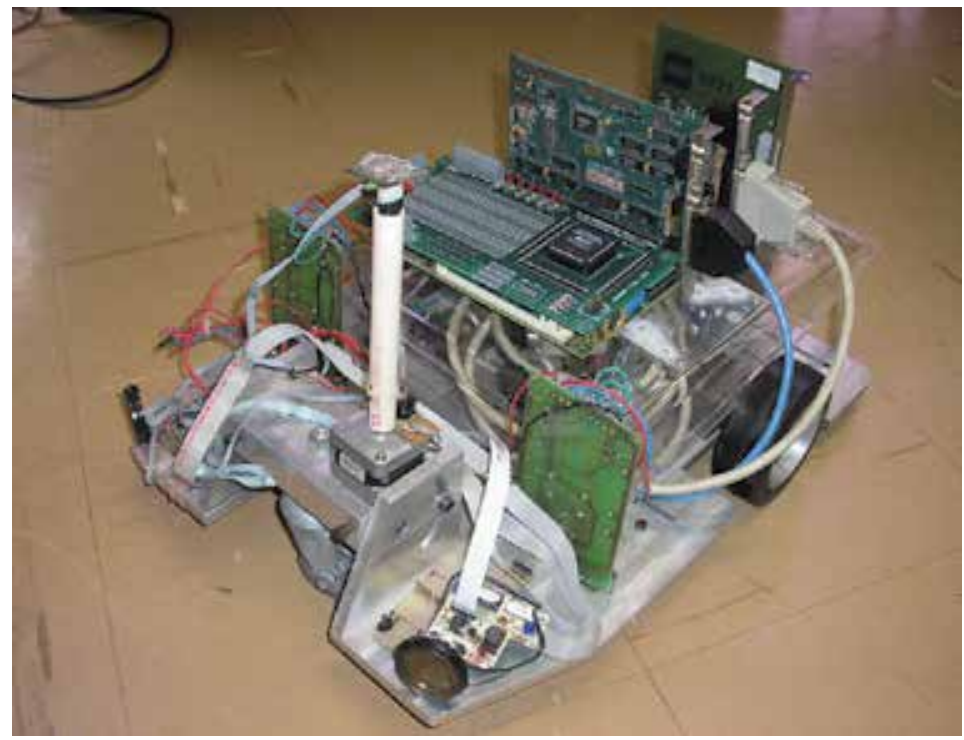

Fig. 1. Mobile robot platform prototype.

An embedded processor, with a dedicated control software, to be used on a platform mobile robotics, is considered (Siciliano et al., 2009). In addition to this platform another one, a commercial platform, coupled to a communication net, is analyzed. The set of platforms, whose objectives are making use of existing communication interfaces and providing an embedded user interface alternative in mobile robot, allows creation of a powerful link with external world. The objective of this platform is to make use of the existing communication interfaces, as well as to provide an embedded user interface alternative in mobile robot. Another aspect considered, is flexibility of hardware project, which allows the expansion of mobile robot facilities. New sensor combinations should be used. Different supervision and control models should equally be used to carry out the mobile robot tasks.

This paper presents the implementation of a virtual environment for project simulation and conception of supervision and control systems for mobile robots and focus on the study of the mobile robot platform, with differential driving wheels mounted on the same axis and a free castor front wheel, whose prototype used to validate the proposal system is depicted in Fig. 1 and Fig. 2 which illustrate the elements of platform.

\section{The control architecture system}

The control architecture system can be visualized at a logical level in the blocks diagram in Fig. 3.

The system was divided into three control levels, organized in the form of different degrees of control strategies. The levels can be described as:

- Supervisory control level: This represents a high level of control. In this level it was possible to carry out the supervision of one or more mobile robots, through the execution of global control strategies. 


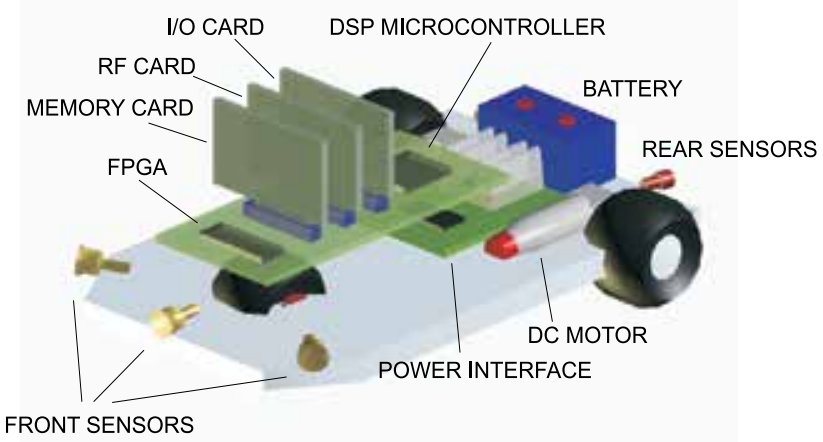

Fig. 2. Illustrative mobile robot platform and elements.

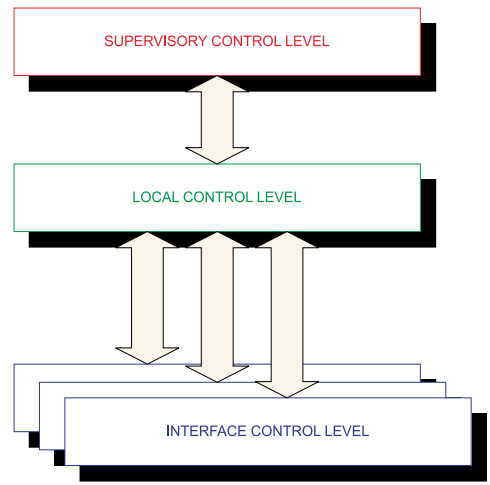

Fig. 3. Different control levels of the proposed system.

- Local onboard control level: In this level control was processed by the mobile robot embedded software implemented in a multicore DSP processor. The control strategies allowed decision making to be done at a local level, with occasional corrections from the supervisory control level. Without communication with the supervisory control level, the mobile robot just carried out actions based on obtained sensor data and on information previously stored in its memory.

- Interface control level: This was restricted to strategies of control associated with the interfaces of the sensor and actuators. The strategies in this level were implemented in hardware, through FPGA(Field-Programmable Gate Array) device.

Figure 4 depicts the control architecture with more details, with the levels controls implemented on the mobile robot platform.

Architecture, from the point of view of the mobile robot, was organized into several independent blocks, connected through the local bus that is composed by data, address and control bus (Fig. 5). A master block manager operates several slave blocks. Blocks associated with the interfaces of sensors and actuators, communication and auxiliary memories were subjected to direct control from the block manager. The advantage of using a common bus was the facility to expand the system. Inside the limitations of resources, it was possible to add new blocks, allowing an adapted configuration of the robot for each task. 


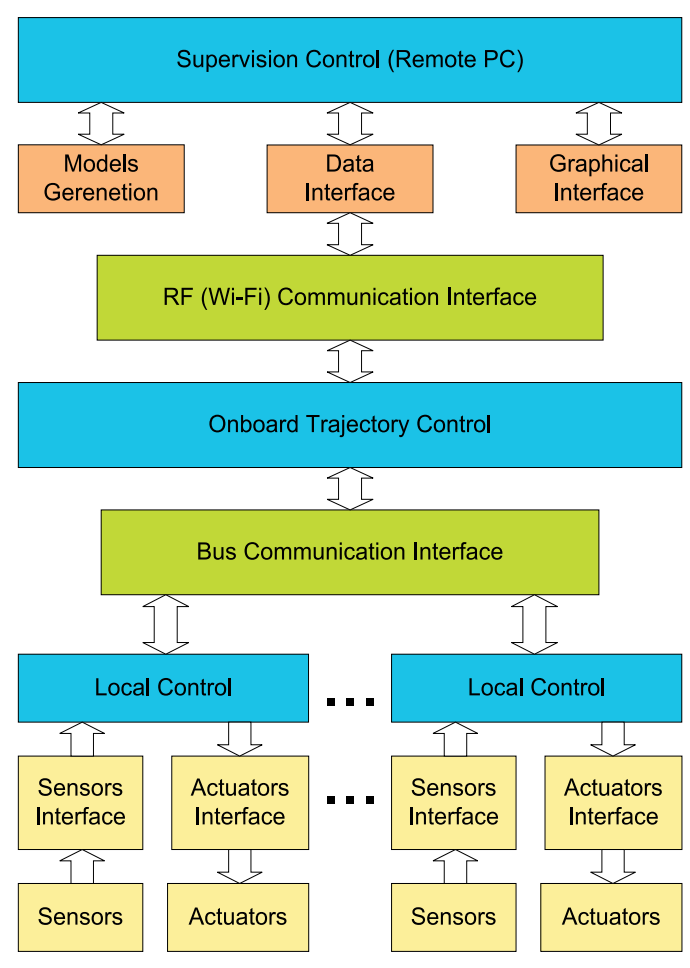

Fig. 4. Mobile Robot control architecture.

\subsection{Control architecture blocks description}

- Supervisory control block: Is the high level of control. In this block is managed the supervision of one or more mobile robots, through the execution of global control strategies. Is implemented in an IBM PC platform and is connected with the local control level, in the mobile robot, through Ethernet wireless WI-FI link. This protocol uses IEEE 802.11a standard for wireless TCP/IP LAN communication. It guarantees up to $11 \mathrm{Mbps}$ in the $2.4 \mathrm{GHz}$ band and requires fewer access points for coverage of large areas. Offers high-speed access to data at up to 100 meters from base station. 14 channels available in the $2.4 \mathrm{GHz}$ band guarantee the expansibility of the system with the implementation of control strategies of multiple robots.

- Master manager block: Responsible for the treatment of all the information received from other blocks, for the generation of the trajectory profile for the local control blocks and for the communication with the external world. In communication with the master manager block, through a serial interface, a commercial platform was used, which implemented external communication using an Ethernet WI-FI wireless protocol. The robot was seen as a TCP/IP LAN point in a communication net, allowing remote supervision through supervisory level. It's implemented with Texas Instrument TMS320C6474 multicore digital signal processor, a 1,2 GHz device delivering up to 10000 million instructions per second (MIPs) with highest performing.

- Sensor interface block: Is responsible for the sensor acquisition and for the treatment of this information in digital words, to be sent to the master manager block. The implementation of that interface through FPGA allowed the integration of information 


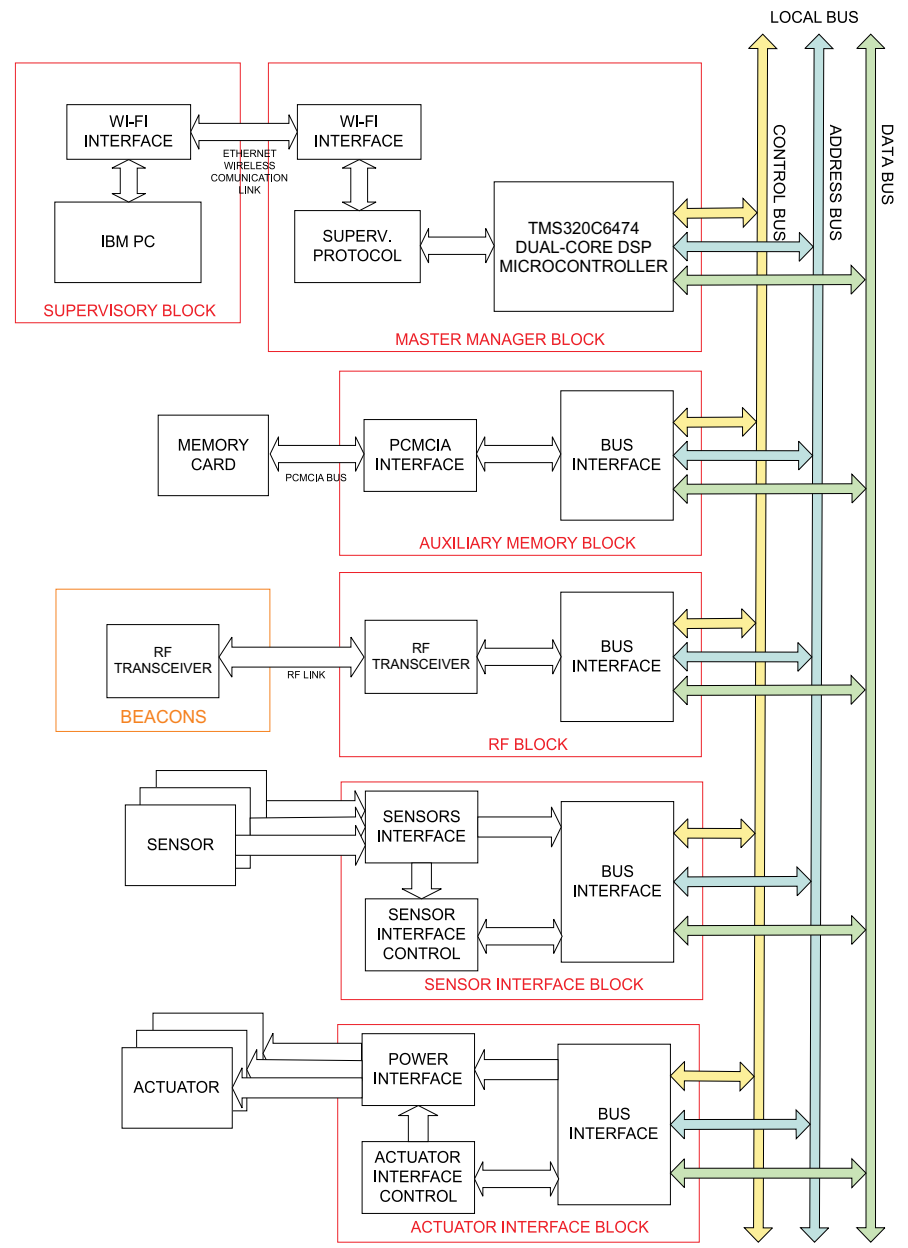

Fig. 5. Hardware architecture block diagram of the proposed system.

from sensors (sensor fusion) locally, reducing manager block demand for processing. In same way, they allowed new programming of sensor hardware during robot operation, increasing sensor treatment flexibility.

- Actuator interface block: This block carried out speed control or position control of the motors responsible for the traction of the mobile robot. The reference signals were supplied through bus communication in the form of digital words. Derived information from the sensor was also used in the controller implemented in FPGA. Due to integration capacity of enormous hardware volume, FPGA was appropriate to implement state machines, reducing the need for block manager processing. Besides the advantage of the integration of the hardware resources, FPGA facilitated the implementation and debugging. The possibility of modifying FPGA programming allowed, for example, changes in control strategies of the actuators, adapting them to the required tasks.

- Auxiliary memory block: This stored the information of the sensor, and operated as a library for possible control strategies of sensors and actuators. Apart from this, it came with an option for operation registration, allowing a register of errors. The best option was 
an interface PCMCIA, because this interface is easily accessible on the market, and being a well adapted for applications in mobile robots, due to low consumption, little weight, small dimensions, high storage capacity and good immunity to mechanical vibrations.

- RF beacons communication block: It allowed the establishment of a bi-directional radio link for beacons data communication. The objective, at the first moment, is establish communication with all beacons in the environment, not at same time, but one by one, recognizing the number of active beacons and their respective codes. At second moment, this RF communication block sends a determinate code and receive back the same code, transmitted from respective beacon. The RF ToF is calculated by DSP processor. To implement this block was used a low power UHF data transceiver module BiM-433-40.

\section{Mobile robot simulator}

The use of the system has begun to gather the main points for generation of the mobile robot trajectory. The idea is to use a system of photographic video camera that catches the image of the environment where the mobile robot navigates. This initial system must be able to identify the obstacles of the environment and to generate a matrix with some strategic points that will be good for input data for the system of trajectory generation. Figure 6 presents a general vision of the considered simulator system.

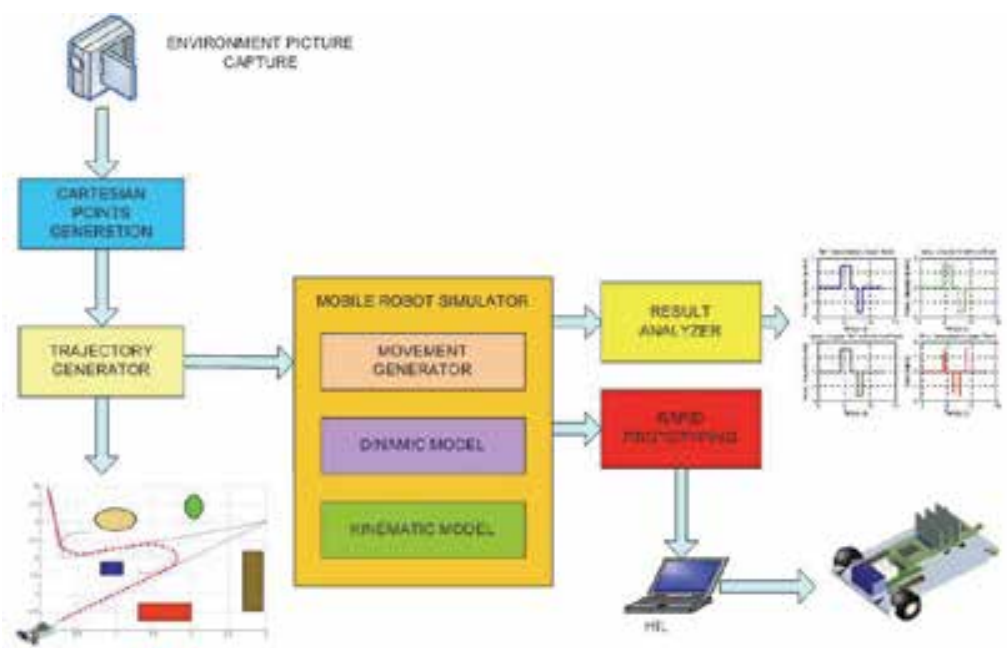

Fig. 6. General vision of the trajectory generator system.

This system is particularly interesting and can be used, for example, in robotic soccer games, where the navigation strategies are made from images of the environment (soccer field) and the obstacles are the other robots players. As it's described follow, with this system, the best trajectory can be defined and traced, respecting always the kinematics holonomics or nonholonomics constraints of the robotic systems in question, and to make all the simulation of the system foreseeing imperfections and analyzing results before the final implementation of the control system in the mobile robot (Melo \& Rosario, 2006).

\subsection{Mobile robot control structure}

The tasks carried through for the mobile robots are based on the independent movement of each degree of freedom, coordinated from a trajectories plan based in its kinematic model. 
In the most of the cases, the tasks programming is planned with anticipation and a map of the environment is loaded in the robot memory board. The mobile robot accomplishes the trajectory with sequence of independent movements of each axle, until reaching the desired final position. From the knowledge of these articulated positions, A generator of references (profile of speeds) based on the kinematic characteristics of each joint is easily implemented (Siciliano et al., 2009).

For accomplishment of tasks in level of cartesian coordinates system and for generation of the reference signals for the position controller of each robotics joint of the mechatronics system in study, the establishment of mathematical model based in the kinematics of the system becomes necessary. Therefore, the control of a robot needs procedures to transform the data of positioning reference, such as the linear speed and the bending radius, in cartesian coordinates, when it is desired to realize the control through a cartesian referential (onim at al., 1995). The Fig. 7 illustrates the mobile robot structure of control with the representu.uve blocks of the trajectory generation, dynamic and kinematic model of the system.

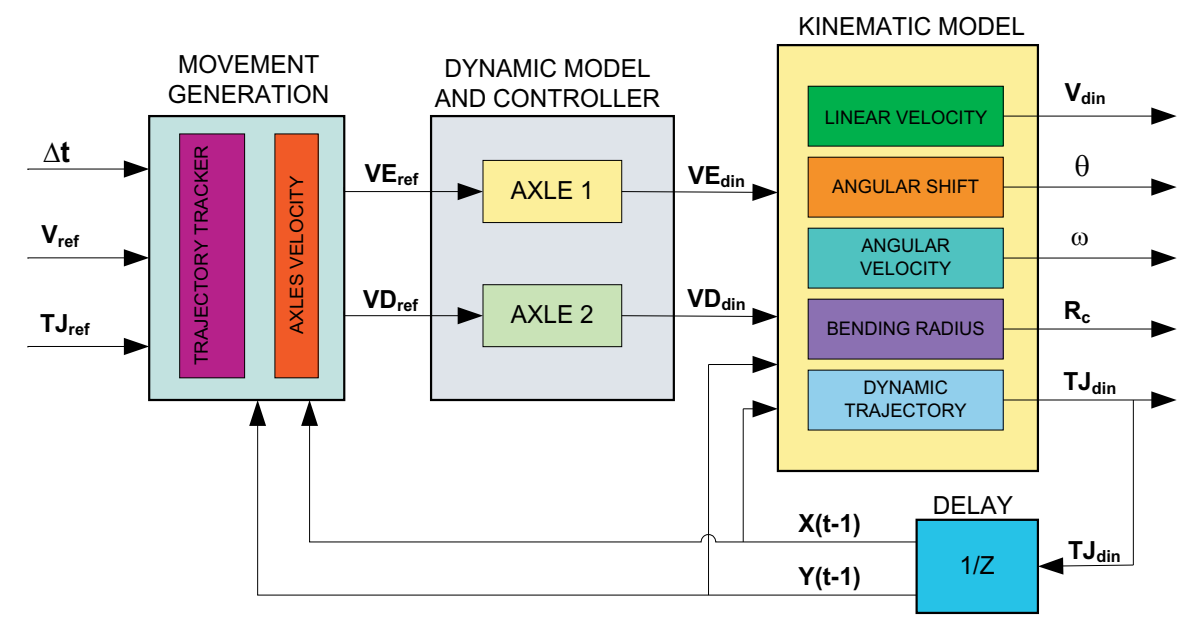

Fig. 7. The mobile robotic control structure.

The trajectory generator receives the references data, such as the positioning vector $\mathbf{X}_{r e f}=$ $\left[\mathbf{x}_{r e f}, \mathbf{y}_{r e f}, \theta_{r e f}\right]$, the robot reference linear speed $V_{r e f}$ and the robot instantaneous trajectory radius $R_{\text {curv }}$, that are converted into $V E_{\text {ref }}$ (linear speed of the left wheel) and $V D_{\text {ref }}$ (linear speed of the right wheel). These differentiated speeds are received by the controller, and in the dynamic model of the system, they are sent to the respective wheels of the robot, through its actuators. Then are generated by the controller the vectors $V E_{\text {din }}$ (dynamic linear speed of the left wheel) and $V D_{\text {din }}$ (dynamic linear speed of the right wheel). Into the block of the kinematic model, these data are converted into the vector final positioning of the robot $\mathbf{X}=[\mathbf{x}, \mathbf{y}, \theta]$.

\subsection{Trajectory embedded control}

The figure 8 illustrates an example of an environment with some obstacles where the robot must navigate. In this environment, the robot is located initially in the P1 point and the objective is to reach the $\mathrm{P} 4$ point. The supervisory generating system of initial cartesian points, must then supply to the module of embedded trajectory generation, the cartesian points P1, $\mathrm{P} 2, \mathrm{P} 3$ and $\mathrm{P} 4$, that are the main points of the traced route. 


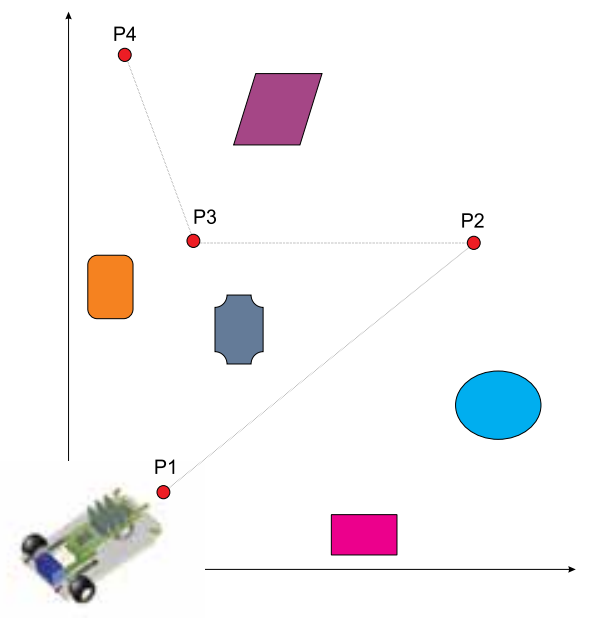

Fig. 8. Example of an environment with some obstacles where the robot must navigate.

The use of the system begin with the captation of main points for generation of the mobile robot trajectory. The idea is to use a system of photographic video camera that catches the image of the environment where the mobile robot will navigate. This initial system must be capable to identify the obstacles of the environment and to generate a matrix with some strategical points that will serve of input for the system of embedded trajectory generation. The mobile robot embedded control system receives initially, through the supervisory system, a trajectory to be executed. These data are loaded in the robot memory that are sent to the module of trajectory generation. At a time the robot starts to execute the trajectory, the dynamic data are returned to the embedded controller, who, with the measurements and sensing, makes the comparisons and due corrections in the trajectory.

The trajectory embedded control system of the mobile robot is formed by three main blocks. The first one is called movements generation block. The second is the block of the controller and dynamic model of the mobile robot. Third is the block of the kinematic model. Figure 12 illustrates the mobile robot control strategy implemented into Matlab Simulink blocks and than loaded in the embedded memory of the DSP processor by HIL(hardware-in-the-loop) technique.

The mobile robot embedded control is implemented with kinematic and dynamic model for axles control and the movement generator modules. Figure 7 illustrates the blocks diagram representing those modules.

The input system variables are:

- $\Delta t$ is the period between one pose point and another.

- $\mathbf{T J}_{r e f}$, is the reference trajectory matrix given by supervisory control block with all the trajectory dots pose coordinates $(x, y, \theta)$.

- $V_{\text {ref }}$, is the robot linear velocity dynamics informed by supervisory control block so that robot can accomplish one particular trajectory.

The embedded system output variables are:

- $\quad \mathbf{T J}_{\text {din }}$, that is the robot dynamic trajectory matrix, given in cartesian coordinate format.

- $\mathbf{V}_{\text {din }}$, is the dynamic linear velocity of the robot. 
- $\mathbf{R}_{c}$, is the mobile robot ICC.

- $\theta$, is the orientation angle.

- $\omega$, is the angular velocity vector.

\subsection{Trajectory Generation Block}

The Trajectory Generation Block of trajectory receives some important points from the camera system so that the trajectory can be traced to be realized by the mobile robot. These points form a cartesian matrix containing more or less points, depending on the complexity of the environment. For testing reasons and for validation of the system, the number of points to be fed the system was fixed in four. Nevertheless, the number of points can be increased depending on the complexity of the environment where the mobile robot will navigate. Another data important to be used by the system have relation with the holonomics constraints of the modeled mobile robot. The bending radius must be informed to the system to be performed in the trajectory. A time that, for a reason or purpose tests, was fixed in four the number of cartesian input points, must supply the radius of the two curves to be executed for the robot. The information of distinct radius makes the system more flexible, making the trajectory able to be traced with different bending radius, depending on the angle of direction displacement and on the robot restrictions.

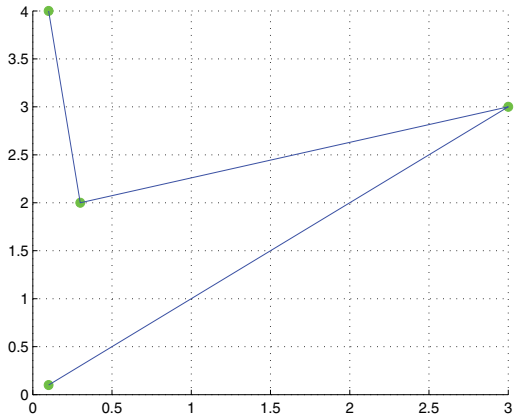

(a) Initial points given to trajectory generation.

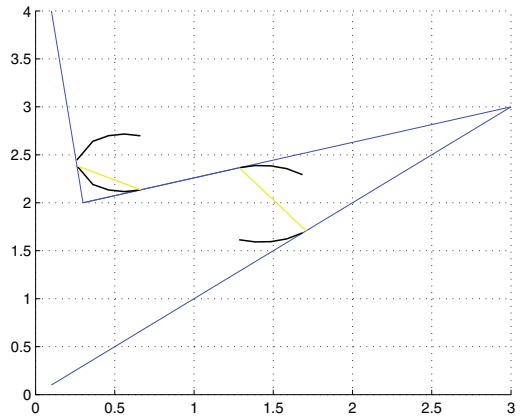

(b) Assay to the delineated trajectory.

Fig. 9. Initial points given by camera system and Assay to the delineated to trajectory.

The graphic of the Fig. 9(a) illustrates the initial points for the trajectory generator. In this example, it was captured from the camera system and transmitted for the simulator the vector $\mathbf{x}=\left[\begin{array}{llll}.1 & 3 & .3 & .1\end{array}\right]$ and the vector $\mathbf{y}=\left[\begin{array}{llll}.1 & 3 & 2 & 4\end{array}\right]$, with the bending radius for first and the second semicircle given by the vector $\mathbf{r}=\left[\begin{array}{ll}.4 & .3\end{array}\right]$. All the measures are in meters. In the Fig. 9(b) it's possible to see the tracing of the straight lines, the semicircles and an intermediate segment of straight line indicating the start and the end of the tracing of each circular movement to be executed by the mobile robot.

The final tracing of the mobile robot trajectory can be observed in the Fig. 10, represented by red spots.

\subsection{The virtual simulator implementation}

Now the main characteristics of a simulator of mobile robotic systems are presented. It was implemented from the kinematic and dynamic models of the mechanical drive systems of the robotic axles, for the simulation of different control techniques in the field of the mobile 


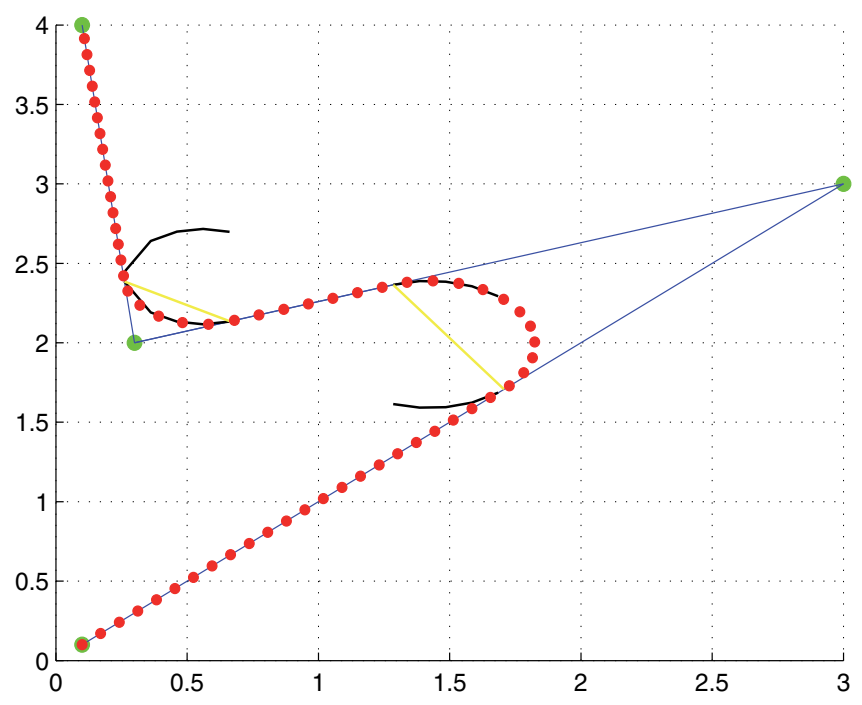

Fig. 10. The final tracing of the mobile robot trajectory.

robotics, allowing to deepen the concepts of navigation systems, trajectories planning and embedded control systems. This simulator, designed in modular and opened architecture, as presented in section 3, allows the direct application of some concepts into of the mobile robotics area, being used for its validation, and as main objective of this study, the model of an prototype of mobile robot with nonholonomic kinematic constraint and differential drive with two degrees of freedom (movement of linear displacement and rotation).

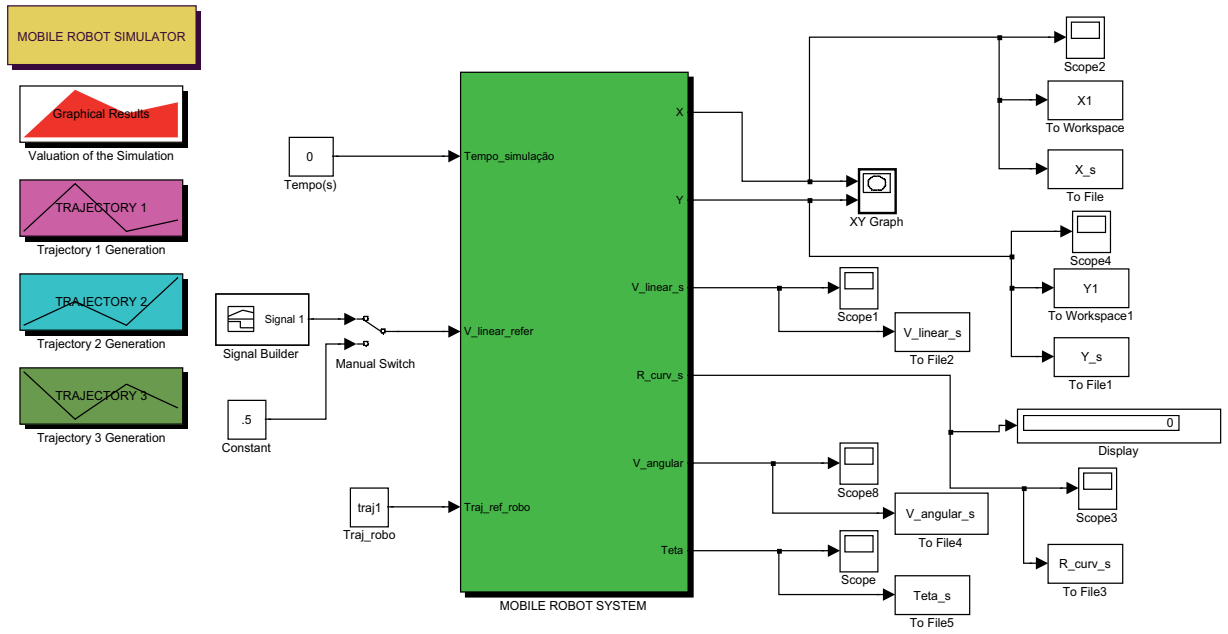

Fig. 11. The Virtual Simulator first page.

For the simulator development, the constructive aspects of the mobile robot prototype had been considered, including the kinematics and dynamics modeling of drive and control systems. The simulator presents the trajectories generating module that is the first block of 
the system and was implemented with the functionality to generate a trajectory for the mobile robot from a matrix of points supplied initially. Another presented block is the controller, implemented in the PID traditional form.

The Fig. 11 shows the initial page of the Virtual Mobile Robot Simulator. The user can choose one of the captured trajectory for analyzing, take a look in the graphical results of the simulation or implement changes in the robot model, by clicking on the mobile robot system (green main block).

The virtual simulator system of the mobile robot is composed of three main blocks. The first one is called movements generation block. The second one is the block of the controller and dynamic model of the mobile robot and the third one is the block of the kinematic model. The Fig. 12 illustrates the mobile robot simulator implemented into Matlab Simulink ${ }^{\circledR}$ blocks.

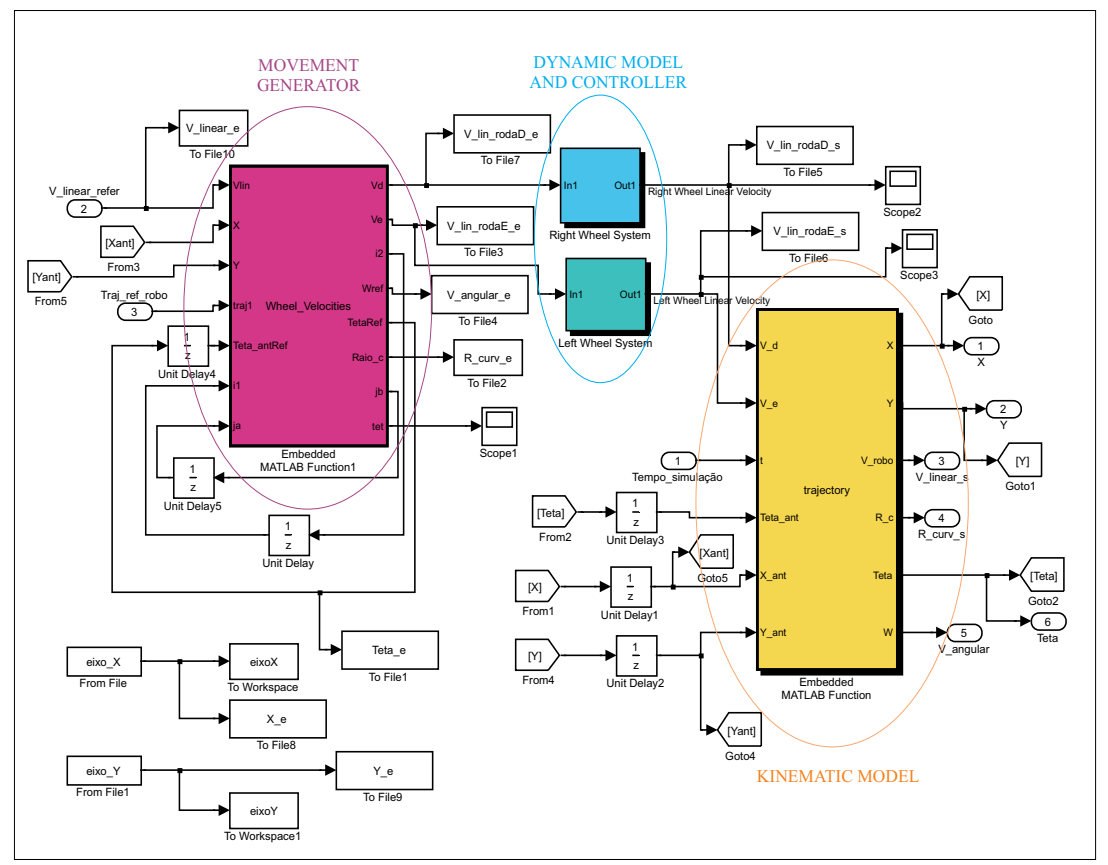

Fig. 12. Mobile robot simulator implemented into Simulink ${ }^{\circledR}$.

\subsection{Results graphical analyzer}

The simulator implemented in Simulink ${ }^{\circledR}$ environment allows the visualization of the inputs and outputs of the system in study.

For a better understanding and analysis of the behavior of the system the implementation of a results graphical analyzer becomes essential. In this way, after realizing the simulations in the domain of the time, timings data archives are obtained corresponding to the study variables (angular and cartesian position, linear and angular speed and control signals), that after convenient treatment, make it possible to verify important results for better analysis of the system behavior. The Fig. 13 illustrates a menu of the graphical analyzer of the mobile robotic system in study with an example of generated graphic. 


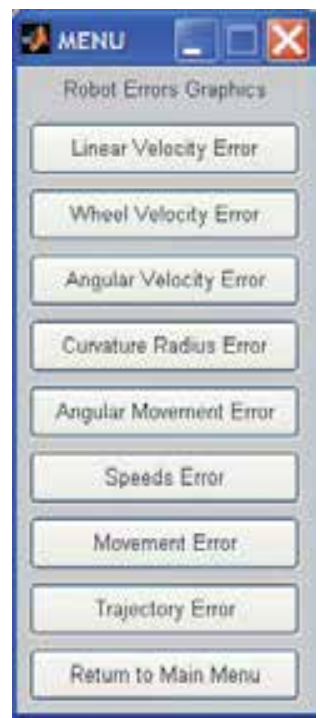

(a) Robot errors analyzer submenu.
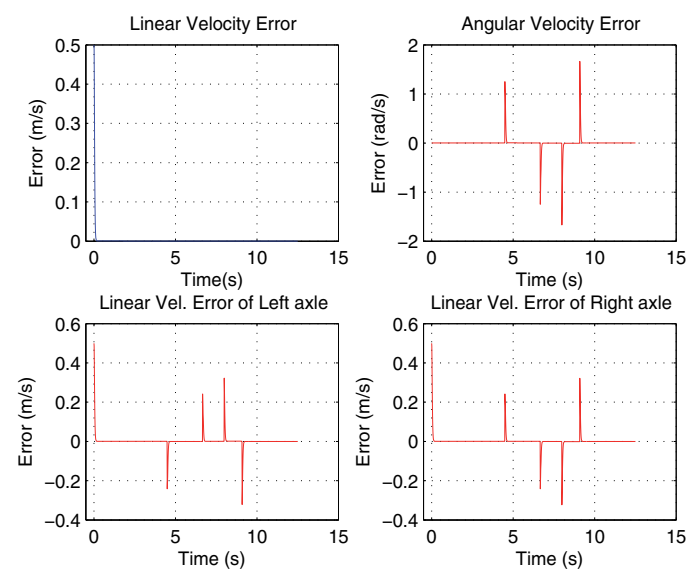

(b) A robot speeds errors graphics.

Fig. 13. Submenu of the mobile robot graphical analyzer with an example of generated graphic.
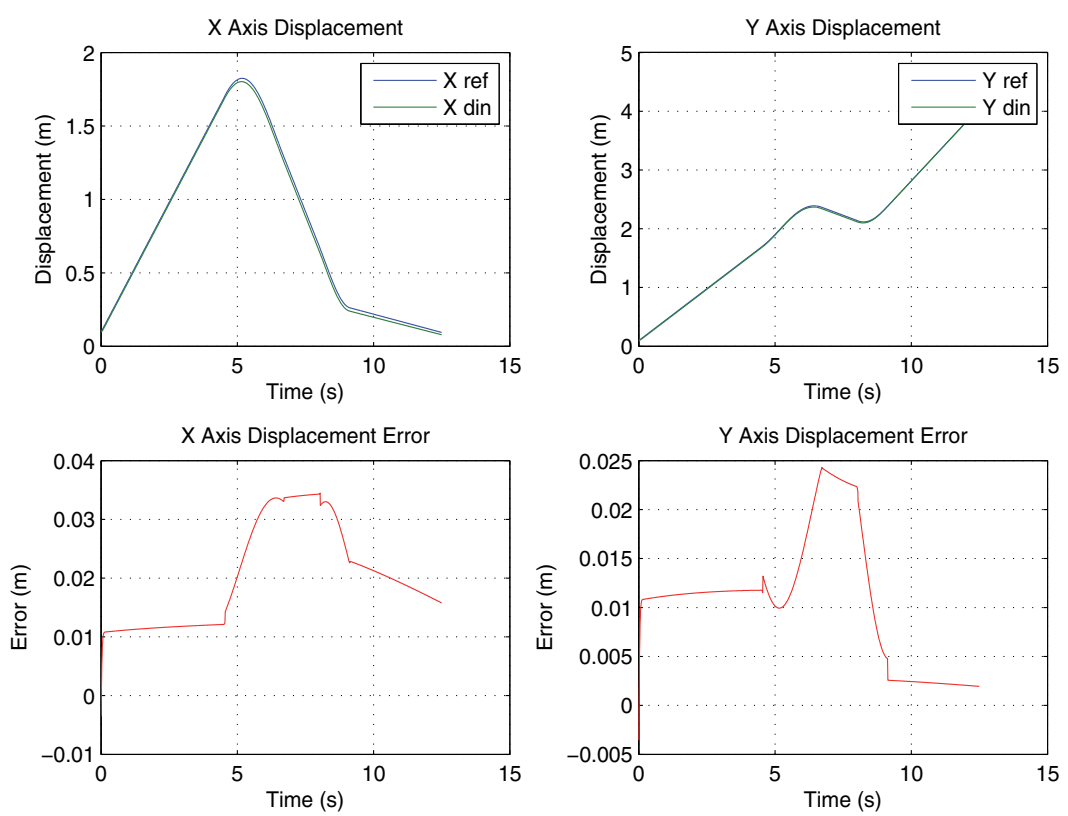

Fig. 14. Dynamic behavior graphics of the robot in the $\mathrm{X}$ and $\mathrm{Y}$ axis with their errors. 
One kind of analysis that is made is in relation to the linear displacement of the robot in axis $\mathrm{X}$ and $\mathrm{Y}$. In Fig. 14, the dynamic behavior of the robot with regard to these parameters, as well as the presented errors is illustrated.

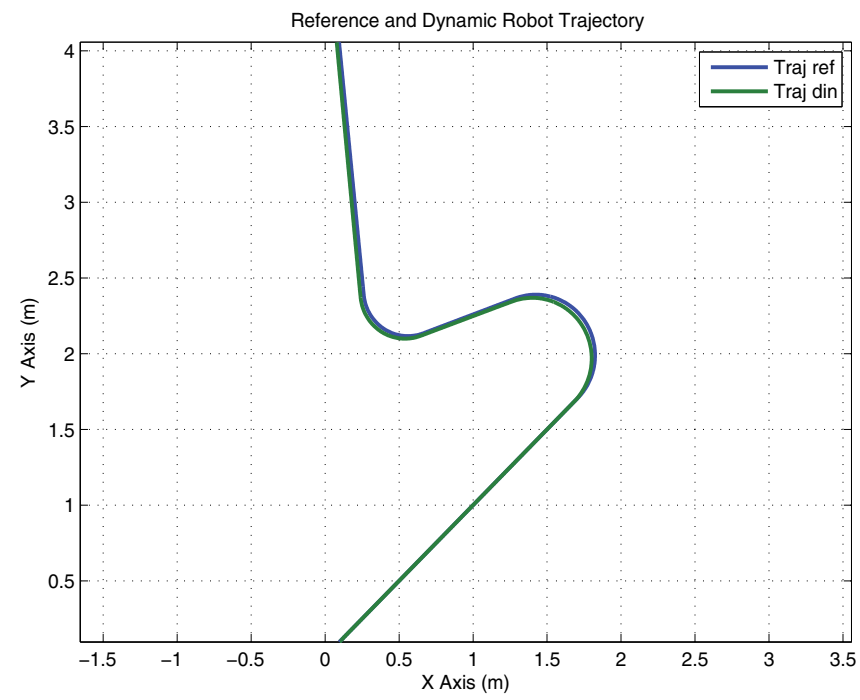

Fig. 15. Cartesian trajectory kinematics and dynamics of the mobile robot.

Another important graphic generated by the system, in the cartesian trajectory sub-menu, that is the graphic of the cartesian trajectory kinematics and dynamics of the mobile robotic system in plan XY. The Fig. 15, shows the dynamic tracing of reference and of the trajectory of the mobile robot.

The Fig. 16 illustrates the graphic of the trajectory error.

\section{Mobile robot rapid prototyping}

In the context of this work, the rapid prototyping is then the methodology that allows the creation of a virtual environment of simulation for the project of a controller for mobile robots. After being tested and validated in the simulator, the control system is programmed in the control board memory of the mobile robot. In this way, an economy of time and material are obtained, firstly validating all the model virtually and later operating the physical implementation of the system.

In this work was used a specific MatLab ${ }^{\circledR}$ tool box that can be used for its real time programming with hardware-in-the-Loop (HIL) techniques, that it is one of the simulation techniques utilized in the rapid prototyping systems for embedded mobile robotic controllers. This new technique of simulation, HIL (previously only available in the aerospace and aeronautical industry), can be used for the development and establishment of parameters of embedded mobile robotic controllers (Ledin, 2001).

\subsection{HIL (Hardware-in-the-loop) simulation}

The HIL technique of simulation is used in development and tests for real time embedded systems. HIL simulations provide a platform accomplish of development for adding the complexity of the plant under control to the tests platform. The control system is enclosed 


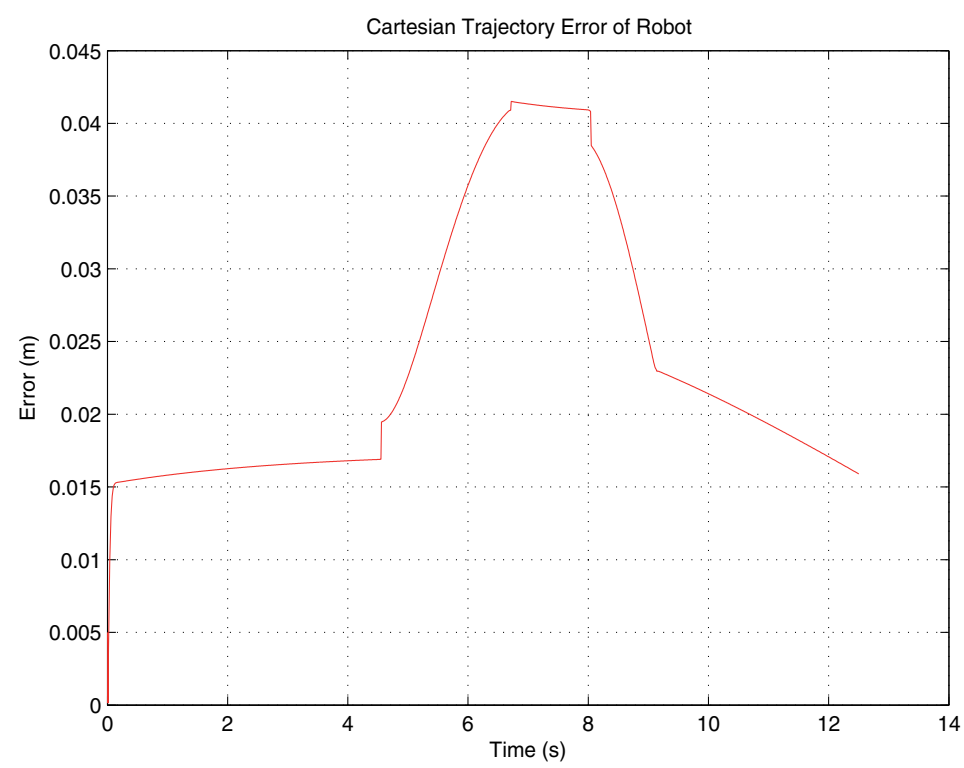

Fig. 16. Error of the kinematics and dynamics trajectory of the mobile robot.

in the tests and developments through its mathematical models representations and all the respective dynamic model (Melo \& Rosario, 2006).

The Fig. 17 illustrates the use of the HIL simulation technique for real time simulation of the considered mobile robotic system.

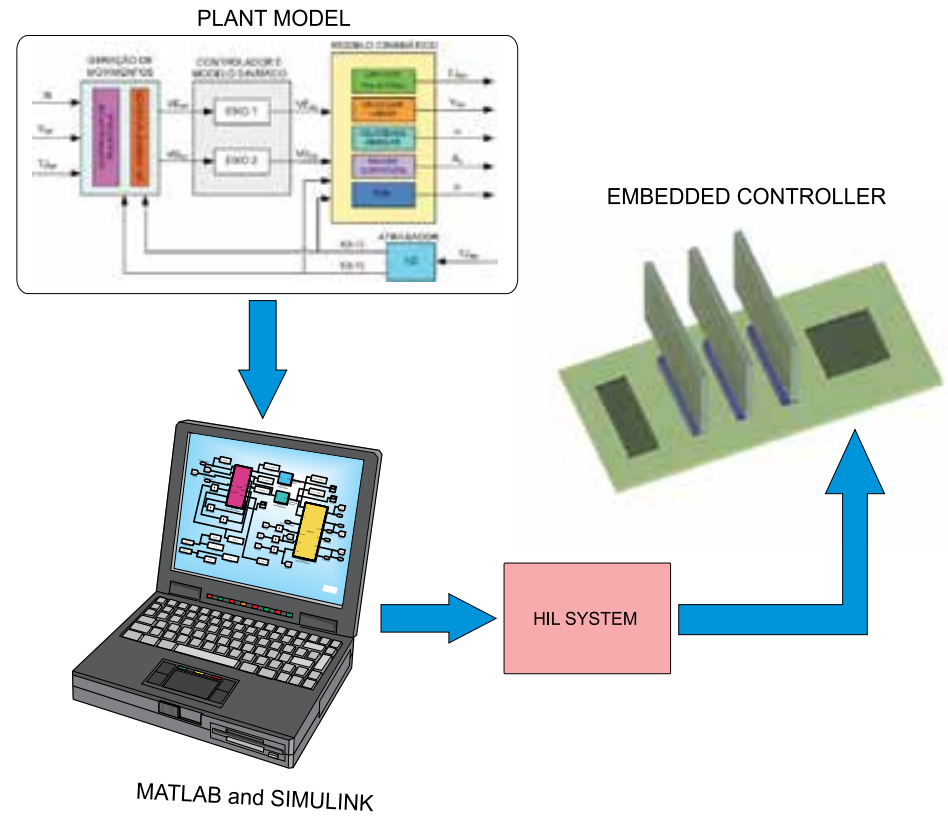

Fig. 17. HIL simulation for mobile robot system. 


\section{Experimental validation}

The Aedromo, a didactic experimental environment for mobile robots, is an ambient used to test and validate the trajectory virtual simulator, the onboard robot control and the rapid prototyping system, all of them describe above. This environment is utilized for many others applications, like robotic soccer game, two or more robots interaction, etc. The supervision and the robots movements coordination are made through a close loop architecture based in a satellite camera over the arena. The information supplied by a video camera is sent for one or two computers for processing. The data obtained from this process are used to generate a sequence of instructions that are sent for the robots. The robots receive the instructions and carry out the actions obeying the predetermined tasks. The instructions are results of developed programmers strategies to execute the tasks and to realize the robot navigation into the environment. The Fig. 18 depicts this environment (Melo \& Mangili, 2009).
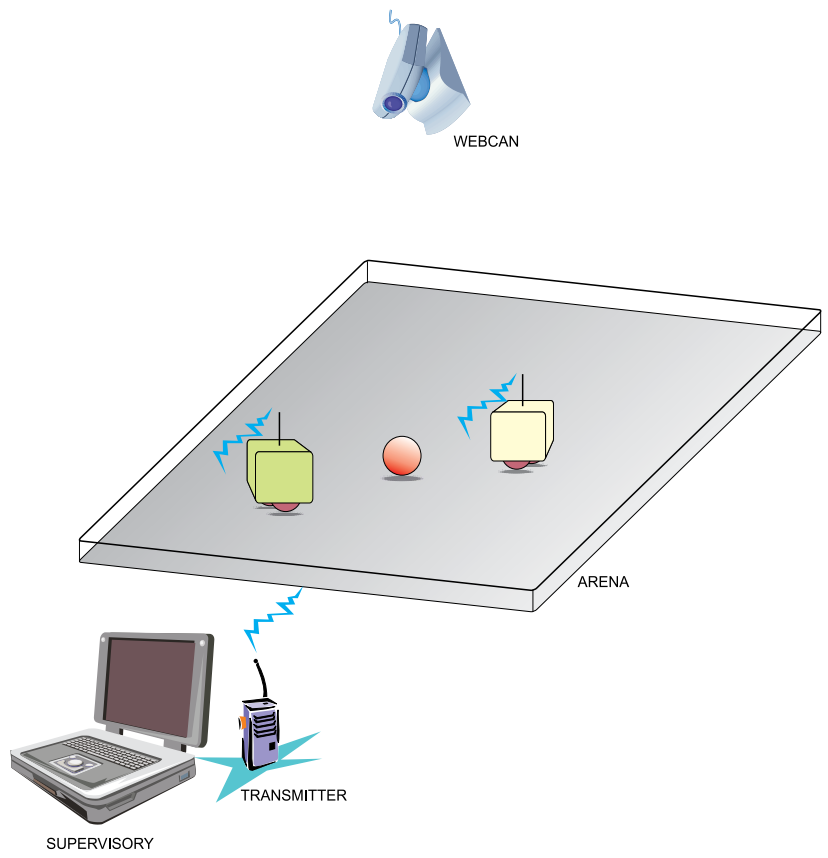

Fig. 18. The Aedromo environment.

The trajectory to be executed by the robot, from trajectory generation software, is illustrated on Fig. 10. This trajectory allows to get parameters of comparison between the real system, represented by de robot prototype, and the virtual system. On the onboard control of the mobile robot a dynamic and kinematic strategy was implemented, in order to find best trajectory, avoiding obstacles on the way, as can be seen in Fig. 8.

The graphic presented on Fig. 19 illustrates the difference of the reference trajectory, showing by the slim line with square blocs (violet) and the dynamic trajectory executed by the robot, showing by the thick line (blue).

Even though the robot executes the proposed trajectory with success, we can see errors between the two trajectory, mainly on curves. The cartesian trajectory kinematics and dynamics of the mobile on simulator was foreseen depicted on Fig. 15 with the trajectory error on Fig. 16. These errors are predictable for the simulator because of the intrinsic dynamic 
characteristic of nonholonomic mobile robotics systems with differential drive wheels, which the prototype are made, and for the PID close-loop controller of wheels velocity axles. The necessity and efficacy of the virtual system, proposed by this work are demonstrated in this example.

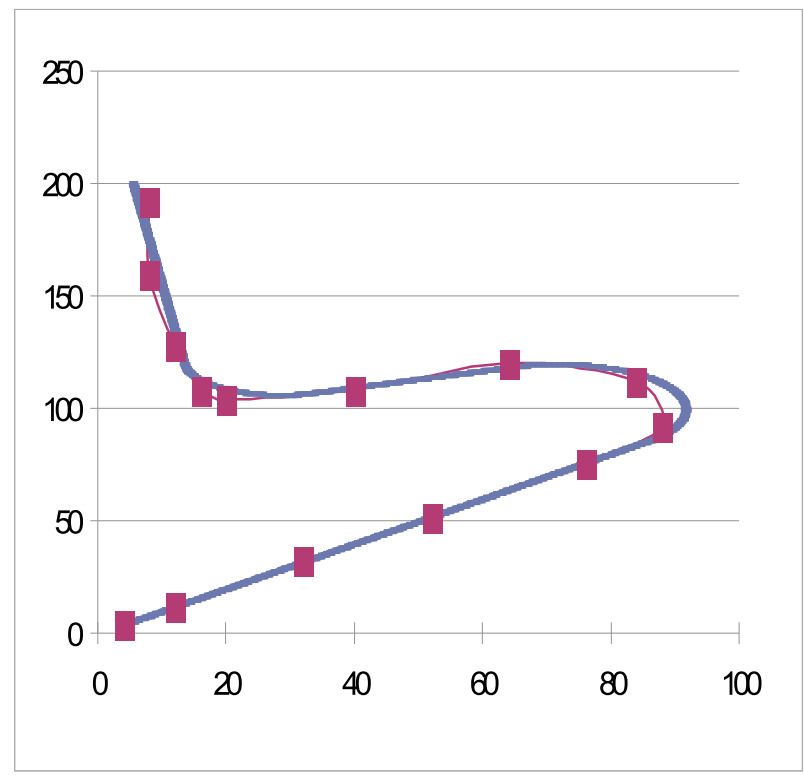

Fig. 19. Reference and dynamic trajectory executed by the robot.

\section{Conclusion}

How can be seen in this work, is evident the advantages of the HIL(hardware-in-the-Loop) and $\mathrm{RCP}$ (Rapid Control Prototyping) simulation techniques for mobile robotic control systems rapid prototyping. This development approach for embedded controllers is made in this work in the way that all the system is tested and approved in the simulator before being implemented at mobile robot. So that for the system has flexibility in its alterations is necessary that its architecture is opened and reconfigurable.

The techniques of rapid prototyping only can be executed if the plant has its kinematic, dynamic and the controller model implemented in a virtual simulator. In this work, MatLab software provides the necessary computational tools so that all system of simulation and rapid prototyping could be implemented.

The main objective of this work was to propose a generic platform for a robotic mobile system, seeking to obtain a support tool for mobile robotic control systems rapid prototyping. In this way, it presents the virtual environment implementation for simulation and design conception of supervision and control systems for mobile robots, which are capable to operate and adapting in different environments and conditions. This came from encountering the growing need to propose to the research that integrates the knowledge acquired in several domains that stimulates teamwork in order to reach a result. Another objective was to to improve knowledge in the mobile robotic area, aiming at presenting practical solutions for industrial problems, such as maintenance, supervision and transport of materials. Some promising aspects of this platform and simulator system are: 
- Flexibility: there is a great variety of possible configurations in the implementation of solutions for several problems associated with mobile robots.

- Great capacity of memory storage allowing implementation of sailing strategies for maps.

- The use of the rapid prototyping technique in mobile robotic systems.

- Possibility of modification of control strategies during the operation of the mobile robot in special mechatronics applications.

\section{References}

Beeson, P., Macmahon, M., Modayil, J., Murarka, A., Kuipers, B. and Stankiewicz, B. (2007). Integrating multiple representations of spatial knowledge for mapping, navigation, and communication. In Proceedings of the AAAI 2007 Spring Symposium on Control Mechanisms for Spatial Knowledge Processing in Cognitive / Intelligent Systems, pp 1203-1201.

Braunl, T. (2008) Embedded Robotics: Mobile Robot Design and Applications with Embedded Systems, 3th. Ed., Springer, Perth, Australia.

Dudek, G. \& Jenkin, M. (2000). Computational Principles of Mobile Robotics, Cambridge University Press, UK.

Ledin, J. (2001). Simulation Engineering: Build Better Embedded Systems Faster. CMP Books, USA.

Graf,B; Wandosell, J. M. H. \& Schaeffer, C. (2001). Flexible Path Planning for Nonholonomic Mobile Robots. Proceedings of Angenommen zur Eurobot'01.pp 456-565, 2001, Stuttgart, Germany.

Melo, L. F.; Lima, C. R. E. \& Rosario, J. M. (2005). A Reconfigurable Control Architecture for Mobile Robots. Proceedings of 2nd. Internacional Symposium on Mutibody and Mechatronics - MuSMe 2005, v. 1. pp. 1-8, september 2005.

Melo, L. F. \& Rosario, J. M. (2006). A Proposal for a hybrid opened archtecture with hardware reconfigurable control applied in mobile robots. Proceedings of IEEE International Conference on Robotic and Bionemetics - ROBIO 2006,v. 1. pp. 1101-1106, october 2006.

Melo, L. F., and Mangili Jr., J. F. (2009). Virtual Simulator with Mobile Robot Rapid Prototyping for Navigation Systems. ICIA 2009. IEEE International Conference on Information and Automation, v. 1. p. 562-568.

Shim, H.-S.; Kim, J.-H. \& Koh, K. (1995). Variable structure control of nonholonomic wheeled mobile robot. Proceedings of IEEE International Conference on Robotics and Automation. vol. 2, pp. 1694-1699, April 1995.

Siciliano, B., Sciavicco, L., Villani, L. and Oriolo, G. (2009) Robotics: Modelling, Planning and Control. Advanced Textbooks in Control and Signal Processing. Spring, Napoli, Italy. 


\title{
ASIP Design and Prototyping for Wireless Communication Applications
}

\author{
Atif Raza Jafri, Amer Baghdadi and Michel Jezequel \\ Telecom Bretagne/Lab-STICC CNRS
}

France

\section{Introduction}

Last three decades can undoubtedly be said as the decades of wireless communications. New state of the art data processing techniques have been developed and used in practical system. These techniques include advanced Error Control Coding (ECC), Bit Interleaved Coded Modulation (BICM), high ordered Quadrature Amplitude Modulations (QAM), Signal Space Diversity (SSD), Multi Input Multi Output (MIMO), Orthogonal Frequency Division Multiplexing (OFDM), and others.

To guide the wireless communication industry, for the adoption of new developed techniques in the commercial devices, diverse wireless communication standards have been evolved rapidly in the domain of cellular telephone network, local/wide wireless area networks and Digital Video Broadcast (DVB). These standards propose best combination of evolved data processing schemes at the transmitter to protect data from destructive channel effects under different transmission conditions. Taking an example of mobile telephony, by using newly developed concepts such as spatial multiplexing feature of MIMO, the evolving 3GPP-LTE standard is aimed at achieving 100 Mega bits per second to support high data rate real-time applications on a mobile terminal. Similar trends are also visible in other public domains of wireless communication such as DVB-NGH (Next Generation Handheld), which is the next generation standard for video broadcasting, providing services for fixed and mobile terminals. The other aspect under consideration is the global roaming or seamless coverage across geographical regions which demands multi-mode radios compatible with different systems and standards to provide seamless services at fixed location.

While translating these requirements on the physical layer of a radio terminal, this can be seen as a flexible high throughput hardware platform which can be configured to the required air interface. The overall flexibility of the radio platform can be achieved through the flexibility of individual components at transmitter side (encoder, interleaver, mapper, etc.) and at receiver side (equalizer, demapper, deinterleaver, decoder, etc.). This emerging flexibility need in digital baseband design constitutes a new major challenge when added to the ever increasing requirements in terms of throughput and area. In addition to technical requirements associated with rapid growth in wireless communication industry, the severe time-to-market constraints compel the designers for adopting the rapid design, validation and prototyping flow to conceive these wireless radio terminals for their successful and timely delivery in the market. In short, the diverse requirements of wireless communication standards imply, between others, two crucial requirements on hardware implementation: (1) 
Hardware platform flexibility for multi-standard support, and (2) Rapid prototyping flow for system validation under different use case scenarios.

\subsection{ASIP and rapid design flow}

Considering the first requirement of flexibility, the very first idea about the flexible platform was presented in the initial work on Software Defined Radio (SDR) (Mitola, 1995). Any reconfiguration of an SDR platform simply corresponds to a change in a software program. The required software does not even need to be stored in the device itself, since it can be downloaded, thereby bringing easy maintenance capability to the radio. In this proposition, off-the-shelf General Purpose Processors (GPP) and Digital Signal Processors (DSP) were presented as programmable Processing Elements (PE) of different functional block of a flexible radio platform. With increasing demand of high throughput and low power requirements, GPP and DSP are no more suitable due to their limited parallelism and huge flexibility which is more than what is required in PEs of functional blocks of future radio platforms and hence causing low throughput and high power consumption.

In this regard, Application Specific Instruction-set Processors (ASIPs) are increasingly used in complex System on Chip (SoC) designs. ASIPs are tailored to particular applications, thereby combining performance and energy efficiency of dedicated hardware solutions with the flexibility of a programmable solution. The main idea is to design a programmable architecture tailored to a specific application, thus preserving only the required flexibility. Coming to the second requirement of rapid design flow, while selecting ASIP as the implementation approach, an ASIP design flow integrating hardware generation and corresponding software development tools (assembler, linker, debugger, etc.) is mandatory. In this regard, by looking at available commercial solutions for ASIP design, it is possible to identify three main classes based on the degree of freedom which is left to the designer:

- Architecture Description Language (ADL) based solutions which can be also defined as ASIP-from-scratch. This approach results in the highest flexibility and efficiency, but on the other hand it requires a significant design effort.

- Template architecture based which allow the designer to add custom instructions to a pre-defined and pre-verified core, thus restricting the degree of freedom with respect to the previous approach to the instruction set definition only.

- Software configurable processors and reconfigurable processors with a fixed hardware, including a specific reconfigurable ISE fabric, which allows the designer to build custom instructions after the fabrication.

CoWare Processor Designer is an ASIP design environment entirely based on LISA (Hoffmann et al., 2001). The language syntax provides a high flexibility to describe the instruction set of various processors, such as SIMD (Single-Instruction Multiple-Data), MIMD (Multiple-Instruction Multiple-Data) and VLIW (Very long instruction word)-type architectures. Moreover, processors with complex pipelines can be easily modeled.

Processor Designer's high degree of automation greatly reduces the time for developing the software tool suite and hardware implementation of the processor, which enables designers to focus on architecture exploration and development. The usage of a centralized description of the processor architecture ensures the consistency of the Instruction-Set Simulator (ISS), software development tools (compiler, assembler, and linker etc.) and RTL (Register Transfer Level) implementation, minimizing the verification and debug effort. Using the Processor 
Designer's automated design and optimization environment which utilizes LISA language description to develop a wide range of processor architectures, like SIMD and VLIW as well as processors with DSP or RISC-specific features. The generation of the software development environment by Processor designer enables to start application software development prior to silicon availability, thus eliminating a common bottleneck in embedded system development.

\subsection{ASIP for wireless communication applications}

Consider the system diagram of Fig. 1, where transmitter includes the channel coding, BICM interleaving, constellation mapping and finally a possibility of SSD and MIMO STC. In the channel encoder there are several flexibility parameters such as input bit in encoder, states of the encoder and trellis construction. BICM interleaver, mapper, SSD and MIMO STCs have their own flexibility parameters.

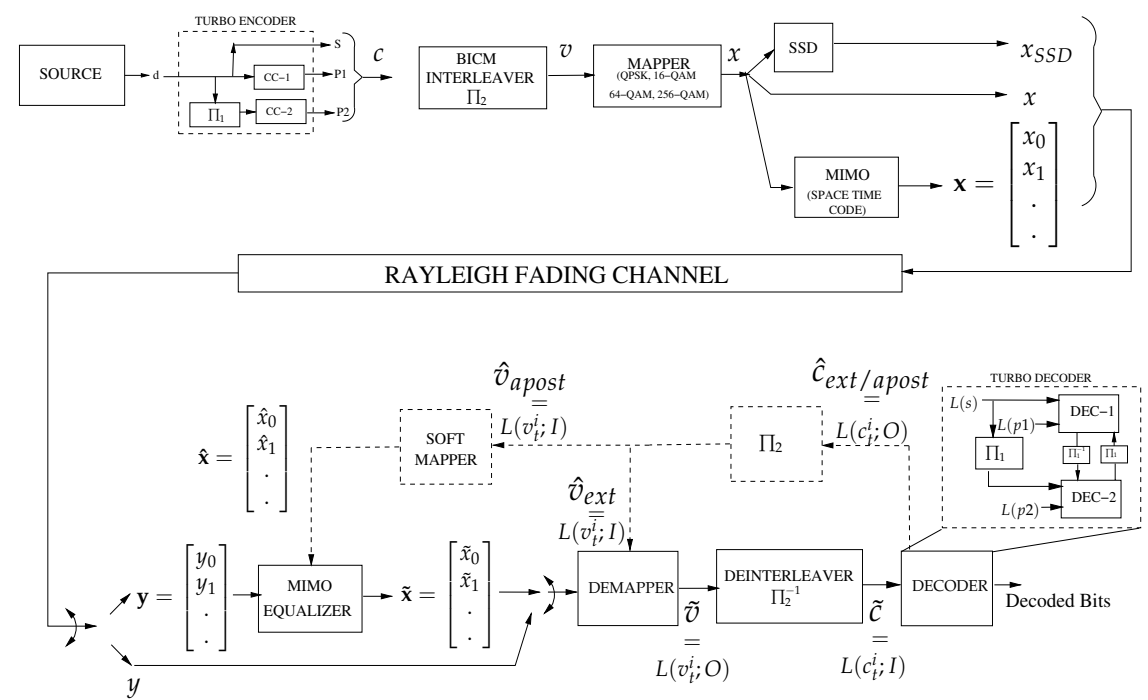

Fig. 1. System Diagram of a Modern Radio Platform

On the receiver side there are blocks such as MIMO equalizer, symbol demapper, deinterleaver and channel decoder. There are also the possibilities of applying iterative/turbo processing to achieve the error rate performance approaching theoretical limits e.g turbo decoding where decoders share the extrinsic information, turbo demodulation where there is an additional feedback exist between decoders and demapper and finally turbo equalization where there is a feedback also to the equalizer. On hardware side, three ASIPs dedicated for turbo decoding, demapping and MIMO equalization functions are required to achieve overall flexibility of the presented receiver. These three ASIPs are named as TurbASIP (Muller et al., 2009), DemASIP (Jafri, Baghdadi \& Jezequel, 2009), and EquASIP (Jafri, Karakolah, Baghdadi \& Jezequel, 2009). The design methodology adopted to conceive these ASIPs is comprised of four steps:

1. Step 1: Analysis of target application and underlined algorithms with respect to flexibility needs of multi-standard requirements.

2. Step 2: Derivation of architectural choices for the target flexibility and parallelism degree.

3. Step 3: Design of basic building blocks of the ASIP. Efficient resource usage and sharing are considered at this step. 
4. Step 4: Design of the complete architecture of the ASIP (including instruction-set, datapath, pipeline stages, memory banks, and I/O interfaces).

To address the severe time to market constraint, a rapid flow for ASIP modeling, validation, and FPGA prototyping is used. This flow is based on Processor Designer Framework from CoWare Inc. The whole process is divided into three abstraction levels where first one is LISA ADL, the second is the HDL level, and finally FPGA/ASIC implementation is last level of this rapid prototyping flow. In this chapter EquASIP is taken as an example to demonstrate the whole approach: (1) Four steps involved in wireless communication ASIP design and (2) Associated three abstraction levels of a rapid validation and FPGA prototyping flow.

\section{EquASIP: ASIP-based MMSE-IC linear equalizer}

The use of multiple antennas is recognized as a key enabling technology in high performance wireless communications. Most of emerging wireless standards, such as IEEE $802.11 \mathrm{~m}$, IEEE 802.16, and 3GPP LTE, propose the use of MIMO systems with different features and parameters. In these standards MIMO techniques such as time diversity and/or spatial multiplexing are specified. Diversity and/or multiplexing achieved through MIMO in different standards are summarized in Table 1. State of the art MIMO detection techniques

\begin{tabular}{|l|c|c|c|}
\hline MIMO Feature & IEEE 802.11n & IEEE 802.16e & 3GPP-LTE \\
\hline Time Diversity(Alamouti) & $\checkmark$ & $\checkmark$ & $\checkmark$ \\
\hline Spatial Multiplexing & $\checkmark$ & $\checkmark$ & $\checkmark$ \\
\hline Golden Code & & $\checkmark$ & \\
\hline $\begin{array}{l}\text { Mixed Diversity/ } \\
\text { Multiplexing }\end{array}$ & & $\checkmark$ & $\checkmark$ \\
\hline
\end{tabular}

Table 1. Multi Standard MIMO Support

can be classified in three categories (Burg et al., 2005): Maximum Likelihood (ML) detection, Sphere Decoding (SD), and linear filtering based detection. The complexity of ML detection increases exponentially with the number of antennas and modulation order. The SD approach has a polynomial complexity. To perform SD, first a QR decomposition of channel matrix is carried out and then tree exploration is performed. This tree search is further categorized as depth-first and breadth-first methods. The depth-first has a reduced area complexity and optimal performance, but has variable throughput with SNR. In breath-first case, the most famous algorithm is the $K$-best in which $K$ best nodes are visited at each level. Hence, the complexity depends on $K$. A large value of $K$ results in high complexity and good performance. Linear filtering based solutions such as Minimum Mean Squared Error Interference cancellation (MMSE-IC), considerably reduce the complexity of the hardware implementation of a MIMO detector. Whereas the compensation for sub-optimality can be achieved using an iterative equalization with the channel decoder.

In linear filtering based solution, matrix inversion implying complex numbered operations is the most demanding computational task. Hence, most of the existing work has been focused on the inversion of variable-sized complex-numbered matrices. Matrix inversion based on QR Decomposition Recursive Least Square (QRD-RLS) algorithm has been proposed (Karkooti et al., 2005). In (Myllyla et al., 2005), authors have proposed a Coordinate Rotation Digital Computer (CORDIC) and Squared Givens Rotation (SGR) based Linear MMSE detector while in (Edman \& Öwall, 2005) a linear array architecture for SGR implementation has been 
introduced. Matrix inversion through block-wise analytical method has been implemented in (Eilert et al., 2007). Two separate MMSE-IC2 equalizers for $4 \times 4$ turbo MIMO SM environment using QPSK and QAM-16 modulations, implementing CORDIC method of QR decomposition, have been proposed in (Boher et al., 2008) for fast fading applications. Using analytic method of matrix inversion, a fully dedicated architecture for MMSE-IC1 LE for $2 \times 2$ turbo MIMO system with pre-coding used in quasi static channel has been proposed in (Karakolah et al., 2009). The other work carried out in (Kim et al., 2008) shows exciting results in terms of throughput for 802.11n MIMO-OFDM application. The implementation is based on a inverse free architecture using square-root MMSE formulation.

To the best of our knowledge all the available implementations target a specific STC with limited modulation support. In the following sections, the process of developing a flexible MMSE-IC equalizer using the ASIP approach and conforming to multi-standard requirements is explained.

\subsection{Step 1: Algorithm analysis and flexibility requirements}

At the inputs of the equalizer, as shown in Fig. 1, the received symbol vector $\mathbf{y}$ is given by the following expression.

$$
\mathbf{y}=\mathbf{H} \mathbf{x}+\mathbf{j}
$$

where $\mathbf{y}$ is a vector of size of number of receive antennas $\left(N_{r}\right), \mathbf{x}$ is a vector of size of number of transmit antennas $\left(N_{t}\right), \mathbf{H}$ is channel matrix of size $N_{r} \times N_{t}$ and the $\mathbf{j}$ is column vector of Additive White Gaussian Noise(AWGN) of size $N_{r}$. The output $\tilde{x}$ of the MMSE-IC equalizer using time invariant approximation as proposed by (Laot et al., 2005) is given by:

$$
\tilde{x}_{k}=\lambda_{k} \mathbf{p}_{k}^{H}\left(\mathbf{y}-\mathbf{H} \hat{\mathbf{x}}+\hat{x}_{k} \mathbf{h}_{k}\right)
$$

where $k=1,2, \ldots N_{t}, \hat{\mathbf{x}}$ is vector of decoded symbols of size $N_{t}$ and $\hat{x}_{k}$ is $k^{t h}$ element of this vector, $\mathbf{h}_{k}$ is $k^{\text {th }}$ column of $\mathbf{H}$ matrix and (.) ${ }^{H}$ is Hermitian operator. The other parameters $\lambda_{k}$, and $\mathbf{p}_{k}$ are given by:

$$
\begin{gathered}
\mathbf{p}_{k}=\mathbf{E}^{-1} \mathbf{h}_{k} \\
\mathbf{E}=\left(\sigma_{x}^{2}-\sigma_{\hat{x}}^{2}\right) \mathbf{H} \mathbf{H}^{H}+\sigma_{w}^{2} \mathbf{I}
\end{gathered}
$$

where $\sigma_{x}^{2}, \sigma_{\hat{x}}^{2}$ and $\sigma_{w}^{2}$ are variances of transmitted symbols, decoded symbols and noise. I is identity matrix.

$$
\lambda_{k}=\frac{\sigma_{x}^{2}}{1+\sigma_{\hat{x}}^{2} \beta_{k}} \text { where } \beta_{k}=\mathbf{p}_{k}^{H} \mathbf{h}_{k}
$$

Equation (2) can be rewritten in the form

$$
\begin{aligned}
\tilde{x}_{k} & =\lambda_{k} \mathbf{p}_{k}^{H}(\mathbf{y}-\mathbf{H} \hat{\mathbf{x}})+\lambda_{k} \mathbf{p}_{k}^{H} \hat{x}_{k} \mathbf{h}_{k} \\
& =\lambda_{k} \mathbf{p}_{k}^{H}(\mathbf{y}-\mathbf{H} \hat{\mathbf{x}})+g_{k} \hat{x}_{k}
\end{aligned}
$$

where $g_{k}=\lambda_{k} \beta_{k}$ is equivalent bias of AWGN noise whose real part is used in demapper.

Once we correlate these equations of MMSE-IC algorithm to the needs of multi-standard requirements, following are the three considered sources in extracting the flexibility parameters:

- MIMO STC supported at the transmitter

- Time diversity of the channel i.e quasi static, block fading and fast fading

- Possibility of iterative equalization in the receiver 


\subsubsection{MIMO STC}

MIMO Spatial multiplexing (SM), Alamouti code and Golden code are the STCs adopted in emerging wireless standards. For MIMO SM with different antenna dimensions, such as $2 \times 2,3 \times 3$ and $4 \times 4$, the expressions ( 3 to 6 ) can directly be implemented using channel matrix and received vector inputs. Hence, a hardware capable of implementing variable sized complex matrix operation involved in the algorithm can address MIMO SM from 2 to 4 antennas. As far as Golden code and Alamouti code are concerned, MMSE-IC algorithm can be used by applying equivalent channel transformations on the inputs prior to their use. In case of $2 \times 2$ Golden code, the equivalent channel transformation is presented in (Cavalec et al., 2008). The idea is to treat two transmitted vectors (each having two elements) as one transmission of four symbols. By applying equivalent channel transformation, the inputs to the MMSE-IC equalizer are $\mathbf{y}$ of four elements and an equivalent channel matrix $\breve{\mathbf{H}}$ of size $4 \times 4$. The equivalent channel transformation of Alamouti code is presented in (BOUVET, 2005) which transforms a $2 \times 1$ channel matrix into a $2 \times 2$ equivalent matrix and $2 \times 2$ channel matrix into a $4 \times 4$ equivalent matrix. Hence, supporting MIMO SM with an additional capability of equivalent channel transformation, addresses this first source of flexibility parameters.

\subsubsection{Time diversity}

The time diversity of the channel decides how frequent the computations of equalization coefficients (Equation 3 to Equation 5) is required. For quasi static channel these coefficients are computed once per iteration whereas for fast fading channel they are computed for each received vector per iteration. In case of block fading, these coefficients are computed for a set of received vectors for which channel matrix is considered as constant.

\subsubsection{Iterative equalization}

The last source of flexibility is the iterative/non-iterative nature of the equalizer. In an iterative context the equalizer must incorporate the a priori information.

\subsection{Step 2: Architectural choices}

In the MMSE-IC algorithm, one can note that the expressions computing equalization coefficients and symbol estimation exhibit similar arithmetic operations. Now considering the flexibility need related to time diversity of the channel, allocating separate resources for equalization coefficients computation will result in an inefficient architecture in case of quasi static and block fading channel. For this reason, and targeting flexibility as well as efficiency, our first architectural choice is based on hardware resource sharing between these two tasks. Out of these two distinctive parts of the algorithm, the one related to equalization coefficient computation is more resource demanding. In fact, in this part of the algorithm, the implied computations can only be done in a serial order. For example, to compute matrix $E^{-1}$ of Equation 3, one need to compute:

- Hermitian of $\mathbf{H}$

- Matrix multiplication $\mathbf{H H}^{\mathbf{H}}$

- Scaler-Matrix multiplication

- Matrix addition

- Matrix inversion 


$$
X=a+b j \quad Y=c+d j
$$
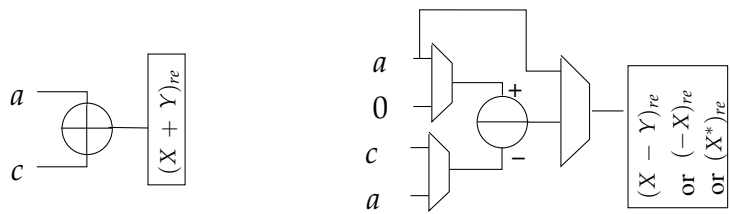

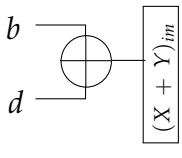

(a)

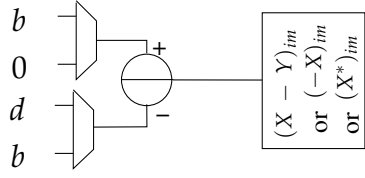

(b)

Fig. 2. Basic components (a) Complex adder (b) Complex subtracter, negater and conjugator

The other metrics (such as $\beta_{k}, \lambda_{k}$ and $g_{k}$ ) are computed with a similar pattern. For this kind of serial computations, temporal parallelism implementation through pipelining can be applied to increase throughput. Now considering the flexibility need related to STC, allocating hardware resources according to the requirements of the most complex STC configuration will result in an inefficient architecture for the low complexity configurations. For this reason, our second architectural choice is based on dimensioning the hardware resources in order to be fully used in all STC configurations. In this regard, the implied complex matrix operations are analyzed and broken down into basic arithmetic operations. Then adequate hardware operators are constructed considering the best tradeoff between flexibility, parallelism and hardware efficiency.

\subsection{Step 3: Design of basic building blocks}

In this section, a bottom-up presentation approach is adopted to explain the proposed hardware architecture capable of performing complex operations through the basic arithmetic operators. In the first part of this section, we will propose the architectures for the basic complex number operators (performing addition, subtraction, negation, conjugation, inversion) which provide maximum resource sharing. Later on, complex matrix operation, achieved through execution of basic complex number operations (performing multiplication, hermitian and inversion) will be presented.

\subsubsection{Complex number operations}

In MMSE-IC algorithms, the complex matrix operations can be broken down into basic complex number operation such as addition, subtraction, negation, conjugation and inversion. To perform each operation the architecture of the operator is detailed below.

\subsubsection{Complex number addition, subtraction, negation and conjugate}

The complex number addition needs two real adders whereas a complex numbered subtraction needs two real subtracters. Using two real subtracters, negation of a complex number can be performed. Similarly, conjugate of a complex number, required in calculating the hermitian of a matrix can also share the real subtracter. Fig. 2(a) shows hardware architecture for addition of two complex numbers $X=a+j b$ and $Y=c+j d$ whereas Fig. 2(b) 


$$
X=a+b j \quad Y=c+d j
$$

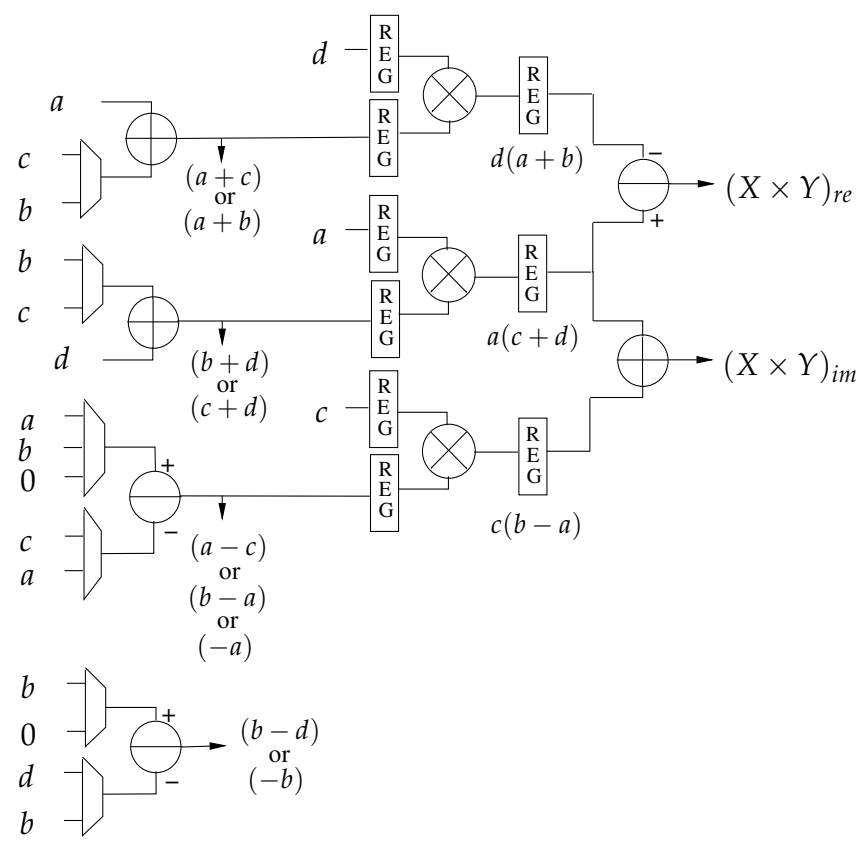

Fig. 3. Combined Complex Adder Subtracter and Multiplier (CCASM)

shows combined architecture of subtraction of $X$ and $Y$ and negation/hermitian of a complex number $X$.

\subsubsection{Complex number multiplication}

By applying the classical formula (7) of multiplication of complex numbers, a complex numbers multiplier must perform 4 real multiplications and 2 real additions/subtractions.

$$
X \times Y=(a+j b)(c+j d)=(a c-b d)+j(a d+b c)
$$

A rearrangement may be proposed to reduce the number of multiplications required, as:

$$
X \times Y=(a+j b)(c+j d)=a(c+d)-d(a+b)+j[a(c+d)+c(b-a)]
$$

By applying this reformulation, a complex number multiplier must perform only three real multiplications and 5 real additions/subtractions. Reducing one real multiplier per complex multiplier at the cost of three adders significantly reduces the complexity of the complex number multiplier. In addition the adders and subtracters of first stage of pipelined multipliers can also be used for complex number addition, subtraction, negation and conjugation. A Combined Complex Adder Subtracter and Multiplier (CCASM) is shown in Fig. 3. This architecture is capable of performing all basic operation of complex number addition, subtraction, negation, conjugation (output at first stage of pipeline) and multiplication (output at third stage of pipeline). 


\subsubsection{Complex number inversion}

The inverse of a complex number can be computed using following expression:

$$
\frac{1}{a+b j}=\frac{a}{a^{2}+b^{2}}-\frac{b}{a^{2}+b^{2}} j
$$

The architecture for this inverter can be obtained by reusing the real multipliers and one adder of the CCASM to compute $a^{2}+b^{2}$. Pre-computed LUT can then be used to find inversion value of $\frac{1}{a^{2}+b^{2}}$. Finally, two real multipliers and one subtracter are required for final result computation.

\subsubsection{Complex matrix operations}

In this subsection we propose the use of basic operators, developed in previous part, to achieve complex numbered matrix operations such as matrix hermitian, multiplication and inversion.

2.3.2.1 Matrix hermitian, addition, subtraction, negation

To perform hermitian operation on a matrix, at first, one need to copy the rows of the matrix into columns of an intermediate matrix. Then by taking complex conjugate of each element of this intermediate matrix, the resultant matrix will be the required hermitian matrix. Using 4 instances of the architecture presented in Fig. 2 with some control logic, provides a fully parallel and flexible architecture to perform Matrix Hermitian, Addition, Subtraction and Negation operations for $2 \times 2$ and $4 \times 4$ matrices. In case of $3 \times 3$ matrix this architecture will be $75 \%$ efficient. Hence, to perform any of these operation on $2 \times 2,3 \times 3$ and $4 \times 4$ matrices, 1,3 and 4 clock cycles will be required.

\subsubsection{Matrix multiplication}

To perform a multiplication of two $2 \times 2$ matrices, 8 complex multiplications are required whereas for $3 \times 3$ and $4 \times 4$ matrices the number of complex multiplications required are 27 and 64 respectively. Use of four CCASM (Fig. 3), can efficiently perform all operations (matrix hermitian, addition, subtraction, negation and multiplication) required for $2 \times 2$ and $4 \times 4$ matrices. For $2 \times 2$ matrix multiplications, two complex adders will be required to sum up the multiplication results whereas in $4 \times 4$ case, in addition to two complex adders, one more adder will be required. The architecture of $2 \times 2$ and $4 \times 4$ matrix multiplications is shown in Fig. 4 . The number of cycles required to perform $2 \times 2,3 \times 3$ and $4 \times 4$ matrix multiplications will be 2,9 and 16 respectively.

\subsubsection{Matrix inversion}

The matrix inversion can be achieved through one of the following methods:

- based on matrix triangulation

- based on analytical method

The first method based on matrix triangulation can realized using systolic architecture through the LU decomposition, Cholesky decomposition or QR decomposition. The method based on QR decomposition is the most interesting due to its numerical stability and its practical feasibility. It consists of decomposing decompose a matrix $\mathbf{A}$ of size $N \times N$ as $\mathbf{A}=\mathbf{Q} \mathbf{R}$ where $\mathbf{Q}$ is an orthogonal matrix $\left(\mathbf{Q} \mathbf{Q}^{H}=\mathbf{I}\right)$ and $\mathbf{R}$ an upper triangular matrix. This decomposition allows to compute the inverse of the matrix $\mathbf{A}$ after a simple inversion of the 


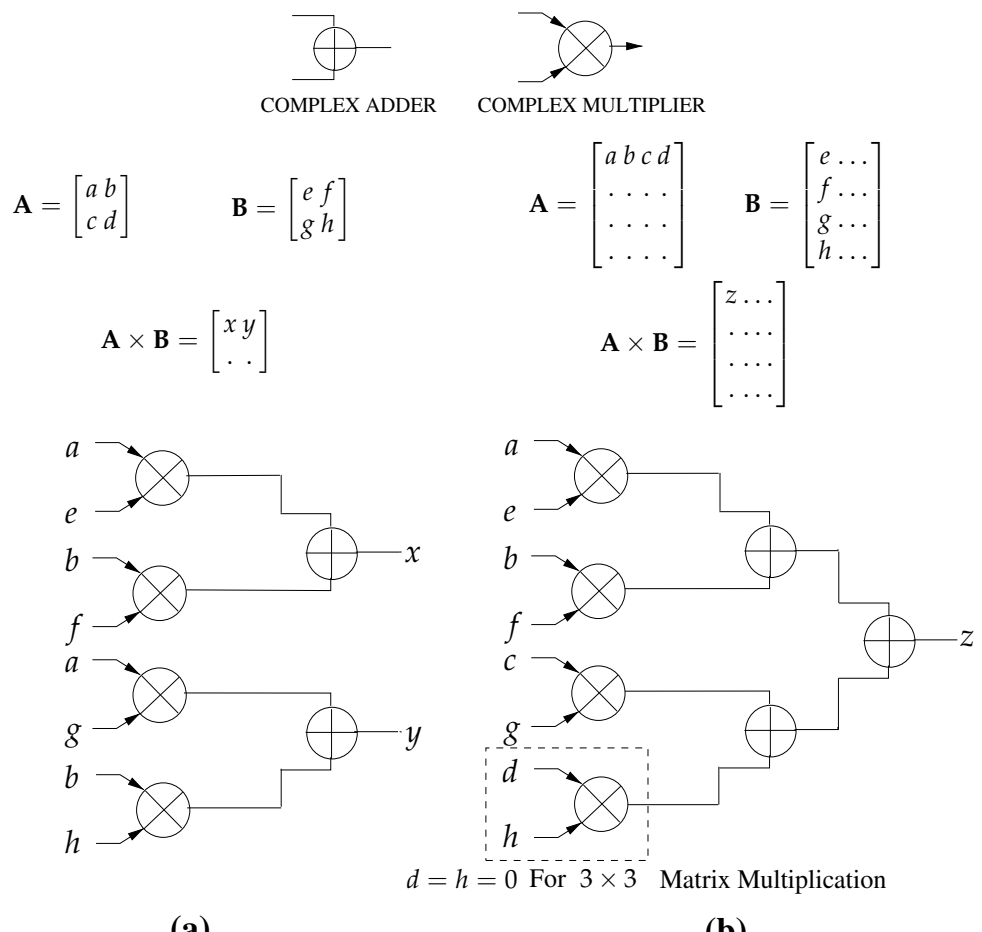

(a)

(b)

Fig. 4. Complex matrix multiplications (a) $2 \times 2$ Matrix multiplication (b) $3 \times 3$ and $4 \times 4$ Matrix multiplication

triangular matrix $\mathbf{R}$ and a matrix multiplication as $\mathbf{A}^{-1}=\mathbf{R}^{-1} \mathbf{Q}$. There are several methods (Golub \& van Van Loan, 1996) to achieve this decomposition, such as the Givens method or the method of Gram-Schmidt. Hardware designers give special attention to the Givens method due to its practical feasibility, its parallelism and its numerical stability (Myllyla et al., 2005)(Edman \& Öwall, 2005). The method of Givens consists of triangularization of matrix A by applying a series of plane rotations called Givens rotations. Each rotation is designed to cancel an element of $\mathbf{A}$. The standard method of Givens uses operations that are not easily implementable, including square root and division. Therefore, there are several variants of this method to avoid these operations. The SGR (Squared Givens Rotations) (Döhler, 1991) and CORDIC method (Volder, 1959) are the best known methods. A comparison between the two approaches: SGR and CORDIC has been made by (Myllyla et al., 2005) through MMSE detector. The results show that the CORDIC-based architecture is more expensive in hardware cost and is 1.5 times slower than those based on SGR. In his thesis work, Edman (Edman, 2006) used SGR method to achieve matrix inversion and studied both triangular and linear architectures. For this type of architecture there are dedicated Processing Elements (PEs) which are used as boundary elements and internal elements of a systolic array or linear array (Edman \& Öwall, 2005). Although linear array architecture is flexible for variable sized matrix inversion, it is dedicated to matrix inversion only.

The analytic method of matrix inversion is good candidate, not only for variable sized matrix inversion but also for resource reuse for other matrix computations. The expression for the 
inversion of $2 \times 2$ matrix through analytical method is given by:

$$
\left[\begin{array}{ll}
a & b \\
c & d
\end{array}\right]^{-1}=\frac{1}{a d-b c}\left[\begin{array}{cc}
d & -b \\
-c & a
\end{array}\right]
$$

To implement Equation 10 the resources required are a complex number negater and a complex divider. For a $4 \times 4$ matrix, the matrix is divided into four $2 \times 2$ matrix and inversion can be achieved block wise.

$$
\left[\begin{array}{ll}
A & B \\
C & D
\end{array}\right]^{-1}=\left[\begin{array}{ll}
W & X \\
Y & Z
\end{array}\right]
$$

where

$$
\begin{aligned}
W & =A^{-1}+A^{-1} B\left(D-C A^{-1} B\right)^{-1} C A^{-1} \\
X & =-A^{-1} B\left(D-C A^{-1} B\right)^{-1} \\
Y & =-\left(D-C A^{-1} B\right)^{-1} C A^{-1} \\
Z & =\left(D-C A^{-1} B\right)^{-1}
\end{aligned}
$$

The inversion of a $3 \times 3$ matrix is performed by extending it to a $4 \times 4$ matrix. This can be done by copying all three rows of $3 \times 3$ matrix into first three rows of $4 \times 4$ matrix and then putting zeros in all elements of fourth row and fourth column where a 1 should be put on the intersection of fourth row and fourth column. The inversion can then be performed using the method mentioned above. The final result lies in first three elements of first three rows (or column). All the expressions involved in the inversion of up to $4 \times 4$ matrix can be achieved through already described matrix operations and will be used in the EquASIP.

\subsubsection{Operator reuse in fixed-point representation}

To find the required data width for fixed-point representation of the parameters involved in MMSE-IC algorithm, long simulations have been conducted for all supported system configurations (STC and modulation type). Results analysis have shown that at maximum 16-bit signed representation with different bits for integer and fractional part is sufficient for all the parameters involved during the different computational steps of MMSE-IC LE algorithm. To enable the reuse of hardware resources for these different computations, involving operands with different fixed-point representations, certain rules have been set. First of all, while reading input data from memories, the data which is represented in less than 16-bits, is sign extended to 16-bit. Secondly, a programmable 33 to 16-bit conversion is performed at the outputs of the multipliers. Last of all, to avoid the hazards caused by overflow/underflow during an arithmetic operation, a control mechanism is provided to fix the output at its maximum/minimum limit.

\subsection{Step 4: Design of the complete architecture of EquASIP}

In this step granularity level of basic building blocks is increased to achieve the data path whereas this datapath is distributed over several pipeline stages to reduce the critical path. Other components such as memory banks are added to achieve complete architecture of the ASIP. The proposed EquASIP architecture is mainly composed of Matrix Register Banks (MRB), Complex Arithmetic Unit (CAU) and Control Unit (CU) besides its memory interfaces. The input to the EquASIP are through "Channel Data Memory" and the soft mapper as 


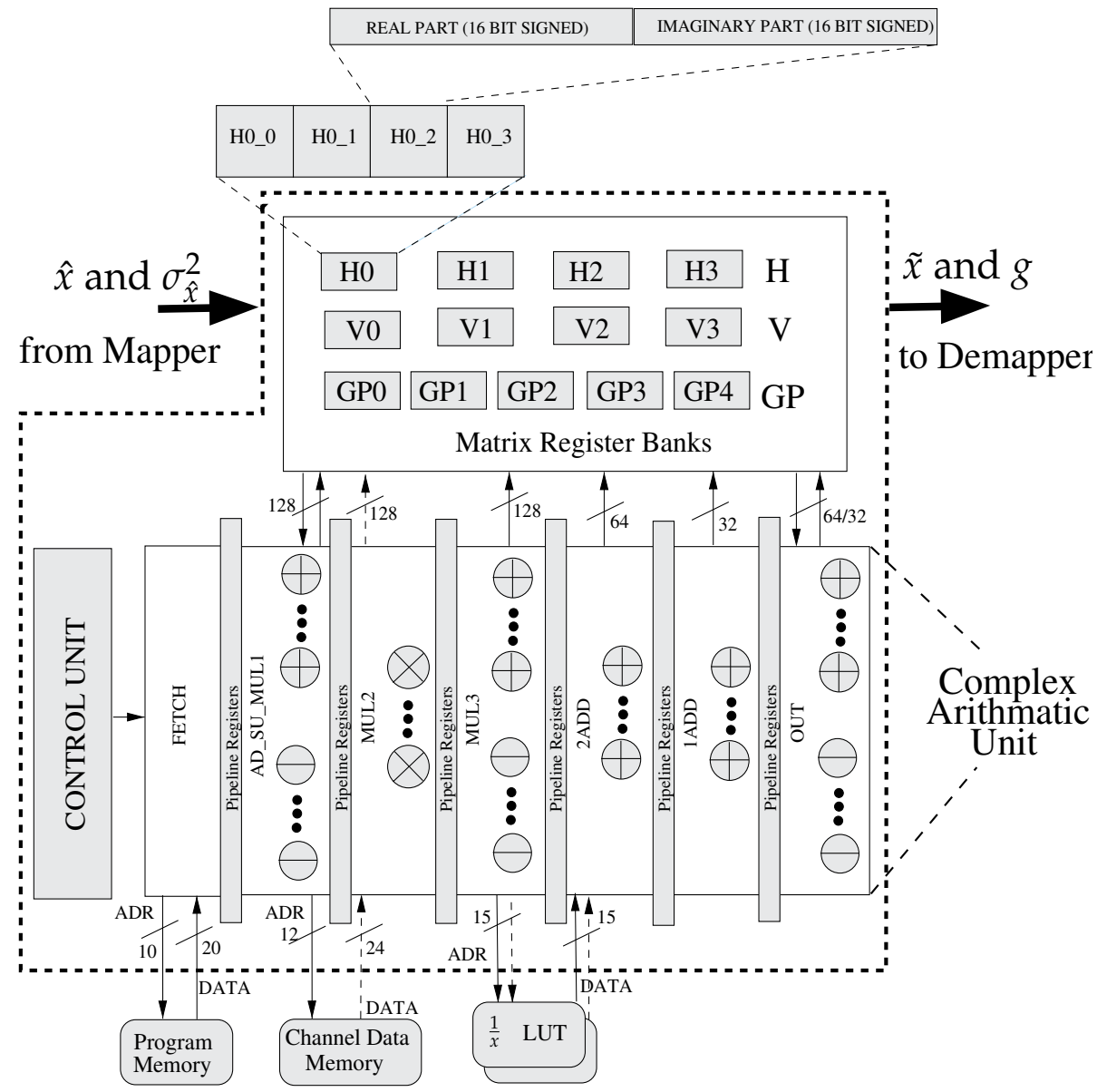

Fig. 5. EquASIP block diagram

shown in Fig. 5. The data bus of all inputs is set to 16 (32 bit for complex number). This provides flexibility to use up to 16 bit data representation and in case of smaller data widths, signed/unsigned extension can be done externally. The ASIP has 7 pipeline stages named as: FETCH, AD_SU_MUL1, MUL2, MUL3, 2ADD, 1ADD and OUT.

\subsubsection{Matrix register banks}

To store a complex number two separate 16-bit registers have been used, one storing the real and the other imaginary part. Based on the requirements of the Equation 6 for a $4 \times 4$ spatially multiplexed MIMO system, 13 MRBs have been proposed, where each MRB can store 4 complex numbers (Fig. 5). H-MRB (H0, H1, H2, and H3) which are connected to the memory, can store 4 rows or columns of Channel Matrix. Four V-MRB (V0, V1, V2, and V3) store 16 entries of $\lambda_{k} p_{k}$. GP0, GP1, GP2, GP3 and GP4 are assigned to the storage of $g_{j}, \hat{x}_{j}, y, g_{j} \hat{x}_{j}$ and the estimated symbols $\tilde{x}$ respectively. Other than this specific use, these GP registers save the intermediate results of equalization coefficients. Among other registers there are three registers to store the variances of noise, modulation symbol and decoded symbols besides pipeline registers and the registers for REPEAT instruction. 


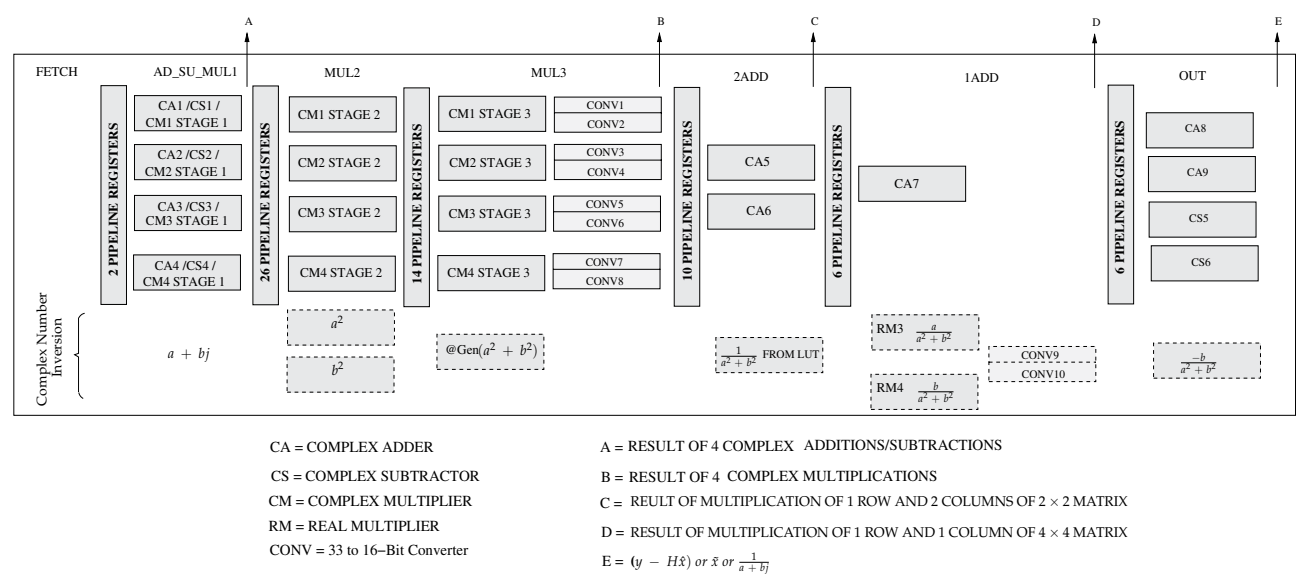

Fig. 6. CAU and pipeline stages

\subsubsection{Complex arithmetic unit}

The computational resources of the Complex Arithmetic Unit (CAU) of EquASIP are shown in Fig. 6. After fetch pipeline stage, 4 CCASM units (Fig. 3) are spread over three pipeline stages to perform 4 concurrent complex additions, complex subtractions/negation, complex conjugation and complex multiplications. The results of complex addition, subtraction, negation and conjugate operations are copied into destination registers in AD_SU_MUL1 pipeline stage. In MUL3 stage, 33-bit to 16-bit transformation is performed according to the information provided in multiply instruction. The results of four complex multiplication (16-bits for each of real and imaginary part of the complex number) are saved in the target registers. To perform $2 \times 2$ matrix multiplication one row/column of first matrix is introduced twice at first input of CCASMs and two columns/rows of second matrix are exposed to the second input of CCASMs. Providing the results of four complex multiplication to two complex adders in 2ADD pipeline stage, the output will give one resultant row/column of multiplication of $2 \times 2$ matrix. In case of $4 \times 4$ matrix multiplication, one row/column from each matrix goes to the inputs of four CCASM. The results of four multiplications are added together using 2 adders of $2 \mathrm{ADD}$ and one adder of $1 \mathrm{ADD}$ pipeline stage to output one element of $4 \times 4$ matrix multiplication. Complex adders/subtracters in last pipeline stage are used in the computation of Equation 6. The inversion process of a complex number in different pipeline stages is shown as dotted area in Fig. 6. For this particular operation, additional resources are required as Look-Up Tables (LUT), two 33 to 16-bit converters, and two real multipliers.

\subsubsection{Control unit}

The EquASIP control unit works as administrator of the 7-stage pipelined CAU as mentioned above and shown in (Fig. 5). It controls the flow of the program instructions over the designed datapath (MRBs, CAU) during the different stages of the pipeline. The functioning of the control unit will be reflected during the instruction set presentation which is detailed in the next section.

\subsubsection{EquASIP instruction set}

The instructions of the proposed ASIP are categorized as follows: 


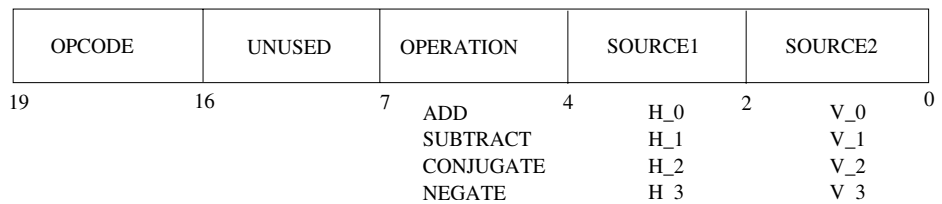

Fig. 7. 20-bit Addition, subtraction, negation and conjugate instructions

\subsubsection{LOAD, MOVE, REPEAT, NOP}

LOAD instruction is used to load channel matrix into H-MRB from memory. While loading data there are possibilities for loading directly or loading after applying conjugation to support equivalent channel transformation. LOAD_CODE instruction is used to initialize the V-MRBs for values which are used in equivalent channel transformation for Golden code. The MOVE instruction is used to transfer data between MRBs whereas REPEAT instruction repeats a block of code as many times as given in REPEAT_SIZE Register. NOP instruction is used to add empty cycles during the execution of the program when required.

2.4.4.2 Matrix addition, subtraction, negation and conjugation instructions

The instruction format for addition, subtraction, negation and conjugation operation is shown in Fig. 7. Besides opcode, the other fields are the "OPERATION" field and two "SOURCE" fields to input two register banks in complex adders and subtracters. The "OPERATION" field of 3-bits indicates the following six different operations: ADD, SUBTRACT, CONJUGATE, NEGATE, MOV_REC and MOV_MOD.

ADD: Using ADD instruction, programmer can select any of the H-MRB as source1 and any of V-MRB as source2. The result of an addition is always saved in GP_0 MRB.

SUBTRACT: Using SUBTRACT instruction, any one of selected H-MRB and any of V-MRB are subtracted and result is always saved in GP_0 MRB.

CONJUGATE/NEGATE: In this single source instruction all four elements of one of the selected H-MRB are conjugated/negated and the results are copied in respective V-MRB i.e $\operatorname{V-MRB}(n)=$ Conjugate $/ \operatorname{Negate}(\mathrm{H}-\mathrm{MRB}(n))$ where $n$ can be any integer from 0 to 3 .

MOV_REV: This instruction copies the elements of $\mathrm{H}-\mathrm{MRB}(0)$, in reverse order, into V-MRB $(0)$ with second element in negative form. This is used to align the elements of $2 \times 2$ matrix (to be inverted) for a multiplication which results in its determinant. For example if $\mathrm{H}-\mathrm{MRB}(0)$ has a matrix $A$ with elements $a, b, c$ and $d$ (Equation 10) then V-MRB(0) will have elements $d,-c, b$ and $a$ after the execution of this instruction. To obtain determinant of $A(\operatorname{det}(A)=$ $a d-b c)$, one can multiply $\mathrm{H}-\mathrm{MRB}(0)$ with $\mathrm{V}-\mathrm{MRB}(0)$ and add the results of first two complex multiplications.

MOV_MOD:This instruction is to copy and rearrange the matrix $A$ (saved in H-MRB(0)) in $\mathrm{V}-\mathrm{MRB}(0)$ to a form required in the inversion of a $2 \times 2$ matrix (Equation 10) i.e. if $\mathrm{H}-\mathrm{MRB}(0)$ has a matrix $A$ with elements $a, b, c$ and $d$ then $\mathrm{V}-\mathrm{MRB}(0)$ will have elements $d,-b,-c$ and $a$ after the execution of this instruction

\subsubsection{MULTIPLY}

This category is the most demanding one in EquASIP instruction set. Different fields of the multiply instruction are detailed in Fig. 8(a). Eight different opcodes fall under this category to use complex multipliers for multiplication of $4 \times 4$ and $2 \times 2$ matrices (MULT4X4 and MULT2X2), multiplication of 4 complex numbers (MULT_CMPLX), 3 different MAC instructions (MAC1, MAC2 and MAC3)and two instructions to compute the output symbols 
(a)

\begin{tabular}{|c|c|c|c|c|}
\hline Opcode & Source 1 & Source 2 & 16Bit Select & Destination \\
\hline 4-Bit & $4-$ Bit & 4-Bit & $4-$ Bit & $4-$ Bit
\end{tabular}
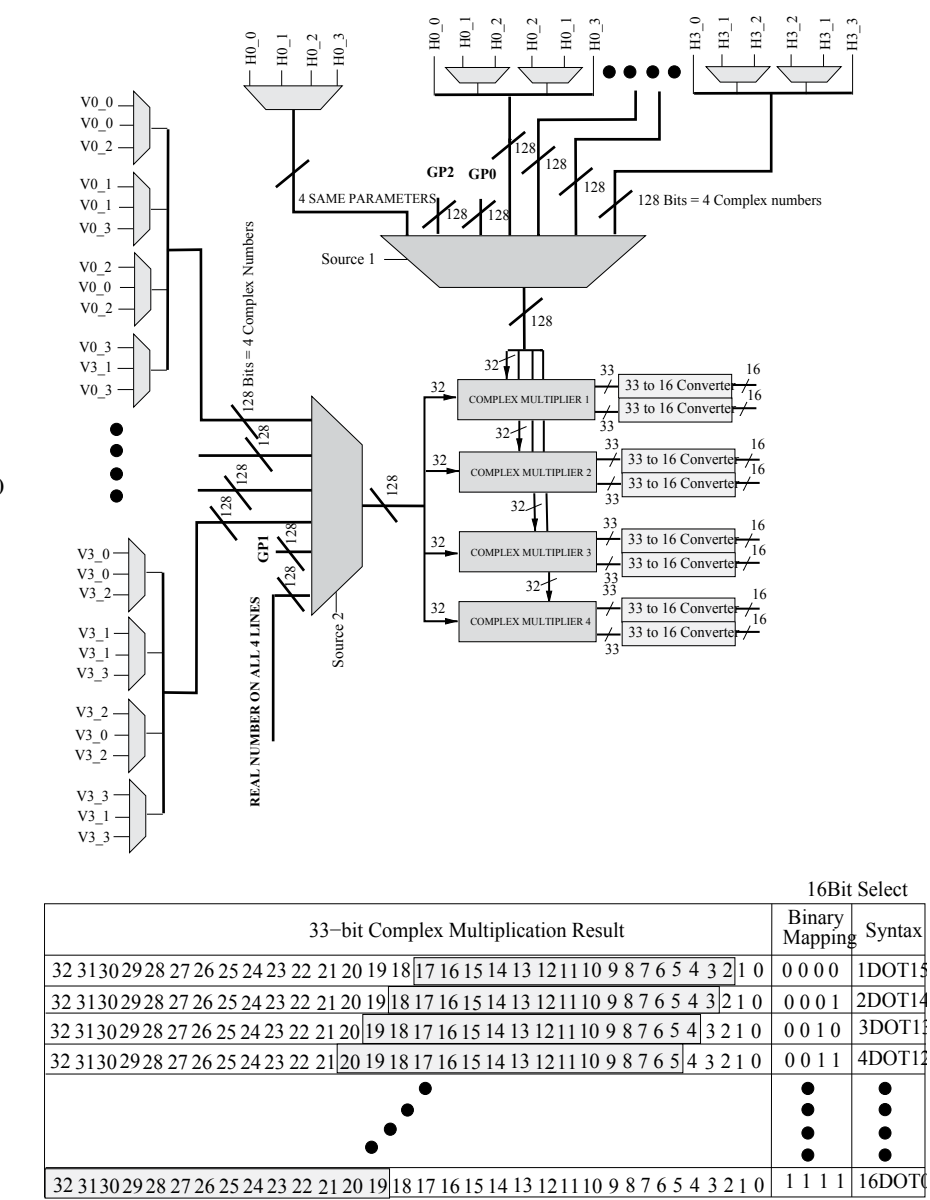

(c)

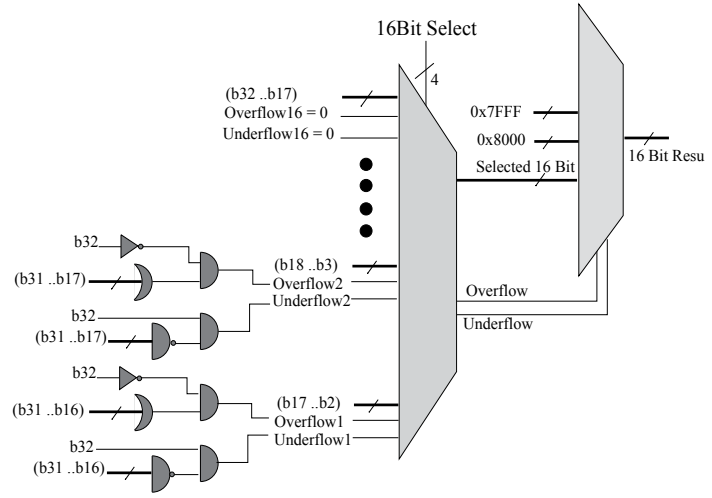

Fig. 8. Complex multiplication datapath: (a) 20-bit Multiply Instruction, (b) Possible inputs to complex multipliers, (c) 33 to 16-bit converter 
$\tilde{x}(\mathrm{OUT} 1$ and OUT2). The $3 \times 3$ matrix multiplication is achieved by $4 \times 4$ matrix multiplication by providing zero at the input lines of fourth CCASM.

Different possible sources to complex multipliers are shown in the Fig. 8(b). Depending upon the fields "Source1" and "Source2" of the instruction, 4 operands are selected as source1 and 4 as source 2 for 4 complex multipliers. To obtain different 16-bit fixed-point representations from 33-bit output of complex multipliers, 33 to 16-bit converters are designed. These converters (Fig. 8(c)) select 16 consecutive bits from 33-bit multiplication result depending upon the "16-Bit Control" field of the instruction. A combinational logic has also been provided to detect overflow/underflow with each choice of bit selection and consequently saturate the output value to maximum/minimum bounds. The "Destination" field of instruction selects the destination for the result.

\subsubsection{DIVIDE}

Two divide instructions have been defined. The first one is the division of a real number while the second one is used to invert a complex number. The first operation during execution of complex number division starts in the third stage of the pipeline to use the real multipliers. LUTs have been used to store the inversion values. The overall operation is shown as dotted area of Fig. 6.

\section{Rapid ASIP FPGA prototyping}

While selecting ASIP as the implementation approach, an ASIP design flow integrating hardware generation and corresponding software development tools (assembler, linker, debugger, etc.) is mandatory. In this chapter we consider the use of Processor Designer framework from Coware Inc. which enables the designer to describe the ASIP at LISA (Hoffmann et al., 2001) abstraction level and automates the generation of RTL model along with software development tools. ASIP design, validation and prototyping flow has been divided into 3 levels of abstraction as shown in Fig. 9 and is detailed in the following subsections.

\subsection{LISA abstraction level}

The first step towards the ASIP implementation is the LISA ADL modeling of the proposed architecture and the application program writing (.asm file) to be executed on the ASIP. To simulate the input data memories the contents of these memories, taken from the software reference model of the target application, are written in different sections of the assembly file as defined in the linker command file. With ADL model of the ASIP, Processor Designer framework generates tools like assembler, linker, processor debugger and simulator. Assembler and linker process the application program (.asm file) to generate the executable file (.out file) which is used in Processor Debugger to verify both the ASIP model and the application program. Once the ASIP is verified, a special utility "lvcdgen" can be used to generate Value Change Dump (VCD) file which store all registers content and ASIP output values during the application program execution. The generated VCD file can be used at lower abstraction levels for verification purpose. The "lvcdgen" utility uses Dynamic Simulator Object and executable file of the application to produce this reference VCD file. The complete flow is shown in Fig. 9(a). 


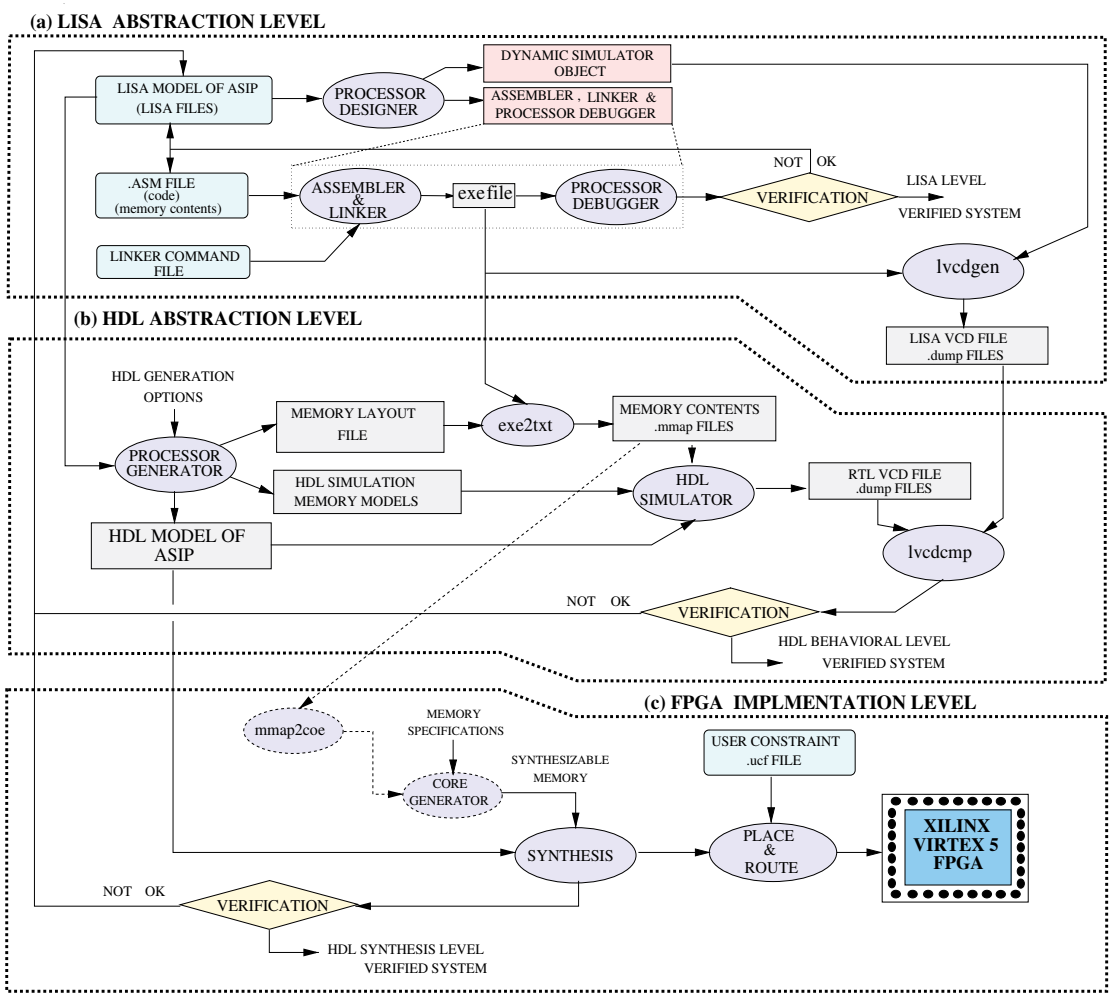

Fig. 9. Prototyping Flow: (a) LISA abstraction level, (b) HDL abstraction level, (c) FPGA implementation level

\subsection{HDL abstraction level}

Processor Designer framework provides the Processor Generator tool which is configured to generate HDL (VHDL/Verilog) model of the ASIP from LISA model, simulation models of memories and the memory layout file as shown in Fig. 9(b). The quality of the generated HDL depends upon the LISA modeling and the configuration options of Processor Generator. It is highly recommended that LISA modeling should be as close as possible to HDL, e.g if in one pipeline stage we want resource sharing, that resource should be declared once. Otherwise, due to inability to detect sharing, resources will be duplicated in HDL. Other issue is the use of high level operators of LISA which may not be produced by the Processor Generator e.g modulo two operation ("variable \% 2" in LISA) should be rather implemented by the LSB manipuation of the considered variable. For memory interface generation, different Memory Interface Definition Files (MIDF) are provided which define the number of ports and latencies. Once memory layout file and executable application program file is available, "exe2bin" utility inputs them to generate the contents of memories in separate .mmap files. With these three inputs (VHDL model, memory model and .mmap files), the VHDL model can be simulated behaviorally using an HDL simulator, e.g ModelSim by Mentor Graphics.

To run HDL simulation, Processor Generator produces ready-to-use Makefile which can be executed to see either the waveforms or to generate VCD file. To verify the generated ASIP HDL model, the VCD file generated through HDL model and the one generated through LISA model (in previous subsection) can be compared using "lvcdcmp" utility. 


\subsection{FPGA implementation level}

At this level, the only missing elements are the synthesizable memory models. Depending upon the FPGA selected, equivalent synthesizable memories are generated through FPGA vendor specific tools and at the same time .mmap memory content files have to be translated, if necessary, in required format for compatibility. With Xilinx devices, "Core Generator" tool can be used to generate the synthesizable memories and "mmaptocoe translator" converts .mmap files into required .coe format. With this complete synthesizable HDL model, synthesis can be performed as shown in Fig. 9(c). After successful synthesis, the placement and routing is performed as per the user constraints file (.ucf file). Inside .ucf file, the user inputs the platform dependent timing and location constraints, e.g the operational frequency and input/output pins. The final step is the generation of the configuration file which can be used to configure the FPGA for the final ASIP prototype model.

\subsection{EquASIP FPGA prototyping}

On board validation is a crucial step in order to demonstrate the feasibility, resolve any eventual system and/or environment issue, and measure the exact performance of the designed architecture. In our case, a logic emulation board (DN9000K10PCI) integrating 6 Xilinx Virtex 5 devices was available and has been used to validate the designed ASIPs. With this board, appropriate communication controllers are available and can be added to the design in order to read/write various output/input memories from a host computer using a USB interface. Using the Xilinx tool suite ISE, a new project was created integrating the ASIP, corresponding memories, and a board communication controller as shown in Fig. 10. The contents of the input memories i.e Channel Data Memory, $\frac{1}{x}$ LUTs and Mapper Output Memory were generated automatically from the fixed-point software reference model in .coe file format along with a reference result file containing the output of the equalizer. In this prototype, except Channel Data Memory and $\frac{1}{x}$ LUT which are synchronous, rest of the memories are asynchronous. Xilinx Virtex 5 device provides two type of memories, Distributed and Block Memories which can be customized for asynchronous and synchronous respectively. In order to record ASIP's results and to compare them with reference result file, a dual port Equalizer Output Memory has been created. One port of this memory is written with equalization results from EquASIP side and the other port is read by external host computer through USB interface. On this host computer, a graphical user interface with adapted parameters is used in order to setup the various parameters of the board and to download the output memory contents for comparison with reference result file.

\section{EquASIP results and performance}

By performing hardware synthesis and executing the application programs, performance of EquASIP is ascertained for different configurations and presented below.

\subsection{Synthesis results}

From the generated RTL description of EquASIP, logic synthesis has been conducted both on ASIC and FPGA. For ASIC target, the processor has been synthesized with Design Compiler tool from Synopsys. For FPGA target, Xilinx ISE tool has been used. In Table 2, the results of synthesis are summarized. 


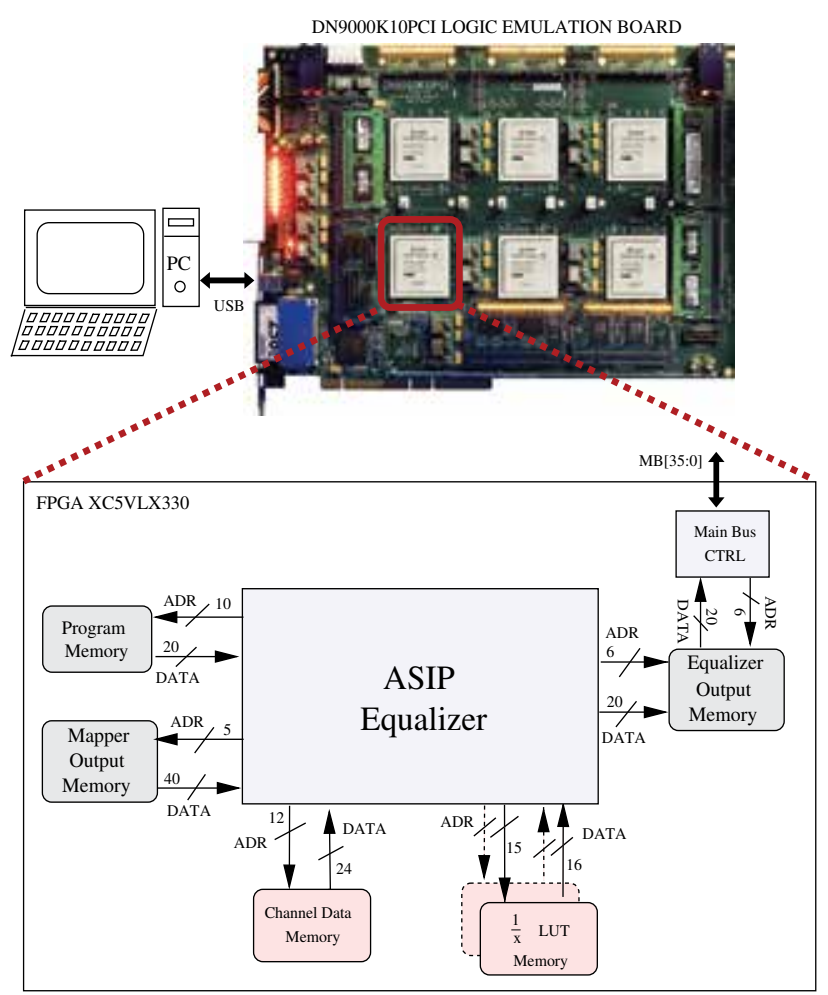

Fig. 10. EquASIP on-board prototype

\begin{tabular}{|l|c|}
\hline ASIC Synthesis Results (Synopsis Design Compiler) \\
\hline \hline Technology & ST $90 \mathrm{~nm}$ \\
\hline Conditions & Worst Case $\left(0.9 \mathrm{~V} ; 105^{\circ} \mathrm{C}\right)$ \\
\hline Area & $0.37 \mathrm{~mm}^{2}(84 \mathrm{~K} \mathrm{Gate})$ \\
\hline Frequency & $546 \mathrm{MHz}$ \\
\hline \hline FPGA Synthesis Results $($ Xilinx Virtex5 xc5vlx330) \\
\hline \hline Slice Registers & 3,174 out of $207,360(1 \%)$ \\
\hline Slice LUTs & 11,299 out of $207,360(5 \%)$ \\
\hline DSP48Es & 14 out of $192(7 \%)$ \\
\hline Frequency & $130 \mathrm{MHz}$ \\
\hline
\end{tabular}

Table 2. EquASIP synthesis results

\subsection{Execution performance}

To estimate the throughput of the EquASIP for different system configurations, the number of cycles required to compute the expressions involved in MMSE-IC1 are summarized in Table 3. Using this information, the user can estimate the throughput of the system under different channel's time diversity conditions and used STC. In case of quasi static conditions, after equalization coefficient computation, the throughput in terms of symbols per clock cycle is described in the last row of Table 3 . For a $3 \times 3$ MIMO SM configuration the symbol throughput is less than a $4 \times 4$ MIMO SM. This is due to the fact that for a $3 \times 3 \mathrm{MIMO}$ SM system $25 \%$ 


\begin{tabular}{|l|c|c|c|}
\hline Expression & $\begin{array}{c}\text { MIMO 2 } \times \mathbf{2} \\
(\text { Cycles })\end{array}$ & $\begin{array}{c}\text { MIMO 3 } \times \mathbf{3} \\
(\text { Cycles })\end{array}$ & $\begin{array}{c}\text { MIMO 4 } \times \mathbf{4} \\
(\text { Cycles })\end{array}$ \\
\hline$E$ (Ref. eq. 4) & 18 & 33 & 50 \\
\hline$E^{-1}$ & 14 & 68 & 68 \\
\hline $\mathbf{p}_{k}$ (Ref. eq. 3) & 12 & 26 & 39 \\
\hline$\beta_{k}$ (Ref. eq. 5) & 7 & 19 & 27 \\
\hline$\lambda_{k}$ (Ref. eq. 5) & 23 & 22 & 23 \\
\hline$\lambda_{j} \mathbf{p}_{k}^{H}, g_{k}$ (Ref. eq. 6) & 7 & 12 & 14 \\
\hline Total & $\mathbf{8 1}$ & $\mathbf{1 8 0}$ & $\mathbf{2 2 1}$ \\
\hline \hline Symbol $\tilde{x}$ Throughput (Ref. eq. 6) & 4 symbols/8 cycles & 3 symbol/11 cycles & 4 symbol/13 cycles \\
ASIC M Symbols/sec (@ 546 MHz) & 273 & 149 & 168 \\
FPGA M Symbols/sec (@ 130 MHz) & 65 & 35.45 & 40 \\
\hline
\end{tabular}

Table 3. EquASIP computation time for MMSE-IC $\mathrm{M}_{1}$ equations

of the resources are not used. This illustrates a typical tradeoff between flexibility, resource utilizations and system performance. The throughput for $2 \times 2$ Golden code is same as $4 \times 4$ SM.

\subsection{Comparison with state of the art}

In Table 4, different architectural parameters of state of the art implementations are summarized and compared with EquASIP implementation results. All of the referenced implementations present dedicated architecture for a specific system configuration except (Eilert et al., 2007) where the proposed architecture supports $2 \times 2$ and $4 \times 4$ matrix inversion. Table 4 is organized in such a way that first of all comparison is made with (Boher et al., 2008), (Kim et al., 2008) and (Karakolah et al., 2009) which provide a complete solution to generate estimated symbol vectors. Then comparison with (Myllyla et al., 2005) (providing solution to compute only the coefficient matrix of Equation 3) is tabulated. Finally, the EquASIP is compared with (Edman \& Öwall, 2005), (Karkooti et al., 2005) and (Eilert et al., 2007) which provide architectures only for matrix inversion. Furthermore, in order to make a fair comparison, the EquASIP was synthesized with the same target technology as used in the implementation with which it is being compared.

The work presented in (Boher et al., 2008) is aimed at achieving fast fading $4 \times 4 \mathrm{MIMO}$ SM using MMSE-IC. This implementation uses $\sigma_{\hat{x}}^{2}=0$ in first iteration and $\sigma_{\hat{x}}^{2}=\sigma_{x}^{2}$ in later iterations to simplify the architecture. However, while using in iterative context this assumption of perfect $\sigma_{\hat{x}}^{2}$ information induces a performance loss. Due to a fully pipelined architecture it outputs a vector containing four estimated symbols at every 38 clock cycle. Hence, the throughput is 1.31 Mega vectors at presented frequency. With EquASIP, working on same configuration, the cycles required for one symbol vector estimation are 234 . This results in a throughput of $0.5 \mathrm{Mega}$ vectors per second at considered frequency. Hence, the flexibility of EquASIP to support 5 different STC comes at the cost of 2.4 times less throughput, $53 \%$ more slice registers and 16 more dedicated multipliers compared to (Boher et al., 2008). In (Kim et al., 2008), the architecture implements $4 \times 4$ MIMO SM detector for $802.11 \mathrm{n}$ standard. In this application the design is made for a worst case scenario where for 48 vectors channel remains constant. To decode a frame of 48 vectors, the work in (Kim et al., 2008) takes 388 clock cycles. Which results in $17.3 \mathrm{M}$ vectors per second at a frequency of $140 \mathrm{MHz}$. When 


\begin{tabular}{|c|c|c|c|c|c|c|c|c|c|c|c|}
\hline \multirow{4}{*}{ Opr. } & \multirow{4}{*}{$\begin{array}{l}\text { System } \\
\text { Config. }\end{array}$} & \multirow{4}{*}{ Ref. } & \multirow{4}{*}{ Algorithm } & \multirow{4}{*}{$\begin{array}{l}\text { Target } \\
\text { Device }\end{array}$} & \multirow{4}{*}{\begin{tabular}{|l} 
Opr. \\
Freq. \\
$(\mathrm{MHz})$
\end{tabular}} & \multicolumn{4}{|c|}{ Hardware Resources } & \multirow{4}{*}{$\begin{array}{l}\text { Clock } \\
\text { Cycle }\end{array}$} & \multirow{4}{*}{$\begin{array}{c}\text { Throughput } \\
\text { (Mega } \\
\text { Operations } \\
\text { per sec) }\end{array}$} \\
\hline & & & & & & \multicolumn{3}{|c|}{ FPGA } & \multirow{3}{*}{\begin{tabular}{|c|} 
ASIC \\
Area \\
(K Gates) \\
\end{tabular}} & & \\
\hline & & & & & & \multicolumn{2}{|c|}{ Slice/LE } & \multirow{2}{*}{$\begin{array}{l}\text { HW } \\
\text { Mul. }\end{array}$} & & & \\
\hline & & & & & & Reg. & LUT & & & & \\
\hline \multirow{6}{*}{$\begin{array}{l}\text { MIMO } \\
\text { Symbol } \\
\text { Vector } \\
\text { Estim. }\end{array}$} & \multirow{2}{*}{\begin{tabular}{|c|}
$4 \times 4 \mathrm{SM}$ \\
Fast Fading \\
\end{tabular}} & (Boher et al., 2008) & QR CORDIC & \multirow{2}{*}{ Startix } & 50 & \multicolumn{2}{|c|}{8670} & 12 & - & 38 & 1.31 \\
\hline & & EquASIP & Analytical & & 120 & \multicolumn{2}{|c|}{13272} & 28 & - & 234 & 0.5 \\
\hline & \multirow{2}{*}{\begin{tabular}{|c|}
$4 \times 4$ SM \\
Block Fading
\end{tabular}} & (Kim et al, 2008) & QR CORDIC & \multirow{2}{*}{ Virtex-II } & 140 & \multicolumn{2}{|c|}{14166} & 103 & - & 388 & 17.31 \\
\hline & & EquASIP & Analytical & & 83 & \multicolumn{2}{|c|}{8477} & 14 & - & 845 & 4.71 \\
\hline & \multirow{2}{*}{\begin{tabular}{|c|}
$2 \times 2$ \\
PC \\
Quasi Static \\
\end{tabular}} & (Karakolah et al., 2009) & \multirow[t]{2}{*}{$\begin{array}{l}\text { Blockwise } \\
\text { Analytical }\end{array}$} & \multirow[t]{2}{*}{ Virtex-V } & 60 & 817 & 2715 & 60 & - & 1 & 120 \\
\hline & & EquASIP & & & 130 & 3174 & 11299 & 14 & - & 3.25 & 40 \\
\hline \multirow{6}{*}{$\begin{array}{c}\mathbf{p}_{k} \\
\text { Eq.3 }\end{array}$} & \multirow{3}{*}{$\begin{array}{c}2 \times 2 \\
\text { MIMO SM }\end{array}$} & \multirow{2}{*}{ (Myllyla et al., 2005) } & QR CORDIC & \multirow{6}{*}{ Virtex-II } & - & \multicolumn{2}{|c|}{11910} & 20 & - & 685 & - \\
\hline & & & QR SGR & & - & \multicolumn{2}{|c|}{6305} & 59 & - & 415 & - \\
\hline & & EquASIP & Analytical & & 83 & \multicolumn{2}{|c|}{8477} & 14 & - & 42 & 1.97 \\
\hline & $4 \times 4$ & (Mvllull al 205) & QR CORDIC & & - & & 805 & 44 & - & 3000 & - \\
\hline & MIMO SM & (Myliyla et al,, 2003) & QR SGR & & - & & - & - & - & - & - \\
\hline & & EquASIP & Analytical & & 83 & & 477 & 14 & - & 157 & 0.53 \\
\hline & & (Edman \& Öwall, 2005) & QR SGR & Virtex-II & 100 & 2224 & 2212 & - & - & 175 & 0.57 \\
\hline & & EquASIP & Analytical & VIILX-10 & 83 & 3177 & 15997 & 14 & - & 68 & 1.2 \\
\hline & & (Karkooti et al., 2005) & QRD-RLS & Virtex-IV & 115 & & 117 & 22 & - & 933 & 0.15 \\
\hline Matrix & $4 \times 4$ & & & Virtex-IV & 100 & 1716 & 2094 & 8 & - & 120 & 0.83 \\
\hline Inv. & Matrix & (Eilert et al., 2007) & Blockwise & $90 \mathrm{~nm}$ & 500 & - & - & - & 43 & 92 & 5.43 \\
\hline & & EquASIP & Analytical & \begin{tabular}{|l} 
Virtex-IV \\
\end{tabular} & 117 & 3232 & 16091 & 14 & - & 68 & 1.7 \\
\hline & & & & $90 \mathrm{~nm}$ & 546 & - & - & - & 85 & 68 & 8.02 \\
\hline
\end{tabular}

Table 4. EquASIP performance comparison

comparing with our work, this EquASIP consumes 221 clock cycles to compute equalization coefficient for a frame and 13 clock cycles for each vector estimation. Hence the total consumed clock cycles for 48 vectors estimation are $221+13 \times 48=845$ which results in a throughput of $4.7 \mathrm{M}$ vectors per second at a frequency of $83 \mathrm{MHz}$. Hence, throughput of the dedicated architecture of (Kim et al., 2008) is almost 3.6 times more at a cost of almost twice the FPGA slice used and 7.5 times more multipliers. Again this implementation is not flexible for variable antenna size, time selectivity of the channel and iterative nature of equalization. The realization of $2 \times 2$ MMSE-IC equalizer in (Karakolah et al., 2009) includes pre-coding (PC). The equivalent channel matrix becomes a $4 \times 4$ matrix shown below:

$$
\mathcal{H}=\left[\begin{array}{cccc}
h_{11} & h_{12} & 0 & 0 \\
h_{21} & h_{22} & 0 & 0 \\
0 & 0 & h_{11} & h_{12} \\
0 & 0 & h_{21} & h_{22}
\end{array}\right]
$$

The inversion of this matrix needs execution of two $2 \times 2$ matrix inversions. Other than this, to map this PC on the EquASIP, one $4 \times 4$ matrix multiplication is required to incorporate the PC matrix. The rest of the computations are same as required in $4 \times 4 \mathrm{MIMO}$ SM. Hence, to compute the equalization coefficients on EquASIP, 197 clock cycles will be consumed. For a target quasi-static environment, the EquASIP takes 197 cycles at $130 \mathrm{MHz}$ as compared to the dedicated architecture taking 20 cycles at $61 \mathrm{MHz}$ (Karakolah et al., 2009). This part is not crucial because it is computed once for a frame. The throughput of EquASIP is 40 Mega 
symbols per second and hence 3 times less than the dedicated architecture. The 3 times faster output of dedicated architecture comes at 5 times multipliers used and this architecture used 4 times less slice registers and LUTs.

The EquASIP is better both in area and performance when compared with (Myllyla et al., 2005). While comparing with (Edman \& Öwall, 2005), (Karkooti et al., 2005) and (Eilert et al., 2007), EquASIP outperforms these architectures in throughput. EquASIP occupies more area as compared to these dedicated implementations for matrix inversion as, besides its flexibility, EquASIP supports all functions required in MMSE-IC equalization algorithm.

In the above analysis, an attempt is made to compare dedicated and flexible architectures for MMSE-based equalization. In the presence of multiple system configurations and different variants of algorithms in the equalizer, EquASIP provides a promising flexible solution compared to dedicated implementations.

\section{Conclusion}

ASIP concept with associated efficient design and prototyping flows is emerging nowadays as a promising implementation solution for wireless communication applications. It is mainly the need to increase the flexibility and the opportunities for modular reuse that is pushing industry to use more and more software-programmable solutions for practically every class of digital components. Trade-offs between performance and flexibility can be tuned to the exact needs of the application, besides the already available design tools enable really efficient hardware synthesis and validation.

This chapter illustrates ASIP design and prototyping approach in wirless communication applications through a detailed example for MIMO detection. The first flexible ASIP implementing an MMSE-IC linear equalizer for turbo equalization application has been presented. Analysis and simulation of mathematical equations involved in MMSE-IC LE allowed to identify potential complex-numbered operations which lead to device the instruction set for the proposed EquASIP. The specific instructions for complex number arithmetic enable to efficiently perform computations on variable sized complex numbered matrices which in turn provide required flexibility in MMSE-IC and promote its reuse for other MMSE-based applications.

Flexibility of the presented EquASIP architecture allows its reuse for each of Alamouti code, Golden code, $2 \times 2,3 \times 3$ or $4 \times 4$ spatially multiplexed turbo MIMO application with BPSK, QPSK, 16-QAM, and 64-QAM. When targeting $90 \mathrm{~nm}$ technology, the proposed architecture enables a maximum throughput of $273 \mathrm{MSymbol} / \mathrm{sec}$ for $2 \times 2,148 \mathrm{MSymbol} / \mathrm{sec}$ for $3 \times 3$ and $168 \mathrm{MSymbol} / \mathrm{sec}$ for $4 \times 4 \mathrm{MIMO}$ systems. The presented original contribution demonstrates promising results using the ASIP approach to implement flexible, yet efficient, MMSE-based iterative MIMO equalizer.

Regarding research perspectives in this domain, the increasing low-power requirement should be considered as another optimizing design dimension to the ASIP solution for wireless communications applications. By inheriting the relevant low-power design techniques from already established low-power implementation schemes for programmable architectures, ASIP design flows can be optimized for power consumption. In the presented ASIP design example an attempt is made to provide maximum flexibility within the equalization application. This upper bound for flexibility implies similar bound for power consumption. Hence by examining the exact required flexibility for a particular system a trade-off can be achieved between flexibility and power demands. 


\section{Acknowledgment}

This work was supported in part by UDEC and TEROPP projects of the French Research Agency (ANR).

\section{References}

Boher, L., Rabineau, R. \& Hélard, M. (2008). Architecture and implmentation of an iterative receiver for MIMO systems, International Symposium on Turbo Codes and Realted Topics.

BOUVET, P.-J. (2005). Récepteurs itératifs pour systèmes multi-antennes, PhD thesis, INSA de Rennes France.

Burg, A., Borgmann, M., Wenk, M., Zellweger, M., Fichtner, W. \& Boelcskei, H. (2005). VLSI Implementation of MIMO Detection Using the Sphere Decoding Algorithm, IEEE Journal on Solid-State Circuits 40(3).

Cavalec, K. A., Sicot, G. \& Leroux, D. (2008). Reduced complexity near-optimal iterative receiver for Wimax full-rate space time code, 5th international symposium on Turbo Codes and related topics.

Döhler, R. (1991). Squared Givens rotation, IMA Journal of Numerical Analysis 11(1): 1-5.

Edman, F. (2006). Digital Hardware Aspects of Multiantenna Algorithms, PhD thesis, Lund University, Department of Electroscience, Lund, Sweden.

Edman, F. \& Öwall, V. (2005). A scalable pipelined complex valued matrix inversion architecture, IEEE International Symposium on Circuits And Systems, ISCAS'05, pp. 4489-4492.

Eilert, J., Wu, D. \& Liu, D. (2007). Efficient complex matrix inversion for MIMO Software Defined Radio, IEEE International Symposium on Circuits and Systems, ISCAS'07 .

Golub, G. H. \& van Van Loan, C. F. (1996). Matrix Computations (3rd Edition), The Johns Hopkins University Press.

Hoffmann, A., Schliebusch, O., Nohl, A., Braun, G., Wahlen, O. \& Meyr, H. (2001). A Methodology for the Design of Application Specific Instruction set Processors (ASIP) Using the Machine Description Language LISA, IEEE/ACM International Conference on Computer Aided Design, ICCAD'2001., pp. 625-630.

Jafri, A. R., Baghdadi, A. \& Jezequel, M. (2009). ASIP-Based Universal Demapper for Multiwireless Standards, IEEE Embedded Systems Letters 1(1): 9-13.

Jafri, A.-R., Karakolah, D., Baghdadi, A. \& Jezequel, M. (2009). ASIP-based Flexible MMSE-IC Linear Equalizer for MIMO Turbo-Equalization Applications, IEEE/ACM Design, Automation and Test in Europe Conference \& Exhibition, DATE'09.

Karakolah, D., Jégo, C., Langlais, C. \& Jézéquel, M. (2009). Design of an iterative receiver for linearly precoded MIMO systems, IEEE International Symposium on Circuits and Systems, ISCAS'09.

Karkooti, M., Cavallaro, J. R. \& Dick, C. (2005). FPGA Implementation of Matrix Inversion Using QRD-RLS Algorithm, Asilomar Conference on Signals, Systems and Computers, pp. 1625-1629.

Kim, H., Zhu, W., Bhatia, J., Mohammad, K., Shah, A. \& Danesrad, B. (2008). A Practical Hardware Friendly MMSE Detector for MIMO-ODFM-Based Systems, EURASIP Journal on Advances in Signal Processing . 
Laot, C., Le Bidan, R. \& Leroux, D. (2005). Low-complexity MMSE turbo equalization: a possible solution for EDGE, IEEE Transactions on Wireless Communications 4(3): 965-974.

Mitola, J. (1995). The Software Radio Architecture, IEEE Communications Magazine 33(5): 26 -38 .

Muller, O., Baghdadi, A. \& Jezequel, M. (2009). From Parallelism Levels to a Multi-ASIP Architecture for Turbo Decoding, IEEE Transactions on Very Large Scale Integration (VLSI) Systems 17(1): 92-102.

Myllyla, M., Hintikka, J.-H., Cavallaro, J., Juntti, M., Limingoja, M. \& Byman, A. (2005). Complexity Analysis of MMSE Detector Architectures for MIMO OFDM Systems, Asilomar Conference on Signals, Systems and Computers, pp. 75 -81.

Volder, J. E. (1959). The Cordic Trigonometric Computing Technique, IEEE Transactions on Electronic Computers 8(3): 330-334. 


\title{
Rapid Prototyping for Evaluating Vehicular Communications
}

\author{
Tiago M. Fernández-Caramés, Miguel González-López, \\ Carlos J. Escudero and Luis Castedo \\ University of A Coruña \\ Spain
}

\section{Introduction}

During the last years, wireless communications between moving vehicles (Vehicle-To-Vehicle, VTV or V2V) and from vehicles to infrastructure (V2I or Roadside-to-Vehicle, RTV) have received a great deal of attention. Vehicular safety (collision prevention systems, accident warnings...) as well as payment and infotainment applications (automatic payment, mobile internet access at high vehicular speeds, traffic jam avoidance...) are increasingly demanded by the automotive industry in their way towards deploying ITS (Intelligent Transport Systems).

Several wireless communication standards targeted to vehicular scenarios have been proposed. Vehicular safety applications require fast exchange of messages in order to obtain a swift reaction from the car or the driver in dangerous situations, such as a sudden slowdown or when two cars approach an intersection. Due to the necessary quick response, transceivers have to send short packets and, therefore, small bandwidths are demanded. Among the different wireless standards, IEEE 802.11p (IEEE, 2010) is the best positioned to act as the reference standard for the PHYsical (PHY) and Medium Access Control (MAC) layer of vehicular communications for safety applications in the near future.

For non-safety vehicular applications, the discussion about which is the most suitable wireless access standard remains an open issue. The most cited candidates are the WiFi standards IEEE 802.11a/b/g and IEEE 802.16e (Mobile WiMAX). Most likely, vehicular communications will take place in the $5 \mathrm{GHz}$ band since both US and European authorities have reserved spectrum for ITS at $5.9 \mathrm{GHz}$. Due to this, the final candidates might be reduced to IEEE 802.11p, IEEE 802.11a and IEEE 802.16e. Other wireless communication standards have been proposed for use in vehicular environments, such as HSDPA (High-Speed Downlink Packet Access), IEEE 802.20 (iBurst) or EDGE Evolution, but the peak data rates they offer for broadband communications (14.4 Mbit/s, $16 \mathrm{Mbit} / \mathrm{s}$ and $1 \mathrm{Mbit} / \mathrm{s}$ ) are lower than those theoretically provided by IEEE 802.11p, IEEE 802.11a or IEEE 802.16e (27 Mbit/s, $54 \mathrm{Mbit} / \mathrm{s}$ and 39.9 $\mathrm{Mbit} / \mathrm{s})$. Also, there are several recently developed standards whose performance in vehicular scenarios has yet to be assessed and whose study will constitute an interesting topic for further research. Such new standards are expected to offer better global performance, but they are either in earlier development stages (e.g. LTE, IEEE $802.16 \mathrm{~m}$ ) or have not been explicitly designed for vehicular applications (e.g. IEEE 802.11n).

One of the most appropriate ways to evaluate the performance of transceivers compliant with a standard consists in using a testbed together with a channel emulator. Testbeds have 
been previously used to assess different wireless communication standards (for instance, IEEE 802.11a (Angelakis et al., 2008), IEEE 802.11n (Nieto et al., 2006), IEEE 802.16e (Hu et al., 2007) and IEEE 802.11p (Fernández-Caramés, 2008)), whilst several channel emulators have been implemented to measure transceiver performance in realistic situations, like those developed for IEEE 802.11n (Dasatti et al., 2005), Dedicated Short-Range Communications (DSRC) (Faseth et al., 2010) or for UHF RFID systems (Arthaber \& Schuberth, 2009).

In this book chapter we present a performance evaluation system made up of a software testbed and a flexible, low-cost, FPGA-based vehicular channel emulator. We detail the way we employed rapid-prototyping techniques for building both the testbed and the channel emulator. In order to decrease the development time required we decided to use MATLAB $^{\circledR}$ and Simulink ${ }^{\circledR}$ for implementing three different transceivers compliant with the standards IEEE 802.16e, IEEE 802.11p and IEEE 802.11a. The vehicular channel emulator was implemented using another rapid-prototyping tool: Xilinx ${ }^{\circledR}$ System Generator ${ }^{\circledR}$. We obtained performance measurements over representative situations of V2V and V2I communications carried out by software-hardware co-simulation: MATLAB/Simulink software transceivers ran on a PC while the vehicular channel emulator was running on an external FPGA.

One of the best ways to increase the transmission capacity with respect to Single-Input Single-Output (SISO) systems (i.e. systems that use one transmit and one receive antenna), consists in building systems that use multiple antennas in transmission (known as MISO (Multiple-Input Single-Output) systems), reception (SIMO (Single-Input Multiple-Output) systems) or in both transmission and reception (MIMO (Multiple-Input multiple-Output) systems) (Foschini, 1998; Telatar, 1999).

Our previous work (Fernández-Caramés, 2010) focused on the performance in vehicular scenarios of IEEE 802.11p using SISO transceivers. As a novelty, we detail herein how we have updated the whole system with the aim of carrying out performance comparisons for multiple-antenna transceivers. We also explain the different optimizations we have performed during the design process of our MIMO channel emulator to deal with the fact that the FPGA computation resources are limited. Finally, we show the obtained performance evaluation results for SISO, SIMO and MIMO transceivers.

The rest of this chapter is organized as follows. Section 2 describes the three wireless standards considered as well as the corresponding transceivers we implemented. Section 3 details the design process of the FPGA-based vehicular channel emulator. Section 4 presents the upgrade of our system to MIMO. Finally, Section 5 details the experiments performed whereas Section 6 is devoted to the conclusions.

\section{Wireless standards and transceivers: IEEE 802.11a, IEEE 802.11p and IEEE 802.16e}

\subsection{IEEE 802.16e (Mobile WiMAX)}

We focused on the PHY layer referred to in the IEEE 802.16e standard as WirelessMAN-OFDMA. Fig. 1 depicts the block diagram of the evaluation system, which shows that Mobile WiMAX has been defined in a similar way to other broadband OFDMA (Orthogonal Frequency-Division Multiple Access) communication systems.

Among the different Mobile WiMAX working modes, in our experiments the transceiver operates in a mode called Downlink PUSC (Partial Usage of Subcarriers). In this mode, the 512 subcarriers are divided into 360 subcarriers for data, 60 for pilots and 92 for the guards and the DC. Each fourteen adjacent subcarriers over two OFDMA symbols constitute a cluster or resource block (24 subcarriers for data and 4 for pilots). Furthermore, each OFDMA symbol 
$P C$ running Matlab/Simulink and System Generator for DSP

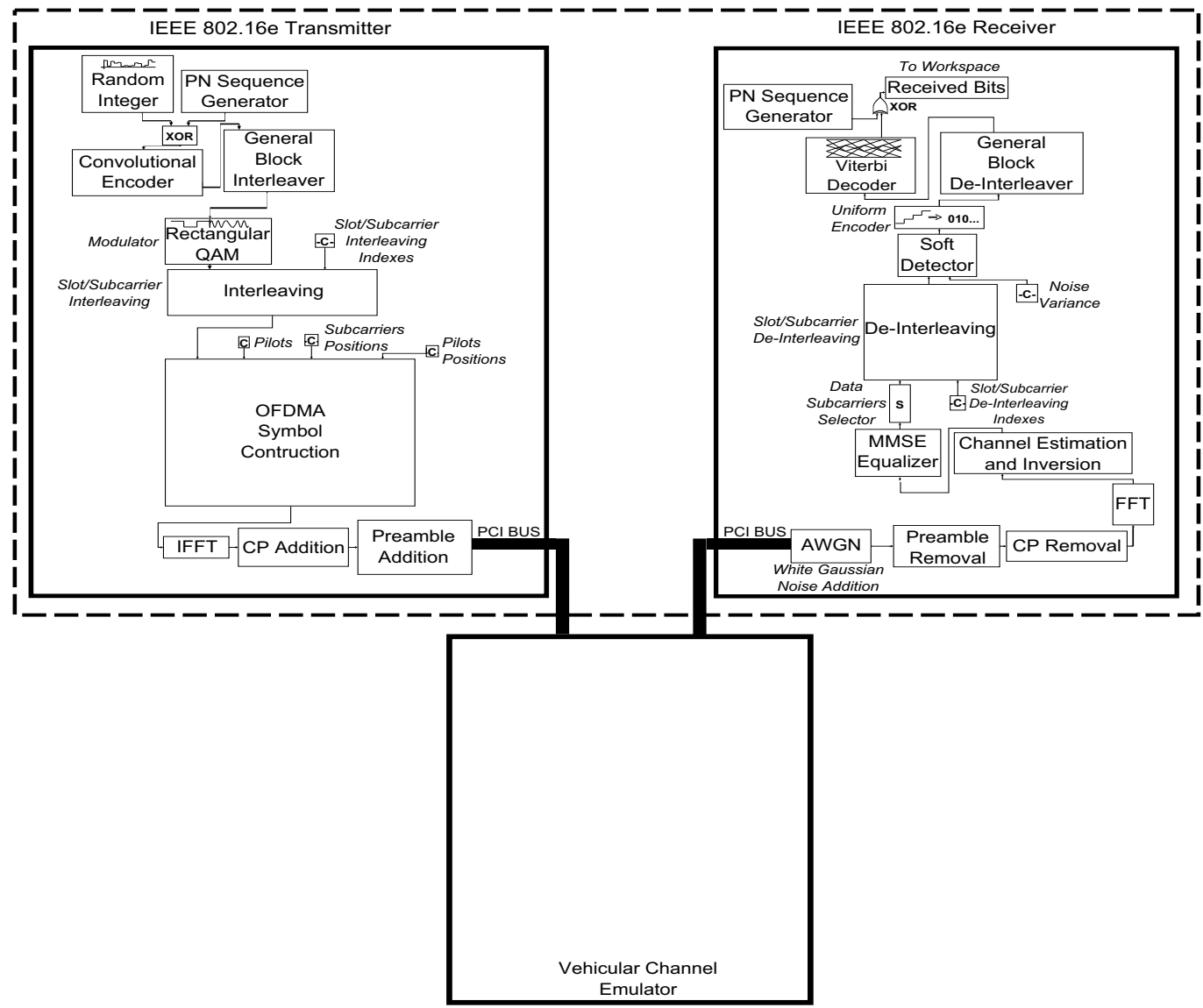

Fig. 1. Evaluation system for IEEE 802.16e.

is divided into fifteen subchannels but, for the sake of simplicity, we assigned all subchannels to a unique user.

\subsubsection{Mobile WiMAX MATLAB/simulink transmitter}

Fig. 1 (left) depicts the transmitter block diagram. We have followed closely the indications given in Section 8.4 of (IEEE, 2009), although we have made modifications in order to simplify the design and reduce simulation time. Such differences are described below.

An IEEE 802.16e transmitter works with slots made up of two consecutive OFDMA symbols. Also, it should be noticed that in our tests we have considered that a fair comparison between the different standards should be performed measuring the FER (Frame Error Rate), considering that a frame corresponds to a FEC (Forward Error Correction) block. Since each FEC block contains 48 data bits and there are 720 data subcarriers for each slot (two OFDMA symbols), using QPSK and a rate $1 / 2$ code, it is concluded that in each slot the transmitter sends 15 FEC blocks.

With respect to the pilots, the standard uses a Pseudo-Random Binary Sequence (PRBS) generator during their modulation. However, since this generator is only used to provide 
additional security, we decided not to include it in our design in order to reduce complexity and simulation time.

Although we always transmit the preamble in order to comply with the requirements of the standard, it is not used in reception since we assume perfect time synchronization. Moreover, the vehicular channel emulator operates in baseband and, therefore, there is no IF (Intermediate Frequency) stage, neither at the transmitter nor at the receiver.

\subsubsection{Mobile WiMAX MATLAB/simulink receiver}

The receiver block diagram is shown on the right of Fig. 1. The first step is the addition of white Gaussian noise in order to obtain BER (Bit Error Rate) and FER curves versus received SNR (Signal-to-Noise Ratio) or $E_{b} / N_{0}$ values. The $E_{b} / N_{0}$ parameter is the received bit energy to noise power spectral density ratio commonly referred to as received SNR per bit.

After removing the preamble and the $\mathrm{CP}$, the FFT is applied to each OFDM symbol and the channel is estimated. We employ a simple channel estimation method consisting in extracting the pilots and divide them by their respective transmitted values (which are known at the receiver), obtaining the estimated channel coefficients for the pilot subcarriers. Such estimates are linearly interpolated to obtain the channel frequency response for the remaining subcarriers. Moreover, to improve channel inversion, we apply an MMSE (Minimum Mean Square Error) equalizer (Rugini, 2005).

The equalized symbols are de-interleaved (at slot and symbol levels) and then sent to a soft detector whose output LLRs are also de-interleaved and decoded using a Viterbi block. Finally, the decoded bits are de-randomized and the final bits are obtained.

\subsection{IEEE 802.11p and IEEE 802.11a}

The standard IEEE 802.11p (IEEE, 2010) is an amendment to IEEE 802.11-2007 (IEEE, 2007) and is technically compatible with the specifications given by ASTM E2213-03 (ASTM, 2003), which addresses the challenges that arise when providing wireless access in vehicular environments. Its MAC and PHY layers are very similar to those used in IEEE 802.11a, but they incur in a lower overhead to allow faster exchanges of safety messages.

In our work we focus on the PHY layer and, at such a level, the main difference between IEEE 802.11a and IEEE 802.11p is that the $20 \mathrm{MHz}$ bandwidth used in IEEE 802.11a reduces to $10 \mathrm{MHz}$ in IEEE 802.11p. Although the mentioned bandwidth reduction results in a loss of data transfer rate, it provides an important advantage when overcoming the effects of vehicular channels: the OFDM symbols are longer in the time domain and the system can deal with larger delay spreads, thus being able to avoid ISI (Inter-Symbol Interference). Therefore, if we ignore the IEEE 802.11p ACR (Adjacent Channel Rejection) and the SEM (Spectrum Emission Mask) requirements, the practical implementation of a basic IEEE 802.11p transceiver is straightforward: it suffices to double all the OFDM timing parameters used by IEEE 802.11a devices.

The design of our IEEE 802.11p/a transceivers (whose key parameters are shown in Table 1) can be found in our previous work (Fernández-Caramés, 2010). They include similar blocks to those present in Fig. 1.

\section{Real-time FPGA-based vehicular channel emulator}

Channel emulation is typically used when evaluating product performance in realistic situations before commercial release. With the aid of a channel emulator the equipment manufacturers avoid unintended interferences, hence the simulation environment can be controlled. Furthermore, the tiresome task of performing successive field measurements is 


\begin{tabular}{|c||c|c|c|}
\hline \multicolumn{1}{|c||}{ Parameter } & 802.11p & 802.11a & 802.16e \\
\hline Carrier Modulation & \multicolumn{3}{|c|}{ BPSK, QPSK, 16-QAM, 64-QAM } \\
\hline Code rate & \multicolumn{3}{|c|}{$1 / 2,2 / 3,3 / 4$} \\
\hline \# subcarriers & 48 data +4 pilots & 360 data +60 pilots \\
\hline OFDM symbol duration & $8 \mu \mathrm{s}$ & $4 \mu \mathrm{s}$ & $64 \mu \mathrm{s}$ \\
\hline Guard time & $1.6 \mu \mathrm{s}$ & $0.8 \mu \mathrm{s}$ & $12.8 \mu \mathrm{s}$ \\
\hline FFT period & $6.4 \mu \mathrm{s}$ & $3.2 \mu \mathrm{s}$ & $51.2 \mu \mathrm{s}$ \\
\hline Total bandwidth & $10 \mathrm{MHz}$ & $20 \mathrm{MHz}$ & $10 \mathrm{MHz}$ \\
\hline Subcarrier spacing & $156.25 \mathrm{KHz}$ & $312.5 \mathrm{KHz}$ & $19.53 \mathrm{KHz}$ \\
\hline
\end{tabular}

Table 1. Feature comparison of wireless standards.

limited to the minimum (to obtain the channel model, if there is none already available) and the rest of the experiments are carried out inside a testing lab.

There are many commercial channel emulators that are manufactured by companies such as Spirent, Rhode \& Schwarz, Azimuth Systems, Agilent... These emulators are usually general-purposed (for instance, Spirent's SR5500 or Rhode's AMU200A), but there are some that are aimed at evaluating a specific technology, like Azimuth's 400WB MIMO Channel Emulator (for IEEE 802.11n and Mobile WiMAX MIMO systems) or Agilent's N5106A PXB MIMO Receiver Tester (with built-in LTE and Mobile WiMAX channels).

All these channel emulators are robust and work great for most applications, but they are normally quite expensive and may not offer enough flexibility to researchers when setting channel configuration parameters. To tackle these issues a number of low-cost ad-hoc channel emulators have recently been proposed.

Due to real-time constraints and suitability, most of the proposed low-cost, easily-reconfigurable ad-hoc emulators are based on FPGA technology. Some examples are described in (Alimohammad, 2008; Ghazel, 2003; Hwang, 2007). In (Ghazel, 2003) an FPGA-based AWGN channel emulator is implemented. The emulator is based on a hardware white Gaussian noise generator that is developed by combining the Box-Muller and Central limit theorems, and designing the whole model in VHDL (Very High Speed Integrated Circuit Hardware Description Language). Similarly, in (Alimohammad, 2008), the authors use a Xilinx Virtex-II Pro to implement a fading channel emulator. The fading process models use sum-of-sinusoids (SOS) algorithms that allow designing and implementing Rician and Rayleigh fading channels.

Finally, (Hwang, 2007) presents a baseband multipath fading channel emulator implemented on a Virtex-IV using the Xilinx XtremeDSP FPGA platform. The emulator is implemented using Simulink models and System Generator IP blocks. The final design is limited to a two-path channel due to the extensive use of FPGA resources; the input/output rate is set to $20 \mathrm{MHz}$; and the Doppler frequency is $5 \mathrm{~Hz}$.

The above mentioned developments have at least two major drawbacks. First, the use of low-level description languages such as VHDL results in slow development stages. Although in most cases VHDL allows obtaining a resource-efficient FPGA design, programming can become a cumbersome task that may consume a large amount of time and economic resources. There are new sophisticated tools like System Generator which allow working with high-level blocks to build complex designs easier and faster.

The second problem is related to the use of high-level tools. These tools facilitate fast prototyping but they usually generate non-optimized large designs that may not fit into the FPGA. For instance, in (Hwang, 2007) the authors only download a two-path channel emulator due to the lack of available hardware resources. Hence, for large designs, optimizations must be made.

The vehicular emulator described in this chapter addresses these issues: we used System Generator to develop the channel emulator faster than using VHDL and we optimized our 
design in order to be able to implement a twelve-path channel emulator, leaving space for future extensions, such as MIMO emulation, also described herein in Section 4.

\subsection{Implemented vehicular channel models}

The implemented channel models are based on the excellent work in (Acosta, 2007b) and (Acosta, 2007a), that is mainly based on a measurement campaign at $5.9 \mathrm{GHz}$ carried out in the spring of 2006 in Atlanta, Georgia. From these measurements the authors obtained six different channel models that cover the most common situations where VTV and RTV communications may take place:

- Urban canyons, with dense and tall buildings, where vehicles move at speeds between $32 \mathrm{Km} / \mathrm{h}$ and $48 \mathrm{Km} / \mathrm{h}$.

- Suburban expressways, with moderately dense, low-story buildings, where the measurement speed was approximately $105 \mathrm{Km} / \mathrm{h}$.

- Suburban surface streets, with moderately dense, low-story buildings, where the driving speed was between $32 \mathrm{Km} / \mathrm{h}$ and $48 \mathrm{Km} / \mathrm{h}$.

Although the measurement campaign was performed at $105 \mathrm{Km} / \mathrm{h}$ in expressways and $32 \mathrm{Km} / \mathrm{h}$ to $48 \mathrm{Km} / \mathrm{h}$ for surface streets, the authors scaled the models to make their Doppler frequencies consistent with vehicle speeds of $140 \mathrm{Km} / \mathrm{h}$ and $120 \mathrm{Km} / \mathrm{h}$, respectively.

\begin{tabular}{|c|c|c|c|c|c|c|c|c|c|}
\hline Vehicular Channel & \begin{tabular}{|c|} 
Distance \\
TX - RX \\
(m)
\end{tabular} & $\begin{array}{l}\text { Speed } \\
(\mathrm{km} / \mathrm{h})\end{array}$ & $\begin{array}{l}\text { Path Modulation } \\
\text { (number of paths) }\end{array}$ & \begin{tabular}{|c|} 
Maximum \\
Delay Spread \\
(ns)
\end{tabular} & $\begin{array}{c}\text { Rician } K \\
\text { (dB) }\end{array}$ & $\begin{array}{c}\text { Overall } \\
K \text { Factor } \\
\text { (dB) }\end{array}$ & \begin{tabular}{|c|} 
Maximum \\
Freq. Shift \\
$(\mathrm{Hz})$
\end{tabular} & \begin{tabular}{|c|} 
Maximum \\
Fading Doppler \\
$(\mathrm{Hz})$
\end{tabular} & \begin{tabular}{|c} 
LOS \\
Doppler \\
$(\mathrm{Hz})$
\end{tabular} \\
\hline VTV-Expressway Oncoming & $300-400$ & 140 & Rician (1) / Rayleigh (10) & 302 & -1.6 & -3.6 & 1466 & 858 & 1452 \\
\hline RTV-Urban Canyon & 100 & 120 & Rician (1) / Rayleigh (11) & 501 & 7.5 & 6.7 & 720 & 994 & 654 \\
\hline RTV-Expressway & $300-400$ & 140 & Rician (1) / Rayleigh (11) & 401 & -5.3 & 4.3 & 769 & 813 & 770 \\
\hline VTV-Urban Canyon Oncoming & 100 & 120 & Rician (1) / Rayleigh (11) & 401 & 4.0 & 3.0 & 1145 & 936 & 1263 \\
\hline RTV-Suburban Street & 100 & 120 & Rician (1) / Rayleigh (11) & 700 & 3.3 & 2.1 & 648 & 851 & 635 \\
\hline VTV-Express. Same Dir. with Wall & $300-400$ & 140 & Rician (2) / Rayleigh (10) & 701 & $23.8,5.7$ & 3.3 & -561 & 1572 & $-60,40$ \\
\hline
\end{tabular}

Table 2. Main characteristics of the vehicular models.

Our channel emulator implements these six vehicular channel models, whose key characteristics are summarized in Table 2. For each model, the following parameters are shown: distance between the transmitter and the receiver, speed of the vehicle, number of paths of the channel and their modulation (Rician or Rayleigh), maximum delay spread, Rician $K$ for the Rician paths, overall $K$ factor (i.e. the ratio of the deterministic power over the total random power of all taps), maximum frequency shift for all paths, maximum fading Doppler (i.e. maximum half-width of the fading spectral shapes of all the paths of each channel) and LOS Doppler of the Rician paths.

\begin{tabular}{|c|c|c|c|c|c|c|c|}
\hline Vehicular Channel & $\begin{array}{c}\text { Coefficient generation } \\
\text { rate [Effective rate] }(\mathrm{Hz})\end{array}$ & $\begin{array}{c}\begin{array}{c}\text { Interpolation } \\
\text { rate }\end{array} \\
\end{array}$ & $\begin{array}{l}\text { Occupied } \\
\text { Slices (\%) }\end{array}$ & \begin{tabular}{|c|} 
Occupied \\
Slice Flip-Flops (\%)
\end{tabular} & $\begin{array}{l}\text { Occupied } \\
\text { LUTs (\%) }\end{array}$ & $\begin{array}{c}\text { Occupied } \\
\text { FIFO16 / RAMB16s (\%) }\end{array}$ & $\begin{array}{r}\text { Occupied } \\
\text { DSP48s (\%) }\end{array}$ \\
\hline VTV-Expressway Oncoming & 3484 [4000] & x2500 & $76 \%$ & $36 \%$ & $50 \%$ & $19 \%$ & $60 \%$ \\
\hline RTV-Urban Canyon & $2194.6[2500]$ & $\mathrm{x} 4000$ & $84 \%$ & $39 \%$ & $57 \%$ & $20 \%$ & $65 \%$ \\
\hline RTV-Expressway & 2168 [2500] & $\mathrm{x} 4000$ & $84 \%$ & $39 \%$ & $57 \%$ & $20 \%$ & $65 \%$ \\
\hline VTV-Urban Canyon Oncoming & 3314 [4000] & x 2500 & $84 \%$ & $39 \%$ & $57 \%$ & $20 \%$ & $65 \%$ \\
\hline RTV-Suburban Street & 1988 [2000] & x5000 & $84 \%$ & $39 \%$ & $57 \%$ & $20 \%$ & $65 \%$ \\
\hline VTV-Express. Same Direction With Wall & 3170 [4000] & $\times 2500$ & $85 \%$ & $40 \%$ & $57 \%$ & $24 \%$ & $65 \%$ \\
\hline
\end{tabular}

Table 3. General parameters and resources occupied by the vehicular channel emulator.

Table 2 also gives an idea of the complexity involved in the implementation of these channels. These high speed and high delay spread scenarios own large Doppler shifts that force the emulator to interpolate and rapidly update each path coefficients. Moreover, although the amount of required FPGA hardware is reduced by working with the baseband IQ components 


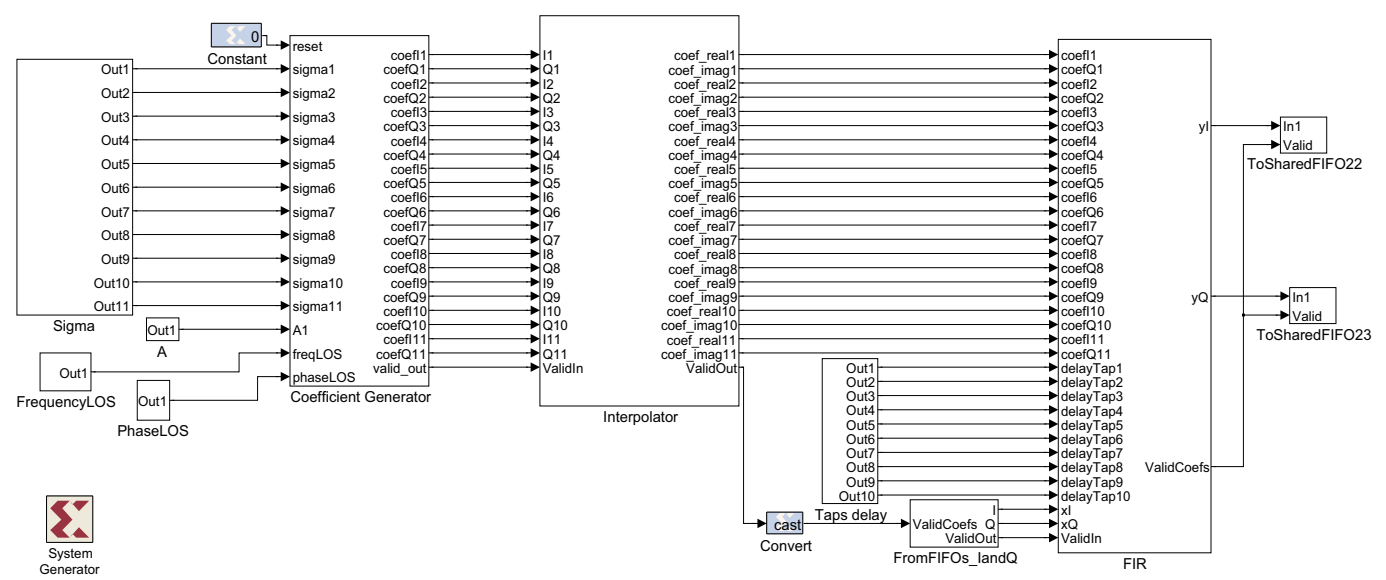

Fig. 2. General view of the System Generator model optimized for the vehicular channel VTV-Expressway Oncoming.

at $10 \mathrm{MHz}$, it is not possible to implement the six channels into our FPGA. Thus, six independent . bit files are generated though, in practice, only three different FPGA designs are needed due to the model similarities:

- One design is exclusively dedicated to the channel VTV-Expressway Oncoming which is the only one with eleven paths.

- Another model is used for VTV-Expressway Same Direction with Wall because it requires the existence of two Rician and ten Rayleigh paths, while the rest of the channels (apart from $V T V$-Expressway Oncoming) consists of just one Rician path and eleven Rayleigh paths.

- One design for the other four channels, which differ in their configuration parameters but share all their FPGA resources.

\subsection{Theoretical channel model}

For the generation of each channel coefficient at the $i$-th path in the time instant $t$, we used the following model

$$
h(i, t)=\sqrt{K_{i} P_{i} /\left(K_{i}+1\right)} \bar{h}(i, t)+\sqrt{P_{i} /\left(K_{i}+1\right)} h_{w}(i, t)
$$

where

- $K_{i}$ and $P_{i}$ are, respectively, the Rice factor and the power of the $i$-th path.

- $h_{w}(i, t)$ represents the contribution of the non-line-of-sight (NLOS) component to the $i$-th path at the time instant $t$. It is a random variable that follows a complex Gaussian distribution with mean zero and unit variance.

- $\bar{h}(i, t)$ : contribution of the line-of-sight (LOS) component to the $i$-th path at the time instant $t$. It is determined by

$$
\bar{h}(i, t)=e^{j\left(2 \pi f_{D, i} \cos \left(\theta_{i}\right) t+\phi_{i}\right)}
$$

where $f_{D, i}, \theta_{i}$ and $\phi_{i}$ are, respectively, the maximum Doppler spread, angle of arrival and phase of the LOS component of the $i$-th path. 
To decrease the number of input configuration parameters, we calculate off-line several of the operations involved in (1) and (2). As it can be viewed in Fig. 2, the emulator only needs five parameter blocks:

- Sigma block contains the power factors of the NLOS components: $\sigma=\sqrt{P_{i} /\left(K_{i}+1\right)}$.

- A block holds the power factors of the LOS components: $A=\sqrt{K_{i} P_{i} /\left(K_{i}+1\right)}$.

- FrequencyLOS block contains part of the exponent of $\bar{h}(i, t): f_{\mathrm{LOS}}=2 \pi f_{D, i} \cos \left(\theta_{i}\right)$.

- Phaselos block is simply $\phi_{i}$.

- Taps delay block holds the normalized delays of the different paths.

\subsection{Hardware and software}

The vehicular channel emulator is implemented on an FPGA using Nallatech's BenADDA-IV development kit which has the following features: it contains a Virtex IV (XC4VSX35-10FF668) that allows using Xtreme-DSP slices of up to $400 \mathrm{MHz}$; includes $4 \mathrm{MB}$ of $166 \mathrm{MHz}$ ZBT-RAM, two 14-bit ADCs able to work up to $105 \mathrm{MS} / \mathrm{s}$ and two 14-bit DACs that can run up to $160 \mathrm{MS} / \mathrm{s}$; provides a dedicated internal clock up to $105 \mathrm{MHz}$, although it can use an external clock; offers the possibility to operate either connected to a PC (via the PCI bus) or in stand-alone mode.

In order to diminish the amount of time required to implement the channel model on the FPGA, we decided to use System Generator for DSP because it enables to design and program our Virtex IV faster. It allows using libraries of high-level blocks and can interact with MATLAB and Simulink. Moreover, another advantage of this software is its ability to exchange data between a design running in the FPGA and a software implementation that is executed on a PC. In fact, for our tests (Section 5) we have run in MATLAB and Simulink the transceivers that interact with the vehicular emulator, which was running on the FPGA.

\subsection{FPGA design overview}

Fig. 2 shows a general view of the hardware design. Several blocks represented in such figure contain sub-blocks which are shown throughout this chapter: the Coefficient Generator block includes Figs. 3 and 4, the Interpolator block contains a number of interpolators like the one shown in Fig. 5.

The design depicted in Fig. 2 has been optimized for a specific channel (VTV-Expressway Oncoming), although the rest of channels models share most of the hardware resources. The design can be divided into different parts that carry out six different major tasks: acquisition of the channel parameters, Gaussian noise generation, Doppler filtering, LOS Doppler generation, interpolation and FIR filtering.

\subsubsection{Acquisition of the channel parameters}

The generation of the configuration parameters of the vehicular channel is performed offline since they remain constant throughout the emulation. The parameters are stored into registers readable by the FPGA. All the parameters equal to zero for a particular channel are removed to save hardware. For example, all the channels but VTV-Expressway Same Direction With Wall have one Rician component, so in these channels we only need one register to store each of the LOS parameters detailed in Section 3.2. 


\subsubsection{White gaussian noise generation}

To obtain the NLOS coefficients, we need to use the System Generator's White Gaussian Noise Generator (WGNG) block that generates i.i.d samples from a Gaussian distribution with zero mean and unit variance. Since the maximum number of complex paths is twelve, we need to obtain twenty-four real-valued of such noise samples that will be filtered depending on the Doppler experienced by each path. Instead of using twenty-four independent WGNG blocks, we multiplex in time the samples produced by only one WGNG block (Fernández-Caramés, 2010). This optimization is crucial since each WGNG block consumes an important amount of FPGA resources. Since each WGNG block runs at $10 \mathrm{MHz}$, using a twenty-five output multiplexor (twenty-five is the integer divider of $10 \mathrm{MHz}$ closest to twenty-four), leads us to generate twenty-four noise samples at a frequency of $400 \mathrm{KHz}$, that still is several orders of magnitude higher than the desired channel coefficient generation effective rates (see Table 3). Therefore, the optimization of the 24-output Gaussian noise generator makes the emulator to produce channel coefficients slower but at a sufficiently high rate, and saving a great deal of FPGA resources.

Using the System Generator's Resource Estimator block, there is a 95\% of saving for all the FPGA resources thanks to this optimization (Fernández-Caramés, 2010). Also, if we needed to reduce the number of occupied resources, it would be possible to avoid using WGNG blocks: the channel coefficients could be generated in MATLAB and then transferred to the FPGA. However, there are important drawbacks in this approach:

- If the channel coefficients were only transmitted from MATLAB during the initialization phase, due to the limited amount of memory on the FPGA, there would be a time when the channel coefficients would have to be used again. Therefore, correlation in the output signal would appear.

- If we transfer a new set of channel coefficients from MATLAB at fixed intervals, we would not be able to use the emulator in stand-alone mode since we always would relay on having a computer running MATLAB linked to the FPGA.

\subsubsection{Doppler filtering}

To generate the actual NLOS components, the generated white Gaussian noise samples have to be filtered according to each path's Doppler spectrum. This spectrum is determined by a fading spectral shape, a frequency shift and a maximum Doppler shift. Table 2 shows these latter two parameters for the considered channel models. Four different spectral shapes are considered: round, flat, classic $3 d B$ and classic $6 d B$ (Acosta, 2007a).

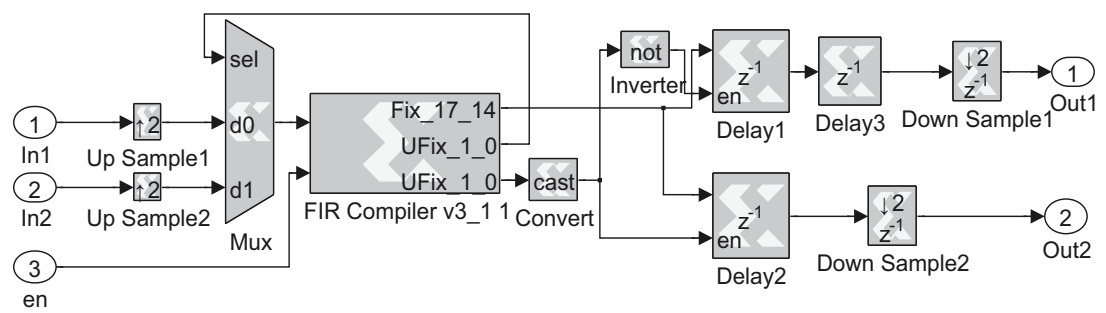

Fig. 3. Optimized blocks for applying the Doppler Spectrum.

Fig. 3 shows the blocks that allow applying the Doppler spectrum to each Rayleigh path. Each Doppler filter consists of 256 coefficients. This filter size provides a good tradeoff between precision and hardware complexity. Since each filter is unique for each path of each vehicular channel, we hard-coded the coefficients in each of the six .bit files. 
To reduce to a half the required hardware, we exploit the fact that the real and the imaginary parts of the filter have to be used twice for each path to perform the complex FIR filtering (see Fig. 4). This optimized block can be seen in Fig. 3, which is contained under the block Doppler Filter shown in Fig. 4.

Table 4 shows some of the resource savings, achieved when reducing to a half the number of filters in a vehicular channel with eleven paths. Although the optimized block uses slightly more slices, the savings occur in the DSP 48 and the RAMB16 blocks, that are reduced by $50 \%$. This is important, since the lack of this type of blocks is a bottleneck to keep on designing the rest of the emulator.

\begin{tabular}{|c||c|c|c|c|}
\hline $\begin{array}{c}\text { Resource } \\
\text { type }\end{array}$ & $\begin{array}{c}\text { Optimized } \\
\text { 24-output Gaussian generator }\end{array}$ & $\begin{array}{c}\text { Non-optimized } \\
\text { 24-output Gaussian generator }\end{array}$ & $\begin{array}{c}\text { Total FPGA } \\
\text { resources }\end{array}$ & $\begin{array}{c}\text { Savings } \\
\text { (\%) }\end{array}$ \\
\hline Slices & 7382 & 7239 & 15360 & $-1.9 \%$ \\
DSP48 blocks & 88 & 176 & 192 & $50 \%$ \\
RAMB16 blocks & 44 & 88 & 192 & $50 \%$ \\
\hline
\end{tabular}

Table 4. Savings due to the optimization of the Doppler filter block

Finally, LOS Doppler has also to be taken into account and must be applied to each Rician path according to Eq. (2). To achieve this, we use the System Generator's DDS (Direct Digital Synthesizer) block that generates a sine and a cosine with the required phase and frequency parameters. Since the angle of arrival of the LOS component has not been considered in (Acosta, 2007b), we always set its value to zero, what means that the received Rician paths arrive straight from the driving direction. This implies that the LOS Doppler is always equal to the path's maximum Doppler spread.

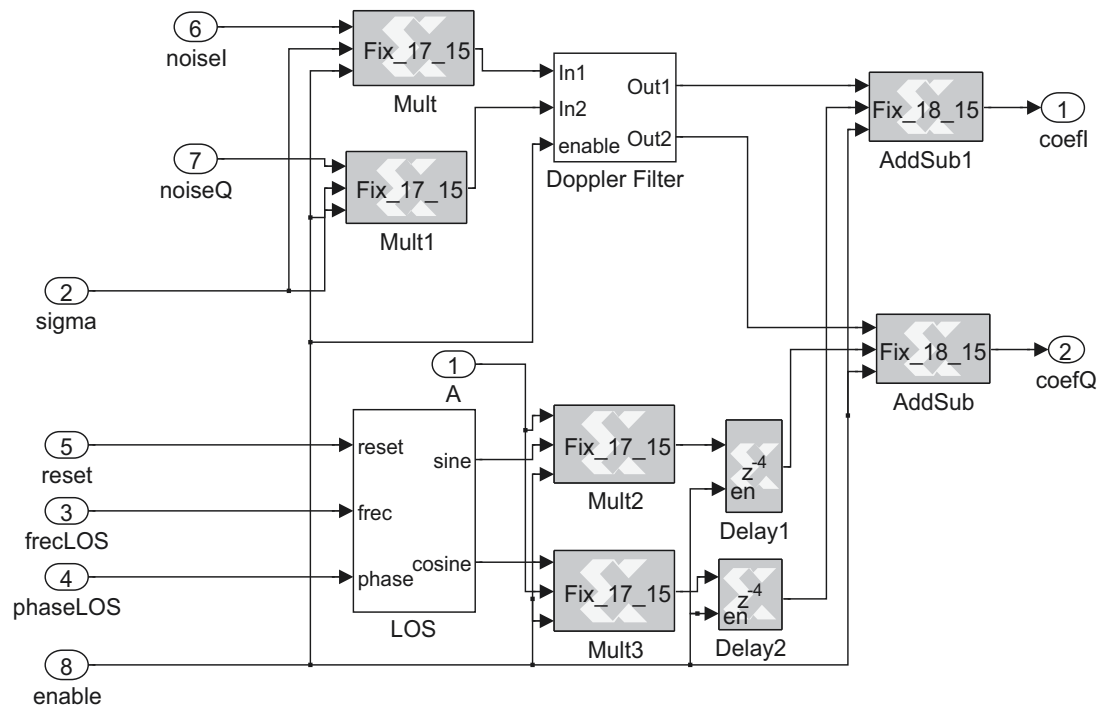

Fig. 4. Generation and addition of the LOS and NLOS components of each path.

\subsubsection{Interpolation and FIR filtering}

After adding the LOS and NLOS components according to Eq. (1) (see Fig. 4), the coefficients must adapt their rate to the rate of the incoming signal (i.e. the signal from our transmitters 


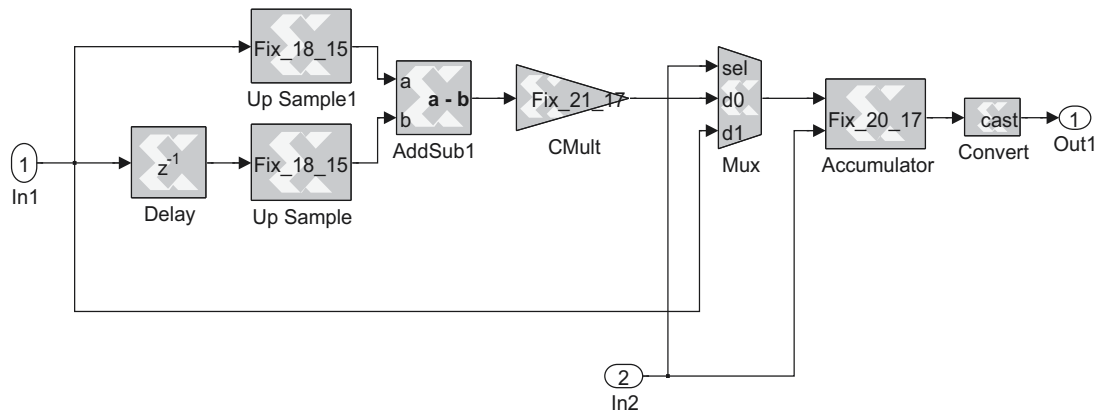

Fig. 5. One path's linear interpolator.

arrives at $10 \mathrm{MHz}$ ). These coefficients are generated at a rate that depends on the maximum Doppler shift and that is much lower than the FPGA's frequency. Indeed, in a specific vehicular channel, the implicit sample rate is twice the maximum Doppler shift of all paths. In the implemented vehicular channel models, this rate fluctuates between $1988 \mathrm{~Hz}$ and $3484 \mathrm{~Hz}$ (see Table 3). To avoid designing a complex resampling stage, instead of using the original coefficient generation rate, we use an effective sample rate that is equal to the nearest superior integer divider of $10 \mathrm{MHz}$. Thus, we only need an interpolator (we actually use two cascaded linear interpolators, whose global interpolation rates are also included in Table 3).

Fig. 5 shows a linear interpolator applied to one of the paths. The way it works is simple:

- At the time instant $t$, the current coefficient and the one generated at the time instant $t-1$ are copied $p$ times, being $p$ the interpolation factor. Hence, we would have two sets of upsampled coefficients: $\mathbf{s}_{t}=[\overbrace{s_{t}, s_{t}, \ldots, s_{t}}^{p}]$ and $\mathbf{s}_{t-1}=[\overbrace{s_{t-1}, s_{t-1}, \ldots, s_{t-1}}^{p}]$. At the time instant 0 , the upsampled coefficients at $t-1$ are all equal to 0 . If the interpolator is currently in a time instant superior to 0 , it is assumed that there exists a previously interpolated value $y_{t-1}$.

- Next, the $n$-th upsampled coefficients from $\mathbf{s}_{t}$ and $\mathbf{s}_{t-1}$ are subtracted and divided by $p$ : $\Delta_{t}=\left(s_{t_{n}}-s_{t-1_{n}}\right) / p$.

- Finally, $\Delta_{t}$ is added or subtracted (depending on its sign) to/from the interpolation value calculated at the time instant $t-1$, obtaining the current output interpolated value: $y_{t}=$ $y_{t-1}+\Delta_{t}$.

Finally, the signal from the transmitter is filtered with the interpolated coefficients. Details about the implemented FIR filter can be found in (Fernández-Caramés, 2010).

\subsection{Emulator basic operation}

The emulator operation can be summarized as follows:

1. The configuration parameters of the vehicular channel are initially read from registers (shown in Fig. 2).

2. The emulator starts to generate channel coefficients, both for the LOS and the NLOS components (illustrated in Figs. 2 to 4 ).

3. The coefficients are interpolated to have their rate adapted to the incoming signal rate, passing each path through linear interpolators (like the one shown in Fig. 5). The interpolation is carried out in two stages, whose interpolation factors depend on the effective generation rates shown in Table 3. For instance, in the channel RTV-Urban Canyon, 
the coefficients have an effective generation rate equal to $2500 \mathrm{~Hz}$. Since the incoming signal rate is $10 \mathrm{MHz}$, the coefficients need to be interpolated with a global factor of 4,000, which can be applied in two stages with interpolation factors $2^{5}$ and $5^{3}$.

4. Finally, the incoming signal is applied to a complex FIR filter that uses the generated channel coefficients.

\section{Upgrading to MIMO}

First, note that the channels modeled in (Acosta, 2007b) are based on SISO measurements, but we use them because they have become the reference for evaluating IEEE 802.11p. Further investigation is still needed to adapt such channels to multiple-antenna environments, but when that occurs, the transceivers and channel emulator model presented in this chapter will continue to be valid, only requiring slight modifications or no modifications at all. Also, for the sake of brevity, regarding MIMO we will restrict ourselves to IEEE 802.11p transceivers.

\subsection{IEEE 802.11p MIMO transceivers}

\subsubsection{Multiple-antenna transmitter}

In the transmitter, the use of several antennas lead us to change our SISO channel estimation and use orthogonal pilots that constitute matrices called OSTPM (Orthogonal Space-Time Pilot Matrices). Specifically, we use Hadamard matrices created using Sylvester's method, which generates a sequence of matrices that are known as Walsh matrices. Such matrices are orthogonal in space and time and, in the case of transmitting with two antennas, they are generated according to:

$$
\mathbf{P}=\left(\begin{array}{cc}
p_{k} & p_{k} \\
p_{k} & -p_{k}
\end{array}\right)
$$

where $p_{k}$ is the BPSK-modulated pilot symbol transmitted at the $k$ subcarrier. Since IEEE 802.11p uses four pilots inside each OFDM symbol, the pilot matrix is generated by replicating Equation (3) to obtain a $2 \times 4$ matrix.

Using this scheme, channel estimation only requires simple linear processing. For instance, in the case of transmitting with two antennas, the received signal at the $k$-th subcarrier for two consecutive OFDM symbols would be:

$$
\begin{aligned}
& y_{1, k}=p_{k} h_{1, k}+p_{k} h_{2, k}+n_{1} \\
& y_{2, k}=p_{k} h_{1, k}-p_{k} h_{2, k}+n_{2}
\end{aligned}
$$

where $n_{1}$ and $n_{2}$ are AWGN samples and $h_{1}$ and $h_{2}$ are the channel coefficients. Thus, the channel coefficient estimations are obtained as:

$$
\hat{h}_{1, k}=\frac{y_{1, k}+y_{2, k}}{2 p_{k}} \quad \hat{h}_{2, k}=\frac{y_{1, k}-y_{2, k}}{2 p_{k}}
$$

Note that this channel estimation method has several limitations. First, it assumes that the channel remains constant over two consecutive pilots, so when the Doppler spread is high, performance will be degraded. The second drawback is related to the pilot generation matrix: it is only possible to use this pilot scheme when the number of transmit antennas is a power of two. Moreover, it requires an even number of transmitted OFDM symbols to be transmitted. In spite of the above mentioned issues, we stick to using this method due to its simplicity and because the maximum Doppler shift of the implemented channels is $1742 \mathrm{~Hz}$, that corresponds to a channel coherence time of $574 \mu \mathrm{s}$, which is clearly higher than the time required to transmit two consecutive OFDM symbols $(16 \mu \mathrm{s})$. 
Apart from the modifications required by the channel estimation step, to exploit space-time diversity, MIMO systems need an additional coding stage. In the case of $2 \times 2$ system Alamouti coding is used (Alamouti, 1998), whereas the $4 \times 4$ transceiver implements a quasi-orthogonal code proposed by Jafarkhani (Jafarkhani, 2000).

\subsubsection{Multiple-antenna receiver}

At the receiver side the main changes with respect to the SISO system are related to support diversity schemes. In the SIMO case the system implements the MRC (Maximum-Ratio Combining) technique, while the MIMO transceiver requires the use of an Alamouti decoder in the $2 \times 2$ case, and a ML (Maximum-Likelihood) detector otherwise. We do not give herein more details about the receivers since they use standard MIMO algorithms and techniques.

\subsection{MIMO vehicular channel emulation}

There are several examples of academic MIMO FPGA-based channel emulators. Some of them are generic (e.g. (Ren, 2010; Wang, 2008; Zhan, 2009)), while others (e.g. (Eslami, 2009)) are specifically oriented towards the implementation of the IEEE $802.11 \mathrm{n}$ reference channel models. However, none of the existing channel emulators has been explicitly developed for recreating VTV or RTV environments.

One of the main problems when implementing MIMO channel emulators in an FPGA is that they require large designs and, therefore, the use of resources has to be optimized. Most of the channel emulators described in the literature are able to implement the whole system into only one FPGA. To fit the design into one FPGA, researchers have to save resources using several clever tricks, being one of the most recurrent the off-line generation of the channel coefficients (Eslami, 2009; Zhan, 2009). Also, some authors (Eslami, 2009) are able to save up to $67 \%$ of the FPGA resources by applying the channel coefficients in the frequency domain. These academic developments present at least three drawbacks. First, the use of low-level description languages such as VHDL slows down the development stage.

The second problem is related to the portability of the channel emulator. A good channel emulator should be able to work in stand-alone mode, i.e. without needing external devices to generate and transfer channel coefficients to the FPGA.

The third drawback is related to scalability. As it can be derived from the results exposed in (Eslami, 2009), when we work with a time-domain based channel emulator, the gate count (i.e. the number of 2-NAND logic gates that would be required to implement the same number and type of logic functions) roughly doubles every time we add a transmit and a receive antenna to the system. Therefore, a scalable solution would have to be able to deal with more inputs and outputs without requiring such important hardware complexity increases.

The vehicular emulator described in this chapter addresses these three drawbacks: we use Xilinx System Generator to develop the channel emulator faster than using an HDL, we optimize our design in order to fit a MIMO twelve-path channel emulator into one FPGA, we design the emulator bearing in mind that it has to be able to work in stand-alone mode with minimal modifications and we propose a time-multiplexing solution that has a very low impact on the emulator design, thus facilitating scalability.

\subsection{Refining the emulator: from SISO to MIMO}

Our first attempt to expand our SISO emulator to accept more input and output antennas consisted in creating a SIMO $1 \times 2$ system by replicating the SISO design. The obtained design was too large to fit into our FPGA, so we proceeded to optimize it. For the sake of brevity, we will only cite the three most important optimizations we carried out, whose savings are 
summarized in Table 5 and where the most complex out of the six designs (in terms of FPGA resources consumed) is used as a reference (VTV-Expressway Same Direction with Wall).

\begin{tabular}{|c||c|c|c|c|c|}
\hline Version & Slices & Slice flip-flops & LUTs & FIFO16/RAMB16 & DSP48 \\
\hline SISO & $85 \%$ & $40 \%$ & $57 \%$ & $24 \%$ & $65 \%$ \\
\hline SIMO 1x2 (V1) & $113 \%$ & $74 \%$ & $110 \%$ & $40 \%$ & $100 \%$ \\
\hline SIMO 1x2 (V2) & $107 \%$ & $71 \%$ & $104 \%$ & $36 \%$ & $100 \%$ \\
\hline SIMO 1x2 (V3) & $99 \%$ & $69 \%$ & $89 \%$ & $37 \%$ & $78 \%$ \\
\hline MIMO 4x4 & $82 \%$ & $43 \%$ & $60 \%$ & $27 \%$ & $66 \%$ \\
\hline
\end{tabular}

Table 5. Resource utilization of different versions of the vehicular channel emulator.

The first optimization reduced the amount of resources dedicated to perform the Doppler filtering stage (i.e. the stage aimed at applying each path's Doppler spectrum) by using a four-output Doppler filter. This filter is a natural evolution of the SISO filter shown in Fig. 3. It makes use of a four-input multiplexor and has a four-output demutiplexor after the FIR Compiler block (see Fig. 6). This is possible since every path uses the same Doppler filters. The resources occupied by this optimized version of the emulator are shown in Table 5 in the row SIMO $1 \times 2(V 1)$.

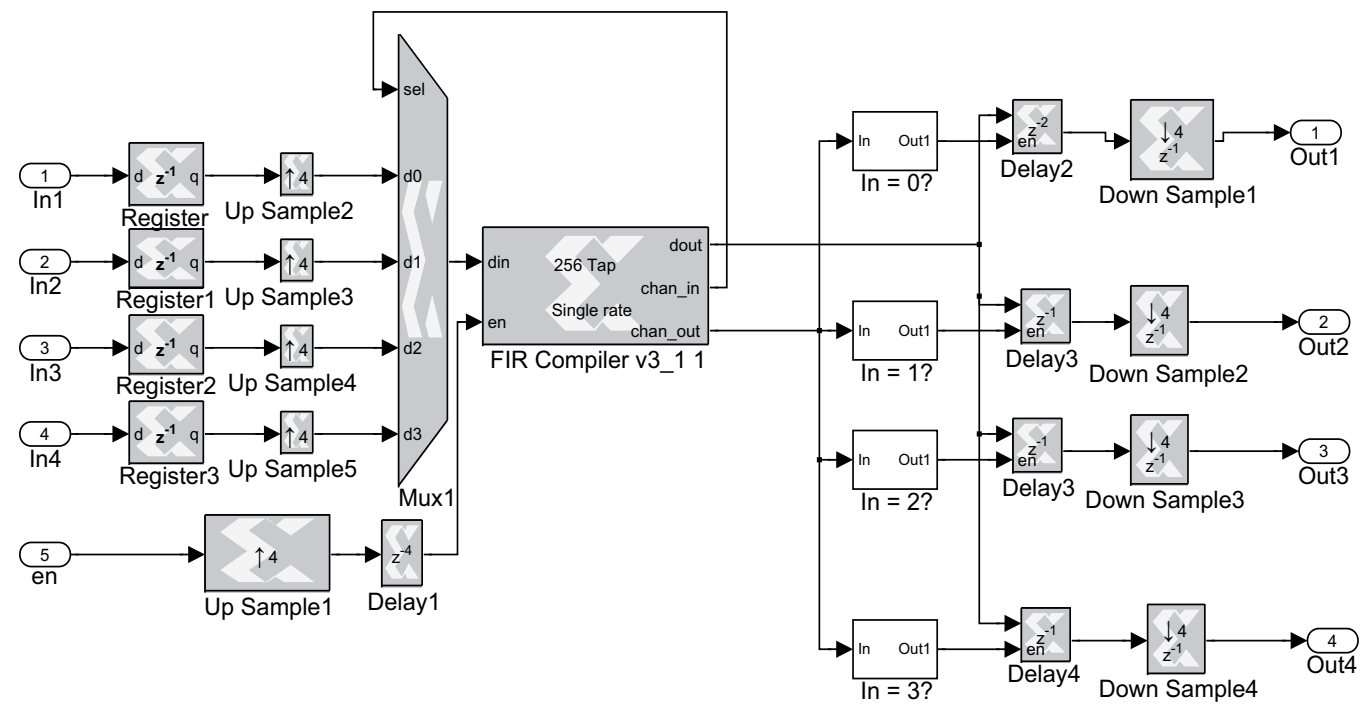

Fig. 6. Doppler filtering stage developed for SIMO $1 \times 2(\mathrm{~V} 1)$.

Our second optimization was related to the high resource consumption of each System Generator's AWGN block, as already mentioned in Section 3.4.2. Thus, we removed one of the two AWGN generators and created a 50-output Gaussian generator (we only need 48 outputs, but 50 is the closest integer divider of $10 \mathrm{MHz}$ ). Note that System Generator's demultiplexors are restricted to use up to 32 outputs, so we had to build the 50-output demultiplexor depicted in Fig. 7. As it can be seen in Table 5, in the row SIMO 1x2 (V2), this optimization allowed us to save $6 \%$ of the slices, $3 \%$ of the slice flip-flops, $6 \%$ of the LUTs and $4 \%$ of the FIFO16/RAMB16 blocks, but it was yet too large to fit into our FPGA.

The third optimization consisted in allowing every path to share Doppler filters, interpolators and FIR filters, being the Gaussian noise generated unique for each path. Hence, we built a multiplexed version of the emulator that buffered incoming signals, switched the Gaussian noise source and applied the channel to each transmitted signal at the proper time instants. As it is shown in the row SIMO $1 \times 2$ (V3) of Table 5, the used resources decreased substantially 


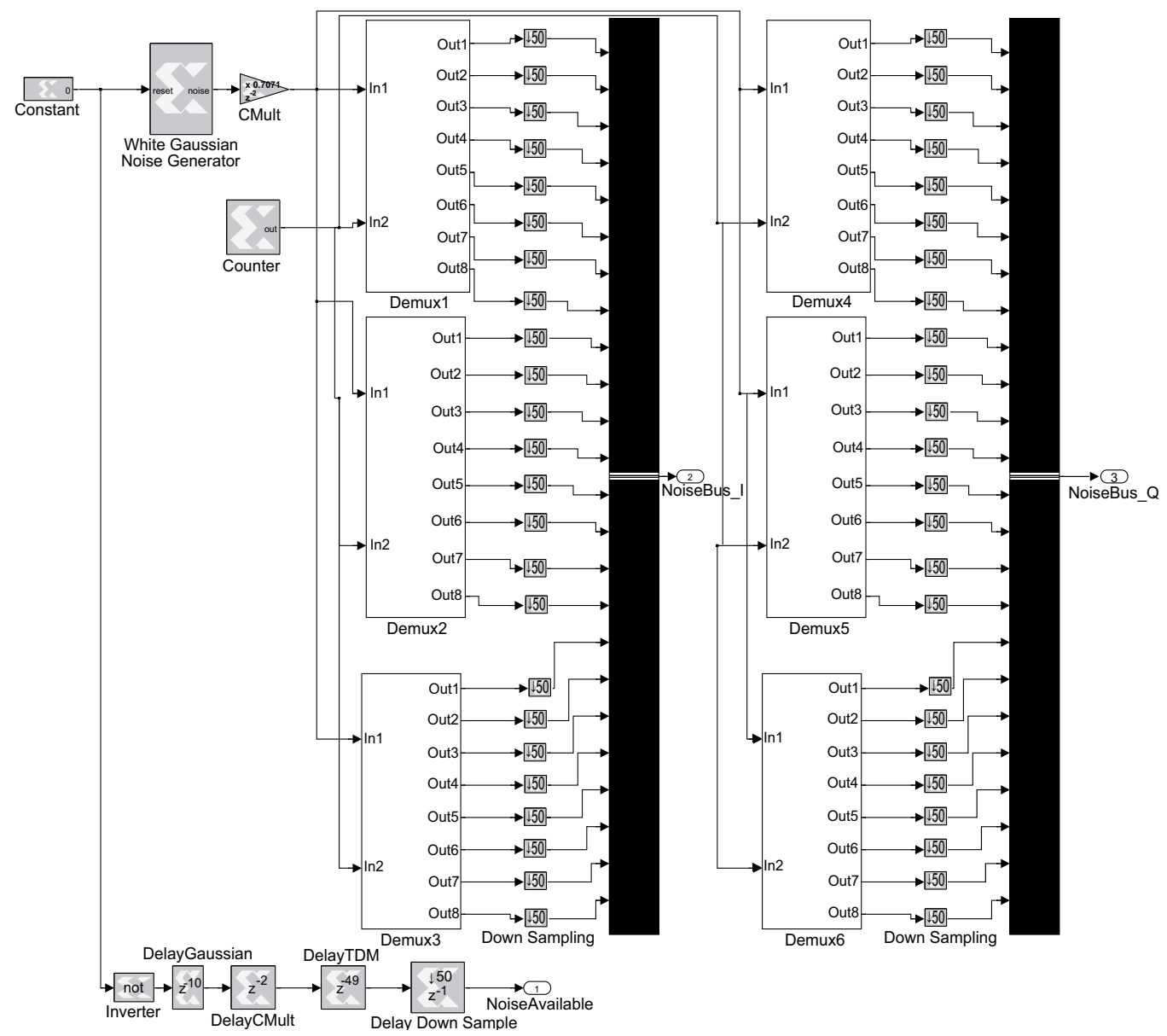

Fig. 7. Gaussian noise generator block developed for SIMO $1 \times 2$ (V2).

with respect to version V2 and permitted to save $8 \%$ of the slices, $2 \%$ of slice flip-flops, $15 \%$ of the LUTs and $22 \%$ of the DSP48 blocks. Thus, the design fitted into our FPGA, but it was clear that it would be very difficult to fit a MIMO $4 \times 4$ system (which has 8 times more paths than an SIMO $1 \times 2$ system) following this optimization strategy.

To solve the space issue we devised different alternatives, but we finally resorted to a scheme whose complexity lays in input and output buffers. Such buffers act similarly to two synchronized parallel-to-serial and serial-to-parallel converters. Each set of signals transmitted from an array of IEEE 802.11p transceivers is stored into a buffer and released at specific time instants, achieving a similar effect as if the parallel transceivers were executed in serial. In Table 5, row MIMO $4 x 4$, it can be seen the important resource savings attained by using the time-multiplexing approach, which even consumes $3 \%$ less slices than our original SISO version thanks to some of the above described optimizations.

We would like to emphasize that although the resources consumed by the time-multiplexed approach have been indicated in Table 5 using the term MIMO $4 \times 4$, such resources would be the same for any system of up to four antennas in transmission and four antennas in reception. It is also important to note that the input buffer has to add zeroes between each pair of signals that were transmitted by different antennas in order to reduce time correlation. In our 
emulator we set the coefficient generation rate to frequencies that range between $2 \mathrm{KHz}$ and $4 \mathrm{KHz}$, so the shortest time the channel remains almost constant would be $500 \mu \mathrm{s}$. Therefore, if the FPGA clock is set to $20 \mathrm{MHz}$, the number of cycles that the channel values remain almost constant would be $500 \mu \mathrm{s} / 50 \mathrm{~ns}=10,000$. To stay safe, we can wait for 100,000 cycles $(5 \mathrm{~ms})$ to guarantee minimal correlation. Hence, we separate each pair of signals by $5 \mathrm{~ms}$.

\section{Experiments}

Performance evaluation of the software transceivers was carried out by passing the signals they produce through the FPGA-based vehicular channel emulator. Taking advantage of the Xilinx Xtreme DSP software kit capabilities, measurements are performed using the co-simulation mode: the transmitter and the receiver run in MATLAB and Simulink, while the channel emulator runs on the FPGA. A maximum of 100,000 48-bit FEC blocks are averaged for each SNR (or $E_{b} / N_{0}$ ) value (the simulation stops when 100 erroneous FEC blocks are detected).

\subsection{Mobile WiMAX Vs. IEEE $802.11 \mathrm{p} / \mathrm{a}$}

In this subsection we present the results of a performance comparison between Mobile WiMAX, IEEE 802.11p and IEEE 802.11a transceivers. For a fair comparison we set the same transmission parameters for every transceiver. A rate 1/2 FEC is used and the subcarriers are filled with QPSK modulated symbols. The receiver assumes perfect time synchronization and, after estimating the channel using a pilot-aided scheme, an MMSE linear equalizer is applied. The remaining transceiver parameters are shown in Table 1. Additionally, for the sake of fairness, instead of comparing performances in terms of PER (Packet Error Rate), we obtained the FEC Frame Error Rate (FER) when all the transceivers make use of the same FEC block size.

\subsubsection{Performance over AWGN and rayleigh fading channels}

In order to obtain a performance reference, we evaluated the implemented transceivers over two non-vehicular environments. Fig. 8.(a) and (b) show, respectively, the transceivers performance over an AWGN channel and a frequency-flat Rayleigh block fading channel whose coefficients were constant during 15 FEC blocks (i.e. one Mobile WiMAX slot).
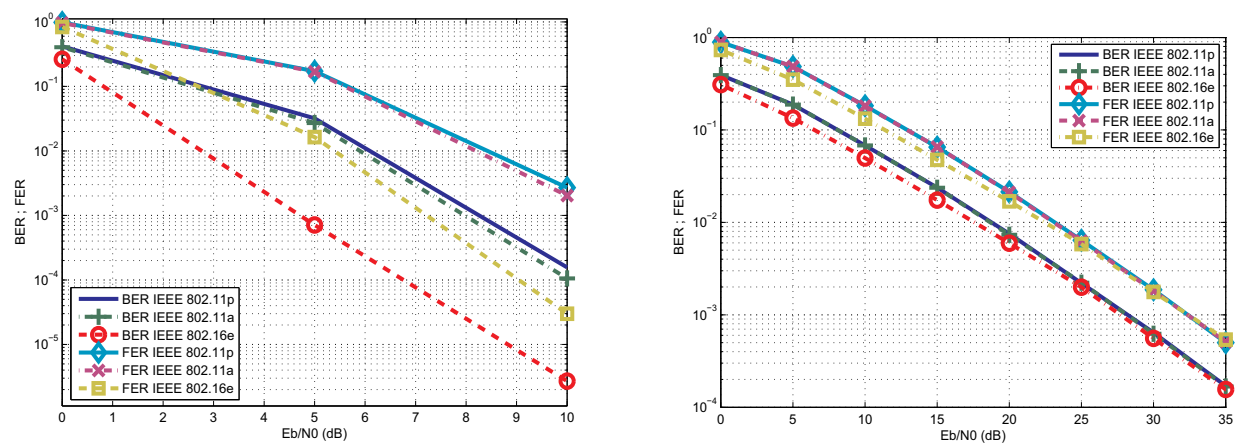

Fig. 8. Performance over (a) AWGN channel (left) and (b) block-fading Rayleigh channel (right). 
For both channels, IEEE 802.11p and IEEE 802.11a produce roughly the same results. This was expected, since the transceiver is the same in all aspects apart from the bandwidth. However, the IEEE 802.16e transceiver yields much better results, especially in the AWGN channel. This is because channel estimation is far more accurate in the case of the IEEE 802.16e transceiver. Indeed, Fig. 9 shows the Mean Squared Error (MSE) between the estimated and the true channel for the IEEE 802.11p (the same results were obtained with IEEE 802.11a) and IEEE 802.16e transceivers when considering an AWGN channel. IEEE 802.16e better estimates the channel thanks to the use of one pilot for each group of six data subcarriers, while IEEE 802.11p makes use of only one pilot for each group of twelve data subcarriers.

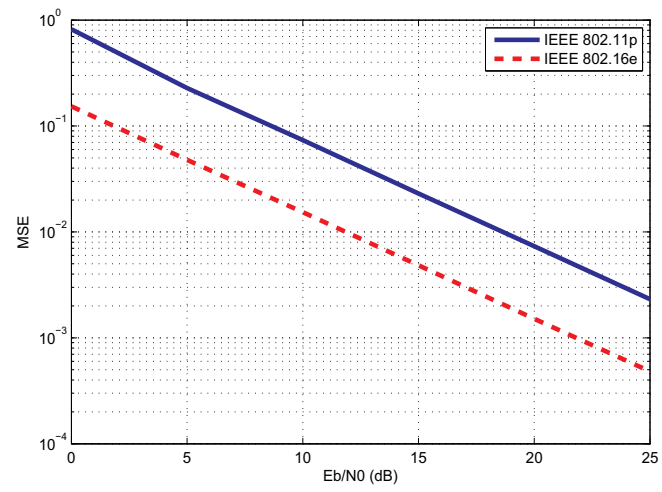

Fig. 9. Channel estimation error when transmitting over an AWGN channel.

\subsubsection{Performance over vehicular channels}

Figs. 10 to 15 depict the BER and FER curves for the three transceivers when transmitting over the six vehicular channels described in Section 3. In general, it can be observed that the IEEE 802.16e transceiver produces better results (both in terms of BER and FER) than IEEE 802.11p, while the IEEE 802.11a transceiver obtains the worst global results.

In urban environments (channels VTV-Urban Canyon Oncoming and RTV-Urban Canyon) Mobile WiMAX outperforms IEEE 802.11p/a in terms of BER and FER for $E_{b} / N_{0}$ values below $20 \mathrm{~dB}$ (see Figs. 10 and 11). Also, notice that Mobile WiMAX requires the lowest $E_{b} / N_{0}$ values to reach a target FER of $10 \%$.

In surface streets (RTV-Suburban Street) Mobile WiMAX also performs better than the other standards (see Fig. 12). For instance, to reach an FER of $10 \%$ Mobile WiMAX requires $8.6 \mathrm{~dB}$, while IEEE 802.11p and IEEE 802.11a need, respectively, $11.1 \mathrm{~dB}$ and $14.6 \mathrm{~dB}$.

In expressways (VTV-Expressway Oncoming, RTV-Expressway, VTV-Expressway Same Direction With Wall) the results depend on the channel. In VTV-Expressway Oncoming (see Fig. 13) IEEE 802.11p clearly outperforms Mobile WiMAX at both low and high values of $E_{b} / N_{0}$. IEEE 802.11p and Mobile WiMAX both exhibit a similar performance when considering RTV-Expressway channels (see Fig. 14): Mobile WiMAX is slightly better than IEEE 802.11p while the situation reverses for high $E_{b} / N_{0}$ values. Finally, in the case of VTV-Expressway Same Direction With Wall, Mobile WiMAX clearly obtains a major gain over IEEE 802.11p/a: for example, it requires an $E_{b} / N_{0}$ of $6.2 \mathrm{~dB}$ less than that of IEEE 802.11p (7.6 dB vs $\left.13.8 \mathrm{~dB}\right)$ to obtain an FER of $10 \%$ (see Fig. 15). 


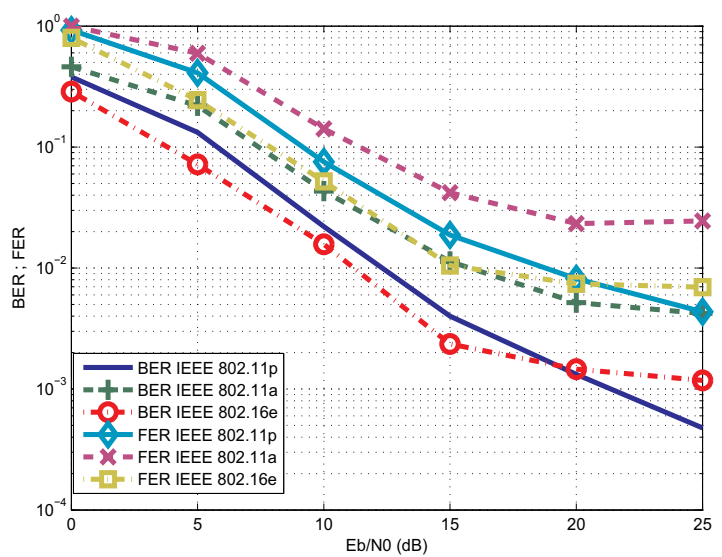

Fig. 10. Performance comparison when transmitting over VTV-Urban Canyon Oncoming.

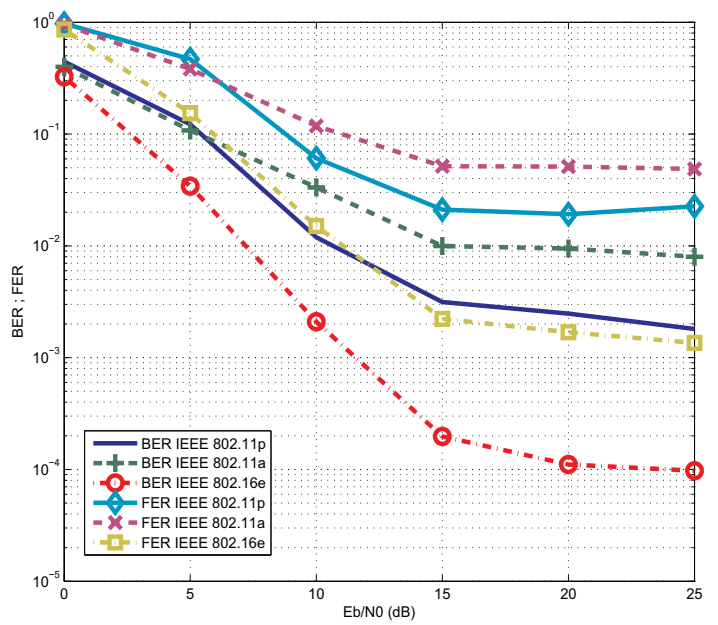

Fig. 11. Performance comparison when transmitting over RTV-Urban Canyon.

\subsubsection{Discussion: Mobile WiMAX or IEEE 802.11p?}

The BER/FER versus $E_{b} / N_{0}$ curves depicted in the previous subsection indicate that the PHY Layer of Mobile WiMAX outperforms that of IEEE 802.11p in most of the reference channel models used as benchmarks in vehicular communications.

An explanation of this behavior is the superior robustness to high channel delay spreads of the Mobile WiMAX PHY Layer. In IEEE 802.11p, assuming a bandwidth of $10 \mathrm{MHz}$ and 64 subcarriers, a $1 / 4$ cyclic prefix will lead to a guard time of $1.6 \mu \mathrm{s}$. In the case of Mobile WiMAX, a transceiver that uses $10 \mathrm{MHz}$ of bandwidth and 512 subcarriers has a $1 / 4$ cyclic prefix that lasts $12.8 \mu \mathrm{s}$. Thus, the OFDM symbols used in Mobile WiMAX can equalize channels with a larger delay spread. Another advantage of the Mobile WiMAX PHY Layer is that the maximum data rate that it can reach is $39.9 \mathrm{Mbits} / \mathrm{s}$ while this value is only $27 \mathrm{Mbits} / \mathrm{s}$ in the case of IEEE 802.11p. 


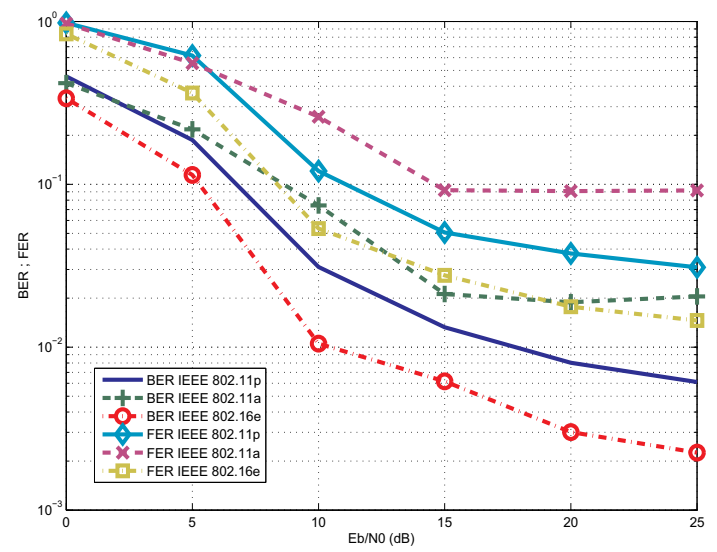

Fig. 12. Performance comparison when transmitting over RTV-Suburban Street.

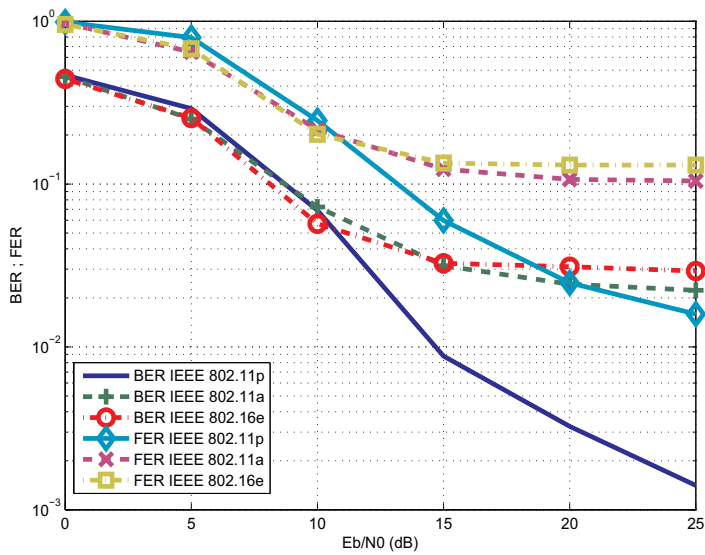

Fig. 13. Performance comparison when transmitting over VTV-Expr. Oncoming.

On the other hand, it should be mentioned that the PHY layer of IEEE 802.11p supports larger vehicle speeds. Indeed, IEEE 802.11p is designed to transmit 1000 byte data packets with an FER lower than $10 \%$ at a maximum Doppler shift of $\pm 2100 \mathrm{~Hz}$, what means that a top speed of roughly $385 \mathrm{Km} / \mathrm{h}$ can be reached when using the $5 \mathrm{GHz}$ band (the transmitter and receiver would drive at almost $193 \mathrm{Km} / \mathrm{h}$ ). This explains the superior performance of IEEE 802.11p over the VTV-Expressway Oncoming, which is the channel with maximum Doppler shift (see Table 2).

\subsection{IEEE 802.11p MIMO measurements}

In order to achieve a fair comparison we have set the same transmission parameters for every transceiver and we have assumed that all of them send signals with the same transmission power. A rate 1/2 FEC code was used and the OFDM subcarriers were filled with QPSK-modulated symbols. The receiver assumed perfect time synchronization and the channel was estimated using the OSTPM-based method described in Section 4.1.1. In SISO systems an MMSE linear equalizer followed by an ML detector was used, whilst SIMO 


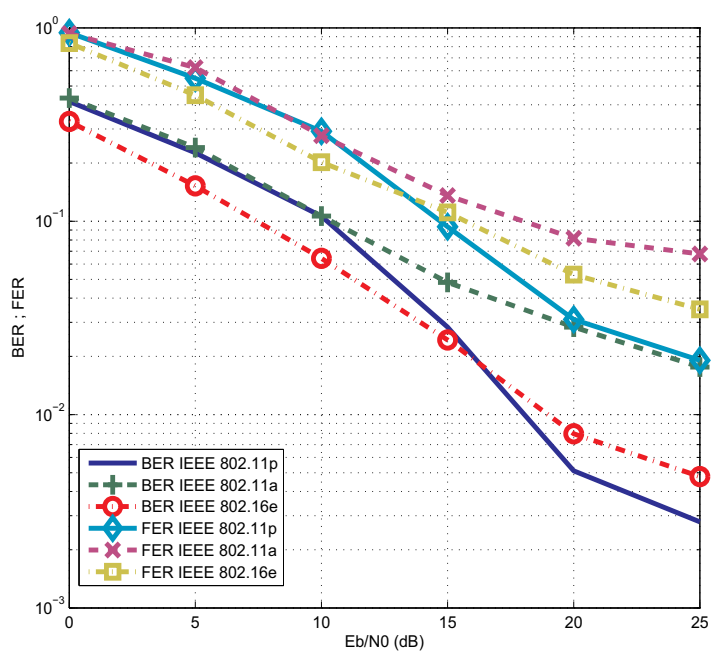

Fig. 14. Performance comparison when transmitting over RTV-Expressway.

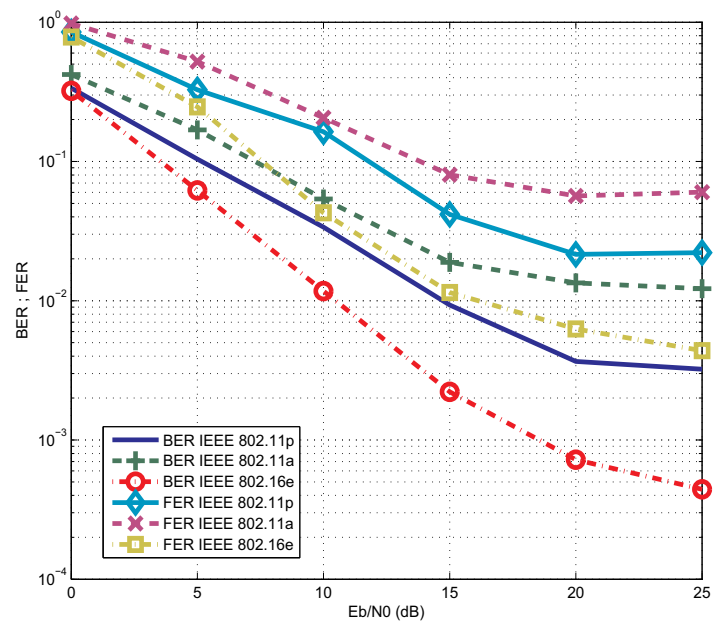

Fig. 15. Performance comparison when transmitting over VTV-Expressway Same Direction with Wall.

transceivers implemented the MRC technique. In the case of MIMO receivers, symbols were decoded if needed (an Alamouti decoder was used for $2 \times 2$ systems) and an ML detector was applied.

A maximum of 10,000 48-bit FEC blocks were averaged for different SNR values (the simulation stopped for each SNR value when 100 erroneous FEC blocks were detected).

\subsubsection{Performance in vehicular channels}

For the sake of space, we only show and compare the results for three different vehicular channels (Figs. 16 to 18), which give a good overview of the performance drawn by the 
implemented multi-antenna IEEE 802.11p transceivers. As expected, it can be observed that SIMO/MIMO transceivers outperform SISO systems.

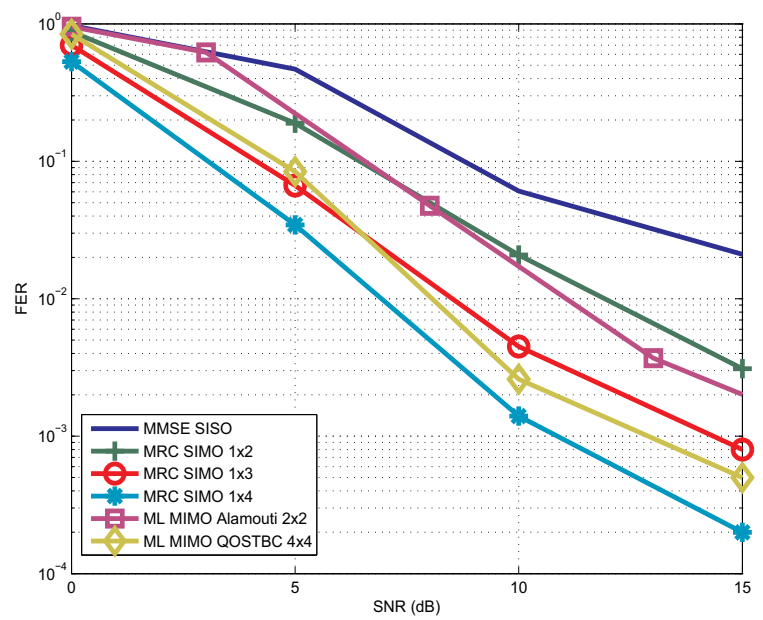

Fig. 16. FER performance for RTV-Urban Canyon.

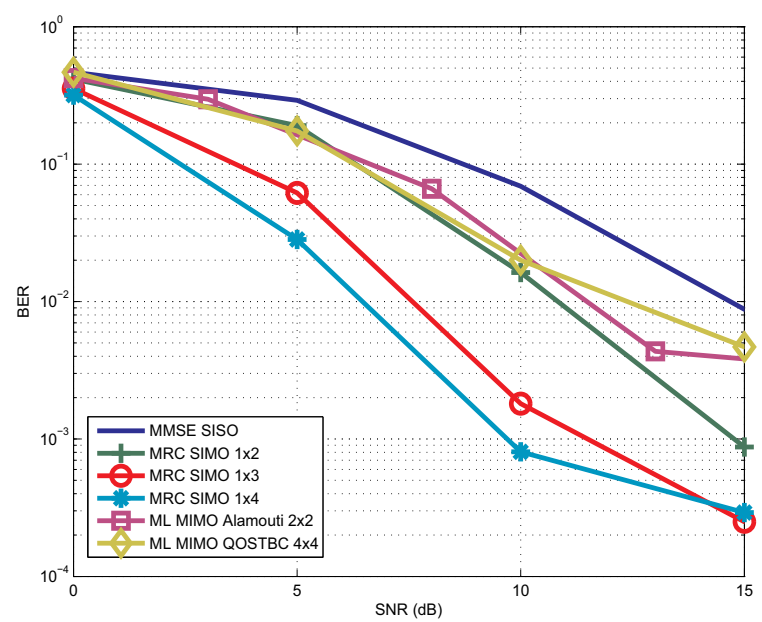

Fig. 17. FER performance for VTV-Expressway Oncoming.

In the emulated conditions, SIMO systems seem to offer the best tradeoff between hardware complexity and performance. In all environments, the SIMO $1 \times 4$ system obtains the best performance and in almost every vehicular channel the SIMO $1 \times 3$ is the transceiver that gets the second best results.

SIMO $1 \times 2$ and MIMO $2 \times 2$ attain similar BER/FER performance, while the results obtained by the MIMO $4 \times 4$ strongly depend on the vehicular channel. Such performance difference is related to two main factors: the channel estimation misbehavior in presence of high Doppler frequencies and the presence of a low overall $K$ factor.

On the one hand, as mentioned in Section 4.1.1, due to the particular channel estimation technique implemented, a high Doppler frequency leads to a bad channel estimation, what is really harmful for the performance of multi-antenna systems. In fact, if we rank the 


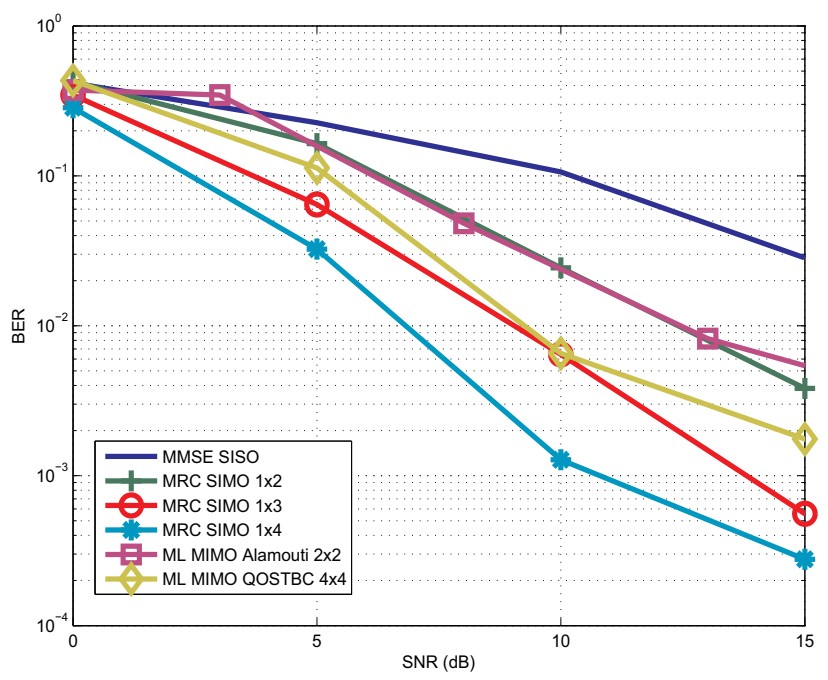

Fig. 18. FER performance for RTV-Expressway.

three channels by their LOS Doppler frequency $(654 \mathrm{~Hz}$ for RTV-Urban Canyon, $770 \mathrm{~Hz}$ for $R T V$-Expressway and $1452 \mathrm{~Hz}$ for VTV-Expr. Oncoming), it is apparent that the lower the Doppler, the better the performance.

On the other hand, if we rank the channels by their overall $K$ factor $(6.7 \mathrm{~dB}$ for RTV-Urban Canyon, $4.3 \mathrm{~dB}$ for RTV-Expressway and $-3.6 \mathrm{~dB}$ for $V T V$-Expr. Oncoming), we can conclude that the higher overall $K$ factor, the better the performance.

Furthermore, the results shown in Table 6, corresponding to the required SNR to obtain a FER of $10 \%$, give a good idea about the performance of each transceiver and confirms our previous statements. The maximum differences in SNR occur when comparing the SISO and the SIMO $1 \times 4$ systems, ranging between $5.73 \mathrm{~dB}$ (for RTV-Urban Canyon) and $8.96 \mathrm{~dB}$ (for $R T V$-Expressway). That is, a SIMO $1 \times 4$ system requires between 4 and 8 times less power than a SISO system to obtain the same FER.

\begin{tabular}{|c|c|c|c|c|c|c|}
\hline Channel & SISO & $\begin{array}{c}\text { SIMO } \\
1 \times 2 \\
\end{array}$ & $\begin{array}{c}\text { SIMO } \\
1 \times 3\end{array}$ & $\begin{array}{c}\text { SIMO } \\
1 \times 4\end{array}$ & $\begin{array}{c}\text { MIMO } \\
2 \times 2\end{array}$ & $\begin{array}{c}\text { MIMO } \\
4 \times 4\end{array}$ \\
\hline & & & & & & 62 \\
\hline $\mathrm{R}$ & 14. & 10 & 7. & 5.7 & 10.0 & 8.62 \\
\hline VTV-Expressway & 13. & & 7.19 & 5.81 & 10.82 & 11.9 \\
\hline
\end{tabular}

Table 6. SNR $(\mathrm{dB})$ required to obtain a FER of $10 \%$ in each vehicular channel.

Finally, it must be pointed out that multiple-antenna transceivers obtain their largest SNR gains when transmitting over channels that assume high vehicular speeds (i.e. the scenarios located in expressways), achieving gains from $7.36 \mathrm{~dB}$ to $8.96 \mathrm{~dB}$ with respect to SISO systems. This is a quite interesting result, since it means that mobile communications performed in high speed scenarios can be greatly improved by placing antenna arrays along the roadside and/or in vehicles and using relatively simple space-time diversity techniques.

\section{Conclusions}

We have presented a performance evaluation tool for wireless standards suitable for vehicular communications. It consists of a software testbed and a flexible, low-cost, FPGA-based 
channel emulator. We have detailed the way we employed rapid-prototyping techniques for building both the testbed and the channel emulator. The resulting evaluation system has been used to assess the performance of the PHY layer of IEEE 802.11e (Mobile WiMAX) and IEEE $802.11 \mathrm{p} / \mathrm{a}$ over representative situations where vehicular communications can take place.

In addition, we have shown how this performance evaluation system has been upgraded for multiple-antenna transceivers. The different hardware optimizations we have performed during the design process of our MIMO channel emulator have been also explained. Finally, we have presented interesting performance evaluation results for SISO, SIMO and MIMO transceivers over reference vehicular channel models.

\section{Acknowledgements}

This work has been supported by Xunta de Galicia through grants 09TIC008105PR and 10TIC003CT, by Ministerio de Ciencia e Innovación of Spain through grant TEC2010-19545-C04-01 (COSIMA project) and by Ministerio de Ciencia e Innovación of Spain and FEDER funds of the EU under grant CSD2008-00010 (COMONSENS project).

\section{References}

Acosta-Marum, G. (2007). Measurement, modelling and OFDM synchronization for the wideband mobile-to-mobile channel. Doctoral Thesis, May 2007.

Acosta-Marum, G.; Ingram, M. A. (2007). Six time- and frequency-selective empirical channel models for vehicular wireless LANs, Proceedings of VTC Fall, ISSN 1090-3038, Baltimore, USA, Oct. 2007.

Alamouti, S. M. (1998) A simple transmitter diversity scheme for wireless communications, IEEE Journal on Selected Areas of Communications, ISSN 0733-8716, vol. 16, No. 8 (1998) 1451-1458.

Alimohammad, A.; Fard, S. F; Cockburn, B. F.; Schlegel, C. (2008). An accurate and compact Rayleigh and Rician fading channel simulator, Proceedings of VTC Spring, ISSN 1550-2252, Singapore, May 2008.

Angelakis, V.; Kossifidis, N.; Papadakis, S.; Siris, V.; Traganitis, A. (2008). The effect of using directional antennas on adjacent channel interference in 802.11a: Modeling and experience with an outdoors testbed, Proceedings of WiOPT2008, ISBN 978-963-9799-18-9, pp. 24-29, 978-963-9799-18-9, Berlin, Germany, April 2008.

Arthaber, H.; Schuberth, C. (2009). A channel emulator for UHF RFID systems, Proceedings of IEEE Radio and Wireless Symposium, ISBN 978-1-4244-2698-0, pp. 518-521, 978-1-4244-2698-0, San Diego, USA, Jan. 2009.

ASTM Intl. (2003). Standard specification for telecommunications and information exchange between roadside and vehicle systems - $5 \mathrm{GHz}$ band Dedicated Short Range Communications (DSRC), Medium Access Control (MAC) and Physical Layer (PHY) specifications, E2213-03, Sep. 2003.

Dassatti, A.; Masera, G.; Nicola, M.; Concil, A.; Poloni, A. (2005). High performance channel model hardware emulator for $802.11 \mathrm{n}$, Proceedings of IEEE International Conference on Field-Programmable Technology, ISBN 0-7803-9407-0, pp. 303-304, Singapore, Dec. 2005.

Eslami, H.; Tran, S. V.; Eltawil, A. M. (2009) Design and implementation of a scalable channel emulator for wideband MIMO systems, IEEE Transactions on Vehicular Technology, ISSN 0018-9545, vol. 58, No. 9 (2009) 4698-4709.

Faseth, T.; Winkler, M.; Schuberth, C.; Arthaber, H.; Magerl, G. (2010). Design and implementation of a wireless link coupled channel emulator for DSRC wireless 
systems, Proceedings of IEEE MTT-S International, ISSN 0149-645X, pp. 1632-1635, Anaheim, USA, May 2010.

Fernández Caramés, T. M.; García Naya, J. A.; González-López, M.; Castedo, L. (2008). FlexVehd: a flexible testbed for vehicular radio interfaces, Proceedings of ITST, ISBN 978-1-4244-2857-1, Phuket, Thailand, Oct. 2008.

Fernández-Caramés, T. M.; González López, M.; Castedo, L. (2010). FPGA-based vehicular channel emulator for real-time performance evaluation of IEEE 802.11p transceivers, EURASIP Journal on Wireless Communications and Networking (2010).

Foschini, G.; Gans, M. (1998). On limits of wireless communications in a fading environment when using multiple antennas, Wireless Personal Communications, ISSN 0929-6212, vol. 6, No. 3 (1998) 311-335.

Ghazel, A.; Boutillon, E.; Danger, J.; Gulak, G.; Laamari, H. (2003). Design and performances analysis of high speed AWGN communication channel emulator, Analog Integrated Circuits and Signal Processing, ISBN 0-7803-7080-5, vol. 34, pp. 133-142, Feb. 2003.

Hu, S.; Wu G. W.; Guan, Y. L.; Law, C. L; Yan, Y.; Li, S. (2007). Development and performance evaluation of mobile WiMAX testbed, Proceedings of IEEE Mobile WiMAX Symposium, ISBN 1-4244-0957-8, pp. 104-107, Orlando, USA, Mar. 2007.

Hwang, J.; Lin, K.; Li, J.; Deng, J. (2007). Fast FPGA prototyping of a multipath fading channel emulator via high-level design, Proceedings of ISCIT, ISBN 978-1-4244-0976-1, Sidney, Australia, Oct. 2007.

IEEE 802.11 (2007), IEEE Standard 802.11: Wireless LAN Medium Access Control (MAC) and Physical Layer (PHY) Specifications, IEEE (2007).

IEEE 802.16 (2009), Air interface for fixed broadband wireless access systems, IEEE, 2009.

IEEE (2010), IEEE Standard Wireless LAN Medium Access Control (MAC) and Physical Layer (PHY) Specifications. Amendment 6: Wireless Access in Vehicular Environments (2010).

Jafarkhani, H. (2000) A quasi-orthogonal space-time block code, IEEE Transactions on Communications, ISSN 0090-6778, vol. 49, No. 1 (2001) 1-4.

Nieto, X.; Ventura, L.M.; Mollfulleda, A. (2006). GEDOMIS: a broadband wireless MIMO-OFDM testbed, design and implementation, Proceedings of TRIDENTCOM2006, ISBN 1-4244-0106-2, Barcelona, Spain, Mar. 2006.

Ren, F.; Zheng, Y. R. (2010) A novel emulator for discrete-time MIMO triply selective fading channels, IEEE Transactions on Circuits and Systems, ISSN 1549-8328, vol. 57, No. 9 (2010) 2542-2551.

Rugini, L.; Banelli, P.; Leus, G. (2005). Simple equalization of time-varying channels for OFDM," IEEE Communications Letters, ISSN 1089-7798, vol. 9, No. 7 (July 2005), 619-621.

Telatar, I. E. (1999). Capacity of multi-antenna Gaussian channels, European Transactions on Telecommunications, ISSN 1541-8251, vol. 10, No. 6 (1999) 585-595.

Wang, T.; Liao, C. H.; Chiueh, T. D. (2007) A real-time digital baseband MIMO channel emulation system, Proceedings of ISCAS, ISBN 1-4244-0920-9, New Orleans, USA, May 2007.

Wang, Y.; Ahmed, A.; Krishnamachari, B.; Psounis, K. (2008) IEEE 802.11p performance evaluation and protocol enhancement, Proceedings of Intl. Conf. on Vehicular Electronics and Safety, ISBN 978-1-4244-2359-0, Columbus, USA, Sep. 2008.

Zhan, Z.; Jun, J.; Ping, Z.; Xin, W. (2009) A generalized hardware implementation of MIMO fading channels, Proceedings of ISCIT, ISBN 978-1-4244-4521-9, Incheon, Korea, Sept. 2009. 


\title{
Position Location Technique in Wireless Sensor Network Using Rapid Prototyping Algorithm
}

\author{
Touati Youcef, Aoudia Hania, Ali-Cherif Arab and Mohamed Demri \\ University of Paris 8 at Vincennes/Saint-Denis \\ Computer Science and Artificial Intelligence Lab. LIASD
}

France

\section{Introduction}

Localization process using Wireless Sensor Network (WSN) is one of the main services that have been prospected in order to ensure assisted people a better mobility and assistance in their life. In this direction, localization methods have been investigated (Röhring, 2009; Vossiek, 2003) and can be classified as the used information, such as: Connectivity information, Angle of Arrival (AOA), Time of Arrival (TOA), Time Difference of Arrival (TDOA), and RSSI method. The latter is the most used one, and it consists to acquire a distance from measuring the received signal strength of the incoming radio signal. The idea behind RSSI is that the configured transmission power at the transmitting device directly affects the receiving power at the receiving device. In practical scenarios, the ideal distribution of the received power is not applicable, because the propagation of the radio signal is interfered with a lot of influencing effects, such as: reflection on metallic objects, superposition of electromagnetic fields, noises and so on. Implementing these models requires parameters identification phase, allowing being closer to sensor model. In reality, it represents just an approximation procedure which doesn't reflect a real world.

Several sensor models have been developed (Tsung-Han, 2008; Awad, 2007; Graefenstein, 2008; Sugano, 2006; Cho, 2007). The most used one can be described by the following equation:

$$
P(d)=P\left(d_{0}\right)-10 \alpha \log \frac{d}{d_{0}}
$$

Where $d_{0}, P\left(d_{0}\right)$ and $\alpha$ are respectively the distance of reference, the received power and an approximation factor $(\approx 3.5)$.

Another methods based on Link Quality Indicator property (LQI) have been proposed too for distance calculation. That property is considered as a measure of force where the quality of received parquets is proportional to RSSI signal. Thus, the distance between nodes can be determined as follows:

$$
P_{i}(x, y)=\frac{\sum_{j=1}^{n}\left(w_{i j} \cdot B_{j}(x, y)\right)}{\sum_{j=1}^{n} w_{i j}}
$$


Where $B(x, y)$ is the position of anchors and $w_{i j}$ is the link weight between nodes, such as:

$$
w_{i j}=\frac{1}{\left(d_{i j}\right)^{\beta}}
$$

Parameter $\beta$ is experimentally estimated.

These methods are very attractive nevertheless they present some drawbacks on requiring theoretical or generic sensory models closer to the real ones. Moreover, these models require a mass of information to achieve higher accuracy leading to increase traffic, sensors energy consumption and a better approximation and optimization of parameters taking into account environment change and signal failures. Thus, it seems to be difficult and less efficient to implement. A good localization algorithm should calculate a position as fast as possible and should be resistant to environmental influences as well as imprecise distances. The challenge here is to prospect others approaches offering more realistic models taking into account these constraints and particularly parameters approximation problem.

Introduced by Zadeh (Zadeh, 1965) in 1965, fuzzy logic concept has been widely applied in different applications such as control and identification process, pattern recognition, graphics analysis and synthesis and robotics (Bezdek, 1981; Dave, 1990; Liang, 2003; Huang, 2003; Lei, 2001) and satisfactory results have been obtained. In contrast, few researches have been directed towards development of fuzzy logic-based approaches for localization in WSN, even if they present advantages in terms of robustness in spite of noise or other sensor failures, ability to handle non-linearity without degradation and the use of linguistic adjectives and relations similar to a natural language for interpretability.

For this purpose, we propose a WSN localization method based on IEEE 802.15.4 standard using fuzzy logic concepts with an evolutionary algorithm for parameters approximation and optimization. The approach allows approximating a realistic model for distance estimation from a set of RSSI measures and taking into account environment influences and signal failures as well as imprecise distances. The approach is based on a FIS optimization methodology combining a Rapid Prototyping (RP) mechanism and Solis and wets' monoagent evolution strategy (Solis, 1981; Glorennec, 1999; Hart, 1994). The methodology allows, in term of probability, a randomized hill-climber with an adaptive step size and a global minima convergence of a coast function. It describes a class of local and global search algorithms with proofs of convergence in the limit of infinite search time.

In contrast to the existing methods for FIS optimization methodology () where a large number of parameters has to be tuned leading to significant computing times requiring high memory capacity (Imai et al., 1998; Jiang et al., 2008; Ramon et al., 2008; Castillo et al., 2008), the proposed approach needs only a few rules for FIS knowledge base optimization, which leads to increased optimization convergence speed and avoids additional criteria such as rules simplification.

The proposed approach takes into account an online and offline aspects and operates in two stages. In the first one, an evolutionary method based on Solis and Wets algorithm is implemented in conjunction with a PA mechanism to estimate online distances between nodes according to a set of RSSI measures. It's based on Glorennec's methodology for FIS data structure optimization and can be implemented off- or on-line and it proceeds as follows: A first set of rules is extracted automatically from training data using RP mechanism Thus, in conjunction with RP mechanism, an evolutionary algorithm based on Solis and Wets method for MFs optimization is then implemented. In the second stage, to 
increase the optimization quality, rules conclusions are updated online using a stochastic gradient method. In order to compute real positions, a triangulation method based on $\mathrm{Al}$ Kashi theory is implemented using data derived from the second phase.

This paper is organized as follows: In Section 2, we present the proposed WSN localization approach based fuzzy logic concepts. Thus, in this context, an evolutionary method based on Solis and Wets algorithm is implemented in conjunction with a PA mechanism to estimate online distances between nodes according to a set of RSSI measures. It's based on Glorennec's methodology for FIS data structure optimization and can be implemented offor on-line. In order to compute real positions, a triangulation method based on Al-Kashi theory is implemented. Section 3 describes the system overview which consists in a smart wheelchair developed in our Lab., called LIASD-WheelChair. In Section 4, experimental and simulation results are presented and discussed. Finally, conclusion and some perspectives will be dressed in section 5 .

\section{Fuzzy localization approach in wireless sensor network}

Implementing our approach requires a FIS architecture which consists on a set of Multiinputs/Single-output (MISO) FIS modules (Cho, 2007). The number of the FIS modules can be determined according to output vector dimension. Each FIS module is a zero-order Takagi-Sugeno Fuzzy Model, also known as TSFM (Takagi \& Sugeno, 1987). The architecture is illustrated in Fig.1.

Each FIS module takes the antecedents $I_{R S S I_{k}}$ at its inputs and produces a distance estimation $d_{f} R S S I_{k}$, where $k=1, \cdots, n$, with $n$, number of FIS modules.

As in TSFM, the architecture implements rules of the following form:

$$
\text { IF } I_{\text {RSSI1 }} \text { is } A(j)\left(I_{R S S I 1}\right) \text { and } \ldots I_{\text {RSSIi }} \text { is } A(j)\left(I_{\text {RSSIi }}\right) \text { THEN d } d_{-} R S S I i=f(j)\left(I_{R S S I}\right)
$$

$I_{R S S I}$ is the normalized input and $A(j)\left(I_{R S S I}\right)$ is a $k$-dimensional fuzzy set of the $j^{\text {th }}$ rule $(j=1, \cdots, m)$.

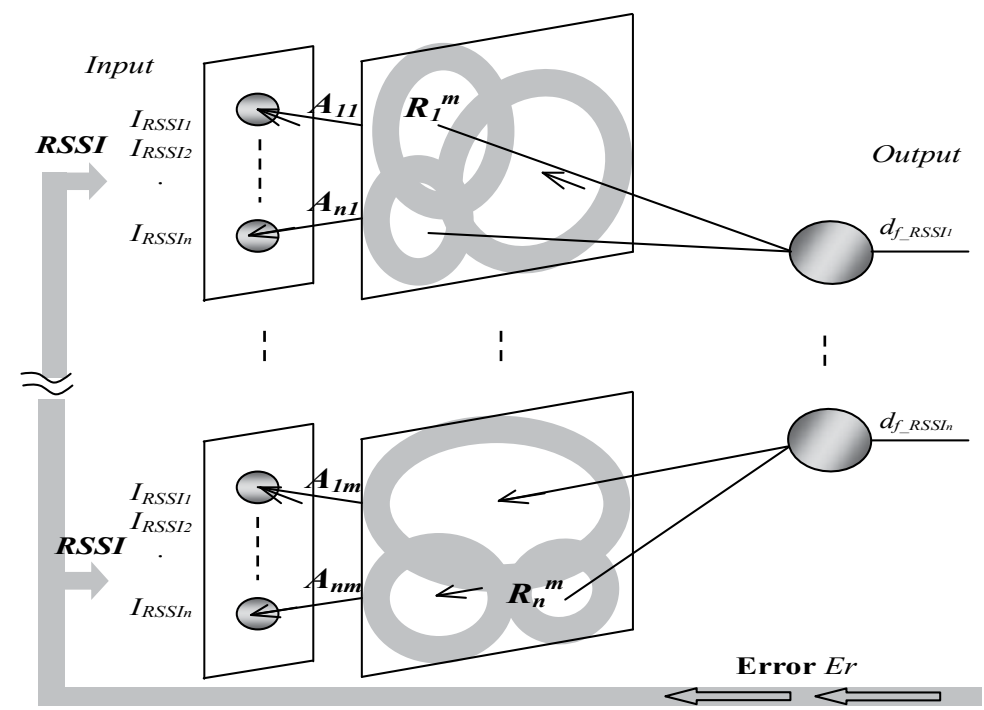

Fig. 1. Takagi-Sugeno fuzzy model 
The output $d_{f_{-} R S S I i}$ which is defined for each rule is computed as a weighed average of the contribution of each rule, so that:

$$
d_{f_{-} R S S I}=\frac{\sum_{j=1}^{L} w^{(j)}\left(I_{R S S I}\right) \cdot y^{(j)}}{\sum_{j=1}^{L} w^{(j)}\left(I_{R S S I}\right)}
$$

$L$ is the number of rules, $w^{(j)}$ and $y^{(j)}$ are the degree of truth and the conclusion of the $j^{\text {th }}$ rule respectively.

For the $i^{\text {th }}$ input variable $I_{R S S I}$, the domain of validity is divided into $k_{i}$ fuzzy sets labelled $A_{i}^{k_{i}}\left(I_{R S S I_{i}}\right)$, as shown in Fig.2. A small value of $k_{i}$ is sufficient, leading to an increased convergence speed of optimization procedure. Here, each fuzzy subset is characterized by a modal value $m_{i, k i}$ with $k_{1}=5$.

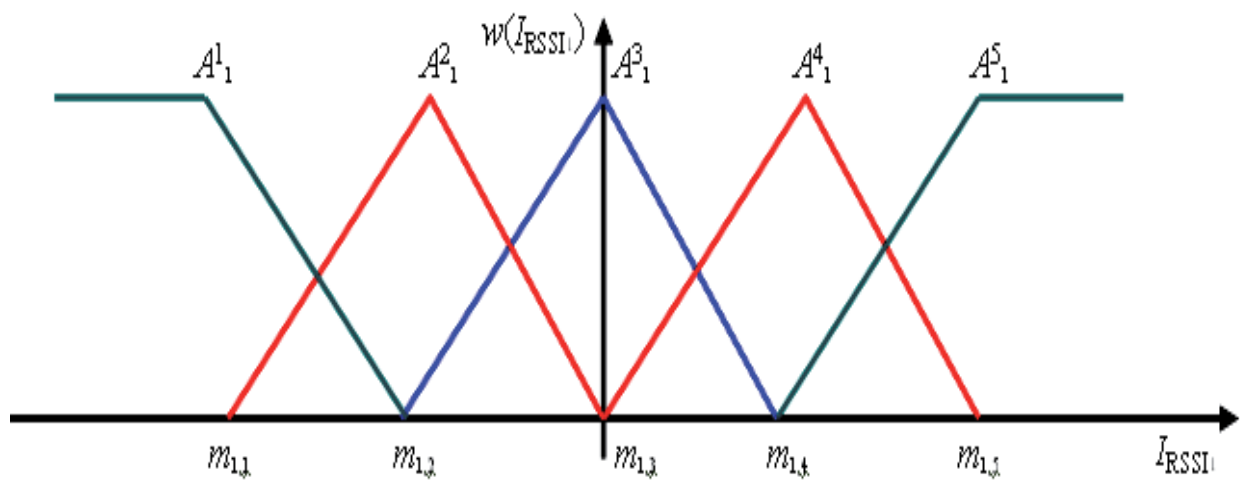

Fig. 2. Fuzzy partitioning

The degree of truth for each input $I_{R S S I i}$ can be written as follows:

$$
w^{(j)}\left(I_{R S S I_{i}}\right)=\max \left(1-\frac{\left|I_{R S S I_{i}}-a_{k_{i}}^{(j)}\right|}{b_{k_{i}}}, 0\right)
$$

So that $a_{k_{i}}^{(j)}=\frac{j-1}{k_{i}-1}$ and $b_{k_{i}}=\frac{1}{k_{i}-1},\left(j=1, \ldots, k_{i}\right)$

Usually $f^{(j)}\left(I_{R S S I}\right)$ is a polynomial function in the input variables $I_{R S S I i}$, but it can be any function as long as it can appropriately describe the output of the system within the fuzzy region specified by the antecedent of the rule. When $f($.$) is a first-order polynomial, the$ resulting FIS is called a first-order Sugeno fuzzy model, which was originally proposed in (Takagi \& Sugeno, 1987). When $f($.$) is a constant, we have a zero-order Sugeno FIS, which is$ similar to a Mamdani FIS case where each rule's consequent is specified by a fuzzy singleton. 


\subsection{Fuzzy estimator for RSSI based-localization}

In this section, an evolutionary method based on Solis and Wets algorithm is implemented in conjunction with a PA mechanism (Solis, 1981; Glorennec, 1999) to estimate online distances between nodes according to a set of RSSI measures. It's based on Glorennec's methodology for FIS data structure optimization (Glorennec, 1999) and can be implemented off- or on-line and it proceeds as follows: A first set of rules is extracted automatically from training data using Rapid Prototyping (RP) mechanism Thus, in conjunction with RP mechanism, an evolutionary algorithm based on Solis and Wets method for MFs optimization is then implemented.

\subsubsection{RP mechanism for initial knowledge base extraction}

The FIS architecture is based on the Takagi-Sugeno (TS) model (Lei, 2001; Takagi, 1987) and consists of several FIS' modules. It's used to learn first the FIS from the measured data set, as it's illustrated in Fig.3.

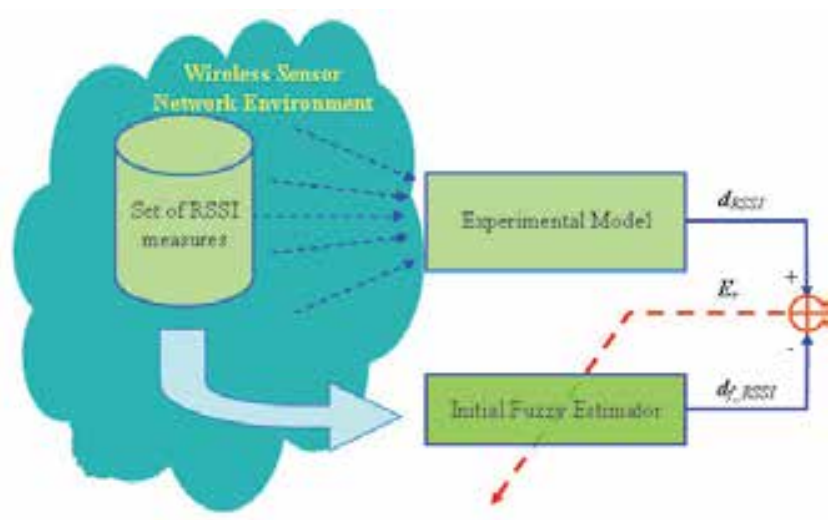

Fig. 3. Initial fuzzy database construction

The first objective is to build a FIS so that for an input $I_{R S S I}$, it produces an output $d_{f_{-} R S S I_{i}}$ that approximates the real measured values $d_{R S S I_{i}}$, obtained from MICAz sensors, minimizing the total Mean Squared Error (MSE) $E_{r}$ as follows:

$$
E_{r}=\frac{1}{2} \sum_{\mu}\left(d_{R S S I i}^{\mu}-d_{f_{-} R S S I i}^{\mu}\right)^{2}
$$

$\mu$ is the index labelling the training samples.

Thus, from an initial learning database which is generated from a set of RSSI measures is then used by a RP mechanism in order to produce initial rules. This algorithm uses a MSE given in (6).

When the learning point is close to a node, the corresponding rule conclusion corresponds to a better approximation of the desired output. Using training data, FIS is initialized availing the property of a strong fuzzy partition, i.e., rule conclusion is the image of one of its nodal vectors.

Thus, if $m^{(j)}$ is a nodal vector corresponding to the $j^{\text {th }}$ rule and $y^{(j)}$ is a conclusion, we have: $F I S(m(j)))=y^{(j)}$ where $F I S(\cdot)$ is the input/output function of the FIS. 
The objective is to find a solution which is already close to the optimal one and to accelerate the training phase. To initialize a vector $y^{(j)}$, we use the continuity of FIS, defined as follows:

$$
\forall j, \lim _{x \rightarrow m^{(j)}} \operatorname{FIS}(x)=y^{(j)}
$$

For a couple of elements $(x, y)$ of training set $S$, the closer $x$ is to $m(j)$, the closer $y$ must be to $y^{(j)}$. This latter, can be approximated by $y^{*}$, so that the vector of the training base $\left(x^{*}, y^{*}\right)$ can be is defined as follows:

$$
x^{*}=\arg \min _{x \in S}\left\|x-m^{(j)}\right\| \equiv \arg \max _{x \in S} w^{(j)}(x)
$$

To limit the noise effects of the learning data, the contribution of the closest points of each node is weighted as follows:

$$
y^{(j)}=\sum_{w^{(j)} \geq s}\left(w^{(j)}(x)\right)^{m} \cdot y(x)
$$

$s$ is a given threshold and $m$ is a positive integer.

The algorithm for initializing rules conclusions and input MFs can be summarized as follows:

For each rule $j$ do:

Search $\left(x^{*}, u_{x}^{*}\right)$ in A so that:

$$
\begin{aligned}
& w_{j}\left(x^{*}\right)=\max _{x \in A} w_{j}(x) \\
& y^{j}=u_{x}^{*}
\end{aligned}
$$

End

To limit the noise effects of the learning data, the contribution of the points closest to each node is weighed by RPA, as follows:

$$
y^{i}=\sum_{w_{i}(x) \geq s} w_{i}^{m}(x) \times u_{x}
$$

Here, $s$ is a data threshold and $m$ an integer higher than 1.

The obtained conclusion remains then in the convex envelope of possible values, which is not guaranteed by gradient descent algorithms.

Thus, once initial knowledge base is extracted, in conjunction with RP mechanism, we implement an evolutionary algorithm based on Solis and Wets method for MFs optimization.

\subsubsection{Evolutionary algorithm based on Solis and Wets method for MFs optimization}

The MFs optimization reduces to the optimization of the nodal values. The input variable for the optimization algorithm is the set of the nodal values for each input. Thus, each FIS module can be coded as:

$$
A=\left(m_{1,1}, \cdots, m_{1, k_{1}}, m_{2,1}, \cdots, m_{2, k_{2}}, \cdots, \cdots, m_{n, 1}, \cdots, m_{n, k_{n}}\right)
$$


Where $m_{i, l}$ represents the $l^{\text {th }}$ nodal value at $i^{\text {th }}$ dimension, with the constraint that for every $i$ : $m_{i, 1}<m_{i, 2}<\cdots<m_{i, k_{i}}$.

Each step of the proposed algorithm starts with some initial point $A^{(0)}$ with mean $M^{(0)}$ and $t=0$. Note $E(x)$ the objective function.

Each step starts with a current point or initial vector $A^{(0)}$ created by dividing each input variable domain into equal parts.

A deviate $G^{(k)}$ is chosen from a normal distribution whose standard deviation is given by parameter $\rho$.

If either $A^{(k)}-G^{(k)}$ or $A^{(k)}+G^{(k)}$ is better, a move is made to the better point and a "success" is recorded. Otherwise a "failure" is recorded. After several successes in a row, $\rho$ is increased to move more quickly. After several failures in a row, $\rho$ is decreased to focus the search. Additionally, a bias term is included to give the search momentum in directions that yield success.

Correspondent FIS is evaluated along learning set using for example, criteria $E_{r}=\frac{1}{2} \sum_{\mu}\left(d_{R S S I i}^{\mu}-d_{f_{-} \text {RSSI }}^{\mu}\right)^{2}$, which depends on $A^{(0)}$, then we have : $E\left(A^{(0)}\right)=E_{r}$.

A Gaussian noise is then added to $A^{(0)}$ components, which lead to modify MF. If the vector doesn't verify the imposed constraints, a new pulling is computed until obtaining of a conformed vector and the evaluation can be restarted.

The objective of vector $M$ is to memorize the good exploration directions in the space. It introduces a bias in the exploration. In the case of failure, this bias is exponentially forgotten: The algorithm tries to follow a valleys to rich the minimum. The Gaussian noise, which is very expansive in term of computation time, is re-used in the direction $A^{(k)}-G^{(k)}$ if the direction $A^{(k)}+G^{(k)}$ is failed. An important feature of this type of local search is that it doesn't rely on gradient information.

The pseudo-algorithm of Solis and Wets local search implementation is given on Fig.4.

\subsection{Fuzzy rules consequences update}

Knowing that conclusions result from a zero-order TS model, a quadratic method can be used for determining the output of the rules $y\left(x_{n}\right)$. The optimization problem can be formulated as follows:

$$
y_{n+1}^{(j)}=y_{n}^{(j)}-\eta \cdot \frac{\partial E r}{\partial y_{n}^{(j)}}
$$

Where $\eta$ and $E_{r}$ respectively the learning rate and the quadratic criterion defined in (6).

The proposed method for distance estimation should ensure a rule base interpretability so that properties of continuity, consistency and completeness are respected (Touati et al., 2006).

Once distances between nodes are estimated, a localization method based on Al-Kashi theory can be then implemented in order to determine the right coordinates in the environment. 


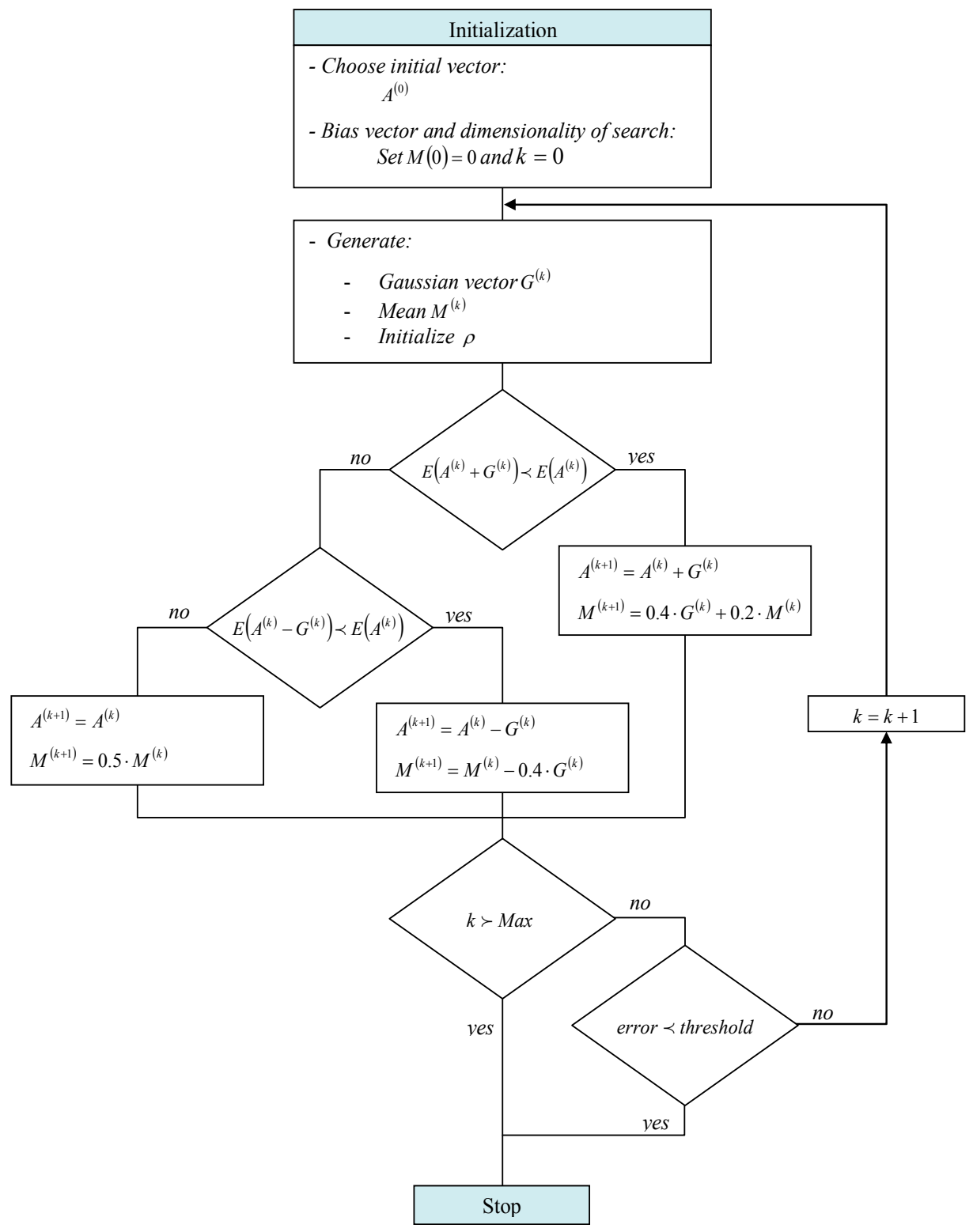

Fig. 4. Solis and Wets Pseudo-algorithm

In order to increase the optimization quality, rules conclusions are updated online using a stochastic gradient method.

\subsubsection{Al-Kashi theory for nodes localization}

Triangulation is the process of finding a distance to a point by calculating the length of one side of a triangle, given measurements of angles and sides of the triangle formed by that point and two other reference points. The implementation requires three reference points whose distances $d_{i}$, between nodes and the base are predefined during estimation phase. In 
this direction, Al-Kashi theory seems to be an efficient tool. The idea is to determine precisely the node position $M_{x}\left(x_{X}, y_{Y}\right)$, localized at distance $d_{1 x}$ from the base $B_{S 1}\left(x_{1}, y_{1}\right)$. In other words, this consists to determine the right orientation angles $\left(\alpha, \alpha_{0}, \beta\right)$, as it's illustrated on Fig.5.

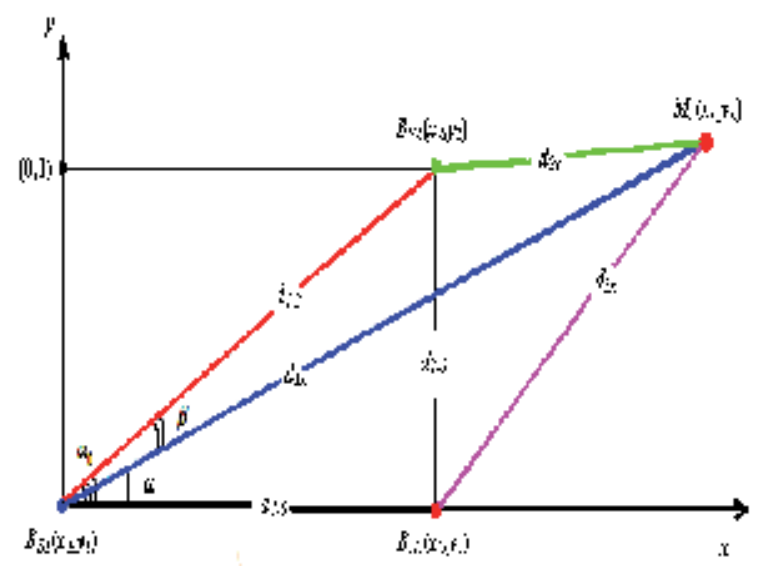

Fig. 5. Triangulation scheme

Thus, having information, i.e., positions and RSSI levels of each node $B_{S i}\left(x_{i}, y_{i}\right)$, the distance between nodes $i$ and $j$ can be computed as follows:

$$
d_{i-j}=\sqrt{\left(x_{j}-x_{i}\right)^{2}+\left(y_{j}-y_{i}\right)^{2}}
$$

From Fig. 6, we can write:

$$
\alpha_{0}=\operatorname{acos}\left(\frac{d_{1-3}}{d_{1-2}}\right)
$$

Based on Al-Kashi theory, we can write:

$$
d_{3 x}^{2}=d_{1 x}^{2}+d_{1-3}^{2}-2 d_{1 x} d_{1-3} \cos (\alpha)
$$

and,

$$
d_{2 x}^{2}=d_{1 x}^{2}+d_{1-2}^{2}-2 d_{1 x} d_{1-2} \cos (\beta)
$$

Thus:

$$
\alpha=\operatorname{acos}\left(\frac{d_{1 x}^{2}+d_{1-3}^{2}-d_{3 x}^{2}}{2 d_{1 x} d_{1-3}}\right)
$$

and,

$$
\beta=a \cos \left(\frac{d_{1 x}^{2}+d_{1-2}^{2}-d_{2 x}^{2}}{2 d_{1 x} d_{1-2}}\right)
$$


The position of any supposed node can be computed according to some cases of angle $\beta$, so that:

$$
\beta=\left(\alpha-\alpha_{0}\right)
$$

Thus:

$$
\left\{\begin{array}{l}
x_{X}=( \pm) d_{1 x} \cos (\alpha) \\
y_{X}=( \pm) d_{1 x} \sin (\alpha)
\end{array}\right.
$$

To resume, knowing distances between nodes after implementing the fuzzy estimator, i.e., distance between mobile node $M_{x}\left(x_{X}, y_{Y}\right)$ and $B_{S 1}\left(x_{1}, y_{1}\right)$, we can determine the right position of $M_{x}\left(x_{X}, y_{Y}\right)$.

\section{Experimental setup}

LIASD-Wheelchair is an adjustable adults' powered wheelchair (Fig.6). It is suitable for indoor or outdoor use and implements wired and wireless networks for communication. The wireless communication is based on IEEE 802.11.4 standard (Touati et al., 2010).

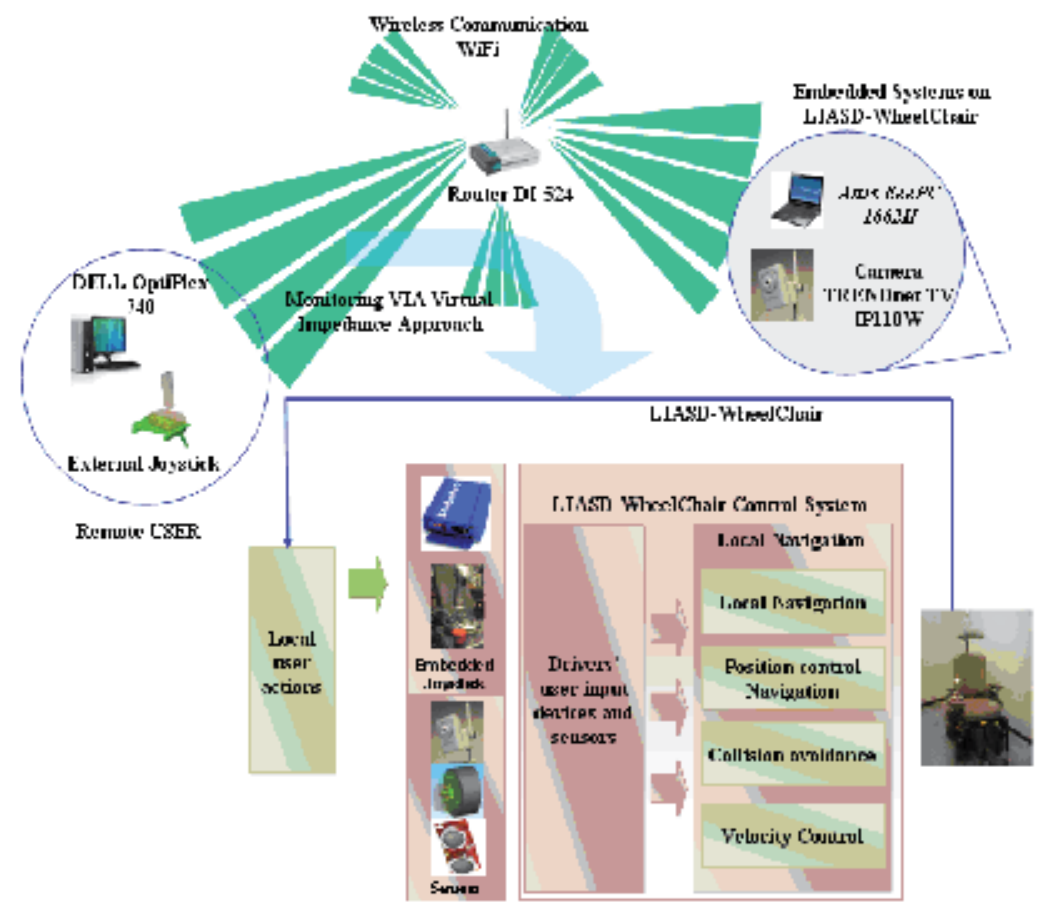

Fig. 6. Global structure of LIASD-WheelChair

The hardware architecture of LIASD-Wheelchair consists of sensory block, control architecture, and communication networks. The presented system includes two optical incremental encoders mounted to a motor, with resolution of 500 Counts per Revolution. Four ultrasonic rangefinders (US SFR08) are used to localize the wheelchair in the 
environment. They have a resolution of $3 \mathrm{~cm}$ and can identify obstacles between $3 \mathrm{~cm}$ and $6 \mathrm{~m}$. The US sensors interact with the computer via TCP/IP server board FMod-TCP DB using an I2C interface. In order to ensure navigation and anti-collision objectives a Wireless Internet Camera Server is mounted on the wheelchair headrest.

The communication network implements an I2C protocol for US sensors management via Fiveco card. USB and RS232 protocols are used to ensure communication between AX2550 controller and embedded laptop Asus-EeePC-1002H.

Knowing that the network architecture is based on an infrastructure mode, the remote user/wheelchair communication is ensured via an access point DI-524 which is able to transfer data at rates of $54 \mathrm{Mbps}$ and $2.4 \mathrm{GHz}$ through its wireless channels.

For the localization process, a WSN working on IEEE 802.15.4 compliant standard is deployed in the environment. Sensors are the latest generation of Motes from Crossbow Technology, called MICAz. They use the Chipcon CC2420 working on $2400 \mathrm{MHz}$, ZigBee ready radio frequency transceiver integrated with an Atmega128L micro-controller and serial flash memory. Fig.7 illustrates the MICAz sensor node with standard antenna and its bloc diagram.
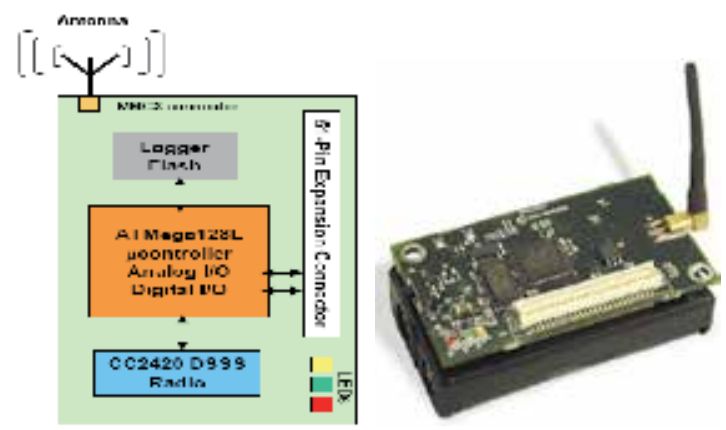

Fig. 7. MICAz sensor node and its architecture

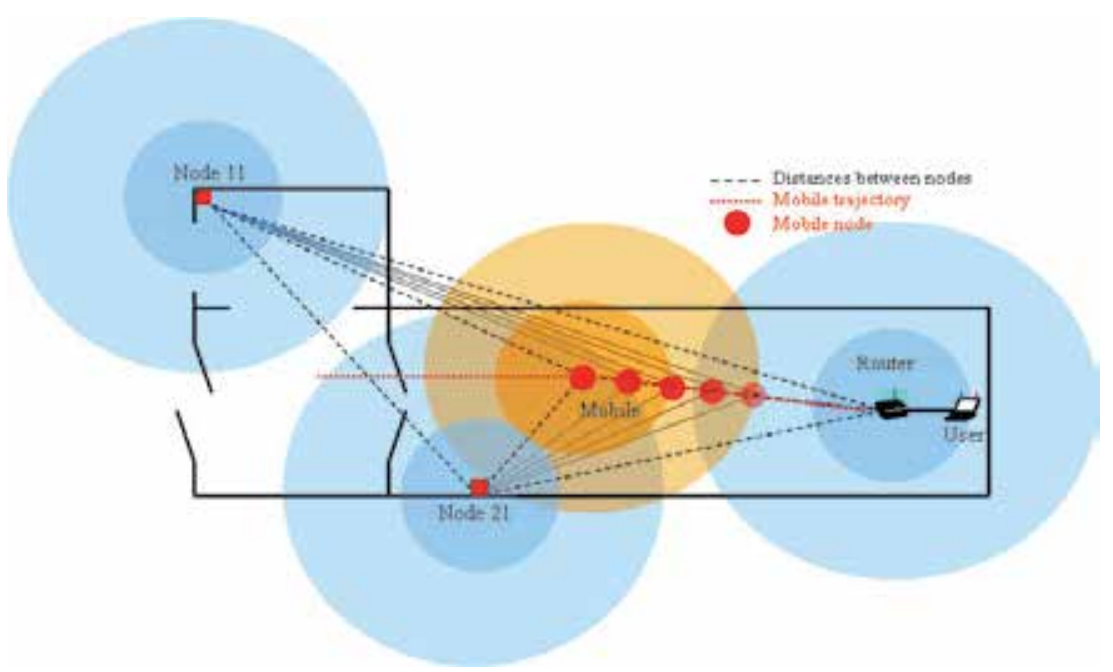

Fig. 8. LIASD-WheelChair moving in WSN environment. 


\section{Experimentation and results}

The proposed localization approach is implemented and tested on an intelligent wheelchair, called LIASD-WheelChair, developed at Computer Science and Artificial Intelligence Lab. LIASD-WheelChair operates in WSN environment. Four Crossbow MICAz nodes are implemented: Two anchors (nodes 11 and 21), one user node and one mobile node representing the wheelchair (Fig.8).

As it's illustrated on Fig.9, the FIS structure conception includes three MISO FIS subsystems, where, $I_{R S S I}=\left(I_{R S S I 11}, I_{R S S I 21}, I_{R S S I M}\right)$ and $d_{R S S I}=\left(d_{R S S I 11}, d_{R S S I 21}, d_{R S S I M}\right)$ represent respectively RSSI measures and distances between nodes and user node.

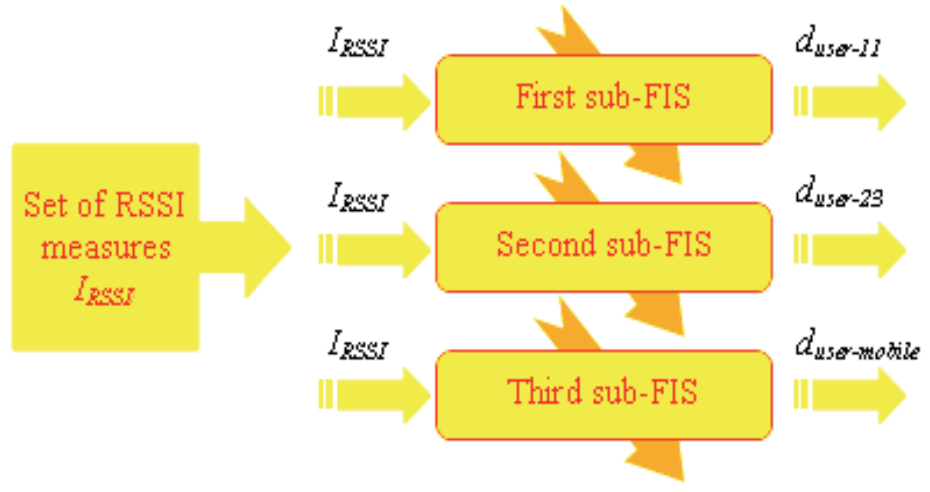

Fig. 9. FIS architecture

For each input variable space $I_{R S S I}$ partitioning, a set of triangular MFs is randomly initialized and the universe of discourse for each variable is divided into $k_{i}$ fuzzy sets.

Knowing that nodes 11 and 21 represent anchors nodes (Fig.8), the associated RSSI values should be approximately constant. Thus, distances between nodes $21-11$, user-21 and user-

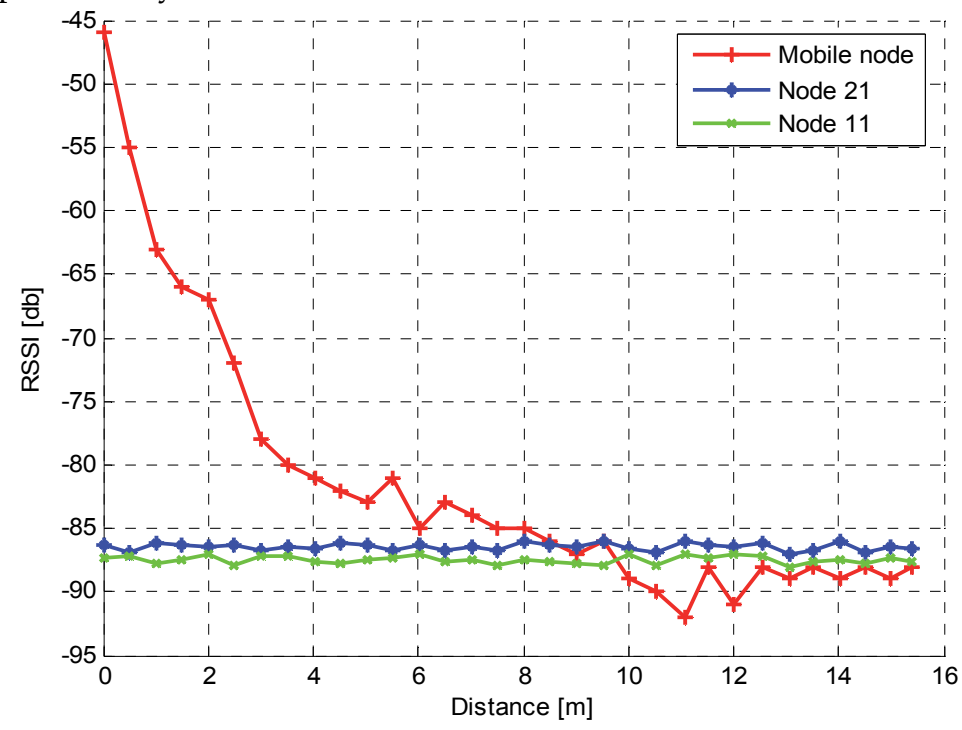

Fig. 10. RSSI measures vs. distances 
11 should approximately be a constant. This leads us to deal only with mobile node $M$, thus associated MISO FIS sub-systems (Third sub-FIS) (Fig.9).

The wheelchair moves in the environment according to specific desired trajectories. In the beginning, experimentation consists to collect a set of RSSI information from different nodes in order to conceive an initial FIS architecture. For this purpose, an initial database $\left(I_{R S S I i}, d_{R S S I i}\right)$ corresponding respectively to RSSI and distances, provided by a mobile node is built, and Fig.10 illustrates the obtained results concerning the whole nodes.

The learning error associated MFs optimization and conclusions update are illustrated respectively on Figures 11 and 12. The results show that the proposed algorithm for distance estimation is very efficient according to obtained error values. It ensures convergence directly at the first steps.

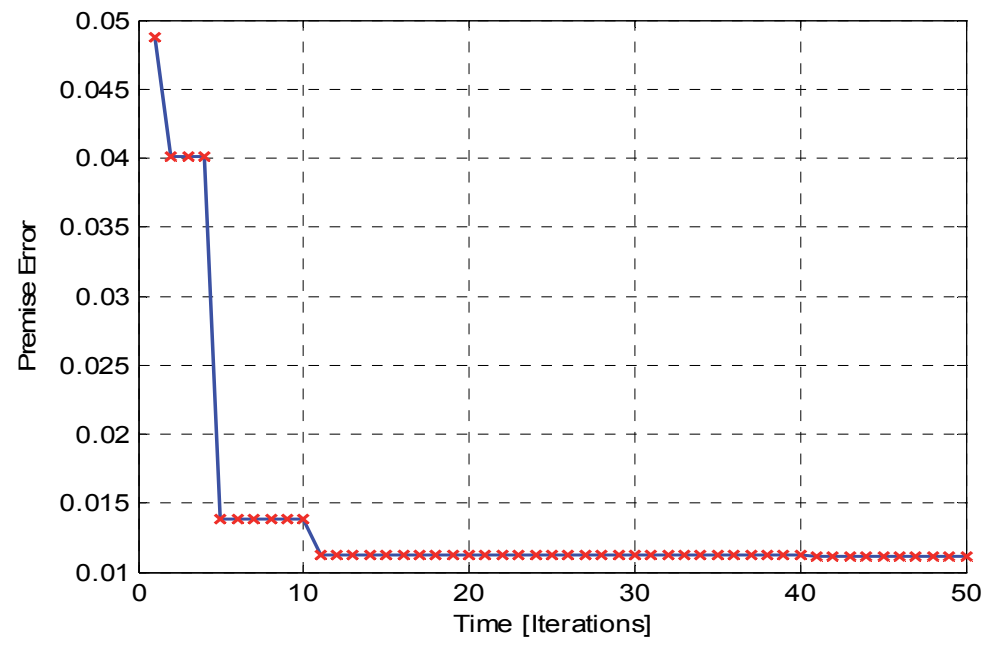

Fig. 11. Membership functions optimization

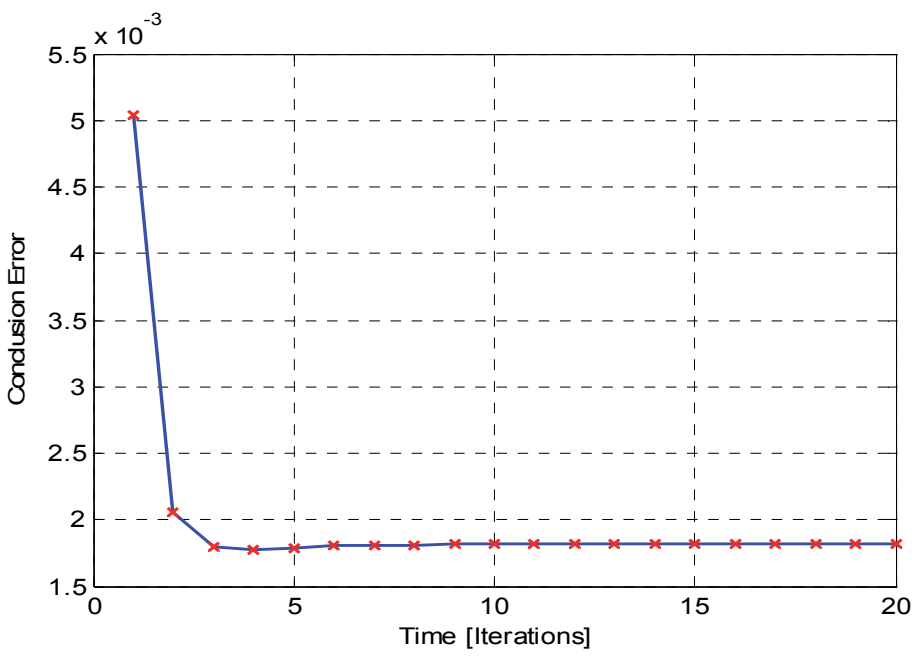

Fig. 12. Conclusions update 
In order to increase performances of the proposed fuzzy estimator, an online computation of distances is implemented according to Solis and Wets method. The obtained results which are illustrated on Fig.13, allows us to conclude that the approach can operates in real time.

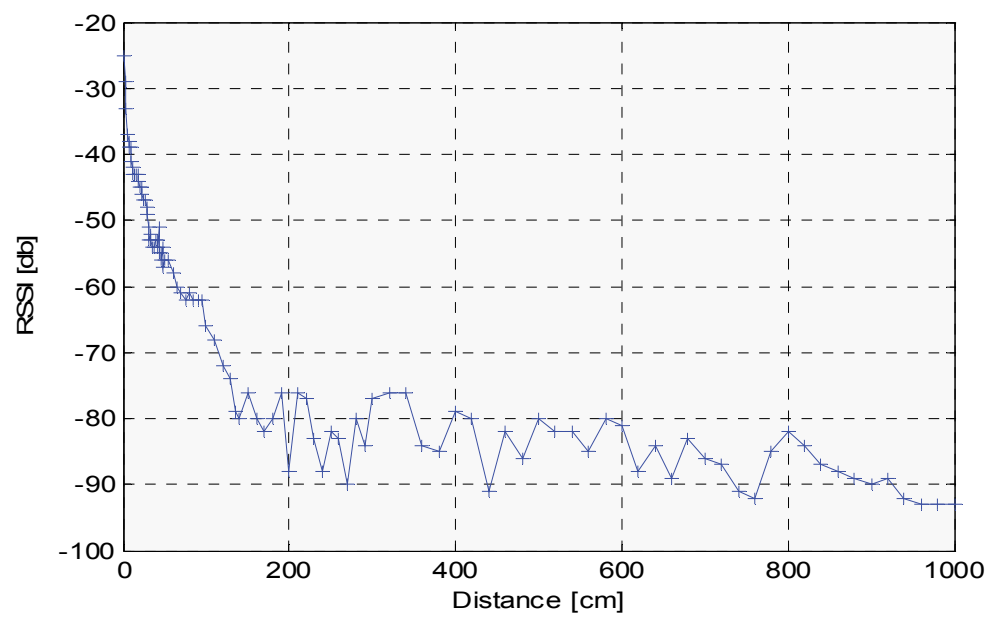

Fig. 13. RSSI measures vs. distance estimation

Fig.14 illustrates wheelchair trajectory operating in WSN environment according to $\mathrm{x}$ - and $\mathrm{y}$-axis.

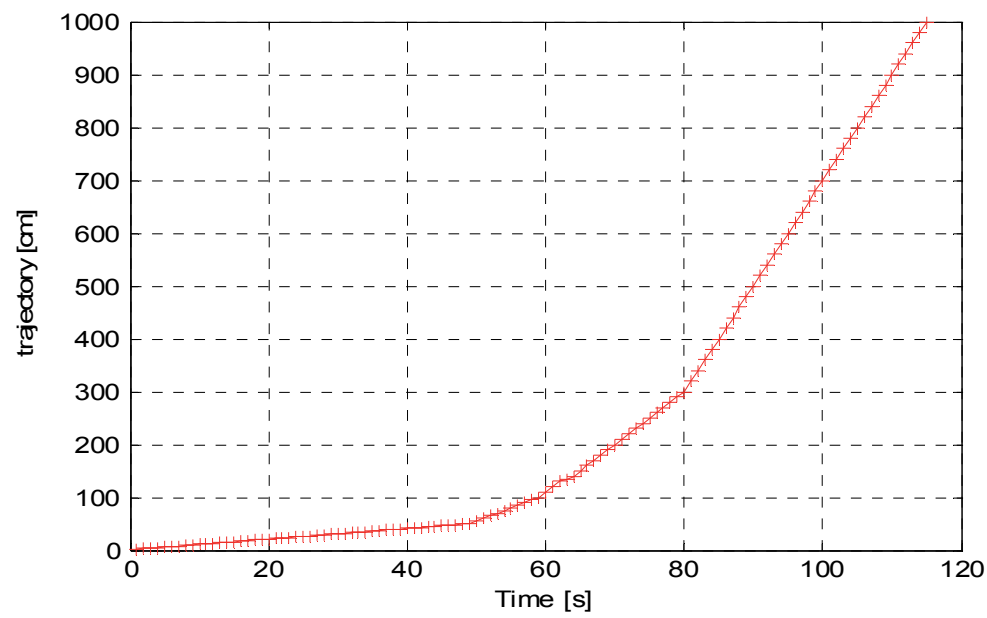

Fig. 14. FIS for online distance estimation

The analysis of obtained results shows that the proposed localization method can approximate nodes distances from RSSI measures and provided by sensor nodes (MICAz). In all cases, the use of fuzzy estimator generates a better estimation than LQI or generic models. Implementing an evolutionary strategy allows a better convergence speed according to classical algorithms requiring an additional phase for conflicts and redundancy treatment. This means that the proposed fuzzy estimator can be embedded on each node and implemented online for distances estimation between nodes. 


\section{Conclusion}

In this paper, we have proposed a WSN localization method based on IEEE 802.15.4 standard using fuzzy logic concepts with an evolutionary algorithm for parameters approximation and optimization. The approach allows approximating a realistic model for distance estimation from a set of RSSI measures and taking into account environment influences and signal failures as well as imprecise distances. The approach is based on a FIS optimization methodology which combines both a Rapid Prototyping mechanism and Solis and wets' mono-agent evolution strategy. The methodology allows, in term of probability, a randomized hill-climber with an adaptive step size and a global minima convergence of a coast function. It describes a class of local and global search algorithms with proofs of convergence in the limit of infinite search time. In contrast to the existing methods for FIS optimization methodology where a large number of parameters has to be tuned leading to significant computing times requiring high memory capacity, the proposed approach needs only a few rules for FIS knowledge base optimization, which leads to increased optimization convergence speed and avoids additional criteria such as rules simplification. The proposed approach takes into account an online and offline aspects and operates in two stages. In the first one, an evolutionary method based on Solis and Wets algorithm is implemented in conjunction with a PA mechanism to estimate online distances between nodes according to a set of RSSI measures. It's based on Glorennec's methodology for FIS data structure optimization and can be implemented off- or on-line and it proceeds as follows: A first set of rules is extracted automatically from training data using RP mechanism Thus, in conjunction with RP mechanism, an evolutionary algorithm based on Solis and Wets method for MFs optimization is then implemented. In the second stage, to increase the optimization quality, rules conclusions are updated online using a stochastic gradient method. In order to compute real positions, a triangulation method based on Al-Kashi theory is implemented using data derived from the second phase. In order to illustrate the efficiency of the proposed approach, experimentation on a smart wheelchair developed in our Lab. called LIASD-WheelChair has been performed.

\section{References}

Röhring, C. \& Spieker, S. (2009). Tracking of transport vehicles for warehouse management using a wireless sensor network. IEEE International Conference on Intelligent Robots and Systems, pp. 3260-3265, Nice, France

Vossiek, M.; Wiebking, L.; Gulden, P.; Wieghardt, J., Hoffmann, C. \& Heide, P. (2003). Wireless local positioning. Microwave magazine, vol.4, $\mathrm{n}^{\circ}$. 4, pp. 77-86

Tsung-Han, L. \& Hei-Ng, I. (2008). A microscopic examination of an rssi-signature-based indoor localization system. The 5th Workshop on Embedded Networked Sensors, Virginia, USA

Awad, A.; Frunzke, T. \& Dressler, F. (2007). Adaptive Distance Estimation and Localization in WSN using RSSI Measures. Proceeding of the 10th Euromicro-Conference on Digital System Design Architectures, Methods and Tools, pp. 471-478

Graefenstein, J. \& Bouzouraa, M.E. (2008). Robust Method for Outdoor Localization of a Mobile Robot Using Received Signal Strength in Low Power Wireless Networks. Proceeding of IEEE International Conference on Robotics and Automation, pp. 33-38, Pasadena, California 
Sugano, M. (2006). Indoor Localization System using RSSI Measurement of Wireless Sensor Network based on ZigBee Standard, Wireless and Optical Communications, ACTA Press, pp. 1-6

Cho, H.; Kang, M.; Park, J.; Park, B. \& Kim, H. (2007). Performance Analysis of Location Estimation Algorithm in ZigBee Networks Using Received Signal Strength. In 21 st International Conference on Advanced Information Networking and Applications Workshops, vol. 2, pp. 203-206, Niagara Falls, Ontario

Zadeh, L.A. (1965). Fuzzy sets. Information \& Control, 8 (3) 338-353, ISSN 0933-5846

Bezdek, J.C. (1981). Pattern recognition with fuzzy objective function algorithms. Plenum Press, ISBN:0306406713, New York

Dave, R.N. (1990). Fuzzy shell-clustering and applications to circle detection in digital images. Journal of General Systems, Vol.16, pp. 343-355, ISSN 0308-1079

Liang, C.Y. \& Su, J.P. (2003). A new approach to the design of a fuzzy sliding mode controller. Fuzzy Sets and Systems, 139(1), pp. 111-124, ISSN 0165-0114

Huang, L.; Ge, S.S. \& Lee, T.H. (2003). Fuzzy unidirectional force control of constrained robotic manipulators. Fuzzy Sets and Systems, 134(1), pp. 135-146, ISSN 0165-0114

Lei, S.; Pallazzolo, A.; Na, U. \& Kascak, A. (2001). Non-linear fuzzy logic control for forced large motions of spinning shafts. Journal of Sound and Vibration, Vol.235, N.3, pp. 435-449

Solis, F. \& Wets, J. (1981). Minimization by random search techniques. Mathematics of Operation Research, Vol.6, ISSN: 0364765X

Glorennec, P.Y. (1999). Learning algorithms for fuzzy inference systems. Hermes Ed. ISBN10: 2746200449

Hart, W.E. (1994). Adaptive Global Optimization with Local Search. dissertation at the University of California at San Diego La Jolla, CA, USA

Takagi, T. \& Sugeno, M. (1987). Fuzzy identification of systems and its applications to modeling and control. IEEE Transaction on Systems, Man and Cybernetics, Vol.1, pp. 116-132, ISBN 0018-9472

Touati, Y. \& Amirat, Y. (2006). Optimization and Design Methodology of Fuzzy Controller for Industrial Robotic Tasks. Proceeding of IEEE International Conference on Robotics and Automation, pp. 3666-3672, Orlando, Florida.

Touati, Y.; Aoudia, H. \& Ali-Chérif, A. (2010). Intelligent Wheelchair localization in wireless sensor network environment: A fuzzy logic approach. $5^{\text {th }}$ IEEE International Conference on Intelligent Systems, pp. 408-413, London, UK

Jiang, H.; Deng, H. \& He, Y. (2008). Determination of Fuzzy Logic Membership Functions Using Extended Ant Colony Optimization Algorithm. Fuzzy Systems and Knowledge Discovery Fourth International Conference on, 1, pp.581-585

Imai , H. \& Tanaka, A. (1998). A method of identifying influential data in fuzzy clustering. IEEE Transaction on Fuzzy Systems, vol. 6, n.1, pp.90-101

Ramon, N.; Castro, C.; Aguilar, L.T.; Castillo, O. \& Rodriguez, A. (2008). Optimizing Type-1 and Type-2 Fuzzy Logic Systems with Genetic Algorithms, Research in Computing Science, vol.39, pp.131-153

Castillo, O., Aguilar, L.T.; Cazarez, N. \& Cardenas, S. (2008). Systematic design of a stable type-2 fuzzy logic controller, Journal of Applied Soft Computing, vol. 8, no. 3, pp. 1274-1279 


\title{
Application of RP and Manufacturing to Water-Saving Emitters
}

\author{
Zhengying Wei \\ $X i^{\prime}$ an Jiaotong University
}

China

\section{Introduction}

\subsection{Introduction of water-saving drip irrigation emitters}

In order to solve the problem of water shortage in agriculture, it's necessary to develop water-saving irrigation (Bralts \& Vincent, 1985). At present, there are more than ten kinds of water-saving irrigation models including the anti-seepage of channels, low pressure irrigation, sprinkler irrigation, micro irrigation, under-membrane irrigation, overmembrane irrigation. Among these irrigation patterns, drip irrigation is the most effective way in arid and semi-arid areas and its utilization rate can reach up to $90 \%$ (Thompson et al., 2009; Lamm F. R. \& Camp C. R., 2007; Onder et al., 2005; Schwankl \& Hanson, 2007; Cetin \& Bilgel, 2002).

Drip irrigation is such an irrigation method that transfers the water under a definite pressure, after filtering, through pipe network into the soil surrounding the root system of plants in drops slowly and uniformly. As the key device in drip irrigation systems, the emitter is to drip the pressured water in the pipeline to the root of the crops evenly and steadily, so as to guarantee the water demand for crop growth. The quality of the emitter has an important effect on the reliability, life span of the drip irrigation system and irrigation quality (Schwankl \& Hanson, 2007).

\subsection{Structure design and flow rate prediction of micro channel emitter}

Usually, the structure of the emitter channel is very complex with a dimension of $0.8 \mathrm{~mm}$ $1.2 \mathrm{~mm}$ (Wei et al., 2007). Emitter's intricate inner channel makes the flow of water have turbulent behavior. As a result, the behavior index will be decreased and the flow rate will be insensitive to the pressure. With all the above advantages, an evened uniform guaranteed irrigation will be improved. So, in the process of design, the channel length will be decreased and the manufacturing cost of the emitter will be reduced. It is the common goal of emitter designer to decrease the flow state index on the basis of simplifying the emitter structure (Li et al., 2005).

In the design of emitter structure, we use 3D parametric CAD software PRO/E to design labyrinth emitter. Fig. 1. shows 4 typical labyrinth channels with their crucial dimensions. According to emitter's hydraulic performance and its requirement for anti-clogging, we can design new channel structures by changing those dimensions.

The irrigation quality of drip irrigation system is verified by emitter's hydraulic performance (Li et al., 2008). In the high-pressure pipeline, the water energy may dissipate 
after flowing through the labyrinth channel and the flow rate can be controlled to meet the water need of the crops. To ensure the emitter's hydraulic performance, before the fabrication of emitter, computational fluid dynamics (CFD) is used to predict emitter's flow rate and analyze its hydraulic performance under various water pressures. If the structure can meet the water flow's requirement very well, then rapid prototyping (RP) is conducted to fabricate the emitter, and also related experiments are performed to test the performance. The simulation process using CFD to predict flow rate is shown in Fig. 2.
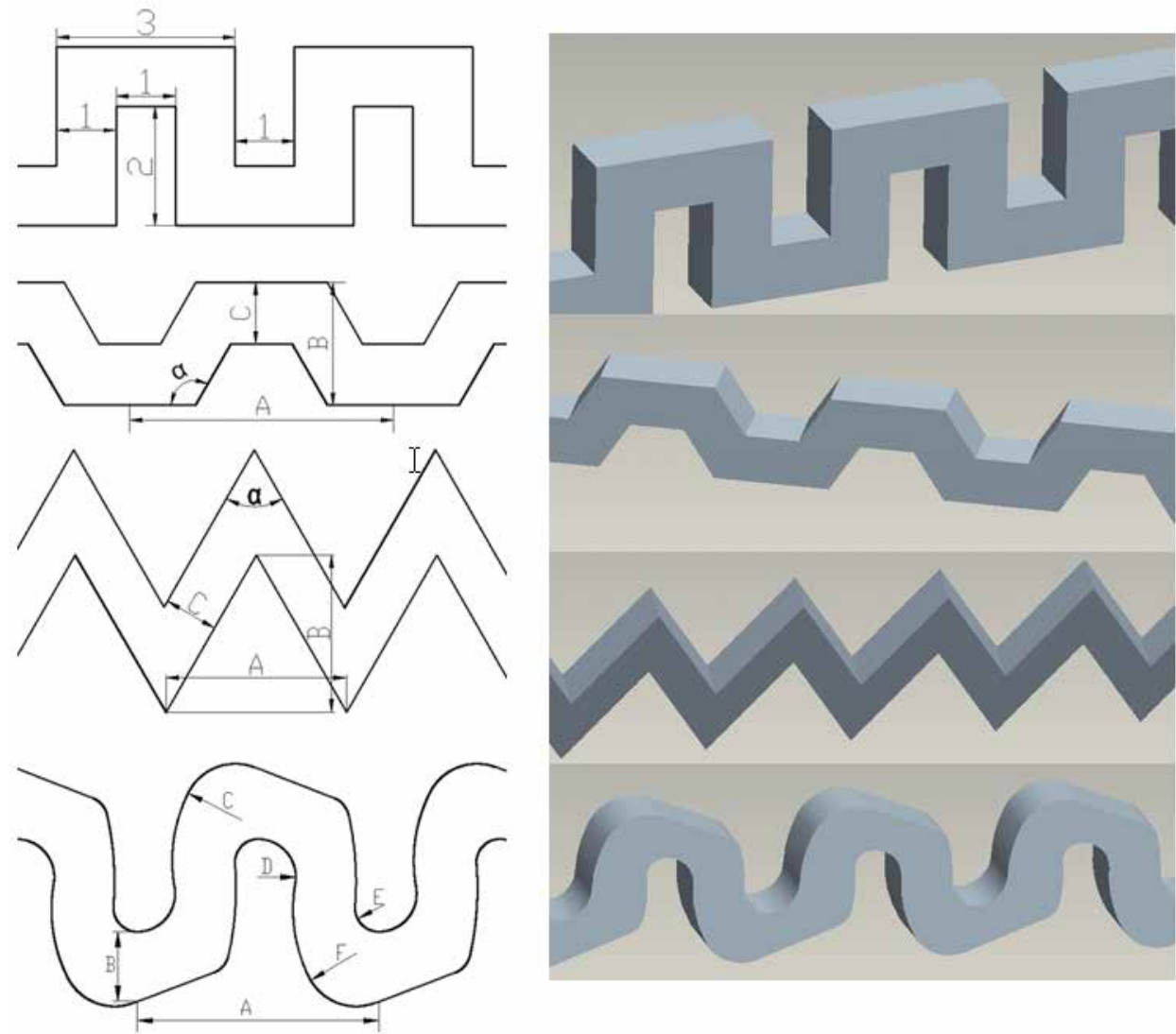

Fig. 1. Structure of four typical labyrinth channel

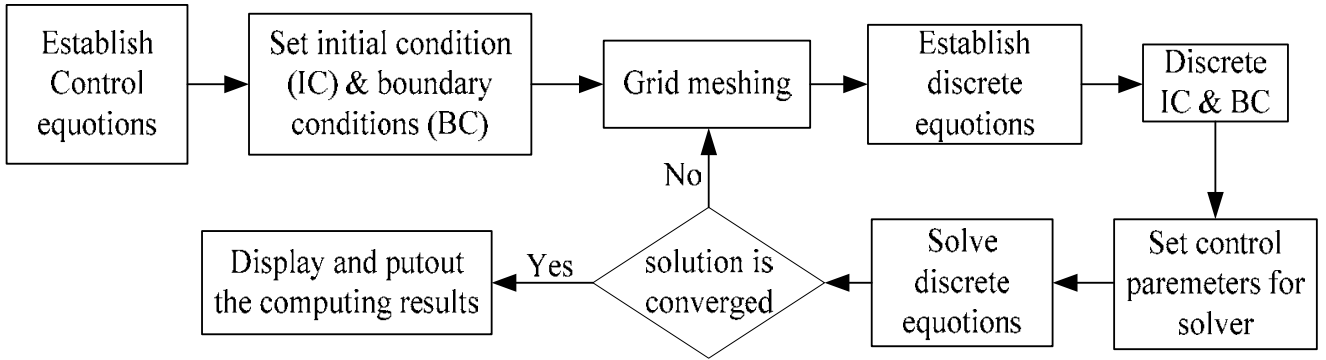

Fig. 2. Flow rate prediction with the use of CFD 


\subsection{Integrating RP manufacture of emitter}

Currently, the traditional method is used in emitter development, the steps are shown in Fig. 3. The period of emitter development is generally 4 to 5 months, leading to high cost and low accuracy. However, taking RP\&M technique into the emitter development can solve these problems conveniently.

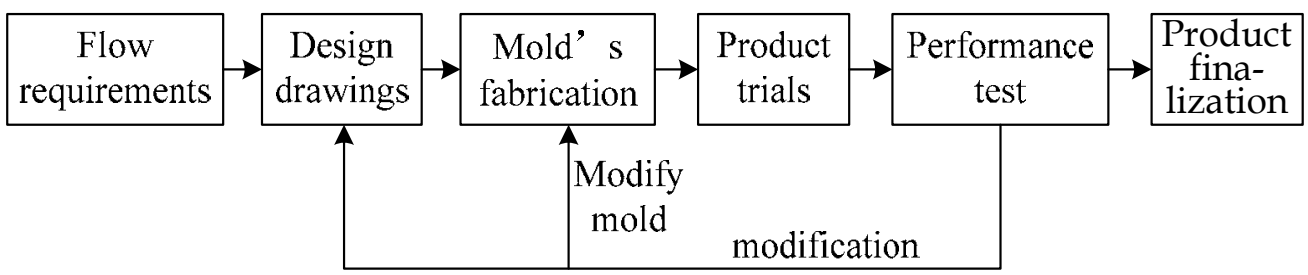

Fig. 3. Development steps of emitter product

The emitter's rapid development process based on RP\&M is shown in Fig. 4. Taking fluid dynamics and integration of RP technique as the core, the closed-loop control of the product development is exercised, and the design and development cycle are greatly reduced, which provide a platform for emitter's design and verification. Finaly the design efficiency is greatly improved (Wei et al., 2007).

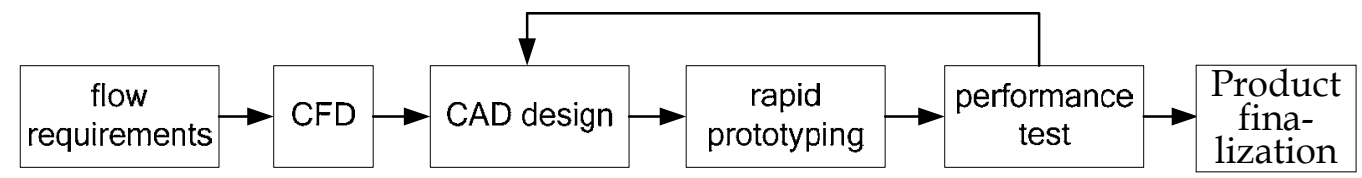

Fig. 4. Rapid development processes of emitter (Wei et al., 2003)

The emitter's performance is directly affected by the structure of the labyrinth channel (Wei $\& \mathrm{Lu}, 2005)$. The more complex the channel boundary is, the smaller the flow state index will be. In order to achieve a low flow rate and control the emitter's flow rate, the viscous resistance of channel to water should be large enough to reduce the water flow rate under a certain pressure. The main factors influencing the channel viscous resistance include the cross-section shape, length and shape of channel. Besides, the channel should be smooth and the mold's fabrication should be considered when designing the emitter. The sharp structure and sudden turning in the connection area of channels should be eliminated to avoid vortexes and stagnation area which will cause particle deposition and microorganism growth and eventually the emitter clogging (Wei et al., 2008).

The length, width and height of the emitter channel are taken as the design parameters and hence a parametric design scheme is formed. Emitter's model is shown in Fig. 5.

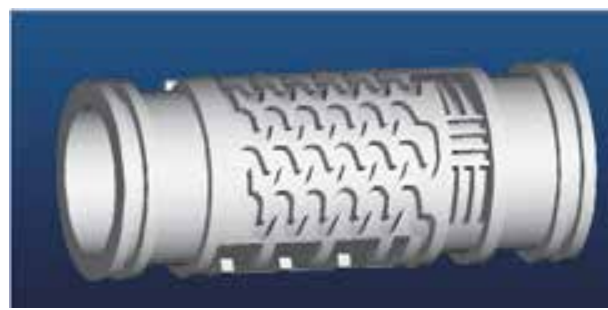

Fig. 5. Emitter's CAD model 
The emitter and the outer tube are designed as a whole by adding an outer tube encapsulating the emitter directly. The integration of 3D CAD model is shown in Fig. 6.

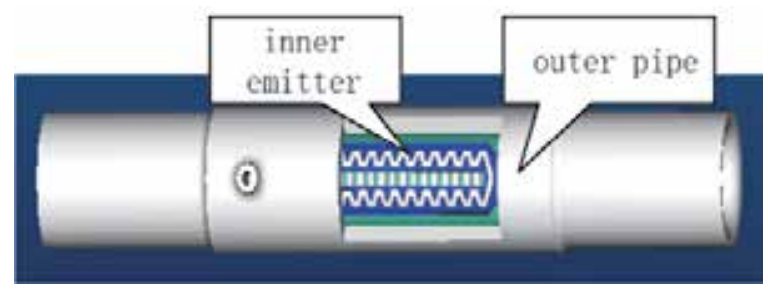

Fig. 6. Integration of experimental three-dimensional solid CAD model of emitter

To finalize the dimensions of the designed emitter as soon as possible, rapid prototyping and manufacturing (RP\&M) is used to develop the emitter. The experimental prototype of the emitter is fabricated. It can be directly used in pressure flow experiments.

\section{Emitter's rapid prototyping manufacturing technology}

$\mathrm{RP}$ of emitter is the key stage in emitter's rapid development. The following contents will introduce the emitter's RP fabricating in detail.

\subsection{Introduction of rapid prototyping}

Rapid Prototyping is a new advanced manufacturing technology developed in early 1980's. The core idea of this technology is based on dimension reduction discrete methods (White, 2001). A complex three-dimensional object is firstly sliced, and then converted into twodimensional planes. The RP machine builds the object layer by layer and finally fabricates the part (Hosni \& Sundaram, 1996).

According to various materials used in RP manufacturing, the RP process can be classified as shown in Fig. 7 (Yan \& Gu, 1996).

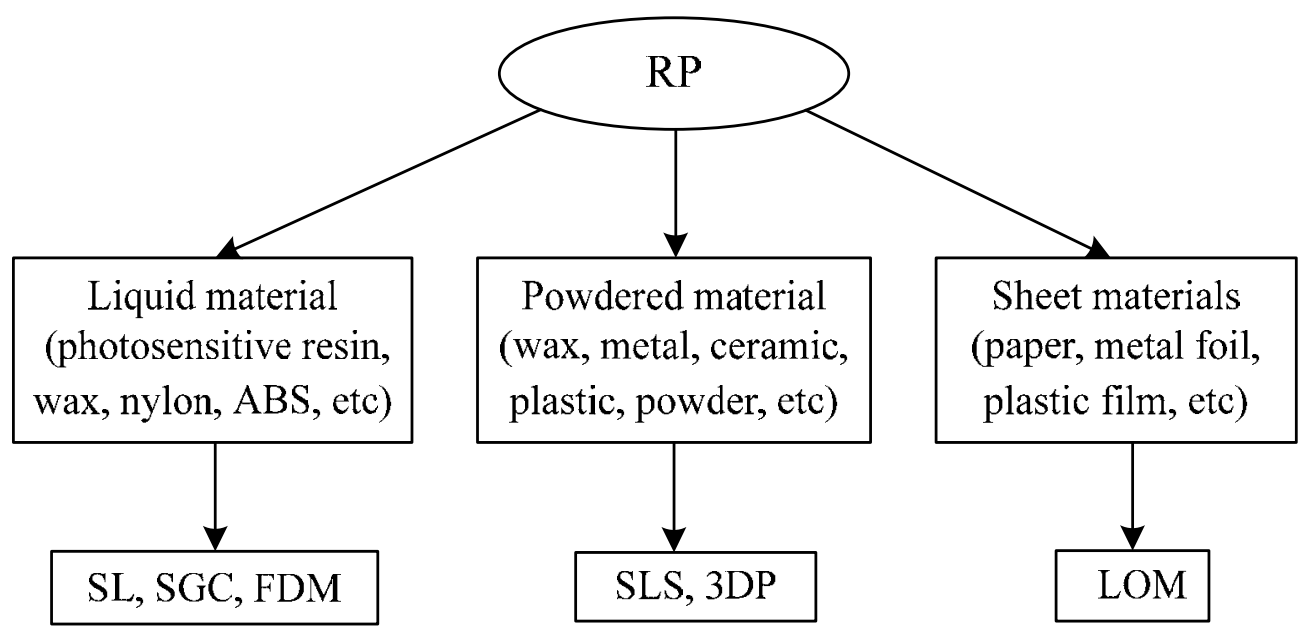

Fig. 7. Classification of RP process 
The products fabricated by SL process have the advantages of better surface quality and higher accuracy (about $0.05 \mathrm{~mm}$ ) and the SL process has higher system resolution (Pham \& Gault, 1998). This method has been widely used in the manufacturing field (White, 2001).

\subsection{Rapid prototyping and fabrication of emitter's integrated model}

SL process can be divided into the following steps: using software to construct threedimensional model or using reverse technology to obtain product's entity (physical models) directly (Choi \& Samavedam, 2002), and then transferring the model's data into STL file, using slicing software to slice the model with the required accuracy, getting a set of contourparallel offset data, and then using software to add support for the contour-parallel offset data. The main purpose of adding support is to facilitate the forming of cavity and suspension, and it can reduce the deformation. The laser is used under the control of scanning system. A thin solidified layer is formed on the photosensitive resin, then the platform sinks until the distance between solidified layer and the resin surface is the thickness of a layer $(0.05 \mathrm{~mm})$, repeat the process, eventually a solid prototype is formed. Fig. 8 is the scheme of emitter's forming process. STL data is the interface of CAD / RP data which is composed of a large number of triangular facets approximating the part's shape. The more the facets are, the higher the accuracy of approximation will be.
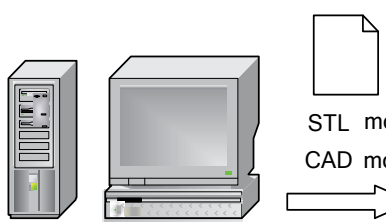

STL model CAD mode

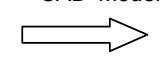

* construct entity

* STL export interface

* other data interface$$
\mid
$$
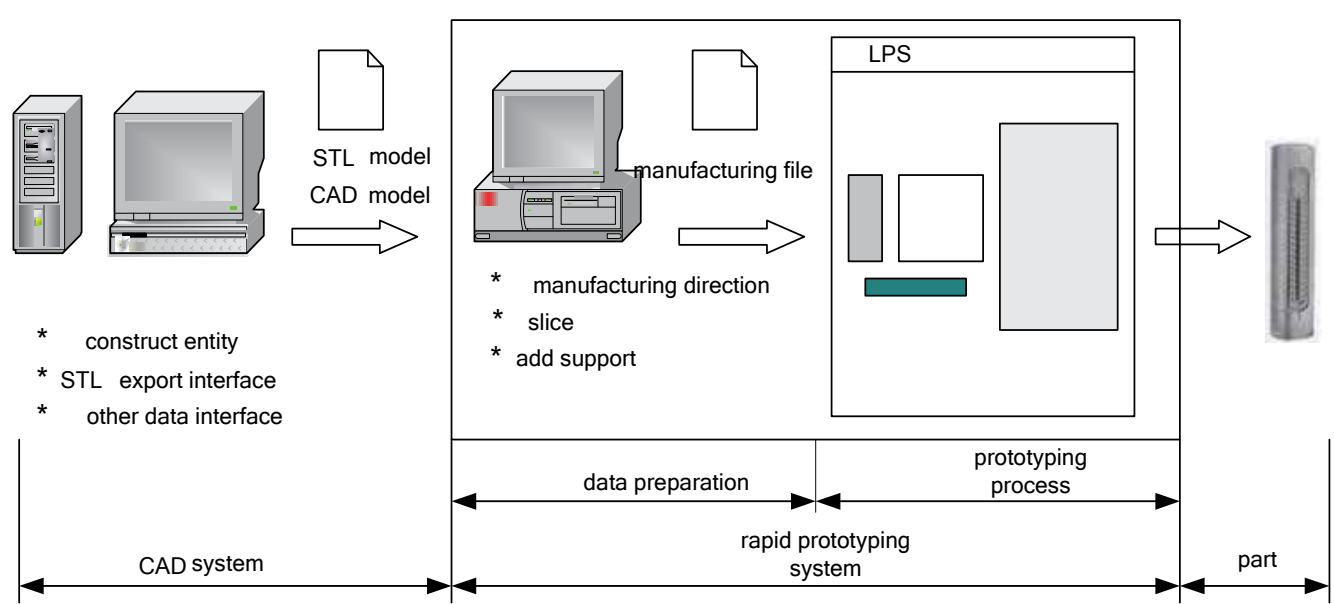

Fig. 8. Scheme of rapid prototyping process

The fabrication process of emitter and its integrating prototyping are as follows, Pro/E is firstly used to design the parameters of emitter and establish the three-dimensional CAD model (as Figure 1-5 and Figure 1-6); then the model is converted into STL files and slicing software is used to cut the model, the cuts can be fabricated in a rapid prototyping machine after adding support. The schematic diagram of detailed production process schematic diagram is shown in Fig. 9 (Wei et al., 2008). The integrating of irrigation emitter and its experimental prototype are shown in Fig. 10.

\subsection{Accuracy analysis and compensation of emitter prototypes}

The emitter prototypes are manufactured by SPS-350B rapid prototyping machine. When using this machine, there are many empirical parameters such as laser sweeping power: 280MW; solidified layer thickness: $0.1 \mathrm{~mm}$; the laser spot size: $0.12 \mathrm{~mm}$; power output of 
post-curing box: $40 \mathrm{~W}$, temperature of post-curing box: $20^{\circ} \mathrm{C}$; sweeping mode: XYSTA, (interlacing sweeping). The deviations in $\mathrm{X}$ and $\mathrm{Y}$ directions are similar.

Emitter channel is built in horizontal direction. Experimental results show that the deviation in this direction is very small ranging from zero to 5 percent. While in the $X-Y$ plane the accuracy is poor, so the accuracy in $\mathrm{X}-\mathrm{Y}$ plane is discussed.

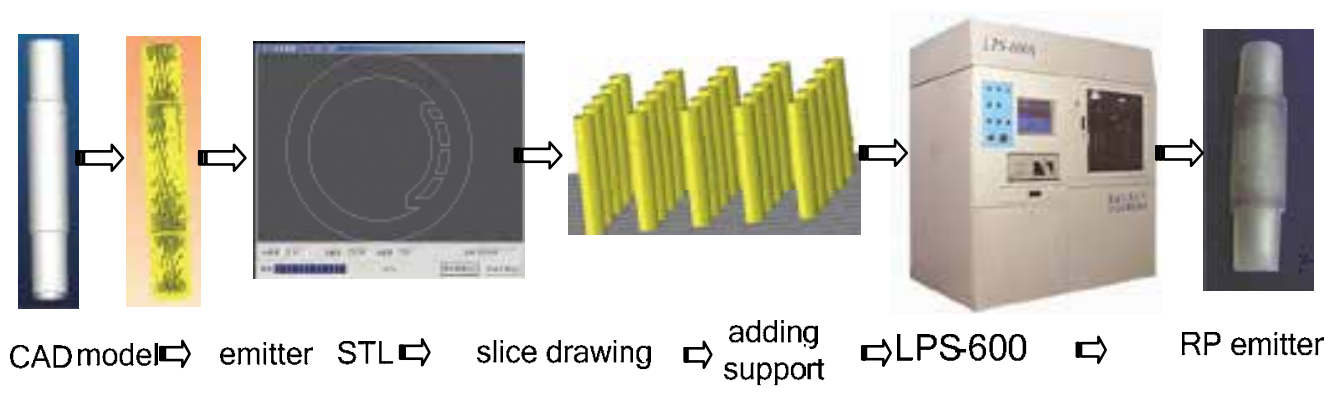

Fig. 9. Manufacture process of integrated emitter prototype
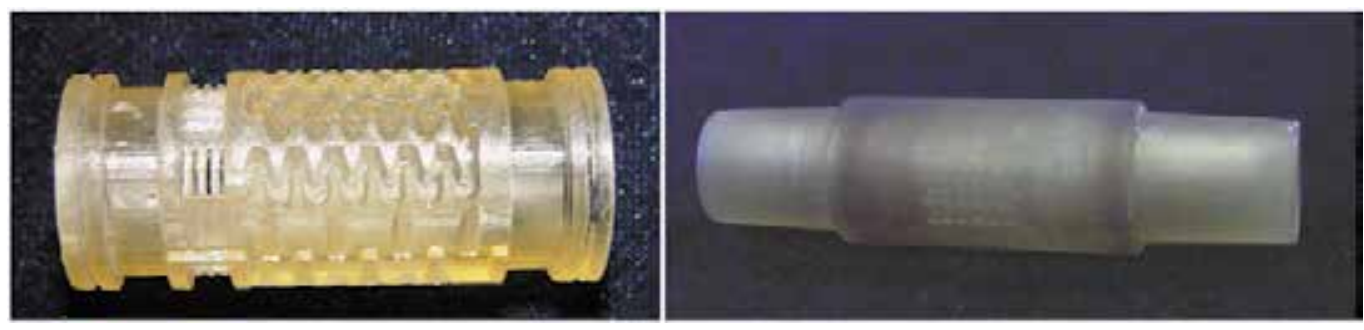

(a) emitter's prototype

(c) emitter's integrated experimental prototype
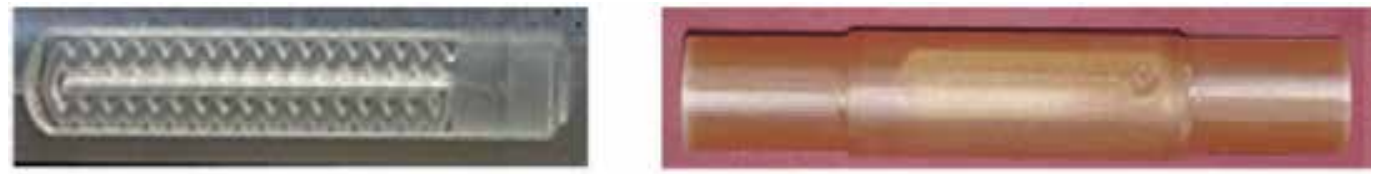

(b) sheet-dropper's prototype

(d) sheet-dropper's integrated experimental prototype

Fig. 10. Experimental prototype and integration of emitter

\subsubsection{Derivation of crucial parameters}

Some parallel grooves on the plate are built to measure $X$ and $Y$ directions' deviation by measuring groove width and the ridge width (the spacing between grooves). The rapid prototyped parts are shown in Fig. 11(a). The size parameters are as follow: groove width: $1 \mathrm{~mm}$; ridge width: $1 \mathrm{~mm}$; groove depth: $1 \mathrm{~mm}$; sample length: $30 \mathrm{~mm}$; sample width: $10 \mathrm{~mm}$; sample thickness: $2 \mathrm{~mm}$.

The process parameters of rapid prototypes are as follows: laser sweeping power: 280MW; layer thickness: $0.1 \mathrm{~mm}$; compensation dimension: $0.12 \mathrm{~mm}$; sweeping mode: XYSTA; power output of post-curing box: $40 \mathrm{~W}$. According to the actual operation situation of RP machine, the approximate processing parameters are determined: sweeping speed: $2000 \mathrm{~mm} / \mathrm{s} \sim 8000 \mathrm{~mm} / \mathrm{s}$; post-curing time: $10 \mathrm{~min} \sim 35 \mathrm{~min}$. During the experiment, the speed are divided into 7 levels: $2000 \mathrm{~mm} / \mathrm{s}, 3000 \mathrm{~mm} / \mathrm{s}, 4000 \mathrm{~mm} / \mathrm{s}, 5000 \mathrm{~mm} / \mathrm{s}, 6000 \mathrm{~mm} / \mathrm{s}$, 
$7000 \mathrm{~mm} / \mathrm{s}, 8000 \mathrm{~mm} / \mathrm{s}$; and the post-curing time is divided into 6 levels: $10 \mathrm{~min}, 15 \mathrm{~min}$, $20 \mathrm{~min}, 25 \mathrm{~min}, 30 \mathrm{~min}, 35 \mathrm{~min}$. These different levels are combined to make different testing samples, and the samples are measured to get the accuracy deviation of the parts.

Compensation is made for arc experimental samples. As Fig. 11(b) shows, in order to distinguish from other samples, single side fillets are designed in the experimental sample. The unit width of the experimental sample is $3 \mathrm{~mm}$, the angle of sharp corner is $60^{\circ}$, a channel is composed of 10 units, the depth of channel is $0.5 \mathrm{~mm}$, the radius of fillet are from $0.1 \mathrm{~mm}$ to $0.5 \mathrm{~mm}$ with an interval of $0.05 \mathrm{~mm}$, the fillets radius of sharp corner and groove are measured to determine the compensation value in both cases.
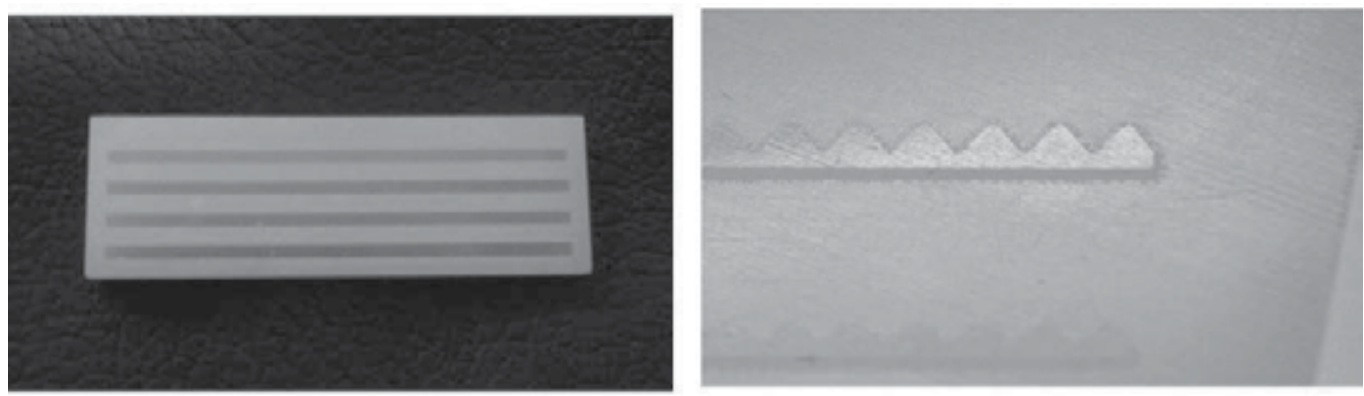

Fig. 11. Rapid prototyped experimental samples

(a) $\mathrm{X}$ and $\mathrm{Y}$ directions deviation testing sample (b) Circular arc compensation testing sample

\subsubsection{Experimental results and accuracy analysis of RP parts}

Microscope system VH-8000 is used for detecting the building accuracy of the experimental samples.

(1) Measurement errors in $X$ and $Y$ directions

The measurement errors in $X$ and $Y$ directions are shown in Table 1 and 2.

\begin{tabular}{|c|c|c|c|c|c|c|c|}
\hline $\begin{array}{l}\text { Post-cured } \\
\text { time } /(\mathrm{min})\end{array}$ & 2000 & 3000 & 4000 & 5000 & 6000 & 7000 & 8000 \\
\hline 10 & 11.76 & 5.29 & 4.90 & 3.14 & 2.16 & 2.55 & 3.53 \\
\hline 15 & 9.81 & 4.30 & 2.65 & 4.51 & 2.84 & 5.39 & 7.89 \\
\hline 20 & 6.97 & 3.93 & 2.16 & 3.43 & 2.35 & 0.59 & 7.26 \\
\hline 25 & 6.37 & 3.83 & 2.35 & 3.14 & 0.98 & 0.68 & 2.16 \\
\hline 30 & 6.28 & 3.73 & 1.18 & 5.88 & 4.61 & 1.18 & 5.20 \\
\hline 35 & 5.49 & 3.04 & 2.55 & 2.55 & 2.45 & 0.10 & 3.24 \\
\hline
\end{tabular}

Table 1. Measurement errors of ridge width under different experimental conditions(\%) 


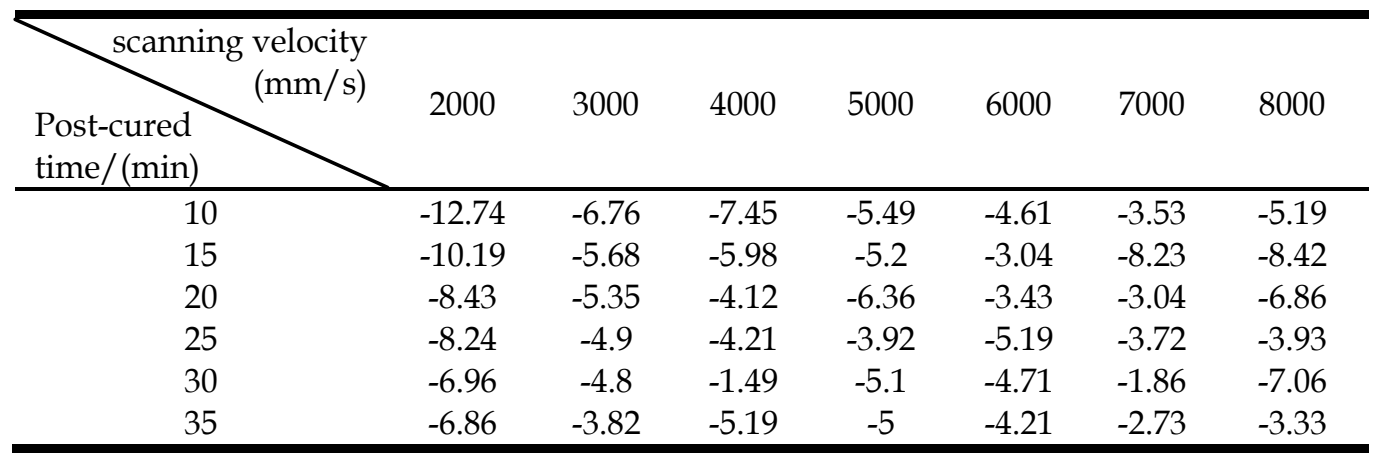

Table 2. Measurement errors of groove width under different experimental conditions(\%)

Analysis of experimental results: The results are shown by the error curve changes with the parameters.

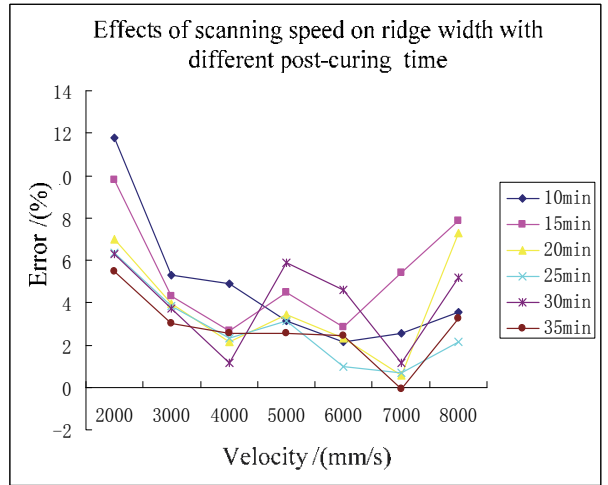

(a) Effects of scanning speed on ridge width

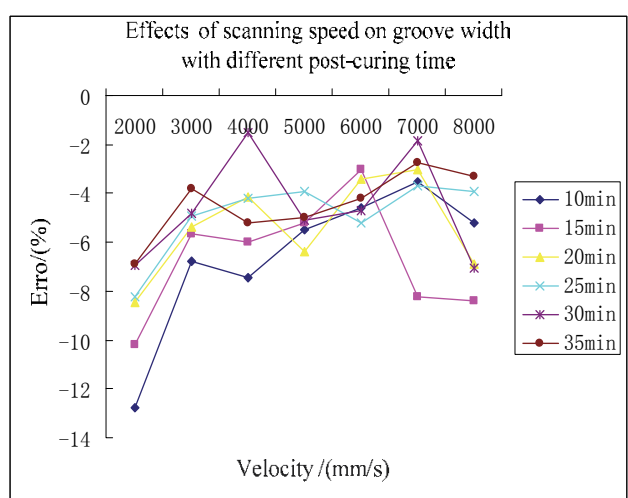

(b) Effects of scanning speed on groove width

Fig. 12. The curves indicate the effect of scanning speed and post-curing times on error
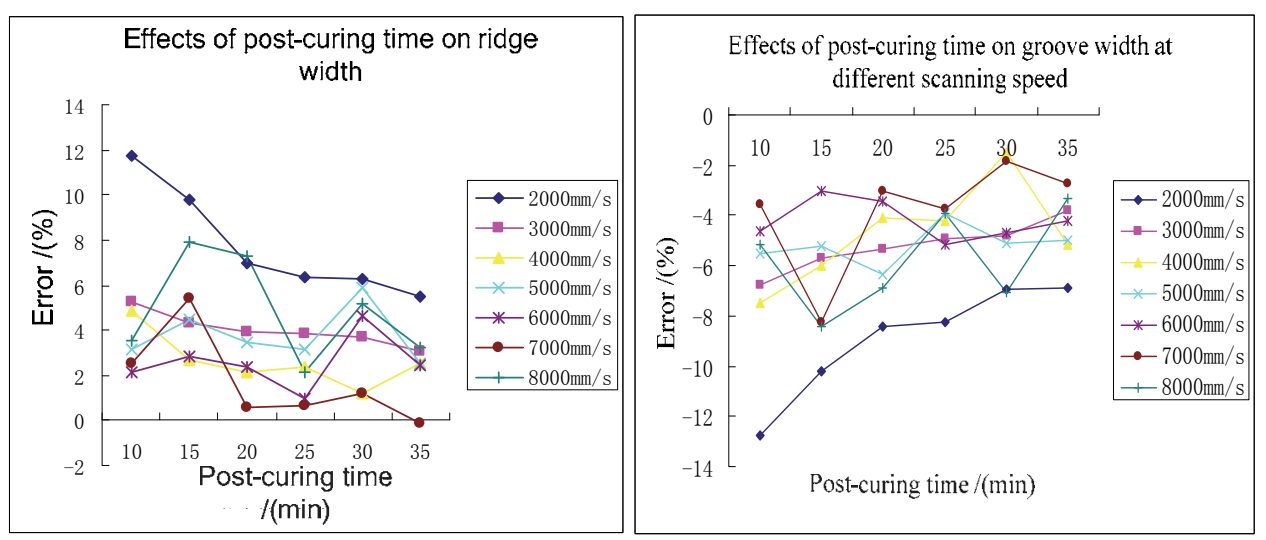

(a) Effects of post-curing time on ridge width (b) Effects of post-curing time on groove width

Fig. 13. The curves indicate the effect of post-curing times and scanning speed on error 
The figures show that the error, at the same scanning speed, is reduced as the post-curing time prolonging and the error changes little after $25 \mathrm{~min}$ post-curing. From the curves of 8 different scanning speeds, we can conclude that after post-curing for $25 \mathrm{~min}$, the error can be ignored.

It was found that the error of ridge width is generally positive while the error of groove width is negative. However, the summation of two is not exactly equal to $2 \mathrm{~mm}$. Under different process conditions, the width errors of the two units are measured to determine the final building parameters.

Table 3 shows that the best experimental result is achieved with a scanning speed of $6000 \mathrm{~mm} / \mathrm{s}$ and post-curing time of $30 \mathrm{~min}$. The overall error is $0.05 \%$ under this process condition. The error of groove width is $4.71 \%$ and the error of ridge width is $4.61 \%$, both are less than $5 \%$, meeting the design requirements.

\begin{tabular}{llllllll}
\hline $\begin{array}{l}\text { scanning velocity/ } \\
\text { Post-cured } \\
\text { time/(min) }\end{array}$ & 2000 & 3000 & 4000 & 5000 & 6000 & 7000 & 8000 \\
\hline 10 & & & & & & & \\
15 & -0.535 & -0.49 & -1.275 & -1.175 & -1.225 & -0.49 & -0.83 \\
20 & -0.73 & -0.19 & -1.665 & -0.345 & -0.1 & -1.42 & -0.265 \\
25 & -0.525 & -0.635 & -0.98 & -1.465 & -0.54 & -1.225 & 0.2 \\
30 & -0.935 & -0.975 & -0.93 & -0.39 & -2.105 & -1.52 & -0.885 \\
35 & -0.34 & -0.435 & -0.155 & 0.39 & -0.05 & -0.34 & -0.93 \\
\hline
\end{tabular}

Table 3. Total measuring error under various experimental conditions(\%)

(2)Accuracy measurement of experimental samples for arc compensation

The measurement results of experimental samples for arc compensation are shown in Table 4.

\begin{tabular}{ccc}
\hline theoretical dimension $/(\mathrm{mm})$ & sharp corner $/(\mathrm{mm})$ & groove $/(\mathrm{mm})$ \\
\hline 0.1 & 0.096 & 0.106 \\
0.15 & 0.138 & 0.167 \\
0.2 & 0.178 & 0.218 \\
0.25 & 0.233 & 0.264 \\
0.3 & 0.278 & 0.310 \\
0.35 & 0.325 & 0.355 \\
0.4 & 0.365 & 0.408 \\
0.45 & 0.429 & 0.453 \\
0.5 & 0.485 & 0.501 \\
\hline
\end{tabular}

Table 4. The results of arc compensation obtained from experiments

From the table, we can get the curve of experimental samples for arc compensation, shown in Fig. 14.

From this curve, the designed values required by actual dimension are obtained. When the radius are ranged from $0.1 \mathrm{~mm}$ to $0.5 \mathrm{~mm}$, the dimensions of experimental samples can get proper compensation. The range of radius in the table can be expanded if needed. 


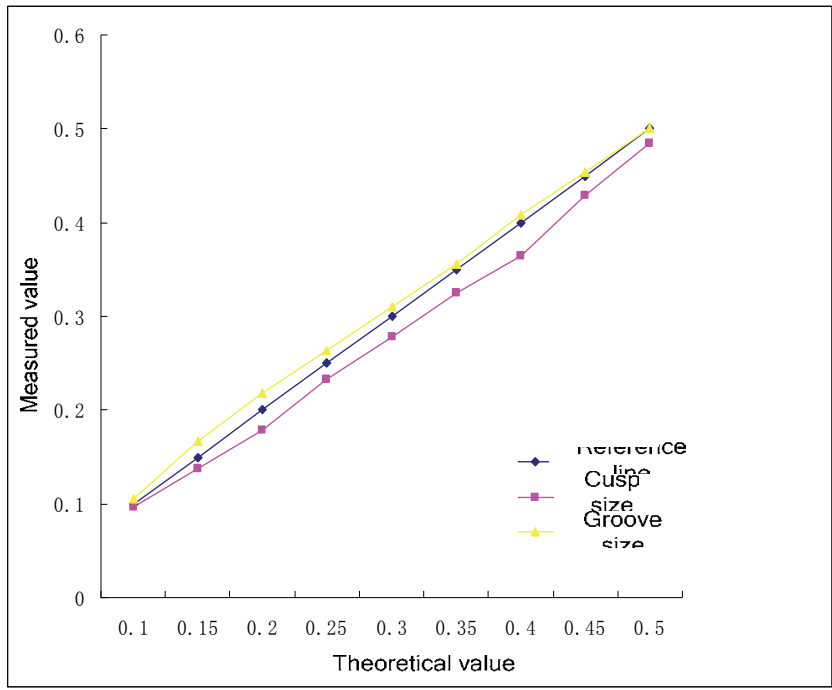

Fig. 14. The curve of experimental samples for arc compensation

The figure shows that the error of groove is obviously less than sharp corner. This is because the contraction of the substrate may affect the actual size of groove and the local contraction of sharp corner may affect the actual size of sharp corner. However, the larger the substrate, the less obvious the contraction. As the size of the substrate increases, the errors of groove and sharp corner first increase and then decrease. When the fillet radius of the groove is larger than $0.35 \mathrm{~mm}$, the dimension of exprimental sample is consistent with the designed value except a very small error.

\section{Analyzing of anti-clogging mechanism and rapid experimental research based on PIV}

The hydraulic behavior and anti-clogging performance are the two key indexes of the emitter (Zhang et al., 2009; Liu et al.,2010; Li et al., 2006; Zhang et al., 2007; Meng et al., 2004; Wei Z. et al., 2008; Wei Q. et al., 2006). In order to analyze the hydraulic behavior and anticlogging performance of these emitters in actual use, rapid prototyping was used to fabricate the experimental samples. Then the visualized experiments with the use of PIV and CFD-based numerical computation were conducted to perform the analysis of the hydraulic behavior and anti-clogging performance. The structural parameters of the emitter were then analyzed and the structure of the labyrinth channel was optimized.

\subsection{PIV visualized experiments and CFD numerical computation \\ 3.1.1 Visualized experimental platform based on PIV}

The visualized experimental platform based on PIV is shown in Fig. 15. The equipment is composed of high speed camera, high pressure Xenon lamp, computer, tracing particles and pressure gauge. The labyrinth channel with an area of $1 \mathrm{~mm}^{2}$ could be observed by a high speed camera and a microscopic len. The camera could be connected with the computer through USB 2.0 interface to analyze and process the measured data of the flow field.

The experimental samples (Fig. 16(c)) were composed of RP parts (the channels) and PMMA plates. The structure of the experimental sample was like a sandwich. Two completely 
identical PMMA plates (Fig. 16(a)) and two RP parts (the channel structure can be obtained if correctly placed, like Fig. 16(b)) were connected by ten bolts. The two PMMA plates used as the channel wall were placed at both sides of the RP parts. The flow behavior in the channel could be visualized due to the transparency of the PMMA plates.
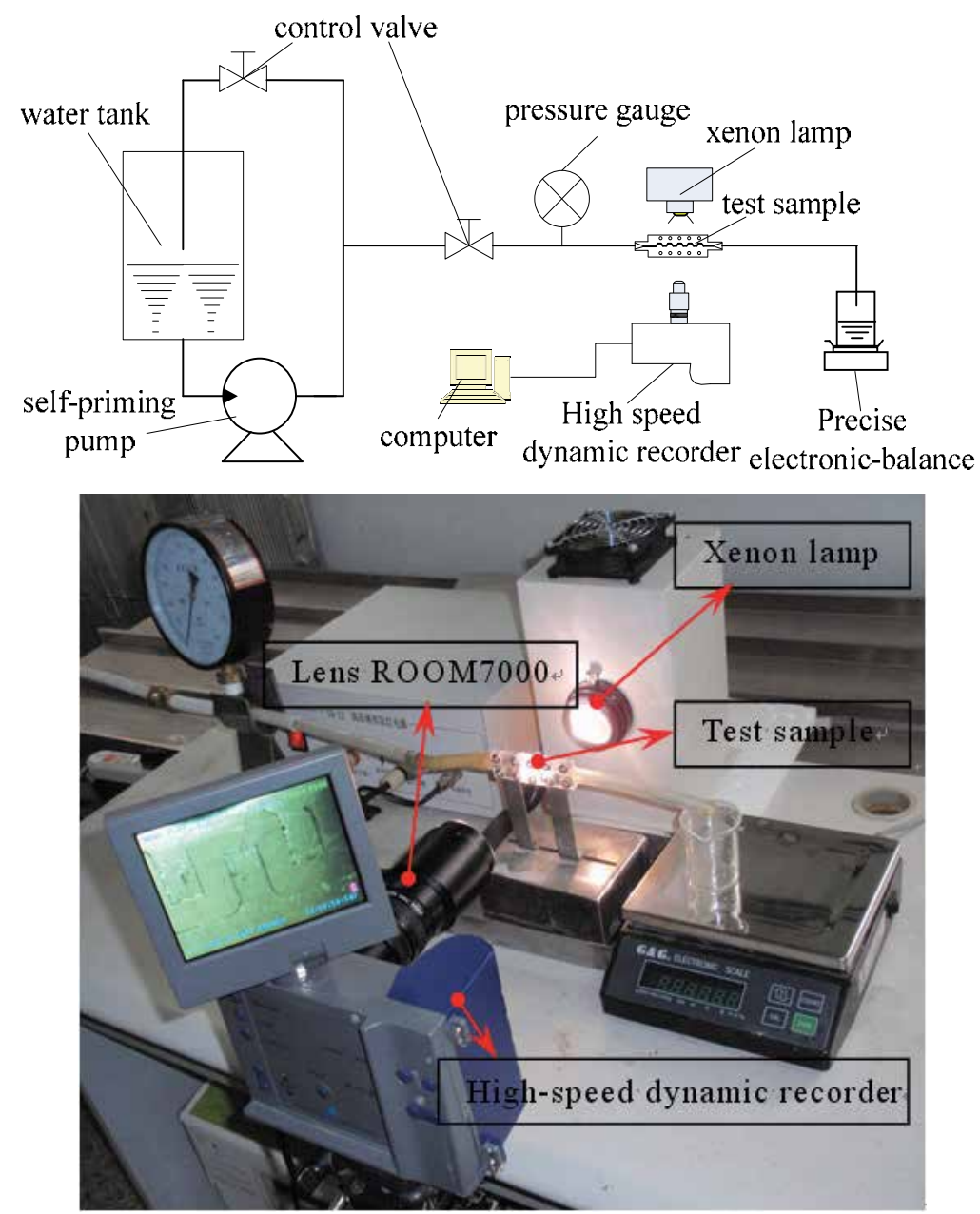

Fig. 15. Scheme of micro-PIV visualized experimental platform

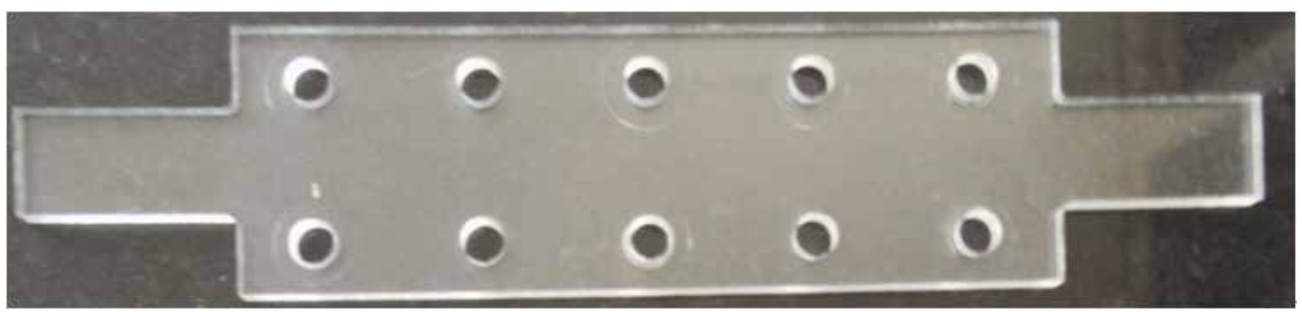

(a) PMMA plate 


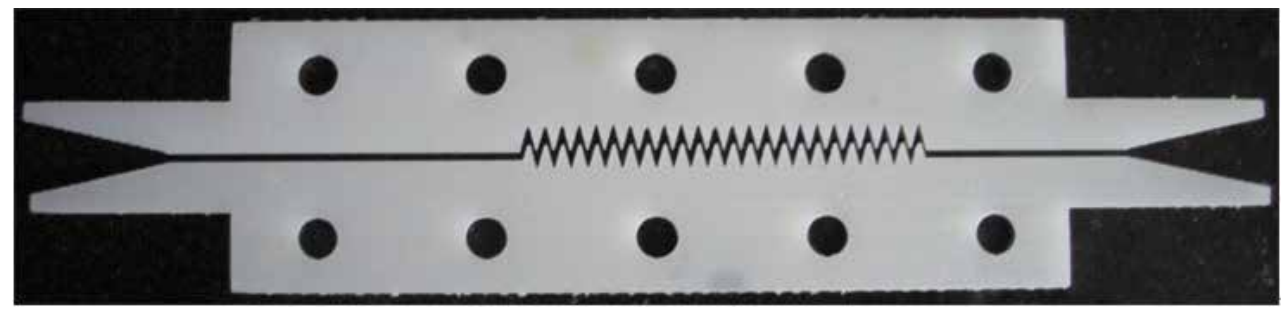

(b) RP sample

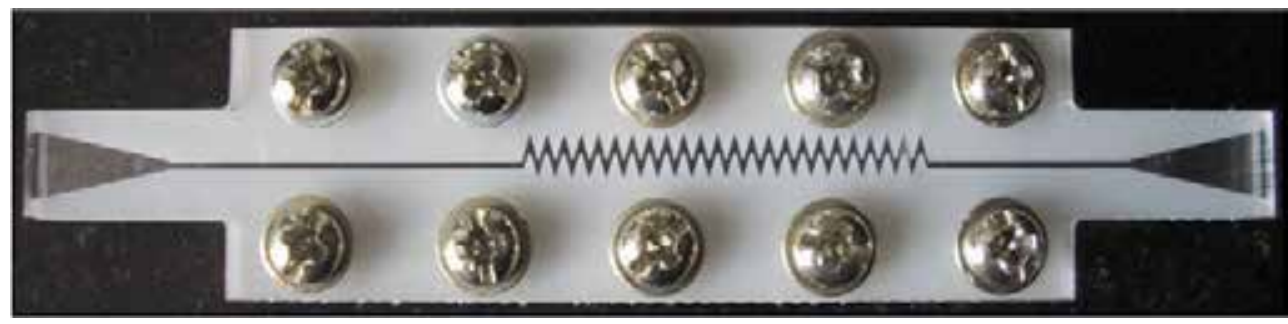

(c) Experimental sample

Fig. 16. Experimental sample and its components

\subsubsection{The CFD numerical theory}

The width of the channel was generally $0.7 \sim 2.0 \mathrm{~mm}$, and the fluid water within the channel was continuous, which met the Navier-Stokes equations and was assumed to be viscous, incompressible and steady at room temperature. The water gravity and surface roughness of the channel wall should be taken into account while the surface tension of the fluid was ignored.

According to the research results of Nishimura (Nishimura et al., 1984a, 1990b), the transition from laminar to turbulent flow occurred when the Reynolds number reached 350 (Kitoh et al., 2005). The channels also had rectangular cross section with a large curvature on the lengthwise section and the Reynolds number was 554 1108 in practical drip irrigation. So the RNG turbulence model and the wall function method were adopted when analyzing the continuous phase water. The equations included mass equation, momentum equation. The working pressure of the emitter was generally $100 \mathrm{KPa}$, so the inlet boundary condition of the model was chosen to be the pressure-inlet with a pressure $\mathrm{Pi}=100 \mathrm{KPa}$, and atmospheric pressure of the outlet was used. The discrete governing equations based on the finite volume method were adopted to carry on the numerical simulation and SIMPLE algorithm was also used.

\subsection{The analysis of the emitter's hydraulic performance based on single phase}

The hydraulic performances of the emitter included macroscopic hydraulic behavior and microscopic hydraulic behavior. The macroscopic hydraulic behavior was mainly indicated by the fluctuation of the flow rate $q$ of the emitter with the water pressure. The microscopic hydraulic behavior was the distribution of the flow field within the labyrinth channels.

Taking the triangle labyrinth channel as an example, the flow behavior in the channel was analyzed. When the inlet pressures were kept at $40 \mathrm{KPa}$ and $150 \mathrm{KPa}$, the Reynolds numbers 
were 600 and 1300 respectively, which were far below the transition Reynolds number 2300 in smooth straight round pipe. According to the pipeline flow theory, with the centrifugal inertial force, the pressure of the fluid at the lateral was obviously higher than that of the inner in a tortuous flow channel, which would generate vortex and secondary flow more easily. The greater the rotational angle was, the greater the strength , the range of vortex and secondary flow would be, meanwhile the time that the eddy vortex and the secondary flow occur would be advanced, which was beneficial to the earlier appearance of the turbulence (Nishimura et al., 1990; Gerolymos et al., 2002; Humphrey et al., 1981; Arnal et al., 1992; Winoto \& Crane, 1980). Fig. 17 shows the flow behavior represented by the streamline in the same flow channel unit at different Reynolds numbers. For the tortuosity of the labyrinth channel was relatively larger, some small circulating vortexes appeared at the sharp corner of the labyrinth channel, but the streamline was still symmetric with the central plane of the channel. With continual rising of the Reynolds number, the inertia force was increasing constantly, which maight generate phase difference between the streamline and the flow channel shape. Meanwhile, the strength and the range of the circulating vortexes were thereby expanding, but the center of them shifted down. When the Reynolds number was 80 , the center of the first vortex moved to midway of the channel, which provided the space for the second vortex to occur. Subsequently, the second vortex gradually enlarges, the whole flow state turns to be three-dimensional because of the oscillation and fluctuation of the shear layer.

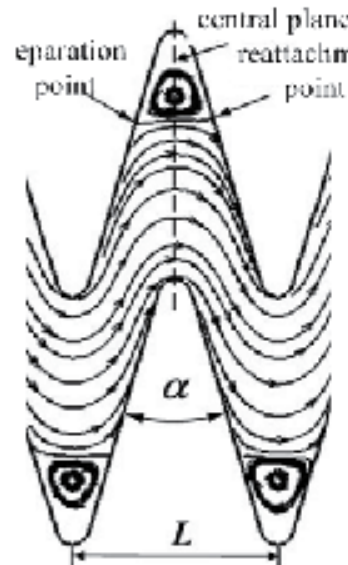

(a) $R e=1$

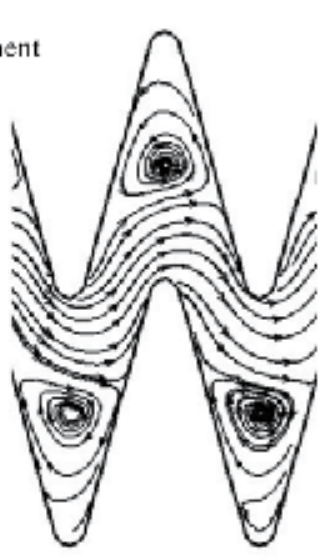

(b) $R e=40$

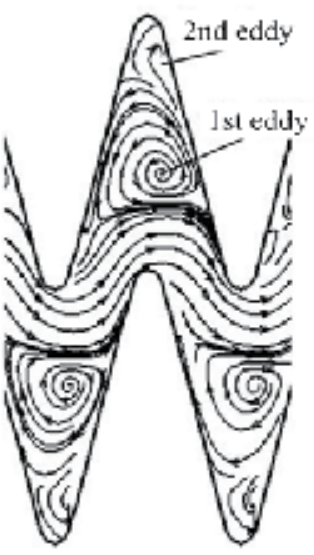

(c) $R e=80$

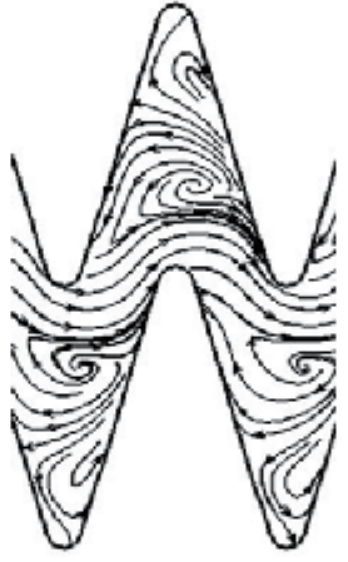

(d) $R e=300$

Fig. 17. Distribution of path lines in labyrinth channel element at different Re

Before the fluid in the tortuous channel arrived at the central plane of the rotational angle, the fluid was to be separated because the pressure of the lateral was relatively higher than that of the inner, then the sign of the pressure gradient would reverse so as to favour the reattachment of fluid and wall, which is shown in Fig. 17(a). Fig. 18 shows the distribution rule between the separation point and the reattachment point under different reynolds conditions. The position of the separation point and the reattachment point would be gradually away from the corner of the channel at the initial stage. However, the position of the separation point was little changed and basically stable near the outlet when the Reynolds Number increased grater than 40, the position of the reattachment point wasn't 
tended to be stable until the Reynolds Number increased to 100. The change of position at the separation and the reattachment point also indicated that the central position of the vortex in the labyrinth channel successively moved to the bottom-right first and then to the bottom-left with the increasing of the Reynolds Number. While the Reynolds Number increased form 250 to 300 , the anomalous change happened to the position of the separation point and the reattachment point. According to the experimental results by Rush et al, the fluid in the channel became unstable as the Reynolds Number went from 250 to 300 (Rush et al., 1999), which was the turning point from laminar flow to turbulent flow. The conclusion showed that the flow were turbulent under the whole working pressure conditions.

Fig. 19 shows the experimental results of the flow rate for two types of emitters in the pressure range $40 \sim 150 \mathrm{KPa}$ and the predicted values on the basis of two kinds of numerical models. The distribution rule of the curves indicated that the numerical calculation was applicable to predict the flow rate of the emitter to a certain extent.

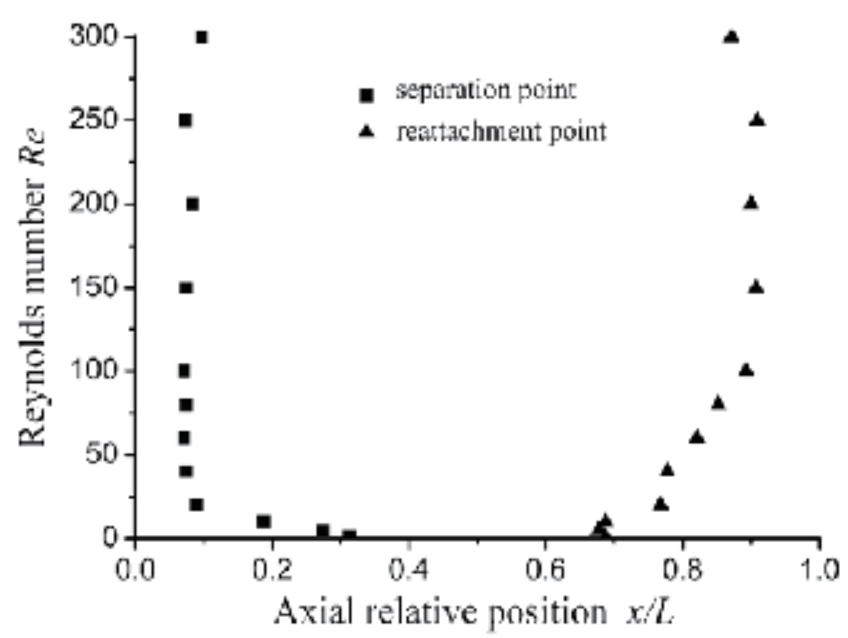

Fig. 18. Distribution of separation points and reattachment points in the labyrinth channel

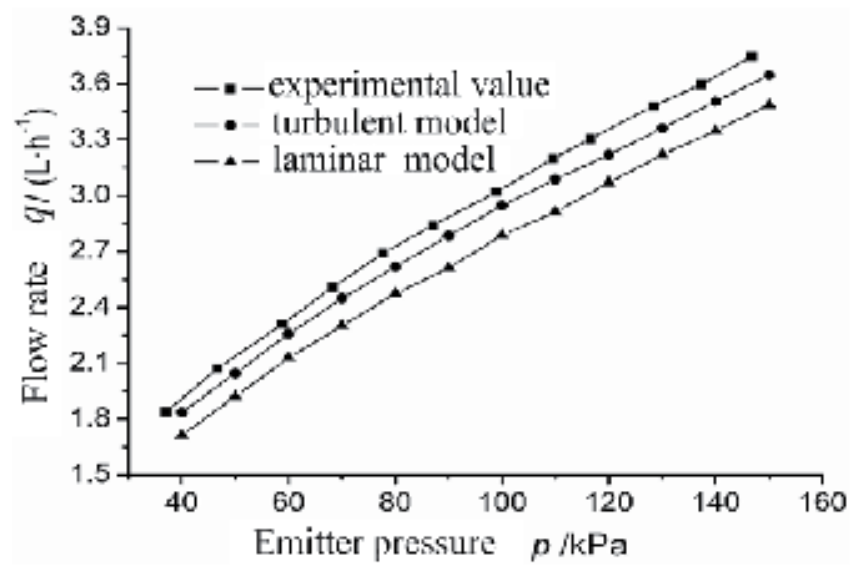

Fig. 19. Distribution of separation points and reattachment points in the labyrinth channel 
The pressure and flow rate obtained by using laminar flow model and turbulent flow model were $\mathrm{q}_{\mathrm{lam}}=0.237 \mathrm{p}^{0.536}$ and $\mathrm{q}_{\mathrm{turb}}=0.27 \mathrm{p}^{0.518}$ respectively. The pressure and flow rate obtained by experiments was $q_{\exp }=0.286 \mathrm{p}^{0.515}$. The average errors by comparing the experimental results with the laminar flow model and the turbulent flow model were $9 \%$ and $4 \%$ respectively. Based on the above-mentioned results, the numerical prediction showed that the result using the turbulent flow model was more reasonable than that using the laminar flow model.

The distribution of the flow filed was researched by using PIV visualized test bed when Reynolds number was 213. The experimental result is shown in Fig. 20(b), which was post processed by image processing software. Fig. 20(a) shows the simulation results of the flow field. The twelfth channel unit, counting from the emitter's inlet, was selected as the object of comparison and analysis. The length of the arrow represented the magnitude of the velocity. Except for a few sparkles, the main flow zone and low-speed zone were obviously displayed. The numerical results and experimental results were in good agreement.

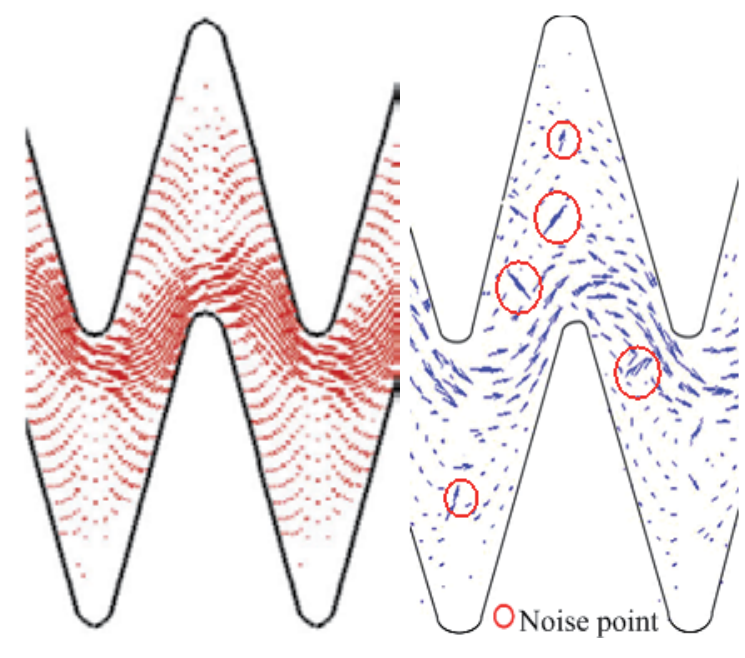

(a)simulating result

(b)experimental result

Fig. 20. Velocity vector graph of the flow field when $R e=213$

For the velocity field was obtained by processing the PIV images, the intuitiveness of the experiment was not obvious. However, the dyeing line method could overcome this shortcoming. Fig. 21 shows the change process of streamline under different Reynolds conditions. The channels in the six figures were composed of three units of the same part in the same test-piece(the twelfth unit from the inlet).

From the above results, we could find that the transition process from laminar flow to turbulent flow in the labyrinth channel was different from that in smooth straight flow channel. For the purpose of improving hydraulic behavior, the turbulent state in labyrinth channel was expected theoretically. From a hydraulic viewpoint, critical Reynolds number was expected to be small. The relationship between the labyrinth-channel structure and Reynolds number was analyzed quantitatively in terms of the distribution features of the separation point and reattachment point, which provided a theoretical basis for the design of the labyrinth-channel structure. 


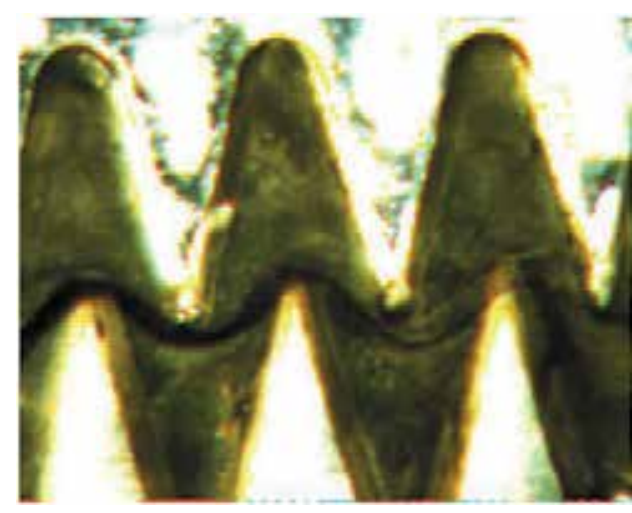

(a) $\operatorname{Re}=22$

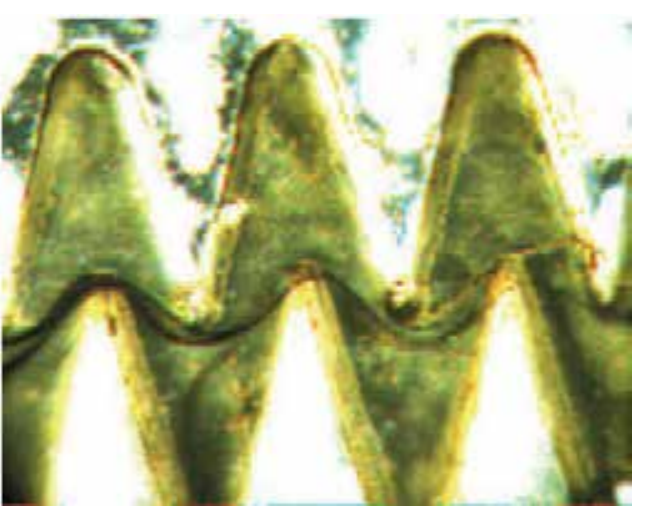

(b) $\operatorname{Re}=57$

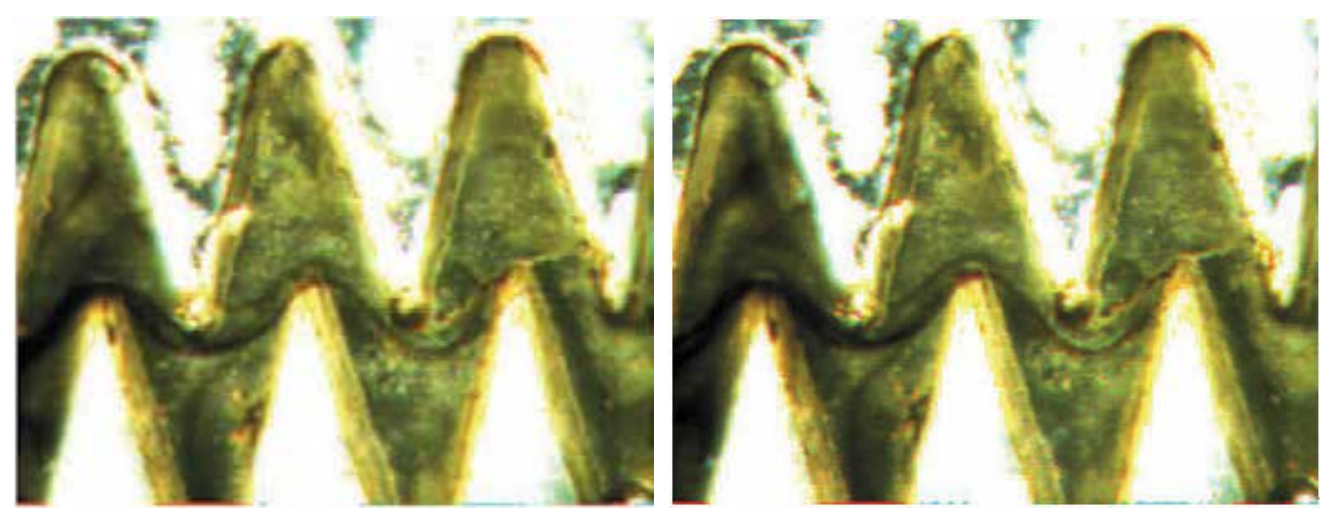

(c) $\operatorname{Re}=93$

$(\mathrm{d}) \operatorname{Re}=131$

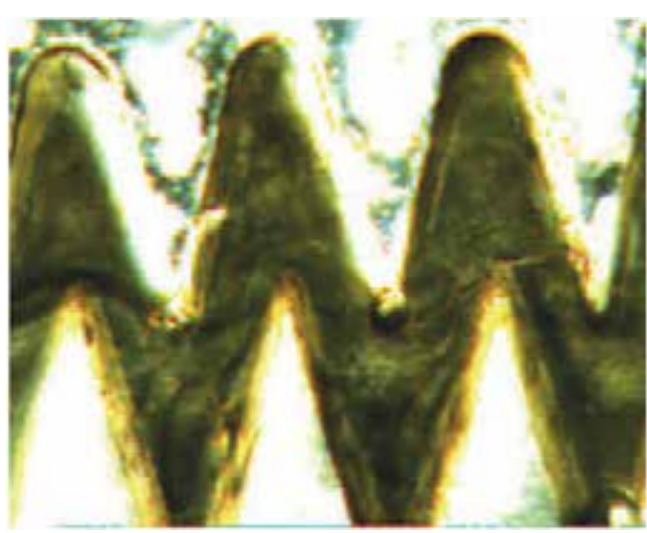

(e) $\operatorname{Re}=277$

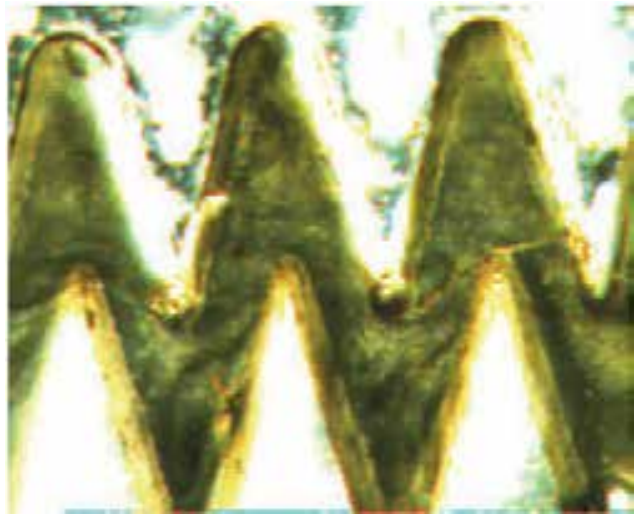

(f) $\operatorname{Re}=368$

Fig. 21. Flow field displayed via dye experiment 


\subsection{Research on the particle-wall collision behavior}

Some micro particles and microorganism will enter the emitter channel even the irrigation water had been filtered, which might lead to deposition and result in clogging if the particles could not pass through the channel smoothly (Wei Z. et al, 2008; Zhang et al., 2007; Padmakumari \& Sivanappan, 1985; Gilbert et al., 1977). In order to analyze the flow state and observe the flow field inside the emitter channel, the CFD (Computational Fluid Dynamics) method was used to conduct numerical simulation of water-sand two phase flow in the micro channel. The flow field distribution of water (continuous phase), and the trajectories of sand (particle phase), were analyzed and the clogging mechanism of micro channel was revealed.

\subsubsection{Collision and rebound theory model}

In order to establish an accurate numerical model of water-sand two phase flow, it is necessary to observe the trajectories of the particles and the collision between particles and the wall in the channel by experiments before doing numerical simulation.

Experimental results showed that some sands in the water are rebounded to main flow zone after collision with the emitter wall while some others moved slowly along the wall or directly adhered to the channel wall. With the deposition of sand, the emitter was clogged. Particle-wall collision is a main cause of emitter clogging. However, when using computational fluid dynamics software to do the numerical simulation of the labyrinth channel, the key parameters (the collision and rebound coefficient of the particle-wall) are usually set according to experiences rather than determined by the experiments. So the following experiments were used to determine the particle-wall collision and rebound coefficients.

The particle-wall collision was shown in Fig. 22. The rebound coefficient is decomposed to normal coefficient $R_{c n}$ and tangential coefficient $R_{c t}$. The normal and tangential rebound coefficients respectively reflect the normal and tangential changes of the particles momentum after collision. Where, $\alpha$ is the incident angle and $\beta$ is the reflect angle.

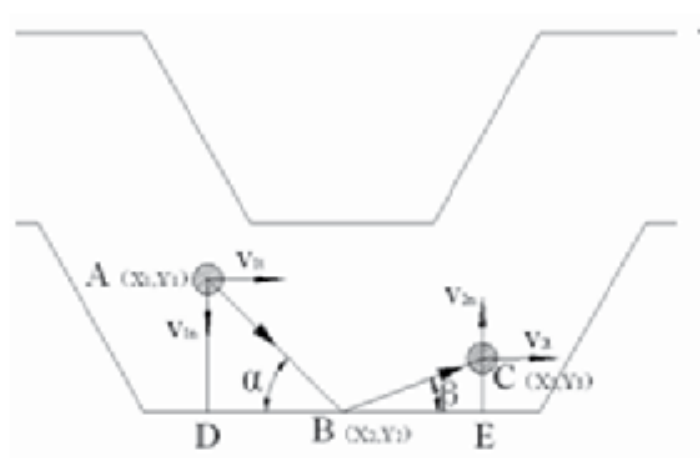

(a) Particle-wall collision

in trapc soidal channel

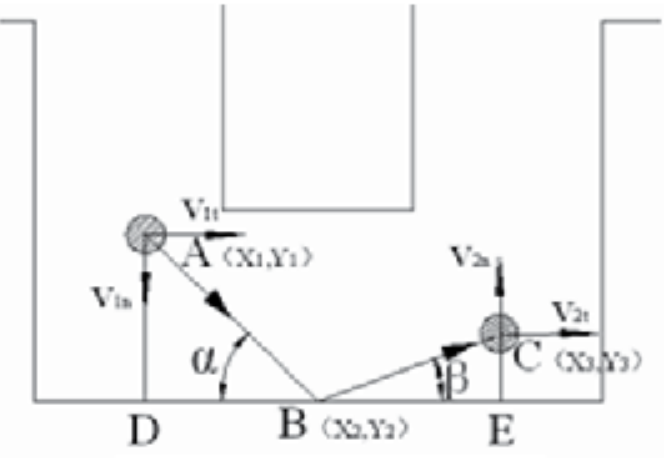

(b) Particle-wall collision in rectangular channel

Fig. 22. Collision model between sand and channel's wall

As shown in Fig. 22, the location of the sand was tracked at different times (at times $t, t+\Delta t$ and $t+2 \Delta t$ ). The lines connecting the adjacent points can be approximately taken as the sand 
trajectory as the time interval $\Delta \mathrm{t}$ was rather small $(0.5 \mathrm{~ms})$. Thus the velocity of particle before collision is approximately $V_{1}=|A B| / \Delta T$, while after the collision $V_{2}=|B C| / \Delta T$. The particle velocity is decomposed tangentially and normally, and the tangential and normal rebound coefficients, incident angle $\alpha$ and reflection angle $\beta$ were obtained according to the geometric relationship.

From the geometric relationship, it can be obtained:

$$
\begin{aligned}
& |A B|=\sqrt{\left(X_{2}-X_{1}\right)^{2}+\left(Y_{2}-Y_{1}\right)^{2}} \\
& |B C|=\sqrt{\left(X_{3}-X_{2}\right)^{2}+\left(Y_{3}-Y_{2}\right)^{2}}
\end{aligned}
$$

Decomposing the particle velocity before and after collision, the tangential velocity before collision is:

$$
V_{1 t}=V_{1} \cos \alpha=\frac{|A B| \cos \alpha}{\Delta T}
$$

The normal velocity before collision is:

$$
V_{1 n}=V_{1} \sin \alpha=\frac{|A B| \sin \alpha}{\Delta T}
$$

The tangential velocity after collision is:

$$
V_{2 t}=V_{2} \cos \beta=\frac{|B C| \cos \beta}{\Delta T}
$$

Thus, the normal rebound coefficient is:

$$
V_{2 n}=V_{2} \sin \beta=\frac{|B C| \sin \beta}{\Delta T}
$$

Thus, the tangential rebound coefficient is:

$$
R_{c t}=\frac{V_{2 t}}{V_{1 t}}=\frac{|B C| \cos \beta \Delta T}{|A B| \cos \alpha \Delta T}=\frac{\sqrt{\left(X_{3}-X_{2}\right)^{2}+\left(Y_{3}-Y_{2}\right)^{2}} \cos \beta}{\sqrt{\left(X_{2}-X_{1}\right)^{2}+\left(Y_{2}-Y_{1}\right)^{2}} \cos \alpha}
$$

The normal rebound coefficient is:

$$
R_{c n}=\frac{V_{2 n}}{V_{1 n}}=\frac{|B C| \sin \beta \Delta T}{|A B| \sin \alpha \Delta T}=\frac{\sqrt{\left(X_{3}-X_{2}\right)^{2}+\left(Y_{3}-Y_{2}\right)^{2}} \sin \beta}{\sqrt{\left(X_{2}-X_{1}\right)^{2}+\left(Y_{2}-Y_{1}\right)^{2}} \sin \alpha}
$$

Where

$$
\sin \alpha=\frac{|A D|}{|A B|}, \sin \beta=\frac{|C E|}{|B C|}, \cos \alpha=\frac{|B D|}{|A B|}, \cos \beta=\frac{|B E|}{|B C|}
$$

Through these above derivation, we can get the collision and rebound coefficients. 


\subsubsection{Experimental measurement of particle-wall reflect coefficients}

Taking trapezoidal labyrinth channel as the example, it is shown how to measure the rebound coefficient. The experimental sample is shown in Fig. 23 and Fig. 24.

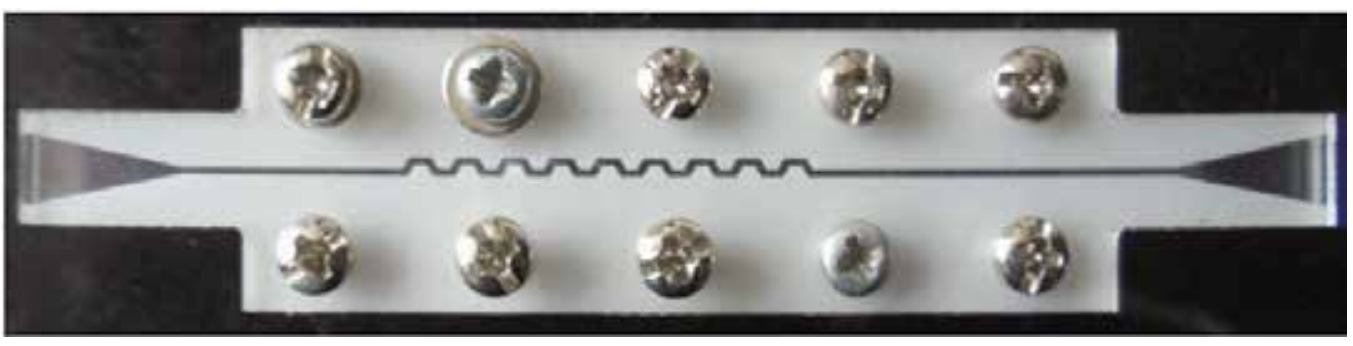

Fig. 23. Experimental sample of labyrinth channel

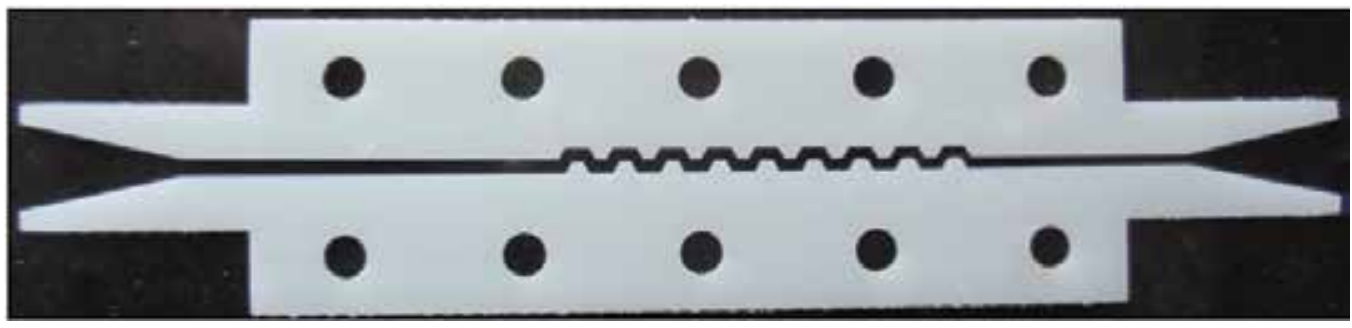

Fig. 24. Experimental sample based on SLA

The collision between sands and emitter wall was observed and recorded by PIV visualized test bed, and then the video segmentation tools were used to pick up all the frames of the video, so the continuous movement images of the sand were obtained. Through processing and analyzing these images based on the computational model of the rebound coefficient, the collision and rebound coefficients in different channels were obtained.

Hydraulic behavior and particle-wall collision of different kinds of labyrinth channel emitters were observed by PIV test bed. The reflect coefficients of 300 grains were measured under the same pressure. The mean value was viewed as the coefficient at this pressure. The relationships between pressure, rebound coefficient and flow rate were shown in Table 5.

\begin{tabular}{ccccc}
\hline Pressure $(\mathrm{kPa})$ & $\mathrm{P}^{*}(\mathrm{kPa})$ & $\mathrm{R}_{\mathrm{ct}}$ & $\mathrm{R}_{\mathrm{cn}}$ & $\mathrm{Q}(\mathrm{L} / \mathrm{h})$ \\
\hline 20 & 0.2857 & 0.768089 & 0.363286 & 3.27 \\
30 & 0.4285 & 0.828028 & 0.440756 & 4.78 \\
40 & 0.5714 & 0.839024 & 0.465534 & 5.94 \\
50 & 0.7142 & 0.866197 & 0.350354 & 6.72 \\
60 & 0.8514 & 0.884615 & 0.258125 & 7.62 \\
70 & 1 & 0.856295 & 0.327147 & 8.70 \\
\hline
\end{tabular}

Table 5. The relationship among pressure, rebound coefficient and flow

Curve fitting of pressure and tangential rebound coefficient:

$$
R_{c t}=0.7584+0.128 P^{*}
$$


Curve fitting of pressure and normal rebound coefficient:

$$
R_{c t}=0.4755-0.168 P^{*}
$$

$\mathrm{P}^{*}$ is the normalized pressure.

Through the above experiments, the rebound coefficient between sand and channel wall can be obtained which can provide theoretical basis for the setting model's boundary conditions for water-sand two phase flow.

\subsection{Studying the clogging problem in emitter}

The clogging mechanism was studied with $\mathrm{SiO}_{2}$ being the tracking particles. The tracking particles density was $2320 \mathrm{~kg} / \mathrm{m}^{3}$ and their average diameter was $67.6 \mu \mathrm{m}$. The experimental conditions were as follows: the inlet pressure was $100 \mathrm{kPa}$, and the particle density was $500 \mathrm{ppm}$. The flow behavior of the tracking particles was recorded by a high speed shooter (shown in Fig. 25 and 26).

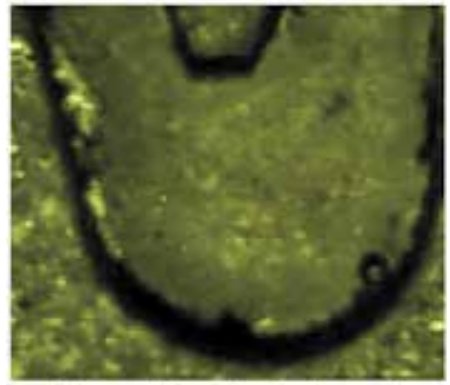

(a) arc labyrinth channel

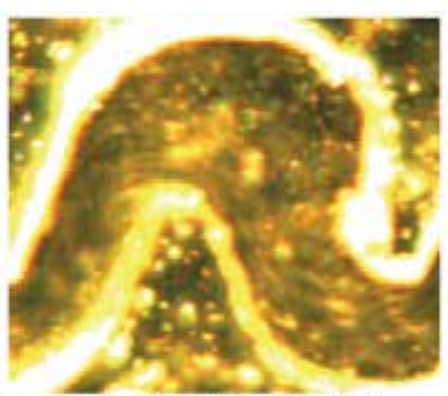

(b) trapezoidal labyrinth channel

Fig. 25. PIV-graph of the water-sand behaviour

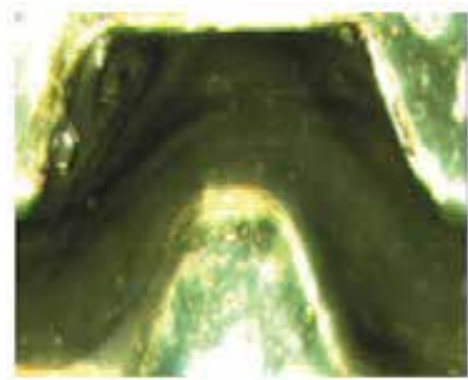

(a) flow behavior displayed by tracking particles

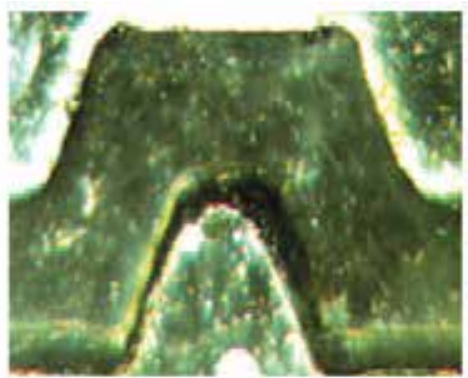

(b) flow behavior displayed by dyeing liquor

Fig. 26. Flow behavior display

The clogging of the micro-channel is shown in Fig. 27. Sand was deposited at the position that has low flow velocity according to the simulation results. Some conclusions could be obtained: the existence of low velocity regions and the vortex regions is the main reason leading to emitter clogging; and the correctness of the CFD analysis is verified, so the CFD analysis can be used in structural optimization designing of the emitter channel. 


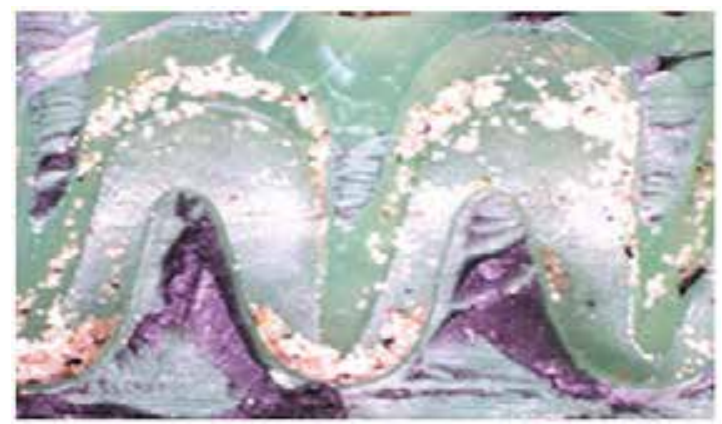

(a) starting stage of clogging

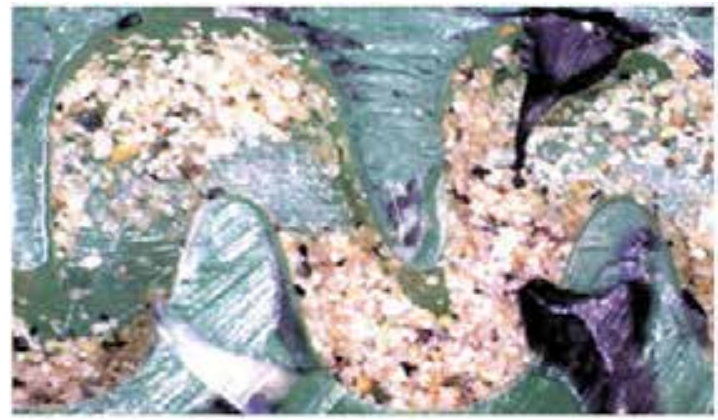

(b) developing stage of clogging

Fig. 27. Clogging status when the sand particles are compulsory entered into the channel

Concluding from the simulation and experimental results, the clogging problem can be resolved by removing the low velocity regions and vortex regions. The flow field and the particle moving trajectory in arc labyrinth channel and rectangular labyrinth channel were analyzed, finally, an optimized channel structure was designed. CFD analysis was implemented on the optimized channel and the results are shown in Fig. 28. The low velocity region and vortex region were mostly eliminated in the optimized channel. This labyrinth channel was named as anti-clogging labyrinth channel.

The optimized emitter was fabricated with rapid prototype technique and the anti-clogging test was performed. Fig. 29 shows the emitter channel after a short period anti-clogging test. Comparing the micro PIV test results with CFD simulation, it is found that the stagnation areas were obviously eliminated and hence the anti-clogging behavior of the emitter was improved.

\section{Emitter rapid tooling CAE and products finalization}

\subsection{Emitter's rapid tooling fabrication and accuracy testing}

At present, the main process of emitter's product-finalization is as follows: first, the prototype sample is fabricated by SL RP process; then, the hydraulic behavior test on the prototype sample is conducted, the structure of the emitter is determined if the experimental results are satisfying, or it needs to be redesigned and the above processes should be repeated. Since layer superposition (thickness $0.1 \mathrm{~mm}$ ) is the principle of rapid prototyping, step effect is obvious in emitter fabrication which cause roughness on the emitter surface. To 
solve this problem, rapid prototyping and $\mathrm{CNC}$ wire cutting were introduced into the emitter development process. CNC wire cutting was used to fabricate the emitter's channel. $\mathrm{RP}$ was used to fabricate the emitter matrix. Then the emitter matrix and emitter channel were assembled to form the prototype of the sheet-emitter.
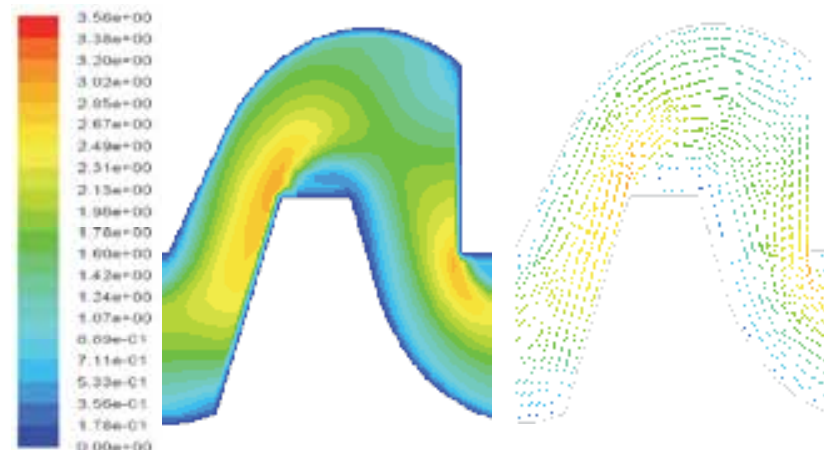

(a) contours of velocity distribution

(b) vectors of velocity distribution

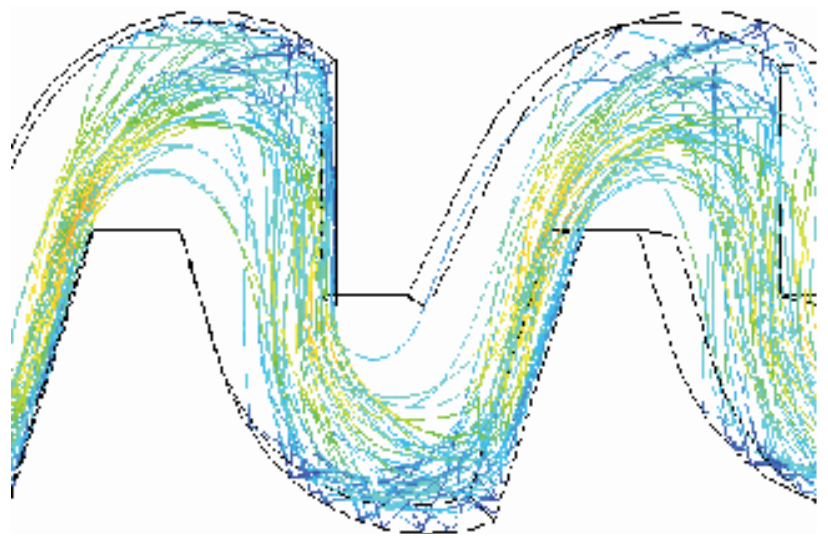

(c) Trajectories of particle

Fig. 28. Analyzing on optimized labyrinth channel

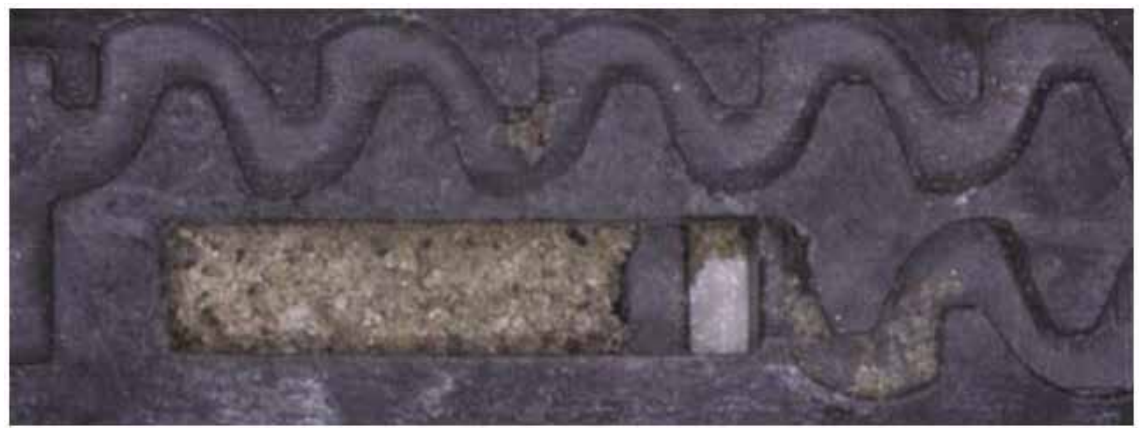

Fig. 29. Clogging status in the optimized emitter 
The process of sheet-emitter small batch producing is shown in Fig. 30.

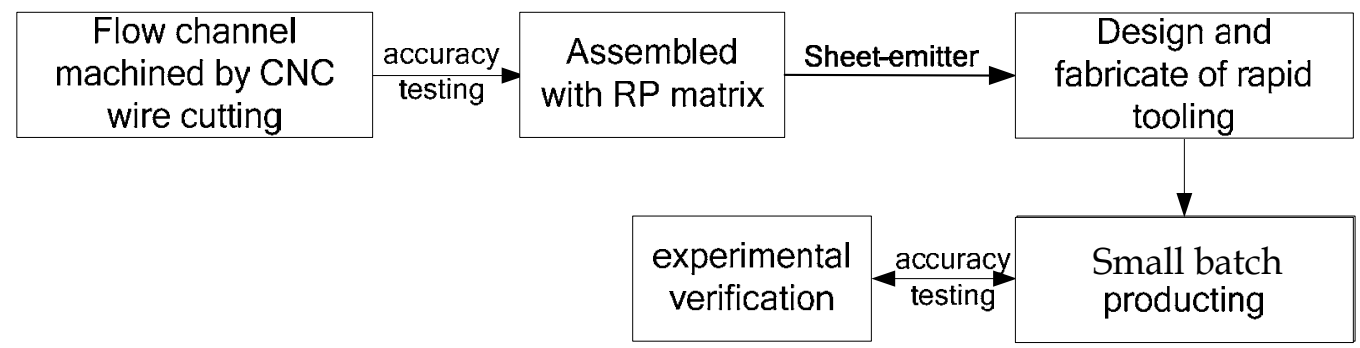

Fig. 30. Manufacturing process of sheet-emitter

When the emitter has small-size channel, the relative size error will be very large which cannot meet the accuracy requirement. In Fig. 31, the width error in certain part of the channel is larger than $5 \%$, and the shape error can reach up to $10 \%$. Those errors will affect the flow behavior, and finally affect the emitter's hydraulic and anti-clogging performance. Under this condition, the hydraulic performance obtained from experiments cannot reflect the actually situation.

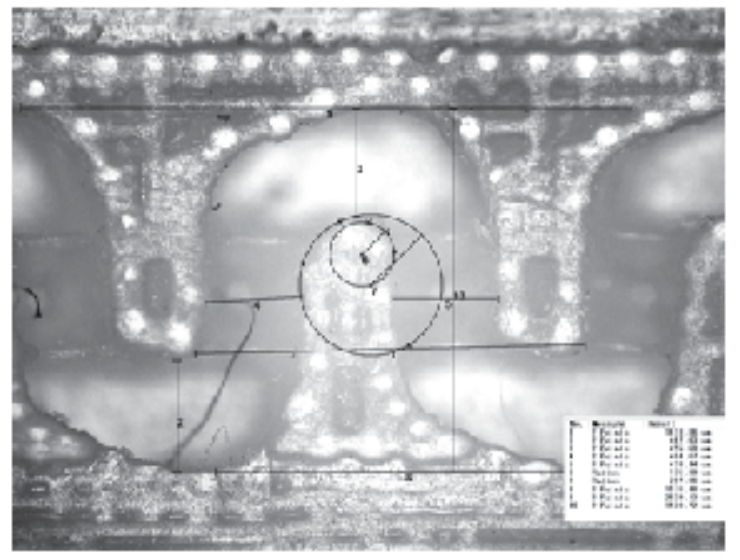

(a) The measurement results of RP sample

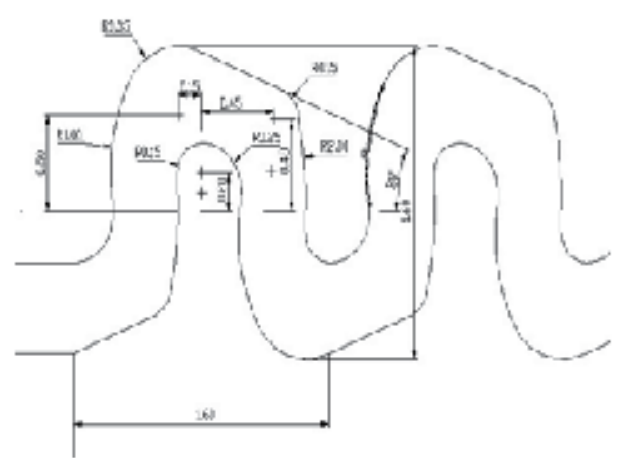

(b) Design size of the channal

Fig. 31. The channel's RP forming accuracy compared with the designed size

Then the forming accuracy of the channel by CNC wire cutting was analyzed. Accuracy measurement had been made on the cutting channel through microscope. The main dimensions of the channel are arc radius and channel width. The design width of channel was $0.4 \mathrm{~mm}$ and the arc diameter was $0.17 \mathrm{~mm}$. From the measurement results, the range of the size error is from $0.24 \%$ to $4.82 \%$, which is less than $5 \%$. Namely, the emitter sample can meet its accuracy requirement.

From the measurement results, it showed that the emitter could meet the accuracy requirements by $\mathrm{CNC}$ wire cutting. With the technology of RP (manufacturing complex samples), the matrix of emitter is fabricated which is assembled with channel fabricated by $\mathrm{CNC}$ wire cutting. The whole model is shown in Fig. 34. 

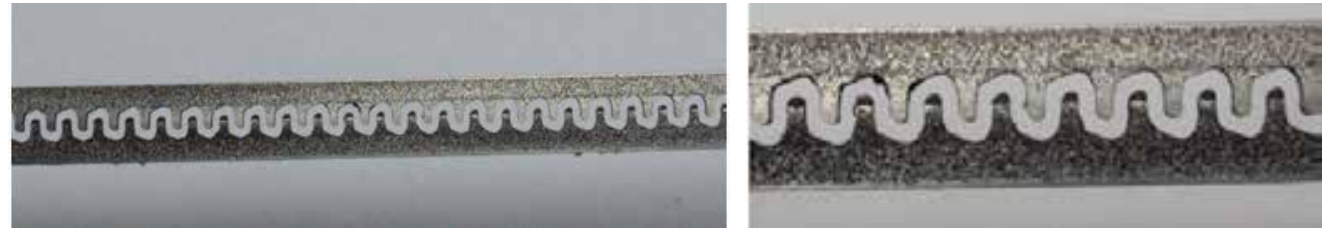

Fig. 32. Oblique teeth channel under wire-cutting process
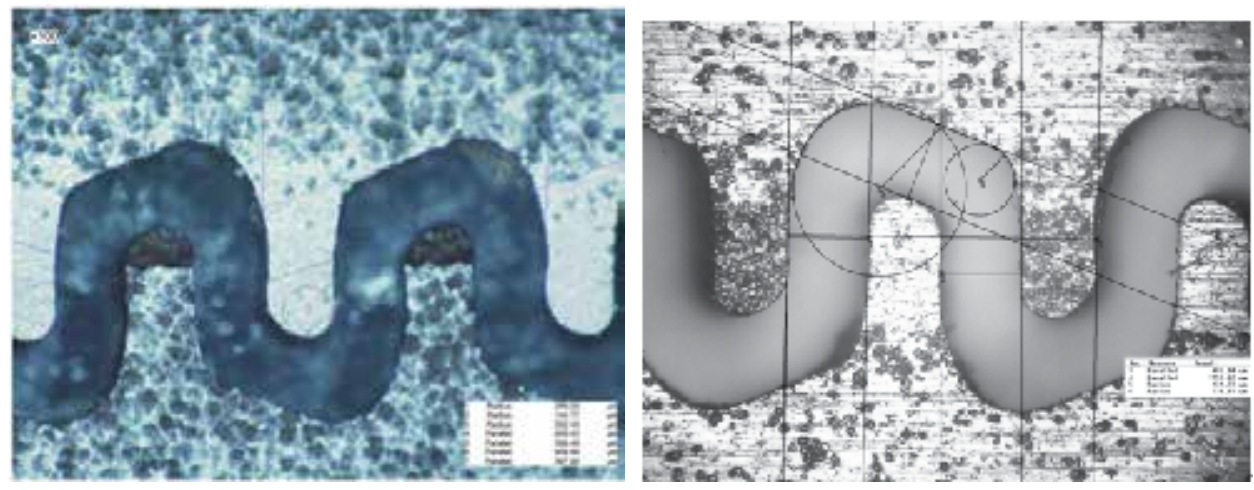

Fig. 33. Accuracy measurement of helical sample under wire-cutting process

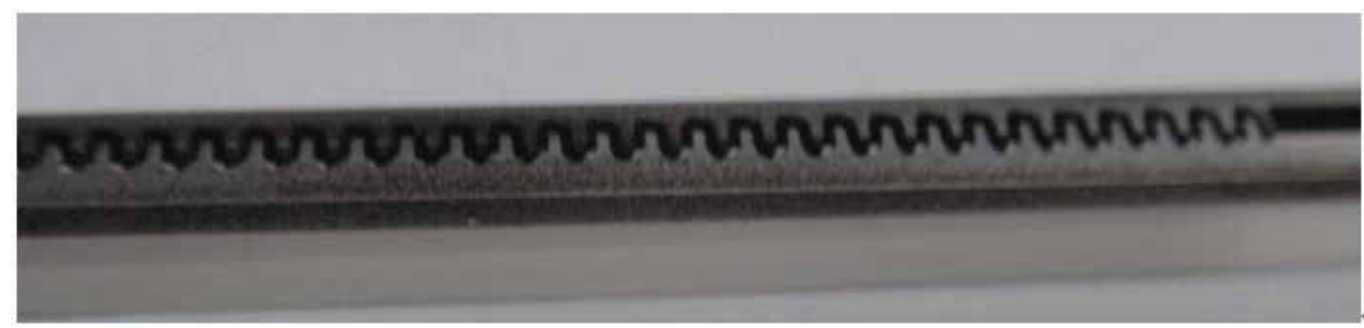

Fig. 34. Oblique teeth channel emitter obtained by assembling the wire-cutting samples and the RP sample

Rapid tooling is fabricated using the assembled sample. With the advantages of perfect simulation effect, high strength and low shrinkage rate, the silicon rubber (PDMS) is chosen to produce rapid tooling. As the silicon rubber doesn't react with the material of RP, no wrinkle is produced in the fabrication process and good surface quality can be obtained. The mold fabricating process of silicon rubber is shown in Fig. 35.

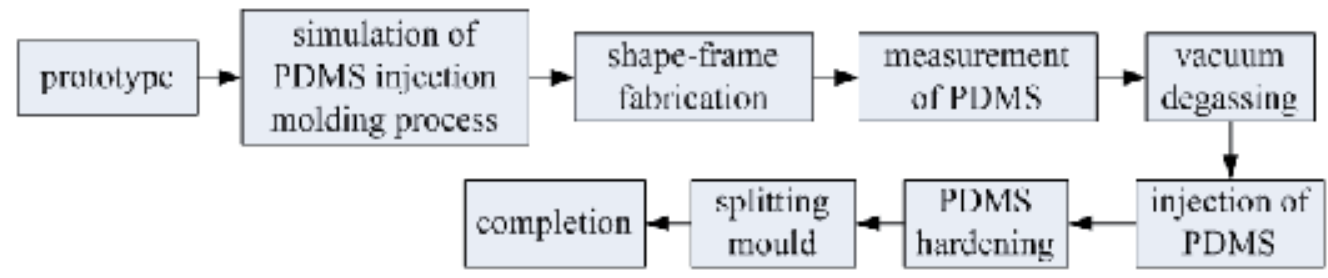

Fig. 35. Manufacturing process flow of PDMS mould 
Before RT fabricating, the software MOLDFLOW was used to determine the best process parameters and the injecting position. Then RT mold (Fig. 36) was produced.
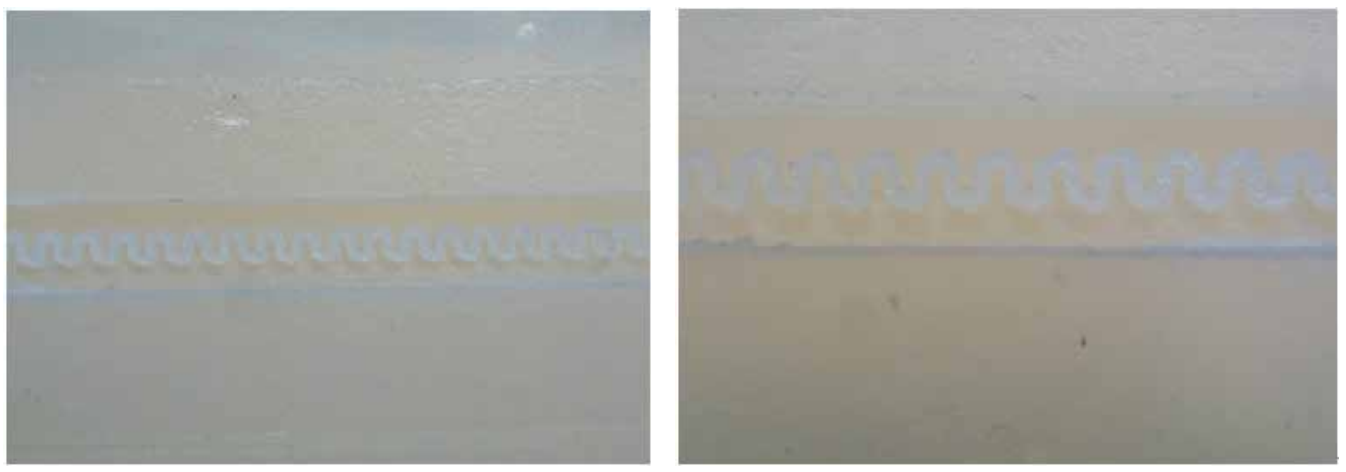

Fig. 36. Silicon rubber mold

Small batch of emitter products were obtained using the silicon rubber mold. One of the products is shown in Fig. 37. Main dimensions of the emitter were measured by a microscope, and the results are shown in Fig. 38. The range of the dimension errors is from $0.23 \%$ to $4.82 \%$, less than $5 \%$. This result can meet design accuracy requirement.
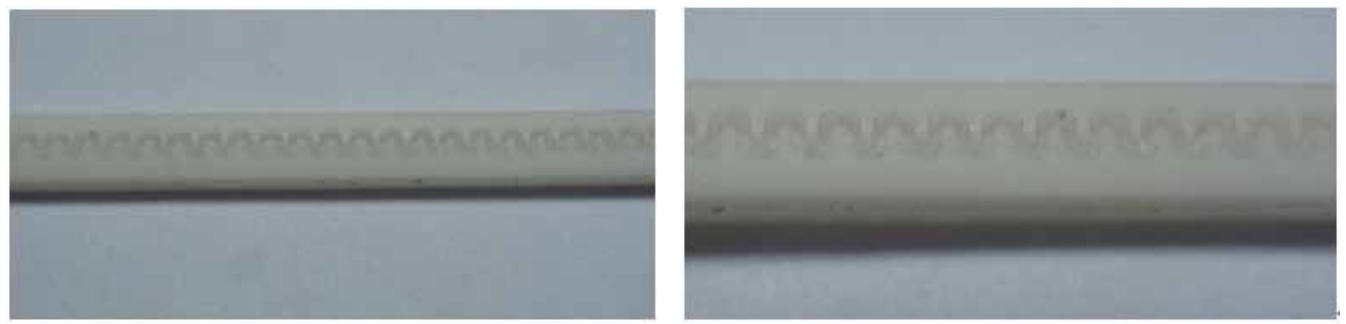

Fig. 37. Oblique teeth channel sheet-dropper products in polyurethane

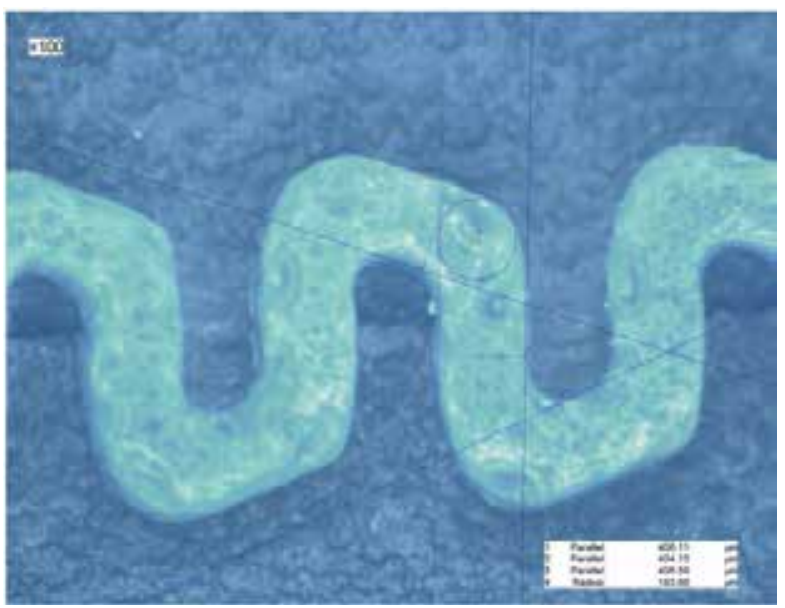

Fig. 38. Dimensions measurement on oblique teeth channel sheet-dropper 
Drops were embedded in polyethylene pipe in the practical application. To avoid additional effect on test, outside of emitter was sealed with polyethylene tape to ensure good sealing. After installation, the result is shown in Fig. 39(a). During the hydraulic performance experiment, ink was injected into the upstream relative to the flow in the channel to test the sealing performance. The result was shown in Fig. 39(b), the sealing performance was perfect as little of the ink was appeared at the outer of the channel. Finally, the pressureflow performance and anti-clogging performance test were conducted on the products.

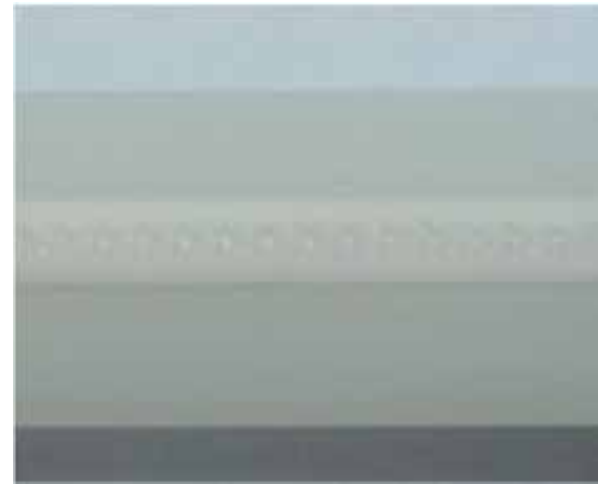

(a) assembled sample

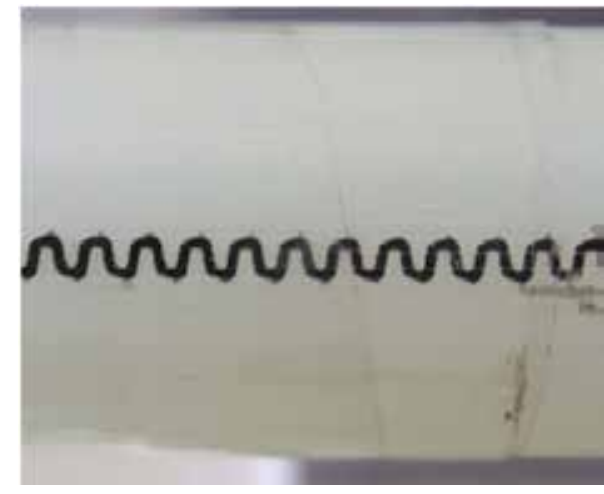

(b) sealing test results

Fig. 39. Oblique teeth channel sheet-dropper's combination and its sealing test

The experimental results and analysis of hydraulic behavior are shown in Fig. 40. Through analyzing the experimental results, the flow rate of oblique-teeth channel sheet-dropper can be obtained: $Q=0.0961 x^{0.5776}$. The flow state index is 0.5776 , which indicates that the oblique-teeth channel is insensitive to pressure changes and has excellent hydraulic behavior.

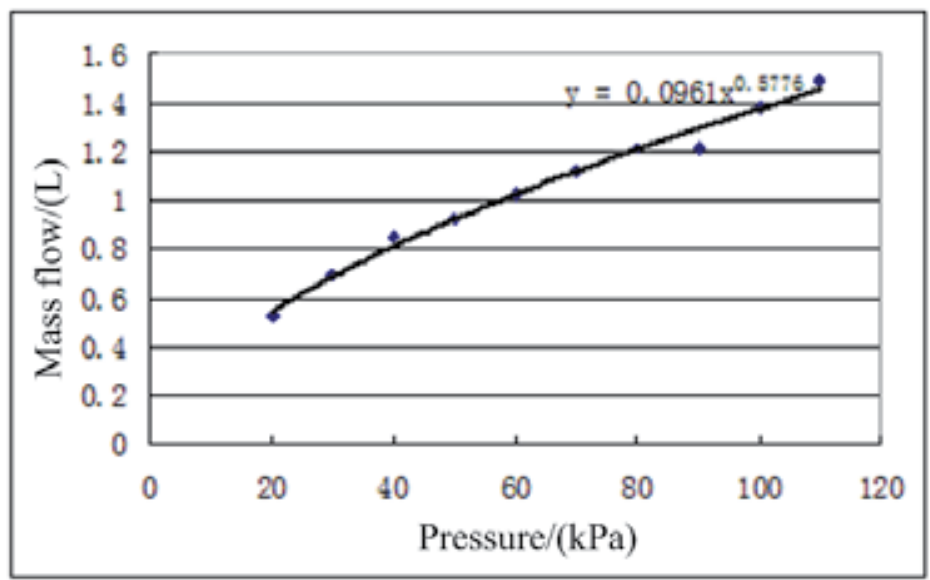

Fig. 40. Hydraulic performance of the oblique-teeth channel sheet-dropper

Then the anti-clogging experiment was conducted. The relationship between emitter's mass flow and time was obtained with inlet pressure being $100 \mathrm{kPa}$. The diameters of tracing particles ranged from F60 to F220. In the experiments, when flow rate was less than, 
compared to the initial average flow, $25 \%$, the emitter was considered completely clogged. The anti-clogging experimental results are shown in Fig. 41. The oblique-teeth channel sheet-dropper was not clogged through the eight stages, which meant the emitter had a good anti-clogging performance.

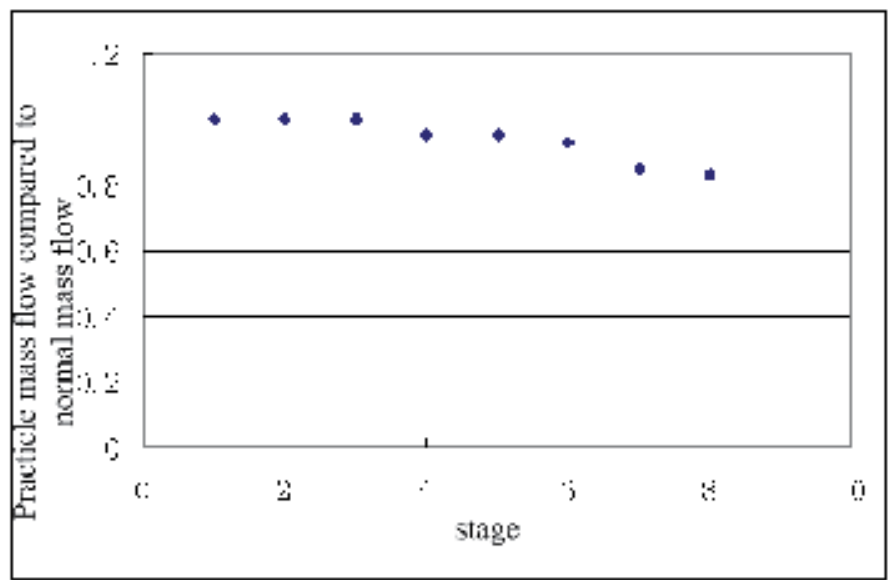

Fig. 41. Anti-clogging performance test on oblique-teeth channel sheet-dropper

With the perfect hydraulic behavior and anti-clogging performance, the test piece could be further applied in development of finalized products.

\subsection{Precision emitter fabrication and product finalization}

When fabricating labyrinth-channel emitters, EDM could be used to form the cavities of channels. But the fabrication of EDM electrode was very difficult. The discharge gaps would affect mold's accuracy. Therefore, the cavity of flow channel should be fabricated independently by $\mathrm{CNC}$ wire cutting as an insert. This method could meet the requirement of "clean-root" and ensure the mold's manufacturing accuracy.
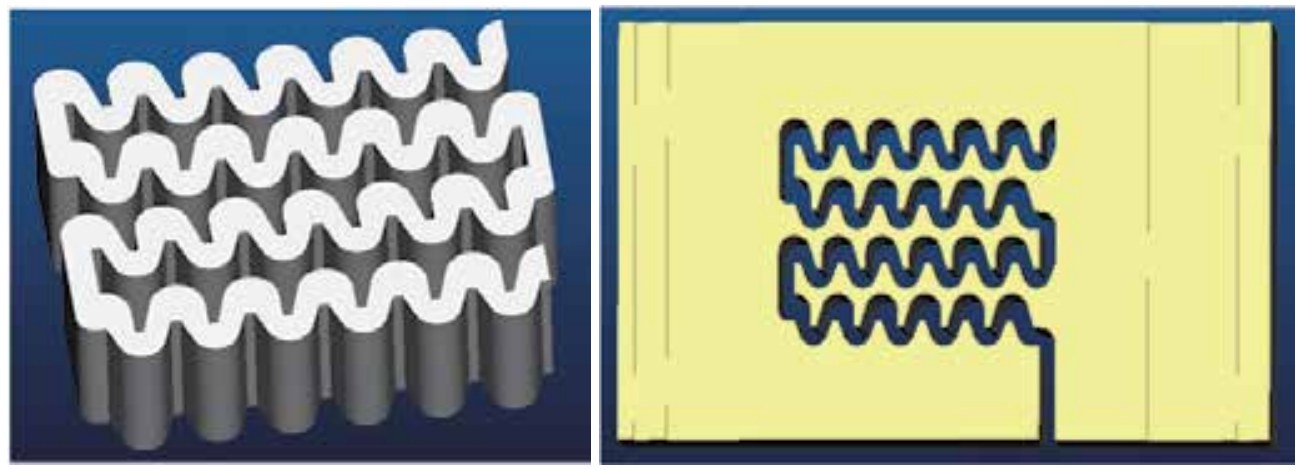

Fig. 42. Diagram of labyrinth grooves and channels inserts pieces

In the calculation of mold's size, the shrinkage of dropper's material should be considered first. The shrinkage of low-density polyethylene was $2.0 \%$. The size of mold cavity was calculated as follows: 


$$
\mathrm{A}_{\text {mold }}=(1+\mathrm{s}) \mathrm{B}_{\text {dropper }} \pm \Delta \text { (tolerance) }
$$

It was difficult to show labyrinth dropper's sophisticated channel structure through twodimensional design drawing, which was involved in the CAD model of this dripper mold cavity. However, dropper mold's opposite CAD model can be used. Pro/E was used to obtain two-dimensional design drawing from dropper mold's opposite CAD model. The design of the mold cavity (ten cavities in one mold) is shown in Fig. 43.

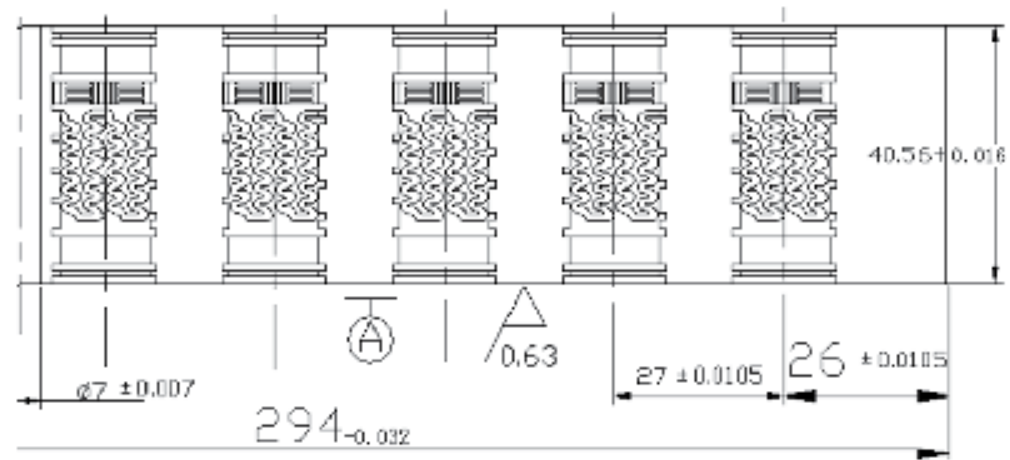

Fig. 43. Mold design of labyrinth channel dropper

In the process of mold design, demoulding convenience should be considered. For the dropper's complex surface and the accuracy requirement, a small core-pulling stroke has to be used to the mold that has a larger molding area, a shallow lateral allowing for the ease of demoulding was adopted in the dropper's demolding process. The outboard cooling scheme was used for the cooling of the mold. Dropper mold assembly is shown in Fig. 44. The structure of the cavity-insert dropper is shown in Fig. 45.

The steel mold and labyrinth dropper products are shown in Fig. 46(a) and (b).

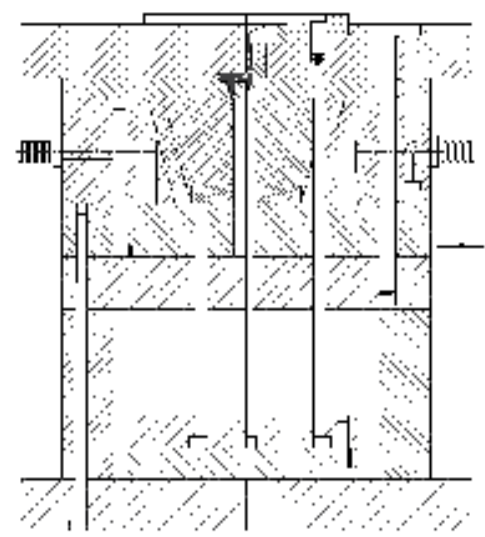

Fig. 44. Dropper mold assembly drawings

Using precision mold manufacturing technology could prevent the existence of dead flow. It also ensured the structure has anti-clogging behavior and high manufacturing accuracy. Insert-embedded dropper with pressure compensating was fabricated based on precision mold manufacturing technology. Mold cavity design was integrated with flow channel and 


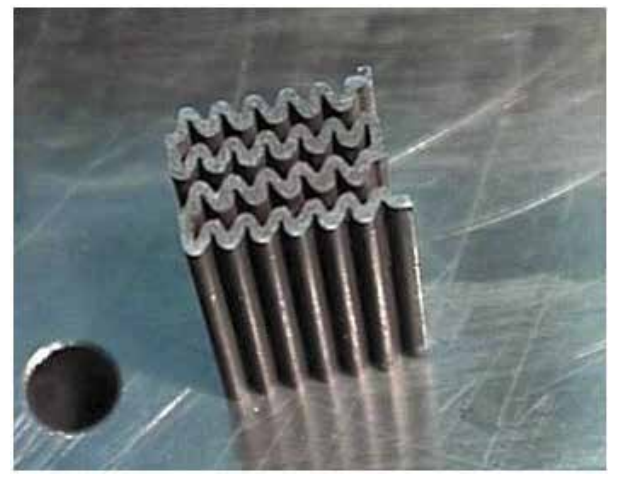

(a) channel body inserts

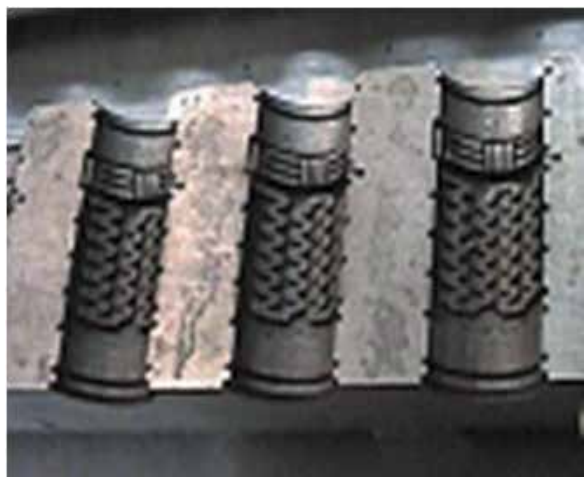

(b) Mold cavity

Fig. 45. Insert cavity mold structure of the dropper
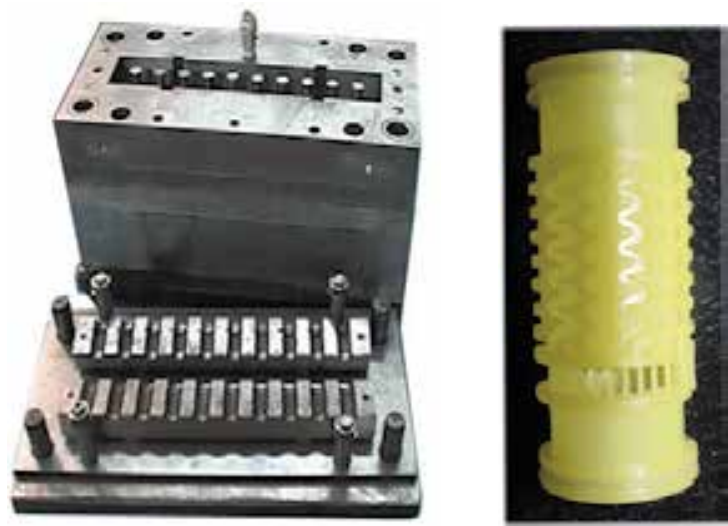

(a) The overall figure of steel mold

(b) labyrinth dropper Products

Fig. 46. Steel injection mold
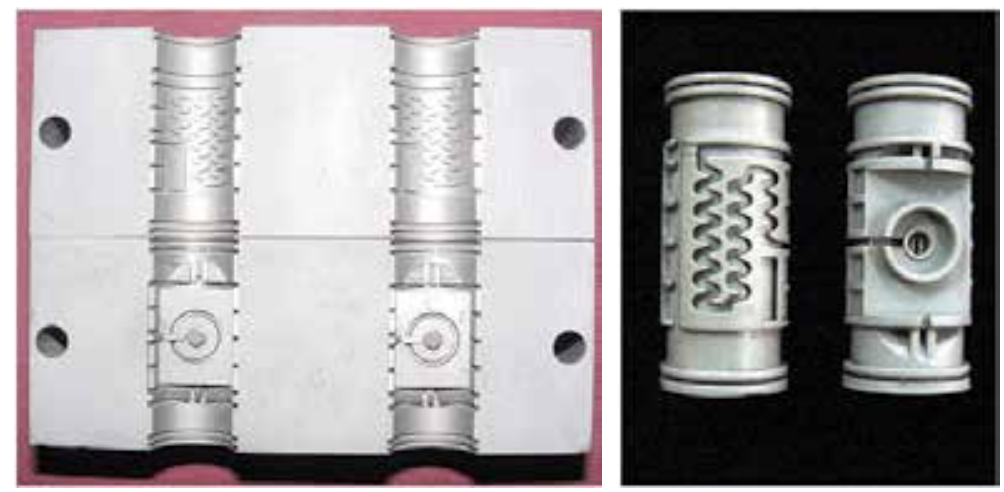

(a) Dropper mold cavity

(b) Pressure compensation dropper

Fig. 47. Pressure compensated labyrinth dropper mold 
inlet grid. The mold cavity is shown in Fig. 47 (a). Pressure regulators were manufactured separately, and then inserted into cavity. On the injection molding machine, pressure compensating dropper can be obtained which is shown in Fig. 47 (b). And two kinds of molds had been developed as shown in Fig. 48.

Through calculations and experiments, the pressure fluctuation of our products is less than $2 \%$; flow rate is $3.956 \mathrm{~L} / \mathrm{h}$; flow fluctuation coefficient is $4.26 \%$, which comply with the standard of the National Quality and Technical Supervision Bureau.

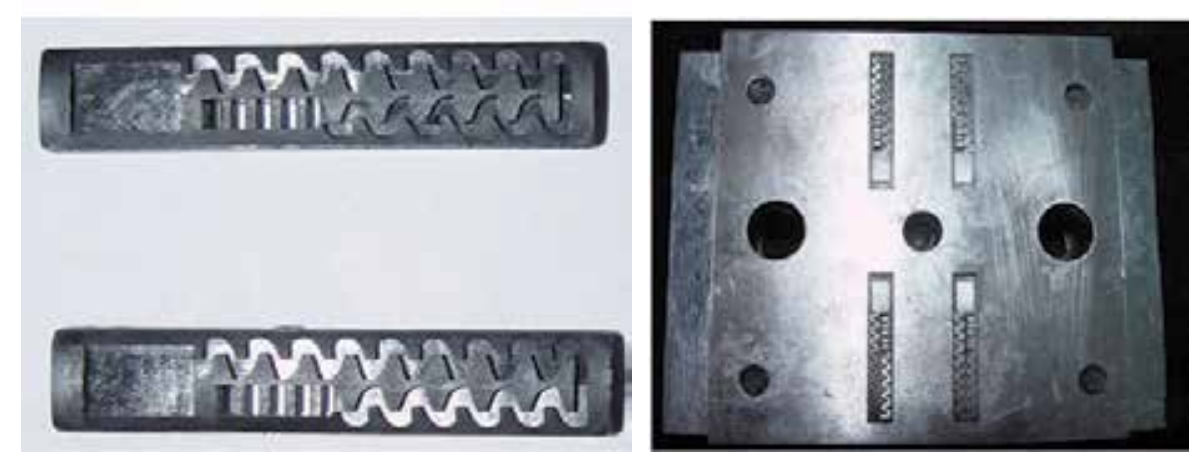

(a) Anti-clogging sheet-dropper $\quad$ (b) diagram of sheet-dropper's mold

Fig. 48. Anti-clogging sheet-dropper's mold

\section{References}

Bralts\& Vincent, F. (1985). Micro Computer Aided Design of Drip/Trickle Irrigation Submain Units, In: Drip/Trickle Irrigation in Action, Proceedings of the Third International Drip/Trickle Irrigation Congress, pp. 452-458, ISBN 091-6150-73-9 Silver Spring, MD, USA. November, 1985

Thompson, T.; Pang, H. \& Li Y. (2009). The Potential Contribution of Subsurface Drip Irrigation to Water-Saving Agriculture in the Western USA. Agricultural Sciences in China Vol. 8, No.7,(July 2009), pp. 850-854, ISSN 1671-2927.

Lamm F. R.\&Camp C. R. (2007). Subsurface drip irrigation, In:Developments in Agricultural Engineering, J. E. A. Freddie R. Lamm, S. N. Francis, (Eds). pp. 473- 551, Elsevier, ISBN 978-0-444-50607-8, Oxford, UK

Onder, S.; Caliskan, M. E.; Onder, D.\&Caliskan, S.(2005). Different irrigation methods and water stress effects on potato yield and yield components. Agricultural Water Management, Vol.73, No.1 (April 2005), pp. 73-86, ISSN 0378-3774

Schwankl L. J. \& Hanson L. R. (2007). Surface drip irrigation, In: Developments in Agricultural Engineering, J. E. A. Freddie R. Lamm, S. N. Francis, (Eds). pp. 431-472, Elsevier, ISBN 978-0-444-50607-8, Oxford, UK

Cetin O. \& Bilgel L.(2002).Effects of different irrigation methods on shedding and yield of cotton. Agricultural Water Management Vol.54, No.1 (March 2002) pp. 1-15, ISSN 0378-3774

Wei Z.; Tang Y.; Zhao W. \& Lu B.(2007). Rapid structural design of drip irrigation emitters based on RP technology. Rapid Prototyping Journal Vol.13, No.5 (2007), pp. 268-275, ISSN 1355-2546 
Li Y.; Li G.; Qiu X. \& Wang J.(2005). Modeling of hydraulic characteristics through labyrinth emitter in drip irrigation using computational fluid dynamics. Nongye Gongcheng Xuebao/Transactions of the Chinese Society of Agricultural Engineering Vol.21, No.3, (March 2005), pp. 12-15, ISSN 1002-6819

Li Y. et al.(2008). CFD and digital particle tracking to assess flow characteristics in the labyrinth flow path of a drip irrigation emitter. Irrigation Science Vol.26, No.5 (March 2008), pp. 427-438, ISSN 0342-7188

Wei Z.; Tang Y. \& Lu B.(2003). A Rapid Manufacturing Method for Water-Saving Emitters for Crop Irrigation Based on Rapid Prototyping and Manufacturing. The International Journal of Advanced Manufacturing Technology Vol.21, No.9 (June 2003), pp. 644-648, ISSN 0268-3768

Wei Z.; Tan Y. g; Zhao W. \& Lu B.(2005). Structure and hydraulic performance experimental research on labyrinth channels of drip irrigation emitters. Nongye Jixie Xuebao/Transactions of the Chinese Society of Agricultural Machinery Vol.36, No.12(December 2005), pp. 51-55, ISSN 1000-1298

Wei Z.; Tang Y.; Wen J. \& Lu B.(2008). Two-phase flow analysis and experimental investigation of micro-PIV and anti-clogging for micro-channels of emitter. Nongye Gongcheng Xuebao/Transactions of the Chinese Society of Agricultural Engineering Vol.24, No6. (June 2008), pp. 1 -9, ISSN 1000-1298

White D. (2001). Rapid tooling technology, In: Encyclopedia of Materials: Science and Technology, K. H. J. Buschow et al. (Eds.), pp. 8003-8009, Elsevier, ISBN 978-0-08043152-9, Oxford, UK

Hosni Y. \& Sundaram R.(1996). Rapid Prototyping and Tooling: A survey and Applications in Current Advances in Mechanical Design and Production VI, E. E. Mohamed, S. W. Professor Abdalla, A. S. Wifi, Prof (Eds). pp. 511-520, Pergamon, Elsevier Science Inc.ISBN 0-08-042140-7, NY, US

Yan X. \& Gu P.(1996). A review of rapid prototyping technologies and systems. ComputerAided Design Vol.28, No.4 (April 1996), pp. 307-318, ISSN 0010-4485

Pham D. T. \& Gault R. S.(1998). A comparison of rapid prototyping technologies. International Journal of Machine Tools and Manufacture Vol.38, No.10-11, (October 1998), pp.1257-1287, ISSN 0890-6955.

Choi S. H.\& S. Samavedam (2002) .Modelling and optimisation of Rapid Prototyping. Computers in Industry, Vol.47, No.1,(January 2002), pp. 39-45, ISSN 0166-3615.

Wei Z.; Tang Y.; Cheng Y. \& Lu B.(2008). New method for rapid design of the integral pressure-compensating emitters. Zhongguo Jixie Gongcheng/China Mechanical Engineering Vol.19, No.12, (June 2008), pp. 1387-1392, ISSN 1004-132X

Zhang J.; Zhao W.; Tang Y. \& Lu B.(2010). Anti-clogging performance evaluation and parameterized design of emitters with labyrinth channels. Computers and Electronics in Agriculture, Vol.74, No1, (October 2010), pp.59-65, ISSN 0168-1699

Liu H.-s. et al.(2010) Flow characteristics in energy dissipation units of labyrinth path in the drip irrigation emitters with DPIV technology. Journal of Hydrodynamics, Ser. B Vol.22, No.1 (February 2010), pp. 137-145, ISSN 1001-6058

Li G. Y.; Wang J. D.; Alam M. \& Zhao Y. F.(2006) Influence of geometrical parameters of labyrinth flow path of drip emitters on hydraulic and anti-clogging performance. Transactions of the ASABE Vol.49, No.3, (April 2006), pp. 637-643, ISSN 0001-2351.

Zhang J.; Zhao W.; Wei Z.; Tang Y. \& Lu B.(2007). Numerical and experimental study on hydraulic performance of emitters with arc labyrinth channels. Computers and Electronics in Agriculture, Vol.56, No.2 (April 2007), pp.120-129, ISSN 0168-1699 
Meng G.; Zhang M.; Zhao W. \& Lu Y. (2004). Numerical flow simulation and optimum channel design of drip irrigation emitter. Hsi-An Chiao Tung Ta Hsueh/Journal of Xi'an Jiaotong University Vol.38, No.9 (September 2004), pp. 920-924, ISSN 0253987X.

Wei Z.; Wen J.; Tang Y. \& Lu B.(2008). Structural design and rapid development platform of emitters under lower water pressure. Nongye Jixie Xuebao/Transactions of the Chinese Society of Agricultural Machinery, Vol.39, No.10 (October 2008), pp. 59-64, ISSN 10001298

Wei Q.; Shi Y.; Dong W.; Lu G.\& Huang S.(2006). Study on hydraulic performance of drip emitters by computational fluid dynamics. Agricultural Water Management Vol.84, No.1-2, (July 2006), pp. 130-136, ISSN 0378-3774

Nishimura T.; Ohori Y. \& Kawamura Y.(1984). Flow Characteristic In A Channel With Symmetric Wavy Wall For Steady Flow. Journal of Chemical Engineering of Japan Vol.17, No. 5 (March 1984), pp.466-471, ISSN 0021-9592.

Nishimura T.; Murakami S.; Arakawa S.\& Kawamura Y. (1990). Flow observations and mass transfer characteristics in symmetrical wavy-walled channels at moderate Reynolds numbers for steady flow. International Journal of Heat and Mass Transfer Vol.33, No.5(May 2009), pp. 835-845, ISSN 0017-9310.

Kitoh O.; Nakabyashi K. \& Nishimura F.(2005). Experimental study on mean velocity and turbulence characteristics of plane Couette flow: low-Reynolds-number effects and large longitudinal vortical structure. Journal of Fluid Mechanics Vol.539, No.00 (September, 2005), pp. 199-227, ISSN 0022-1120

Gerolymos G. A.; Neubauer J.; Sharma V. C. \& Vallet I.(2002). Improved prediction of turbomachinery flows using near-wall reynolds-stress model. Journal of Turbomachinery Vol.124, No.1(January 2002), pp. 86-99, ISSN 0889-504X

Humphrey J. A. C.; Whitelaw J. H. \& Yee G.(1981). Turbulent flow in a square duct with strong curvature. Journal of Fluid Mechanics Vol.103, No.00 (February 1981), pp. 443463, ISSN 0022-1120

Arnal M. P.; Goering D. J. \& Humphrey J. A. C.(1992). Unsteady laminar flow developing in a curved duct. International Journal of Heat and Fluid Flow Vol.13, No.4, (December 1992), pp. 347-357, ISSN 0142-727X.

Winoto S. H. \& Crane R. I. (1980) Vortex structure in laminar boundary layers on a concave wall. International Journal of Heat and Fluid Flow Vol.2, No.4, (December 1980), pp. 221-231, ISSN 0142-727X.

Rush T. A.; Newell T. A. \& Jacobi A. M.(1999). An experimental study of flow and heat transfer in sinusoidal wavy passages. International Journal of Heat and Mass Transfer Vol.42, No.9 (May, 1999), pp. 1541-1553, ISSN 0017-9310.

Zhang J.; Wei G.; Zhao W.; Wei Z. \& Lu B.(2007). Numerical analysis on liquid-solid twophase flows in arc-type channel of the emitter. Zhongguo Jixie Gongcheng/China Mechanical Engineering Vol.18, No.5 (March 2007), pp. 589-593, ISSN 1004-132X

Padmakumari O. \& Sivanappan R. K.(1985). Sudy of Clogging of Emitters In Drip Systems. In: Drip/Trickle Irrigation in Action, Proceedings of the Third International Drip/Trickle Irrigation Congress, pp. 80-86, ISBN 091-6150-73-9 Silver Spring, MD, USA. November, 1985.

Gilbert R. G.; Nakayama F. S. \& Bucks D. A.(1977). Trickle Irrigation: Prevention Of Clogging. Transactions of the ASAE Vol.22, No.3, (June 1979), pp. 514-519, ISSN $0001-2351$ 


\title{
The Use of the Rapid Prototyping Method for the Manufacture and Examination of Gear Wheels
}

\author{
Grzegorz Budzik \\ Rzeszow University of Technology, Faculty of Mechanical Engineering and Aeronautic
} Poland

\section{Introduction}

Rapid prototyping systems make it possible to manufacture prototypes of complex shapes, including gear wheels prototypes. RP systems are increasingly employed in gear wheels testing. The use of RP systems is particularly vital to the process of manufacturing gear wheels of non-standard (non-involute) tooth profile. For this kind of gear wheels, it is not possible to use traditional tools for tooth cutting; therefore the prototypes of such gear wheels are made by the RP methods directly on the basis of the 3D-CAD model.

The chapter is composed of four parts. Part 1 provides an analysis of RP methods in terms of application for the manufacture of gear wheels. Part 2 describes the method for preparing geometric data for the RP process. Part 3 discusses the determination of the real geometric accuracy of gear wheels prototypes made by means of selected RP methods. Part 4 shows the possibility of using RP systems for gear wheels testing, including tooth contact analysis (TCA) and fatigue tests of non-involute gear wheels.

\section{An analysis of RP methods in terms of application for the manufacture of gear wheels}

The subject matter of his chapter centers around RP techniques utilizing processes of shaping the model through the increment of material, consisting in its gradual addition until the moment of obtaining the required shape. The manufacture of the model involves the conversion of the object computer imaging into its physical form, with a minimal manual intervention and in the shortest time possible (fig. 1). The first step in the rapid prototyping process is to define the object as a computer-generated CAD-3D model. The achieved image is then converted into a specific form of data set by means of various formats of data conversion, suitable for RP systems (Budzik, 2009).

Almost all available rapid prototyping methods are based on the same rule of dividing the model into horizontal layers, from which the physical prototype of the object is built in the proper order. In this way, the incremental shaping of objects becomes very effective in the case of piece or small-batch production.

On account of high dynamics of changes in the field of RP systems, the percentage of individual RP methods in the market is also changing rapidly. RP processes can be classified according to a wide range of division criteria, including: 
- the kind of input data,

- the physical or chemical principle of operation of the RP method,

- the initial state of the processed materials,

- the preferred RP applications.
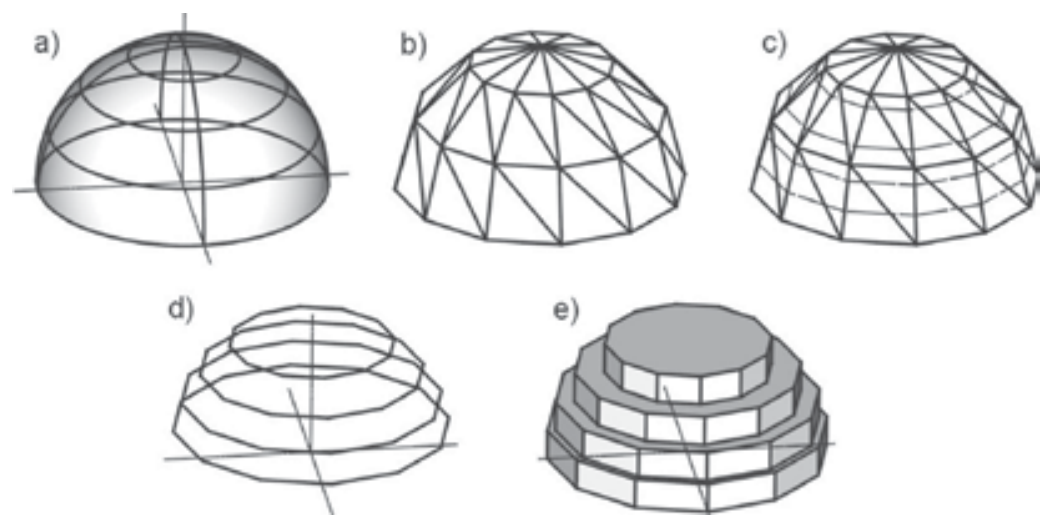

Fig. 1. RP process: a) 3D-CAD model, b) 3D-STL model, c) STL model divided into layers, d) layer model, e) physical prototype

$\mathrm{RP}$ processes and methods are most often classified according to the initial state of the processed materials. There is no classification that would encompass all known RP methods, as the applied techniques and materials used in them are constantly evolving.

Analyzing individual incremental RP technologies, it is possible to define the criteria of method selection, divided as follows:

- the accuracy of the prototype fabrication,

- the prototype material properties,

- the time of the prototype fabrication,

- the cost of the prototype fabrication.

The isolation of the individual criteria is a complex task, as for certain methods, individual criteria may be mutually exclusive. Also, within groups of starting materials and the assigned RP methods based on a definite physical or chemical principle, it is possible to specify a variety of rapid prototyping processes (Budzik, 2009).

\subsection{The JS method}

The method of layer jetting of liquid photopolymer (JS - Jetting Systems) consists in the application of a layer of polymer from the print head and subsequent hardening of the polymer layer by means of UV light emitted by a lamp integrated with the print head. In this system there are two materials applied on the working platform during the construction of the model: the model material and the material supporting the model. Models are built on a working platform moving along the vertical axis $(z)$ of the working space. Polymer layers are applied one by one by the print head on the parallel plane of the working platform $(x, y)$. The Jetting System methods of the ProJet (3D Systems) and PolyJet (OBJET) systems enable the application of resin materials for the manufacture of prototypes of various mechanical properties. Figure 2 presents gear elements produced by ProJet methods (fig. $2 \mathrm{a}$ - an adjustable spanner worm gear) and PolyJet methods (fig. $2 \mathrm{~b}-\mathrm{a}$ gear wheel of a cycloidal profile). 


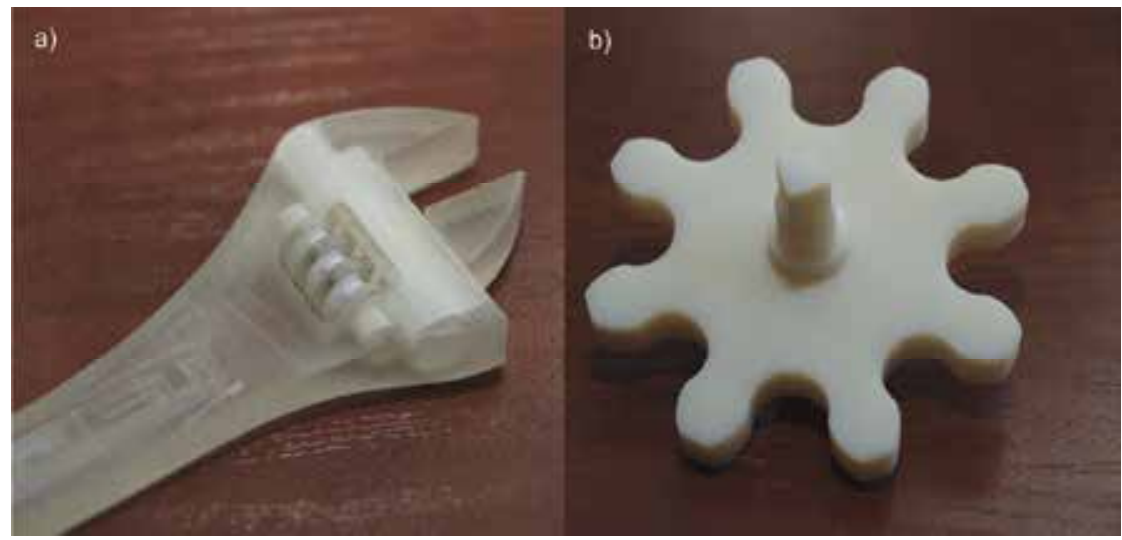

Fig. 2. Gear elements produced by JS method: a) gear manufactured by ProJet technique, b) gear wheel of cycloidal profile manufactured by PolyJet method

\subsection{The SLS method}

The Selective Laser Sintering (SLS) method consists in hardening the surface of powdered material by means of a laser beam. The physical model is constructed on the basis of 3D-RP geometry saved in e.g. the STL format.

The SLS method involves the use of a powdered material, which is spread in layers by means of a shaft on the working platform. The laser beam causes the sintering of the layer in accordance with the defined boundaries and filling of the solid cross-section. After the hardening, the working element is lowered by the value of the layer thickness and powder is spread again. During the sintering of the next layer, the previous one gets partially melted, thanks to which a uniform model solid is formed. Once the complete model is produced, the powder which has not been sintered is removed.

Being built, the model is supported by the powder, which fills the working space, hence no separate propping structures are necessary. The received model has a porous structure. Depending on the function of the model, it can be impregnated with other materials, which leads to the improvement of its mechanical properties. Figure 3 shows models of gear wheels made by the SLS method: a gear wheel made from polyamide - EOS Formiga P100 device (fig. 3a) and gear wheels made from TiAl6V4 powder - EOSINT device (fig. 3b, c).

\subsection{The SLA method}

Stereolithography (SLA) is one of the oldest rapid prototyping methods. The commencement of building the model and its mounting on the platform require the construction of supporting structures. The supports can be automatically or manually generated and it is possible to correct their shape. The method necessitates the propping of both the whole first layer of the model, the out-of-plumb elements and the fragments of the model, the building of which starts at a certain height. Once one cross-section has been hardened, the working platform is lowered, together with the manufactured part of the model, which is placed inside a container filled with liquid resin, having the thickness of the layer. Figure 4 shows models of gear wheels made by the SLA method.

The finishing of the model - the so-called Post Processing - includes the removal of the supports, the washing of the model with the use of acetone or isopropanol (cleaning the 
liquid resin remnants off the surface) and additional UV irradiation for the purpose of completing the polymerization in the whole volume of the model.

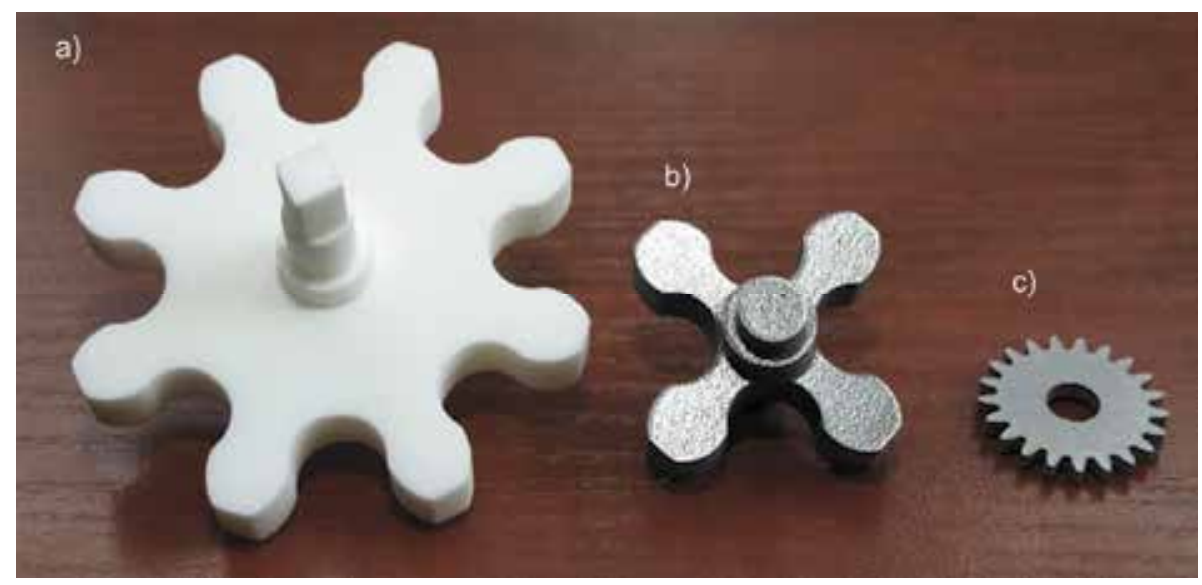

Fig. 3. Gear wheels made by SLS method: a) gear wheel made from PA2200 polyamide, b), c) gear wheels made from TiAl6V4 titanium

Considering the fact that different powders can be utilized in the SLS technique (e.g. powders of plastics and mixtures of metal and ceramic powders), this method is suitable for the manufacture of metal and ceramic tools.

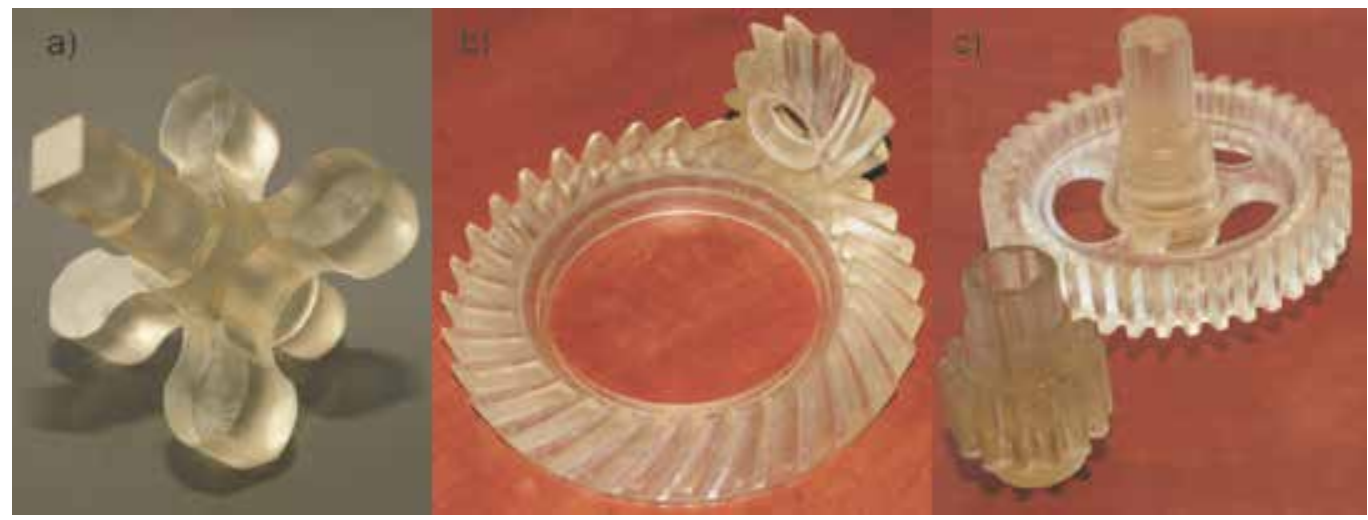

Fig. 4. Gear wheels made by SLA method: a) gear wheel of cycloidal profile, b) bevel gear wheels, c) cylindrical gear wheels

\subsection{The FDM method}

The FDM method consists in the building of the model in the form of layers with the use of a material heated by the head and fluidized, which is pumped through a nozzle also equipped with a mechanism controlling and cutting off the material supply. The nozzle is mounted in a holder having the possibility of moving horizontally and vertically, which enables the placement of a material layer in the working space in accordance with the defined geometry of the cross-section. The applied layer solidifies as soon as it flows out of the nozzle binding to the layer applied before. During the construction of the model, 
another nozzle pumps out a material supporting the proper model. This material also serves the purpose of joining the proper model with the working platform. The supporting structures generated together with the model are removed once the complete object has been built. It is possible to construct supports using a water-soluble material, which makes it easier to remove them.

Several kinds of materials can be applied in the FDM method, such as waxes, alkylbenzenesulfonates and polycarbonates as well as bicompatible materials. Figure 5 presents models of gear wheels produced by the FDM method.

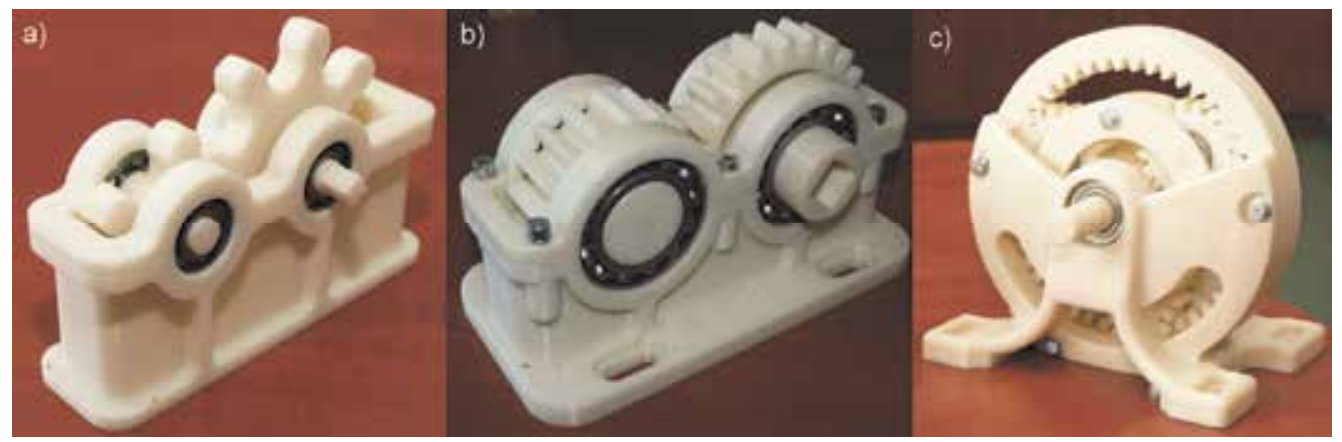

Fig. 5. Gears made by FDM method: a) single-stage gear with wheels of cycloidal profile, b) single-stage gear with wheels of involute profile, c) planetary gear

\subsection{The 3DP method}

The method of three-dimensional printing (3DP) consists in combining a powdered material in layers by means of a binding agent applied by the print head. The printing process begins with the application of a powder layer from the supplementary container. A measured amount of the powder is dosed by a system moving the piston in the cylinder of the device and then spread (distributed) with the use of the roller on the surface of the working platform. The binding agent is applied onto the prepared powder layer in accordance with the defined cross-section of the solid. The agent binds the powdered material, thus creating a layer of the model, after which the working platform is lowered by the value of the layer thickness (e.g. $0.1 \mathrm{~mm}$ ) and the cycle is repeated.

This method makes it possible to print out geometric models (fig. 6a) as well as moulds (fig. 6b) for casting alloys of pouring temperature up to $1100^{\circ} \mathrm{C}$ (fig. 6c).

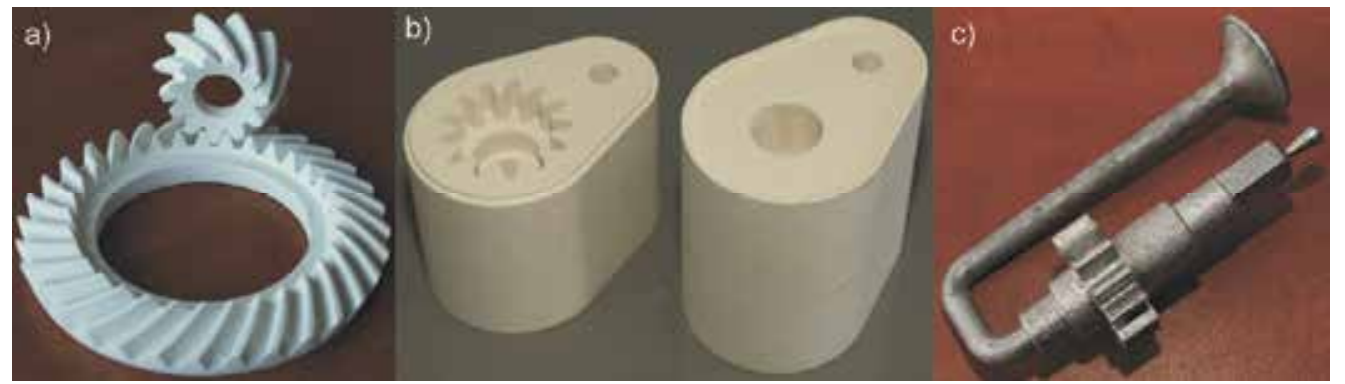

Fig. 6. Models produced by 3DP method: a) gear wheels, b) gear wheel casting mould, c) cast made in the mould from $\mathrm{Al}$ alloy 
Some devices also enable three-dimensional printing in a 24-bit pallet of colors (e.g. Z 510 made by $Z$ Corporation), which makes it possible to map paint covering or reconstruct the appearance of objects made from several different materials. In the working space of devices designed for the 3DP method, it is possible to produce a few models at the same time or generate a model consisting of a few different-colored elements (Budzik et al. 2010).

\section{A description of the method for preparing geometric data for the RP process}

Physical prototypes made by incremental or decremental RP techniques are based on the 3D-CAD model. Depending on the RP method, proper computer data processing is required. The generation of the prototype is possible on the basis of a geometric (volumetric) CAD model. Computer processing begins in the CAD environment, from which the model is exported into the proper format of the RP system.

It is also possible to fabricate the prototype on the basis of data directly from the RE process. It should be remembered, however, that data of this type is less accurate than data processed in 3D-CAD programs. Programs dedicated to RP devices verify the correctness of models. Further file processing comes down to defining the right parameters of the device operation (the layer thickness for the incremental systems or the path for the $\mathrm{CNC}$ decremental systems). It is the so-called program post-processing, which consists in the preparation of the final data, essential to the construction of the physical model (files in the RP device format).

The process of data preparation has a considerable influence on the accuracy of the model generated in the rapid prototyping process. The parameters of data exported from CAD systems should always be higher than the accuracy of the RP device. Thanks to this, in the process of the model construction, the RP device does not repeat program errors.

The process of data preparation has a significant influence on the accuracy of the model received in the rapid prototyping process. Computer-aided design systems have a certain accuracy of mapping the 3D-CAD model geometry (e.g. the linear accuracy of the model made in the CATIA system is $0.001 \mathrm{~mm}$ ). At the current stage of technological development, this accuracy is higher than the accuracy of mapping the geometry of the prototype produced with the use of rapid prototyping systems. The parameters of geometric data exported from CAD systems should be adjusted to the assumed accuracy of the physical prototype fabrication. In general, the accuracy of the 3D-CAD model should be higher than the accuracy of the mapping of the RP device geometry. Thanks to this, in the process of the physical model construction, the RP device does not repeat program errors (Budzik et al. 2010).

\subsection{Data formats for the CAD/RP process}

Rapid prototyping systems require that the files of 3D-CAD digital models be saved in the proper formats. The most popular 3D-RP formats include: STL format (Stereolithography Language), SLC format (Sliced Layer Contour), CLI format (Common Layer Interface), HPGL format (Hewlett Packard Graphic Language) and The MGX format- a format of the Magic RP program.

In the process of data processing by means of 3D-CAD/3D-RP there are also universal 3DCAD formats applied, such as STEP, IGES or 3DS. By way of example, ZPrint, a program for operating three-dimensional printers, makes it possible to read in the CAD model with 
colored texture saved in the 3DS format. The universal Magisc RP program allows for the reading in of files in most formats applied in CAD systems, depending on the installed translators. However, in practice, the most commonly used formats are STEP and IGES.

Comparing the possible applications of the STEP and IGES formats in the process of 3D$\mathrm{CAD} / 3 \mathrm{D}-\mathrm{RP}$ data processing, it should be noted that, whereas in most cases the use of the STEP format enables accurate mapping of the CAD model shape, mapping errors often occur in the IGES format, such as defective or lost surfaces of the model. In the case of gear wheels, such faults may emerge on the surface (fig. 7).

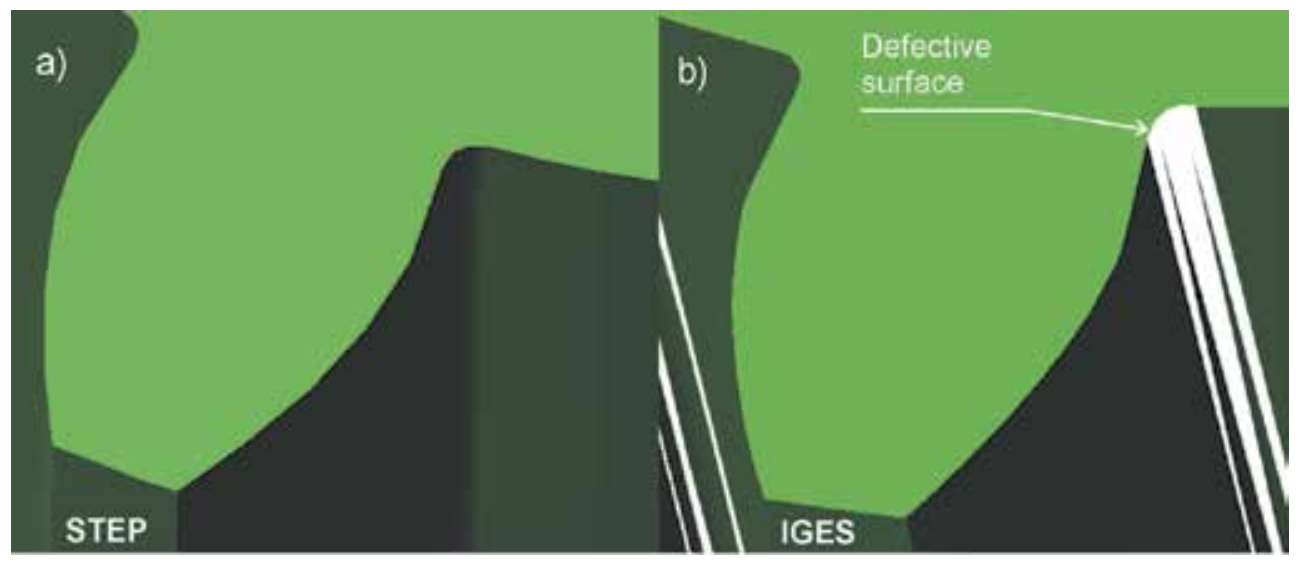

Fig. 7. 3D-CAD gear wheel models saved in: a) STEP format and b) IGES format with visible surface defects

Faults of this kind are most often transferred in further data processing in the RP process, which, for example in the STL model, takes the form of lost surfaces (Budzik et al. 2008).

In such case, it is not possible to build the physical prototype by an incremental method. However, an attempt can be made to correct the STL model by using proper program tools (e.g Magics RP).

Formats for saving files are constantly being developed by manufacturers of rapid prototyping devices. This development requires a cooperation with the producers of CAD software. Because of the application of the STL format, a vast majority of CAD programs have the possibility of exporting data in this format. These programs include: AutoCAD, Alibre, CATIA, I-DEAS, IronCAD, Mechanical Desktop, ProE, Rhino, SolidDesigner, SolidEdge, SolidWorks, Think3, NX and 3D Studio Max.

Data export is usually realized through the "save as *.stl" command, sometimes the command of export to STL. On rare occasions, there is a separate command entered in the program dialog window (e.g. "amstlout" in Mechanical Desktop). Depending on the program, it is possible to determine the accuracy of the model mapping. Some programs allow for a very detailed definition of the parameters of the STL file (e.g. Mechanical Desktop). The STL format is used to describe and approximate the shapes of various surfaces by means of flat triangular surface flakes. Figure 8 shows a single flat triangular unitary surface.

The description of the single surface includes a normal vector (facet normal) and coordinates of three vertexes. In addition, for each surface, the normal vector (facet normal) $N$ is defined, directed outside the approximated solid. 

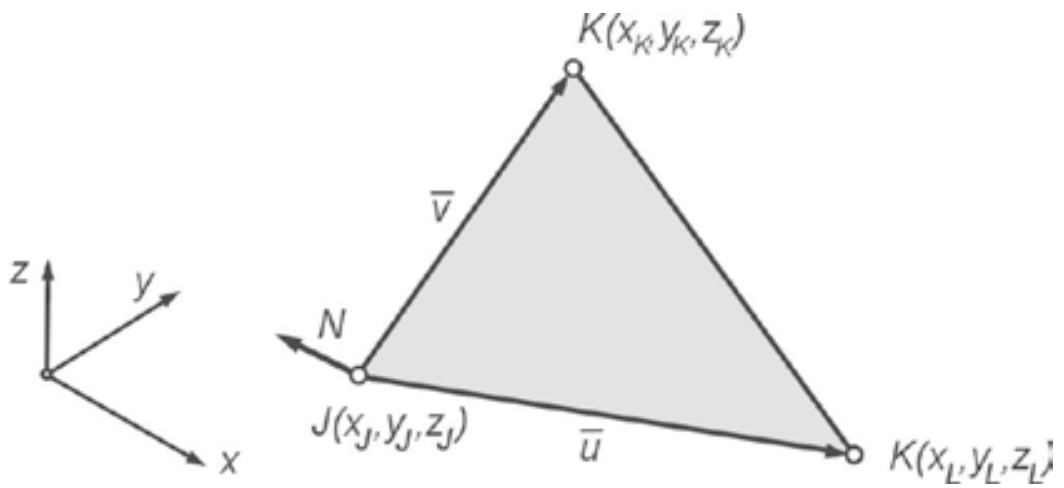

Fig. 8. Single flat triangular unitary surface of STL format

\subsection{The accuracy of the model mapping in the STL format}

The process of converting the 3D-CAD model into the 3D-CAD-RP form (STL format) is called tessellation. It is a process in which three-dimensional model geometry is approximated by means of triangular surfaces (Budzik et al. 2007). The size and shapes of the triangles can be regulated by setting the proper parameters of data export. The accuracy of the approximation depends on the model surface shape Flat surfaces are fully mapped by triangles (Campbell, 2008). The mapping of curvilinear surfaces causes the emergence of the so-called chord error, which occurs between the model surface and the triangle net which approximates it . The error is measured perpendicularly to the triangle surface, and its value depends on the assumed approximation parameters (fig. 9a).

The error of the $f$ chord is also responsible for the accuracy of the construction of convex and concave surfaces having different radii of curvature. The accuracy of approximation can also be described by means of the approximation angle - $\alpha$ (fig. 9b).
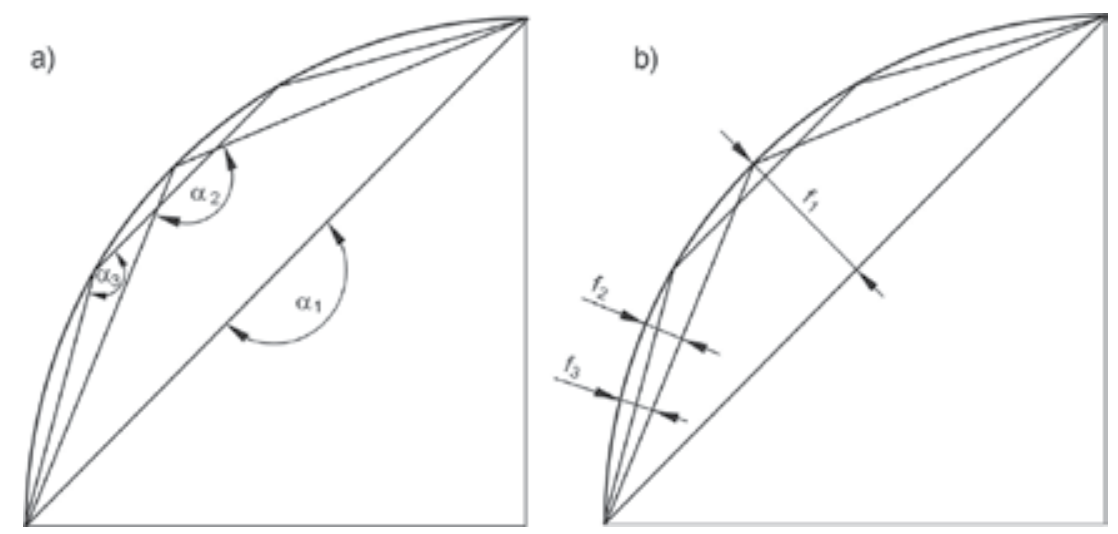

Fig. 9. Mapping error between CAD model spherical surface and approximating triangle: a) angle deviation, b) chord deviation

The parameters of geometric data export from 3D-CAD systems should be dictated by the assumed accuracy of mapping the physical model built by the RP device. Increasing the surface approximation accuracy in the process of tessellation causes an increase in the number of triangles describing the model surfaces (fig. 10) and a greater size of the STL file. 
a)

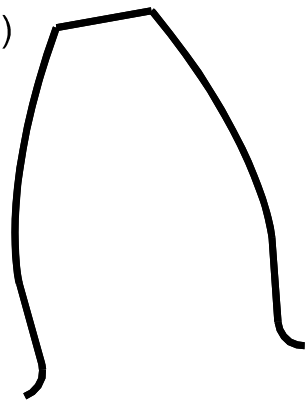

b)

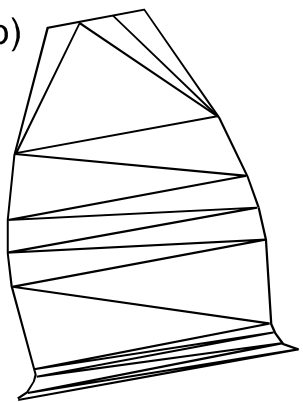

c)

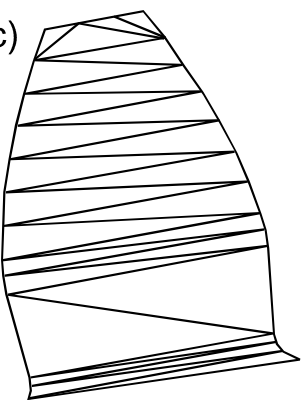

Fig. 10. Gear wheel model tooth profile: a) nominal profile (theoretical - CAD), b) STL model profile (2540 triangles of the whole gear), c) STL model profile (2724 triangles)

In the case of simple geometric models, the size of the generated STL file is not large. It is much greater for models of complex curvilinear surfaces, such as e.g. gear wheels.

The production of accurate RP gear wheels models necessitates the application of the proper tolerance of data export to the STL format. The tessellation parameters are defined in 3DCAD systems by means of various algorithms, which determine mapping accuracy and are responsible for the occurrence of geometry mapping errors. The STL format is also a format of saving data received in the 3D scanning process. In this case, the geometry of the model saved in the STL format virtually always contains errors in the form of noises, missing surface fragments or surface discontinuity.

\subsection{Mapping errors in the STL format}

During the conversion of 3D-CAD files into the STL format, there may occur geometry description errors. These errors result from the inaccuracy of the model processing by tessellation algorithms in the translators of CAD systems. The most common STL model errors include: the reversal of normal vectors, gaps and interspaces between the triangles describing the surface, warped surface, losses of whole surfaces or their fragments, overlapping triangles, the assignment of a single edge to more than one triangle.

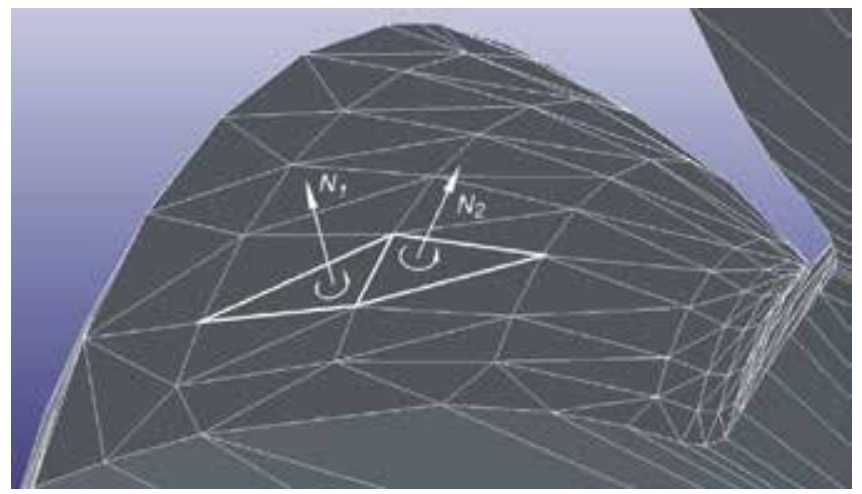

Fig. 11. Correct triangles with normal vectors oriented outwards (STL pinion model)

The correct geometry description with the use of the STL format must meet the condition of all normal vectors of unitary surfaces being directed outside the approximated solid. In some CAD systems, errors occur during the export to the STL file, involving the reversal of 
the normal vector. The most common source of the error is the wrong order of providing the triangle vertexes. The sequence depends on the sense (turn) of the normal vector, defined by the right-handed screw method (fig. 11). Such an error may apply to whole surfaces or individual triangles.

Another error is the 'loss' of a triangle or triangles. Blanks (triangles) can occur individually or as combinations of a few triangles. Empty triangles can also appear when the radii of curvatures are changed, e.g. in the area (fig. 12).

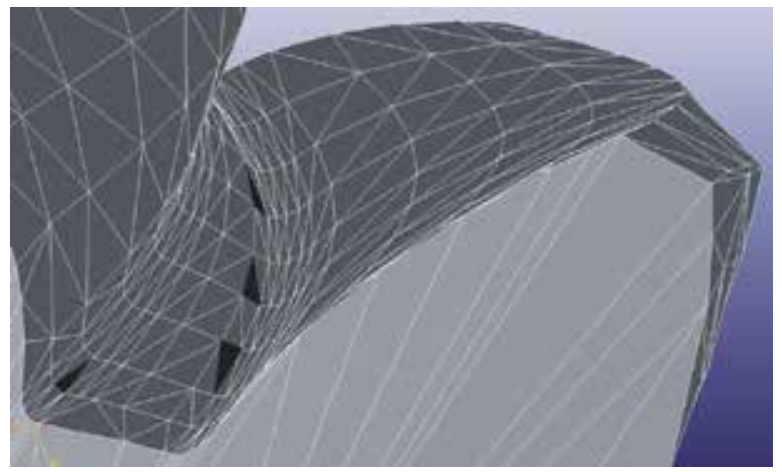

Fig. 12. Empty triangles of gear wheel STL model (marked in black)

Having surface discontinuity, such a model is impossible to build by means of RP devices. This kind of error is most often generated by low-quality modelers of CAD systems. The correction of such an error, i.e. 'patching' the gap created by the 'lost' (not generated) flake of surface geometry requires the application of geometric program operations. In such case, the STL file should be opened in a program supporting STL formats (e.g. Magisc RP), the coordinates of the vertexes of the surface flake should be read, the normal vector $N$ to the surface should be determined and such a surface should be saved in the STL file.

In the case of models of complex shapes, geometric errors can occur, which are not allowed for by the algorithms used for tessellation. These phenomena are the source of errors of STL models. These errors usually emerge in the places of joining surface flakes into one object fig. 13). Such an error may result in the lack of a common edge of the surface flakes being joined.

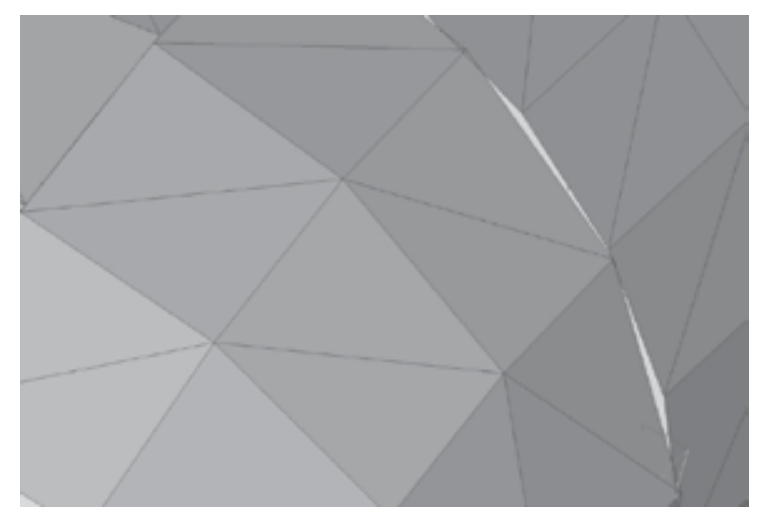

Fig. 13. Warps at the joint of surfaces in STL model 
It is possible to correct some of the errors in CAD programs, using program options. If the surfaces of the model are built with the use of parametric curves and surfaces, the correction of the error comes down to the modification of the values of curves and surfaces parameters.

In the case of free surfaces modeling, the correction is possible through the modification of the control points of curves or surfaces.

There are also mapping errors in the form of disappearance of whole surface flakes (fig. 14). Errors of this type usually occur during geometric data export from the IGES to the STL format when there is no source file in the format of the program in which the 3D-CAD model has been created. Such errors can be corrected with the aid of specialized software (Magics RP) equipped with a module of data export in the IGES format.

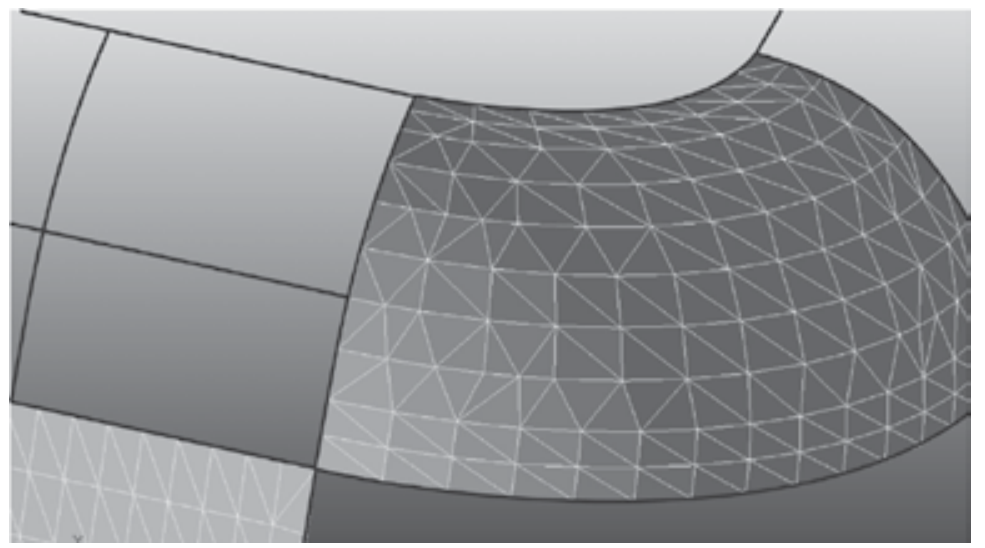

Fig. 14. Lost surface flakes in STL model

The model geometric data in the STL file is described by triangles with $\mathrm{x}, \mathrm{y}, \mathrm{z}$ coordinates for each of the triangle vertexes. The coordinates are described in the global system of Cartesian coordinates. Errors of data generated during the creation of the STL file result from the tessellation process. Program correction of errors is based on mathematical transformations of the triangular surfaces in 3D space.

\subsection{Data processing in CAD systems}

Most programs for three-dimensional modeling have a module of data export to the STL format. Depending on the program, the modules make it possible to receive data of different degrees of accuracy. Some programs do not have the possibility of determining the parameters of the STL file accuracy, others limit the scope of the defined accuracy to e.g. merely providing the length of the side of the triangle describing the solid surface or specifying a general accuracy level (Inventor).

Just like Inventor, Mechanical Desktop (MDT) is a program available in the software package of AutoDesk. However, it offers much greater possibilities in terms of creating elements of complex shapes and saving them in the STL format. The MDT program has a separate command for data saving in the STL format: AMSTLOUT. The options of this command make it possible to set the accuracy of the STL model mapping by means of four parameters, defined as: angle tolerance, constant of proportionality, intervals between vertexes, surface tolerance (fig. 15). 


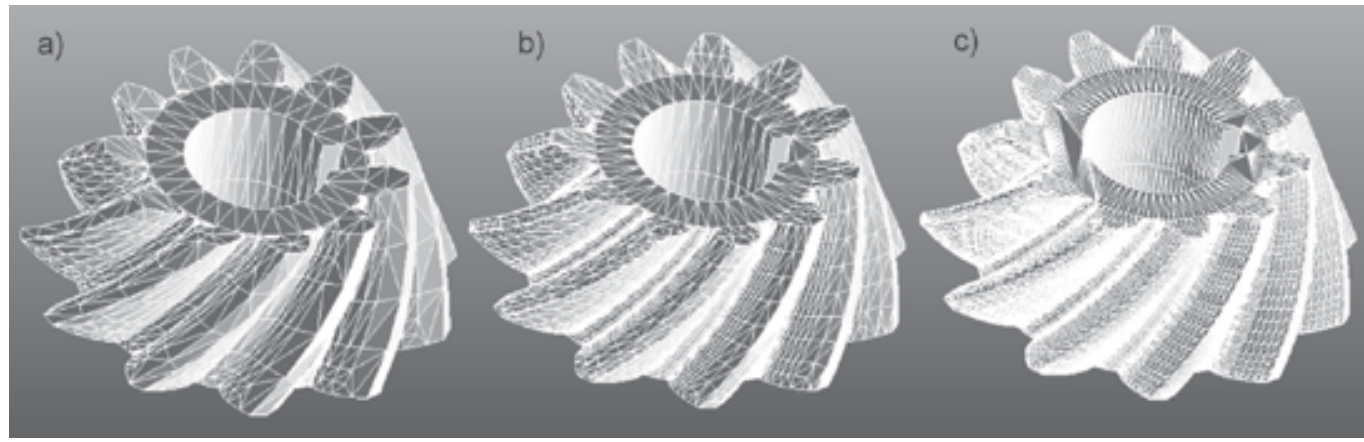

Fig. 15. STL model being exported from Mechanical Desktop with defined surface accuracy: $\mathrm{a}=0,5 \mathrm{~mm}, \mathrm{~b}=0,1 \mathrm{~mm}, \mathrm{c}=0,05 \mathrm{~mm}$

In CATIA V5R18 it is possible to save a file in the STL format in the Part Design module. However, the geometric parameters of such a model of standard settings cannot be used for the manufacture of accurate gear wheels. In order to increase the accuracy of the STL model, the Machining $\rightarrow$ STL Rapid Prototyping module should be activated in CATIA. Next, in this module, the Tessellation command should be selected and the accuracy of the STL model should be defined by specifying two parameters: sag and step (fig. 16).

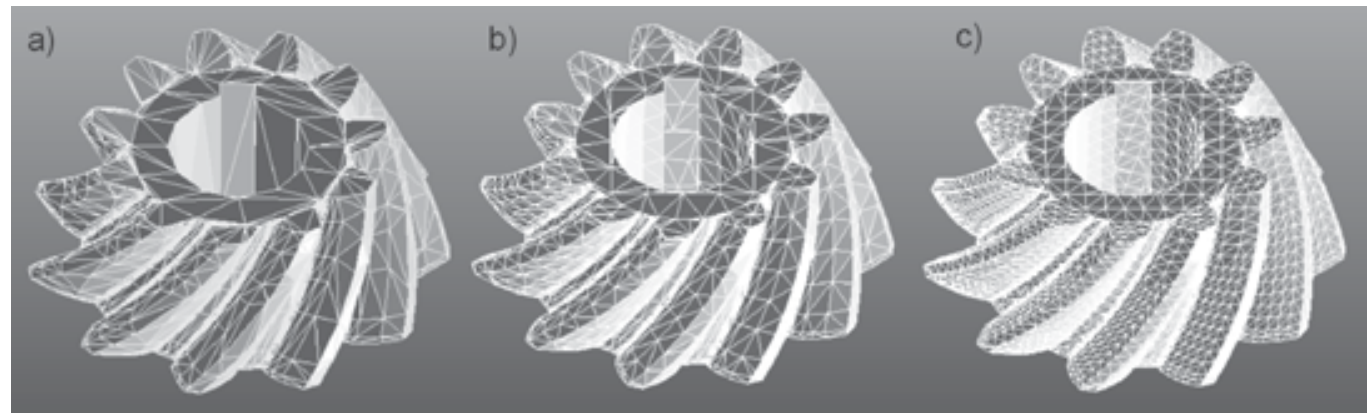

Fig. 16. STL model being exported from CATIA: a) sag $=10 \mathrm{~mm}$, step $=6 \mathrm{~mm}$,

b) sag $=0,2 \mathrm{~mm}$, step $=6 \mathrm{~mm}, \mathrm{c}$ ) $\mathrm{sag}=0,2 \mathrm{~mm}$, step $=3 \mathrm{~mm}$

The accuracy of data depends on its source. The CAD model has an accuracy determined by the accuracy of the system in which the model has been created (for CATIA the linear accuracy is $0.001 \mathrm{~mm}$ ). The constructor has an influence on the model accuracy at the stage of file export into the format of RP devices (e.g. STL). Depending on the program capabilities, the constructor can define the export parameters (e.g. angle tolerance, surface tolerance, constant of proportionality, intervals between triangles vertexes - MDT). Some programs make it possible to define (assume) the length of the triangle side in the STL format.

\subsection{Data processing in RP systems - magics RP software}

Magics RP is a universal program for the processing of files used in processes of data preparation for rapid prototyping. The basic version of the program makes it possible to read and process STL and MGX files.

Additionally, the program capabilities can be expanded by modules for the export of data saved in the following formats: MGX, IGES, VDA, VRLM, Catia V4, Catia V5, STEP, NX, 
ProE and Pointcloud. During the import, the source file should be selected and the accuracy of the STL model mapping should be defined in the window of parameter settings. In the course of the import, a window pops up showing the progress of the performed operation. Magics RP enables the edition of models, the correction of geometry errors of the model saved in 3D-CAD and 3D-RE formats, such as overlapping edges, missing triangles or surface fragments occurring in the triangulation process (fig. 17).

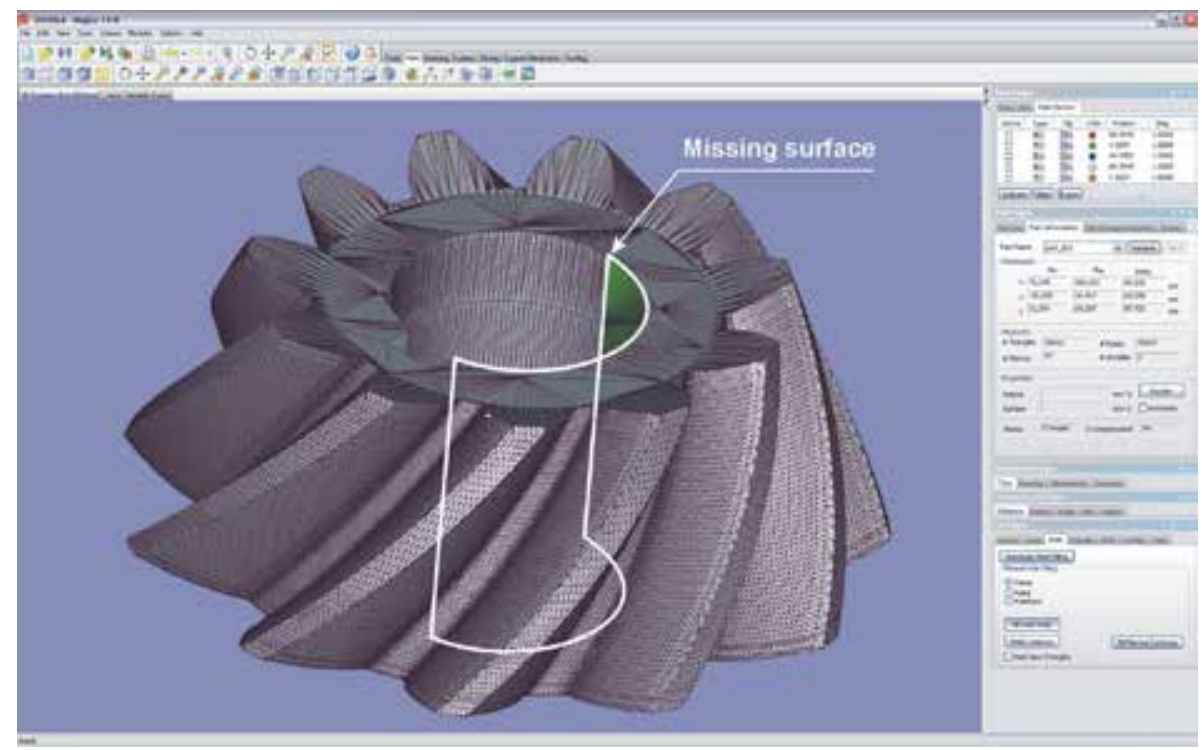

Fig. 17. View of missing surface - error generated during triangulation of STEP model

Some of STL models errors can be corrected with the aid of program tools (fig. 18a) by using automatic options. This, however, can lead to further errors in the form of the model shape change on the reconstructed surface and, sometimes, on the adjacent surfaces (fig. 18b).

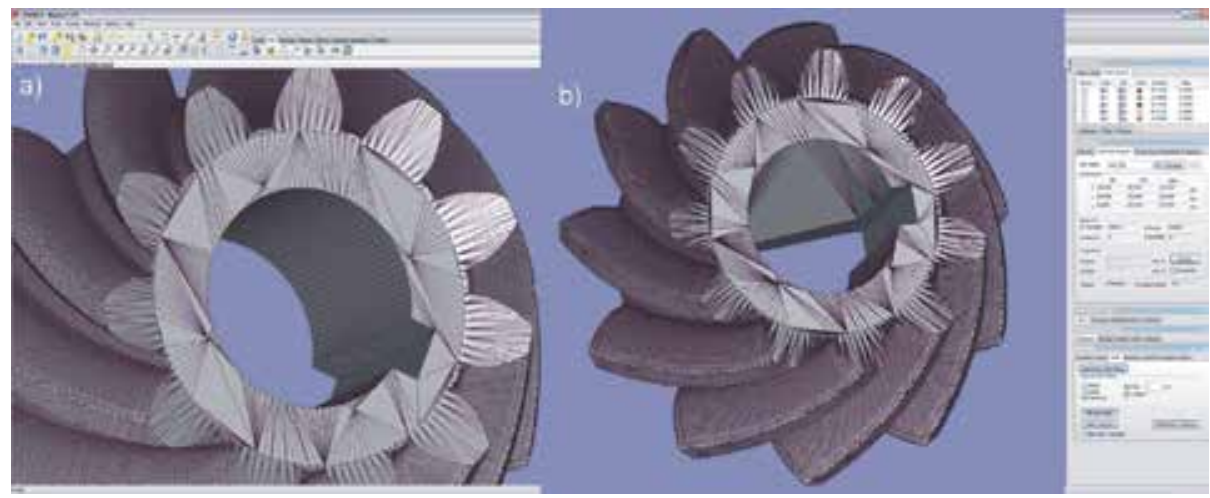

Fig. 18. Correction of model error: a) correct model, b) wrongly corrected model

In order to obtain a correct STL model, it is recommended that an individual approach be adopted towards the process of correction of geometry errors for each processed object (fig. 19a). The program allows for the optimization of the STL model by changing the parameters 
of the triangles mapping the object geometry. One of such operations is the reduction of the number of the triangles (fig. 19b). However, in this case, it should be ensured that the reduction of the triangles has not caused a change in the model geometry, which will affect the manufacture of the prototype.

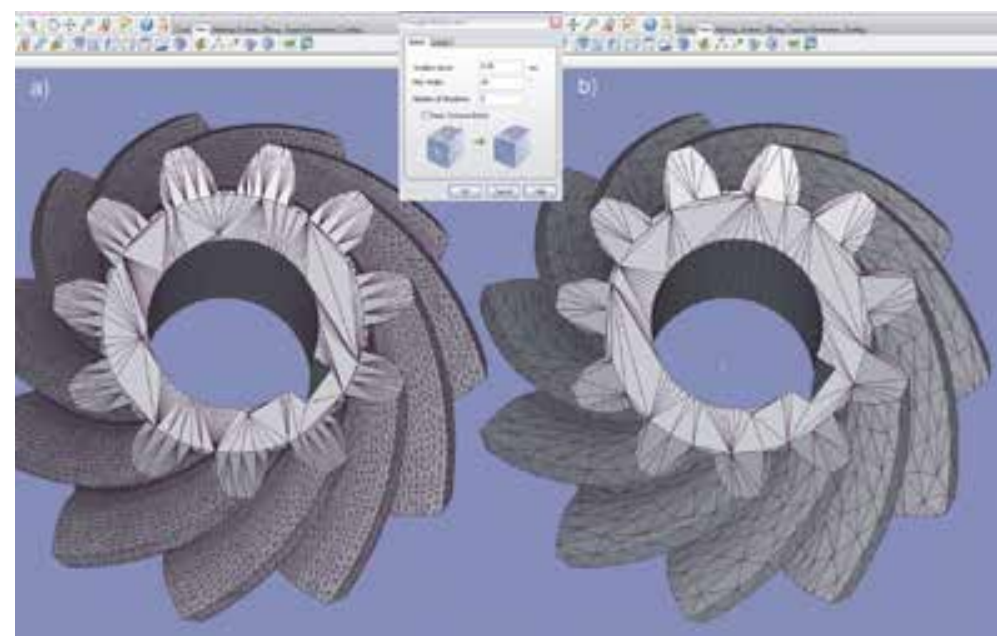

Fig. 19. Reduction of the number of triangles describing STL model surface: a) gear wheel starting model, b) model after reduction

Magics RP also makes it possible to process data coming from 3D-CAD systems as well as geometry received in the process of $3 \mathrm{D}$ scanning (fig. 20a). The program also enables the performance of simple 3D modeling operations (e.g. making holes or closing the scanned surfaces - fig. 20b). Thanks to this, it is possible to quickly generate the STL model, on the basis of which the physical prototype can be fabricated.

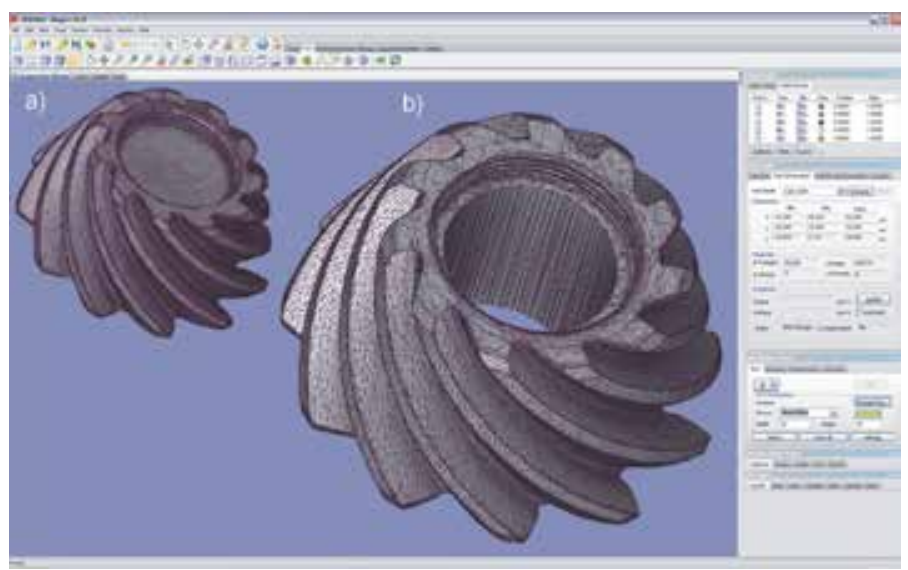

Fig. 20. Data processing in Magics RP: a) surface of scanned gear wheel received in scanning process, b) gear wheel STL model after program processing

Another important benefit of the program is a library of rapid prototyping devices. It is here that data can be prepared for the processes of most RP devices manufactured by companies, 
such as 3D Systems, Stratasys, OBJET, ZCorporation. The library of devices is expanded as new machines and technologies are introduced into the market.

Magics RP is a supplementation of programs sold together with rapid prototyping devices. It allows for data processing in the process of technology preparation for rapid prototyping systems, and, above all, makes it possible to correct some errors of the model, difficult to eliminate when there are no original 3D-CAD files.

Magics RP also enables the division of large models into parts, the size of which does not exceed the dimensions of the RP device working chamber. During the division, the program makes it possible to insert elements joining the individual fragments of the model.

\section{An analysis of geometric accuracy of gear wheels models}

Test prototypes of cycloidal gear wheels have been generated by incremental rapid prototyping methods (JS, SLA, SLS, FDM, 3DP), on the basis of the same 3D-CAD model. In order to eliminate the impact of errors of data processing on the physical prototype, the CAD model was exported to the STL format and a high accuracy of the parameters of the model geometry mapping was assumed. Test models were also used for studies of the determination of the real geometric accuracy of gear wheel prototypes made by the analyzed RP methods.

\subsection{The measurement of test prototypes with the use of CMM}

The objective of the tests was to make an analysis of the geometric accuracy of gear wheels models produced by the rapid prototyping techniques described above with reference to the CAD model (fig. 21a). The measurements were conducted with the use of the coordinate measuring machine - WENZEL LH 87 (fig. 21b). The measurements were carried out in the scanning mode along the measuring path. The shape of the path, the direction of measurement direction and the starting point are shown in figure 21c.
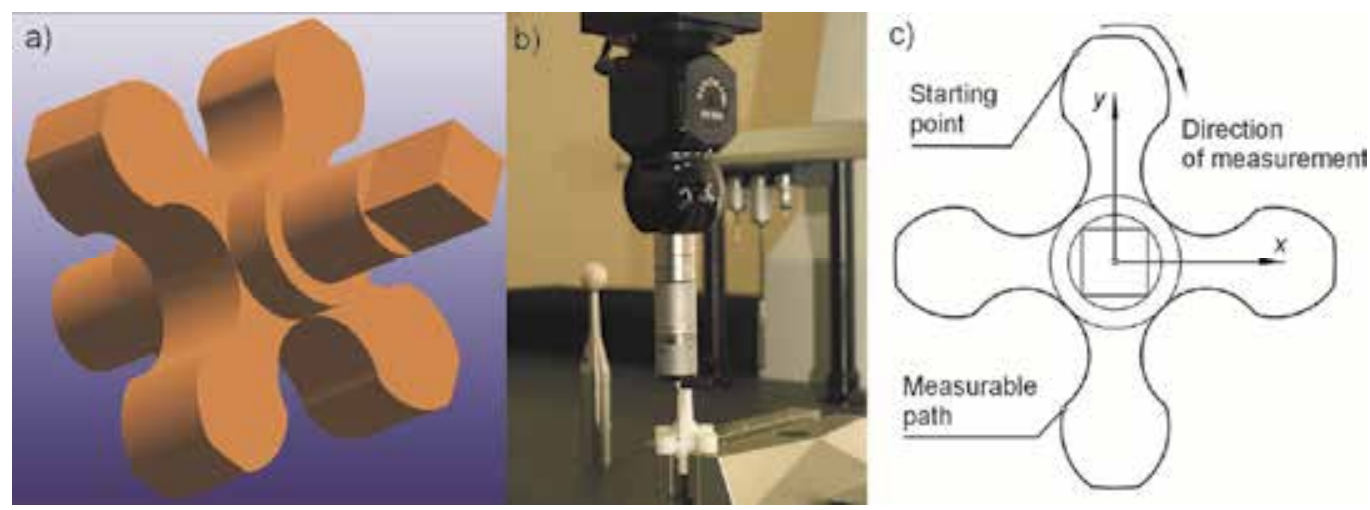

Fig. 21. Measurement preparation: a) gear wheel CAD model, b) measuring machine WENZEL LH87, c) measurable path

The preparation of the coordinate measuring machine involved actions typically performed in compliance with the coordinate measurements procedure. Measurements were conducted on crude (not having been subjected to the finishing abrasive machining) prototypes built by means of incremental RP methods: JS-PolyJet, SLS, SLA, FDM and 
3DP. The results of the measurements in the form of selected protocols have been presented in figures 22-24 respectively.

The fabrication of gear wheels prototypes requires the most accurate rapid prototyping methods. An analysis has been made of models of test blades produced by the JS-PolyJet, SLS, FDM and 3DP methods. In the first place, a gear wheel produced by the JS-PolyJet technology was measured. This model has a high accuracy in the majority of the measuring points, the deviations having a positive value, which does not go beyond the assumed tolerance range of $0.1 \mathrm{~mm}$ or only slightly exceeds it (fig. 22a).

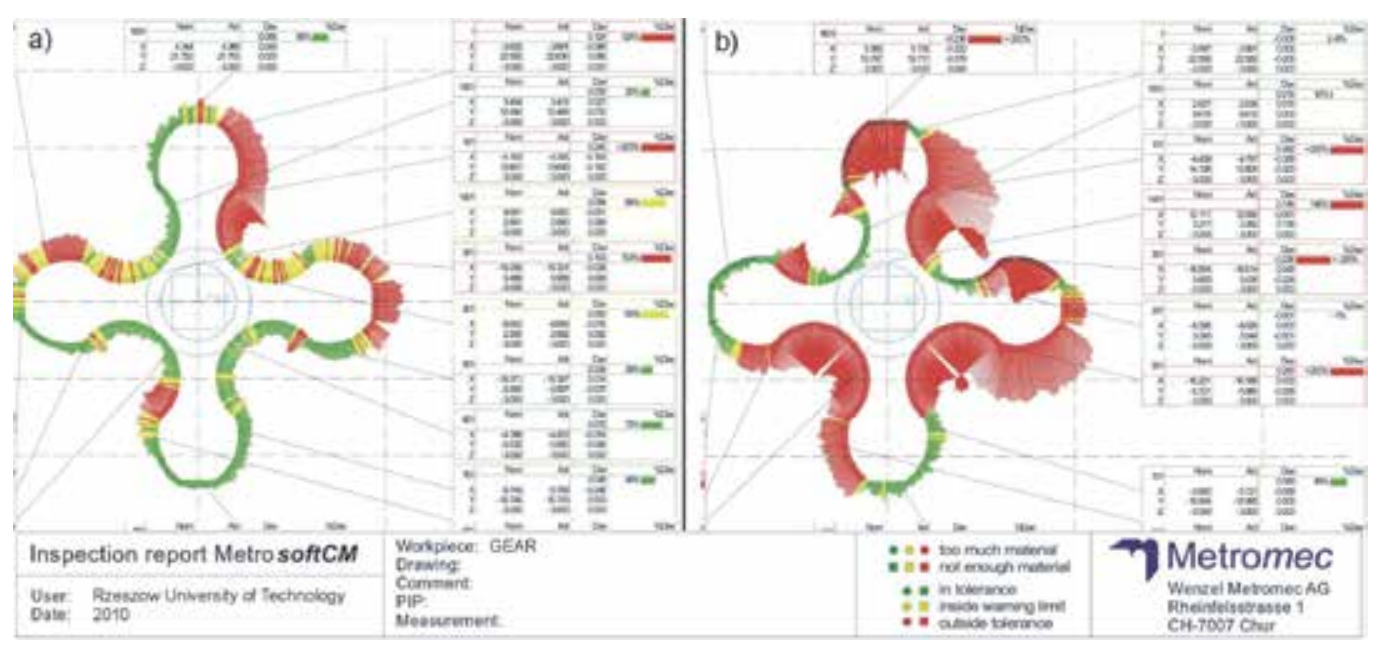

Fig. 22. Measurement protocol of gear: a) JS-PolyJet (Vero material), SLS (PA 2200 material)

Often the geometric accuracy of models also depends on the material the model is made from. The SLS model has been made from polyamide, which exhibits considerable (for RP techniques) processing shrinkage reaching 3\%, causing deformations of the model which exceed the assumed tolerance range (fig. 22a).

The highest accuracy is presented by the SLA model (fig. 23a). In the case of the FDM models, gear wheels made from the ABS material have been measured, with a layer thickness of $0.254 \mathrm{~mm}$. The model has a fairly high accuracy (fig. 23b). As a result of the measurements, the accuracy of the 3DP model has also been determined (fig. 24). For this technique, the model geometry immediately after the print-out may differ from the model geometry after the infiltration process. The difference value will be dependent on the shrinkage of the applied infiltrator and the depth of the infiltration.

\subsection{The measurement of test prototypes with the application of ATOS GOM system}

The coordinate measuring technique enables the measurement of gear wheels by means of contact measuring machines, with the application of advanced specialized measuring software. Gear wheels of any gear tooth forms and profiles manufactured in the RP technology can be measured with the use of optical coordinate scanners, for which the accuracy of identification of the measuring points depends on the applied optical system, i.e. measuring field (Budzik et al 2010).

The application of optical scanners as well as the methodology and algorithms devised for the needs of the measurement of gear wheels allows for the metrologically correct 
measurement of the characteristic geometric qualities determining the accuracy of the fabrication of the measured elements (fig. 25a). A gear wheel and a pinion made in the FDM (ABS) and SLA (SL5170) technologies have been measured. An analysis of accuracy has been made with the use of the same CAD models (fig. 25b), which served as the basis for the manufacture of example wheels of the bevel gear of a circular arc line of Gleason's tooth. The results of the analyses discussed above are presented in figures 26 and 27.

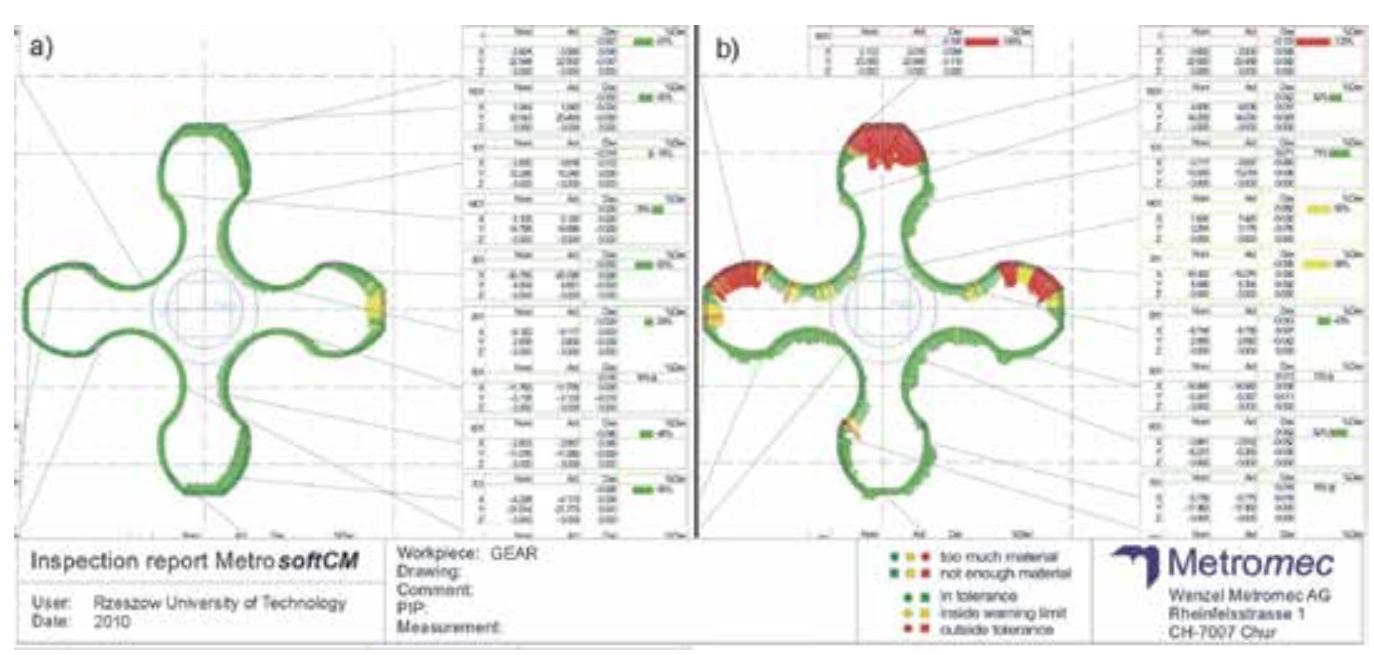

Fig. 23. Measurement protocol of gear: a) SLA (SL5170 material), b) FDM (ABS material)

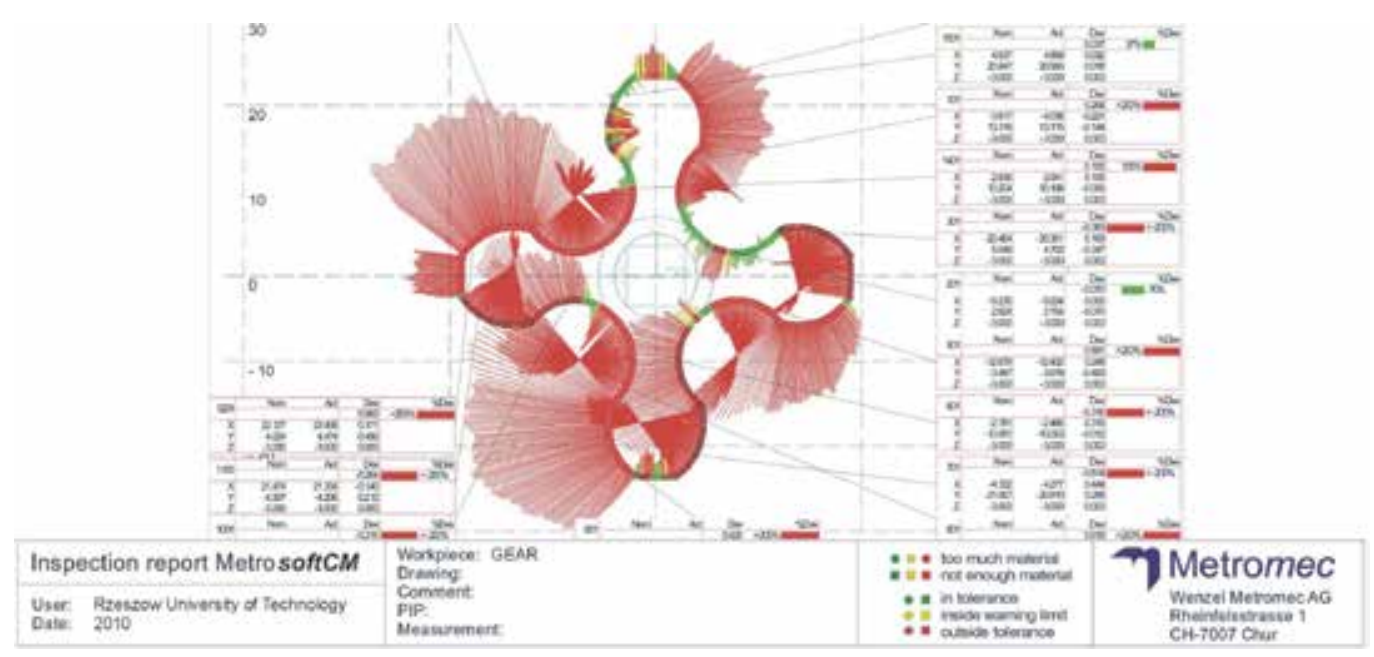

Fig. 24. Measurement protocol of 3DP gear wheel (ZP131 + Z-Max Epoxy material) 

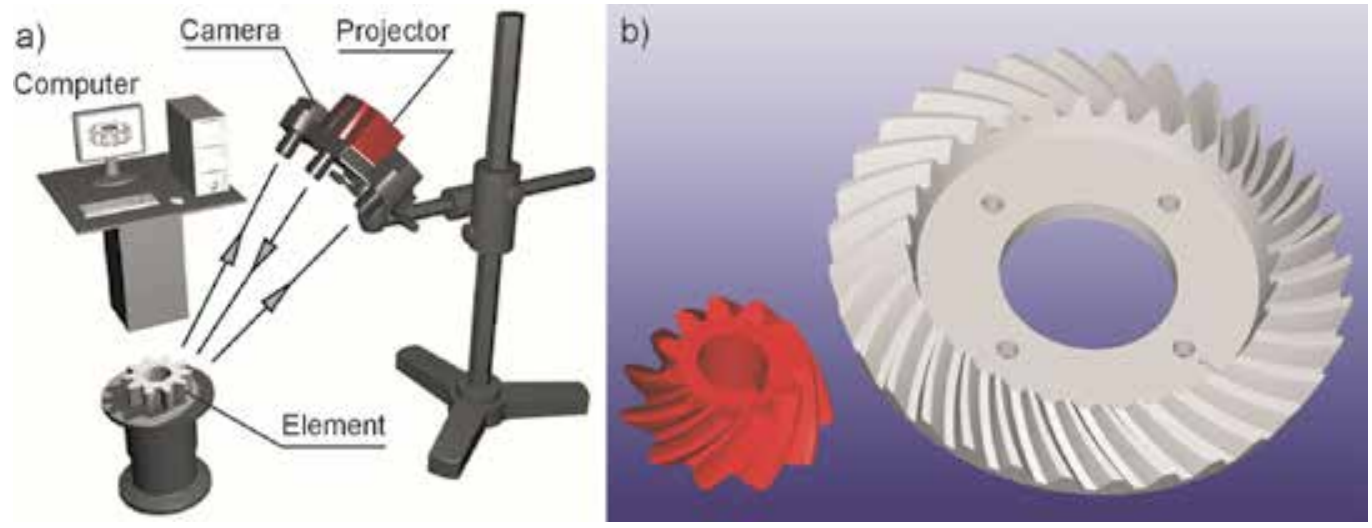

Fig. 25. Measurement of prototypes by means of ATOS GOM system: a) test stand, b) 3D-CAD models of measured gear wheels
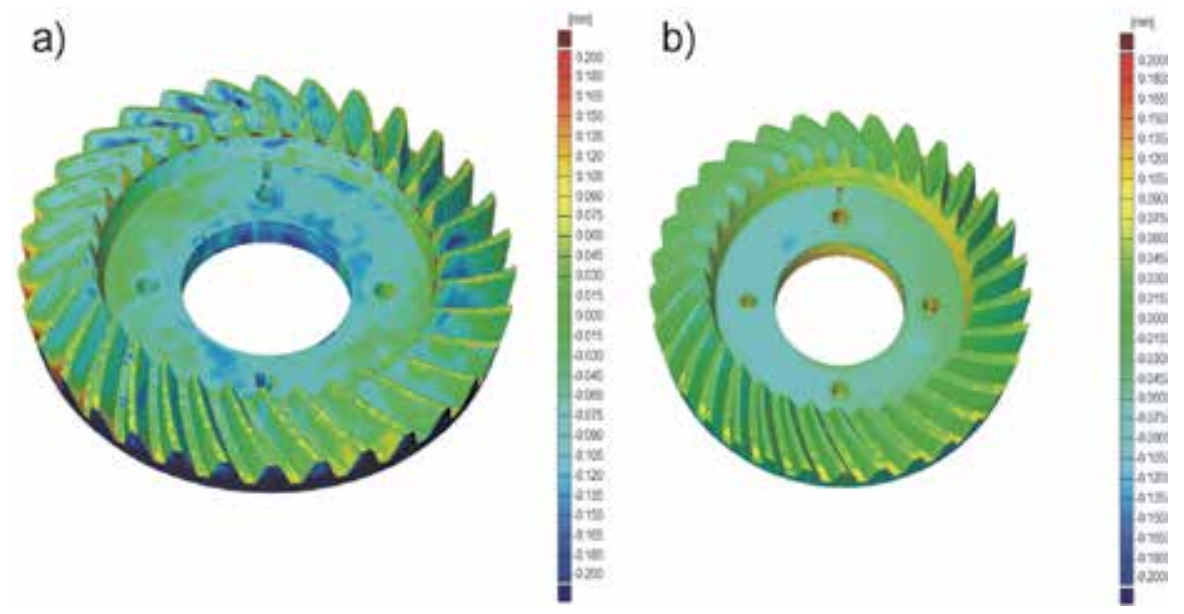

Fig. 26. Deviations of gear wheels prototypes: a) FDM, b) SLA
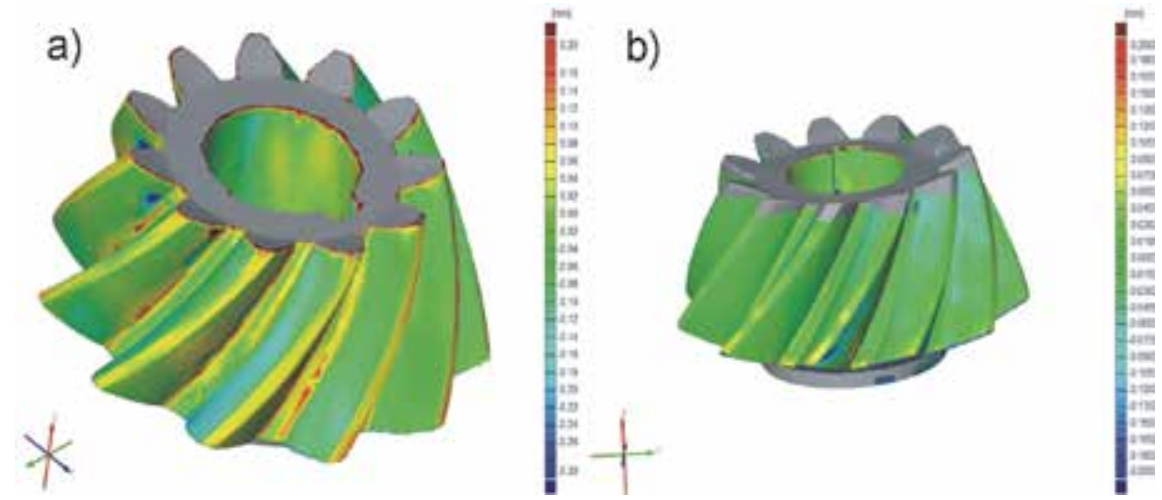

Fig. 27. Deviations of pinion prototypes: a) FDM, b) SLA 
Based on the analysis of the deviation values of the gear wheel and the pinion in all the measured points, in comparison with the CAD model, it can be observed that gear wheels made in the SLA technology (SL5170) are characterized by low values of limit deviations. Local deviations of positive values are caused by discontinuity, lack of measuring points or too few measuring points in the places of occurrence of very small radii of transition surfaces.

An analysis of the real deviation values of gear wheels made from different materials, by means of various rapid prototyping technologies, and especially their characteristic distribution, may serve a the basis for the creation of correction maps of the gear wheel CAD model to obtain an element corresponding to the assumed model in very narrow tolerances. The measurements of gear wheels conducted with the application of two incremental RP methods (SLA and FDM) allowed for the determination of the real accuracy of the manufacture of the bevel gear prototype.

The models of the gear wheels were oriented on the working platform in such a way that the axis of rotation was parallel to the axis of the RP device. This made it possible to increase the accuracy of the produced gear wheels. The primary source of geometric deviations and surface roughness (especially in the direction of the axis of the RP device) is the layer structure of models in incremental methods.

The accuracy of fabrication of gear wheels and their tests remain a major technological problem. The application of the coordinate measuring techniques in combination with the $\mathrm{CAD} / \mathrm{CAM} / \mathrm{RP}$ methods in the manufacture of gear wheels aims at improving the process of design, construction and technology (Oleksy et al. 2010).

The production of gear wheels prototypes with the application of RP methods is based on the 3D-CAD model. Therefore, it was possible to determine the values of deviations with reference to the nominal 3D-CAD model. In the case of measurements with the use of coordinate optical methods, the shape of the gear wheels or the tooth form are not particularly significant for the measuring process.

Coordinate measuring methods make it possible to determine the real accuracy of models made with the application of rapid prototyping techniques. The measurement and analysis of the geometric parameters of gear wheels allow for the compensation of errors and the determination of the correction value for RP devices.

The accuracy of RP models can be corrected at various stages of the technological process. First of all, the correction can be made during the construction of the model, the processing of numerical data and the export of data to one of the RP formats, e.g. STL or SLC. This is particularly important for gear wheels, the tooth forms of which are generated in the process of processing simulation. The numerical model errors may occur at this stage of modeling. Such errors are impossible to determine for the STL model. They emerge at the last stage of the manufacturing process, while the layers of the mesh profile are being built. It is especially visible in the stereolithography method, the software of which (3D Lightyear) is sensitive to this type of program errors.

\section{The possibility of RP systems application for gear wheels testing}

Rapid prototyping methods are increasingly applied in the manufacture of test prototypes (Liou, 2008). In the case of gear drives tests, RP techniques can be employed for the production of gear wheels used for mating area analysis, resistance (including photoelasticity) and fatigue tests. For tests of the mating area, its observation is possible through 
the transparent structure of the prototype (e.g. SLA or PolyJet). Tests of photo-elasticity are also based on the use of transparent materials. Here, prototypes can be prepared directly by incremental methods or indirectly through transparent resin casting by means of the Vacuum Casting technique, for example. Resistance fatigue tests can be conducted for models made by any RP method, with the proviso that the criteria of model similarity are earlier specified with the application of e.g. the FEM analysis (Pacana et al. 2010).

\subsection{Tooth Contact Analysis (TCA)}

The design of gears with new atypical profiles is a complex process, requiring a lot of geometric and strength computations. The production of atypical gears frequently requires the making of expensive and atypical tools. Launching new types of gears, especially those with gears of atypical profiles, requires also adequate research into the transmission stand test. The process of designing may become significantly reduced with such aids as the computer aided design (CAD), the finite elements method (FEM) and rapid prototyping methods (RP). Thanks to the CAD technique application, it is possible to model a gear geometrically, the FEM technique allows for initial verification of strength, whereas preliminary testing may be carried out on gears made with a rapid prototyping method, e.g. stereolithography (Sobolak \& Budzik 2008).

Instantaneous contact area is one of the determinants of correct gear mating. Instantaneous contact area is the area on the tooth flank, which contacts the mating surface of another tooth at a specific moment in time. The shape and size of that instantaneous contact area, as well as its changes as the gears turn, affect the correctness of gear mating and the kinematical precision of any gearbox (Sobolak \& Budzik 2008).

The contact between tooth surfaces in an ideally geometric gearbox occurs in a point or line but, because of deformability of the mating teeth flanks, in reality, it is always a certain surface.

At a specific moment in time, a contact area may exist between several pairs of teeth. It is the so-called total instantaneous contact area. In experimental studies, the mating area, which is a Boolean sum of instantaneous contact areas on tooth flanks, is usually determined. The mating print determination is frequently used in industrial practice, while that of the instantaneous contact area in not practiced because of the inherent difficulties.

The transparency of the gear material is of key importance for the determination of contact area in the method described here. The idea of contact area determination consists in the observation of the contact area between mating surfaces through a transparent material. Among other materials, the SL5170 resin has transparency characteristics suitable for the stereolithography method, but other transparent materials can also be used for such testing. Stereolithography requires a 3D geometrical model of the produced object. It also possible to cast gear models from transparent materials in silicon molds (Vacuum Casting method). Silicon molds may be made on the basis of existing gears. Such a method allows for the determination of the cause of abrasion and damage in the existing gearbox.

The tested gearbox may become loaded with torque, properly selected to suit a specific plastic it is made of (using the model similarity rule (Sobolak \& Budzik 2008), or may be tested without load with the use of a specially selected liquid. The liquid shall create meniscus that facilitates observation.

The meniscus that is created depends on the type of liquid whereas the liquid layer thickness, $\delta$, may be determined experimentally with the use of suitable stereolithographic 
models. The meniscus thickness may be determined from the formula (1). The thickness of the liquid layer, $\delta$, may be determined using fragments of two spheres (Fig. 28):

$$
\delta=r_{1}+r_{2}-\sqrt{r_{1}^{2}-\frac{a^{2}}{4}}-\sqrt{r_{2}^{2}-\frac{a^{2}}{4}}
$$

where:

$\delta$ - thickness of liquid layer (meniscus),

a - diameter of observed contact area,

r1, r2 - radii of spheres.

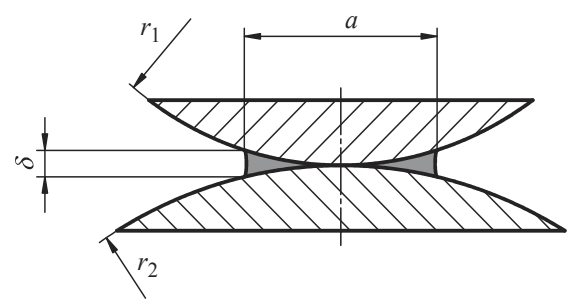

Fig. 28. Diagram for determining approximate thickness of liquid for the sphere-sphere case

In both cases (with loading or with liquid) the distance, $\delta$, equivalent to the gear deformation value in the contact area is determined.

Tests that were carried out with fragments of spheres of various radii showed that the thickness of meniscus, $\delta$ formed for stereolithographic models depended on the type of the liquid used only. Quite importantly, it does not depend on curving radii. In gearboxes, especially those of complicated gear geometry, gear teeth have variable curvature (e.g. involute tooth profile, arc-wise tooth line).

\subsubsection{The making of stereolithographic model of gears}

Geometric CAD models of Gleason SGM bevel gears with a circular arc tooth line, as presented (Sobolak \& Budzik 2008), are used in this example (Fig. 29a). Gears were made with tool mapping by the simulation technique. Thus, they are representative of gears made as a result of actual machining. Among others, they contain machining marks left by the tool. The technique of tool mapping simulation in the CAD environment consists in the simulation of machining, wherein both the tool and the workpiece are 3D models. The tool
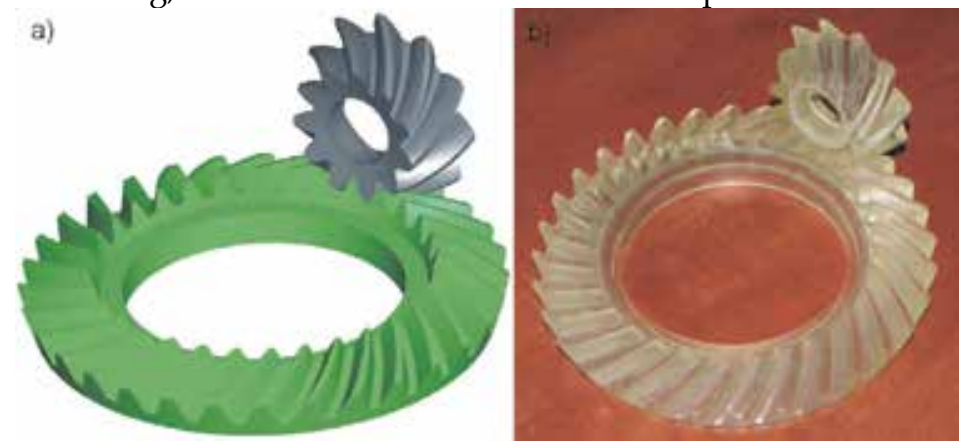

Fig. 29. Test models: a) 3D-CAD model, b) SLA model 
performs discrete motions in respect to the workpiece in accordance with the kinematics of actual machining. In each of the consecutive positions from a workpiece-representing body, a common part (interference) of tool and workpiece is removed.

The CAD models were prepared in a manner which enables direct observation of the mating area. For this purpose, suitable recesses were made with CAD system in 3D gear models, so as to facilitate a constant observation of the contact area.

CAD models of gears and fixture were created using the Mechanical Desktop software, from where they were exported to the STL format. In the STL format the 3D bodies are described with the use of flat, triangular surface flakes. Compared to CAD, the surface precision of the STL model is assumed as $0.001 \mathrm{~mm}$. This is dedicated accuracy for the export of CAD to the STL file for the gear tooth. Then, the process of stereolithographic model making on the 3D Systems SLA-250 apparatus was prepared (Fig. 29b).

\subsubsection{Stand tests of the contact area}

Gear wheels were compared in a special test stand (fig. 30), which enables the examination of pairs of gear wheels in various configurations. Static as well as dynamic determination of the mating area is possible. The stand is equipped with elements fixing gear wheels (1), a powder brake for imposing a load on the gear (2), a torque meter (3), a stepper motor (4) for propelling the gear and a steering console (5) with a computer.

The contact area is visible on the inside of the pinion through transparent resin (fig. 31a). Its shape is somewhat similar to an extended ellipse. Noticeable irregularities of the contact area contour are caused by machining marks, as would be left by a real tool on tooth flanks (in fact the analyzed gears are machined by gear generating, as it was duly taken into account in building the CAD models of pinion and gear wheel - fig. 31b).

Observation-facilitating conical recess inside the pinion is also visible. In order to visualize the contact area, the gears were moistened with distilled water with detergents added. The

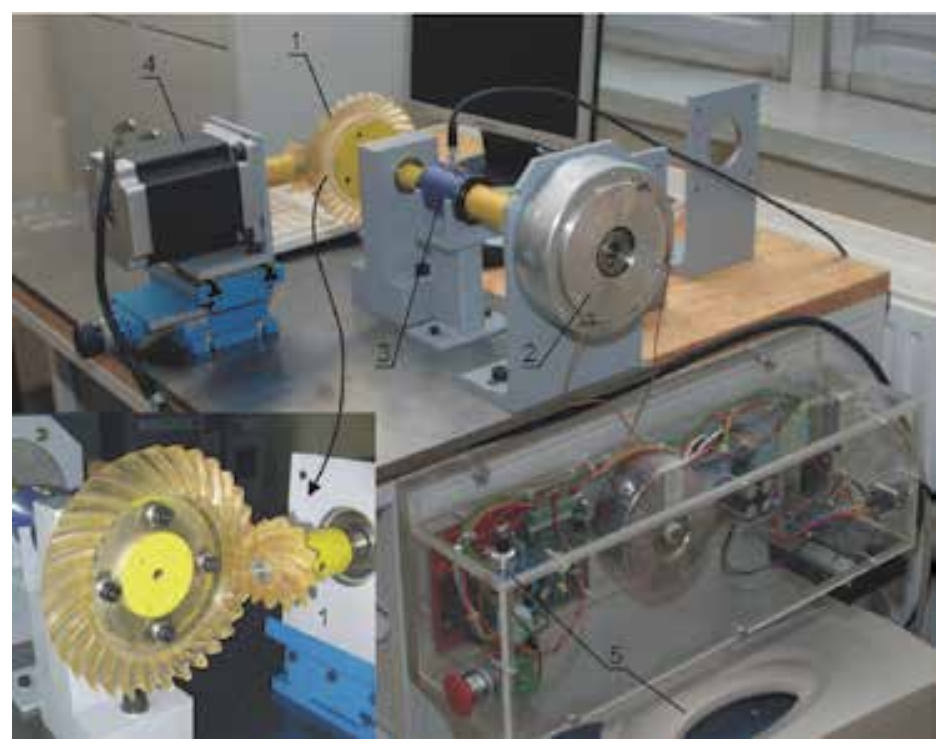

Fig. 30. Stand for testing the contact area: 1-gear, 2-brake, torque meter, 4-stepper motor, 5-sterring console 
detergents were selected experimentally so that they form meniscus of thickness similar to the typical thickness of the layer of ink used in gear inspection machines, i.e. $0.00635 \mathrm{~mm}$. The contact area obtained corresponds well with that geometrically determined with the use of 3D models in the CAD system. The determination of the geometric instantaneous contact area in the CAD system consists in a mutual gear infeed by a preset amount equal to, say, the thickness of the layer of ink used in gear inspection machines and in the determination of interference. The spatial curve that defines the interference volume is treated as the instantaneous contact area of mating gears.

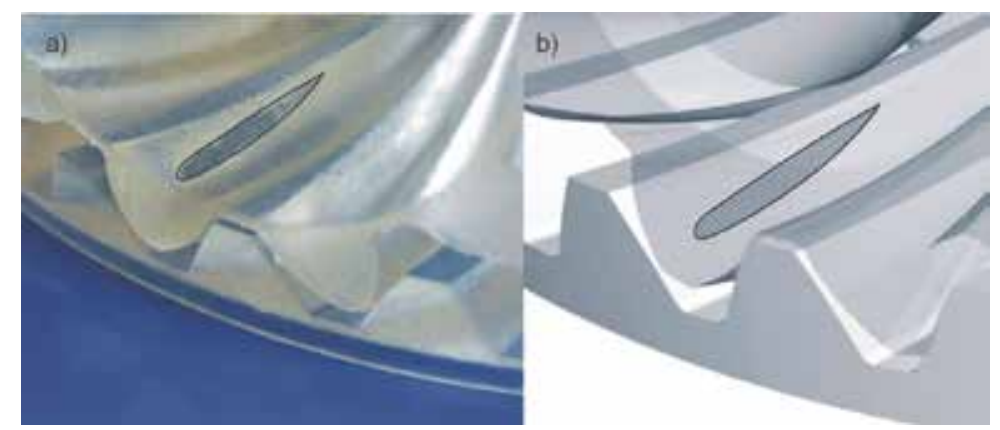

Fig. 31. Contact area: a) visible on internal side of SLA model, b) visible on CAD model

The deliberate application of stereolithography and the use of the suitable polymer (e.g. transparent resin, SL5170) produced a test gearbox, in which the instantaneous contact area may be determined through direct observation. The determination of the instantaneous contact area and, in particular, its changes as the gears turn by one division of graduation, enables conclusions to be drawn about the correct gearbox designing. The application of the ink method is also possible for mating marks determination.

The application of rapid prototyping techniques, (such as stereolithography, applied in the example) allows for a considerable acceleration of research into new designs of gears and gearboxes, as there is no need to make a real gearbox of metal, which would require an extended time for design as well as testing, and would involve a higher cost. The results of the stand testing correspond with the analyses performed in the CAD environment and with analytical methods.

\subsection{Fatigue tests of non-involute gear wheels}

In the engineering design of toothed gears, the need often arises to verify the correctness or accuracy of solutions achieved by means of analytical methods.

The design and implementation of new tooth profiles of gear wheels require a long calculation and testing process. It is possible to speed up the process by verifying the results of analytical calculations with the aid of the CAE/FEM numerical analysis and experimental tests of prototypes made by RP methods (Pacana et al. 2010).

The subject of such tests were the objectives of numerous projects, including R03 02102 and POIG.0101.02-00-015/08). During the realization of the assignments of the research projects, a wide range of innovative tooth profiles of gear wheels were designed. The designed wheels were subjected to analysis with the application of FEM. As a result of the analysis, tooth profiles were selected for the construction of test prototypes by RP methods and stand tests (fig. 32). 
The selection of the proper profiles for gear wheels intended for tests was also preceded by calculations and analysis of bending stresses at the tooth base for four consecutive teeth in the mesh. Thanks to the juxtaposition of graphs depicting the successive teeth in one chart and their determination in time, it is possible to define the work character and the load course for the whole gear. These graphs can be used to observe the mutual locations of onepair and two-pair mating areas for consecutive tooth pairs in the mesh.

Once the FEM analysis was completed, test prototypes of selected pairs of gear wheels were produced with the application of the SLA and FDM methods.

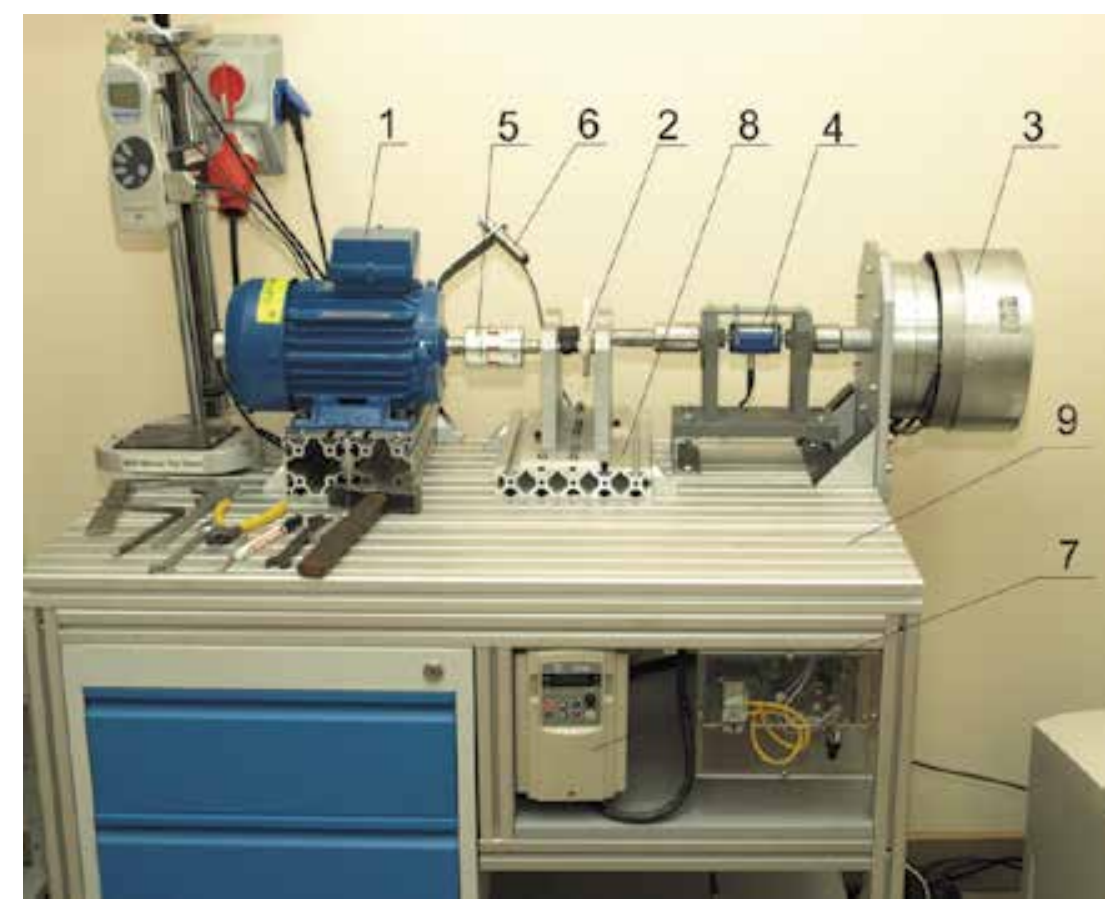

Fig. 32. Stand for gear tests: 1-Motor, 2-Reducer, 3-Brake, 4- Torque meter, 5- Clutch, 6- Pyrometer, 7-Inverter, 8-Base, 9-Stand table

A three-phase induction motor (1), controlled by an inverter (7) through a clutch (5) drives the tested stand gear. The gear is loaded with a brake (3), which introduces into the system a stressing moment, measured by means of a torque meter (4). The position of the gear shafts is digitally determined by location converters. The gear temperature is measured with the application of a contactless method, utilizing temperature radiation, by means of pyrometers (6). All data obtained during the fatigue tests are read by the measuring system and transferred to the computer.

The stand is universal, with the possibility of changing the configuration of the test system, and its modular structure enables the examination of single units and pairs of gear wheels of the individual pair of gears. For this reason, the stand base was designed in such a way as to allow for the mounting of individual components in various configurations.

The conducted quality analysis of various tooth profiles was aimed at determining the values of bending stresses at the tooth base for gear wheels having different parameters of the toothed wheel rim. Calculations were made for gear wheels of the following tooth 
profiles: involute, arc, sinusoidal and BBW circular arc. For all of the analyzed profiles, the same boundary conditions were adopted for calculations and tests. This allowed for the verification of the correctness of the received calculations results with the aid of stand tests of real gears with wheels made by means of RP methods.

Based on the realized tests, it can be concluded that non-involute gear wheels have the highest resistance. Minor differences were observed between the MES analysis and the stand tests of prototypes. This confirms the validity of the adopted research assumptions and the assumptions of the model similarity criteria. The slight discrepancy between the results of the FEM calculations and the findings of the stand tests may further be reduced by entering the data obtained in the research process into the FEM model in the course of the next approximations.

\section{Conclusion}

Rapid prototyping methods are widely applied in many fields of technology. One of the essential benefits of RP techniques is the possibility of generating prototypes of complex, virtually unlimited internal and external geometry, such as gear wheels. This article discusses selected aspects of the manufacture and testing of gear wheels with the application of rapid prototyping methods in the theoretical and practical aspects.

The analysis presented in chapter 1 has a general nature and concerns selected RP techniques, most commonly used in practice. Chapter 2 describes the practical aspects of one of the most significant stages in the rapid prototyping process - program data processing. This phase has a major influence on the geometric accuracy of prototypes irrespective of the applied RP system. The next chapter discusses the tests results of the geometric accuracy of gear wheels prototypes produced with the application of coordinate measuring methods. The last part demonstrates the possible applications of prototypes manufactured by means of incremental techniques in laboratory and industrial tests.

The subject of the application of RP methods for the production and testing of gear wheels goes beyond the scope of this article. Therefore, the information presented herein includes selected issues of research work realized at the Department of Machine Design of Rzeszow University of Technology as part of many projects, such as "Modern Material Technologies in Aerospace Industry", No POIG.0101.02-00-015/08.

\section{Acknowledgment}

Financial support of Structural Funds in the Operational Programme - Innovative Economy (IE OP) financed from the European Regional Development Fund - Project "Modern Material Technologies in Aerospace Industry", No POIG.0101.02-00-015/08 is gratefully acknowledged.

\section{References}

Budzik, G. (2009). The Analysis of Geometrical Data Preparation Process of Aircraft Engines Parts Manufacturing with RP Systems Using, Combustion Engines, Vol. 2009-SC1, pp. 295-300.

Budzik, G., Oleksy, M., Grzelka, M., Wieczorowski, M., Magniszewski, M., Slota, J. (2010). The Application of Optical Measurements for the Determination of Accuracy of 
Gear Wheels Casts Manufactured in the RT/RP Process, Archives of Foundry Engineering, Vol. 10, No. 1/2010, pp. 395-398.

Budzik, G., Kozik, B., Pacana, J., Żmuda, B. (2010). Modelling and Prototyping of Aeronautical Planetary Gear Demonstrator, Journal of KONES Powertrain and Transport, Vol. 17, No. 3/2010, pp. 49-54.

Budzik, G., Markowski, T., Sobolak M. (2008). Analysis of Surface Roughness of Transmission Gear Teeth made by Different Rapid Prototyping Methods, Journal of KONES Powertrain and Transport, Vol. 15, No. 2/2008, pp. 29-34.

Budzik, G., Markowski, T., Sobolak M. (2007). Hybrid Foundry Patterns of Bevel Gears, Archives of Foundry Engineering, Vol. 7, No. 1/2007, pp. 131-134.

Campbell, I.R (2008). Designing a New Range of Products to Capitalize on Rapid Manufacturing, Additive Layered Manufacturing: From Evolution to Revolution, Faculty for Mechanical Engineering, Maribor 2008, pp. 123-132

Grzelka, M., Gapiński, B. (2006). Recommendations on the Measurement of Cylindrical Gears with Coordinate Measuring Machines, Book of Summaries XVIII IMEKO World Congress, (September 2006), Rio de Janeiro, Brazil, pp. 138-143).

Liou, W. (2008). Rapid Prototyping and Engineering Applications - a Toolbox for Prototype Development, Taylor E Francis Group, 2008.

Modern Material Technologies in Aerospace Industry, Project No POIG.0101.02-00-015/08, European Regional Development Fund.

Oleksy, M., Budzik, G., Heneczkowski, M. (2010). Hybrid Polymer Composites for Rapid Prototyping of Gears, POLIMERY No. 2010/55/5, pp. 403-407

Pacana, J., Kozik, B., Budzik, G. (2010). Defining Instantaneous Contact Track of Aeronautical Bevel Gear Applying Finite Elements Method and Rapid Prototyping Method, Journal of KONES Powertrain and Transport, Vol. 17, No. 2/2010, pp. 379-386

Sobolak, M., Budzik, G. (2008). Experimental Method of Tooth Contact Analysis (TCA) with Rapid Prototyping (RP) Use, Rapid Prototyping Journal, Vol. 14, No. 4, 2008, pp. 197201.

Sobolak, M., Budzik, G. (2008). Visualization of Internal Stress Pattern in Gears in Power Transmission System Using Rapid Prototyping (RP), Journal of KONES Powertrain and Transport, Vol. 15, No. 2/2008, pp. 457-461. 

